January 26, 2011

TUM-EFT 11/10

CLNS 10/2066

ANL-HEP-PR-10-44

Alberta Thy 11-10

CP3-10-37

FZJ-IKP-TH-2010-24

INT-PUB-10-059

\title{
Heavy quarkonium: progress, puzzles, and opportunities
}

\begin{abstract}
N. Brambilla ${ }^{* \dagger},{ }^{1}$ S. Eidelman ${ }^{* \dagger},{ }^{2,3}$ B. K. Heltsley ${ }^{* \dagger}{ }^{\dagger}$ R. ${ }^{4 o g t}{ }^{* \dagger},{ }^{5,6}$ G. T. Bodwin ${ }^{\dagger},{ }^{7}$ E. Eichten ${ }^{\dagger}, 8$ A. D. Frawley ${ }^{\dagger},{ }^{9}$ A. B. Meyer ${ }^{\dagger},{ }^{10}$ R. E. Mitchell ${ }^{\dagger},{ }^{11}$ V. Papadimitriou ${ }^{\dagger},{ }^{8}$ P. Petreczky ${ }^{\dagger},{ }^{12}$ A. A. Petrov ${ }^{\dagger},{ }^{13}$ P. Robbe ${ }^{\dagger},{ }^{14}$ A. Vairo ${ }^{\dagger},{ }^{1}$ A. Andronic, ${ }^{15}$ R. Arnaldi, ${ }^{16}$ P. Artoisenet, ${ }^{17}$ G. Bali, ${ }^{18}$ A. Bertolin, ${ }^{19}$ D. Bettoni,${ }^{20}$ J. Brodzicka, ${ }^{21}$ G. E. Bruno, ${ }^{22}$ A. Caldwell, ${ }^{23}$ J. Catmore, ${ }^{24}$ C.-H. Chang, ${ }^{25,}{ }^{26}$ K.-T. Chao ${ }^{27}$ E. Chudakov, ${ }^{28}$ P. Cortese, ${ }^{16}$ P. Crochet,${ }^{29}$ A. Drutskoy, ${ }^{30}$ U. Ellwanger, ${ }^{31}$ P. Faccioli, ${ }^{32}$ A. Gabareen Mokhtar, ${ }^{33}$

X. Garcia i Tormo ${ }^{34}$ C. Hanhart ${ }^{35}$ F. A. Harris,${ }^{36}$ D. M. Kaplan, ${ }^{37}$ S. R. Klein, ${ }^{38}$ H. Kowalski, ${ }^{10}$ J.-P. Lansberg, ${ }^{39,}{ }^{40}$ E. Levichev ${ }^{2}$ V. Lombardo, ${ }^{41}$ C. Lourenço, ${ }^{42}$ F. Maltoni, ${ }^{43}$ A. Mocsy ${ }^{44}$ R. Mussa, ${ }^{16}$ F. S. Navarra, ${ }^{45}$ M. Negrini, ${ }^{20}$ M. Nielsen,${ }^{45}$ S. L. Olsen,${ }^{46}$ P. Pakhlov, ${ }^{47}$ G. Pakhlova, ${ }^{47}$ K. Peters,${ }^{15}$
\end{abstract} A. D. Polosa, ${ }^{48}$ W. Qian, ${ }^{49,14}$ J.-W. Qiu, ${ }^{12,}{ }^{50}$ G. Rong, ${ }^{51}$ M. A. Sanchis-Lozano, ${ }^{52}$ E. Scomparin, ${ }^{16}$ P. Senger ${ }^{15}$ F. Simon, ${ }^{23,}{ }^{53}$ S. Stracka, ${ }^{41,54}$ Y. Sumino, ${ }^{55}$ M. Voloshin, ${ }^{56}$ C. Weiss, ${ }^{28}$ H. K. Wöhri, ${ }^{32}$ and C.-Z. Yuan ${ }^{51}$

${ }^{1}$ Physik-Department, Technische Universität München, James-Franck-Str. 1, 85748 Garching, Germany

${ }^{2}$ Budker Institute of Nuclear Physics, Novosibirsk 630090, Russia

${ }^{3}$ Novosibirsk State University, Novosibirsk 630090, Russia ${ }^{4}$ Cornell University, Ithaca, $N Y 14853$, USA

${ }^{5}$ Physics Division, Lawrence Livermore National Laboratory, Livermore, CA 94551, USA

${ }^{6}$ Physics Department, University of California at Davis, Davis, CA 95616, USA

${ }^{7}$ High Energy Physics Division, Argonne National Laboratory, 9700 South Cass Avenue, Argonne, IL 60439, USA

${ }^{8}$ Fermi National Accelerator Laboratory, P.O. Box 500, Batavia, IL 60510, USA

${ }^{9}$ Physics Department, Florida State University, Tallahassee, FL, 32306-4350, USA

${ }^{10}$ Deutsches Elektronen-Synchrotron DESY, Hamburg, Germany

${ }^{11}$ Indiana University, Bloomington, IN 47405, USA

${ }^{12}$ Physics Department, Brookhaven National Laboratory, Upton, NY 11973-5000, USA

${ }^{13}$ Department of Physics and Astronomy, Wayne State University, Detroit, MI 48201, USA

${ }^{14}$ Laboratoire de l'Accélérateur Linéaire, IN2P3/CNRS and Université Paris-Sud 11, Centre Scientifique d'Orsay, BP 34, F-91898 Orsay Cedex, France

${ }^{15}$ GSI Helmholtzzentrum für Schwerionenforschung, D-64291 Darmstadt, Germany

${ }^{16}$ INFN Sezione di Torino, Via P. Giuria 1, I-10125 Torino, Italy

${ }^{17}$ Department of Physics, The Ohio State University, Columbus, OH 43210, USA

${ }^{18}$ Institut für Theoretische Physik, Universität Regensburg, 93040 Regensburg, Germany

${ }^{19}$ INFN Sezione di Padova, Via Marzolo 8, I-35131 Padova, Italy

${ }^{20}$ Università di Ferrara and INFN Sezione di Ferrara, Via del Paradiso 12, I-44100 Ferrara, Italy

${ }^{21}$ Institute of Nuclear Physics Polish Academy of Sciences, Kraków, Poland

${ }^{22}$ Università di Bari and INFN Sezione di Bari, Via Amendola 173, I-70126 Bari, Italy

${ }^{23}$ Max Planck Institute for Physics, München, Germany

${ }^{24}$ Department of Physics, Lancaster University, Lancaster, LA1 $4 Y B$, UK

${ }^{25}$ CCAST (World Laboratory), P.O.Box 8730, Beijing 100190, China

${ }^{26}$ Institute of Theoretical Physics, Chinese Academy of Sciences, Beijing 100190, China

${ }^{27}$ Department of Physics, Peking University, Beijing 100871, China

${ }^{28}$ Thomas Jefferson National Accelerator Facility, 12000 Jefferson Ave., Newport News, VA 23606, USA

${ }^{29}$ Clermont Université, Université Blaise Pascal, CNRS-IN2P3, LPC, BP 10448, F-63000 Clermont-Ferrand, France

${ }^{30}$ University of Cincinnati, Cincinnati, OH 45221, USA

${ }^{31}$ Laboratoire de Physique Théorique, Unité mixte de Recherche - CNRS - UMR 8627,

Université de Paris-Sud, F-91405 Orsay, France

${ }^{32}$ LIP, Av. Elias Garcia 14, 1000-149 Lisbon, Portugal 


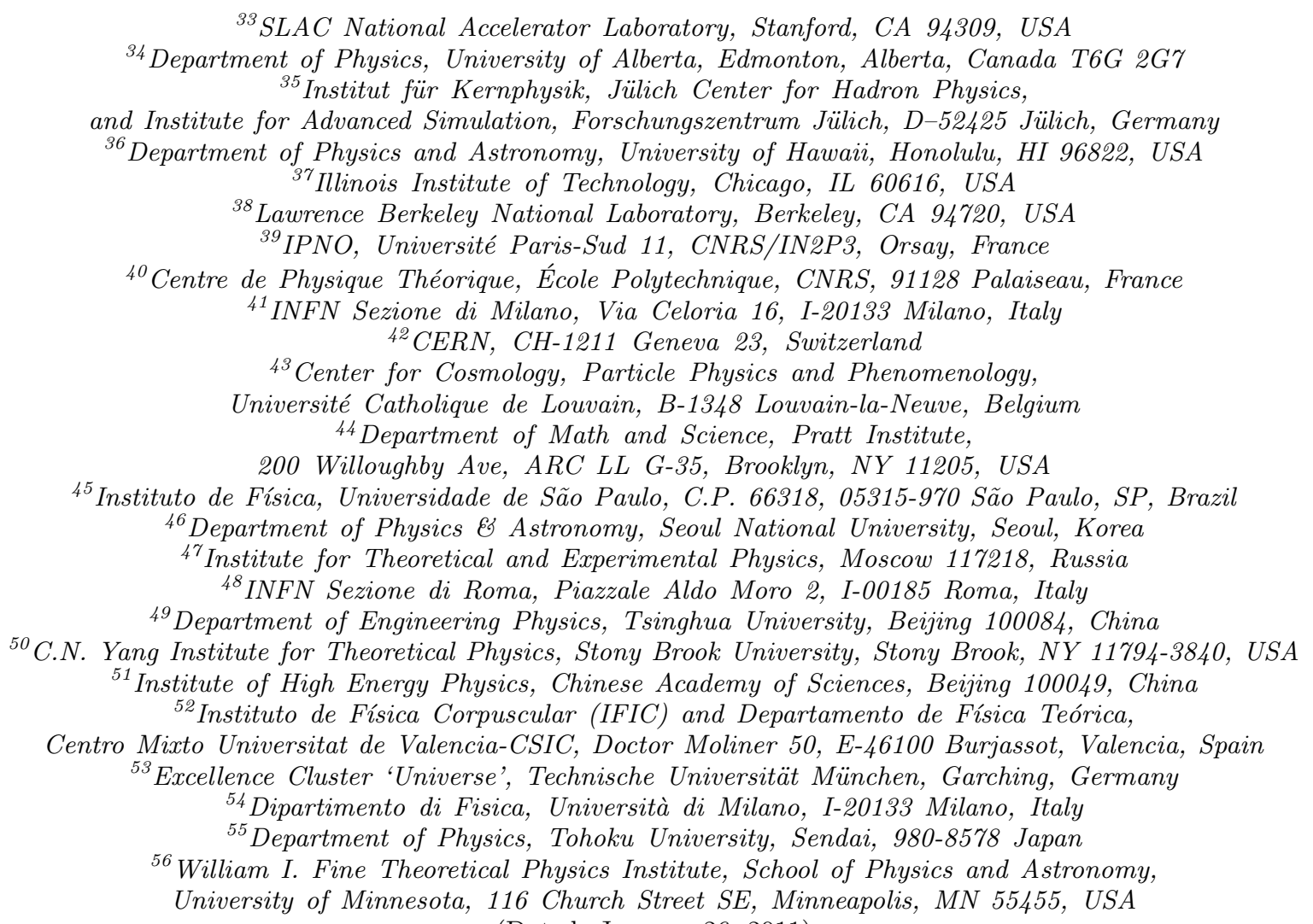

(Dated: January 26, 2011)

\begin{abstract}
A golden age for heavy quarkonium physics dawned a decade ago, initiated by the confluence of exciting advances in quantum chromodynamics (QCD) and an explosion of related experimental activity. The early years of this period were chronicled in the Quarkonium Working Group (QWG) CERN Yellow Report (YR) in 2004, which presented a comprehensive review of the status of the field at that time and provided specific recommendations for further progress. However, the broad spectrum of subsequent breakthroughs, surprises, and continuing puzzles could only be partially anticipated. Since the release of the YR, the BESII program concluded only to give birth to BESIII; the $B$-factories and CLEO-c flourished; quarkonium production and polarization measurements at HERA and the Tevatron matured; and heavy-ion collisions at RHIC have opened a window on the deconfinement regime. All these experiments leave legacies of quality, precision, and unsolved mysteries for quarkonium physics, and therefore beg for continuing investigations at BESIII, the LHC, RHIC, FAIR, the Super Flavor and/or Tau-Charm factories, JLab, the ILC, and beyond. The list of newly-found conventional states expanded to include $h_{c}(1 P), \chi_{c 2}(2 P), B_{c}^{+}$, and $\eta_{b}(1 S)$. In addition, the unexpected and still-fascinating $X(3872)$ has been joined by
\end{abstract}

\footnotetext{
* Editors

$\dagger$ Section coordinators

¥ Corresponding author: bkh2@cornell.edu
}

more than a dozen other charmonium- and bottomonium-like " $X Y Z$ " states that appear to lie outside the quark model. Many of these still need experimental confirmation. The plethora of new states unleashed a flood of theoretical investigations into new forms of matter such as quark-gluon hybrids, mesonic molecules, and tetraquarks. Measurements of the spectroscopy, decays, production, and in-medium behavior of $c \bar{c}, b \bar{b}$, and $b \bar{c}$ bound states have been shown to validate some theoretical approaches to QCD and highlight lack of quantitative success for others. Lattice QCD has grown from a tool with computational possibilities to an industrialstrength effort now dependent more on insight and innovation than pure computational power. New effective field theories for the description of quarkonium in different regimes have been developed and brought to a high degree of sophistication, thus enabling precise and solid theoretical predictions. Many expected decays and transitions have either been measured with precision or for the first time, but the confusing patterns of decays, both above and below open-flavor thresholds, endure and have deepened. The intriguing details of quarkonium suppression in heavy-ion collisions that have emerged from RHIC have elevated the importance of separating hotand cold-nuclear-matter effects in quark-gluon plasma studies. This review systematically addresses all these matters and concludes by prioritizing directions for ongoing and future efforts. 


\section{Contents}

\section{Dedication}

\section{Introduction}

2. Spectroscopy ${ }^{2}$

2.1. Conventional vectors above open-flavor threshold 9 2.1.1. Vectors decaying to open charm 9 2.1.2. Vectors decaying to open bottom 12

2.2. Newly found conventional quarkonia 12 2.2.1. Observation of $h_{c}(1 P) \quad 13$

2.2.2. Observation of $\eta_{c}(2 S)$

2.2.3. Observation of $\chi_{c 2}(2 P)$

2.2.4. Observation of $B_{c}^{+}$

2.2.5. Observation of $\eta_{b}(1 S)$

2.2.6. Search for $h_{b}(1 P)$

2.2.7. Observation of $\Upsilon\left(1^{3} D_{2}\right)$

2.3. New unanticipated states

2.3.1. $X(3872)$, the enduring exotic

2.3.2. Unconventional vector states

2.3.3. Other positive $C$-parity states

2.3.4. Charged exotic mesons: the $Z$ 's

2.4. Characteristics of quarkonium systems

2.5. Nonrelativistic effective field theories

2.5.1. Physics at the scale $m$ : NRQCD

2.5.2. Physics at the scales $m v, m v^{2}$ : pNRQCD 32

2.5.3. $m v \gg \Lambda_{\mathrm{QCD}}$ : weakly-coupled pNRQCD 32

2.5.4. $m v \sim \Lambda_{\mathrm{QCD}}$ : strongly-coupled pNRQCD 33

2.6. Lattice QCD spectrum calculations

2.6.1. Relativistic heavy quark actions $\quad 35$

2.6.2. NRQCD lattice calculations 35

2.6.3. pNRQCD lattice calculations 36

2.7. Predictions for the $\eta_{b}(1 S)$ mass 36

2.8. Standard Model parameter extractions 36

2.8.1. $\alpha_{\mathrm{s}}$ determinations $\quad 37$

2.8.2. Determinations of $m_{b}$ and $m_{c} \quad 38$

2.8.3. $m_{t}$ determination 39

2.9. Exotic states and states near or above threshold 39

2.9.1. Gluonic excitations 40

2.9.2. Spectrum with light quarks 40

2.9.3. Molecular states $\quad 42$

2.9.4. Tetraquark states $\quad 45$

2.9.5. Hadrocharmonium 47

2.9.6. QCD sum rules 48

2.9.7. Theoretical explanations for new states $\quad 52$

2.10. Beyond the Standard Model 52

2.10.1. Mixing of a light CP-odd Higgs and $\eta_{b}(n S)$ resonances

2.10.2. Supersymmetric quarkonia

2.10.3. Invisible decays of $\psi$ and $\Upsilon$

3. Decay $^{8}$

3.1. Radiative transitions

3.1.1. Theoretical status

3.1.2. Study of $\psi(1 S, 2 S) \rightarrow \gamma \eta_{c}(1 S)$

3.1.3. Observation of $h_{c}(1 P) \rightarrow \gamma \eta_{c}(1 S)$

3.1.4. Nonobservation of $\psi(2 S) \rightarrow \gamma \eta_{c}(2 S)$

3.1.5. $\psi(2 S) \rightarrow \gamma \gamma J / \psi$ through $\chi_{c} J$

3.1.6. Higher-order multipole amplitudes

3.1.7. Observation of $\psi(3770) \rightarrow \gamma \chi_{c J}(1 P)$

3.1.8. Observation of $\Upsilon(2 S, 3 S) \rightarrow \gamma \eta_{b}(1 S)$

3.2. Radiative and dileptonic decays

3.2.1. Theoretical status
3.2.2. Measurement of $\psi, \Upsilon \rightarrow \gamma g g$

63

3.2.3. Observation of $J / \psi \rightarrow \gamma \gamma \gamma$

3.2.4. Nonobservation of $\psi(2 S), \Upsilon(1 S) \rightarrow \gamma \eta$

3.2.5. Two-photon widths of charmonia

3.2.6. Dileptonic widths in the $\psi$ family

3.2.7. Dileptonic widths in the $\Upsilon$ family

3.3. Hadronic transitions

3.3.1. Theoretical status

3.3.2. Branching fractions for $\psi(2 S) \rightarrow X J / \psi$

3.3.3. Observation of $\psi(2 S) \rightarrow \pi^{0} h_{c}(1 P) \quad 73$

3.3.4. Nonobservation of $\eta_{c}(2 S) \rightarrow \pi^{+} \pi^{-} \eta_{c}(1 S) 73$

3.3.5. Observation of $\psi(3770) \rightarrow X J / \psi \quad 73$

3.3.6. Observation of $\Upsilon(2 S) \rightarrow \eta \Upsilon(1 S) \quad 73$

3.3.7. Observation of $\chi_{b 1,2}(2 P) \rightarrow \omega \Upsilon(1 S)$

3.3.8. Dipion transitions from $\Upsilon(2 S, 3 S) \quad 73$

3.3.9. Observation of $\Upsilon\left(1^{3} D_{J}\right) \rightarrow \pi^{+} \pi^{-} \Upsilon(1 S) \quad 76$

3.3.10. Dipion and $\eta$ transitions from $\Upsilon(4 S) \quad 76$

3.3.11. Dipion transitions near $\Upsilon(5 S) \quad 76$

3.3.12. Observation of $\chi_{b J}(2 P) \rightarrow \pi \pi \chi_{b J}(1 P) \quad 77$

3.4. Hadronic decays 77

3.4.1. The $12 \%$ rule and $\rho \pi$ puzzle $\quad 77$

3.4.2. Observation of $h_{c}(1 P) \rightarrow 2\left(\pi^{+} \pi^{-}\right) \pi^{0} \quad 78$

3.4.3. $\chi_{c J}(1 P)$ hadronic decays 78

3.4.4. Non- $D \bar{D} \psi(3770)$ hadronic decays $\quad 78$

3.4.5. Observation of $\Upsilon(1 S) \rightarrow$ antideuteron $+X 80$

3.4.6. Observation of $\Upsilon, \chi_{b J} \rightarrow$ open charm $\quad 81$

3.4.7. Observation of $\chi_{b J}(1 P, 2 P) \rightarrow$ hadrons $\quad 81$

\section{Production $^{9}$}

4.1. Introduction to theoretical concepts $\quad 82$

4.1.1. Momentum scales and factorization 82

4.1.2. The color-singlet model 82

4.1.3. The color-evaporation model 83

4.1.4. The NRQCD factorization approach 83

4.1.5. The fragmentation-function approach 84

4.1.6. Relationship of the fragmentation-function approach to the NRQCD factorization approach

84

4.1.7. Difficulties in establishing NRQCD factorization

4.1.8. $k_{T}$ factorization

4.1.9. Factorization in exclusive quarkonium production

4.1.10. Factorization in quarkonium decays $\quad 86$

4.1.11. Future opportunities 86

4.2. Production at the Tevatron, RHIC and the LHC 86

4.2.1. Channels at higher-order in $\alpha_{s} \quad 87$

4.2.2. Phenomenology, including QCD corrections

4.2.3. Quarkonium polarization: a key observable

89

4.2.5. Future opportunities

4.3. ep collisions $\quad 98$

4.3.1. Phenomenology of the cross section, including NLO corrections

99

4.3.2. Polarization 100

4.3.3. Future opportunities 102

4.4. Fixed-target production 102

4.4.1. Phenomenology of fixed-target production 102

4.4.2. Future opportunities 103

4.5. Exclusive production in $e^{+} e^{-}$collisions 103

4.5.1. Theory vs. experiment 103

4.5.2. Future opportunities 105 
4.6. Inclusive production in $e^{+} e^{-}$collisions

4.6.1. Experiments and LO theoretical expectations

4.6.2. $e^{+} e^{-} \rightarrow J / \psi+c \bar{c}$ at $\mathrm{NLO}$

4.6.3. $e^{+} e^{-} \rightarrow J / \psi+g g$ at $\mathrm{NLO}$

4.6.4. Color-octet process $e^{+} e^{-} \rightarrow J / \psi\left({ }^{3} P_{J}^{[8]},{ }^{1} S_{0}^{[8]}\right)+g$

4.6.5. $\mathrm{J} / \psi$ production in $\gamma \gamma$ collisions

4.6.6. Future opportunities

4.7. $B_{c}$ production

4.7.1. Experimental progress

4.7.2. Calculational schemes

4.7.3. Phenomenology

4.7.4. Future opportunities

5. In medium ${ }^{19}$

5.1. Quarkonia as a probe of hot and dense matter

5.2. Cold-nuclear-matter effects

5.3. Quarkonium in hot medium

5.3.1. Spectral properties at high temperature

5.3.2. Color screening and deconfinement

5.3.3. Quarkonium spectral functions and quarkonium potential

5.3.4. Dynamical production models

5.3.5. Summary of hot-medium effects

5.4. Recent results at SPS energies

5.4.1. $J / \psi$ production in $p A$ collisions

5.4.2. Anomalous $J / \psi$ suppression

5.5. Recent hadroproduction results from RHIC

5.5.1. Charmonium from $p p$ collisions

5.5.2. Charmonium from $\mathrm{Cu}+\mathrm{Cu}$ collisions

5.5.3. Bottomonium production

5.5.4. $J / \psi$ production from $\mathrm{d}+\mathrm{Au}$ collisions

5.5.5. $\mathrm{J} / \psi$ production from $\mathrm{Au}+\mathrm{Au}$ collisions

5.6. Anomalous suppression: SPS vs RHIC

5.7. Photoproduction in nuclear collisions

6. Experimental outlook ${ }^{21}$

6.1. BESIII

6.1.1. Spin singlets: $h_{c}(1 P), \eta_{c}(1 S, 2 S)$

6.1.2. Vectors above $\psi(3770): \psi$ 's and $Y$ 's

6.1.3. Hadronic decays 131

6.1.4. Excited $C$-even charmonium states $\quad 132$

6.1.5. Decays of $\chi_{c J}(1 P)$

6.1.6. Prospects 132

6.2. ALICE

6.2.1. $\mathrm{J} / \psi$ production from $\mathrm{Pb}+\mathrm{Pb}$ collisions

6.2.2. $J / \psi$ production from $p p$ collisions

6.2.3. $J / \psi$ production from $b$-hadron decays 134

6.2.4. Production of $\chi_{c J}(1 P)$ and $\psi(2 S)$

6.2.5. $\Upsilon$ production 135

6.2.6. First LHC high-energy running 135

6.3. ATLAS 135

6.3.1. Trigger considerations 135

6.3.2. Event selection 137

6.3.3. Prompt quarkonium polarization 138

6.3.4. Early $7 \mathrm{TeV}$ LHC running 139

6.4. CMS

6.4.1. Quarkonium production 139

6.4.2. Quarkonium polarization 141

6.5. $\mathrm{LHCb}$

6.5.1. Charmonium physics

6.5.2. $B$ physics

6.5.3. Bottomonium physics 144
6.6. RHIC 145

6.7. Super Flavor Factories 146

6.8. PANDA 147

6.8.1. Experimental technique 147

6.8.2. Detector 148

6.8.3. Charmonium and open charm physics 148

6.9. CBM at FAIR 152

6.10. Tau-charm factory in Novosibirsk 152

6.11. Charmonium photoproduction facilities $\quad 153$

6.11.1. $\mathrm{J} / \psi$ photoproduction at high energy 153 6.11.2. $J / \psi$ photoproduction at low energy 155

6.12. Proposed $\bar{p} p$ project at Fermilab 156

6.13. Future linear collider 157

7. Conclusions and priorities 159

$\begin{array}{ll}\text { Acknowledgments } & 163\end{array}$

References

164 


\section{Dedication}

We dedicate this review to the memories of three friends of the Quarkonium Working Group. Richard Galik, building on his leadership role in quarkonium physics at CLEO, supported and guided our efforts from the start. His relentless focus, unyielding objectivity, and insistent collegiality continue to inspire those who knew him. Beate Naroska pioneered quarkonium measurements at HERA and enthusiastically advocated for the 2007 QWG meeting at DESY as a member of the local organizing committee. A visionary scientist and dedicated teacher, she made invaluable and enduring contributions to our field. Andrzej Zieminski had a longstanding devotion to the study of quarkonium production in hadron collisions. He worked energetically to ensure the success of the inaugural QWG meeting in 2002 as a convener of the QCD Tests Working Group, and continued his sustaining commitment as liason to the $\mathrm{D} \varnothing$ Collaboration. We remember Rich, Beate, and Andrzej with fondness and gratitude.

\section{INTRODUCTION}

Heavy quarkonium is a multiscale system which can probe all regimes of quantum chromodynamics (QCD). At high energies, a perturbative expansion in the strong coupling constant $\alpha_{\mathrm{s}}\left(Q^{2}\right)$ is possible. At low energies, nonperturbative effects dominate. In between, the approximations and techniques which work at the extremes may not succeed, necessitating more complex approaches. Hence heavy quarkonium presents an ideal laboratory for testing the interplay between perturbative and nonperturbative QCD within a controlled environment. To do so in a systematic manner requires the intersection of many avenues of inquiry: experiments in both particle physics and nuclear physics are required; perturbative and lattice QCD calculations must be performed in conjunction with one another; characteristics of confinement and deconfinement in matter must be confronted; phenomenology should be pursued both within the Standard Model and beyond it. Above all, experiments must continue to provide measurements which constrain and challenge all aspects of QCD, and theory must then guide experiment toward the next important observables.

Effective field theories ${ }^{1}$ (EFTs) describing quarkonium processes have continued to develop and now provide a unifying description as well as solid and versatile tools yielding well-defined predictions. EFTs rely on higherorder perturbative calculations and lattice simulations. Progress on both fronts has improved the reach and pre-

\footnotetext{
${ }^{1}$ EFTs such as HQEFT, NRQCD, pNRQCD, SCET, ..., are described elsewhere in this article.
}

cision of EFT-based predictions, enabling, e.g., the increasingly precise determinations of several fundamental parameters of the Standard Model (i.e., $\alpha_{\mathrm{s}}, m_{c}$, and $m_{b}$ ).

Several experiments operating during this era, primarily BABAR at SLAC and Belle at KEK), CLEO-III and CLEO-c at CESR, CDF and D $\varnothing$ at Fermilab, and BESII and BESIII at IHEP have, in effect, operated as quarkonium factories, vastly increasing the available data on quarkonia spectra and decays. Over the same period, investigations of quarkonium production in fixed target experiments at Fermilab and CERN, HERA-B at DESY, and PHENIX and STAR at RHIC have vastly increased the knowledge base for cold- and hot-medium studies. The resulting variety of collision types, energy regimes, detector technologies, and analysis techniques has yielded quarkonium-related physics programs that are both competitive and complementary. Taken together, the experimental programs provide the confirmations and refutations of newly observed phenomena that are crucial for sustained progress in the field as well as the breadth and depth necessary for a vibrant quarkonium research environment.

The Quarkonium Working Group (QWG) was formed in 2002 as a dedicated and distinct effort to advance quarkonium studies by drawing sometimes disparate communities together in this common cause. QWG activities bring experts in theory and experiment together to discuss the current status and progress in all the relevant subfields. Subsequent participant interactions are intended to synthesize a consensus of progress and priorities going forward. Periodic QWG meetings have been effective in achieving this function. The exhaustive $C E R N$ Yellow Report [1], the first document produced by QWG detailing the state of quarkonium physics and suggestions for future efforts, was released in 2004 to embody such a synthesis. Since that report appeared, much has been accomplished in theory and experiment, warranting an updated review.

This review provides a comprehensive exploration of heavy quarkonium physics applicable to the landscape of 2010 , with particular emphases on recent developments and future opportunities. The presentation is organized into five broad and frequently overlapping categories:

- Spectroscopy (Sect. 2), which focuses on the existence, quantum numbers, masses, and widths of heavy quarkonium (or quarkonium-like) bound states;

- Decay (Sect. 3), an examination of the patterns and properties of quarkonia transitions and decays, with special attention given to the decay dynamics and exclusive final-state branching fractions;

- Production (Sect. 4), the study of heavy quarkonium creation in $e^{+} e^{-}, p \bar{p}, \ell p, \gamma p$, and $p p$ collisions;

- In medium (Sect. 5), the investigation of deconfinement and formation of quark-gluon plasma in 
heavy-ion collisions via measurement of quarkonium suppression;

- Experimental outlook (Sect. 6), the status and physics reach of new and planned experimental facilities.

Below we briefly introduce and motivate each of these sections.

Heavy quarkonium spectroscopy examines the tableau of heavy-quark bound states, thereby providing the starting point for all further investigations. Which states exist? Why? What are their masses, widths, and quantum numbers? Which states should exist but have not yet been observed? Does QCD fully explain the observed terrain? If not, why? New experimental and theoretical efforts over the last decade have provided some answers to these questions, while also raising new ones. Some long-anticipated states have, at last, been measured (e.g., $h_{c}(1 P), \eta_{c}(2 S)$, and $\eta_{b}(1 S)$ ), while many unanticipated states (e.g., $X(3872)$ and $Y(4260)$ ) also appeared. Does the underestimation of the $\eta_{b}(1 S)$ hyperfine splitting by some QCD calculations indicate faults in application of theory, inaccuracy of measurements, or the presence of new physics? Have we observed mesonic molecules? Tetraquarks? Quark-gluon hybrids? How would we know if we had? How many of the new states are experimental artifacts? Do $X(3872)$ decay patterns comport with those of any conventional quarkonium? Is $X(3872)$ above or below $D^{* 0} \bar{D}^{0}$ threshold? Is the $e^{+} e^{-}$ hadronic cross section enhancement near $10.86 \mathrm{GeV}$ simply the $\Upsilon(5 S)$ resonance or does $\Upsilon(5 S)$ overlap with a new $Y_{b}$ state, as suggested by recent dipion transition data? These questions, among many others, animate continuing theoretical and experimental spectroscopic investigations.

For states away from threshold, theory provides a description, at the level of the binding-energy scale, in the form of an EFT called pNRQCD. Precise and accurate calculation of the $\eta_{b}(1 S)$ hyperfine splitting remains a challenge for both perturbative and lattice calculations. With one exception, no EFT description has yet been constructed nor have the appropriate degrees of freedom been clearly identified for most new states close to threshold. The exception is $X(3872)$, which displays universal characteristics due to its proximity to $D^{* 0} \overrightarrow{D^{0}}$ threshold, thus prompting a plethora of calculations based on a single elegant formalism. Spectroscopy has advanced from both direct and EFT-formulated lattice calculations. In general, however, the threshold regions remain troublesome for the lattice as well as EFTs, excited-state lattice calculations have been only recently pioneered, and the full treatment of bottomonium on the lattice remains a challenge.

A substantial challenge in the realm of quarkonium decay is for theory to keep pace with the large number of new measurements. These include increasingly precise measurements of prominent decay modes (e.g., dilepton branching fractions and widths of $J / \psi$ and $\Upsilon$, branching fractions for and dynamical descriptions of dipion transitions from $\psi(2 S)$ and $\Upsilon(n S)$ ), and first measurements or important refinements of previously low-statistics results (e.g., $J / \psi \rightarrow 3 \gamma ; J / \psi \rightarrow \gamma \eta_{c}(1 S) ; \Upsilon(4 S) \rightarrow$ $\left.\pi^{+} \pi^{-} \Upsilon(1 S, 2 S, 3 S)\right)$, and the burgeoning lists of exclusive hadronic decay modes $\left(e . g ., \eta_{c}(1 S)\right.$ and $\left.\chi_{b J}\right)$. Some previously puzzling situations (e.g., theory-experiment disagreements for higher-order multipoles in $\psi(2 S) \rightarrow$ $\left.\gamma \chi_{c J}, \chi_{c J} \rightarrow \gamma J / \psi\right)$ have been resolved by improved measurements while others (e.g., the $\rho \pi$ puzzle, suppressed $\psi(2 S)$ and $\Upsilon(1 S)$ decays to $\gamma \eta)$ remain. Has the twopeak dipion mass structure in $\Upsilon(3 S) \rightarrow \pi^{+} \pi^{-} \Upsilon(1 S)$ been explained? What exactly is the source of the distorted photon lineshape in $J / \psi \rightarrow \gamma \eta_{c}(1 S)$ ? Does the $\psi(3770)$ have non- $D \bar{D}$ decay modes summing to more than $\sim 1 \%$ ? Our review of decays details new measurements and addresses these and related questions.

For a quarkonium with a small radius, an EFT description of radiative magnetic dipole transitions has been recently obtained, replacing the now-outdated model description; its extension to electric dipole transitions and to states with larger radius is needed. Steady improvement in NRQCD inclusive decay-width calculations has taken place in higher-order expansions in the velocity and strong coupling constant as well as in the lattice evaluation of matrix elements. Predictions match measurements adequately at the level of ratios of decay widths. Further improvements would require the lattice calculation or data extraction of the NRQCD matrix elements and perturbative resummation of large contributions to the NRQCD matching coefficients. The new data on hadronic transitions and hadronic decays pose interesting challenges to the theory.

The pioneering measurements of quarkonium production at the Tevatron were carried out in the early 1990s. Soon after, NRQCD factorization became the standard tool for theoretical calculations. Since then, the Tevatron, $B$-factories, and HERA have all performed important measurements, some of which have given rise to inconsistencies, puzzles, and new challenges for both theory and experiment. Among these are apparent inconsistencies in quarkonium polarization at the Tevatron between Run I and Run II for the $J / \psi$, between CDF and D $\varnothing$ for the $\Upsilon$, and between experiment and NRQCD factorization predictions for both. At least as surprising was the observation at the $B$-factories that close to $60 \%$ of $e^{+} e^{-}$ collisions that contain a $J / \psi$ also include a charm meson pair. Photoproduction measurements at HERA revealed discrepancies with LO NRQCD factorization predictions. In response to these and other challenges, the theory of quarkonium production has progressed rapidly.

NRQCD factorization is the basis for much of the current theoretical work on quarkonium production. Factorization in exclusive quarkonium production has recently been proven to all orders in perturbation theory for both double-charmonium production in $e^{+} e^{-}$annihilation and $B$-meson decays to charmonium and a light meson. NRQCD factorization for inclusive quarkonium pro- 
duction has been shown to be valid at NNLO. However, an all-orders proof of factorization for inclusive quarkonium production remains elusive. This is a key theoretical issue, as a failure of factorization at any order in perturbation theory would imply that there are large, non-factorizing contributions, owing to the presence of soft-gluon effects.

Corrections to hadroproduction have been calculated at NLO, and, in the case of the color-singlet channel, partially at NNLO, even though just a few years ago these calculations were thought to be barely possible. The new calculations show that, because of kinematic enhancements, higher-order corrections can be orders of magnitude larger than the Born-level contributions. In the case of double-charmonium production in $e^{+} e^{-}$collisions, relativistic and perturbative corrections increased the predicted cross sections by more than a factor of four, bringing them into agreement with experiment. New NRQCD factorization calculations of quarkonium photoproduction to NLO at HERA have also moved predictions into agreement with experiment. The importance of higher-order corrections has raised the issue of the convergence of the perturbation series. New methods to address this issue are on the horizon.

New observables have been proposed that may help us to understand the mechanisms of quarkonium production. For example, alternative methods for obtaining information about the polarization of produced quarkonia have been suggested. The associated production of quarkonia may also be an important tool in understanding new states. The production characteristics of the $X(3872)$ may shed light on its exotic nature. The improved theoretical landscape will soon be confronted with the first phase of running at the LHC, where charmonium and bottomonium production will be measured with high statistics in a greatly extended kinematic range.

The study of quarkonium in medium has also undergone crucial development. The large datasets from heavy-ion collisions at RHIC suggest that the quarkgluon plasma is actually more like a liquid than a plasma. The suppression of quarkonium production in a hot medium was proposed as a clean probe of deconfined matter. However, the use of quarkonium yields as a diagnostic tool of the hot medium has turned out to be quite challenging. Indeed, quarkonium production was already found to be suppressed by cold-nuclear-matter effects in proton-nucleus collisions. Such effects require dedicated experimental and theoretical attention themselves. In high-energy environments such as at heavy-ion colliders, where more than one $Q \bar{Q}$ pair may be produced in a collision, coalescence of $Q$ and $\bar{Q}$ can lead to secondary quarkonium production, requiring understanding of the transport properties of the medium to separate primary and secondary quarkonium production. The interpretation of in-medium hot matter effects requires understanding the $Q \bar{Q}$ interaction in terms of finite temperature $(T)$ QCD. The successful Hard Thermal Loop effective theory integrates over the hardest momenta proportional to
$T$ for light quark and gluon observables. To extend the Hard Thermal Loop theory to heavy quarkonium at finite temperature, the additional scales introduced by the bound state must be taken into account. Recently there has been significant progress in constructing a perturbative EFT description of quarkonium at finite $T$, resulting in a clearly defined potential. This potential displays characteristics that are considerably different from the phenomenological, lattice-inspired description used up to now with well-defined phenomenological implications, as we further discuss. The higher energy of the heavy-ion collisions at the LHC will expand the study of quarkonium in media to bottomonia production and suppression. These studies will be crucial for arriving at a uniform description of heavy quarkonia in cold and hot nuclear matter.

Lastly, we turn our attention to a discussion of the experimental outlook in the near term as well as longerterm prospects. For the LHC, the future is now, with the first quarkonium data presented this year. While the preliminary data are encouraging, the full potential for LHC quarkonium studies is still to come. There is a future in low energy quarkonium hadroproduction studies as well, including two experiments at GSI in Darmstadt, Germany. PANDA will make precision spectroscopy studies in $\bar{p} p$ and $\bar{p} A$ interactions, while the CBM detector will make fixed-target studies of $p A$ and $A A$ interactions to further the understanding of quarkonium production and suppression in high baryon-density matter. Quarkonium physics goals at the currently-running BESIII, as well as at proposed super flavor and tau-charm factories are also discussed. Measurements of quarkonium photoproduction offer important insight into the gluon generalized parton distribution (GPD) in nuclei, the role of color correlations, and the color-dipole nature of quarkonia undergoing elastic scattering at high energies. These investigations can be performed at JLab, CERN, and the EIC in the medium term at lower energies, whereas higher energy studies will have to await the ENC, EIC, or LHeC. Important top quark measurements with high precision can be performed at a future $e^{+} e^{-}$linear collider (ILC or CLIC) in the region just above $t \bar{t}$ threshold. Overall, an extremely active and ambitious future lies ahead for the study of heavy quarkonia with new facilities. 


\section{SPECTROSCOPY ${ }^{2}$}

Spectroscopy is, in part, bump-hunting in mass spectra. Of late, progress has occurred mostly at $e^{+} e^{-}$colliding beam facilities (BES at BEPC, CLEO at CESR, BABAR at PEP-II, Belle at KEKB, KEDR at VEPP$4 \mathrm{M})$, but other venues have gotten into the game as well, including E835 at Fermilab ( $\bar{p} p$ gas-jet target) and $\mathrm{CDF}$ and $\mathrm{D} \varnothing$ at the Tevatron $p \bar{p}$ collider. Tevatron searches target inclusive production of a fully reconstructed state, and can best succeed when the presence of leptons (e.g., $J / \psi \rightarrow \mu^{+} \mu^{-}$) or displaced vertices (e.g., $B$-decay) can suppress backgrounds and when there is no need to observe photons. The main strength of $e^{+} e^{-}$colliders is the capability to obtain large datasets at or near charmonium and/or bottomonium vector state masses with well-known initial-state quantum numbers and kinematics. Modern $e^{+} e^{-}$detectors feature precision charged particle trackers, electromagnetic calorimeters, Cherenkov-radiation imagers or time-of-flight taggers, and muon filters, which together allow measurement of the individual decay remnants: $\gamma, e^{ \pm}, \mu^{ \pm}, \pi^{ \pm}, K^{ \pm}$, $p(\bar{p})$. These capabilities in $e^{+} e^{-}$collisions are exploited using the following techniques:

Full event reconstruction: Datasets taken onresonance at vector quarkonium masses allow full reconstruction of cascade transitions involving another state $\left(\right.$ e.g., $\psi(2 S) \rightarrow \pi^{0} h_{c}, h_{c} \rightarrow \gamma \eta_{c}(1 S)$ or $Y(4260) \rightarrow$ $\left.\pi^{+} \pi^{-} J / \psi\right)$.

Inclusive spectra: One or more final state particles are selected in each event. The measured four-momenta are then used to search directly for mass peaks, or indirectly via missing mass; i.e., the mass recoiling against the particle(s) selected. Two examples are an inclusive photon or $\pi^{0}$ momentum spectrum to identify transitions (e.g., $\Upsilon(3 S) \rightarrow \gamma \eta_{b}$ or $\psi(2 S) \rightarrow \pi^{0} h_{c}(1 P)$ ), which typically have small signals on very large backgrounds. In the continuum reaction $e^{+} e^{-} \rightarrow X J / \psi$ with $J / \psi \rightarrow \ell^{+} \ell^{-}$ (double-charmonium production), the unmeasured particle(s) $X$ can be identified via peaks in the missing mass spectrum.

Energy scan: Scans in $e^{+} e^{-}$center-of-mass energy $(\sqrt{s})$ can map out vector resonances via either inclusive hadronic-event counting $(R)$ and/or exclusive final states (e.g., $\left.D \bar{D}^{*}\right)$. This does not use machine time efficiently, however, because accelerators work best when operated at a single energy for a long time so tuning can optimize the instantaneous luminosity. Competing priorities usually limit the duration of such scans.

\footnotetext{
${ }^{2}$ Contributing authors: N. Brambilla ${ }^{\dagger}$, B. K. Heltsley ${ }^{\dagger}$, A. A. Petrov ${ }^{\dagger}$, G. Bali, S. Eidelman, U. Ellwanger, A. Gabareen Mokhtar, X. Garcia i Tormo, R. Mussa, F. S. Navarra, M. Nielsen, S. L. Olsen, P. Pakhlov, G. Pakhlova, A. D. Polosa, M. A. Sanchis-Lozano, Y. Sumino, and M. Voloshin
}

$\underline{\boldsymbol{\gamma} \gamma \text {-fusion: }}$ The process $e^{+} e^{-} \rightarrow e^{+} e^{-} \gamma^{(*)} \gamma^{(*)} \rightarrow$ $e^{+} e^{-} X$ allows searches for a large range of masses for $X$, but $X$ is restricted to having spin-0 or 2 and positive $C$-parity $\left(e . g ., \eta_{c}(2 S)\right.$ or $\left.\chi_{c 2}(2 P)\right)$. The outgoing $e^{+} e^{-}$ tend to escape the detector at small angles, leaving $X$ with very small momentum transverse to the beamline.

ISR: Initial-state radiation (ISR) allows access to all vector states with masses below the $\sqrt{s}$ value of the $e^{+} e^{-}$collision. The effective luminosity per unit of radiative photon energy (or the mass recoiling against it) is well-known, allowing for well-normalized exposures at all masses. The ISR photon tends to escape the detector at small angles, leaving the recoiling state with small momentum transverse to the beam but large momentum along it. While the rate for ISR production at any fixed $\sqrt{s}$ is small per unit luminosity, factorysized datasets at $B A B A R$ and Belle make this a viable tool (e.g., $\left.e^{+} e^{-} \rightarrow \gamma Y(4260), e^{+} e^{-} \rightarrow \gamma D \bar{D}^{*}\right)$, and the simultaneous exposure of all allowed masses recoiling against the ISR photon contrasts with the discrete points available from a direct $e^{+} e^{-}$scan.

$\boldsymbol{B}$-decays: Large $B$-factory datasets at the $\Upsilon(4 S)$ make it possible to utilize two-body kinematics to search for exclusive decays of $B$-mesons (e.g., $B \rightarrow K Z_{i}^{+}$).

It is worth emphasizing here that the key tenet of experimental science is that discoveries must be reproducible and verified by independent parties as a prerequisite for general acceptance. This is no small point in a period such as the present when the world has been bombarded with more than one new state found per year. It is worth pondering spectra which initially were thought to indicate new states in the heavy quarkonium mass region but were later proven otherwise by independent measurements. Such occurrences happen to the best of experiments and most often can be attributed to fluctuations and/or extremely subtle systematic effects, not overt analysis blunders. Figure 1 highlights two such examples. Figures 1(a) and (b) show dielectron mass distributions observed [2] inclusively in $400 \mathrm{GeV}$ proton collisions on beryllium. A $J / \psi$ peak is clearly seen in (a) while there is an apparent peak near $6 \mathrm{GeV}$ in (b). The authors estimated a $2 \%$ probability that the $6 \mathrm{GeV}$ peak was due to background fluctuations, explicitly cautioned that confirmation was needed, and suggested that the name " $\Upsilon$ " be given to this or any subsequently confirmed high-mass dilepton peak. The $6 \mathrm{GeV}$ phenomenon was not confirmed several months later in a dimuon version [3] of the same experiment. The same authors discovered the true $\Upsilon(1 S)$ shortly thereafter [4]. Figures 1(c) and (d) show an inclusively selected photon-energy distribution in $\psi(2 S)$ decays [5]. The size of the peak near $91 \mathrm{MeV}$ represents a branching fraction of $0.8 \%$ and has statistical significance of $>6 \sigma$. The peak position corresponds to a mass recoiling against the photon of $3594 \pm 5 \mathrm{MeV}$ and a width $\Gamma<8 \mathrm{MeV}$. The result stood as an $\eta_{c}(2 S)$ candidate for twenty years. It was finally refuted [6] as having $\mathcal{B}<0.2 \%$ at $90 \% \mathrm{CL}$ (confidence level). Incidents such as these (and many others) have led many experi- 

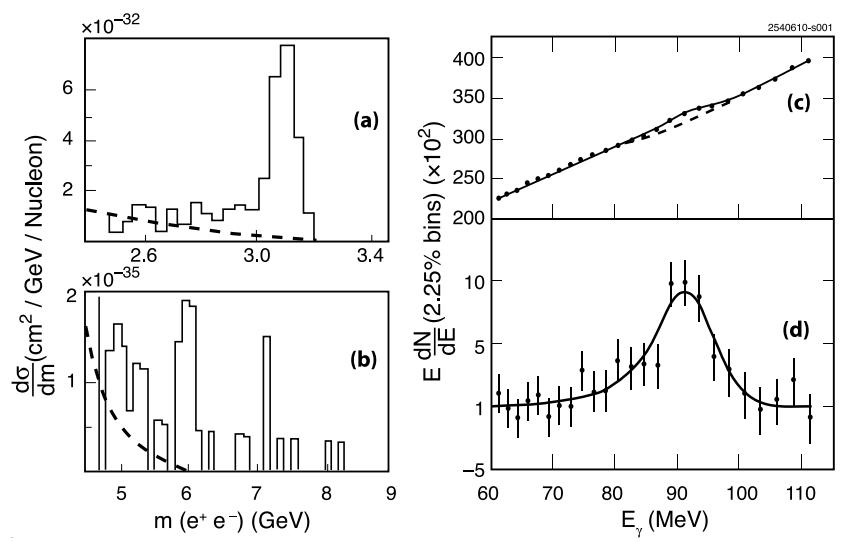

FIG. 1: (a), (b) Dielectron mass distributions from inclusively selected proton-on-beryllium collisions [2], in which dashed curves indicate estimated backgrounds. (c) Inclusive photon energy distribution in $\psi(2 S)$ decays [5] (points with error bars), showing a fit (solid line) to a signal and a smooth background (dashed line). Part (d) is the same as (c) but with the smooth backgrounds subtracted. Both peaks were later refuted. Adapted from [2, 5] with kind permission, copyrights (1976, 1982), respectively, The American Physical Society

ments to adopt more stringent criteria and procedures for establishing signals. These include requiring a threshold of " $5 \sigma$ " statistical significance for claiming "observation", allowing systematic variations to reduce the reported significance, tuning of selection criteria on small subsamples of data not used in the signal search, and the intentional obscuring of signal regions until cuts are frozen (so-called "blind" analysis). However, every potential signal deserves independent confirmation or refutation.

This section will first focus on recent measurements: What is the current status of each state? How were the measurements performed? Which need verification? Which are in conflict? Then the theoretical issues will be addressed.

\subsection{Conventional vectors above open-flavor threshold}

Here we describe recent measurements relevant to the determinations of mass, width, and open-charm content of the four known vector charmonia above open-charm threshold. These states were first observed thirty years ago in $e^{+} e^{-}$annihilation as enhancements in the total hadronic cross section [7-11]. No update of their parameters was made until 2005, when a combined fit to the Crystal Ball [12] and BES [13] $R$-measurements was performed by Seth [14]. Even more recently, BES [15] reported new parameter values for the $\psi$ resonances. A plethora of open charm cross section measurements has become available and is discussed in what follows. Finally, recent studies of resonant structures just above the open-bottom threshold are described.

\subsubsection{Vectors decaying to open charm}

The total cross section for hadron production in $e^{+} e^{-}$ annihilation is usually parametrized in terms of the ratio $R$, defined as

$$
R=\frac{\sigma\left(e^{+} e^{-} \rightarrow \text { hadrons }\right)}{\sigma\left(e^{+} e^{-} \rightarrow \mu^{+} \mu^{-}\right)}
$$

where the denominator is the lowest-order QED cross section,

$$
\sigma\left(e^{+} e^{-} \rightarrow \mu^{+} \mu^{-}\right)=\frac{4 \pi \alpha^{2}}{3 s} .
$$

Away from flavor thresholds, measured $R$ values are consistent with the three-color quark model predictions plus terms governed by QCD and the running of $\alpha_{\mathrm{s}}\left(Q^{2}\right)$. Resonant states in the vicinity of flavor thresholds can be studied with fits of measured $R$ distributions. As a part of a study of open charm cross sections in the region from 3.97-4.26 GeV, CLEO [16] published radiatively corrected $R$-values as shown in Fig. 2. These are in good agreement with earlier measurements $[12,13]$, which are also shown, demonstrating that in this energy range $R$ values are reasonably well-vetted experimentally.

The extraction of resonance parameters from such $R$ measurements, however, has evolved in complexity, causing systematic movement in some of the parameters over time. The latest BES [15] fit to their $R$-scan data is more sophisticated than previous efforts and includes the effects of interference and relative phases, as shown in Fig. 3 and Table 1. To take into account interference, BES relied on model predictions for branching fractions of $\psi$ states into all possible two-body charm meson final states. Thus the measured parameters from this fit still

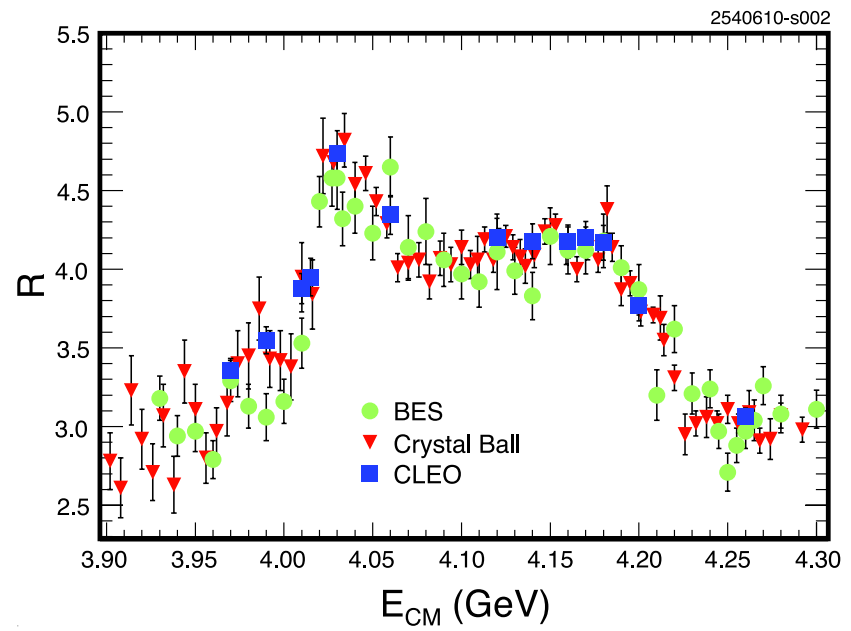

FIG. 2: Measurements of $R$, including radiative corrections, in the open charm region. From Crystal Ball [12], BES [13], and CLEO [16]. Adapted from [16] with kind permission, copyright (2009) The American Physical Society 


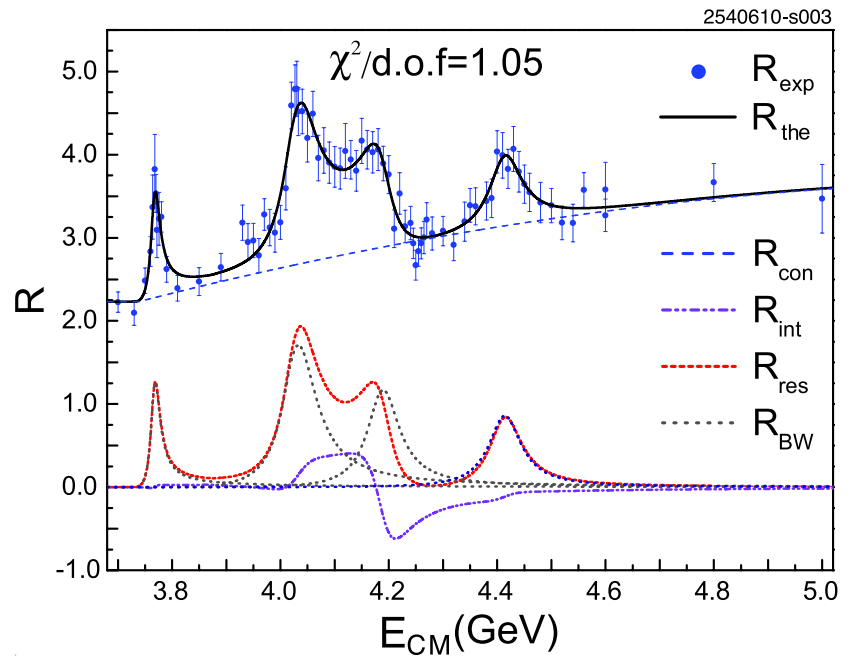

FIG. 3: From BES [15], measured $R$ values from data (dots with error bars) and curves showing the best fit (solid) and the contributions of its components: continuum background (long dashed), individual resonance (dotted), interference (dash-dotdot), and the summation of the nonbackground curves (short dashed). Adapted from [15] with kind permission, copyright (2008) Elsevier

include some model uncertainties which are difficult to estimate. Other systematic uncertainties are estimated using alternative choices and combinations of Breit-Wigner forms, energy dependence of the full width, and continuum charm background. It was found that the results are sensitive to the form of the energy-dependent total width but are not sensitive to the form of background.

In a separate analysis, BES [19] fit their $R$ data from 3.65-3.90 GeV, finding a $7 \sigma$ preference for two interfering lineshapes peaked near 3763 and $3781 \mathrm{MeV}$ relative to a single such shape for the $\psi(3770)$, although other sources for the observed distortion of a pure $D$ wave Breit-Wigner are possible (see also Sect. 3.4.4). A very recent preliminary analysis of KEDR $[20] e^{+} e^{-}$scan data near the $\psi(3770)$ applies an extended vector dominance model and includes interference with the tail of the $\psi(2 S)$ resonance, concluding that the latter interference causes a significant shift upward in the fitted peak of the $\psi(3770)$ as compared to most previous fits, including those of BES. The KEDR measurements are not consistent with the two-peak distortion seen by BES.

For determination of the resonance parameters in the open charm region, inclusive hadronic cross section measurements appear not to supply enough information to determine the relative strength of different decay channels. More data and more reliable physical models appear to be needed in order to make further progress. The PDG-supplied parameters in Table 1 bypass these issues and provide parameters under the simplest of assumptions, which may or may not turn out to be correct.

Detailed studies of the open-charm content in the charmonium region were not undertaken until large datasets
TABLE 1: The resonance parameters of the high-mass charmonia from the BES global fit [15] together with the values from PDG04 [17], Seth [14], and PDG08 [18]

\begin{tabular}{ccccc}
\hline \hline Resonance & $m(\mathrm{MeV})$ & $\Gamma_{\text {tot }}(\mathrm{MeV})$ & $\delta\left(^{\circ}\right)$ & Ref. \\
\hline & $3769.9 \pm 2.5$ & $23.6 \pm 2.7$ & & PDG04 [17] \\
$\psi(3770)$ & $3771.1 \pm 2.4$ & $23.0 \pm 2.7$ & & Seth [14] \\
& $3772.0 \pm 1.9$ & $30.4 \pm 8.5$ & 0 & BES [15] \\
& $3772.92 \pm 0.35$ & $27.3 \pm 1.0$ & & PDG08 [18] \\
\hline \multirow{4}{*}{$\psi(4040)$} & $4040 \pm 1$ & $52 \pm 10$ & & PDG04 [17] \\
& $4039 \pm 1.0$ & $80 \pm 10$ & & Seth [14] \\
& $4039.6 \pm 4.3$ & $84.5 \pm 12.3$ & $130 \pm 46$ & BES [15] \\
\hline \multirow{4}{*}{$\psi(4160)$} & $4159 \pm 20$ & $78 \pm 20$ & & PDG04 [17] \\
& $4153 \pm 3$ & $103 \pm 8$ & & Seth [14] \\
& $4191.7 \pm 6.5$ & $71.8 \pm 12.3$ & $293 \pm 57$ & BES [15] \\
\hline \multirow{4}{*}{$\psi(4415)$} & $4415 \pm 6$ & $43 \pm 15$ & & PDG04 [17] \\
& $4421 \pm 4$ & $62 \pm 20$ & & Seth [14] \\
& $4415.1 \pm 7.9$ & $71.5 \pm 19.0$ & $234 \pm 88$ & BES [15] \\
\hline \hline
\end{tabular}

were obtained by CLEO at discrete energy points and by the $B$-factory experiments using radiative returns to obtain a continuous exposure of the mass region. The picture that has emerged is complex due to the many thresholds in the region, nine of which are two-body final states using allowed pairs of $D^{0}, D^{+}, D^{* 0}, D^{*+}, D_{s}^{+}$, and $D_{s}^{*+}$. Moreover, distinguishing genuine two-body from "multibody" decays (e.g., $D \bar{D}^{*}$ from $D^{0} D^{-} \pi^{+}$) poses a challenge. Experimentally, the data are consistent where measurements overlap; significant discrepancies with some predictions mean that theoretical work remains.

Exclusive $e^{+} e^{-}$cross sections for hadronic final states containing charm mesons were studied by several groups. In the $\sqrt{s}=3.7-5 \mathrm{GeV}$ energy region, Belle [21-26], and $B A B A R$ [27-29] used initial-state radiation (ISR) to reach the charmonium region, and CLEO used its large data sample taken at the $\psi(3770)$ peak [30] and its scan over $\sqrt{s}=3.97-4.26 \mathrm{GeV}$ [16]. Some of these results can be seen in Table 2 and Figs. 4 and 5. Measurements of the neutral to charged $D \bar{D}$ cross section ratio at the $\psi(3770)$ peak show consistency but the PDG08 [18] world average, $1.260 \pm 0.021$, is dominated by the CLEO [30] value. The $D \bar{D}$ cross sections across the entire charm energy range from Belle [21] and BABAR [27] appear in Fig. 4 and are consistent with one another. Both observe a structure in the ISR $D \bar{D}$ cross section (Figs. 4(a) and (b)), known as $G(3900)$, which must be taken into account to describe both the $D \bar{D}$ cross section and $R$ in the region between $\psi(3770)$ and $\psi(4040)$. The $G(3900)$ is not considered to be a specific $c \bar{c}$ bound state, as it is qualitatively consistent with a prediction from a coupledchannel model [34]. The $D^{+} D^{*-}$ cross sections from Belle [22] and BABAR [28] exhibit a single broad peak near threshold whereas $D^{*+} D^{*-}$ results $[22,28]$ feature several local maxima and minima across this energy range. 


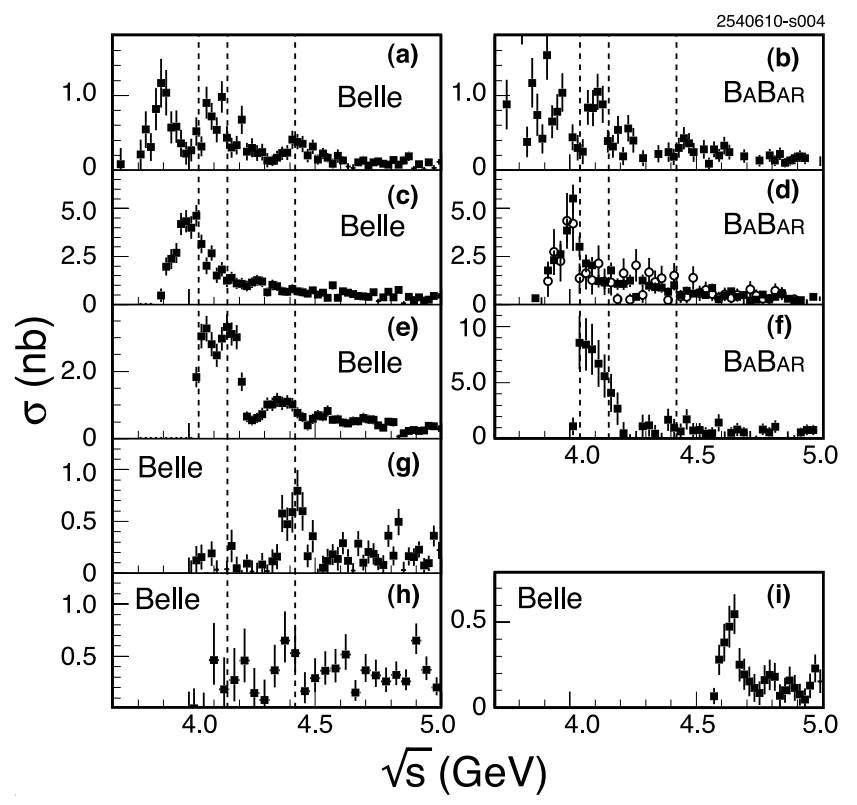

FIG. 4: Measured $e^{+} e^{-}$exclusive open-charm meson- or baryon-pair cross sections for $\sqrt{s}=3.7-5.0 \mathrm{GeV}$ from Belle and BABAR, showing (a) $D \bar{D}$ [21]; (b) $D \bar{D}$ [27]; (c) $D^{+} D^{*-}[22] ;$ (d) $D \bar{D}^{*}$ for $D=D^{0}$ (solid squares) and $D=D^{+}$(open circles) [28]; (e) $D^{*+} D^{*-}$ [22]; (f) $D^{*} \bar{D}^{*} \quad[28] ; \quad$ (g) $D^{0} D^{-} \pi^{+} \quad[23] ; \quad$ (h) $D^{0} D^{*-} \pi^{+} \quad$ [24]; (i) $\Lambda_{c}^{+} \Lambda_{c}^{-}$[25]. Vertical dashed lines indicate $\psi$ masses in the region. Adapted from [21-25, 27, 28] with kind permission, copyrights $(2008,2007,2008,2009,2008,2007,2008)$, respectively, The American Physical Society

The $e^{+} e^{-} \rightarrow \Lambda_{c}^{+} \Lambda_{c}^{-}$cross section measured by Belle [25], shown in Fig. 4(i), exhibits a substantial enhancement just above threshold near $4.6 \mathrm{GeV}$ (addressed below).

$B A B A R$ [28, 29] performed unbinned maximum likelihood fits to the $D \bar{D}, D \bar{D}^{*}, D^{*} \bar{D}^{*}$, and $D_{s}^{(*)+} D_{s}^{(*)-}$ spectra. The expected $\psi$ signals were parametrized by $P$-wave relativistic Breit-Wigner (RBW) functions with their parameters fixed to the PDG08 values [18]. An interference between the resonances and the nonresonant contributions was required in the fit. The computed ratios of the branching fractions for the $\psi$ resonances to nonstrange open-charm meson pairs and the quark model predictions are presented in Table 2. The BABAR results deviate from some of the theoretical expectations. The BABAR [29] cross sections for $D_{s}^{(*)+} D_{s}^{(*)-}$ production show evidence for $\psi(4040)$ in $D_{s}^{+} D_{s}^{-}$and $\psi(4160)$ in $D_{s}^{*+} D_{s}^{-}$, and are consistent with the CLEO [16] results where they overlap.

The $e^{+} e^{-} \rightarrow D^{0} D^{-} \pi^{+}$cross section measured by Belle [23] is shown in Fig. 4(g) and exhibits an unambiguous $\psi(4415)$ signal. A study of the resonant structure shows clear signals for the $\bar{D}_{2}^{*}(2460)^{0}$ and $D_{2}^{*}(2460)^{+}$ mesons and constructive interference between the neutral $D^{0} \bar{D}_{2}^{*}(2460)^{0}$ and the charged $D^{-} D_{2}^{*}(2460)^{+}$decay amplitudes. Belle performed a likelihood fit to the $D \bar{D}_{2}^{*}(2460)$ mass distribution with a $\psi(4415)$ signal

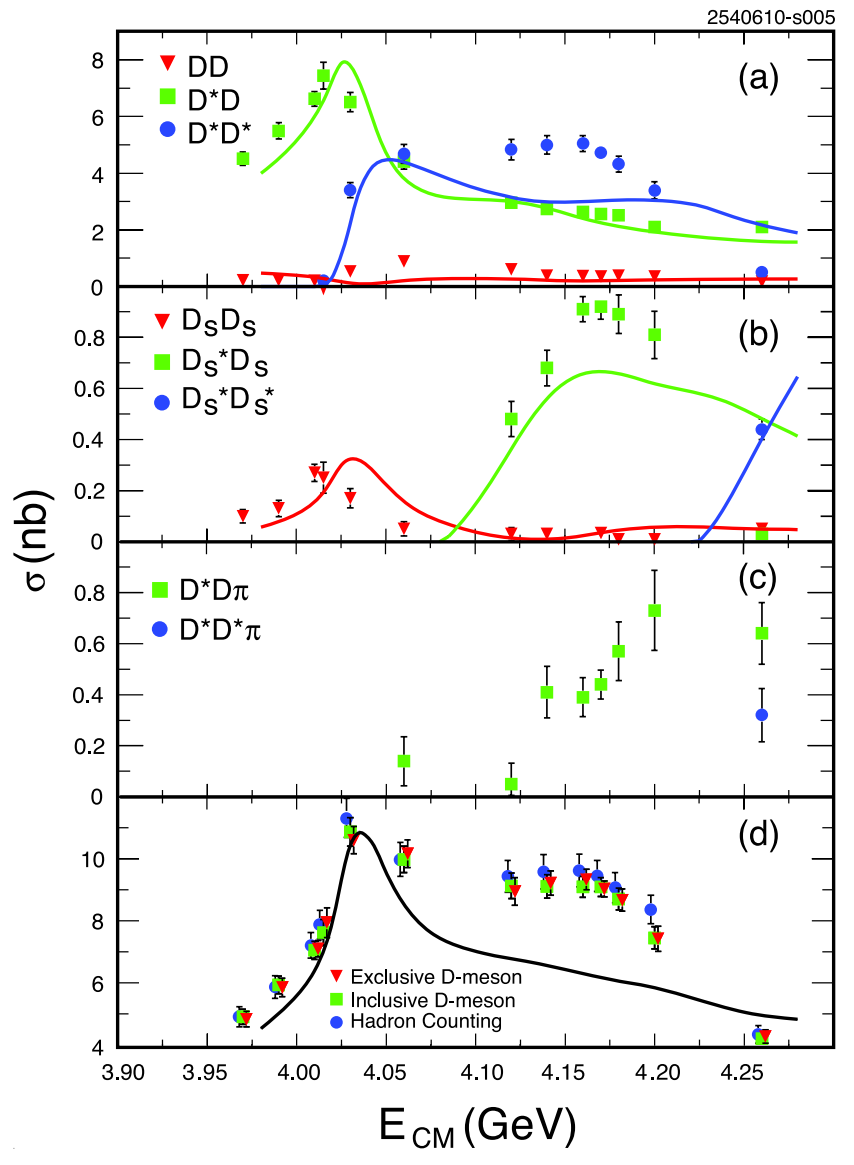

FIG. 5: From CLEO [16], cross sections, without radiative corrections, for $e^{+} e^{-}$annihilation into: (a)-(c) the exclusive open-charm meson-pairs shown; and (d) for two methods of open-charm counting added onto the extrapolated $u d s$ cross section compared to an all-flavor hadron-counting cross section. Solid lines show updated potential model predictions [34]. Adapted from [16] with kind permission, copyright (2009) The American Physical Society

parametrized by an $S$-wave RBW function. The significance of the signal is $\sim 10 \sigma$ and the peak mass and total width are in good agreement with the PDG06 [35] values and the BES fit results [15]. The branching fraction for $\psi(4415) \rightarrow D \bar{D}_{2}^{*}(2460) \rightarrow D \bar{D} \pi^{+}$was found to be between $10 \%$ and $20 \%$, depending on the $\psi(4415)$ parametrization. The fraction of $D \bar{D}_{2}^{*}(2460) \rightarrow D \bar{D} \pi^{+}$final states composed of nonresonant $D^{0} D^{-} \pi^{+}$was found to be $<22 \%$. Similarly, the $D^{0} D^{*-} \pi^{+}$content of $\psi(4415)$, shown in Fig. 4(h), has been determined by Belle [24]; a marginal signal is found $(3.1 \sigma)$, and its branching fraction was limited to $<10.6 \%$. Belle [26] has also reported a preliminary spectrum of $e^{+} e^{-} \rightarrow$ $D_{s}^{(*)+} D_{s}^{(*)-}$ cross sections from $\sqrt{s}=3.8-5 \mathrm{GeV}$ using ISR from a data sample of $967 \mathrm{fb}^{-1}$ in $40 \mathrm{MeV}$ bins; the values are consistent with but higher-statistics and more finely binned than those of BABAR [29].

The CLEO exclusive cross sections [16] in the top three frames of Fig. 5 are not directly comparable to those 
TABLE 2: From BABAR [28], ratios of branching fractions for the $\psi(4040), \psi(4160)$ and $\psi(4415)$ resonances. The first error is statistical, the second systematic. Theoretical expectations are from models denoted ${ }^{3} P_{0}[31], C^{3}$ [32], and $\rho K \rho$ [33]

\begin{tabular}{cccccc}
\hline \hline State & Ratio & Measured & ${ }^{3} P_{0}$ & $C^{3}$ & $\rho K \rho$ \\
\hline$\psi(4040)$ & $D \bar{D} / D \bar{D}^{*}$ & $0.24 \pm 0.05 \pm 0.12$ & 0.003 & & 0.14 \\
& $D^{*} \bar{D}^{*} / D \bar{D}^{*}$ & $0.18 \pm 0.14 \pm 0.03$ & 1.0 & 0.29 \\
\hline \multirow{2}{*}{$(4160)$} & $D \bar{D} / D^{*} \bar{D}^{*}$ & $0.02 \pm 0.03 \pm 0.02$ & 0.46 & 0.08 & \\
& $D \bar{D}^{*} / D^{*} \bar{D}^{*}$ & $0.34 \pm 0.14 \pm 0.05$ & 0.011 & 0.16 & \\
\hline \multirow{2}{*}{$(4415)$} & $D \bar{D} / D^{*} \bar{D}^{*}$ & $0.14 \pm 0.12 \pm 0.03$ & 0.025 & & \\
& $D \bar{D}^{*} / D^{*} \bar{D}^{*}$ & $0.17 \pm 0.25 \pm 0.03$ & 0.14 & & \\
\hline \hline
\end{tabular}

from BABAR and Belle as they are not radiatively corrected, but generally seem to reflect consistency. The updated potential model predictions of Eichten [16, 34] shown in Fig. 5 fail to describe many features of the data. The CLEO total cross section determinations, shown in Fig. $5(\mathrm{~d})$, reveal that, within the measurement accuracy of $5-10 \%$, two- and three-body modes with open charm saturate the yield of all multihadronic events above the extrapolated $u d s$ contribution.

\subsubsection{Vectors decaying to open bottom}

The current generation of $B$-factories have scanned the energy range above open bottom threshold. BABAR [36] performed a comprehensive low-luminosity $\left(25 \mathrm{pb}^{-1}\right.$ per point), high-granularity $(\approx 5 \mathrm{MeV}$ steps) scan between 10.54 and $11.2 \mathrm{GeV}$, followed by an eight-point scan, $0.6 \mathrm{fb}^{-1}$ total, in the proximity of the $\Upsilon(6 S)$ peak. Belle [37] acquired $\approx 30 \mathrm{pb}^{-1}$ for just nine points over 10.80-11.02 GeV, as well as $8.1 \mathrm{fb}^{-1}$ spread over seven additional points more focused on the $\Upsilon(5 S)$ peak. The BABAR scan is shown in Fig. 6. Both scans suggest instead that the simple Breit-Wigner parametrization, previously used to model the peaks observed in the CLEO [38] and CUSB [39] scans, is not adequate for the description of the complex dynamics in the proximity of the $B^{(*)} \bar{B}^{(*)}$ and $B_{s}^{(*)} \bar{B}_{s}^{(*)}$ thresholds. Data points on $R_{b}=\sigma(b \bar{b}) / \sigma(\mu \mu)$ are better modeled assuming a flat $b \bar{b}$ continuum contribution which interferes constructively with the $5 S$ and $6 S$ Breit-Wigner resonances, and a second flat contribution which adds incoherently. Such fits strongly alter the PDG results on the $5 S$ and $6 S$ peaks, as shown in Table 3. Strong qualitative agreement is observed between the experimental behavior of the $R_{b}$ ratio and the theory predictions based on the coupled-channel approach [40].

Additional insight can be provided by the exclusive decomposition of the two-body (i.e., $B \bar{B}, B \bar{B}^{*}, B^{*} \bar{B}^{*}$ ) and many-body decay modes. Results from $e^{+} e^{-}$collisions have been given by Belle [41] using a data sample of $23.6 \mathrm{fb}^{-1}$ acquired at the $\Upsilon(5 \mathrm{~S})$. Charged $B$-mesons were

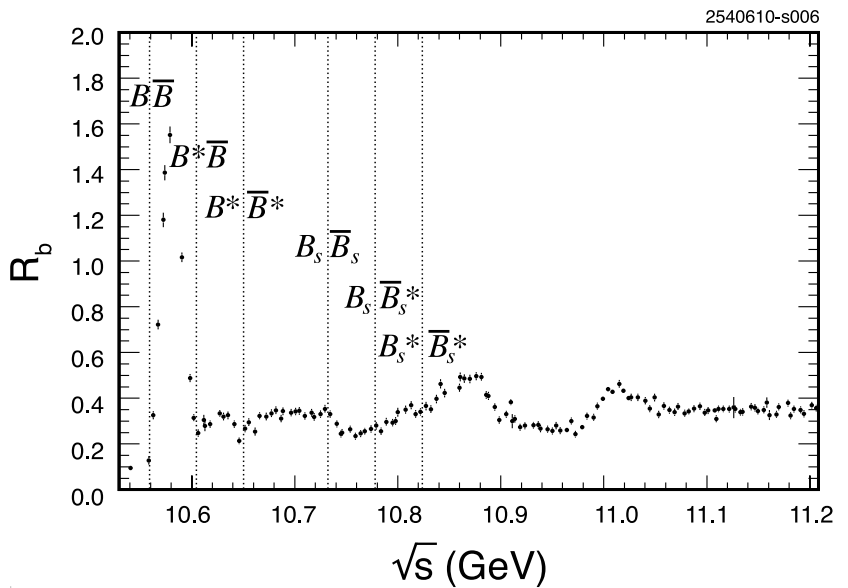

FIG. 6: From BABAR [36], measured values of the hadronic cross section attributable to $b$-flavored states, normalized to the point muon pair cross section, from a scan of the center-ofmass energy region just below the $\Upsilon(4 S)$ to above the $\Upsilon(6 S)$. Dashed vertical lines indicate the various $B \bar{B}$ mass thresholds. Adapted from [36] with kind permission, copyright (2009) The American Physical Society

reconstructed in two decay channels, $K^{ \pm} J / \psi$ and $D^{0} \pi^{ \pm}$ (with $J / \psi \rightarrow l^{+} l^{-}$and $D^{0} \rightarrow K \pi, K \pi \pi \pi$ ). Neutral $B$ mesons were reconstructed in $K^{* 0} J / \psi$ and $D^{ \pm} \pi^{\mp}$, with $D^{ \pm} \rightarrow K^{ \pm} \pi^{ \pm} \pi^{\mp}$. The $B^{*}$ mesons were reconstructed via their radiative transition. Belle observes a large fraction (about $16.4 \%$ of the total $b \bar{b}$ pairs) from 3 - and 4-body decay modes, i.e., $B^{(*)} \bar{B}^{(*)} \pi, B^{(*)} \bar{B}^{(*)} \pi \pi$. A significant fraction of these events can actually be expected from ISR production of $\Upsilon(4 S)$. Theory predictions on multibody decays at $\Upsilon(5 S)$ range from $0.03 \%$ [42] to $0.3 \%$ [43].

\subsection{Newly found conventional quarkonia}

Table 4 lists properties of new conventional heavy quarkonium states. The $h_{c}$ is the ${ }^{1} P_{1}$ state of charmonium, singlet partner of the long-known $\chi_{c J}$ triplet ${ }^{3} P_{J}$. The $\eta_{c}(2 S)$ is the first excited state of the pseudoscalar

TABLE 3: New BABAR and Belle results on masses and widths of the $\Upsilon(5 S)$ and $\Upsilon(6 S)$ resonances, compared to PDG averages. The angle $\phi$ parametrizes the phase of interfering continuum

\begin{tabular}{ccccl}
\hline \hline$\Upsilon$ & $m(\mathrm{MeV})$ & $\Gamma(\mathrm{MeV})$ & $\phi(\mathrm{rad})$ & Ref. \\
\hline \multirow{5}{5}{$\mathrm{S}$} & $10876 \pm 2$ & $43 \pm 4$ & $2.11 \pm 0.12$ & BABAR [36] \\
& $10879 \pm 3$ & $46_{-7}^{+9}$ & $2.33_{-0.24}^{+0.26}$ & Belle [37] \\
& $10865 \pm 8$ & $110 \pm 13$ & - & PDG08 [18] \\
\hline \multirow{2}{*}{$6 \mathrm{~S}$} & $10996 \pm 2$ & $37 \pm 3$ & $0.12 \pm 0.07$ & BABAR [36] \\
& $11019 \pm 8$ & $79 \pm 16$ & - & PDG08 [18] \\
\hline \hline
\end{tabular}


TABLE 4: New conventional states in the $c \bar{c}, b \bar{c}$, and $b \bar{b}$ regions, ordered by mass. Masses $m$ and widths $\Gamma$ represent the weighted averages from the listed sources. Quoted uncertainties reflect quadrature summation from individual experiments. In the Process column, the decay mode of the new state claimed is indicated in parentheses. Ellipses (...) indicate inclusively selected event topologies; i.e., additional particles not required by the Experiments to be present. For each Experiment a citation is given, as well as the statistical significance in number of standard deviations (\# $\sigma)$, or "(np)" for "not provided". The Year column gives the date of first measurement cited, which is the first with significance of $>5 \sigma$. The Status column indicates that the state has been observed by at most one (NC!-needs confirmation) or at least two independent experiments with significance of $>5 \sigma(\mathrm{OK})$. The state labelled $\chi_{c 2}(2 P)$ has previously been called $Z(3930)$

\begin{tabular}{|c|c|c|c|c|c|c|c|}
\hline State & $m(\mathrm{MeV})$ & $\Gamma(\mathrm{MeV})$ & $J^{P C}$ & Process (mode) & Experiment $(\# \sigma)$ & Year & Status \\
\hline$h_{c}(1 P)$ & $3525.45 \pm 0.15$ & $\begin{array}{c}0.73 \pm 0.53 \\
(<1.44)\end{array}$ & $1^{+-}$ & $\begin{array}{l}\psi(2 S) \rightarrow \pi^{0}\left(\gamma \eta_{c}(1 S)\right) \\
\psi(2 S) \rightarrow \pi^{0}(\gamma \ldots) \\
p \bar{p} \rightarrow\left(\gamma \eta_{c}\right) \rightarrow(\gamma \gamma \gamma) \\
\psi(2 S) \rightarrow \pi^{0}(\ldots)\end{array}$ & $\begin{array}{c}\text { CLEO [44-46] (13.2) } \\
\text { CLEO [44-46] }(10.0), \text { BES [47] (18.6) } \\
\text { E835 [48] }(3.1) \\
\text { BESIII }[47](9.5)\end{array}$ & 2004 & OK \\
\hline$\eta_{c}(2 S)$ & $3637 \pm 4$ & $14 \pm 7$ & $0^{-+}$ & $\begin{array}{l}B \rightarrow K\left(K_{S}^{0} K^{-} \pi^{+}\right) \\
e^{+} e^{-} \rightarrow e^{+} e^{-}\left(K_{S}^{0} K^{-} \pi^{+}\right) \\
e^{+} e^{-} \rightarrow J / \psi(\ldots)\end{array}$ & $\begin{array}{c}\text { Belle [49] (6.0) } \\
\text { BABAR [50] (4.9), CLEO [51] (6.5), } \\
\text { Belle [52] (6) } \\
\text { BABAR [53] (np), Belle [54] (8.1) }\end{array}$ & 2002 & OK \\
\hline$\chi_{c 2}(2 P)$ & $3927.2 \pm 2.6$ & $24.1 \pm 6.1$ & $2^{++}$ & $e^{+} e^{-} \rightarrow e^{+} e^{-}(D \bar{D})$ & Belle [55] (5.3), BABAR [56] (5.8) & 2005 & OK \\
\hline$B_{c}^{+}$ & $6277.1 \pm 4.1$ & - & $0^{-}$ & $\bar{p} p \rightarrow\left(\pi^{+} J / \psi\right) \ldots$ & $\mathrm{CDF}[57](8.0), \mathrm{D} \varnothing[58](5.2)$ & 2007 & OK \\
\hline$\eta_{b}(1 S)$ & $9390.7 \pm 2.9$ & $?$ & $0^{-+}$ & $\begin{array}{l}\Upsilon(3 S) \rightarrow \gamma+(\ldots) \\
\Upsilon(2 S) \rightarrow \gamma+(\ldots)\end{array}$ & $\begin{array}{c}\text { BABAR [59] (10), CLEO [60] (4.0) } \\
\text { BABAR [61] }(3.0)\end{array}$ & 2008 & NC! \\
\hline$\Upsilon\left(1^{3} D_{2}\right)$ & $10163.8 \pm 1.4$ & $?$ & $2^{--}$ & $\begin{array}{l}\Upsilon(3 S) \rightarrow \gamma \gamma(\gamma \gamma \Upsilon(1 S)) \\
\Upsilon(3 S) \rightarrow \gamma \gamma\left(\pi^{+} \pi^{-} \Upsilon(1 S)\right)\end{array}$ & $\begin{array}{l}\text { CLEO [62] }(10.2) \\
\text { BABAR [63] (5.8) }\end{array}$ & 2004 & OK \\
\hline
\end{tabular}

ground state $\eta_{c}(1 S)$, lying just below the mass of its vector counterpart, $\psi(2 S)$. The first $B$-meson seen that contains charm is the $B_{c}$. The ground state of bottomonium is the $\eta_{b}(1 S)$. And the $\Upsilon(1 D)$ is the lowest-lying $D$-wave triplet of the $b \bar{b}$ system. All fit into their respective spectroscopies roughly where expected. Their exact masses, production mechanisms, and decay modes provide guidance to their descriptions within QCD.

\subsubsection{Observation of $h_{c}(1 P)$}

Two experiments reported $h_{c}(1 P)$ sightings in 2005, with CLEO [44, 45] reporting an observation at $>6 \sigma$ in the isospin-forbidden decay chain $e^{+} e^{-} \rightarrow \psi(2 S) \rightarrow$ $\pi^{0} h_{c}, h_{c} \rightarrow \gamma \eta_{c}(1 S)$, and E835 [48] found $3 \sigma$ evidence in $p \bar{p} \rightarrow h_{c}, h_{c} \rightarrow \gamma \eta_{c}(1 S), \eta_{c}(1 S) \rightarrow \gamma \gamma$. CLEO [46] later updated its measurements with a larger dataset, refining its mass measurement to a precision of just over $0.2 \mathrm{MeV}$, finding a central value slightly more accurate than that of E835, which has an uncertainty of just under $0.3 \mathrm{MeV}$. CLEO utilized two detection methods. The first was a semi-inclusive selection that required detection of both the transition $\pi^{0}$ and radiative photon but only inferred the presence of the $\eta_{c}(1 S)$ through kinematics. The second employed full reconstruction in fifteen different $\eta_{c}(1 S)$ decay modes, five of them previ- ously unseen. The two methods had some statistical and almost full systematic correlation for the mass measurement because both rely on the $\pi^{0}$ momentum determination. As the parent $\psi(2 S)$ has precisely known mass and is produced nearly at rest by the incoming $e^{+} e^{-}$ pair, the mass of the $h_{c}(1 P)$ is most accurately determined by fitting the distribution of the mass recoiling against the $\pi^{0}$, as shown for the exclusive analysis in Fig. 7. CLEO's two methods had comparable precision and gave consistent masses within their uncorrelated uncertainties. Statistical uncertainties from the numbers of signal (background) events in the exclusive (inclusive) analysis are larger than the systematic errors attributable to calorimeter energy resolution. The E835 measurement relies on knowledge of the initial center-of-mass energy of the $p \bar{p}$ for each event during a scan of the $h_{c}(1 P)$ mass region as well as upon reconstruction of all three photons with kinematics consistent with the production and decay hypothesis. Unlike the CLEO result, backgrounds are negligible. Mass measurement accuracy was limited equally by statistics ( 13 signal events with a standard deviation in center-of-mass energy of $0.07 \mathrm{MeV}$ ) and systematics of $\bar{p}$ beam energy stability. Using a sample of $106 \mathrm{M} \psi(2 S)$, in 2010 BESIII [47] reported a mass result using the $\pi^{0} \gamma$ inclusive method, matching CLEO's precision. The spin-averaged centroid of the triplet states, $\left\langle m\left(1^{3} P_{J}\right)\right\rangle \equiv\left[m\left(\chi_{c 0}\right)+3 m\left(\chi_{c 1}\right)+5 m\left(\chi_{c 2}\right)\right] / 9$, is ex- 


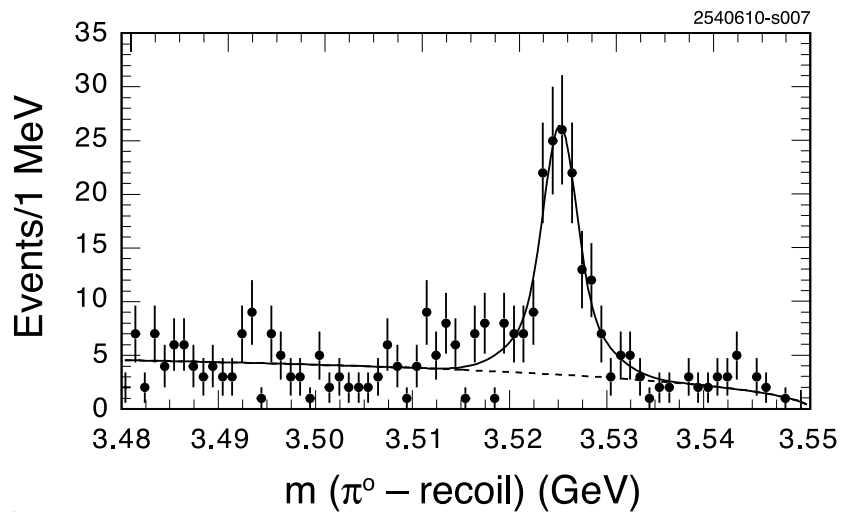

FIG. 7: From CLEO [46], the mass recoiling against the $\pi^{0}$ in the $\psi(2 S) \rightarrow \pi^{0} h_{c}, h_{c} \rightarrow \gamma \eta_{c}(1 S)$ exclusive sample in which the $\pi^{0}, \gamma$, and $\eta_{c}(1 S)$ are all explicitly reconstructed in the detector. Adapted from [46] with kind permission, copyright (2008) The American Physical Society

pected to be near the $h_{c}(1 P)$ mass, making the hyperfine mass splitting, $\Delta m_{\mathrm{hf}}\left[h_{c}(1 P)\right] \equiv\left\langle m\left(1^{3} P_{J}\right)\right\rangle-m\left[h_{c}(1 P)\right]$, an important measure of the spin-spin interaction. The $h_{c}$-related quantities are summarized in Table 5; mass measurements are consistent. It could be a coincidence [46] that $\Delta m_{\mathrm{hf}}\left[h_{c}(1 P)\right]_{\exp } \approx 0$, the same as the lowest-order perturbative QCD expectation, because the same theoretical assumptions lead to the prediction

$$
\frac{m\left(\chi_{c 1}\right)-m\left(\chi_{c 0}\right)}{m\left(\chi_{c 2}\right)-m\left(\chi_{c 1}\right)}=\frac{5}{2}
$$

whereas measured masses [18] yield a value of 2.1, 20\% smaller than predicted.

\subsubsection{Observation of $\eta_{c}(2 S)$}

The search for a reproducible $\eta_{c}(2 S)$ signal has a long and checkered history. There were hints in early $e^{+} e^{-} \rightarrow c \bar{c}$ data for a purported $\eta_{c}(2 S)$ with mass near $3455 \mathrm{MeV}$ in $\psi(2 S) \rightarrow \gamma \gamma J / \psi$ events [64] and in inclusive radiative $\psi(2 S)$ decays $[65,66]$. A possible signal [67] near $3591 \mathrm{MeV}$ was reported in 1978 in $\psi(2 S) \rightarrow \gamma \gamma J / \psi$. Crystal Ball ruled out that result in 1982 [5] and also reported an $\eta_{c}(2 S)$ signal in inclusive radiative $\psi(2 S)$ decays with a mass of $3592 \pm 5 \mathrm{MeV}$. The latter result persisted, in limbo, unconfirmed and unrefuted, for twenty years, until Belle [49] found a signal in $B \rightarrow K \eta_{c}(2 S)$ in the exclusive $\eta_{c}(2 S) \rightarrow K_{S}^{0} K^{-} \pi^{+}$decay mode (a favorite all-charged final state for $\eta_{c}(1 S)$ ), at $3654 \pm 6 \pm 8 \mathrm{MeV}$. Since then measurements of $\eta_{c}(2 S)$ in that mass region have been reported by BABAR [50] (see Fig. 8), CLEO [51], and Belle [52] in $\gamma \gamma$-fusion to $K \bar{K} \pi$ final states and by BABAR [53] and Belle [54] in double charmonium production. With this plethora of independent measurements
TABLE 5: Width and mass measurements of the $h_{c}(1 P)$. $\left\langle m\left(1^{3} P_{J}\right)\right\rangle$ and $\Delta m_{\mathrm{hf}}$ are defined in the text

\begin{tabular}{ccc}
\hline \hline Quantity & Value $(\mathrm{MeV})$ & Ref. $\left(\chi^{2} /\right.$ d.o.f. $)$ \\
\hline Width & $0.73 \pm 0.45 \pm 0.28$ & BES [47] \\
& $<1.44 @ 90 \% \mathrm{CL}$ & BES [47] \\
\hline Mass & $3525.8 \pm 0.2 \pm 0.2$ & E835 [48] \\
& $3525.28 \pm 0.19 \pm 0.12$ & CLEO [46] \\
& $3525.40 \pm 0.13 \pm 0.18$ & BES [47] \\
& $3525.45 \pm 0.15$ & Avg $^{3}(2.2 / 2)$ \\
\hline$\left\langle m\left(1^{3} P_{J}\right)\right\rangle$ & $3525.30 \pm 0.07$ & PDG08 [18] \\
$\Delta m_{\mathrm{hf}}$ & $-0.15 \pm 0.17$ & \\
\hline \hline
\end{tabular}

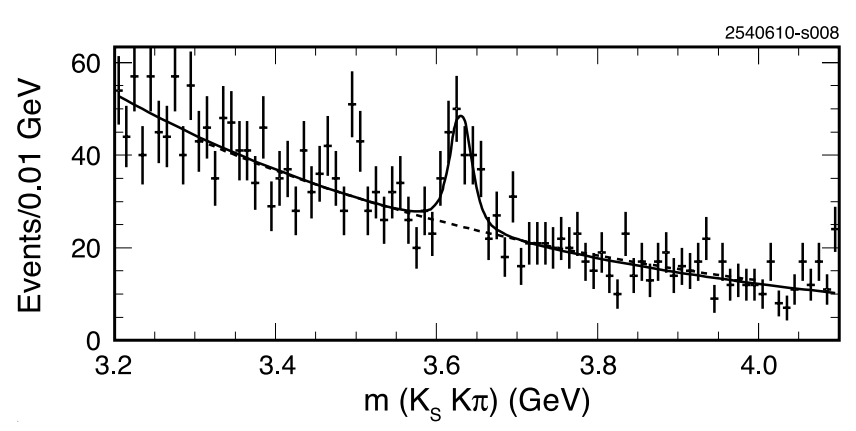

FIG. 8: From BABAR [50], the $K \bar{K} \pi$ invariant mass distribution from selected $e^{+} e^{-} \rightarrow e^{+} e^{-} K_{S}^{0} K^{+} \pi^{-}$events, data (points with error bars) overlaid with a fit (solid line) having two components, a smooth background (dashed line) and an $\eta_{c}(2 S)$ signal. Adapted from [50] with kind permission, copyright (2004) The American Physical Society

in three different production mechanisms and two methods of mass reconstruction (fully reconstructed exclusive decay to $K \bar{K} \pi$ and missing mass), it might have been reasonable to expect clarity and cohesion to have emerged. However, complete experimental unity eludes us because, while the mass values are all in the same vicinity, when averaged they have a PDG $S$-factor of 1.7 , the factor by which the weighted-average uncertainty is inflated. The two most precise measurements, both from $\gamma \gamma \rightarrow \eta_{c}(2 S)$, disagree by $2.5 \sigma$; the two least precise, both by Belle, disagree by $2.2 \sigma$; the two double-charmonium results disagree by $1.6 \sigma$; and the two $B A B A R$ results disagree by $1.4 \sigma$. There are no easily identifiable outliers to discard. The lesson here may be that statistics in all the meth-

\footnotetext{
3 A note concerning tables in this section: where the label "Avg" is attached to a number, it signifies an inverse-square-errorweighted average of values appearing directly above, for which all statistical and systematic errors were combined in quadrature without accounting for any possible correlations between them. The uncertainty on this average is inflated by the multiplicative factor $S$ if $S^{2} \equiv \chi^{2} /$ d.o.f. $>1$
} 
ods utilized are hard to come by, or that background shapes are more complicated than assumed, or that these measurements have been plagued by extraordinary bad luck. In any case, further exploration is clearly merited. CLEO [68] attempted to find exclusive $\eta_{c}(2 S)$ decays in radiative $\psi(2 S)$ decays, guided by the success of such methods for $\eta_{c}(1 S)$ [69], but found no clear signals in its sample of $25 \mathrm{M} \psi(2 S)$.

Just prior to submission of this article, Belle [70] announced a preliminary observation of $\eta_{c}(2 S)$, produced in two-photon fusion, in three new decay modes $\left(3\left(\pi^{+} \pi^{-}\right)\right.$, $K^{+} K^{-} 2\left(\pi^{+} \pi^{-}\right)$, and $\left.K_{S}^{0} K^{-} \pi^{+} \pi^{-} \pi^{+}\right)$. These modes will offer more concrete avenues of approach to $\eta_{c}(2 S)$ in order to better measure its properties.

\subsubsection{Observation of $\chi_{c 2}(2 P)$}

In 2005 Belle [55] observed an enhancement in the $D \bar{D}$ mass spectrum from $e^{+} e^{-} \rightarrow e^{+} e^{-} D \bar{D}$ events with a statistical significance of $5.3 \sigma$. Properties are shown in Table 6 . It was initially dubbed the $Z(3930)$, but since has been widely ${ }^{4}$ (if not universally) accepted as the $\chi_{c 2}(2 P)$. The analysis selects fully reconstructed $D$ meson pairs with at most one $\pi^{0}$ and at most six pions/kaons per event, using the decays $D^{0} \rightarrow K^{-} \pi^{+}, K^{-} \pi^{+} \pi^{0}$, and $K^{-} \pi^{+} \pi^{+} \pi^{-}$, and $D^{+} \rightarrow K^{-} \pi^{+} \pi^{+}$. The outgoing $e^{+} e^{-}$were presumed to exit the detector at small angles. This $\gamma \gamma$-fusion signature was enforced by requiring small transverse momentum with respect to the beam direction in the $e^{+} e^{-}$center-of-mass frame and restricting the $D \bar{D}$ longitudinal momentum to kinematically exclude $e^{+} e^{-} \rightarrow \gamma D \bar{D}$. Figure 9 shows the resulting $D \bar{D}$ mass and angular distributions; the latter are consistent with

TABLE 6: Properties of the $\chi_{c 2}(2 P)$ (originally $Z(3930)$ )

\begin{tabular}{ccc}
\hline \hline Quantity & Value & Ref. $\left(\chi^{2} /\right.$ d.o.f. $)$ \\
\hline Mass $(\mathrm{MeV})$ & $3929 \pm 5 \pm 2$ & Belle [55] \\
& $3926.7 \pm 2.7 \pm 1.1$ & BABAR [56] \\
& $3927.2 \pm 2.6$ & $\operatorname{Avg}^{3}(0.14 / 1)$ \\
\hline Width $(\mathrm{MeV})$ & $29 \pm 10 \pm 2$ & Belle [55] \\
& $21.3 \pm 6.8 \pm 3.6$ & BABAR [56] \\
& $24.1 \pm 6.1$ & $\operatorname{Avg}^{3}(0.37 / 1)$ \\
\hline$\Gamma_{\gamma \gamma} \times \mathcal{B}(D \bar{D})$ & $0.18 \pm 0.05 \pm 0.03$ & Belle [55] \\
$(\mathrm{keV})$ & $0.24 \pm 0.05 \pm 0.04$ & $B A B A R[56]$ \\
& $0.21 \pm 0.04$ & $\operatorname{Avg}^{3}(0.46 / 1)$ \\
\hline \hline
\end{tabular}

${ }^{4}$ Lattice calculations [71] suggest that the $\chi_{c 2}(2 P)$ (i.e., the $2{ }^{3} P_{2}$ $c \bar{c}$ state) and the $1^{3} F_{2}$ state could be quite close in mass, so that perhaps the $Z(3930)$ is not the $2^{3} P_{2}$ but rather the $1^{3} F_{2}$.
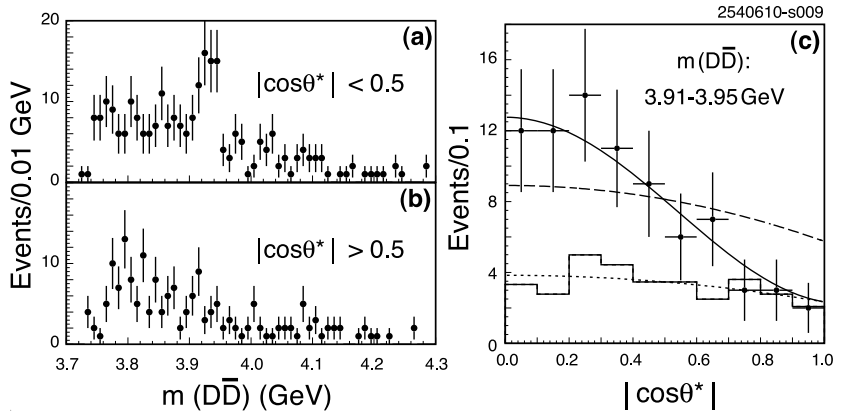

FIG. 9: From the Belle [55] observation of $\chi_{c 2}(2 P) \rightarrow D \bar{D}$. (a) and (b) $m(D \bar{D})$ distributions from selected $e^{+} e^{-} \rightarrow$ $e^{+} e^{-} D \bar{D}$ events, for the $\left|\cos \theta^{*}\right|$ regions indicated, where $\theta^{*}$ is the polar angle of a $D$ momentum vector in the $\gamma \gamma$ centerof-mass frame. Part (c) shows the corresponding $\left|\cos \theta^{*}\right|$ distributions for the $m(D \bar{D})$ region indicated, from data (points with error bars) and background (solid line histogram). Also shown are expected distributions for the spin-2 (helicity-2) (solid curve) and spin-zero (dashed curve) hypotheses, both of which include background (dotted curve). Adapted from [55] with kind permission, copyright (2006) The American Physical Society

the spin-2, helicity-2 hypothesis but disagree with spin- 0 . $B A B A R$ [56] confirmed the Belle observation in $\gamma \gamma$-fusion with significance of $5.8 \sigma$ and found properties consistent with those from Belle.

\subsubsection{Observation of $B_{c}^{+}$}

Unique among mesons is the $B_{c}^{+}$because it is the lowest-lying (and only observed) meson composed of a heavy quark and a heavy antiquark of different flavors. As such, its mass, lifetime, decay, and production mechanisms garner attention so as to constrain and cross-check QCD calculations similar to those used for other heavy quarkonia. Since its mass is well above $6 \mathrm{GeV}$, production of pairs $B_{c}^{+} B_{c}^{-}$at the $e^{+} e^{-} B$-factories, which take most of their data near the $\Upsilon(4 S)$, has not been possible. Although a hint from OPAL [72] at LEP and then suggestive evidence from CDF [73] for the existence of $B_{c}^{+}$were published in 1998 , it was not until a decade later that two confirming observations in excess of $5 \sigma$ significance were made in Run II at the Tevatron. Both mass measurements used the decay chain $B_{c}^{+} \rightarrow J / \psi \pi^{+}$, $J / \psi \rightarrow \mu^{+} \mu^{-}$, and obtain for mass and statistical significance the values

$$
\begin{aligned}
& m\left(B_{c}^{+}\right)=6275.6 \pm 2.9 \pm 2.5 \mathrm{MeV} \quad(8 \sigma) \mathrm{CDF}[57] \\
& =6300 \pm 14 \pm 5 \mathrm{MeV} \quad(5.2 \sigma) \mathrm{D} \varnothing[58] \text {. }
\end{aligned}
$$

The CDF $B_{c}^{+}$mass plot is shown in Fig. 10. Their weighted average (Table 4 ) is about $2 \sigma$ lower than the lattice QCD prediction [74] of $6304 \pm 12_{-0}^{+18} \mathrm{MeV}$. The only observed decay modes for $B_{c}^{+}$are $J / \psi \pi^{+}$and $J / \psi \ell^{+} \nu_{\ell}$. The semileptonic mode has been used by both 


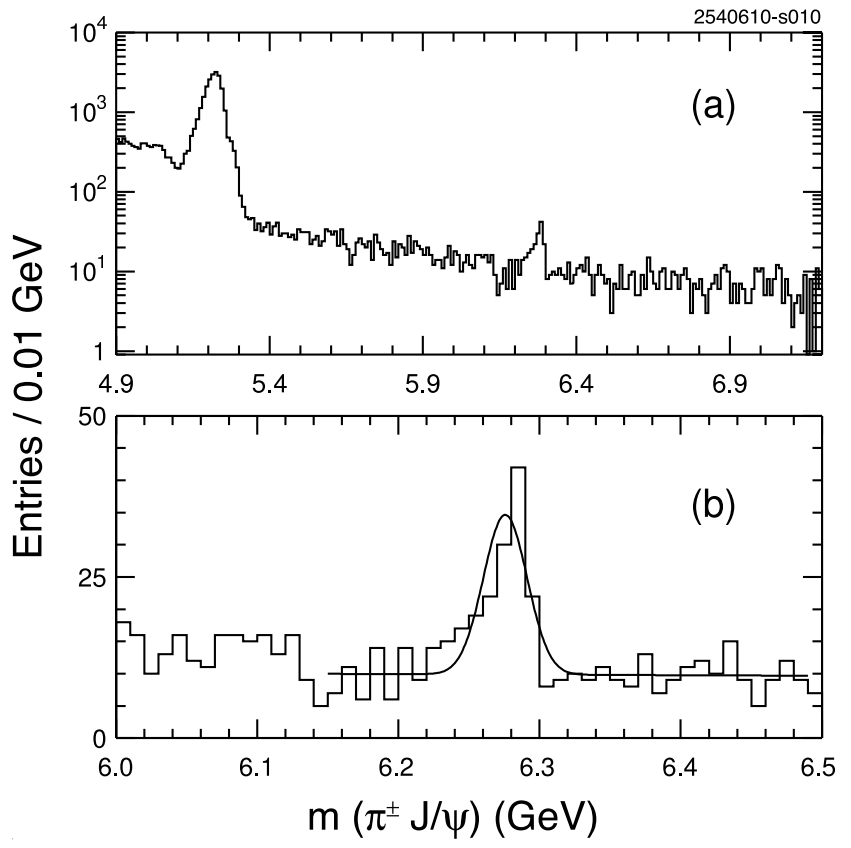

FIG. 10: From CDF [57], (a) $J / \psi \pi^{+}$invariant mass combinations from selected $\bar{p} p \rightarrow \pi^{+} J / \psi X$ events. The bump near $5.2 \mathrm{GeV}$ is due to $B^{+} \rightarrow K^{+} J / \psi$ decays with a pion mass assignment for the kaon. (b) As in (a), but zoomed in on the 6.0-6.5 GeV mass region; the solid curve indicates the projection of the $B_{c}^{+}$maximum likelihood fit to the data. Adapted from [57] with kind permission, copyright (2008) The American Physical Society

CDF $[75,76]$ and $\mathrm{D} \varnothing[77]$ to measure the $B_{c}^{+}$lifetime. Their results are consistent with each other and have a weighted average [76] of $0.46 \pm 0.04$ ps. (See also Sect. 4.7 for discussion of $B_{c}^{+}$).

\subsubsection{Observation of $\eta_{b}(1 S)$}

Nonobservation of the bottomonium ground state was an annoying thorn in the side of heavy quarkonium spectroscopy until 2008, when BABAR [59, 61] succeeded in observing the $\eta_{b}(1 S)$ where previous efforts had failed. The hyperfine mass-splitting of singlet-triplet states, $\Delta m_{\mathrm{hf}} \equiv m\left(1^{3} S_{1}\right)-m\left(1^{1} S_{0}\right)$, probes the spin-dependence of bound-state energy levels, and, once measured, imposes constraints on theoretical descriptions. The $\eta_{b}$ remained elusive for a variety of reasons. Branching fractions for transitions from the $\Upsilon(n S)$ states are small and no low-multiplicity, high-rate "golden" decay modes analogous to $\eta_{c}(1 S) \rightarrow K \bar{K} \pi$ appear to exist for $\eta_{b}$. This left inclusive $\Upsilon(n S) \rightarrow \gamma \eta_{b}$ as the first line of attack.

$B A B A R$ 's success was mainly due to large data samples obtained just prior to shutdown of the experiment. For the express objective of $\eta_{b}$-discovery (among others), BABAR accumulated $122 \mathrm{M} \Upsilon(3 S)$ and $100 \mathrm{M} \Upsilon(2 S)$ decays, compared to CLEO $(9 \mathrm{M} \Upsilon(2 S)$ and $6 \mathrm{M} \Upsilon(3 S))$ and Belle $(11 \mathrm{M} \Upsilon(3 S))$. Even with such large data samples and a high-performance cesium iodide crystal calorimeter, BABAR's task was far from trivial: the expected photon line was buried under a sea of $\pi^{0}$-decay photons even after all photon candidates that combine with any other photon to form a $\pi^{0}$ were vetoed. The $\eta_{b}$ photon line was also obscured by two other physics processes, each inducing structure in $E_{\gamma}$, the photon energy in the $\Upsilon(n S)$ rest frame. The $\eta_{b}$ photon line lies in the high energy tail of the three Doppler-smeared and merged $\chi_{b J}(n P) \rightarrow \gamma \Upsilon(1 S)$ peaks and adjacent to that of the radiative return process, $e^{+} e^{-} \rightarrow \gamma \Upsilon(1 S)$. BABAR introduced a method to suppress nonresonant "continuum" photons and thereby enhance experimental signalsquared-to-background ratio $\left(S^{2} / B\right)$, noting that such backgrounds tend to follow initial parton (jet) directions, whereas the $\eta_{b}$ decay products will have direction uncorrelated with that of the transition photon. The angle $\theta_{T}$ was defined to be the angle between each transition photon candidate and the thrust axis [78] of the rest of the event. (The thrust axis is the direction that maximizes the sum of absolute values of momenta projected upon it, and, on a statistical basis, follows the axis of two-jet events.) The thrust angle associated with each candidate radiative photon was calculated and required to satisfy $\left|\cos \theta_{T}\right|<0.7$, the criterion found by $B A B A R$ to maximize $S^{2} / B$. The analysis extracted a signal by fitting the $E_{\gamma}$ distribution to four components: an empirically determined smooth background, merged $\chi_{b J}$ peaks, a monochromatic ISR photon line, and an $\eta_{b}$ signal. The resulting $E_{\gamma}$ spectrum from the BABAR [59] $\Upsilon(3 S)$ analysis is shown in Fig. 11, with an $\eta_{b}$ signal of significance of $>10 \sigma$. A few months after this discovery, BABAR [61] announced confirmation of their signal with a nearly identical analysis of their $\Upsilon(2 S)$ data, albeit with smaller signficance $(3.0 \sigma)$. To avoid bias, these analyses established procedures while "blind" to the $\eta_{b}$ signal region in $E_{\gamma}$.

Initially, there was some worry that the BABAR results were in mild conflict with earlier nonobservation upper limits from a CLEO [79] analysis, which had as its primary focus a detailed study of the dipole transitions $\Upsilon(n S) \rightarrow \gamma \chi_{b J}(m P)$. However, CLEO [60] later corrected errors and omissions in that analysis and announced new results consistent with but less precise than $B A B A R$ 's, including $4 \sigma$ evidence for $\Upsilon(3 S) \rightarrow \gamma \eta_{b}$ and a larger upper limit on $\mathcal{B}\left(\Upsilon(2 S) \rightarrow \gamma \eta_{b}\right)$. In addition to including the initially omitted ISR peak in the fit to $E_{\gamma}$ and assuming a more reasonable width, $\Gamma\left(\eta_{b}\right)=10 \mathrm{MeV}$, for the signal, CLEO exploited an $E_{\gamma}$ resolution slightly better than $B A B A R$, parametrized the observed photon lineshape more accurately than before, and added a new twist to the $B A B A R$-inspired thrust-angle restriction. Instead of simply rejecting a high-background region of thrust angle, CLEO accumulated three $E_{\gamma}$ distributions, one for each of three $\left|\cos \theta_{T}\right|$ bins, the middle one being $0.3<\left|\cos \theta_{T}\right|<0.7$. Hence no statistical power was wasted by throwing any data away, and an improved $S^{2} / B$ in the combined $\left|\cos \theta_{T}\right|<0.3$ and $0.3-0.7$ bins 


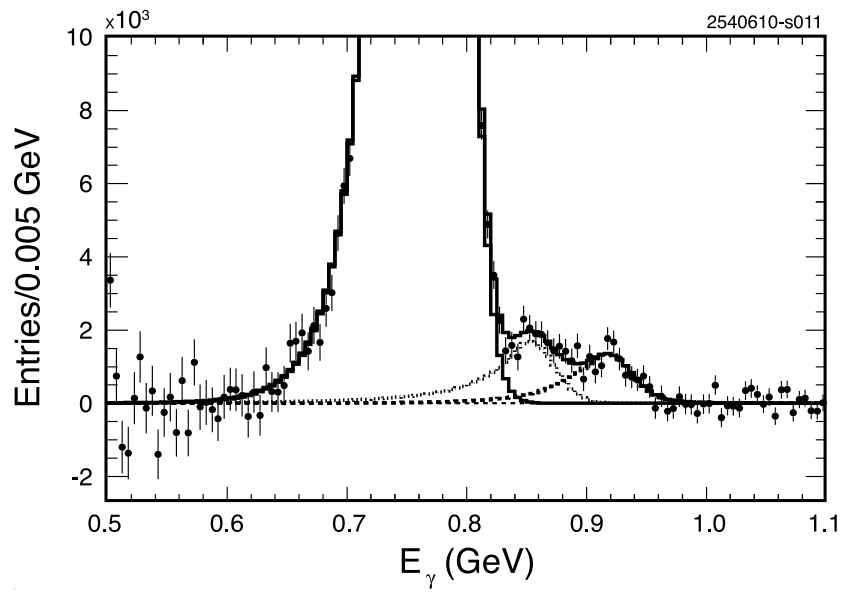

FIG. 11: From BABAR [59], the inclusive photon energy spectrum in the $e^{+} e^{-}$center-of-mass frame $\Upsilon(3 S)$ data after subtraction of the smooth background. The solid curve shows the best fit, and the peaks correspond to, from left to right, $\chi_{b 1,2} \rightarrow \gamma \Upsilon(1 S)$, ISR production of $\Upsilon(1 S)$, and $\Upsilon(3 S) \rightarrow \gamma \eta_{b}$. Adapted from [59] with kind permission, copyright (2008) The American Physical Society

relative to $<0.7$ was exploited. A $B A B A R$-like fit to the measured $E_{\gamma}$ distributions in all three $\left|\cos \theta_{T}\right|$ bins simultaneously extracted the $\eta_{b}$ signal. CLEO left the photon selection criteria from the original analysis unchanged and quoted a final mass, rate, and significance which were each the mean from an ensemble of fits with reasonable confidence levels, not on any arbitrarily chosen individual fit. The fit ensemble contained many variations, each specifying a different background parametrization, $E_{\gamma}$ range, and/or logarithmic or linear $E_{\gamma}$ scale.

Tables 4 and 7 summarize the experimental $\eta_{b}$ results, which together yield

$$
\Delta m_{\mathrm{hf}}\left[\eta_{b}(1 S)\right]_{\exp }=69.6 \pm 2.9 \mathrm{MeV} .
$$

Belle is poised to search for $\eta_{b}$ using its $11 \mathrm{M} \Upsilon(3 S)$ events and recently augmented $160 \mathrm{M} \Upsilon(2 S)$ dataset.

Theoretical predictions for $\eta_{b}(1 S)$ hyperfine splitting are discussed Sects. 2.5.3, 2.6.2, 2.7, 2.8.1, and 2.10.1.

\subsubsection{Search for $h_{b}(1 P)$}

A preliminary analysis from $B A B A R$ [80] describes two searches for $h_{b}(1 P)$ in a sample of $122 \mathrm{M} \Upsilon(3 S)$ decays. The first search employs a method similar to the CLEO $h_{c}(1 P)$ inclusive search (see Sect. 2.2.1) by selecting $\Upsilon(3 S)$ decays with both a soft $\pi^{0}$ and a radiative photon, looking for the decay chain $\Upsilon(3 S) \rightarrow \pi^{0} h_{b}(1 P)$, $h_{b}(1 P) \rightarrow \gamma \eta_{b}(1 S)$. With the radiative photon restricted to the range allowed for the transition to $\eta_{b}(1 S)$, the mass recoiling against the soft $\pi^{0}$ is plotted and scanned for a peak above a smooth background. BABAR sees a $2.7 \sigma$ effect at $m\left(\pi^{0}-\right.$ recoil $)=9903 \pm 4 \pm 1 \mathrm{MeV}$. In a second
TABLE 7: Measured $\eta_{b}(1 S)$ properties. The value quoted for the weighted average of $\Delta m_{\mathrm{hf}}$ includes all three measurements

\begin{tabular}{cccc}
\hline \hline Quantity & $\Upsilon(2 S) \rightarrow \gamma \eta_{b}$ & $\Upsilon(3 S) \rightarrow \gamma \eta_{b}$ & Ref. ( $\chi^{2} /$ d.o.f. $)$ \\
\hline$E_{\gamma}$ & $610.5_{-4.3}^{+4.5} \pm 1.8$ & $921.2_{-2.8}^{+2.1} \pm 2.4$ & BABAR [59,61] \\
$(\mathrm{MeV})$ & - & $918.6 \pm 6.0 \pm 1.8$ & CLEO [60] \\
\hline$m\left(\eta_{b}\right)$ & $9392.9_{-4.8}^{+4.6} \pm 1.8$ & $9388.9_{-2.3}^{+3.1} \pm 2.7$ & BABAR [59,61] \\
$(\mathrm{MeV})$ & - & $9391.8 \pm 6.6 \pm 2.0$ & CLEO [60] \\
\hline$\Delta m_{\mathrm{hf}}$ & $67.4_{-4.5}^{+4.8} \pm 1.9$ & $71.4_{-3.1}^{+2.3} \pm 2.7$ & BABAR [59,61] \\
$(\mathrm{MeV})$ & - & $68.5 \pm 6.6 \pm 2.0$ & CLEO [60] \\
& & $69.6 \pm 2.9$ & $\operatorname{Avg}(0.6 / 2)$ \\
\hline $\mathcal{B} \times 10^{4}$ & $4.2_{-1.0}^{+1.1} \pm 0.9$ & $4.8 \pm 0.5 \pm 1.2$ & BABAR [59,61] \\
& $<8.4$ & $7.1 \pm 1.8 \pm 1.1$ & CLEO [60] \\
& $<5.1$ & $<4.3$ & CLEO [79] \\
\hline \hline
\end{tabular}

search, an upper limit of

$$
\begin{array}{r}
\mathcal{B}\left(\Upsilon(3 S) \rightarrow \pi^{+} \pi^{-} h_{b}(1 P)\right)<2.5 \times 10^{-4} \\
\text { for } 9.88<m\left(h_{b}(1 P)\right)<9.92 \mathrm{GeV} \text { at } 90 \% \mathrm{CL}
\end{array}
$$

is set.

\subsubsection{Observation of $\Upsilon\left(1^{3} D_{2}\right)$}

CLEO [62] made the first of two observations of $\Upsilon(1 D)$, using the four-photon cascade shown in Fig. 12:

$$
\begin{aligned}
& \Upsilon(3 S) \rightarrow \gamma \chi_{b J}(2 P), \\
& \chi_{b J}(2 P) \rightarrow \gamma \Upsilon(1 D), \\
& \Upsilon(1 D) \rightarrow \gamma \chi_{b J}(1 P), \\
& \chi_{b J}(1 P) \rightarrow \gamma \Upsilon(1 S), \\
& \Upsilon(1 S) \rightarrow \ell^{+} \ell^{-},
\end{aligned}
$$

where $\ell^{ \pm} \equiv e^{ \pm}$or $\mu^{ \pm}$. The largest background source of four soft photons and an $\Upsilon(1 S)$ is $\Upsilon(3 S) \rightarrow \pi^{0} \pi^{0} \Upsilon(1 S)$, which was suppressed by vetoing events with two photonpairings that are both consistent with $\pi^{0}$ masses. The next-most pernicious background is the quite similar four-photon cascade through $\Upsilon(2 S)$ instead of $\Upsilon(1 D)$ (also shown in Fig. 12); the softer two photons overlap the signal photons within the experimental resolution. This latter background was suppressed by kinematically constraining each event to a $\Upsilon(1 D)$ hypothesis with unknown $\Upsilon(1 D)$ mass and including all $J$ possibilities for the intermediate $\chi_{b J}(n P)$ states, and then requiring a good fit quality, $\chi^{2}(1 D)$. Masses from the surviving candidates are shown in Fig. 13, with the mass recoiling against the softest two photons in (a) and the value obtained from the minimum $\chi^{2}(1 D)$ combination in (b). Both give consistent masses for an $\Upsilon\left(1^{3} D_{2}\right)$, and the latter has an inconclusive $1.9 \sigma$ hint of a second peak 


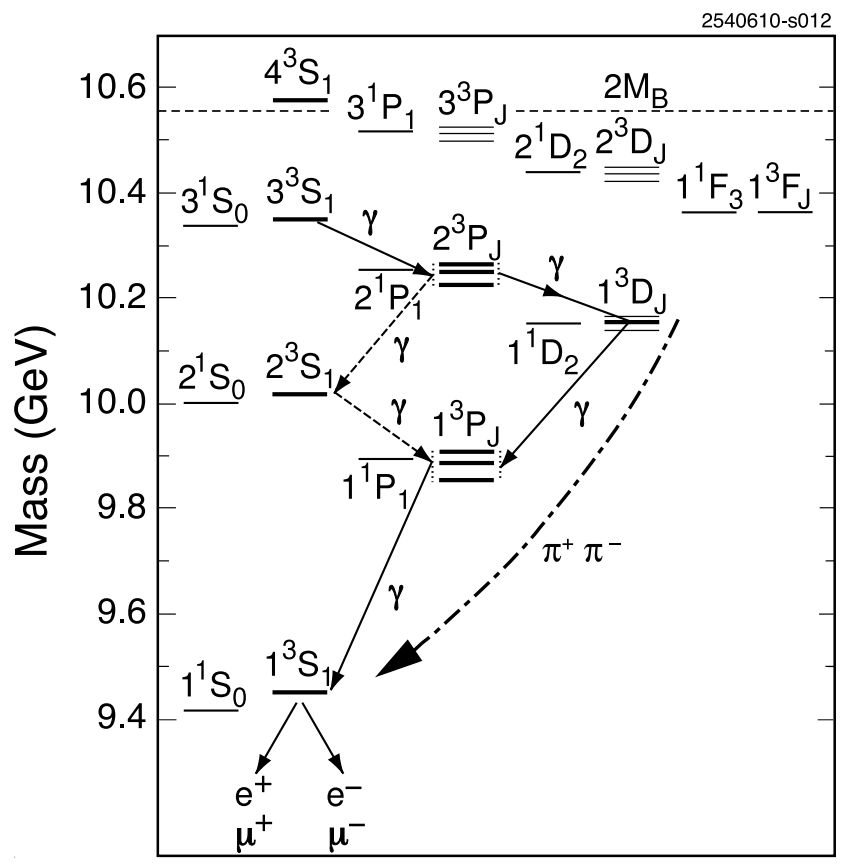

FIG. 12: Expected $b \bar{b}$ bound-state mass levels. The fourphoton transition sequence from the $\Upsilon(3 S)$ to the $\Upsilon(1 S)$ via the $\Upsilon(1 D)$ states is shown (solid lines). An alternative route for the four-photon cascade via the $\Upsilon(2 S)$ state is also displayed (dashed lines). The hadronic dipion transition from $\Upsilon(1 D)$ to $\Upsilon(1 S)$ is indicated by the dot-dash curve. Adapted from [62] with kind permission, copyright (2004) The American Physical Society

$13 \mathrm{MeV}$ above the primary one, which could be an indication of the corresponding $\Upsilon\left(1^{3} D_{3}\right)$ state. The observed $34.5 \pm 6.4$ signal events in the central peak correspond to a statistical significance of $10 \sigma$, most of which are attributed to cascades involving the $\chi_{b 1}(n P)$ for both $n=1$ and 2 and to production of an $\Upsilon\left(1^{3} D_{2}\right)$. The product branching fraction for the entire cascade was found to be $(2.5 \pm 0.7) \times 10^{-5}$. Upper limits on other possible decays relative to the four-photon cascade were also set to be $<0.25$ for $\Upsilon(1 D) \rightarrow \eta \Upsilon(1 S)$ and $<1.2$ for $\pi^{+} \pi^{-} \Upsilon(1 D)$, both at $90 \%$ CL.

Belle has an $\Upsilon(3 S)$ dataset slightly larger than CLEO and therefore could mount a comparable $\Upsilon(1 D)$ search. $B A B A R$ has twenty times more $\Upsilon(3 S)$ than CLEO, and therefore has the capability to search for other decay chains and to explore hyperfine mass structure of the allowed $\Upsilon(1 D)$ spin states. While neither Belle nor BABAR has yet explored the four-photon cascade, $B A B A R$ has observed [63] $\Upsilon\left(1^{3} D_{2}\right)$ produced from a two-photon cascade from $\Upsilon(3 S)$ decay as does CLEO, but then undergoing a charged dipion transition to the $\Upsilon(1 S)$, which then decays to $\ell^{+} \ell^{-}$. The $\pi^{+} \pi^{-} \ell^{+} \ell^{-}$invariant mass distribution from such events, restricted to those with dilepton masses near that of the $\Upsilon(1 S)$, is shown in Fig. 14. In addition to confirming the $\Upsilon\left(1^{3} D_{2}\right)$ signal at $5.8 \sigma$, the $\Upsilon\left(1^{3} D_{1}\right)$ and $\Upsilon\left(1^{3} D_{3}\right)$ states are seen at $1.8 \sigma$ and $1.6 \sigma$, respectively. The $\Upsilon\left(1^{3} D_{2}\right)$ mass is somewhat larger than but

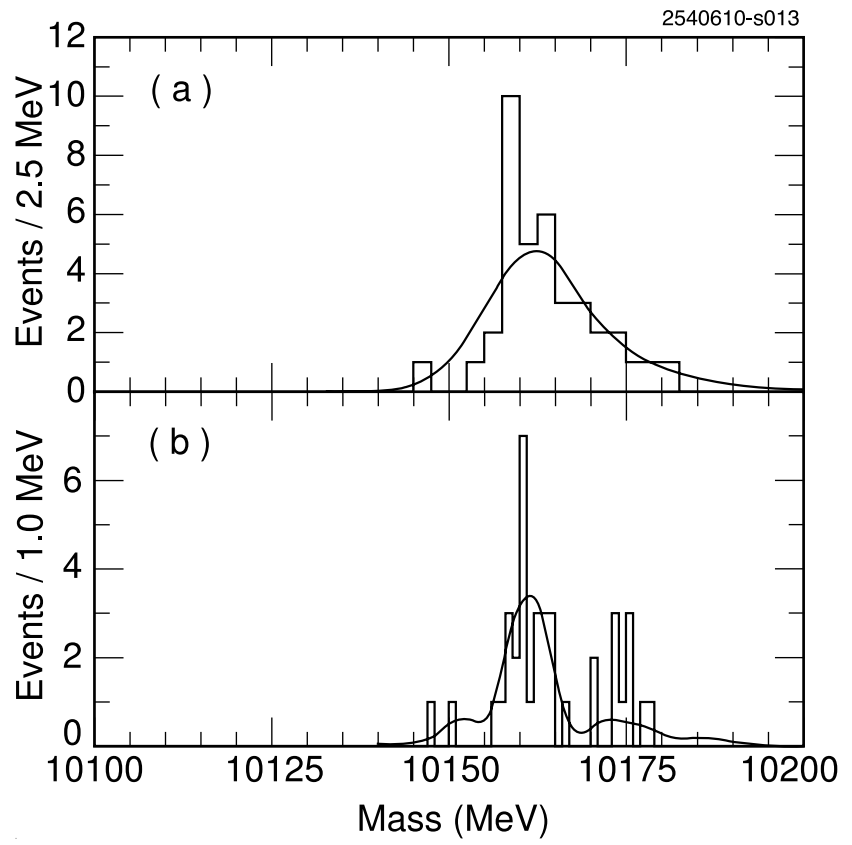

FIG. 13: From CLEO [62], distributions of (a) mass recoiling against the softest two photons, and (b) mass that produces the smallest $\chi^{2}(1 D)$ (see text) per event, from $\Upsilon(3 S) \rightarrow \gamma \gamma \gamma \gamma \ell^{+} \ell^{-}$events, selected to be consistent with a four-photon cascade through $\chi_{b J}(2 P), \Upsilon(1 D)$, and $\chi_{b J}(1 P)$ to $\Upsilon(1 S)$. The solid line histogram represents data, and the curves represent the CLEO fits. Adapted from [62] with kind permission, copyright (2004) The American Physical Society

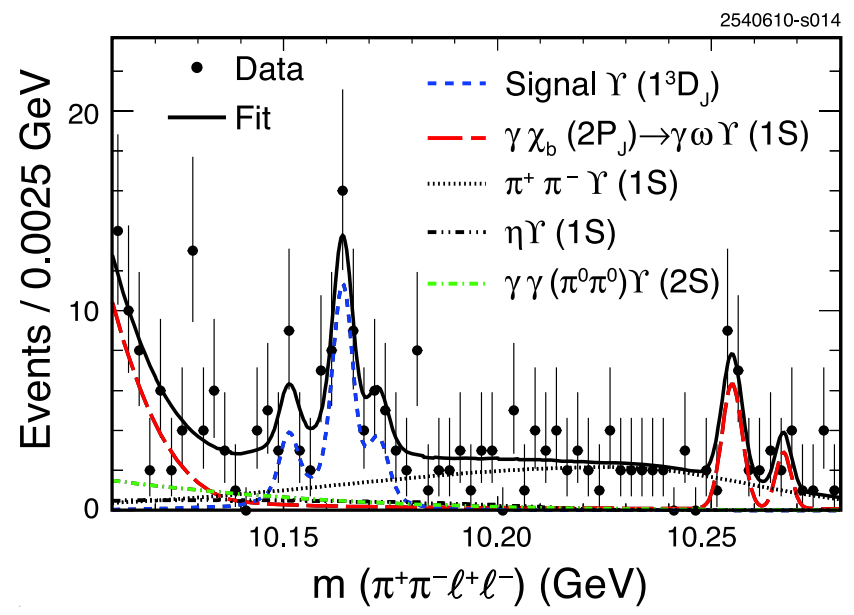

FIG. 14: From BABAR [63], the $\pi^{+} \pi^{-} \ell^{+} \ell^{-}$invariant mass restricted to those with dilepton masses near that of the $\Upsilon(1 S)$. Curves represent $\Upsilon\left(1^{3} D_{J}\right)$ signals only (short dash), the total fit (solid) which includes four backgrounds it (others). Adapted from [63] with kind permission, copyright (2010) The American Physical Society

consistent with the CLEO value, as shown in Table 8 . The BABAR analysis also concludes first, that the dipion invariant mass distribution is in substantially better agreement with that predicted for a $\Upsilon\left(1^{3} D_{J}\right)$ than an $S$ 
TABLE 8: Measured mass values of the $\Upsilon\left(1^{3} D_{2}\right)$

\begin{tabular}{ccc}
\hline \hline Decay & Value (MeV) & Ref. ( $\chi^{2} /$ d.o.f.) \\
\hline$\Upsilon(3 S) \rightarrow \gamma \gamma \gamma \gamma \Upsilon(1 S)$ & $10161.1 \pm 0.6 \pm 1.6$ & CLEO [62] \\
$\Upsilon(3 S) \rightarrow \gamma \gamma \pi^{+} \pi^{-} \Upsilon(1 S)$ & $10164.5 \pm 0.8 \pm 0.5$ & $B A B A R[63]$ \\
Both of above & $10163.8 \pm 1.4$ & $\operatorname{Avg}^{3}(3.1 / 1)$ \\
\hline \hline
\end{tabular}

or $P$ state, and second, that angular distributions of the $\Upsilon\left(1^{3} D_{2}\right)$ signal events are consistent with the quantum number assignments of $J=2$ and $P=-1$.

\subsection{New unanticipated states}

\subsection{1. $X(3872)$, the enduring exotic}

The $X(3872)$ occupies a unique niche in the menagerie of unanticipated states listed in Table 9 as both the first and the most intriguing. At this point it is widely studied, yet its interpretation demands much more experimental attention. Its apparent quantum numbers, mass, and decay patterns make it an unlikely conventional charmonium candidate, and no consensus explanation has been found.

In 2003, while studying $B^{+} \rightarrow K^{+} \pi^{+} \pi^{-} J / \psi$, Belle [85] discovered an unexpected enhancement in the $\pi^{+} \pi^{-} J / \psi$ invariant mass spectrum near $3872 \mathrm{MeV}$. This sighting of the $X$ in $B$-decays was later confirmed by BABAR [87]. The $X \rightarrow \pi^{+} \pi^{-} J / \psi$ decay was also observed inclusively in prompt production from $\bar{p} p$ collisions at the Tevatron by both CDF [88-90] and DØ [91]. CDF [89] studied the angular distributions and correlations of the $\pi^{+} \pi^{-} J / \psi$ final state, finding that the dipion was favored to originate as a $\rho^{0}$, and that only $J^{P C}$ assignments of $1^{++}$and $2^{-+}$ explained their measurements adequately. Belle [92] reported evidence for the $\gamma J / \psi$ decay, which $B A B A R[97,98]$ confirmed at $4 \sigma$ significance. The radiative decay verifies the positive $C$-parity assignment of CDF. It also bolsters the $1^{++}$assignment because a $2^{-+}$state would have to undergo a high-order multipole transition which would be more strongly suppressed than the observed rates allow.

From the beginning, the proximity of the $X$ mass to $D^{* 0} \bar{D}^{0}$ threshold was conspicuous, and eventually decays to $D^{* 0} \bar{D}^{0}$ were observed by BABAR [96] and Belle [95]. Interest in the relationship of $X$ to $D^{* 0} \bar{D}^{0}$ fueled improvements in measurements of its mass, as shown in Table 10, and of the $D^{0}$ mass, as shown in Table 11. The $X$ mass measurements based upon the $\pi^{+} \pi^{-} J / \psi$ decay are consistent with one another. The world-average $X$ mass, restricted to measurements using $\pi^{+} \pi^{-} J / \psi$ decays, is dominated by the CDF [90] inclusive result, illustrated in Fig. 15. The CDF systematic uncertainty on the mass was obtained from studies of $\psi(2 S) \rightarrow \pi^{+} \pi^{-} J / \psi$ decays, which have a similar topology and a well-known $\psi(2 S)$

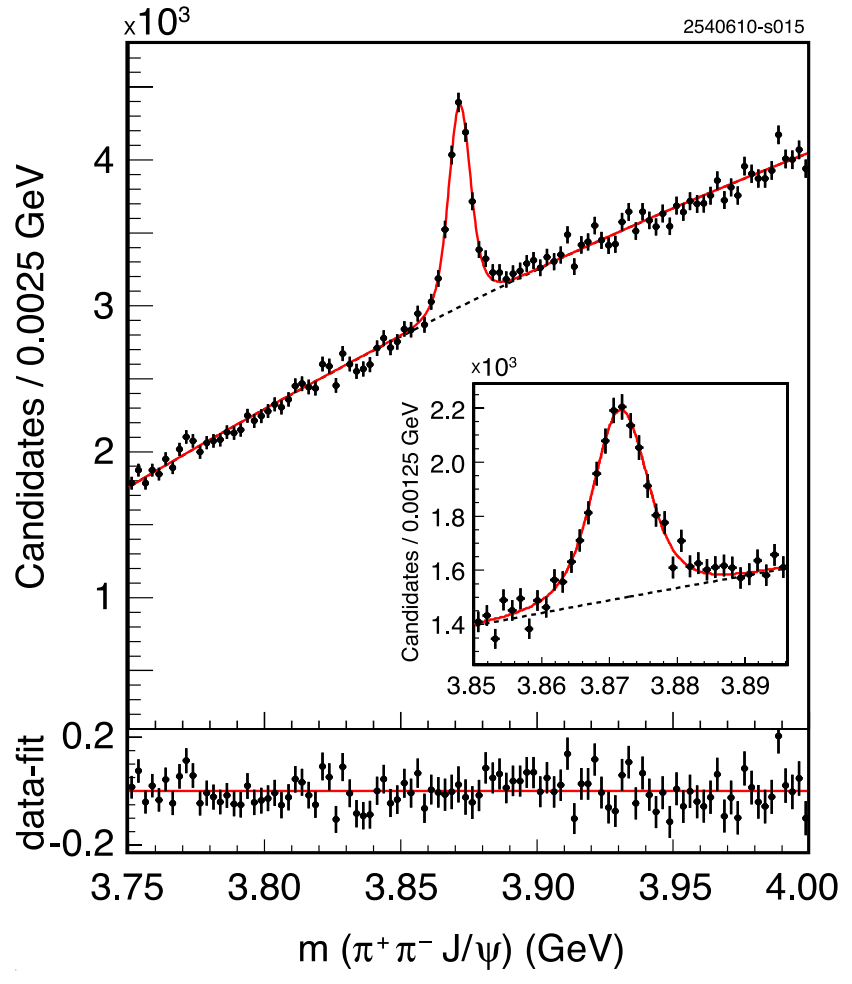

FIG. 15: From CDF [90], the $\pi^{+} \pi^{-} J / \psi$ invariant mass distribution for $X(3872)$ candidates, showing the data (points), the projection of the unbinned maximum-likelihood fit (solid line) and its smooth background component (dashed line), and the inset which enlarges the peak region with finer binning. The lower panel shows residuals of the data with respect to the fit. Adapted from [90] with kind permission, copyright (2009) The American Physical Society

mass to match. The measured mass discrepancy was extrapolated from the $\psi(2 S)$ mass to the $X$ mass to obtain error estimates. The world-average $D^{0}$ mass precision is dominated by a CLEO [119] measurement that uses the decay chain $D^{0} \rightarrow \phi K_{S}^{0}, \phi \rightarrow K^{+} K^{-}, K_{S}^{0} \rightarrow \pi^{+} \pi^{-}$, and is limited by statistics. Despite all these advances, the $D^{* 0} \bar{D}^{0}$ mass threshold test remains ambiguous, with $m[X(3872)]-\left[m\left(D^{* 0}\right)+m\left(D^{0}\right)\right]=-0.42 \pm 0.39 \mathrm{MeV}$. This limits the hypothetical $D^{* 0} \bar{D}^{0}$ binding energy to be $<0.92 \mathrm{MeV}$ at $90 \% \mathrm{CL}$ and does not foreclose the possibility that the $X(3872)$ is above $D^{* 0} \bar{D}^{0}$ threshold. Further clarity here would require much more precise mass measurements for both the $X$ and the $D^{0}$.

Both Belle and BABAR have reported $X(3872)$ signals in the $D^{* 0} \bar{D}^{0}$ final state with branching fractions about ten times higher than for $\pi^{+} \pi^{-} J / \psi$. Both used $D^{* 0} \rightarrow D^{0} \pi^{0}$ and $D^{0} \gamma$ decays, both selected and kinematically constrained a $D^{* 0}$ candidate in each event, and both performed unbinned maximum likelihood fits to the $D^{* 0} D^{0}$ mass. (Belle's fit is two-dimensional, the second dimension being a $B$-meson-consistency kinematic variable; $B A B A R$ cuts on $B$-meson consistency.) Both results appear in Table 10. (An earlier Belle publication [94] used a dataset smaller by one-third than in [95], made 
TABLE 9: As in Table 4, but for new unconventional states in the $c \bar{c}$ and $b \bar{b}$ regions, ordered by mass. For $X(3872)$, the values given are based only upon decays to $\pi^{+} \pi^{-} J / \psi . X(3945)$ and $Y(3940)$ have been subsumed under $X(3915)$ due to compatible properties. The state known as $Z(3930)$ appears as the $\chi_{c 2}(2 P)$ in Table 4. See also the reviews in [81-84]

\begin{tabular}{|c|c|c|c|c|c|c|c|}
\hline State & $m(\mathrm{MeV})$ & 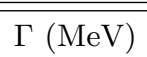 & $\overline{J^{P C}}$ & Process (mode) & Experiment $(\# \sigma)$ & Year & Status \\
\hline$X(3872)$ & $3871.52 \pm 0.20$ & $\begin{array}{l}1.3 \pm 0.6 \\
(<2.2)\end{array}$ & $1^{++} / 2^{-+}$ & $\begin{array}{l}B \rightarrow K\left(\pi^{+} \pi^{-} J / \psi\right) \\
p \bar{p} \rightarrow\left(\pi^{+} \pi^{-} J / \psi\right)+\ldots \\
B \rightarrow K(\omega J / \psi) \\
B \rightarrow K\left(D^{* 0} \bar{D}^{0}\right) \\
B \rightarrow K(\gamma J / \psi) \\
B \rightarrow K(\gamma \psi(2 S))\end{array}$ & $\begin{array}{l}\text { Belle [85, 86] (12.8), BABAR [87] (8.6) } \\
\text { CDF [88-90] (np), DØ [91] (5.2) } \\
\text { Belle [92] (4.3), BABAR [93] (4.0) } \\
\text { Belle [94, 95] (6.4), BABAR [96] (4.9) } \\
\text { Belle [92] (4.0), BABAR [97, 98] (3.6) } \\
\text { BABAR [98] (3.5), Belle [99] (0.4) }\end{array}$ & 2003 & OK \\
\hline$X(3915)$ & $3915.6 \pm 3.1$ & $28 \pm 10$ & $0 / 2^{?+}$ & $\begin{array}{l}B \rightarrow K(\omega J / \psi) \\
e^{+} e^{-} \rightarrow e^{+} e^{-}(\omega J / \psi)\end{array}$ & $\begin{array}{c}\text { Belle [100] (8.1), BABAR [101] (19) } \\
\text { Belle [102] (7.7) }\end{array}$ & 2004 & OK \\
\hline$X(3940)$ & $3942_{-8}^{+9}$ & $37_{-17}^{+27}$ & $?++$ & $\begin{array}{l}e^{+} e^{-} \rightarrow J / \psi\left(D \bar{D}^{*}\right) \\
e^{+} e^{-} \rightarrow J / \psi(\ldots)\end{array}$ & $\begin{array}{c}\text { Belle [103] }(6.0) \\
\text { Belle [54] (5.0) }\end{array}$ & 2007 & NC! \\
\hline$G(3900)$ & $3943 \pm 21$ & $52 \pm 11$ & $1^{--}$ & $e^{+} e^{-} \rightarrow \gamma(D \bar{D})$ & BABAR [27] (np), Belle [21] (np) & 2007 & OK \\
\hline$Y(4008)$ & $4008_{-49}^{+121}$ & $226 \pm 97$ & $1^{--}$ & $e^{+} e^{-} \rightarrow \gamma\left(\pi^{+} \pi^{-} J / \psi\right)$ & Belle [104] (7.4) & 2007 & $\mathrm{NC} !$ \\
\hline$Z_{1}(4050)^{+}$ & $4051_{-43}^{+24}$ & $82_{-55}^{+51}$ & $?$ & $B \rightarrow K\left(\pi^{+} \chi_{c 1}(1 P)\right)$ & Belle [105] (5.0) & 2008 & $\mathrm{NC!}$ \\
\hline$Y(4140)$ & $4143.4 \pm 3.0$ & $15_{-7}^{+11}$ & $?^{?+}$ & $B \rightarrow K(\phi J / \psi)$ & $\mathrm{CDF}[106,107](5.0)$ & 2009 & NC! \\
\hline$X(4160)$ & $4156_{-25}^{+29}$ & $139_{-65}^{+113}$ & $?^{?+}$ & $e^{+} e^{-} \rightarrow J / \psi\left(D \bar{D}^{*}\right)$ & Belle [103] (5.5) & 2007 & $\mathrm{NC!}$ \\
\hline$Z_{2}(4250)^{+}$ & $4248_{-45}^{+185}$ & $177_{-72}^{+321}$ & $?$ & $B \rightarrow K\left(\pi^{+} \chi_{c 1}(1 P)\right)$ & Belle [105] (5.0) & 2008 & $\mathrm{NC!}$ \\
\hline$Y(4260)$ & $4263 \pm 5$ & $108 \pm 14$ & $1^{--}$ & $\begin{array}{l}e^{+} e^{-} \rightarrow\left(\pi^{+} \pi^{-} J / \psi\right) \\
e^{+} e^{-} \rightarrow\left(\pi^{0} \pi^{0} J / \psi\right)\end{array}$ & $\begin{array}{c}\text { BABAR }[108,109](8.0) \\
\text { CLEO [110] }(5.4) \\
\text { Belle [104] }(15) \\
\text { CLEO [111] }(11) \\
\text { CLEO [111] (5.1) }\end{array}$ & 2005 & OK \\
\hline$Y(4274)$ & $4274.4_{-6.7}^{+8.4}$ & $32_{-15}^{+22}$ & $?^{?+}$ & $B \rightarrow K(\phi J / \psi)$ & CDF [107] (3.1) & 2010 & NC! \\
\hline$X(4350)$ & $4350.6_{-5.1}^{+4.6}$ & $13.3_{-10.0}^{+18.4}$ & $0,2^{++}$ & $e^{+} e^{-} \rightarrow e^{+} e^{-}(\phi J / \psi)$ & Belle [112] (3.2) & 2009 & NC! \\
\hline$Y(4360)$ & $4353 \pm 11$ & $96 \pm 42$ & $1^{--}$ & $e^{+} e^{-} \rightarrow \gamma\left(\pi^{+} \pi^{-} \psi(2 S)\right)$ & BABAR [113] (np), Belle [114] (8.0) & 2007 & OK \\
\hline$Z(4430)^{+}$ & $4443_{-18}^{+24}$ & $107_{-71}^{+113}$ & $?$ & $B \rightarrow K\left(\pi^{+} \psi(2 S)\right)$ & Belle $[115,116](6.4)$ & 2007 & NC! \\
\hline$X(4630)$ & $4634_{-11}^{+9}$ & $92_{-32}^{+41}$ & $1^{--}$ & $e^{+} e^{-} \rightarrow \gamma\left(\Lambda_{c}^{+} \Lambda_{c}^{-}\right)$ & Belle [25] (8.2) & 2007 & $\mathrm{NC!}$ \\
\hline$Y(4660)$ & $4664 \pm 12$ & $48 \pm 15$ & $1^{--}$ & $e^{+} e^{-} \rightarrow \gamma\left(\pi^{+} \pi^{-} \psi(2 S)\right)$ & Belle [114] (5.8) & 2007 & $\mathrm{NC!}$ \\
\hline$Y_{b}(10888)$ & $10888.4 \pm 3.0$ & $30.7_{-7.7}^{+8.9}$ & $1^{--}$ & $e^{+} e^{-} \rightarrow\left(\pi^{+} \pi^{-} \Upsilon(n S)\right)$ & Belle $[37,117](3.2)$ & 2010 & $\mathrm{NC} !$ \\
\hline
\end{tabular}

no $D^{* 0}$-mass constraint, and measured a mass value of $3875.2 \pm 0.7_{-1.6}^{+0.3} \pm 0.8 \mathrm{MeV}$.) Belle [95] fit to a conventional Breit-Wigner signal shape convolved with a Gaussian resolution function. BABAR [96] fit the data to an ensemble of MC samples, each generated with different plausible $X$ masses and widths and assuming a purely $S$-wave decay of a spin-1 resonance. The BABAR $X$ mass from $D^{* 0} \overline{D^{0}}$ decays is more than $3 \mathrm{MeV}$ larger than the world average from $\pi^{+} \pi^{-} J / \psi$, which engendered speculation that the $D^{* 0} \bar{D}^{0}$ enhancement might be a different state than that observed in $\pi^{+} \pi^{-} J / \psi$, but the smaller value observed by Belle in $D^{* 0} \bar{D}^{0}$ seems to make that possibility unlikely. The two $X$ mass measurements us- ing $D^{* 0} \overline{D^{0}}$ decays are inconsistent by $2.2 \sigma$, and are $1.8 \sigma$ and $4.7 \sigma$ higher than the $\pi^{+} \pi^{-} J / \psi$-based mass. However, important subtleties pointed out by Braaten and co-authors [121, 122] appear to explain at least qualitatively why masses extracted in this manner are larger than in $\pi^{+} \pi^{-} J / \psi$.

Measuring the $X$ mass with the $D^{* 0} \bar{D}^{0}$ decay is considerably more challenging than with $\pi^{+} \pi^{-} J / \psi$ for several reasons [121, 122]. If conceived as a bound or virtual $D^{* 0} \overline{D^{0}}$ state [123], the $X$ lineshape in this decay mode is determined by the binding energy, the $D^{* 0}$ natural width, and the natural width of the $X$ itself, which is at least as large as the $D^{* 0}$ width [121]. Because the 
TABLE 10: $X(3872)$ mass and width measurements by decay mode and experiment. The $\chi^{2} /$ d.o.f. values given in parentheses refer to weighted averages of the masses only. The lines marked $\left(B^{ \pm}\right)$and $\left(B^{0}\right)$ represent mass values quoted by $B A B A R$ in charged and neutral $B$-decays, respectively

\begin{tabular}{cccc}
\hline \hline Mode & $\begin{array}{c}\text { Mass } \\
(\mathrm{MeV})\end{array}$ & $\begin{array}{c}\text { Width } \\
(\mathrm{MeV})\end{array}$ & $\begin{array}{c}\text { Ref. } \\
\left(\chi^{2} / \text { d.o.f. }\right)\end{array}$ \\
\hline$\pi^{+} \pi^{-} J / \psi$ & $3871.46 \pm 0.37 \pm 0.07$ & $1.4 \pm 0.7$ & Belle [86] \\
$\left(B^{ \pm}\right)$ & $3871.4 \pm 0.6 \pm 0.1$ & $1.1 \pm 1.5 \pm 0.2$ & BABAR [87] \\
$\left(B^{0}\right)$ & $3868.7 \pm 1.5 \pm 0.4$ & - & BABAR [87] \\
& $3871.8 \pm 3.1 \pm 3.0$ & - & $\mathrm{D} \varnothing[88]$ \\
& $3871.61 \pm 0.16 \pm 0.19$ & - & $\mathrm{CDF}[90]$ \\
& $3871.52 \pm 0.20$ & $1.3 \pm 0.6$ & $\mathrm{Avg}^{3}(2.1 / 4)$ \\
\hline$D^{* 0} \bar{D}^{0}$ & $3875.1_{-0.5}^{+0.7} \pm 0.5$ & $3.0_{-1.4}^{+1.9} \pm 0.9$ & $B A B A R[96]$ \\
& $3872.9_{-0.4}^{+0.6+0.4}$ & $3.9_{-1.4}^{+2.8+0.2}$ & $\operatorname{Belle}_{-0.1}^{+0.1}$ \\
& $3874.0 \pm 1.2$ & $3.5_{-1.0}^{+1.6}$ & $\mathrm{Avg}^{3}(4.7 / 1)$ \\
\hline \hline
\end{tabular}

TABLE 11: Mass measurements relevant to the $X(3872)$. We define $\delta m_{0} \equiv m\left(D^{* 0}\right)-m\left(D^{0}\right)$ and $\Delta m_{t h r} \equiv m[X(3872)]-$ $\left[m\left(D^{* 0}\right)+m\left(D^{0}\right)\right]$

\begin{tabular}{ccc}
\hline \hline Quantity & Mass $(\mathrm{MeV})$ & Ref. $\left(\chi^{2} /\right.$ d.o.f. $)$ \\
\hline$m\left(D^{0}\right)$ & $1864.6 \pm 0.3 \pm 1.0$ & ACCMOR [118] \\
& $1864.847 \pm 0.150 \pm 0.095$ & CLEO [119] \\
& $1865.3 \pm 0.33 \pm 0.23$ & KEDR [120] \\
& $1864.91 \pm 0.16$ & Avg $^{3}(1.2 / 2)$ \\
\hline$\delta m_{0}$ & $142.12 \pm 0.07$ & PDG08 [18] \\
\hline $2 m\left(D^{0}\right)+\delta m_{0}$ & $3871.94 \pm 0.33$ & - \\
$m[X(3872)]$ & $3871.52 \pm 0.20$ & Table $10\left(\pi^{+} \pi^{-} J / \psi\right)$ \\
$\Delta m_{t h r}$ & $-0.42 \pm 0.39$ & - \\
& $\in[-0.92,0.08]$ & $@ 90 \% \mathrm{CL}$ \\
\hline \hline
\end{tabular}

binding energy of the $X$ is less than $1 \mathrm{MeV}$, whether or not its mass peak is below $D^{* 0} \bar{D}^{0}$ threshold, substantial fractions of the lineshape will lie both above and below that threshold. The portion of the $X$ lineshape below $D^{* 0} \bar{D}^{0}$ threshold, by definition, cannot decay to $D^{* 0} \bar{D}^{0}$. However, $D^{0} \bar{D}^{0} \pi^{0}$ and $D^{0} \bar{D}^{0} \gamma$ final states are possible from decays of a bound, effectively off-shell, $D^{* 0}$, as there is adequate phase space available above $D^{0} \bar{D}^{0} \pi^{0}$ threshold. Due to imperfect experimental resolution, these final states are indistinguishable from $D^{* 0} \bar{D}^{0}$ even though the $D^{* 0}$ decay products have masses below that of $D^{* 0}$. Furthermore, the analysis procedure which mass-constrains a $D^{* 0}$ candidate in each event distorts the purported $X$ mass distribution for below-threshold decays. Conversely, that portion of the $X$ lineshape above $D^{* 0} \bar{D}^{0}$ threshold can, of course, decay to $D^{* 0} \bar{D}^{0}$, but the $D^{* 0} \bar{D}^{0}$ mass distribution should, by definition, be exactly zero below threshold. Therefore the kinematic constraint on the reconstructed $D^{0} \pi^{0}$ to the $D^{* 0}$-mass, as carried out by Belle and $B A B A R$, results in a broad $D^{* 0} \bar{D}^{0}$ mass peak above threshold that should not be misconstrued as the true $X$ lineshape: neither the mass nor width results from $D^{* 0} \bar{D}^{0}$ reflect the true mass or width of the $X$. Rather, the lineshapes for $\pi^{+} \pi^{-} J / \psi, D^{* 0} \bar{D}^{0}$, and $D^{0} \bar{D}^{0} \pi^{0}$ (and $D^{0} \bar{D}^{0} \gamma$ ) final states are related but slightly different from one another, as shown in Fig. 16. More data and more sophisticated analyses are required to fully exploit what $D^{0} \bar{D}^{0} \pi^{0}$ and $D^{0} \overline{D^{0}} \gamma$ decays can reveal about the nature of the $X$.

Branching-fraction-related measurements for $X(3872)$ appear in Table 12 . Note that $X \rightarrow D^{* 0} \bar{D}^{0}$ decays are an order of magnitude more prevalent than $X \rightarrow \pi^{+} \pi^{-} J / \psi$, and that experimental information concerning the radiative decay $X \rightarrow \gamma \psi(2 S)$ has recently become murky; Belle's preliminary upper limit [99] is inconsistent with the BABAR [98] measurement. Belle [92] found $4.3 \sigma \mathrm{ev-}$ idence for $X \rightarrow \pi^{+} \pi^{-} \pi^{0} J / \psi$ in $B$-decays, with the $3 \pi$ invariant mass clustered near the kinematic endpoint, which is almost one full $\Gamma_{\omega}$ below the $\omega$ mass peak. This suggests the decay $X \rightarrow \omega J / \psi, \omega \rightarrow \pi^{+} \pi^{-} \pi^{0}$ on the $\omega$ low-side tail. Despite this apparent phase-space suppression, the rate for $X \rightarrow \omega J / \psi$ was found to be comparable to that of $\pi^{+} \pi^{-} J / \psi$. In 2010 BABAR [93] reported corroborating evidence $(4.0 \sigma)$ of an $X(3872)$ peak in $B^{0,+} \rightarrow\left(J / \psi \pi^{+} \pi^{-} \pi^{0}\right) K^{0,+}$ decays using their full data sample, also finding a decay rate comparable to

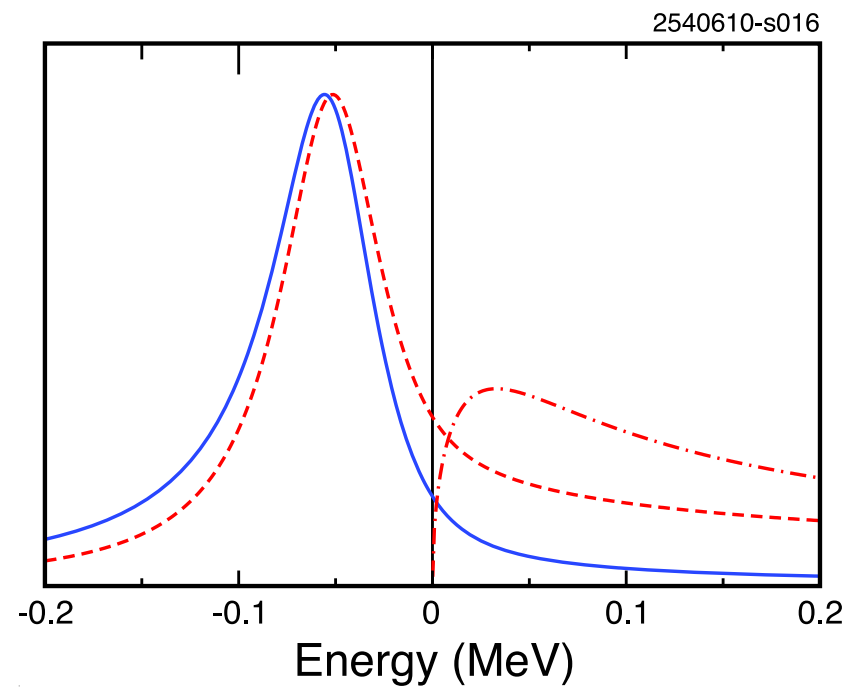

FIG. 16: From Braaten and Stapleton [122], the $X(3872)$ lineshapes extracted from a fit to the Belle [95] $D^{* 0} \bar{D}^{0}$ events, unfolding the effects of experimental resolution, for $\pi^{+} \pi^{-} J / \psi$ (solid curve), $D^{0} \bar{D}^{0} \pi^{0}$ (dashed), and, when always constraining one $D^{0} \pi^{0}$ pairing per event to a $D^{* 0}$ mass, $D^{* 0} \bar{D}^{0}(d o t$ dashed). The horizontal axis is the invariant mass of the decay products relative to $D^{* 0} \bar{D}^{0}$ threshold, and the solid and dashed curves are normalized so as to have the same peak height. Adapted from [122] with kind permission, copyright (2010) The American Physical Society 
TABLE 12: For $X(3872)$, measured branching fractions and products thereof, in units of $10^{-6}$ :

$\mathcal{B}_{B^{+}} \equiv \mathcal{B}\left(B^{+} \rightarrow K^{+} X\right)$,

$\mathcal{B}_{\gamma 1} \equiv \mathcal{B}\left(B^{+} \rightarrow K^{+} X\right) \times \mathcal{B}(X \rightarrow \gamma J / \psi)$,

$\mathcal{B}_{\gamma 2} \equiv \mathcal{B}\left(B^{+} \rightarrow K^{+} X\right) \times \mathcal{B}(X \rightarrow \gamma \psi(2 S))$,

$\mathcal{B}_{0} \equiv \mathcal{B}\left(B^{0} \rightarrow K X\right) \times \mathcal{B}(X \rightarrow f)$,

$\mathcal{B}_{+} \equiv \mathcal{B}\left(B^{+} \rightarrow K X\right) \times \mathcal{B}(X \rightarrow f)$, and

$\mathcal{B}_{0+} \equiv\left(\mathcal{B}_{0}+\mathcal{B}_{+}\right) / 2$ for final state $f$.

Branching fraction ratios are defined as:

$R_{0+} \equiv \mathcal{B}\left(B^{0} \rightarrow K^{0} X\right) / \mathcal{B}\left(B^{+} \rightarrow K^{+} X\right)$,

$r_{D D \pi} \equiv \mathcal{B}_{0+}\left(D^{0} \bar{D}^{0} \pi^{0}\right) / \mathcal{B}_{0+}\left(\pi^{+} \pi^{-} J / \psi\right)$,

$r_{\omega} \equiv \mathcal{B}(X \rightarrow \omega J / \psi) / \mathcal{B}\left(X \rightarrow \pi^{+} \pi^{-} J / \psi\right)$,

$r_{\gamma 1} \equiv \mathcal{B}(X \rightarrow \gamma J / \psi) / \mathcal{B}\left(X \rightarrow \pi^{+} \pi^{-} J / \psi\right)$, and

$r_{\gamma 2} \equiv \mathcal{B}(X \rightarrow \gamma \psi(2 S)) / \mathcal{B}\left(X \rightarrow \pi^{+} \pi^{-} J / \psi\right)$

\begin{tabular}{|c|c|c|c|}
\hline What & Mode & Value & Ref. ( $\chi^{2} /$ d.o.f. $)$ \\
\hline $\mathcal{B}_{B^{+}}$ & & $<320$ & BABAR [124] \\
\hline $\mathcal{B}_{\gamma 1}$ & $\gamma J / \psi$ & $\begin{array}{c}1.8 \pm 0.6 \pm 0.1 \\
2.8 \pm 0.8 \pm 0.1 \\
2.2 \pm 0.5 \\
\end{array}$ & $\begin{array}{c}\text { Belle [92] } \\
B A B A R[98] \\
\operatorname{Avg}^{3}(1.0 / 1) \\
\end{array}$ \\
\hline $\mathcal{B}_{\gamma 2}$ & $\gamma \psi(2 S)$ & $\begin{array}{c}<3.4 \\
0.8 \pm 2.0^{a} \\
9.5 \pm 2.7 \pm 0.6 \\
3.8 \pm 4.1 \\
\end{array}$ & $\begin{array}{c}\text { Belle [99] } \\
\text { Belle [99] } \\
\text { BABAR [98] } \\
\mathrm{Avg}^{3}(6.4 / 1) \\
\end{array}$ \\
\hline $\mathcal{B}_{\gamma 2} / \mathcal{B}_{\gamma 1}$ & $\gamma \psi(2 S) / \gamma J / \psi$ & $4.3 \pm 1.6$ & Values above \\
\hline $\begin{array}{c}\mathcal{B}_{0} \\
\mathcal{B}_{+} \\
\mathcal{B}_{0} \\
\mathcal{B}_{+} \\
\mathcal{B}_{0+} \\
\end{array}$ & $D^{* 0} \bar{D}^{0}$ & $\begin{array}{c}167 \pm 36 \pm 47 \\
222 \pm 105 \pm 42 \\
97 \pm 46 \pm 13 \\
77 \pm 16 \pm 10 \\
90 \pm 19 \\
\end{array}$ & $\begin{array}{c}\text { BABAR [96] } \\
\text { BABAR [96] } \\
\text { Belle [95] } \\
\text { Belle [95] } \\
\operatorname{Avg}^{3}(3.6 / 3)\end{array}$ \\
\hline $\mathcal{B}_{0+} / \mathcal{B}_{B^{+}}$ & $D^{* 0} \bar{D}^{0}$ & $>28 \%$ & Above \\
\hline $\begin{array}{l}\mathcal{B}_{0} \\
\mathcal{B}_{+} \\
\mathcal{B}_{0} \\
\mathcal{B}_{+} \\
\mathcal{B}_{0+}\end{array}$ & $\pi^{+} \pi^{-} J / \psi$ & $\begin{array}{c}3.50 \pm 1.90 \pm 0.40 \\
8.40 \pm 1.50 \pm 0.70 \\
6.65 \pm 1.63 \pm 0.55 \\
8.10 \pm 0.92 \pm 0.66 \\
7.18 \pm 0.97\end{array}$ & $\begin{array}{c}\text { BABAR [87] } \\
\text { BABAR [87] } \\
\text { Belle [86] } \\
\text { Belle [86] } \\
\operatorname{Avg}^{3}(4.9 / 3) \\
\end{array}$ \\
\hline $\mathcal{B}_{0+} / \mathcal{B}_{B^{+}}$ & $\pi^{+} \pi^{-} J / \psi$ & $>2.2 \%$ & Above \\
\hline$R_{0+}$ & $\begin{array}{c}\pi^{+} \pi^{-} J / \psi \\
\pi^{+} \pi^{-} J / \psi \\
D^{* 0} \bar{D}^{0} \\
D^{* 0} \bar{D}^{0} \\
\text { Both }\end{array}$ & $\begin{array}{c}0.82 \pm 0.22 \pm 0.05 \\
0.41 \pm 0.24 \pm 0.05 \\
1.26 \pm 0.65 \pm 0.06 \\
1.33 \pm 0.69 \pm 0.43 \\
0.70 \pm 0.16\end{array}$ & $\begin{array}{c}\text { Belle [86] } \\
\text { BABAR [87] } \\
\text { Belle [95] } \\
B A B A R[96] \\
\operatorname{Avg}^{3}(3.0 / 3) \\
\end{array}$ \\
\hline$r_{D^{*} D}$ & $D^{* 0} \bar{D}^{0}$ & $12.5 \pm 3.1$ & Ratio of avgs \\
\hline $\begin{array}{l}\mathcal{B}_{0} \\
\mathcal{B}_{+} \\
\mathcal{B}_{0} \\
\mathcal{B}_{+} \\
\mathcal{B}_{0+}\end{array}$ & $\omega J / \psi$ & $\begin{array}{c}3.5 \pm 1.9 \pm 0.7 \\
8.5 \pm 1.5 \pm 1.7 \\
6 \pm 3 \pm 1 \\
6 \pm 2 \pm 1 \\
5.8 \pm 1.2 \\
\end{array}$ & $\begin{array}{c}\text { Belle [92] } \\
\text { Belle [92] } \\
\text { Belle [93] } \\
\text { Belle [93] } \\
\operatorname{Avg}^{3}(2.7 / 3) \\
\end{array}$ \\
\hline$r_{\omega}$ & $\omega J / \psi$ & $\begin{array}{c}1.0 \pm 0.4 \pm 0.3 \\
0.8 \pm 0.3 \\
0.85 \pm 0.26\end{array}$ & $\begin{array}{c}\text { Belle [92] } \\
\text { BABAR [93] } \\
\operatorname{Avg}^{3}(0.1 / 1)\end{array}$ \\
\hline $\begin{array}{l}r_{\gamma 1} \\
r_{\gamma 2}\end{array}$ & $\begin{array}{c}\gamma J / \psi \\
\gamma \psi(2 S)\end{array}$ & $\begin{array}{l}0.31 \pm 0.08 \\
0.53 \pm 0.57\end{array}$ & $\begin{array}{l}\text { Values above } \\
\text { Values above }\end{array}$ \\
\hline $\begin{array}{l}\mathcal{B}_{0} \\
\mathcal{B}_{+} \\
\mathcal{B}_{0+}\end{array}$ & $\pi^{+} \pi^{0} J / \psi$ & $\begin{array}{c}-5.7 \pm 4.9 \\
2.0 \pm 3.8 \\
-0.9 \pm 3.7\end{array}$ & $\begin{array}{l}B A B A R[125] \\
B A B A R[125] \\
\operatorname{Avg}^{3}(1.5 / 1)\end{array}$ \\
\hline
\end{tabular}

${ }^{a}$ Belle only quotes an upper limit for this preliminary result. From the information presented in [99], we have extracted an approximate central value and error for this table that of $\pi^{+} \pi^{-} J / \psi$, as shown in Table 12. Their analysis was able to identify the three-pion decay as coming from an $\omega$-meson decay by weighting the entries based on the pion opening angles in the $\omega$ rest-frame: phasespace weighting results in no net signal. In a comparison of the observed $m(3 \pi)$ mass distribution to that of MC simulations, BABAR also found that the inclusion of one unit of orbital angular momentum in the $J / \psi \omega$ system, with its consequent negative parity, substantially improves the description of the data. Hence the $X(3872)$ quantum number assignment [89] of $J^{P C}=2^{-+}$is preferred somewhat over the $1^{++}$hypothesis in the $B A B A R$ analysis, leading $B A B A R$ to conclude that the $X(3872)$ can be interpreted as an $\eta_{c 2}(1 D)$ charmonium state [81, 126]. However, the $1^{++}$assignment cannot be ruled out as unlikely by this analysis, just less likely than $2^{-+}$. In addition, it has been shown [127] that a $2^{-+}$assignment is not consistent with other properties of the $X(3872)$.

$B A B A R$ [125] searched for a charged partner state in the decay $X^{+} \rightarrow \rho^{+} J / \psi$, finding the results in Table 12 . The average from charged and neutral $B$-decays should be compared with the isospin-symmetry prediction, which is double the rate for $\rho^{0} J / \psi$. These rates disagree by more than $4 \sigma$, making it most likely that the $X$ is an isosinglet. The BABAR [124] upper limit on $\mathcal{B}_{B^{+}} \equiv \mathcal{B}\left(B^{+} \rightarrow K^{+} X\right)$ permits an inferred lower limit on $\mathcal{B}\left(X \rightarrow \pi^{+} \pi^{-} J / \psi\right)$, which, when combined with the relative rates of $D^{* 0} \bar{D}^{0}$ and $\pi^{+} \pi^{-} J / \psi$, yields $2.2 \%<\mathcal{B}\left(X \rightarrow \pi^{+} \pi^{-} J / \psi\right)<10.5 \%$ and $28 \%<\mathcal{B}(X \rightarrow$ $\left.D^{* 0} D^{0}\right)<94.2 \%$. Belle [86] has studied the question of whether or not the $X$, like conventional charmonia, tends to be produced more strongly in $B^{0} \rightarrow K^{* 0} X$ relative to nonresonant $(\mathrm{NR}) B^{0} \rightarrow\left(K^{+} \pi^{-}\right)_{\mathrm{NR}} X$. Using $X \rightarrow \pi^{+} \pi^{-} J / \psi$ decays, they limit the $K^{* 0} /\left(K^{+} \pi^{-}\right)_{\mathrm{NR}}$ ratio to be $<0.5$ at $90 \% \mathrm{CL}$, contrasted with ratios closer to 3 for other charmonium states.

The possibility that the $X$ enhancement in $\pi^{+} \pi^{-} J / \psi$ is composed of two different narrow states, $X_{L}$ and $X_{H}$, was addressed by CDF [90]. By analyzing the observed lineshape, $X_{L}$ and $X_{H}$ were found to have masses closer than $3.2(4.3) \mathrm{MeV}$ for a relative production fraction of unity $(20 \%)$. Any mass difference between the $X$ appearing in charged and neutral $B$-decays has also been limited to $<2.2 \mathrm{MeV}$, as detailed in Table 13 .

Taking the totality of experimental information on the $X(3872)$ at face value, the $X$ is a narrow resonant structure with the most probable quantum numbers $J^{P C}=1^{++}$and $I=0$, and has mass within $1 \mathrm{MeV}$ of $D^{* 0} \bar{D}^{0}$ threshold. It may have comparable decay rates to $\gamma \psi(2 S)$ and (often-slightly-below-threshold) $D^{* 0} \bar{D}^{0}$, but has an order of magnitude smaller rate to both $\omega J / \psi$ and $\rho^{0} J / \psi$. Decays to $\gamma J / \psi$ occur at roughly a quarter of the $\gamma \psi(2 S)$ rate. If there are two components of the observed enhancements, they must be closer in mass than a few $\mathrm{MeV}$. It is produced and observed in Tevatron $p \bar{p}$ collisions with a rate similar to conventional charmonia, and at the $B$-factories in $B \rightarrow K X$ decays. Unlike conventional charmonia, $B \rightarrow K^{*} X$ is suppressed with 
respect to $B \rightarrow K \pi X$.

The summarized properties of $X(3872)$ do not comfortably fit those of any plausible charmonium state. Prominent decays to $D^{* 0} \bar{D}^{0}$ and proximity to $D^{* 0} \bar{D}^{0}$ mass threshold naturally lead to models [123] which posit the $X$ to be either a weakly bound molecule of a $D^{* 0}$ and a $\bar{D}^{0}$ slightly below threshold or a virtual one slightly unbound. Accommodating the large radiative decay rates and substantial $\pi^{+} \pi^{-} J / \psi$ rate in such models creates challenges because the $D^{* 0}$ and $D^{0}$ would be spatially separated by large distances, suppressing the probability of overlap for annihilation. This has led to the hypothesis of mixing with a charmonium state having the same quantum numbers. Models of a tightly bound diquarkdiantiquark system $c u \bar{c} \bar{u}$ feature two neutral $(c d \bar{c} \bar{d}, c d \bar{c} \bar{s})$ and one charged $(c u \bar{c} \bar{d})$ partner state, which are limited by the corresponding mass difference and null search measurements. Better understanding of the $X(3872)$ demands more experimental constraints and theoretical insight.

\subsubsection{Unconventional vector states}

$\underline{Y(4260), Y(4360), Y(4660), \text { and } Y(4008)}$

The first observation of an unexpected vector charmonium-like state was made by BABAR [108] in ISR production of $Y(4260) \rightarrow \pi^{+} \pi^{-} J / \psi$, which was later updated [109] with twice the data, as shown in Fig. 17. CLEO [110] and Belle [104] confirmed the BABAR result, but Belle also found a smaller, broader structure at $4008 \mathrm{MeV}$, as seen in Fig. 18. Aside from the lower mass state, for which the updated BABAR [109] analysis placed an upper limit, the three sets of measurements were quite consistent in mass and width, as shown in Table 14, but only roughly so in strength. BABAR [113] found one more apparent enhancement, $Y(4360)$, in $\pi^{+} \pi^{-} \psi(2 S)$, which Belle [114] measured with somewhat larger mass and smaller width, as seen in Table 15. Belle also found a second structure near $4660 \mathrm{MeV}$, as seen in Fig. 19. (A combined fit [128] to Belle and BABAR $\pi^{+} \pi^{-} \psi(2 S)$ data found

TABLE 13: For $X(3872), \Delta m_{0+}$ (in $\mathrm{MeV}$ ), the difference between the $X(3872)$ mass obtained from neutral and charged $B$ decays; and $\Delta m_{L H}$ (in $\mathrm{MeV}$ ), the difference in mass of two $X$ states produced with equal strength in $p \bar{p}$ collisions

\begin{tabular}{cccc}
\hline \hline Quantity & Mode & Value & Ref. ( $\chi^{2} /$ d.o.f.) \\
\hline$\Delta m_{0+}$ & $\pi^{+} \pi^{-} J / \psi$ & $2.7 \pm 1.6 \pm 0.4$ & BABAR [87] \\
& & $0.18 \pm 0.89 \pm 0.26$ & Belle [86] \\
& & $0.79 \pm 1.08$ & $\operatorname{Avg}^{3}(1.8 / 1)$ \\
& & $<2.2$ at $90 \%$ CL & Above \\
\hline$\Delta m_{L H}$ & $\pi^{+} \pi^{-} J / \psi$ & $<3.2$ at $90 \%$ CL & CDF [90] \\
\hline \hline
\end{tabular}

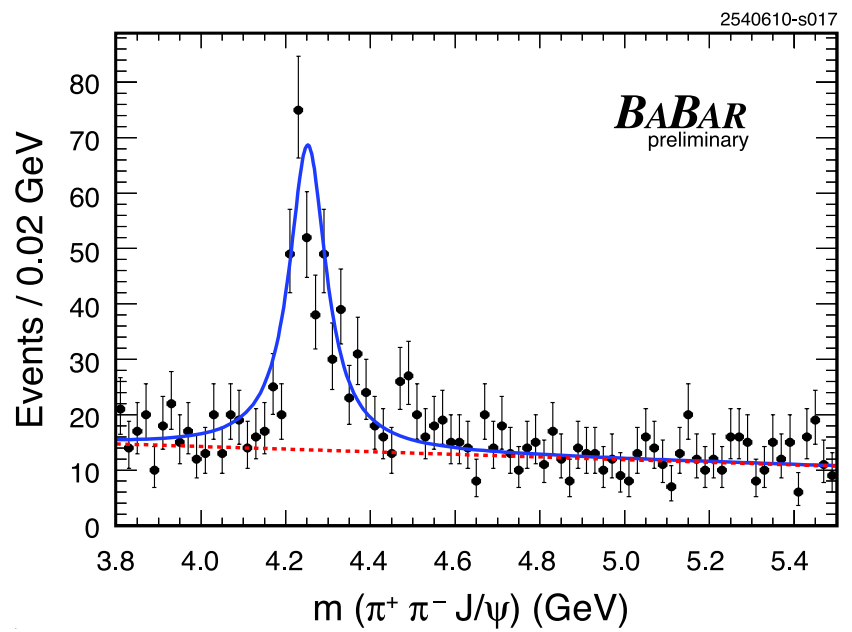

FIG. 17: From BABAR [109], the invariant mass of $\pi^{+} \pi^{-} J / \psi$ candidates produced in initial-state radiation, $e^{+} e^{-} \rightarrow$ $\gamma_{I S R} \pi^{+} \pi^{-} J / \psi$. Points with error bars represent data, and the curves show the fit (solid) to a signal plus a linear background (dashed)

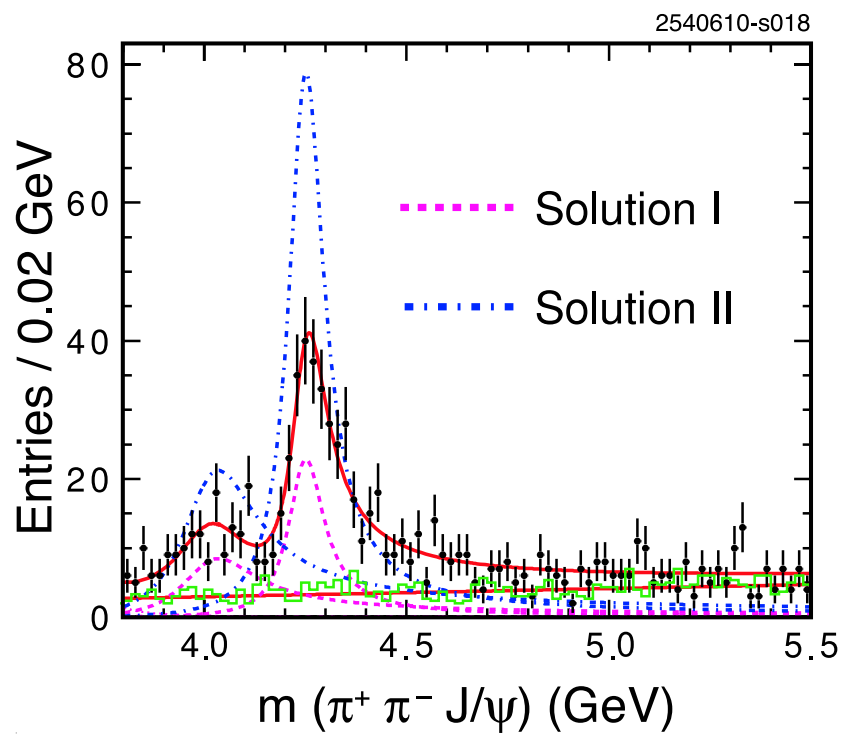

FIG. 18: From Belle [104], the invariant mass of $\pi^{+} \pi^{-} J / \psi$ candidates produced in initial-state radiation, $e^{+} e^{-} \rightarrow$ $\gamma_{I S R} \pi^{+} \pi^{-} J / \psi$, with $J / \psi$-sidebands already subtracted, unlike Fig. 17. Points with error bars represent data, the solid curve shows the best fits to the data to two resonances including interference with a floating phase, and the dashed and dashed-dot curves show the two pairs of individual resonance contributions for the two equally probable best-fit phases. Adapted from [104] with kind permission, copyright (2007) The American Physical Society

them to be consistent with one other.) Because dipion transitions between vector quarkonia are commonplace for charmonium and bottomonium, it was natural, then, that the first inclination was to ascribe the $Y$ 's to excited vector charmonia. A number of additional features of these states contradicted this hypothesis, however. Only 
TABLE 14: Measured properties of the decay $Y(4260) \rightarrow$ $\pi^{+} \pi^{-} J / \psi$, including its mass $m$, width $\Gamma$, and branching fraction $\mathcal{B}$. The Belle [104] single-resonance fit result is quoted to allow for comparison to the other two

\begin{tabular}{ccc}
\hline \hline Quantity & Value & Ref. $\left(\chi^{2} /\right.$ d.o.f. $)$ \\
\hline$m$ & $4259 \pm 8_{-6}^{+2}$ & BABAR [109] \\
$(\mathrm{MeV})$ & $4263 \pm 6$ & Belle [104] \\
& $4284_{-16}^{+17} \pm 4$ & CLEO [110] \\
& $4263 \pm 5$ & $\operatorname{Avg}^{3}(1.8 / 2)$ \\
\hline$\Gamma$ & $88 \pm 23_{-4}^{+6}$ & BABAR [109] \\
& $126 \pm 18$ & Belle [104] \\
$(\mathrm{MeV})$ & $73_{-25}^{+39} \pm 5$ & CLEO [110] \\
& $108 \pm 15$ & Avg ${ }^{3}(2.4 / 2)$ \\
\hline $\mathcal{B} \times \Gamma$ ee & $5.5 \pm 1.0_{-0.7}^{+0.8}$ & BABAR [109] \\
$(\mathrm{eV})$ & $9.7 \pm 1.1$ & Belle [104] \\
& $8.9_{-3.1}^{+3.9} \pm 1.8$ & CLEO [110] \\
& $8.0 \pm 1.4$ & Avg $^{3}(6.1 / 2)$ \\
\hline \hline
\end{tabular}

TABLE 15: Measured properties of the two enhancements found in the $\pi^{+} \pi^{-} \psi(2 S)$ mass distribution: $Y(4360)$ and $Y(4660)$. Liu et al. [128] performed a binned maximum likelihood fit to the combined Belle and BABAR cross section distributions (Fig. 19)

\begin{tabular}{ccc}
\hline \hline Quantity & Value & Ref. ( $\chi^{2} /$ d.o.f. $)$ \\
\hline$m$ & $4324 \pm 24$ & BABAR [113] \\
$(\mathrm{MeV})$ & $4361 \pm 9 \pm 9$ & Belle [114] \\
& $4353 \pm 15$ & $\operatorname{Avg}^{3}(1.8 / 1)$ \\
& $4355_{-10}^{+9} \pm 9$ & Liu [128] \\
\hline$\Gamma$ & $172 \pm 33$ & BABAR [113] \\
$(\mathrm{MeV})$ & $74 \pm 15 \pm 10$ & Belle [114] \\
& $96 \pm 42$ & Avg $^{3}(6.8 / 1)$ \\
& $103_{-15}^{+17} \pm 11$ & Liu [128] \\
\hline$m$ & $4664 \pm 11 \pm 5$ & Belle [114] \\
$(\mathrm{MeV})$ & $4661_{-8}^{+9} \pm 6$ & Liu [128] \\
\hline$\Gamma$ & $48 \pm 15 \pm 3$ & Belle [114] \\
$(\mathrm{MeV})$ & $42_{-12}^{+17} \pm 6$ & Liu [128] \\
\hline \hline
\end{tabular}

one, $Y(4660)$, is remotely near a predicted $1^{--} c \bar{c}$ state $\left(3^{3} D_{1}\right)$. The $Y(4260)$ and $Y(4360)$ did not show up in inclusive hadronic cross section $(R)$ measurements as seen in Fig. 3, as would be expected of such states. (There is no fine-grained $R$-scan data near $Y(4660)$.)

A comparison of the measured $\pi^{+} \pi^{-} J / \psi$ and total hadronic cross sections in the $\sqrt{s} \simeq 4260 \mathrm{MeV}$ region yields a lower bound for $\Gamma\left(Y \rightarrow \pi^{+} \pi^{-} J / \psi\right)>508 \mathrm{keV}$ at $90 \% \mathrm{CL}$, an order of magnitude higher than expected

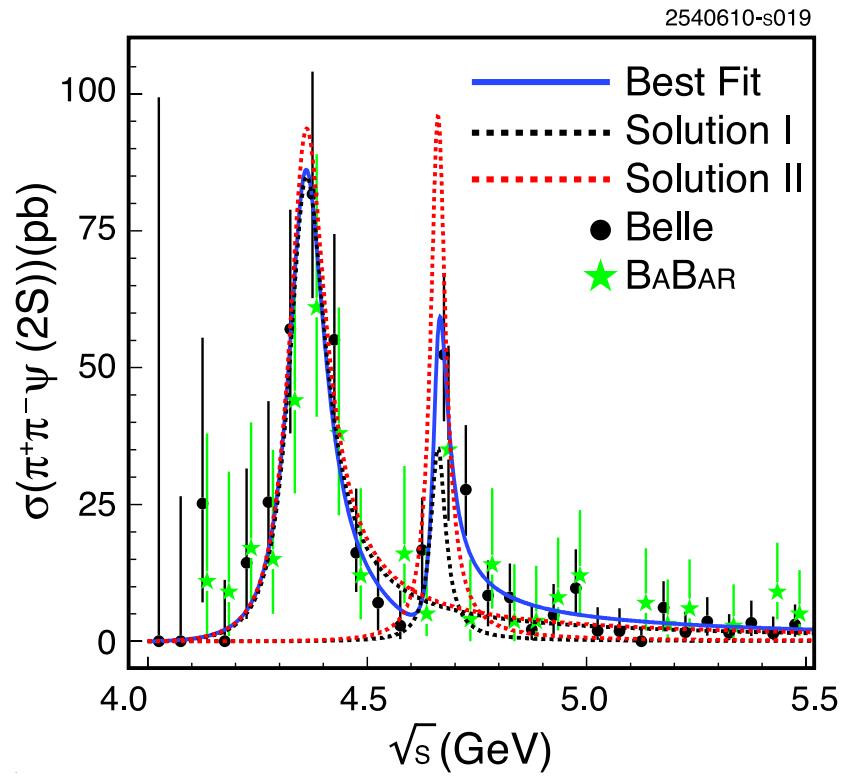

FIG. 19: From [128], the $\pi^{+} \pi^{-} \psi(2 S)$ cross section as a function of $\sqrt{s}$, showing the result of a binned maximum-likelihood fit of combined Belle and BABAR data, The solid circles and stars show the Belle and BABAR data, respectively. The solid curve shows the best fits to the data to two resonances including interference with a floating phase, and the dashed curves show the two pairs of individual resonance contributions for the two equally probable best-fit phases. Adapted from [128] with kind permission, copyright (2008) The American Physical Society

TABLE 16: Upper limits at 90\% CL on the ratios $\sigma\left(e^{+} e^{-} \rightarrow\right.$ $Y \rightarrow T) / \sigma\left(e^{+} e^{-} \rightarrow Y \rightarrow \pi^{+} \pi^{-} J / \psi\right)$ at $E_{\text {c.m. }}=4.26 \mathrm{GeV}$ $(\mathrm{CLEO}[16])$ and $\mathcal{B}(Y \rightarrow T) / \mathcal{B}\left(Y \rightarrow \pi^{+} \pi^{-} J / \psi\right)$ (for $Y(4260))$, and $\mathcal{B}(Y \rightarrow T) / \mathcal{B}\left(Y \rightarrow \pi^{+} \pi^{-} \psi(2 S)\right)$ (for $Y(4360)$ and $Y(4660)$ ), from BABAR [27-29] and Belle [22], where $T$ is an open charm final state

\begin{tabular}{lccc}
\hline \hline \multicolumn{1}{c}{$T$} & $Y(4260)$ & $Y(4360)$ & $Y(4660)$ \\
\hline$D \bar{D}$ & $4.0[16], 7.6[27]$ & & \\
$D \bar{D}^{*}$ & $45[16], 34[28]$ & & \\
$D^{*} \bar{D}^{*}$ & $11[16], 40[28]$ & & \\
$D \bar{D}^{*} \pi$ & $15[16], 9[22]$ & $8[22]$ & $10[22]$ \\
$D^{*} \bar{D}^{*} \pi$ & $8.2[16]$ & & \\
$D_{s}^{+} D_{s}^{-}$ & $1.3[16], 0.7[29]$ & & \\
$D_{s}^{+} D_{s}^{*-}$ & $0.8[16], 44[29]$ & \\
$D_{s}^{*+} D_{s}^{*-}$ & $9.5[16]$ & \\
\hline \hline
\end{tabular}

for conventional vector charmonium states [129]. Charmonium would also feature dominant open charm decays, exceeding those of dipion transitions by a factor expected to be $\gtrsim 100$, since this is the case for $\psi(3770)$ and $\psi(4160)$. As summarized in Table 16, no such evidence has been found, significantly narrowing any window for either charmonia or, in some cases, quark-gluon hybrid 
interpretations. CLEO [111] studied direct production of $Y(4260)$ in $e^{+} e^{-}$collisions; verified the production cross section; and identified the only non- $\pi^{+} \pi^{-} J / \psi$ decay mode seen so far, $\pi^{0} \pi^{0} \mathrm{~J} / \psi$, occuring at roughly half of the $\pi^{+} \pi^{-} J / \psi$ rate.

Any explanation for these vector states will have to describe their masses, widths, and manifest reluctance to materialize in open charm or unflavored light meson final states. The dipion invariant mass spectra exhibit curious structures, as seen for $Y(4260)$ in Fig. 20 [109], $Y(4360)$ in Fig. 21(a) [114], and $Y$ (4660) in Fig. 21(b) [114]. The first shows a distinctly non-phase-space double-hump structure which is qualitatively confirmed by Belle [104], the second exhibits a plurality of events at higher masses, and the third indicates a quite dominant $f_{0}(980)$ component.

\section{$X(4630)$}

The $e^{+} e^{-} \rightarrow \Lambda_{c}^{+} \Lambda_{c}^{-}$cross section was measured by Belle [25] using ISR and partial reconstruction (Fig. 4(i)). A clear peak is evident near the threshold, and corresponds to

$$
\begin{gathered}
\mathcal{B}\left(X(4630) \rightarrow e^{+} e^{-}\right) \times \mathcal{B}\left(X(4630) \rightarrow \Lambda_{c}^{+} \Lambda_{c}^{-}\right)= \\
(0.68 \pm 0.33) \times 10^{-6} .
\end{gathered}
$$

The nature of this enhancement remains unclear. Although both mass and width of the $X(4630)$ (see Table 9) are consistent within errors with those of the $Y(4660)$, this could be coincidence and does not exclude other interpretations.

\section{$Y_{b}(10888)$}

A recent Belle scan above the $\Upsilon(4 S)$ was motivated by an earlier observation [117] of anomalously large $\pi^{+} \pi^{-} \Upsilon(n S)(n=1,2,3)$ cross sections near the $\Upsilon(5 S)$

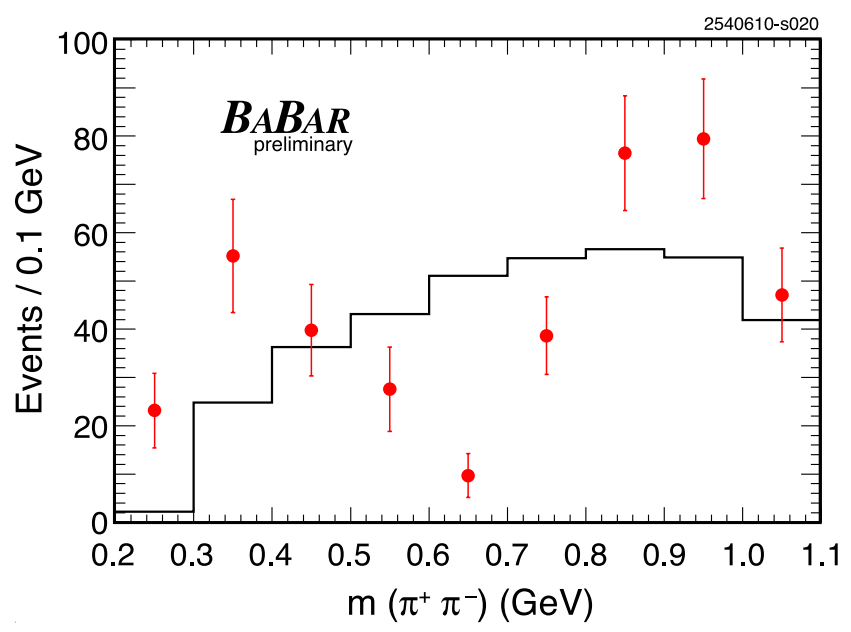

FIG. 20: From BABAR [109], the dipion invariant mass distribution of ISR-produced $Y(4260) \rightarrow \pi^{+} \pi^{-} J / \psi$ decays, where points represent data and the line histogram is phase-space MC simulation

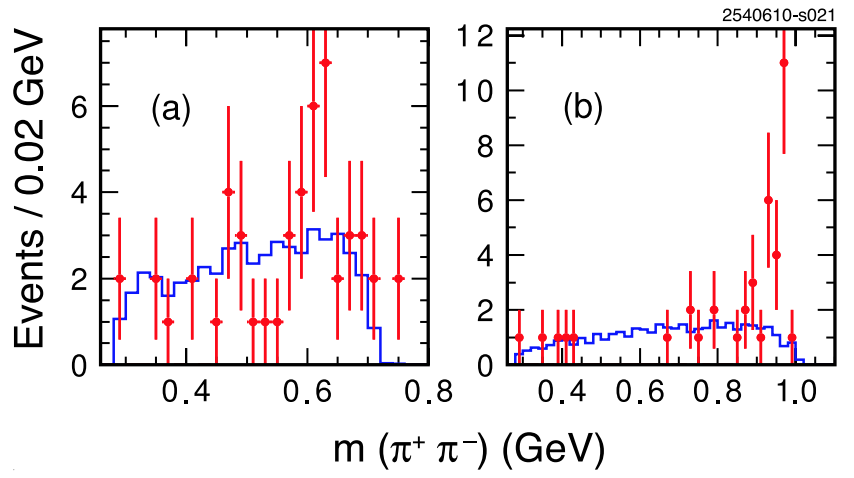

FIG. 21: From Belle [114], the dipion invariant mass distribution of ISR-produced (a) $Y(4360) \rightarrow \pi^{+} \pi^{-} \psi(2 S)$ and (b) $Y(4660) \rightarrow \pi^{+} \pi^{-} \psi(2 S)$, where points represent data and the line histograms show phase-space MC simulations. Adapted from [114] with kind permission, copyright (2007) The American Physical Society

peak energy. These new data allowed independent determinations of the $\Upsilon(5 S)$ lineshape and that of the $\pi^{+} \pi^{-} \Upsilon(n S)$ enhancement [37]. A simultaneous fit to all three measured $\pi^{+} \pi^{-} \Upsilon(n S)$ cross sections to a single Breit-Wigner function represents the data well; this lineshape has somewhat ${ }^{5}$ higher mass and narrower width (see Table 9) than does the $\Upsilon(5 S)$ resonance measured with loosely selected hadronic events in the same experiment. This suggests that the enhancement $\pi^{+} \pi^{-} \Upsilon(n S)$ could be a $1^{--} Y_{b}$ state distinct from $\Upsilon(5 S)$ and perhaps of a similar origin as $Y(4260)$. The relevant cross sections and lineshapes are shown in Fig. 22. See also the discussion in Sect. 3.3.11.

\subsubsection{Other positive $C$-parity states}

Of the multitude of new charmonium-like states, a puzzling cluster of them from different production mechanisms and/or decay chains gather near a mass of $3940 \mathrm{MeV}(Z(3930), Y(3940), X(3940), X(3915))$ and have positive $C$-parity. Four others $(Y(4140), X(4160)$, $Y(4274)$, and $X(4350))$ also have $C=+$ and have related signatures. Definitive determination of whether some of these are distinct from others and whether any can be attributed to expected charmonia requires independent confirmations, more precise mass and branching fraction measurements, and unambiguous quantum number assignments.

\footnotetext{
${ }^{5}$ When Belle [117] adds the cross sections from $\pi^{+} \pi^{-} \Upsilon(n S)$ events at each energy scan point to the loosely selected hadronic events for a fit to a single resonance, the quality of the fit degrades by $2.0 \sigma$ (where systematic uncertainties are included) relative to the hadronic events alone. That is, the $\pi^{+} \pi^{-} \Upsilon(n S)$ and hadronic events are consistent with a single enhancement within two standard deviations.
} 


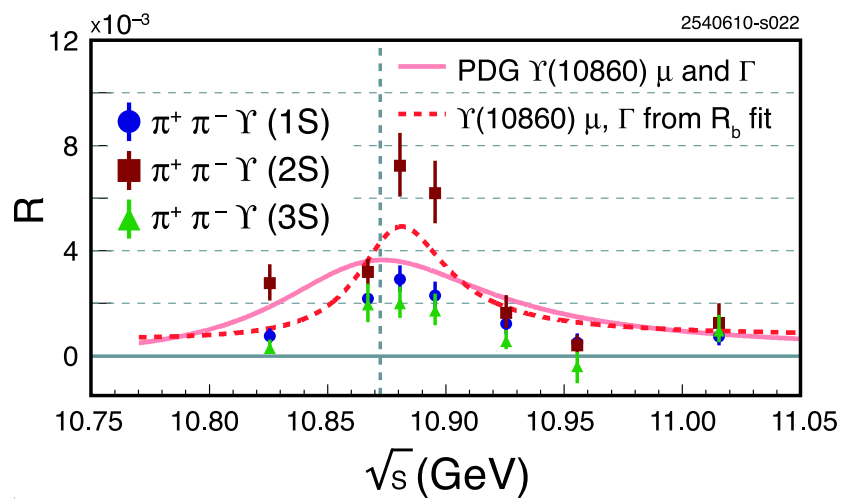

FIG. 22: From Belle [37], results of an energy scan near the $\Upsilon(5 S)$. The points with error bars indicate the three sets of cross sections for the $\pi^{+} \pi^{-} \Upsilon(n S) \quad(n=1,2,3)$, normalized to the point cross section. The solid and dashed curves show the $\Upsilon(5 S)$ lineshape from PDG08 [18] and Belle [37], respectively

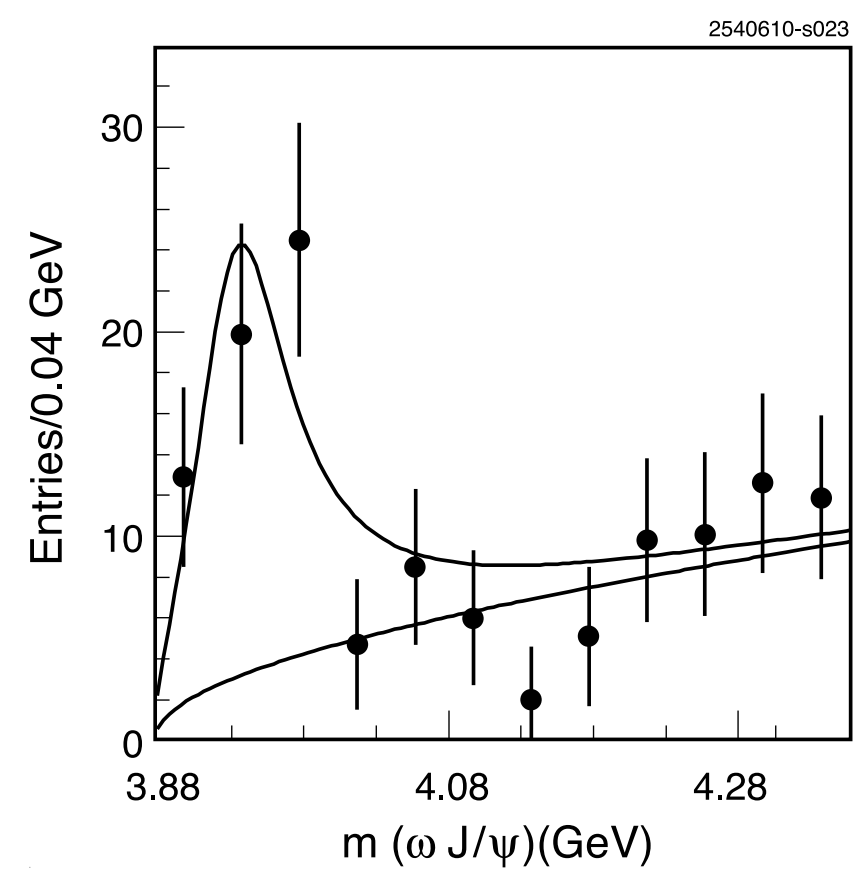

FIG. 23: From Belle [100], the $\omega J / \psi$ mass distribution in $B^{+} \rightarrow K^{+} \omega J / \psi$ decays. The upper curve is the total fit function, the lower one is the contribution of the phase-space-like threshold function. Adapted from [100] with kind permission, copyright (2005) The American Physical Society

\section{$Z(3930), Y(3940)$, and $X(3915)$}

Some of this fog is clearing as more measurements appear. As described previously, the state decaying to $D \bar{D}$, previously known as $Z(3930)$, has been identified as the $\chi_{c 2}(2 P)$. The $Y(3940) \rightarrow \omega J / \psi$ enhancement initially found by Belle [100] in $B^{+} \rightarrow K^{+} Y(3940)$ decays is shown in Fig. 23. It was confirmed by BABAR [101] with more statistics, albeit with somewhat smaller mass, as shown in Fig. 24. But Belle [102] also found a statis-

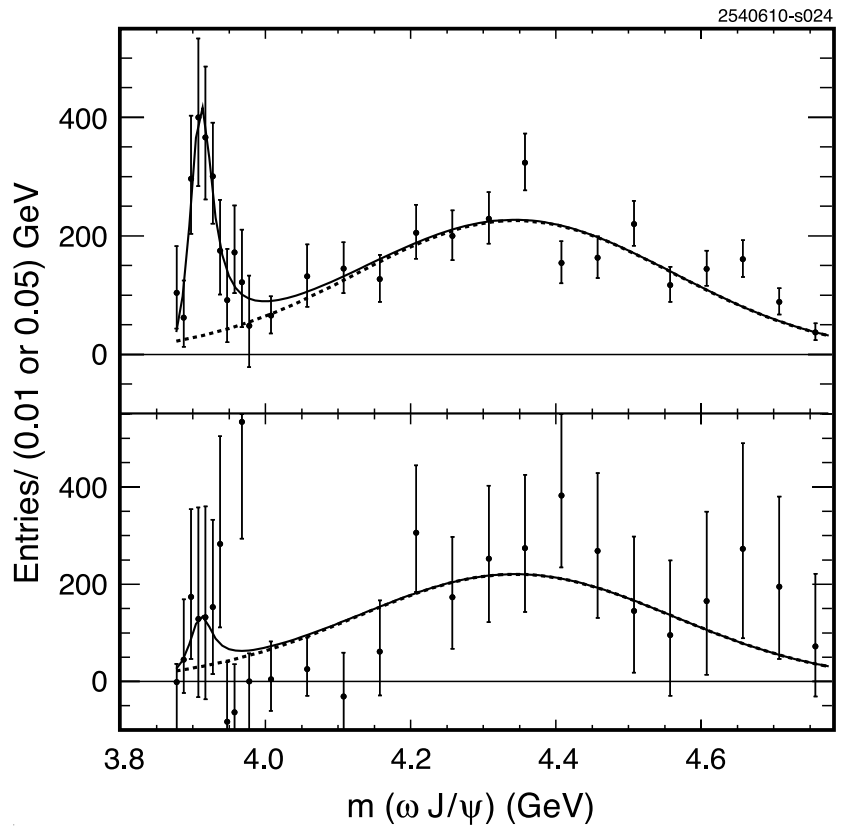

FIG. 24: From BABAR [101], the $\omega J / \psi$ mass distribution in $B^{+} \rightarrow K^{+} \omega J / \psi$ (upper) and $B^{0} \rightarrow K^{0} \omega J / \psi$ (lower) decays. The solid (dashed) curve represents the total fit (background) function. Adapted from [101] with kind permission, copyright (2008) The American Physical Society

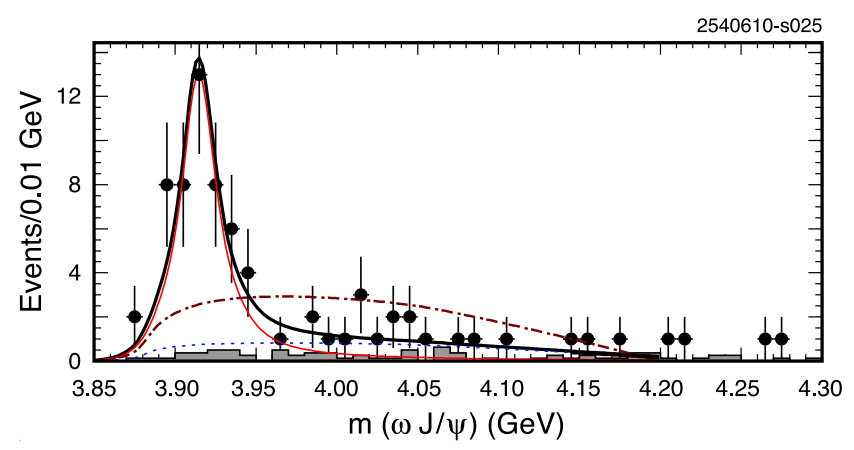

FIG. 25: From Belle [102], the $\omega J / \psi$ invariant mass distribution for the $\gamma \gamma \rightarrow \omega J / \psi$ data (points) and background from scaled non- $\omega J / \psi$ sidebands (shaded). The bold solid, thinner solid, and dashed curves are the total, resonance, and background contributions, respectively. The dot-dashed curve is the best fit with no resonance included. Adapted from [102] with kind permission, copyright (2009) The American Physical Society

tically compelling $(7.7 \sigma)$ resonant structure $X(3915)$ in $\gamma \gamma$ fusion decaying to $\omega J / \psi$, as seen in Fig. 25. As the higher-mass Belle $B \rightarrow K Y(3940)(\rightarrow \omega J / \psi)$ sighting shares the same production and decay signature as that of BABAR's $Y$ (3940), which has mass and width consistent with the $X(3915)$, the simplest interpretation is that the $Y(3940)$ and $X(3915)$ are the same state, and that the latter name should prevail as the mass is closer to 
TABLE 17: Measured properties of the $X(3915) \rightarrow \omega J / \psi$ (subsuming what has previously been called $Y(3940))$. Here $\mathcal{B}_{B} \times \mathcal{B}_{X} \equiv \mathcal{B}\left(B^{+} \rightarrow K^{+} X\right) \times \mathcal{B}(X \rightarrow \omega J / \psi)$ and $\Gamma_{\gamma \gamma} \times \mathcal{B}_{X} \equiv$ $\Gamma(X \rightarrow \gamma \gamma) \times \mathcal{B}(X \rightarrow \omega J / \psi)$

\begin{tabular}{cccc}
\hline \hline Quantity & Value & Decay & Ref. $\left(\chi^{2} /\right.$ d.o.f. $)$ \\
\hline$m$ & $3942 \pm 11 \pm 13$ & $B^{+} \rightarrow K^{+} X$ & Belle [100] \\
$(\mathrm{MeV})$ & $3914.6_{-3.4}^{+3.8} \pm 2.0$ & $B^{+} \rightarrow K^{+} X$ & BABAR [101] \\
& $3915 \pm 3 \pm 2$ & $\gamma \gamma \rightarrow X$ & Belle [102] \\
& $3915.6 \pm 3.1$ & Both & Avg $^{3}(2.7 / 2)$ \\
\hline$\Gamma$ & $87 \pm 22 \pm 26$ & $B^{+} \rightarrow K^{+} X$ & Belle [100] \\
$(\mathrm{MeV})$ & $34_{-8}^{+12} \pm 5$ & $B^{+} \rightarrow K^{+} X$ & BABAR [101] \\
& $17 \pm 10 \pm 3$ & $\gamma \gamma \rightarrow X$ & Belle [102] \\
& $28 \pm 10$ & Both & Avg ${ }^{3}(4.6 / 2)$ \\
\hline $\mathcal{B}_{B} \times \mathcal{B}_{X}$ & $7.1 \pm 1.3 \pm 3.1$ & $B^{+} \rightarrow K^{+} X$ & Belle [100] \\
$\left(10^{-5}\right)$ & $4.9_{-0.9}^{+1.0} \pm 0.5$ & $B^{+} \rightarrow K^{+} X$ & BABAR [101] \\
& $5.1 \pm 1.0$ & $B^{+} \rightarrow K^{+} X$ & Avg ${ }^{3}(0.4 / 1)$ \\
\hline$\Gamma_{\gamma \gamma} \times \mathcal{B}_{X}$ & $61 \pm 17 \pm 8$ & $\gamma \gamma \rightarrow X\left(0^{+}\right)$ & Belle [102] \\
$(\mathrm{keV})$ & $18 \pm 5 \pm 2$ & $\gamma \gamma \rightarrow X\left(2^{+}\right)$ & Belle [102] \\
\hline \hline
\end{tabular}

$3915 \mathrm{MeV}$. It is this reasoning which motivates grouping them together in Tables 9 and 17. The $X(3915)$ clearly has $C=+$, but $J^{P}$ remains to be determined.

What more can be gleaned from the existing measurements? The production rate and total width determinations summarized in Table 17 are useful in testing whether the $X(3915)$ behaves like other charmonia. Existing measurements for product branching fractions in $B^{+} \rightarrow K^{+} \psi$ decays, where $\psi=\eta_{c}(1 S), J / \psi, \chi_{c J}$, $h_{c}(1 P), \eta_{c}(2 S)$, and $\psi(2 S)$, indicate that such states appear with branching fractions of $10^{-3}$ or below. If the $X(3915)$ behaved at all similarly, this would, in turn, imply that $\Gamma(X(3915) \rightarrow \omega J / \psi) \gtrsim 1 \mathrm{MeV}$, whereas $\psi(2 S)$ and $\psi(3770)$ have hadronic transition widths at least ten times smaller. Similarly, the $\gamma \gamma$-fusion results in Table 17 imply a much larger $\Gamma(X(3915) \rightarrow \gamma \gamma)$ than is typical of the few-keV two-photon widths of $\chi_{c 0,2}$, unless $\omega J / \psi$ completely dominates its width (which would also be surprising for a $c \bar{c}$ state). In agreement with this pattern, $X(3915)$ does not appear to have prominent decays to $\gamma J / \psi[97], D \bar{D}[130]$, or $D \bar{D}^{*}[95]$. Hence any conventional $c \bar{c}$ explanation for $X(3915)$ would likely have trouble accommodating these quite uncharmonium-like features. More data and more analysis, especially of angular distributions, are necessary to firmly establish these conclusions.

$\underline{X(3940)}$

The situation for masses near $3940 \mathrm{MeV}$ gets even messier when Belle's analyses of resonances in $e^{+} e^{-} \rightarrow$ $J / \psi(\ldots)$ [54] and $e^{+} e^{-} \rightarrow J / \psi D \bar{D}^{*}$ [103] are considered. The former, as shown in Fig. 26, which examined mass recoiling against a $J / \psi$ in inclusive production, was con- firmed by the latter partial reconstruction analysis with the mass and width shown in Table 9. The latter analysis reconstructed the $J / \psi$ and a $D$ or $D^{*}$, and then used the missing-mass spectrum to isolate events consistent with the desired topology. The event was then kinematically constrained to have the requisite missing mass $\left(D^{*}\right.$ or $D)$, improving resolution on the missing momentum. That four-momentum was then combined with that of the reconstructed $D^{(*)}$ to form the $D \bar{D}^{*}$ invariant mass, as illustrated in Fig. 27, yielding a signal for $X(3940)$ with a significance of $6.0 \sigma$. From mass measurements alone, this state appears distinct from the $X(3915)$ by $3.1 \sigma$. Bolstering this notion, explicit searches for a state with the appropriate mass in $B$-decays (to $D \bar{D}^{*}[95]$ ) or double-charmonium production (decaying to $\omega J / \psi[103]$ ) were negative, yielding incompatible $\omega J / \psi / D \bar{D}^{*}$ relative branching fractions for the two production mechanisms.

$\underline{X(4160)}$

Belle [103] extended the $e^{+} e^{-} \rightarrow J / \psi D \bar{D}^{*}$ analysis to also search for resonances decaying to $D^{*} \bar{D}^{*}$, and found a broad enhancement $X(4160)$ just above $D^{*} \bar{D}^{*}$ threshold with mass and width shown in Table 9 . Double vectorcharmonium production, which can occur through two intermediate virtual photons instead of one, has not yet been observed, so it seems unlikely that this state could be $\psi(4160)$. And if $e^{+} e^{-} \rightarrow J / \psi X(4160)$ is produced via annihilation, its $C$-parity would necessarily be positive.

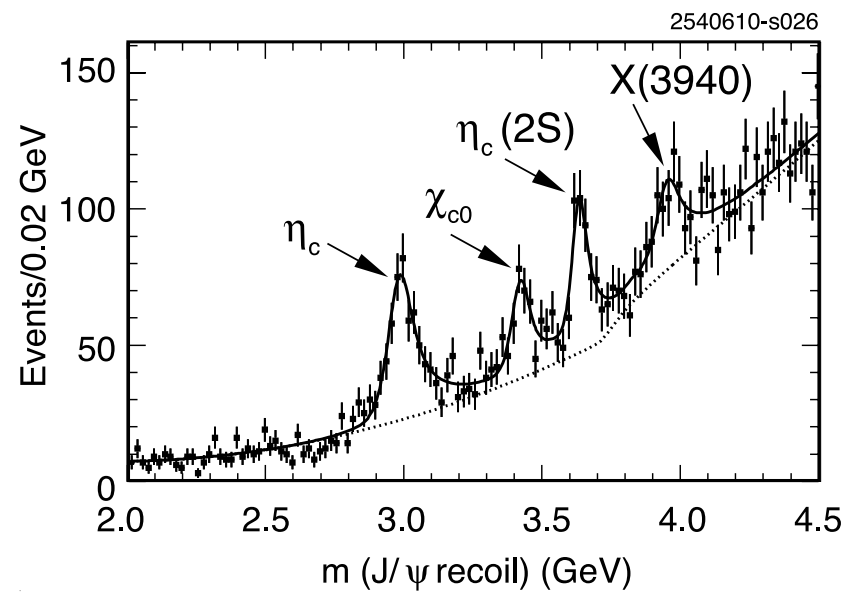

FIG. 26: From Belle [54], the distribution of mass recoiling against the $J / \psi$ in $e^{+} e^{-} \rightarrow J / \psi(\ldots)$ events (points with error bars). Results of the fit are shown by the solid curve, the dashed curve corresponds to the expected background distribution. Adapted from [54] with kind permission, copyright (2007) The American Physical Society 


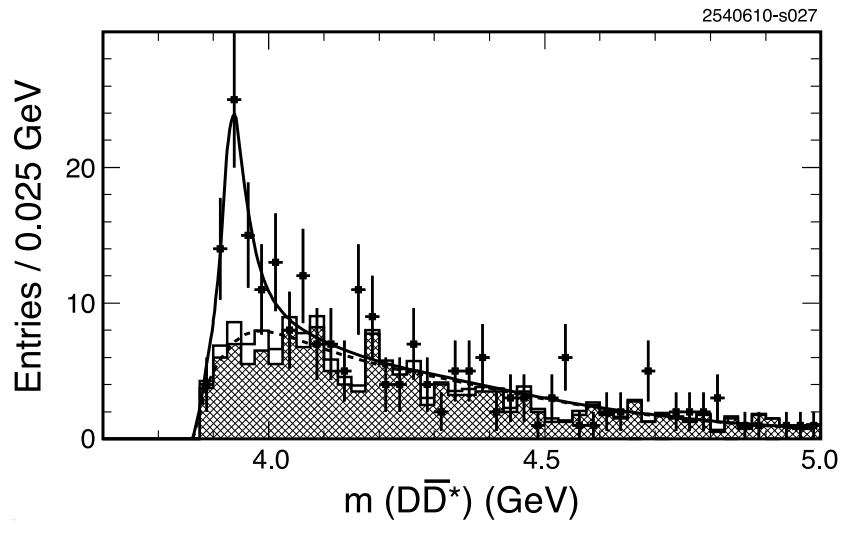

FIG. 27: From Belle [103], the distribution of $D \bar{D}^{*}$ mass recoiling against the $J / \psi$ (points with error bars). Results of the full (background only) fit are shown by the solid (dashed) curve, and the hatched histogram is from the scaled $D^{(*)}$ sidebands. Adapted from [103] with kind permission, copyright (2008) The American Physical Society

$Y(4140), Y(4274)$, and $X(4350)$

New measurements from CDF [106, 107] indicate at least one more $C=+$ state seen in $B$ decays and decaying to two vectors, one being a $J / \psi$, near threshold. In inclusively selected $B^{+} \rightarrow K^{+} \phi J / \psi$ decays, two enhancements in the $\phi J / \psi$ mass spectrum, with masses and widths as shown in Table 9, are $Y(4140)$ and $Y(4274)$. The analysis requires both that the final state be kinematically consistent with $B$-decay, but also uses the CDF particle identification system to require three charged kaons in the decay. The signal significances are $5.0 \sigma$ for $Y(4140)$ and $3.1 \sigma$ for $Y(4274)$. Both remain unconfirmed. However, Belle [112] searched for production of $Y(4140)$ in two-photon fusion, $e^{+} e^{-} \rightarrow e^{+} e^{-} Y(4140)$, $Y(4140) \rightarrow \phi J / \psi$, and found no evidence for it, obtaining a limit of $\Gamma_{\gamma \gamma} \times \mathcal{B}(\phi J / \psi)<40 \mathrm{eV}$ for $J^{P}=0^{+}$ and $<5.9 \mathrm{eV}$ for $2^{+}$at $90 \% \mathrm{CL}$. In that same analysis, Belle reported a $3.2 \sigma$ enhancement, $X(4350) \rightarrow \phi J / \psi$, with mass and width as in Table 9 and a production rate measured to be $\Gamma_{\gamma \gamma} \times \mathcal{B}(\phi J / \psi)=6.7_{-2.4}^{+3.2} \pm 1.1 \mathrm{eV}$ for $J^{P}=0^{+}$and $1.5_{-0.6}^{+0.7} \pm 0.3 \mathrm{eV}$ for $2^{+}$.

\subsubsection{Charged exotic mesons: the Z's}

The charmonium-like charged $Z$ states, seen by Belle in $Z^{-} \rightarrow \psi(2 S) \pi^{-}$and $\chi_{c 1} \pi^{-}$in $B \rightarrow Z^{-} K$ decays, are of special interest. If these states are mesons, they would necessarily have a minimal quark substructure of $c \bar{c} u \bar{d}$ and therefore be manifestly exotic. In a manner similar to the first unearthing of $X(3872), Z^{-}$states were found in exclusively reconstructed $B$-decays in which a conventional charmonium state is a decay product of the $Z^{-}$. Here, a single charged pion accompanies either a $\psi(2 S)$ or $\chi_{c 1}$ (compared to a $\pi^{+} \pi^{-}$pair accompanying a $J / \psi$ in the case of $X(3872))$. The $\psi(2 S)$ is found via either $\psi(2 S) \rightarrow \pi^{+} \pi^{-} J / \psi$ followed by $J / \psi \rightarrow \ell^{+} \ell^{-}$or from direct dileptonic decay, $\psi(2 S) \rightarrow \ell^{+} \ell^{-}$. The $\chi_{c 1}$ is tagged by its decay $\chi_{c 1} \rightarrow \gamma J / \psi, J / \psi \rightarrow \ell^{+} \ell^{-}$. Statistics are gained in the $Z(4430)^{-}$analysis by using both charged and neutral $B$ decays, combining each $Z^{-}$candidate with either a neutral $\left(K_{S}^{0} \rightarrow \pi^{+} \pi^{-}\right)$or charged kaon candidate, if present. In all cases, background not from $B$-decays is small after the usual $B$-selection criteria on expected $B$-candidate energy and mass have been applied, and is well-estimated using appropriately scaled sidebands in those variables. Each experiment finds consistency in various subsets of its own data (e.g., from charged and neutral $B$ mesons and in different $\psi(2 S)$ and $J / \psi$ decay modes) and thereby justifies summing them for final results. Belle and BABAR have comparable mass resolution and statistical power for studying these decays.

Belle found [115] the first $Z^{-}$state by observing a sharp peak near $M\left(\psi(2 S) \pi^{-}\right)=4430 \mathrm{MeV}$ with statistical significance of $>6 \sigma$. The largest backgrounds are $B \rightarrow \psi(2 S) K_{i}^{*}, K_{i}^{*} \rightarrow K \pi^{-}$, where $K_{i}^{*} \equiv K^{*}(892)$ or $K_{2}^{*}(1430)$. Hence $K \pi^{-}$mass regions around $K^{*}(892)$ and $K_{2}^{*}(1430)$ were excised in this Belle analysis. However, as interference between different partial waves in the $K \pi^{-}$system can produce fake "reflection" peaks in the $M\left(\psi(2 S) \pi^{-}\right)$distribution, further attention is warranted. In the kinematically allowed $K \pi^{-}$mass range for this three-body $B$-decay, only $S$-, $P$ - and $D$-partial waves in $K \pi^{-}$are significant. Belle found that no combination of interfering $L=0,1,2$ partial waves can produce an artificial mass peak near $4430 \mathrm{MeV}$ without also producing additional, larger structures nearby in $M\left(\psi(2 S) \pi^{-}\right)$. Such enhancements are absent in the Belle data, ruling out such reflections as the origin of the apparent signal.

In recognition of the role $K \pi^{-}$dynamics can play in the background shape and in response to the BABAR nonconfirming analysis described below, Belle released a second analysis [116] of $Z(4430)^{-}$in their data. Here Belle modeled the $B \rightarrow \psi(2 S) \pi^{-} K$ process as the sum of several two-body decays, with a $B \rightarrow Z(4430)^{-} K$ signal component and, for background, $B \rightarrow \psi(2 S) K_{i}^{*}$, where $K_{i}^{*}$ denotes all of the known $K^{*} \rightarrow K \pi^{-}$resonances that are kinematically accessible. Results of this second analysis are depicted in Fig. 28, which is an $m^{2}\left(\psi(2 S) \pi^{-}\right)$ Dalitz plot projection with the prominent $K^{*}$ bands removed. The data (points with error bars) are compared to the results of the fit with (solid histogram) or without (dashed histogram) the $Z(4430)^{-}$resonance; the former can be seen to be strongly favored over the latter, a $6.4 \sigma$ effect. This Dalitz plot fit yields the mass and width shown in Table 9 as well as the product branching fraction, $\mathcal{B}\left(B^{0} \rightarrow Z^{-} K\right) \times \mathcal{B}\left(Z^{-} \rightarrow \psi(2 S) \pi^{-}\right)=$ $\left(3.2_{-0.9}^{+1.8}{ }_{-1.6}^{+9.6}\right) \times 10^{-5}$. These values for the mass, width, and rate are consistent with the corresponding measurements reported in the initial Belle publication, but have larger uncertainties, which is indicative of the larger set of systematic variations in both signal and background 


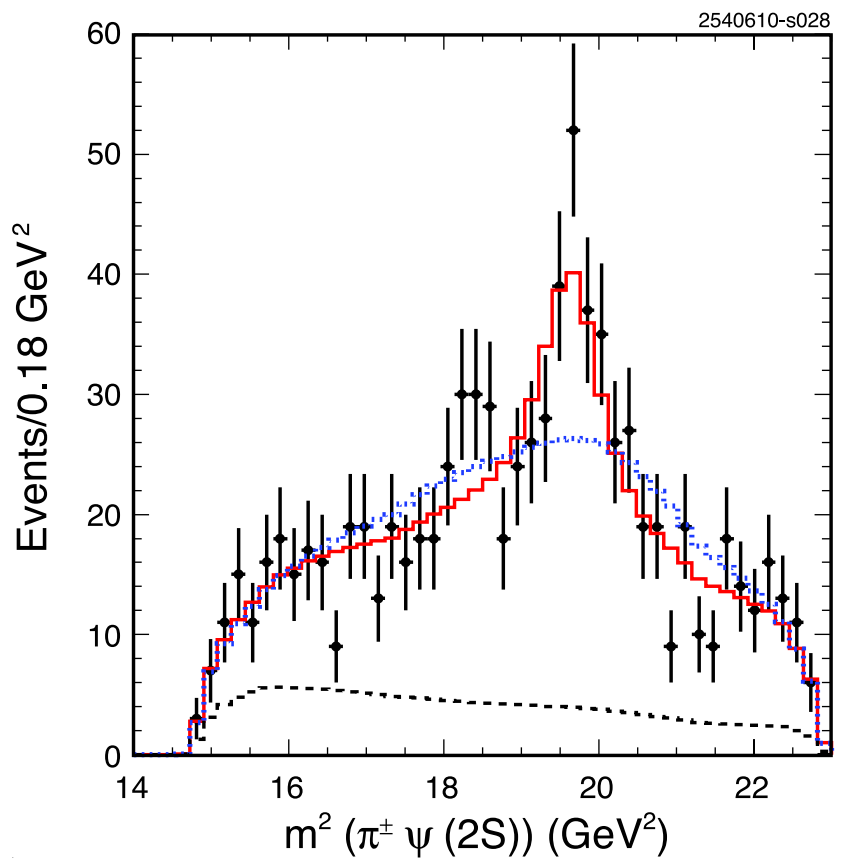

FIG. 28: From Belle [116], for $B \rightarrow K \pi^{-} \psi(2 S)$ candidates, the data points show the $m^{2}\left(\psi(2 S) \pi^{-}\right)$projection of the Dalitz plot with the $K_{i}^{*}$ bands removed. The solid (dotted) histogram shows the corresponding projections of the fits with (without) a $Z(4430)^{-} \rightarrow \psi(2 S) \pi^{-}$resonance term. The dashed histogram represents non- $B$-decay background estimated from energy-difference sidebands. Adapted from [116] with kind permission, copyright (2009) The American Physical Society

properties that were considered. In the default fit, the $Z(4430)^{-}$resonance was assumed to have zero spin. Variations of the fit included a $J=1$ assignment for the $Z(4430)^{-}$, models with hypothetical $K^{*} \rightarrow K \pi^{-}$resonances with floating masses and widths, and radically different parametrizations of the $K \pi^{-} S$-wave amplitude.

The corresponding BABAR search [131] added a decay mode $Z(4430)^{-} \rightarrow J / \psi \pi^{-}$that was not considered in either Belle study. Its inclusion increases statistics for two purposes: first, potentially for more signal, since it is entirely reasonable to expect this mode to occur with at least as large a branching fraction as the discovery mode; and second, to study the $K \pi^{-}$resonance structure in the background, since the $J / \psi$ modes contain about six times more events than those with the $\psi(2 S)$. The BABAR analysis exploits this more copious $B$-decay mode with exhaustive studies of $K \pi^{-}$partial wave dynamics, including fine-grained determination of angular distributions and selection efficiencies over all regions of the Dalitz plot. The data were fit with floating $S$-, $P$-, and $D$-wave intensities. For both $J / \psi \pi^{-} K$ and $\psi(2 S) \pi^{-} K$ samples, good fits are obtained, as shown in the projections in Fig. 29. Hence both $J / \psi \pi^{-}$and $\psi(2 S) \pi^{-}$mass distributions from $B A B A R$ are well-represented by simulations with no extra resonant structure near $M\left(\psi(2 S) \pi^{-}\right)=4430 \mathrm{MeV}$. At the same time, if the $B A B A R M\left(\psi(2 S) \pi^{-}\right)$distribution
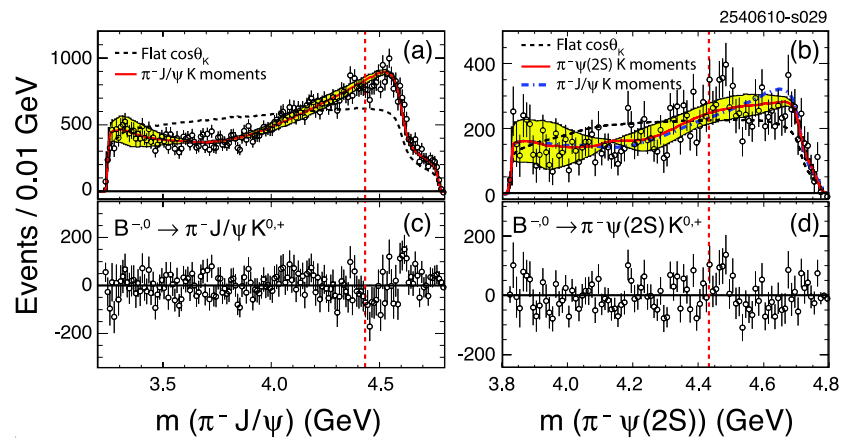

FIG. 29: From BABAR [131], the $\psi \pi^{-}$mass distributions for (a) $B \rightarrow J / \psi \pi^{-} K$ and (b) $B \rightarrow \psi^{\prime} \pi^{-} K$. The points show the data (integrated over all $K \pi^{-}$regions) after efficiency correction and background subtraction. The dashed curves show the $K \pi^{-}$reflection expected for a uniform decay angle $\left(\cos \theta_{K}\right)$ distribution, while the solid curves show the result when accounting for the measured angular variation. The shaded bands represent the effect of statistical uncertainty on the normalized moments. In (b), the dot-dashed curve indicates the result expected if the $K \pi^{-}$properties measured for $J / \psi \pi^{-} K$ are used. The dashed vertical lines indicate the value of $m\left(\psi \pi^{-}\right)=4433 \mathrm{MeV}$. In (c) and (d) appear the residuals (data-solid curve) for (a) and (b), respectively, after the solid curves are subtracted from the data. Adapted from [131] with kind permission, copyright (2009) The American Physical Society

with a $K^{*}(892)$ and $K_{2}^{*}(1430)$ veto (as done by Belle) is fit for the presence of a $Z(4430)^{-}$at the same mass and width found by Belle, a signal with $2 \sigma$ statistical significance is found, indicating a statistical consistency in the corresponding Belle and BABAR mass distributions. This latter finding was verified with a direct bin-by-bin comparison between the Belle and BABAR $M\left(\psi(2 S) \pi^{-}\right)$distributions after the $K^{*}(892)$ and $K_{2}^{*}(1430)$ veto: the two samples were found to be statistically equivalent. That is, while no statistically significant signal for $Z(4430)^{-}$in the $B A B A R$ data has been found, neither does the $B A B A R$ data refute the positive Belle observation of $Z(4430)^{-}$.

Belle has also found [105] signals, dubbed $Z_{1}(4050)^{-}$ and $Z_{2}(4250)^{-}$, in the $\chi_{c 1} \pi^{-}$channel, again using $B \rightarrow$ $Z^{-} K$ decays. Here the kinematically allowed mass range for the $K \pi^{-}$system extends beyond the $K_{3}^{*}(1780) F$ wave resonance. Thus $S$-, $P$ -,$D$ - and $F$-wave terms for the $K \pi^{-}$system are all included in the model. A Dalitz fit with a single resonance in the $Z^{-} \rightarrow \chi_{c 1} \pi^{-}$channel is favored over a fit with only $K_{i}^{*}$ resonances and no $Z^{-}$by $>10 \sigma$. Moreover, a fit with two resonances in the $\chi_{c 1} \pi^{-}$ channel is favored over the fit with only one $Z^{-}$resonance by $5.7 \sigma$. Fitted mass values appear in Table 9 . The product branching fractions have central values similar to that for the $Z(4430)^{-}$but with large errors. Figure 30 shows the $m\left(\chi_{c 1} \pi^{-}\right)$projection of the Dalitz plot with the $K^{*}$ bands excluded and the results of the fit with no $Z^{-} \rightarrow \chi_{c 1} \pi^{-}$resonances and with two $Z^{-} \rightarrow \chi_{c 1} \pi^{-}$ resonances. 


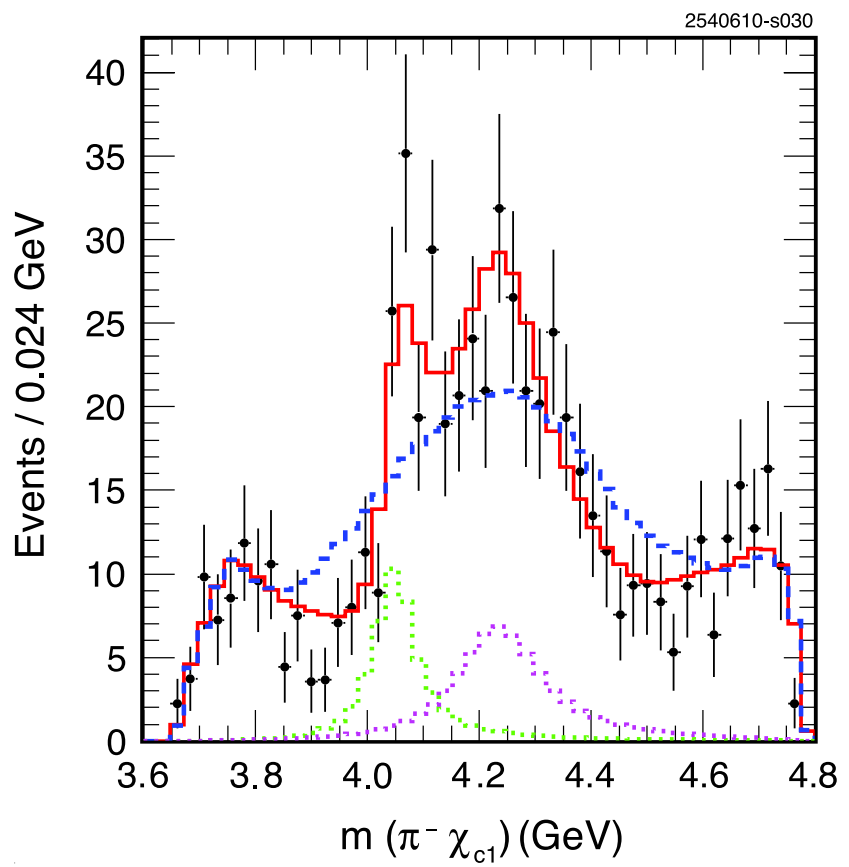

FIG. 30: From Belle [105], for $B \rightarrow K \pi^{-} \chi_{c 1}$ candidates, the data points show the $m\left(\pi^{-} \chi_{c 1}\right)$ projection of the Dalitz plot with the $K^{*}$ bands removed. The solid (dashed) histogram shows the corresponding projection of the fit with (without) the two $Z_{i}^{-} \rightarrow \chi_{c 1} \pi^{-}$resonance terms. The dotted histograms show the fitted contributions of the two resonances. Adapted from [105] with kind permission, copyright (2008) The American Physical Society

Thus, although two experiments have explored these new states, we are left in the less than satisfying situation of three claims of definitive observations and one nonobservation which does not exclude the positive measurement. If any or all of the three charged $Z^{-}$states reported by Belle are in fact meson resonances, they would be "smoking guns" for exotics. It is therefore crucial that these states be confirmed or refuted with independent measurements. In particular, $B A B A R$ should search for $Z_{1}(4050)^{-}$and $Z_{2}(4250)^{-}$in the $\chi_{c 1} \pi^{-}$channel and Belle should search for $Z(4430)^{-}$in the $J / \psi \pi^{-}$channel. That the purported $Z(4430)^{-}$might decay copiously to $\psi(2 S) \pi^{-}$but barely or not at all to $J / \psi \pi^{-}$is a theoretical puzzle worth addressing. The D $\varnothing$ and CDF experiments at the Tevatron could also search for inclusive production of $Z(4430)^{-}$.

\subsection{Characteristics of quarkonium systems}

Heavy quarkonia are systems composed of two heavy quarks, each having mass $m$ much larger than the QCD confinement scale $\Lambda_{\mathrm{QCD}}$. Because the system is nonrelativistic, quarkonium is characterized by the heavy-quark bound-state velocity, $v \ll 1,\left(v^{2} \sim 0.3\right.$ for $c \bar{c}, v^{2} \sim 0.1$ for $b \bar{b}, v^{2} \sim 0.01$ for $t \bar{t}$ ) and by a hierarchy of energy scales: the mass $m$ (hard scale, $\mathrm{H}$ ), the relative momentum $p \sim m v$ (soft scale, S), and the binding energy $E \sim m v^{2}$ (ultrasoft scale, US). For energy scales close to $\Lambda_{\mathrm{QCD}}$, perturbation theory breaks down and one has to rely on nonperturbative methods. Regardless, the nonrelativistic hierarchy of scales,

$$
m \gg p \sim 1 / r \sim m v \gg E \sim m v^{2},
$$

where $r$ is the typical distance between the heavy quark and the heavy antiquark, also persists below the scale $\Lambda_{\mathrm{QCD}}$. Since $m \gg \Lambda_{\mathrm{QCD}}, \alpha_{\mathrm{s}}(m) \ll 1$, and phenomena occuring at the scale $m$ may be always treated perturbatively. The coupling may also be small if $m v \gg \Lambda_{\mathrm{QCD}}$ and $m v^{2} \gg \Lambda_{\mathrm{QCD}}$, in which case $\alpha_{\mathrm{s}}(m v) \ll 1$ and $\alpha_{\mathrm{s}}\left(m v^{2}\right) \ll 1$, respectively. This is likely to happen only for the lowest charmonium and bottomonium states (see Fig. 31). Direct information on the radius of the quarkonia systems is not available, and thus the attribution of some of the lowest bottomonia and charmonia states to the perturbative or the nonperturbative soft regime is at the moment still ambiguous. For $t \bar{t}$ threshold states even the ultrasoft scale may be considered perturbative.

This hierarchy of nonrelativistic scales separates quarkonia [1] from heavy-light mesons, the latter of which are characterized by just two scales: $m$ and $\Lambda_{\mathrm{QCD}}[132,133]$. This makes the theoretical description of quarkonium physics more complicated. All quarkonium scales get entangled in a typical amplitude involving a quarkonium observable, as illustrated in Fig. 32. In particular, quarkonium annihilation and production take place at the scale $m$, quarkonium binding takes place at the scale $m v$ (which is the typical momentum exchanged inside the bound state), while very low-energy gluons and light quarks (also called ultrasoft degrees of freedom) are

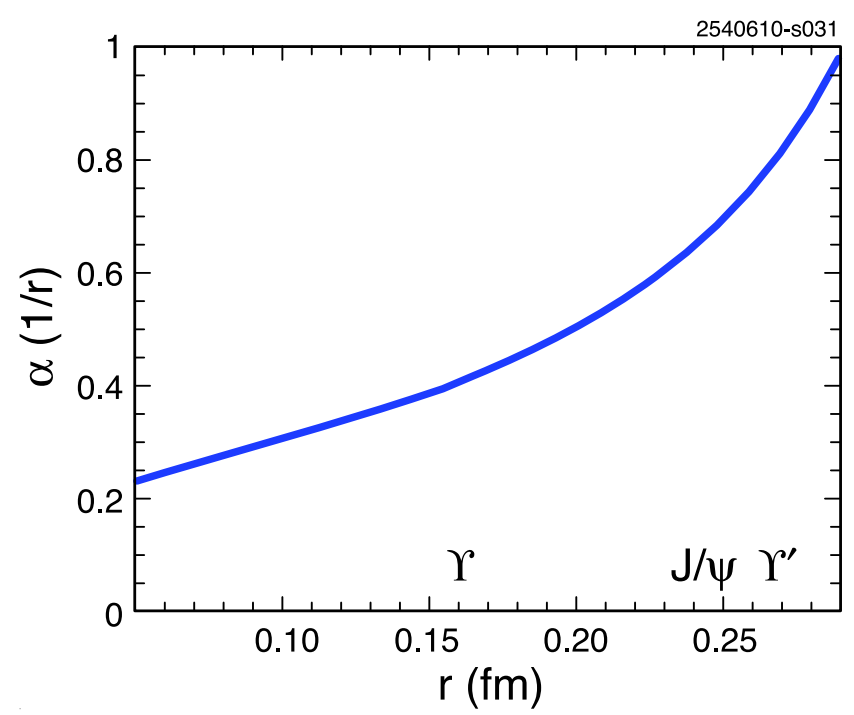

FIG. 31: The strong coupling constant, $\alpha_{\mathrm{s}}$, at one loop, as a function of quarkonium radius $r$, with labels indication approximate values of $m v$ for $\Upsilon(1 S), J / \psi$, and $\Upsilon(2 S)$ 


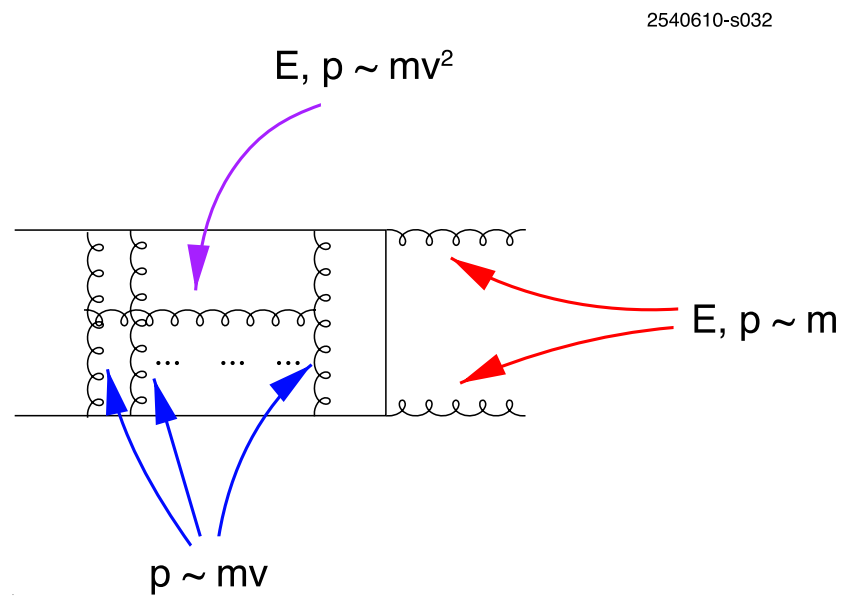

FIG. 32: Typical scales appearing in a quarkonium annihilation diagram

sufficiently long-lived that a bound state has time to form and therefore are sensitive to the scale $m v^{2}$. Ultrasoft gluons are responsible for phenomena like the Lamb shift in QCD. The existence of several scales complicates the calculations. In perturbative calculations of loop diagrams the different scales get entangled, challenging our abilities to perform higher-order calculations. In lattice QCD, the existence of several scales for quarkonium sets requirements on the lattice spacing $(a<1 / m)$ and overall size $\left(L a>1 /\left(m v^{2}\right)\right)$ that are challenging to our present computational power.

However, it is precisely the rich structure of separated energy scales that makes heavy quarkonium particularly well-suited to the study of the confined region of QCD, its interplay with perturbative QCD, and of the behavior of the perturbation series in QCD: heavy quarkonium is an ideal probe of confinement and deconfinement. Quarkonia systems with different radii have varying sensitivies to the Coulombic and confining interactions, as depicted in Fig. 33. Hence different quarkonia will dissociate in a medium at different temperatures, providing, e.g., a thermometer for the plasma, as discussed in Sect. 5.3.

\subsection{Nonrelativistic effective field theories}

The modern approach to heavy quarkonium is provided by Nonrelativistic Effective Field Theories (NR EFTs) [134]. The idea is to take advantage of the existence of a hierarchy of scales to substitute simpler but equivalent NR EFTs for QCD. A hierarchy of EFTs may be constructed by systematically integrating out modes associated with high-energy scales not relevant for the quarkonium system. Such integration is performed as part of a matching procedure that enforces the equivalence between QCD and the EFT at a given order of the expansion in $v$. The EFT realizes factorization between the high-energy contributions carried by the matching

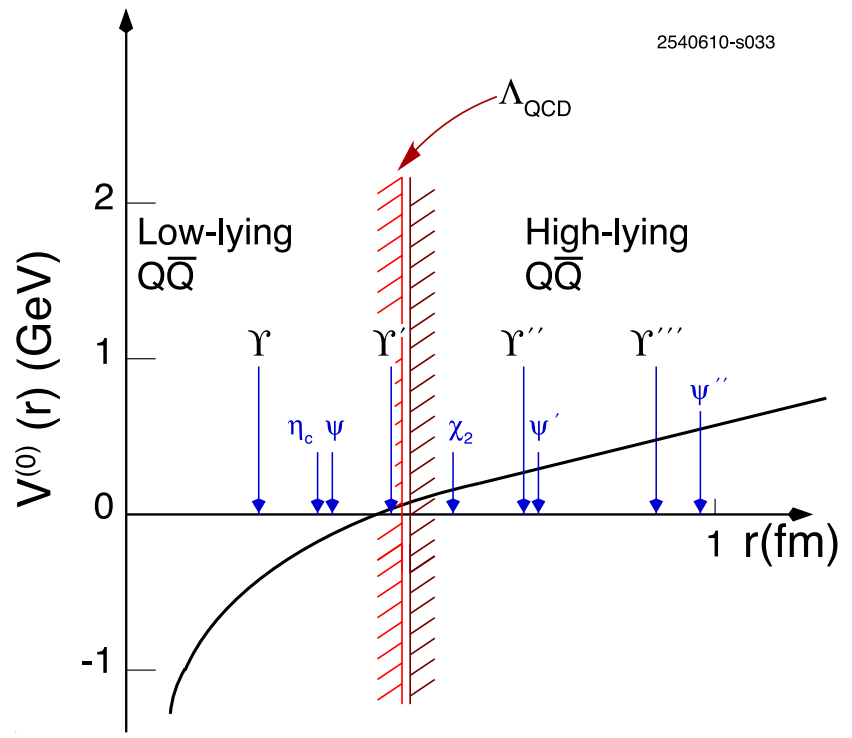

FIG. 33: Static $Q \bar{Q}$ potential as a function of quarkonium radius $r$

coefficients and the low-energy contributions carried by the dynamical degrees of freedom at the Lagrangian level. The Poincaré symmetry remains intact at the level of the NR EFT in a nonlinear realization that imposes exact relations among the EFT matching coefficients $[135,136]$.

\subsubsection{Physics at the scale $m$ : NRQCD}

Quarkonium annihilation and production occur at the scale $m$. The suitable EFT is Nonrelativistic QCD [137, 138], which follows from QCD by integrating out the scale $m$. As a consequence, the effective Lagrangian is organized as an expansion in $1 / m$ and $\alpha_{\mathrm{s}}(m)$ :

$$
\mathcal{L}_{\mathrm{NRQCD}}=\sum_{n} \frac{c_{n}\left(\alpha_{\mathrm{s}}(m), \mu\right)}{m^{n}} \times O_{n}\left(\mu, m v, m v^{2}, \ldots\right)
$$

where $O_{n}$ are the operators of NRQCD that are dynamical at the low-energy scales $m v$ and $m v^{2}, \mu$ is the NRQCD factorization scale, and $c_{n}$ are the Wilson coefficients of the EFT that encode the contributions from the scale $m$ and are nonanalytic in $m$. Only the upper (lower) components of the Dirac fields matter for quarks (antiquarks) at energies lower than $m$. The low-energy operators $O_{n}$ are constructed out of two or four heavyquark/antiquark fields plus gluons. The operators bilinear in the fermion (or antifermion) fields are the same ones that can be obtained from a Foldy-Wouthuysen transformation of the QCD Lagrangian. Four-fermion operators have to be added. Matrix elements of $O_{n}$ depend on the scales $\mu, m v, m v^{2}$ and $\Lambda_{\mathrm{QCD}}$. Thus operators are counted in powers of $v$. The imaginary part of the coefficients of the four-fermion operators contains the information on heavy quarkonium annihilation. The 
NRQCD heavy quarkonium Fock state is given by a series of terms, where the leading term is a $Q \bar{Q}$ in a colorsinglet state, and the first correction, suppressed in $v$, comes from a $Q \bar{Q}$ in an octet state plus a gluon. Higherorder terms are subleading in increasing powers of $v$. NRQCD is suitable for spectroscopy studies on the lattice. The latest results on charmonium, bottomonium, and $B_{c}$ spectroscopy are reported in Sect. 2.6.2. For the latest results on NRQCD inclusive decay amplitudes, see Sect. 3.2.1.

\subsubsection{Physics at the scales $m v, m v^{2}: p N R Q C D$}

Quarkonium formation occurs at the scale $m v$. The suitable EFT is pNRQCD [139, 140], which follows from NRQCD by integrating out the scale $m v \sim r^{-1}$. The soft scale $m v$ may or may not be larger than the confinement scale $\Lambda_{\mathrm{QCD}}$ depending on the radius of the quarkonium system. When $m v^{2} \sim \Lambda_{\mathrm{QCD}}$, we speak about weaklycoupled pNRQCD because the soft scale is perturbative and the matching of NRQCD to pNRQCD may be performed in perturbation theory. When $m v \sim \Lambda_{\mathrm{QCD}}$, we speak about strongly-coupled pNRQCD because the soft scale is nonperturbative and the matching of NRQCD to pNRQCD is not possible in perturbation theory. Below we will review recent results and applications of the two EFTs.

\subsection{3. $m v \gg \Lambda_{\mathrm{QCD}}$ : weakly-coupled $p N R Q C D$}

The effective Lagrangian is organized as an expansion in $1 / m$ and $\alpha_{\mathrm{s}}(m)$, inherited from NRQCD, and an expansion in $r$ [140]:

$$
\begin{gathered}
\mathcal{L}_{\mathrm{pNRQCD}}=\int d^{3} r \sum_{n} \sum_{k} \frac{c_{n}\left(\alpha_{\mathrm{s}}(m), \mu\right)}{m^{n}} \\
\times V_{n, k}\left(r, \mu^{\prime}, \mu\right) r^{k} \times O_{k}\left(\mu^{\prime}, m v^{2}, \ldots\right),
\end{gathered}
$$

where $O_{k}$ are the operators of pNRQCD that are dominant at the low-energy scale $m v^{2}, \mu^{\prime}$ is the pNRQCD factorization scale and $V_{n, k}$ are the Wilson coefficients of the EFT that encode the contributions from the scale $r$ and are nonanalytic in $r$. The degrees of freedom that make up the operators $O_{k}$ are $Q \bar{Q}$ states, color-singlet $S$, color-octet $O_{a} T^{a}$, and (ultrasoft) gluons. The operators are defined in a multipole expansion. In the equations of motion of pNRQCD, we may identify $V_{n, 0}=V_{n}$ with the $1 / m^{n}$ potentials that enter the Schrödinger equation and $V_{n, k \neq 0}$ with the couplings of the ultrasoft degrees of freedom that provide corrections to the Schrödinger equation. Since the degrees of freedom that enter the Schrödinger description are, in this case, both $Q \bar{Q}$ color singlet and $Q \bar{Q}$ color octets, both singlet and octet potentials exist.

The bulk of the interaction is contained in potentiallike terms, but non-potential interactions, associated with the propagation of low-energy degrees of freedom are, in general, present as well and start to contribute at NLO in the multipole expansion. They are typically related to nonperturbative effects. Matrix elements of $O_{n, k}$ depend on the scales $\mu^{\prime}, m v^{2}$ and $\Lambda_{\mathrm{QCD}}$.

If the quarkonium system is small, the soft scale is perturbative and the potentials can be entirely calculated in perturbation theory [134]. They are renormalizable, develop a scale dependence, and satisfy renormalization group equations that eventually allow resummation of potentially large logarithms.

\section{Progress in the perturbative calculation of the potentials}

\section{- $Q \bar{Q}$ singlet and octet potentials}

There has been much progress in the computation of the perturbative corrections to the potential between a static quark and a static antiquark in a color-singlet configuration. The subleading logarithmic term coming from ultrasoft effects has been computed [141]. The resummation of those subleading ultrasoft logarithms has also been performed [142], along with a comparison of the static energy to lattice data [142]. These calculations confirm that perturbation theory can reproduce the lattice very accurately at short distances (up to $0.25 \mathrm{fm}$ ) once the cancellation of the leading renormalon singularity is implemented.

The three-loop correction to the static potential is now completely known: the fermionic contributions to the three-loop coefficient [143] first became available, and, more recently, the remaining purely gluonic term has been obtained $[144,145]$. The value found for the $n_{f^{-}}$ independent three-loop coefficient is much lower than the previous, widely-used Padé estimate and within the range obtained by comparing to lattice data [142]. The future implementation of the three-loop result may improve the precision of some mass and strong-coupling determinations. In particular, the recently obtained theoretical expression [146] for the complete QCD static energy at next-to-next-to-next-to-leading-logarithmic accuracy (NNNLL) has been used to determine $r_{0} \Lambda_{\overline{\mathrm{MS}}}$ by comparison with available lattice data, where $r_{0}$ is the lattice scale and $\Lambda_{\overline{\mathrm{MS}}}$ is the QCD scale, obtaining

$$
r_{0} \Lambda_{\overline{\mathrm{MS}}}=0.622_{-0.015}^{+0.019}
$$

for the zero-flavor case. This extraction was previously performed at the NNLO level (including an estimate at NNNLO) in [147]. The same procedure can be used to obtain a precise evaluation of the unquenched $r_{0} \Lambda_{\overline{\mathrm{MS}}}$ value after short-distance unquenched lattice data for the $Q \bar{Q}$ exist.

At three-loop order a violation of the Casimir scaling for the static QQbar potential is found, see [148].

The static octet potential is known up to two loops [149]. Relativistic corrections to the static singlet potential have been calculated over the years and are summarized in [134]. 


\section{- $Q Q Q$ and $Q Q q$ static potentials}

The three-quark static potential has been calculated in perturbation theory at next-to-leading order in the singlet, octet, and decuplet channels [150, 151]. Mixing between the octet representations has been found already at tree level. At next-to-next-to-leading order, the subset of diagrams producing three-body forces has been identified in Coulomb gauge and their contribution to the potential calculated. Combining it with the contribution of the two-body forces, which may be extracted from the quark-antiquark static potential, the complete next-tonext-to-leading order of the three-quark static potential in the color-singlet channel has been obtained in [150]. These results may be important for accurate calculations of the lowest $Q Q Q$ states as well as for comparison and study of the $Q Q Q$ lattice static energies in the domain of small interquark distance.

The same pNRQCD description is also possible for $Q Q$ states, which are relevant for doubly-charmed baryons [151, 152]. The $Q Q$ antitriplet static potential, relevant for the $Q Q q$ states, has been calculated at nextto-next-to-leading order [150]. First lattice calculations of the $Q Q q$ potential have also become available [153$155]$.

\section{Progress on the lowest spectra calculation}

For systems with small radii, precision calculations are possible. In such cases, quarkonium may become a benchmark for our understanding of QCD, in particular for the transition region between perturbative and nonperturbative QCD, and for the precise determination of Standard Model parameters (e.g., heavy quark masses, $\alpha_{\mathrm{s}}$, as described in Sect. 2.8). When the soft scale is perturbative, the energy levels are given by the expectation value of the perturbative potentials, calculated to the appropriate order of the expansion in $\alpha_{\text {s }}$ plus nonperturbative terms that are not part of the potential, which start to contribute to the energy levels at order $m \alpha_{\mathrm{s}}{ }^{5}$. They enter energy levels and decay calculations in the form of local or nonlocal electric and magnetic condensates [134, 156-159]. A precise and systematic knowledge of such nonperturbative, purely glue-dependent objects is still lacking. It is therefore important that these condensates be fixed, either by dedicated lattice determinations or extracted from the data. Within pNRQCD it is possible to relate the leading electric and magnetic nonlocal correlators to the gluelump masses and to some existing lattice (quenched) determinations [134].

However, since the nonperturbative contributions are suppressed in the power counting, it is possible to obtain precise determinations of the masses of the lowest quarkonium resonances within purely perturbative calculations, in the cases in which the perturbative series is convergent (i.e., after the appropriate subtractions of renormalons have been performed), and large logarithms are resummed [160-163].

Once the potentials are known, energy levels can be computed. The lowest heavy quarkonium states are suit- able to obtain precise determinations of the $b$ and $c$ mass. Such determinations are competitive with those coming from different systems and different approaches, as has been discussed at length [1]. An update of recent result on mass extractions is given in Sect. 2.8.

Once the quark masses have been obtained, it is possible to obtain the energy levels of the lowest resonances. However, which quarkonium state belongs to which regime is an open issue and no clear-cut method exists to decide this a priori, since we lack a direct method to determine the quarkonium radius [164, 165]. Typically the lowest states $\Upsilon(1 S), \eta_{b}, B_{c}$, and possibly $J / \psi$ and $\eta_{c}$ are assumed to be in the weakly-coupled regime. The $S$-wave energy levels are known in perturbation theory at next-to-next-to-next-to-leading order $\left(m \alpha_{\mathrm{s}}{ }^{5}\right)$ [156, 157, 166-169].

A prediction of the $B_{c}$ mass has been obtained [170]. The NNLO calculation with finite charm mass effects $[171,172]$ predicts a mass of $6307(17) \mathrm{MeV}$, in agreement with the CDF measurement [173] and the lattice determination [74]. A NLO calculation reproduces, in part, the $\chi_{b J}(1 P)$ fine splitting [174]. The same procedure seems to work at NNLO even for higher-mass bottomonium states (i.e., measured masses match the predictions within the respective theory errors, which are larger for higher-mass states) [171]. Including logs resummation at NLL, it is possible to obtain a prediction [175]

$$
\Delta m_{\mathrm{hf}}\left[\eta_{b}(1 S)\right]=41 \pm 11(\mathrm{th})_{-8}^{+9}\left(\delta \alpha_{\mathrm{s}}\right) \mathrm{MeV}
$$

in which the second error comes from the uncertainty in $\alpha_{\mathrm{s}}$. This value is consistent with another perturbative prediction [176] but both undershoot the average experimental value from Eq. (5) by about $2 \sigma$. This discrepancy with experiment remains a challenge for theory. (There are further discussions of the $\eta_{b}(1 S)$ hyperfine splitting in Sects. 2.2.5, 2.6.2, 2.7, 2.8.1, and 2.10.1, the last of which offers the possibility of new physics becoming manifest in $\eta_{b}(n S)$ mass splittings.) Similar calculations yield a predicted $B_{c}$ hyperfine separation [177]

$$
m\left(B_{c}^{*}\right)-m\left(B_{c}\right)=50 \pm 17_{-12}^{+15} \mathrm{MeV} .
$$

\subsection{4. $m v \sim \Lambda_{\mathrm{QCD}}:$ strongly-coupled $p N R Q C D$}

When $m v \sim \Lambda_{\mathrm{QCD}}$ the soft scale is nonperturbative and the matching from NRQCD to pNRQCD cannot be performed in perturbation theory. Then the potential matching coefficients are obtained in the form of expectation values of gauge-invariant Wilson-loop operators. In this case, heavy-light meson pairs and heavy hybrids develop a mass gap of order $\Lambda_{\mathrm{QCD}}$ with respect to the energy of the $Q \bar{Q}$ pair, the second circumstance being apparent from lattice simulations. Thus, away from threshold, the quarkonium singlet field $S$ is the only low-energy dynamical degree of freedom in the pNRQCD Lagrangian (neglecting ultrasoft corrections coming from pions and 
other Goldstone bosons). This pNRQCD Lagrangian may be expressed as [134, 178, 179]:

$$
\mathcal{L}_{\mathrm{pNRQCD}}=S^{\dagger}\left(i \partial_{0}-\frac{\mathbf{p}^{2}}{2 m}-V_{S}(r)\right) S .
$$

The singlet potential $V_{S}(r)$ can be expanded in powers of the inverse of the quark mass; static, $1 / m$ and $1 / m^{2}$ terms were calculated long ago [178, 179]. They involve NRQCD matching coefficients (containing the contribution from the hard scale) and low-energy nonperturbative parts given in terms of static Wilson loops and fieldstrength insertions in the static Wilson loop (containing the contribution from the soft scale). In this regime of pNRQCD, we recover the quark potential singlet model. However, here the potentials are calculated in QCD by nonperturbative matching. Their evaluation requires calculations on the lattice or in QCD vacuum models.

Recent progress includes new, precise lattice calculations of these potentials obtained using the Lüscher multi-level algorithm (see Sect. 2.6.3 for more details).

Then, away from threshold, all the masses can be obtained by solving the Schrödinger equation with such potentials. Some applications of these results to the spectrum calculation are ongoing [180].

A trivial example of application of this method is the mass of the $h_{c}(1 P)$. The lattice data show a vanishing long-range component of the spin-spin potential. Thus the potential appears to be entirely dominated by its short-range, delta-like, part, suggesting that the ${ }^{1} P_{1}$ state should be close to the center-of-gravity of the ${ }^{3} P_{J}$ system. Indeed, the measurements described in Sect. 2.2.1 and summarized in Table 5 are consistent with this expected value.

If we explicitly consider light quarks, each quarkonium state may develop a width due to decay through pion emission. The neavy-light states develop a mass gap of order $\Lambda_{\mathrm{QCD}}$ with respect to quarkonium which can be absorbed into the definition of the potentials or of the (local or nonlocal) condensates [181].

\subsection{Lattice QCD spectrum calculations}

In quarkonia, the ultrasoft scale $m v^{2}$ is often of a similar size as the scale $\Lambda_{\mathrm{QCD}}$, where non-perturbative effects become important. For all charmonium and many bottomonium states the soft scale $m v \sim 1 / r$ is not much larger than $\Lambda_{\mathrm{QCD}}$ either. The non-perturbative contributions can be evaluated via computer simulations of lattice-regularized QCD (Lattice QCD), where the lattice spacing $a$ provides a hard ultraviolet cut-off on the available momenta in a Euclidean space-time volume.

Light sea quarks are particularly expensive to simulate numerically since the computational effort increases as a large inverse power of the corresponding pseudoscalar mass $m_{\mathrm{PS}}$. The spatial lattice extent, $L a$, should be much larger than $m_{\mathrm{PS}}^{-1}$ to control finite-size effects, necessitating large volumes. Only very recently have the first simulations near the physical pion mass $m_{\mathrm{PS}} \approx m_{\pi}$ become possible [182-185]. In the first reference a variant of the staggered fermion action was applied. These fermions are usually only defined for $n_{f}$ mass-degenerate fermions, where $n_{f}$ is a multiple of four. This restriction was circumvented by replacing the determinant of the $n_{f}=8+4$ staggered action by a fourth root that may then correspond to $n_{f}=2+1$. However, it remains controversial whether this ad hoc prescription recovers a unitary, local quantum field theory and thus the correct continuum limit. Moreover, there are claims that the additional so-called taste symmetry cannot be completely restored in the continuum limit [186, 187], some aspects of which have been refuted [188], and others of which have yet to be clearly established [189].

The lattice regularization of QCD is not unique, and many different discretized actions can be constructed that should yield the same results after removing the cutoff (continuum limit extrapolation: $a \rightarrow 0$ ). While the Wilson action is subject to $O(a)$ discretization effects, other actions are $O(a)$ improved, e.g., chiral actions fulfilling the Ginsparg-Wilson relation, staggered actions, twisted-mass QCD or the nonperturbatively-improved Sheikholeslami-Wilson (clover) action. Ideally, lattice simulations are repeated at several values of the lattice spacing, $a \ll \Lambda_{\mathrm{QCD}}^{-1}$, and the results then extrapolated to the continuum limit. Lattice artifacts will be large if physics at scales $q \nless a^{-1}$ becomes important, spoiling the continuum limit extrapolation. In this respect, the charm quark mass, $m_{c}$, and, even more so, the bottom quark mass, $m_{b}$, pose challenges. By exploiting or ignoring the multiscale nature of quarkonium systems, different routes can be taken.

One way to proceed is to integrate out $m$ as well as $m v$ and to evaluate the resulting potential nonperturbatively in lattice simulations of pNRQCD [139, 140]. Subsequently, the energy levels can be obtained by solving a Schrödinger equation. This directly relates lattice simulations to QCD vacuum models and to potential models. However, at present this approach is only semiquantitative since no calculations of the matching of lattice pNRQCD, where rotational symmetry is broken, to QCD is available.

Another approach, which is more common, is to integrate out only $m$ and to simulate NRQCD [137, 138] on the lattice [190]. In this case, the lattice spacing, $a^{-1}$, plays the role of the soft matching scale, $\mu$, used above. Unless this is restricted to $m \gtrsim a^{-1} \gtrsim m v$, the NRQCD matching coefficients will become uncontrollably large, and the continuum limit cannot be taken. Therefore $a$ must be sufficiently fine for discretization effects to be smaller than the neglected higher-order terms in the heavy-quark expansion. This restricts the applicability of NRQCD methods to systems containing $b$ quarks.

Relativistic heavy quark actions can also be used because the inequality $a^{-1} \gg m$ usually still holds so that spin-averaged level-splittings can be obtained. To a lesser extent, reliable results on the fine structure can 
be achieved as well. The Fermilab effective field theory interpretation of the heavy quark action [191, 192] smoothly connects the region $m \sim a^{-1}$ with the continuum limit $a^{-1} \gg m$, allowing charm and bottom systems to be treated in the same set up.

The following sections survey the present state-of-theart. We start with results obtained using a relativistic heavy-quark action, continue with NRQCD, and conclude with lattice pNRQCD results.

\subsubsection{Relativistic heavy quark actions}

One way of limiting the computational cost of small lattice spacings, i.e., of a large number of lattice points, is the use of anisotropic actions, with a temporal lattice spacing, $a_{\tau}$, smaller than the spatial lattice spacing, $a_{\sigma}=\xi a_{\tau}$, where $\xi>1$. The spatial lattice extent, $L_{\sigma} a_{\sigma}$, still needs to be sufficiently large to accommodate the quarkonium state, which has a size of order $r \simeq(m v)^{-1}$. In the presence of light sea quarks, one would additionally wish to realize $L_{\sigma} a \gg m_{\mathrm{PS}}^{-1}$. With sufficiently large $a_{\sigma}$, it is possible to limit the number of lattice points, $\propto L_{\sigma}^{3}$. It is then easy to realize $a_{\tau}<m^{-1}$, where $m$ is the particle mass. Naively, such simulations are cheaper by a factor $\xi^{3}$ relative to the isotropic case. This method was successfully explored in the quenched approximation [193, 194], and is reviewed in [1].

At tree level, it can be arranged that the lattice spacing errors are $O\left[\left(m a_{\tau}\right)^{n}\right]$, where $n \in\{1,2\}$ depends on the heavy-quark action, but care is needed to ensure it [195]. One-loop corrections may lead to $O\left[\alpha_{\mathrm{s}}\left(m a_{\sigma}\right)^{n}\right]$ terms: to the extent that $\alpha_{\mathrm{s}} \xi^{n}$ is small, the leading-order lattice effects can be regarded as $O\left[\left(m a_{\tau}\right)^{n}\right]$. The anisotropy parameter $\xi$ must be determined consistently for the quark and gluon contributions to the QCD action. Within the quenched approximation, where the feedback of quark fields onto the gluons is neglected, this problem factorizes. The tuning is much harder to achieve and numerically more costly in QCD with sea quarks. Nevertheless such a program was pursued very successfully by the Hadron Spectrum Collaboration [196]. It should be noted, however, that, in this case, the $O(a)$ improvement is not nonperturbatively accurate.

Such anisotropic configurations have been employed to calculate electromagnetic transition rates from excited charmonium states, high-spin states, and exotics [71, 197, 198] with small volumes. The lattice spacing and sea quark mass dependencies will be investigated in the near future. The same holds for an exploratory study of the Regensburg group, using the isotropic clover action for $n_{f}=2$ sea quarks (generated by the QCDSF Collaboration [199]) and charm quarks, using a lattice cutoff $a^{-1} \approx 1.7 \mathrm{GeV}$ [200]. While the spin-averaged splittings are in qualitative agreement with experiment, the fine structure is underestimated due to unphysicallyheavy sea quarks and the missing continuum limit extrapolation. Another study with improved Wilson fermions was performed by PACS-CS with $n_{f}=2+1$ sea quark flavours at the physical $\pi$ mass [183], focusing only on very few $J^{P C}$ channels. In this case, the $J / \psi-\eta_{c}(1 S)$ splitting is underestimated by about $10 \%$ relative to experiment, which can probably be attributed to the finite lattice spacing. Note that valence quark annihilation channels were omitted in all these studies.

To this end, mixing effects with noncharmed mesons and flavor-singlet contributions to the spectrum were evaluated [201] and found to be smaller than $10 \mathrm{MeV}$ in the pseudoscalar channel. The latter effect was also investigated by the MILC Collaboration [202], with similar results. It should be noted that valence quark annihilation diagrams were neglected in all other unquenched studies. Finally, mixing effects between radially-excited $c \bar{c}$ states and four-quark molecules (or tetraquarks) were investigated at a sea pion mass, $m_{\mathrm{PS}}<300 \mathrm{MeV}$, at $a^{-1} \approx 2.4 \mathrm{GeV}$ [203]. Indications of attraction and mixing were found in the $1^{++}$channel, supporting the interpretation of the $X(3872)$ as a $D^{* 0} \bar{D}^{0}$ bound state with a $\chi_{c 1}(2 P)$ admixture. Other charmed tetraquark studies [204, 205] were performed in the quenched approximation and did not take into account valence-annihilation diagrams. A recent review on the tetraquark topic is presented in [206].

At present, all lattice studies of heavy quarkonium spectroscopy in which a continuum limit is taken are based on $n_{f} \approx 2+1$ configurations generated by the MILC Collaboration [207] using the so-called AsqTad improved staggered sea-quark action [208]. Together with the Fermilab Lattice Collaboration, charmonium and bottomonium spectra were investigated using the clover action for the heavy quarks at four different lattice spacings ranging from $(1.2 \mathrm{GeV})^{-1}$ down to $(2.2 \mathrm{GeV})^{-1}$, and various light quark masses [209]. Only $S$ - and $P$ waves were studied. After extrapolating to the physical limit, all spin-averaged splittings, with the exception of the charmonium $2 \bar{S}-1 \bar{S}$ splitting, which is muddled with threshold effects, are in agreement with experiment. Moreover, the $1 S$ and $1 P$ fine structures are compatible with the experimental values. The continuum limit extrapolation was essential to achieve this. The same combination of AsqTad sea-quark action and clover charmquark action was also used in a recent calculation of singly- and doubly-charmed baryons [210].

\subsubsection{NRQCD lattice calculations}

As discussed above, lattice NRQCD is a suitable method for bottomonia studies, for which $a m_{b}>1$ and $\Lambda_{\mathrm{QCD}} /\left(v m_{b}\right) \ll 1$. However, the precision of the results for fine-structure splittings is limited here by the fact that the QCD matching coefficients are typically taken at tree level only in such lattice simulations.

The HPQCD and UKQCD Collaborations presented calculations [211] of the bottomonium spectrum using the $n_{f}=2+1$ MILC gauge configurations [207] described 
above, expanding to order- $v^{4}$ in the heavy-quark velocity $v$. Another lattice calculation for bottomonium [212] that closely followed the methods of [211] was later released, but using the $n_{f}=2+1$ configurations provided by the RBC and UKQCD Collaborations [213]) with the chiral domain-wall sea-quark action for a single lattice spacing $a^{-1} \approx 1.7 \mathrm{GeV}$, also expanding to order $v^{4}$. Both calculations [211, 212] found agreement with experiment for the spin-averaged splittings. The spin-dependent splitting was seen to be systematically underestimated in [212], possibly due to the omission of relativistic, radiative, and discretization corrections (see Sect. 2.7 for more on $\left.\Delta m_{\mathrm{hf}}\left[\eta_{b}(1 S)\right]\right)$. The same method was then used to calculate the spectrum of other states containing one or two $b$-quarks, including baryons [212, 214]. Again, the underestimation of the experimental fine structure might be due to either the coarse lattice spacing or the imprecise matching between lattice NRQCD and QCD.

Large scale simulations of the spectrum of $\Upsilon$ states and $B_{c}$, which include sea quark mass and continuum limit extrapolations, were performed by the HPQCD collaboration $[215,216]$. They employed an NRQCD action for the $b$ quark and combined this with the relativistic HISQ (highly improved staggered quark) action for the charm quark. Their findings are very similar to those of [209]; it will be very interesting to perform a detailed comparison between results from a relativistic $b$-quark action and NRQCD on the same ensemble of gauge configurations.

The mass of the triply-heavy baryon $\Omega_{b b b}$ has been calculated [217] in lattice QCD with $2+1$ flavors of light sea quarks. The $b$-quark is implemented with improved lattice NRQCD. Gauge field ensembles from both the RBC/UKQCD and MILC collaborations with lattice spacings in the range from $0.08-0.12 \mathrm{fm}$ are used. The final result for the mass, which includes an electrostatic correction, is

$$
\begin{aligned}
m\left(\Omega_{b b b}\right)=14.371 & \pm 0.004 \text { (stat.) } \pm 0.011 \text { (syst.) } \\
& \pm 0.001 \text { (exp.) GeV. }
\end{aligned}
$$

The hyperfine splitting between the physical $J=\frac{3}{2}$ state and a fictitious $J=\frac{1}{2}$ state is also presented [217].

\subsection{3. $p N R Q C D$ lattice calculations}

Another approach is offered by pNRQCD [178, 179]. Unfortunately, lattice pNRQCD also suffers from the fact that the matching coefficients between lattice NRQCD and QCD are only known at tree level. pNRQCD bridges the gap between QCD and potential models. Implemented on the lattice, it amounts to calculating static potentials and spin- and velocity-dependent corrections. Recent and not so recent progresses in this area include the calculation of string breaking in the static sector [218], which offers one entry point into the study of threshold states, the calculation of potentials in baryonic static-static-light systems [155, 219], and the calculation of interaction energies between two static-light mesons [220]. New very precise results on all leading relativistic corrections to the static $Q \bar{Q}$ potential have also been calculated [221-223].

\subsection{Predictions for the $\eta_{b}(1 S)$ mass}

The calculation described above in Sect. 2.5.3 with result in Eq. (13) gives a numerical result for $\Delta m_{\mathrm{hf}}\left[\eta_{b}(1 S)\right]$ $(41 \pm 14 \mathrm{MeV})$ that is typical of perturbative calculations (e.g., $44 \pm 11 \mathrm{MeV}$ is given by [176]). These values are somewhat smaller than those obtained from lattice NRQCD as described in Sect. 2.6.2 above (e.g., $61 \pm$ $14 \mathrm{MeV}$ in [211] and $52 \pm 1.5$ (stat.) $\mathrm{MeV}$ in [212]). However, it has been argued [224] that additional short-range corrections of $\delta^{\text {hard }} \Delta m_{\mathrm{hf}}\left[\eta_{b}(1 S)\right] \approx-20 \mathrm{MeV}$ would lower these unquenched lattice results to the level of the perturbative predictions.

Very recently a newer lattice prediction [225] performed at order $v^{6}$ in the NRQCD velocity expansion has been obtained at tree level for the NRQCD matching coefficients and with domain-wall actions for sea quarks, including the spin splittings, and based on the RBC/UKQCD gauge field ensembles. This approach [225] addresses the concerns in [224] (namely, that radiative contributions in the calculation are missing because the NRQCD matching coefficients are calculated at tree level) by calculating appropriate ratios of spin splittings and thereafter normalizing to a measured value. With this method, and using the experimental result for the $1 P$ tensor splitting as input, a $1 S$ bottomonium hyperfine splitting of

$$
\begin{aligned}
\Delta m_{\mathrm{hf}}\left[\eta_{b}(1 S)\right]=60.3 & \pm 5.5 \text { (stat.) } \\
& \pm 5.0 \text { (syst.) } \\
& \pm 2.1 \text { (exp.) } \mathrm{MeV} \\
=60.3 & \pm 7.7 \mathrm{MeV}
\end{aligned}
$$

is determined. This value is slightly smaller $(1.1 \sigma)$ than but consistent with the experimental measurements

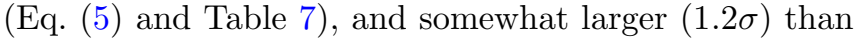
but consistent with earlier pQCD calculations [175, 176] (see Eq. (13)). However, this still leaves the weaklycoupled pNRQCD (perturbative) and experimental values $2.0 \sigma$ apart, and it is not clear why; the lattice NRQCD lies in between. More study and better precision in the predictions are required to gain confidence in both perturbative and nonperturbative calculations. (See further discussions of the $\eta_{b}(1 S)$ hyperfine splitting in Sects. 2.2.5, 2.5.3, 2.8.1, and 2.10.1).

\subsection{Standard Model parameter extractions}

Given the progress made in the effective field theories formulation, in the calculation of high order perturbative contributions and in the lattice simulations, quarkonium 
appears to be a very suitable system for precise determination of Standard Model parameters like the heavy quark masses and $\alpha_{\mathrm{s}}$. Below we report about recent determinations. For a review and an introduction to the procedure and methods used in this section, see [1].

\subsection{1. $\alpha_{\mathrm{s}}$ determinations}

Below we review several extractions of the strong coupling constant related to observables in heavy quarkonium physics. All values for $\alpha_{\mathrm{s}}$ are quoted in the $\overline{\mathrm{MS}}$ scheme with $n_{f}=5$, unless otherwise indicated.

\section{$\alpha_{\mathrm{s}}$ from quarkonia masses on the lattice}

A precise determination of $\alpha_{\mathrm{s}}$ from lattice simulations has been presented by the HPQCD Collaboration [182]. The mass difference $m[\Upsilon(2 S)]-m[\Upsilon(1 S)]$, the masses $m[\Upsilon(1 S)], m\left[\eta_{c}(1 S)\right]$, and light meson masses were used to tune the bare parameters. Several short-distance quantities, mainly related to Wilson loops, were computed to obtain the original result [182]

$$
\alpha_{\mathrm{s}}\left(m_{Z^{0}}\right)=0.1183 \pm 0.0008 \text {. }
$$

An independent implementation of a similar lattice-based approach, using the same experimental inputs as [182], yields [226]

$$
\alpha_{\mathrm{s}}\left(m_{Z^{0}}\right)=0.1192 \pm 0.0011 .
$$

The HPQCD Collaboration updated their original result of Eq. (18), superseding it with [227]

$$
\alpha_{\mathrm{s}}\left(m_{Z^{0}}\right)=0.1184 \pm 0.0006 .
$$

This value is not independent of the result in Eq. (19). The two results in Eqs. (18) and (19) are expected to be nearly identical, as they use the same inputs and different calculations of the same theoretical effects.

Another new determination [227, 228] of $\alpha_{\mathrm{s}}$ uses moments of heavy-quark correlators calculated on the lattice and continuum perturbation theory. The same references also provide a determination of the $c$ - and $b$-quark masses, described in Sect. 2.8.2. The result is [227]

$$
\alpha_{\mathrm{s}}\left(m_{Z^{0}}\right)=0.1183 \pm 0.0007 .
$$

These extractions of $\alpha_{\mathrm{s}}$, based on lattice calculations and quarkonia masses, are the most precise individual determinations among the many methods and measurements available [229], and will thus tend to dominate any average over different $\alpha_{\mathrm{s}}$ determinations such as that in [229]. $\alpha_{\mathrm{s}}$ from quarkonium radiative decays

The CLEO [230] measurement of the inclusive radiative decay $\Upsilon(1 S) \rightarrow \gamma X$ (see Sect. 3.2.2), together with a theoretical description of the photon spectrum [231], has made it possible to obtain a precise determination of $\alpha_{\mathrm{s}}$ from $\Upsilon(1 S)$ decays [232]. A convenient observable is the parton-level ratio

$$
R_{\gamma} \equiv \frac{\Gamma(V \rightarrow \gamma g g)}{\Gamma(V \rightarrow g g g)}
$$

for decays of heavy vector meson $V$. The wave function of $V=\Upsilon(1 S)$ at the origin and the relativistic corrections cancel at order $v^{2}$ in this ratio. Furthermore, one also needs to include color-octet contributions in the decay rates, requiring an estimation of the color-octet NRQCD matrix elements. The two color-octet matrix elements that appear in the numerator of $R_{\gamma}$ at order $v^{2}, O_{8}\left({ }^{1} S_{0}\right)$ and $O_{8}\left({ }^{3} P_{0}\right)$, also appear in the denominator, which includes $O_{8}\left({ }^{3} S_{1}\right)$, thus decreasing the theoretical uncertainty associated with the estimation of those matrix elements in the $\alpha_{\mathrm{s}}$ extraction. The theoretical expression for $R_{\gamma}$ at order $v^{2}$ is

$$
R_{\gamma}=\frac{36}{5} \frac{e_{b}^{2} \alpha}{\alpha_{\mathrm{s}}} \frac{1+C_{\gamma i} \mathcal{R}_{i}}{1+C_{i} \mathcal{R}_{i}}
$$

Here $e_{b}$ is the $b$-quark charge, $\alpha$ is the fine structure constant, and $C_{\gamma i} \mathcal{R}_{i}$ and $C_{i} \mathcal{R}_{i}$ represent the order $v^{2}$ corrections to the numerator and denominator, respectively. The $v^{2}$ corrections [232] account for radiative, relativistic, and octet effects. Experimental values of $R_{\gamma}$ for $V=\Upsilon(1 S, 2 S, 3 S)$ appear in Table 24. The value of $R_{\gamma}$ for $\Upsilon(1 S)$ obtained by CLEO [230] using the Garcia-Soto (GS) QCD calculation [231] for the photon spectrum ${ }^{6}$ is

$$
R_{\gamma}[\Upsilon(1 S)]_{\exp }=0.0245 \pm 0.0001 \pm 0.0013
$$

where the first error is statistical and the second is systematic. The value of $\alpha_{\mathrm{s}}$ obtained from Eq. (23) using Eq. (24) as input is [232]

$$
\begin{aligned}
\alpha_{\mathrm{s}}\left(m_{\Upsilon(1 S)}, n_{f}=4\right) & =0.184_{-0.014}^{+0.015}, \\
\alpha_{\mathrm{s}}\left(m_{Z^{0}}\right) & =0.119_{-0.005}^{+0.006} .
\end{aligned}
$$

The recent lattice [233] and continuum [234] estimates of the octet matrix elements are used to obtain this result. The experimental systematic uncertainty from Eq. (24) dominates the error in $\alpha_{\mathrm{s}}$ shown in Eq. (25).

There are also CLEO measurements of $R_{\gamma}[J / \psi]$ [235] and $R_{\gamma}[\psi(2 S)]$ [236] (see Sect. 3.2.2 and Table 25). One could, in principle, extract $\alpha_{\mathrm{s}}$ in the same way as for $\Upsilon(1 S)$ above. However, the relativistic and octet corrections are more severe than for the $\Upsilon(1 S)$, so terms of

\footnotetext{
${ }^{6}$ A theoretical description of the photon spectrum is needed to extrapolate to the experimentally inaccessible, low-energy part of the spectrum.
} 
higher order than $v^{2}$ may not be small enough to ignore. The effects due to the proximity of $\psi(2 S)$ to open-charm threshold are difficult to estimate [237].

\section{$\alpha_{\mathrm{s}}$ from bottomonium hyperfine splitting}

The observation of $\eta_{b}(1 S)$ by $B A B A R[59,61]$ and CLEO [60] allows $\alpha_{\mathrm{s}}$ to be extracted from the singlettriplet hyperfine mass splitting,

$$
\Delta m_{\mathrm{hf}}\left[\eta_{b}(1 S)\right] \equiv m[\Upsilon(1 S)]-m\left[\eta_{b}(1 S)\right] .
$$

The theoretical expression used for the hyperfine splitting includes a perturbative component and a nonperturbative one. The perturbative component is given by the expression of [175], which includes order $\alpha_{\mathrm{s}}$ corrections to the leading order (LO) term,

$$
\Delta m_{\mathrm{hf}}\left[\eta_{b}(1 S)\right]_{\mathrm{LO}}=\frac{C_{F}^{4} \alpha_{\mathrm{s}}{ }^{4} m_{b}}{3},
$$

and resummation of the logarithmically-enhanced corrections up to the subleading logarithms (which are of the form $\alpha_{s}^{n} \ln ^{n-1} \alpha_{s}$ ). The non-perturbative part is parametrized in terms of the dimension-four gluon condensate, which is fixed according to [238].

Using the average experimental value in Table 7 and Eq. (5) the resulting value of $\alpha_{\mathrm{s}}$ is [239]

$$
\alpha_{\mathrm{s}}\left(m_{Z^{0}}\right)=0.125 \pm 0.001 \pm 0.001 \pm 0.001,
$$

where the first error is experimental, the second is associated with the gluon condensate and the third accounts for the $b$-quark mass uncertainty. This $\alpha_{\mathrm{s}}$ value is slightly more than $3 \sigma$ higher than the updated HPQCD [227] lattice result in Eq. (20).

\subsubsection{Determinations of $m_{b}$ and $m_{c}$}

Below we review recent extractions of the heavy quark masses related to observables in heavy quarkonium physics.

\section{$\underline{\text { Sum rules }}$}

\section{- Moments}

The determination of the heavy quark masses from a sum-rule analysis requires theoretical predictions for the $n^{\text {th }}$ moments of the cross section for heavy-quark production in $e^{+} e^{-}$collisions. The theoretical expression for the moments is related to derivatives of the vacuum polarization function at $q^{2}=0$. Four-loop $\left[\mathcal{O}\left(\alpha_{\mathrm{s}}{ }^{3}\right)\right]$ results for the vacuum polarization function have appeared for the first moment [240-242], second moment [243], third moment [244], and approximate results for higher moments [245, 246]. All those four-loop results are used in the most recent low-momentum sum-rule determinations of the heavy-quark masses reported below.

- Low-n sum rules
The most recent determination [247, 248] of the $c$ - and $b$-quark masses using low-momentum sum rules incorporates four-loop results for the derivatives of the vacuum polarization function along with the most recent experimental data. The results are [248]

$$
\begin{aligned}
m_{c}^{\overline{\mathrm{MS}}}(3 \mathrm{GeV}) & =0.986 \pm 0.013 \mathrm{GeV}, \\
m_{c}^{\overline{\mathrm{MS}}}\left(m_{c}^{\overline{\mathrm{MS}}}\right) & =1.279 \pm 0.013 \mathrm{GeV},
\end{aligned}
$$

and

$$
\begin{aligned}
m_{b}^{\overline{\mathrm{MS}}}(10 \mathrm{GeV}) & =3.610 \pm 0.016 \mathrm{GeV} \\
m_{b}^{\overline{\mathrm{MS}}}\left(m_{b}^{\overline{\mathrm{MS}}}\right) & =4.163 \pm 0.016 \mathrm{GeV} .
\end{aligned}
$$

For a critical discussion of the error attached to these determinations and a new (preliminary) mass determination using low-momentum sum rules see [249].

- Large-n sum rules

A determination of the $b$-quark mass using nonrelativistic (large- $n$ ) sum rules, including resummation of logarithms, has been performed [250]. It incorporates next-to-next-to leading order results along with the complete next-to-leading logarithm resummation (and partial next-to-next-to-leading logarithm resummation). Including logarithm resummation improves the reliability of the theoretical computation. The value of the $\overline{\mathrm{MS}}$ mass is [250]

$$
m_{b}^{\overline{\mathrm{MS}}}\left(m_{b}^{\overline{\mathrm{MS}}}\right)=4.19 \pm 0.06 \mathrm{GeV} .
$$

The $c$-quark mass has also been determined from a nonrelativistic sum-rules analysis [251], with the result

$$
m_{c}^{\overline{\mathrm{MS}}}\left(m_{c}^{\overline{\mathrm{MS}}}\right)=1.25 \pm 0.04 \mathrm{GeV} .
$$

\section{- Alternative approaches}

A determination of the $c$ - and $b$-quark masses which uses moments at $q^{2} \neq 0$ and includes the dimension-six gluon condensate (also determined from the sum rules) has been reported [252]:

$$
\begin{aligned}
& m_{c}^{\overline{\mathrm{MS}}}\left(m_{c}^{\overline{\mathrm{MS}}}\right)=1.260 \pm 0.018 \mathrm{GeV} \\
& m_{b}^{\overline{\mathrm{MS}}}\left(m_{b}^{\overline{\mathrm{MS}}}\right)=4.220 \pm 0.017 \mathrm{GeV}
\end{aligned}
$$

which employ an estimate of the four-loop contribution to the $q^{2} \neq 0$ moments.

\section{Quark masses from the lattice}

A determination of the $b$-quark mass in full (unquenched) lattice QCD using one-loop matching to continuum QCD, finds [211]

$$
m_{b}^{\overline{\mathrm{MS}}}\left(m_{b}^{\overline{\mathrm{MS}}}\right)=4.4 \pm 0.3 \mathrm{GeV} .
$$

The $c$-quark mass was calculated by comparing lattice determinations of moments of heavy-quark correlators to 
four-loop continuum perturbation theory [227, 228]. A $b$-quark mass calculation is also included in [227]. Due to the use of continuum, rather than lattice, perturbation theory, a higher-order perturbative calculation can be used, achieving very precise results [227]:

$$
\begin{aligned}
m_{c}^{\overline{\mathrm{MS}}}(3 \mathrm{GeV}) & =0.986 \pm 0.006 \mathrm{GeV}, \\
m_{c}^{\overline{\mathrm{MS}}}\left(m_{c}^{\overline{\mathrm{MS}}}\right) & =1.273 \pm 0.006 \mathrm{GeV},
\end{aligned}
$$

and

$$
\begin{aligned}
m_{b}^{\overline{\mathrm{MS}}}(10 \mathrm{GeV}) & =3.617 \pm 0.025 \mathrm{GeV} \\
m_{b}^{\overline{\mathrm{MS}}}\left(m_{b}^{\overline{\mathrm{MS}}}\right) & =4.164 \pm 0.023 \mathrm{GeV} .
\end{aligned}
$$

\subsection{3. $m_{t}$ determination}

Determination of the top-quark mass $m_{t}$ at a future $e^{+} e^{-}$linear collider from a $t \bar{t}$ line-shape measurement (see Sect. 6.13) requires good theoretical knowledge of the total $t \bar{t}$ production cross section in the threshold region. The threshold regime is characterized by $\alpha_{\mathrm{s}} \sim v$. The $\mathrm{N}^{k} \mathrm{LO}$ result includes corrections of order $\alpha_{\mathrm{s}}{ }^{n} v^{m}$ with $n+m=k$. The $\mathrm{N}^{2} \mathrm{LO}$ result has been known for some time now. Several contributions to the $\mathrm{N}^{3} \mathrm{LO}$ result have been calculated. Those include corrections to the Green functions and wave function at the origin [166, 167, 253255]; matching coefficients of the effective theory currents [256, 257]; electroweak effects in NRQCD [258, 259]; and corrections to the static potential (see Sect. 2.5.3).

Renormalization-group-improved expressions, which sum terms of the type $\alpha_{\mathrm{s}} \ln v$, are also necessary to reduce the normalization uncertainties of the cross section and improve the reliability of the calculation. Those resummations, which were originally only done in the framework of velocity NRQCD, have now been calculated within pNRQCD [260]. The terms at next-to-nextto-leading logarithmic accuracy are not yet completely known [261]. Consistent inclusion of all effects related to the instability of the top quark is needed. Some recent progress in this direction has been made [262264]. It is expected that a linear collider will provide an $m_{t}$ determination with uncertainties at the level of $100 \mathrm{MeV}$ (see Sect. 6.13). For comparison, the Tevatron Electroweak Working Group has reported a best-current value of $m_{t}=173.1 \pm 1.3 \mathrm{GeV}$ [265].

At the LHC, top quarks will be produced copiously. It has been pointed out [266] that in the threshold region of $t \bar{t}$ production at LHC, a significant amount of the (remnant of) color-singlet $t \bar{t}$ resonance states will be produced, unlike at the Tevatron where the coloroctet $t \bar{t}$ states dominate. In fact, there appears the $1 S$ peak in the $t \bar{t}$ invariant mass distribution below $t \bar{t}$ threshold, even after including the effects of initial-state radiation and parton-distribution function, and the position of this $1 S$ peak is almost the same as that in $e^{+} e^{-}$collisions [267, 268]. Namely, theoretically there is a possibility of extracting the top quark mass with high accuracy from this peak position, although experimentally it is quite challenging to reconstruct the $t \bar{t}$ invariant mass with high accuracy.

Recently, a theoretical framework to compute the fullydifferential cross sections for top quark production and its subsequent decays at hadron colliders has been developed, incorporating the bound-state effects which are important in the $t \bar{t}$ threshold region [269]. A Monte Carlo event generator for LHC has been developed and various kinematical distributions of the decay products of top quarks have been computed. In particular, it was found that a bound-state effect deforms the $\left(b W^{+}\right)$$\left(\bar{b} W^{-}\right)$double-invariant-mass distribution in a correlated manner, which can be important in the top event reconstruction.

\subsection{Exotic states and states near or above threshold}

For states away from threshold, it has been shown that appropriate EFTs to describe the quarkonium spectrum can be constructed. In particular, in pNRQCD, the relevant degrees of freedoms are clearly identified: the leading order description coincides with the Schrödinger equation, the potentials are the pNRQCD matching coefficients, and the energy levels are calculable in a welldefined procedure. Close to threshold, the situation changes drastically [270, 271]. As described earlier in this section, the region close to and just above threshold is presently the most interesting, with a wealth of newly discovered states. Most new states do not fit potentialmodel expectations. This is to be expected, as we have seen that a potential model description of quarkonium (strongly-coupled pNRQCD) emerges only for binding energies smaller than $\Lambda_{\mathrm{QCD}}$. Since the open heavy-flavor threshold is at the scale $\Lambda_{\mathrm{QCD}}$ in HQET, a potential model description of states above that threshold cannot provide a reasonable approach. On the other hand, from a QCD point of view, a plethora of new states are expected. NRQCD is still a good EFT for states close to and just above threshold, at least when their binding energies remain much smaller than the heavy-flavor mass. The heavy quarks move slowly in these states, and the static limit should remain a good starting point.

Below we examine how things change close to threshold and the new degrees of freedom that emerge. First, Sect. 2.9.1 considers the case in which there are only quarkonium and gluonic excitations. Away from threshold, the gluonic excitations have been integrated out to obtain strongly-coupled pNRQCD and the QCD nonperturbative potentials are the pNRQCD matching coefficients. Close to threshold the gluonic excitations no longer develop a gap with respect to quarkonium and they have to be considered as dynamical degrees of freedom. Next, Sect. 2.9.2 considers the situation with dynamical ultrasoft light quarks and we discuss all the new degrees of freedom that may be generated. No QCD based theory description is yet possible in this situation, 
apart from systems like the $X(3872)$ that display universal characteristics and may be treated with EFTs methods. Models for the description of states close to threshold just pick up some of the possible degrees of freedom and attribute to them some phenomenological interaction. These models will be described and their predictions contrasted. Third, Sect. 2.9.6 will summarize the predictions of sum rules, a method that allows calculation of the masses of the states once an assumption on the operator content is made. Lastly, all the new unconventional states will be summarized along with possible interpretations in Sect. 2.9.7.

\subsubsection{Gluonic excitations}

First, consider the case without light quarks. Here the degrees of freedom are heavy quarkonium, hybrids and glueballs. In the static limit, at and above the $\Lambda_{\mathrm{QCD}}$ threshold, a tower of hybrid static energies (i.e., of gluonic excitations) must be considered on top of the $Q \bar{Q}$ static singlet energy $[272,273]$. The spectrum has been thoroughly studied on the lattice [274]. At short distances, it is well described by the Coulomb potential in the color-singlet or in the color-octet configurations. At short distances, the spectrum of the hybrid static energies is described in the leading multipole expansion of pNRQCD by the octet potential plus a mass scale, which is called gluelump mass [140, 275]. At large distances the energies rise linearly in $r$. The first hybrid excitation plays the role of the open heavy-flavor threshold, which does not exist in this case. If the Born-Oppenheimer approximation is viable, many states built on each of the hybrid potentials are expected. Some of these states may develop a width if decays to lower states with glueball emission (such as hybrid $\rightarrow$ glueball + quarkonium) are allowed. The states built on the static potential (ground state) are the usual heavy quarkonium states.

Consider, for example, the $Y(4260)$, for which many interpretations have been proposed, including a charmonium hybrid [276-278]. If the $Y(4260)$ is interpreted as a charmonium hybrid, one may rely on the heavy-quark expansion and on lattice calculations to study its properties. Decays into $D^{(*)} \bar{D}^{(*)}$ should be suppressed since they are forbidden at leading order in the heavy-quark expansion [277] (see also [279]). This is in agreement with the upper limit on $Y \rightarrow D \bar{D}$ reported by BABAR (see Table 16). The quantum numbers of the $Y(4260)$ are consistent with those of a pseudoscalar $0^{-+}$fluctuation $|\phi\rangle$, belonging to the family of $m v^{2}$ fluctuations around the gluonic excitation between a static quark and a static antiquark, with quantum numbers $1^{+-}$, also known as $\Pi_{u}$,

$$
|Y\rangle=\left|\Pi_{u}\right\rangle \otimes|\phi\rangle .
$$

It is suggestive that, according to lattice calculations [274], $\Pi_{u}$ is the lowest gluonic excitation between a static quark and a static antiquark above the quark-antiquark color singlet. $|\phi\rangle$ is a solution to the Schrödinger equation with a potential that is the static energy of $\Pi_{u}$. Fitting the static energy of $\Pi_{u}$ at short and intermediate distances, one finds

$$
E_{\Pi_{u}} r_{0}=\text { constant }+0.11 \frac{r_{0}}{r}+0.24\left(\frac{r}{r_{0}}\right)^{2},
$$

as illustrated in Fig. 34. Solving the corresponding Schrödinger equation,

$$
m_{Y}=(2 \times 1.48+0.87+0.53) \mathrm{GeV}=4.36 \mathrm{GeV},
$$

where $1.48 \mathrm{GeV}$ is the charm mass in the RS scheme [280] and $0.87 \mathrm{GeV}$ is the $\Pi_{u}$ gluelump mass in the same scheme [275].

\subsubsection{Spectrum with light quarks}

Once light fermions have been incorporated into the spectrum, new gauge-invariant states appear beside the heavy quarkonia, hybrids, and glueballs. On the one hand, we have the states with no heavy quark content. Due to chiral symmetry, there is a mass gap, of $\mathrm{O}\left(\Lambda_{\chi}\right)$, between the Goldstone bosons, which are massless in the chiral limit, and the rest of the spectrum. The Goldstone bosons are considered as ultrasoft degrees of freedom and $\Lambda_{\chi} \sim \Lambda_{\mathrm{QCD}}$, so that away from threshold the rest of the spectrum should be integrated out. Besides these, there are also bound states made of one heavy quark and light quarks, i.e., the $\bar{Q} q-Q \bar{q}$ system. The energy of this system is, according to the HQET counting rules, $m_{\bar{Q} q}+m_{Q \bar{q}}=2 m+2 \bar{\Lambda}$. Therefore, since the heavylight binding energy $\bar{\Lambda} \sim \Lambda_{\mathrm{QCD}}$, away from threshold these states have to be integrated out. Close to threshold the situation is different. In this case, there is no mass gap between the heavy quarkonium and the creation of a $\bar{Q} q-Q \bar{q}$ pair. Thus, for study of heavy quarkonium near threshold, these degrees of freedom must be included in the spectrum, even if the mixing between the heavy

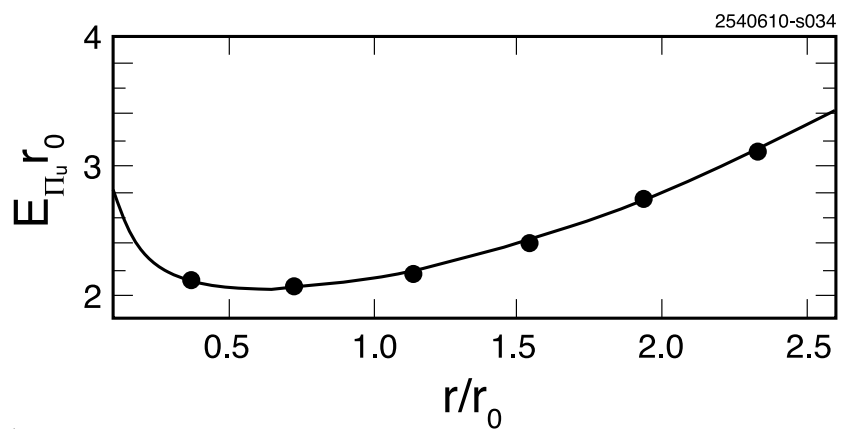

FIG. 34: The hybrid static potential $\Pi_{u}$ at short and intermediate distances, $r_{0} \approx 0.5 \mathrm{fm}$. The solid circles are the lattice data[274] and the smooth curve traces out Eq. (43) 
quarkonium and the $\bar{Q} q-Q \bar{q}$ is expected to be small, being suppressed in the large Nc counting. Summarizing, light fermions contribute within this picture in three ways:

- Hard light fermions, which are encoded into the matching coefficients of the NRQCD Lagrangian and obtained from the computation of perturbative Feynman diagrams at the scale $m$.

- Soft light fermions, a term that denotes, in a generic way, all the fermions that are incorporated in the potentials. It is expected that their main effects can be simulated by a variation of the value of the parameters in the potentials. They can be evaluated nonperturbatively via unquenched lattice calculation of the potentials.

- Ultrasoft light fermions, which are the ones that will become pions and, since they are also ultrasoft degrees of freedom, they should be incorporated in the effective Lagrangian together with the heavy quarkonium.

So the general picture is as follows: The inclusion of light quarks does not remove any states predicted in the no-light-quarks scenario, but the availability of decays via pion emission does increase the width of each such state in the spectrum. Moreover, in addition to the regular quarkonium states, new states built using the lightquark quantum numbers may form. States made of two heavy and light quarks include those built on pairs of heavy-light mesons $(D \bar{D}, B \bar{B}, \ldots)$, like hadronic molecular states [33, 281]; molecular states composed of the usual quarkonium states (built on the static potential); and light hadrons (hadro-quarkonium [282] ); pairs of heavy-light baryons [283]; tetraquark states [284]; and likely many others. It would be particularly interesting to have the spectrum of tetraquark potentials, or at least their ground states, from lattice QCD, since a tetraquark interpretation of some of the newly discovered states has been advocated [285, 286] (see Sect. 2.9.4). If, again, the Born-Oppenheimer approximation is a reasonable approach, many states built on each of the tetraquark potentials may be expected, many developing (large) widths due to decays by emission of a pion (or other light hadron).

How these different kinds of states "talk to each other" is an important issue [287]. Results on crosstalk of the static potential with a pair of heavy-light mesons on the lattice have recently been reported (see Sect. 2.6). This explains why, from the QCD point of view, so many states of a new nature appear in this region of the spectrum. However, a systematic QCD description of these states has not yet been developed. For the time being, models are developed in order to obtain more detailed information on these systems. Exceptional cases, e.g., those for which the state is extremely close to a threshold (e.g., $X(3872)$ ), allow for an effective field theory treatment [288] (see also Sect. 2.9.3).
Results from some of the above-mentioned models follow: There are differences among models involving fourquark fields, two heavy and two light. Given four-quark fields of the type, e.g., $c \bar{c} q \bar{q}$ (where $q$ represents a generic light quark), three quark-pair configurations are possible. All of them have been exploited in the literature. However, the resulting models are not equivalent because different dynamics are attributed to different configurations. Due to the absence of further theoretical input from QCD, many tetraquark studies rely on phenomenological models of the tetraquark interaction unless some special hierarchy of dynamical scales may be further exploited on the top of the nonrelativistic and perturbative expansions discussed so far. In [289, 290], it is assumed that

$$
X \sim(c \bar{c})_{S=1}^{8}(q \bar{q})_{S=1}^{8},
$$

i.e., that the dominant Fock-space component of the $X(3872)$ contains a $c \bar{c}$ pair and a $q \bar{q}$ pair in a color-octet configuration with spin 1 . Calculations have been based on a phenomenological interaction Hamiltonian. In [285], it is assumed that

$$
X \sim(c q)_{S=1}^{\overline{3}}(\bar{c} \bar{q})_{S=0}^{3}+(c q)_{S=0}^{\overline{3}}(\bar{c} \bar{q})_{S=1}^{3} .
$$

Here the clustering of quark pairs in tightly-bound colortriplet diquarks is not induced by a scale separation as it would happen in baryons with two heavy quarks [151], but is a dynamical assumption of the model. In [291294], it is assumed that

$$
\begin{aligned}
X & \sim(c \bar{q})_{S=0}^{1}(q \bar{c})_{S=1}^{1}+(c \bar{q})_{S=1}^{1}(q \bar{c})_{S=0}^{1} \\
& \sim D^{0} \bar{D}^{* 0}+D^{* 0} \bar{D}^{0}
\end{aligned}
$$

i.e., that the dominant Fock-space component of the $X(3872)$ is a $D^{0} \bar{D}^{* 0}$ and $D^{* 0} \bar{D}^{0}$ molecule. Small shortrange components of the type

$$
(c \bar{c})_{S=1}^{1}(q \bar{q})_{S=1}^{1} \simeq J / \psi \rho(\text { or } \omega)
$$

are included as well. Predictions depend on the phenomenological Hamiltonian, which typically contains short-range $\left(\sim 1 / \Lambda_{\mathrm{QCD}}\right)$, potential-type interactions among the quarks and long-range $\left(\sim 1 / m_{\pi}\right)$ one-pion exchange. In [288, 295-297], it is assumed not only that the $X(3872)$ is a $D^{0} \bar{D}^{* 0}$ and $\bar{D}^{0} D^{* 0}$ molecule but also that it is loosely bound, i.e., that the following hierarchy of scales is realized:

$$
\Lambda_{\mathrm{QCD}} \gg m_{\pi} \gg \frac{m_{\pi}^{2}}{m_{D^{0}}} \approx 10 \mathrm{MeV} \gg E_{\mathrm{b}}
$$

Indeed, the binding energy, $E_{\mathrm{b}}$, which may be estimated from $m_{X}-\left(m_{D^{* 0}}+m_{D^{0}}\right)$, is, as Table 13 shows, very close to zero, i.e., much smaller than the natural scale $m_{\pi}^{2} / m_{D_{0}}$. Systems with a short-range interaction and a long scattering length have universal properties that may be exploited: in particular, production and decay amplitudes factorize into short-range and long-range component, where the latter depends only on a single parameter, the scattering length (see Sect. 2.9.3). 


\subsubsection{Molecular states}

Loosely bound hadronic molecules

The $X(3872)$ resonance (see Sect. 2.3.1) cannot be easily explained as a standard charmonium excitation [298]. The close proximity of its mass to $D^{* 0} \bar{D}^{0}$ threshold suggested that it could be a good example of a hadronic molecule with $J^{P C}=1^{++}$quantum numbers. A $D^{* 0} \bar{D}^{0}$ molecule would be characterized by an extremely small binding energy, as small as $E_{\mathrm{b}} \approx 0.1 \mathrm{MeV}$. Indeed, the $B \rightarrow K X$ Belle production mechanism allows the formation of a nearly-at-rest $D^{* 0} \bar{D}^{0}$ system which could be very weakly bound. Several other bound states with similar properties have also been discovered.

The threshold proximity of many of the new states implies that, regardless of the binding mechanism, there should be a significant component of a hadronic molecule in the wave function of the state. The small binding energy of this molecular component indicates that the $D^{* 0} \bar{D}^{0}$ scattering length, $a$, is unnaturally large. This leads to some simplifications in the description of the properties of the molecule, as its binding energy, $E_{\mathrm{b}}$, and wave function are in fact largely determined by $a$, a phenomenon known as low-energy universality. In the limit of a very shallow bound state, the scattering length is

$$
a=\frac{1}{\sqrt{2 m_{\mathrm{r}} E_{\mathrm{b}}}},
$$

where

$$
m_{\mathrm{r}}=\frac{m_{D^{0}} m_{D^{* 0}}}{m_{D^{0}}+m_{D^{* 0}}}
$$

is the reduced mass. Clearly, the scattering length $a \simeq$ $10 \mathrm{fm}$ is much larger than the natural length scale $R$ for a molecular state bound by pion exchange, $R \simeq 1 / m_{\pi}=$ $1.5 \mathrm{fm}$. There is a universal prediction for a wave function of the $S$-wave molecular state,

$$
\psi(r)_{\mathrm{mol}}=\frac{1}{\sqrt{2 \pi a}} \frac{e^{-\frac{r}{a}}}{r},
$$

where $r$ is the separation of the constituents in the molecule's rest frame, which is correct up to terms of order $R / a$. A proper investigation of near-threshold resonances is needed to address the appearance of this new length scale, the possibility of distinguishing between an "elementary" particle ( $q \bar{q}$, hybrid, or compact tetraquark) and a composite state (hadronic molecule), and how to estimate the admixture of the composite. It was suggested by Weinberg [299-301] that the admixture fraction can be determined model-independently for such near-threshold bound states. In this scheme, the admixture of a nonmolecular component is parametrized in terms of a single parameter, $0 \leq \xi^{2} \leq 1$, which measures the probability of finding the molecular component in the physical wave function of the state of interest. For $\xi<1$, Eq. (52) becomes

$$
\psi(r)_{\mathrm{mol}}=\frac{\xi^{2}}{\sqrt{2 \pi a}} \frac{e^{-\frac{r}{a}}}{r} .
$$

Accordingly, the expression for the scattering length, Eq. (50), is then

$$
a=\frac{2 \xi^{2}}{1+\xi^{2}} \frac{1}{\sqrt{2 m_{\mathrm{r}} E_{\mathrm{b}}}} .
$$

The expression acquires corrections of order $R$. A simultaneous measurement of both binding energy and scattering length can extract the value of the parameter $\xi$, and the nature of the state becomes an observable. When this formalism [299-301] was applied to the deuteron, it was shown that, indeed, the deuteron is a proton-neutron molecule. The method just described can only be applied if the particles forming the molecule are in a relative $S$-wave and if the state studied is sufficiently close to threshold, i.e., if $k \simeq \sqrt{2 m_{\mathrm{r}} E_{\mathrm{b}}}$ is the smallest momentum scale in the problem. The approach was generalized [302-304] to include inelastic channels, as well as an above-threshold resonance. It is stressed in [302, 303] that the relevant quantity to be studied is the effective coupling constant squared, or, equivalently, the residue at the bound-state pole that parametrizes the coupling strength of this state to the relevant continuum channel, which can be shown to be proportional to $\xi^{2}$ with a known factor of proportionality. Thus this coupling constant, which is an observable, measures the amount of molecular admixture in the sense defined in [299-301]. A related approach uses pole-counting [305], which studies the structure of the near-threshold singularities of the scattering amplitude. It appears that the state is mostly elementary if there are two nearby poles in the scattering amplitude, whereas composite particle corresponds to a single, near-threshold pole. While these methods provide a diagnostic tool for identifying near-threshold molecular states, they do not provide information on the binding mechanism. Some of these states might be interpreted as hadrocharmonia, discussed in detail in Sect. 2.9.5.

The analysis sketched above has been applied to various states. Evidence supporting the identification of $Y(4660)$ as a $\psi(2 S) f_{0}(980)$ [306] bound system has been found. In addition, it has been proposed that $X(3872)[33,281,307,308]$ is a $D^{* 0} \bar{D}^{0}$ bound system, and that $D_{s}(2317)$ and $D_{s}(2460)$ [309-312] are bound states of $K D$ and $K D^{*}$, respectively. One may use unitarization schemes to investigate corresponding states not located near thresholds; e.g., the $Y(4260)$ was suggested to be a $J / \psi f_{0}(980)$ bound system [313], which would make it a close relative of $Y(4660)$.

\section{Molecules and effective field theories}

Effective field theory (EFT) techniques can be used to study the dynamical properties of a threshold molecular state independent of any particular model. This is possible due to the multitude of scales present in QCD. 
The small binding energy suggests that this state can play the role of the deuteron in meson-antimeson interactions. Thus methods similar to those developed for the deuteron may be employed, with the added benefit of heavy-quark symmetry. A suitable effective Lagrangian describing the $X(3872)$ contains only heavy-meson degrees of freedom with interactions approximated by local four-boson terms constrained only by the symmetries of the theory. While the predictive power of this approach is somewhat limited, several model-independent statements can be made. For instance, existence of $D^{* 0} \bar{D}^{0}$ molecule does not necessarily imply existence of $D^{* 0} \bar{D}^{* 0}$ or $D^{0} \bar{D}$ molecular states [314]. First steps towards the development of a systematic EFT for the $X(3872)$ have been taken [314-317].

Effective field theories can be used to study formation [318] and decays [319] of $X(3872)$ and other molecular states. In particular, it can be employed to study lineshapes $[121,320]$. Those studies reveal that the spectral shape of the resonances located near thresholds is the relevant observable. This is a direct consequence of the importance of the effective coupling constant for the nature of the state. In the case of the $Y(4660)$, the spectrum shows a visible deviation from a symmetric distribution [306], which, in the molecular picture, can be traced to the increasing phase space available for the $\psi(2 S) f_{0}(980)$ system. (Alternatively, the asymmetry might originate from interference of the resonance signal with that of the lower-lying $Y(4360)$ [321] — see also the discussion in Sect. 2.9.4.) Only the mass of the $Y$ and the overall normalization were left as free parameters in a fit to the experimental mass spectrum; the width can be calculated from the effective coupling constant under the assumption that the $Y(4660)$ is indeed a $\psi(2 S) f_{0}(980)$ bound system. This fit [306] gives a mass of $m_{Y}=\left(4665_{-5}^{+3}\right) \mathrm{MeV}$. From this fit, the effective coupling constant was found to be in the range 11-14 GeV. This, in turn, allowed a prediction of the width of $\Gamma_{Y}=(60 \pm 30) \mathrm{MeV}$. The current quality of data allows for additional decay channels, e.g., $\Lambda_{c}^{+} \Lambda_{c}^{-}$. To double check that the analysis is sensible, a second fit to the experimental mass spectrum is performed in which the effective coupling is allowed to float in addition to $m_{Y}$ and the overall normalization. This second fit calls for a coupling constant of $13 \mathrm{GeV}$. This result is interpreted in [306] as strong evidence in favor of a molecular interpretation for the $Y(4660)$. Under this interpretation, employing heavy-spin symmetry allows one to predict a close relative, $Y_{\eta}$, to the $Y(4660)$, namely a bound state of $\eta_{c}(2 S)$ and $f_{0}(980)$ [322]. The mass difference between this state and $Y(4660)$ is predicted to match that between $\psi(2 S)$ and $\eta_{c}(2 S)$ up to corrections of order $\left(\Lambda_{\mathrm{QCD}} / m_{c}\right)^{2}$, which gives $m_{Y_{\eta}}=4616_{-6}^{+5} \mathrm{MeV}$. The width and spectral shape in the $\eta_{c}(1 S) \pi \pi$ channel are predicted to be equal to those of the $Y(4660)$, respectively. Further systematic studies are necessary to put these conjectures on firmer ground. This modelindependent scheme has been extended to states with one unstable constituent [323]. Thus the method can now be applied to many more states in the spectrum. Measurements with higher statistics are needed to test these predictions. For example, an improved spectral shape measurement for $Y(4660)$ could determine if the large predicted coupling to $\psi(2 S) f_{0}(980)$ is present. Another test is a search for the decay $B^{+} \rightarrow K^{+} Y_{\eta}$, which has been estimated [322] to have a branching fraction of $\sim 10^{-3}$. After accounting for the possibility of final state interactions in this picture, it has also been proposed [324] that the $X(4630)$, which decays to $\Lambda_{c}^{+} \Lambda_{c}^{-}$, could be the same state as the $\psi(2 S) f_{0}(980)$ molecule $Y(4660)$, which could be tested with measurements of $B^{+} \rightarrow K^{+} \Lambda_{c}^{+} \Lambda_{c}^{-}$decays.

$\underline{X(3872) \text { as a } D^{* 0} \bar{D}^{0} \text { molecule }}$

Analyses of published $X(3872)$ data in both the $D^{* 0} \overline{D^{0}}$ and $\pi^{+} \pi^{-} J / \psi$ channels have shed light on the nature of the $X$. As for the $Y(4660)$, the lineshape contains the important information. One approach [325], using then-existing data, concluded that the $X(3872)$ is indeed generated by nonperturbative $D^{* 0} D^{0}$ interactions, which are not sufficiently strong to form a bound state, but only to produce a virtual state very close to $D^{* 0} \overline{D^{0}}$ threshold. A different approach [320] stressed that, if the $X(3872)$ is a bound state, there will be a resonant $D^{0} \bar{D}^{0} \pi^{0}$ peak below the $D^{* 0} \bar{D}^{0}$ threshold attributable to the nonzero width of the $D^{* 0}$. This latter approach, also using the initial lineshape measurements, identified the $X$ as a molecule, although a virtual state was not excluded. Later, using the same formalism as [325] and additional data that had become available, a fit to the measured lineshape found [326] that a significant admixture of a compact component inside the $X$ wavefunction is required. As discussed in Sect. 2.3.1 and illustrated in Fig. 16, it was pointed out [122] that the experimental lineshapes for these $D^{0} \bar{D}^{0} \pi^{0}$ events had been generated by constraining the measured particle momenta so that the $D^{0} \pi^{0}$ (or $\bar{D}^{0} \pi^{0}$ ) candidates would have the $D^{* 0}$ mass. If the $X(3872)$ were a loosely bound molecule, such an analysis procedure would cause the resonant invariant mass peak of $D^{0} \bar{D}^{0} \pi^{0}$ that is actually below $D^{* 0} \bar{D}^{0}$ threshold to erroneously appear above the threshold and broadened. Taking this effect into account, the analysis [122] found the data to be consistent with the identification of the $X(3872)$ as a bound state with mass below $D^{* 0} \bar{D}^{0}$ threshold. If future measurements have more statistics in all relevant decay modes of $X(3872)$ and/or improve upon measured mass resolutions, while simultaneously avoiding the kinematic constraint to the $D^{* 0}$ mass, more definitive statements on the molecular nature of the $X(3872)$ could be made.

Another avenue for studying the molecular nature of $X(3872)$ could become available at the LHC. In particular, an EFT description [327] of $X(3872)$ scattering off $D^{0}$ or $D^{* 0}$ mesons has been developed, and proposed for testing with either $B_{c}$ or $B \bar{B}$ decays with final-state interactions. 
Questioning the $X(3872)$ molecular interpretation

The production cross section of $X(3827)$ at the Tevatron is a potential discriminant for its molecular interpretation. Neither CDF nor D $\varnothing$ have reported such a measurement because it is not a trivial one. However, based on published and unpublished-but-public CDF documents, the product of the cross section of $\mathrm{X}(3872)$ and its branching fraction into $\pi^{+} \pi^{-} J / \psi$ can be estimated. The inclusive, prompt ${ }^{7}$ production rate of $X \rightarrow \pi^{+} \pi^{-} J / \psi$ relative to $\psi(2 S) \rightarrow \pi^{+} \pi^{-} J / \psi$, both with $J / \psi \rightarrow \mu^{+} \mu^{-}$, for the same transverse momentum $\left(p_{\mathrm{T}}\right)$ and rapidity $(y)$ restrictions, assuming equal selection efficiencies, is estimated to be [328]

$$
\begin{array}{r}
\sigma(p \bar{p} \rightarrow X+\text { any })_{\text {prompt }} \times \mathcal{B}\left(X \rightarrow \pi^{+} \pi^{-} J / \psi\right) \\
\sigma(p \bar{p} \rightarrow \psi(2 S)+\text { any })_{\text {prompt }} \\
=(4.7 \pm 0.8) \%
\end{array}
$$

This value is used in conjunction with a CDF [329] measurement of the absolute cross section for inclusive $\psi(2 S)$ production as a function of $p_{\mathrm{T}}$ for central rapidity to obtain [328]

$$
\begin{array}{r}
\sigma(p \bar{p} \rightarrow X+\text { any })_{\text {prompt }} \times \mathcal{B}\left(X \rightarrow \pi^{+} \pi^{-} J / \psi\right) \\
=(3.1 \pm 0.7) \mathrm{nb} \\
\text { for } p_{\mathrm{T}}>5 \mathrm{GeV} / c \text { and }|y|<0.6
\end{array}
$$

assuming $X$ and $\psi(2 S)$ have the same rapidity distribution. Since the unknown branching fraction satisfies $\mathcal{B}\left(X \rightarrow \pi^{+} \pi^{-} J / \psi\right)<1$, Eq. (56) also provides a lower limit on the prompt $X$ production cross section for the transverse momentum and rapidity restrictions given.

The large magnitude of the prompt production cross sections measured in $p \bar{p}$ collisions at the Tevatron came as a surprise to many. The original Monte Carlo (MC) studies, based in part on the generators HERWIG [330] and PYTHIA [331], suggested that formation of loosely bound $D^{* 0} \bar{D}^{0}$ molecules in this environment fall far short of the observed rates. Further theoretical work coupled with MC studies [328] reinforced this viewpoint, which was then challenged by an independent examination [332] of the issues involved. Both approaches allow formation of a molecule if its constituents, after their initial production by the underlying generator, have relative momentum up to a value $k_{\max }$. The two approaches differ markedly in the values of $k_{\max }$ that are permitted. In [328], $k_{\max }$ is chosen to be comparable to the binding momentum $k_{\mathrm{b}}=\sqrt{2 m_{\mathrm{r}} E_{\mathrm{b}}}$, whereas [332] argues that a value larger by an order of magnitude, and correspondingly even larger prompt production cross section, is more appropriate due to constituent rescattering effects.

\footnotetext{
7 The adjective prompt refers to $\mathrm{X}$ particles that are produced by
} QCD interactions and not by the weak decays of $b$-hadrons.
Further justification for the choices in [332] was given in [333], in which deuteron production is taken as a case study to judge the efficacy of the arguments. In addition, it allowed tuning of the underlying generator for production of the molecule constituents in the required pairs. It is argued that the prescription in [328] for the value of $k_{\max }$ used in MC generation, as applied to deuterons, is flawed for both fundamental and empirical reasons. Fundamentally, a loosely bound $S$-wave molecule does not satisfy the minimum uncertainty principle, $\Delta r \Delta k \sim 1$. Instead, it maximizes the uncertainty, satisfying $\Delta r \Delta k \gg 1$ with $\Delta r \sim k_{\mathrm{b}}^{-1}$ and $\Delta k \gg k_{\mathrm{b}}$. Empirically, the MC technique in [328] underpredicts the CLEO [334] measurements of antideuterons in $\Upsilon(1 S)$ decays (see Sect. 3.4.5).

A rebuttal to [333] has been made [335]. It argues that the deuteron and $X(3872)$ are not comparable. First, the discrepancy obtained between data and $\mathrm{MC}$ using a small $k_{\max }$ (based mostly on the uncertainty principle, which limits the reasonable variations of $k$, and therefore $k_{\text {max }}$, to be of order $k_{\mathrm{b}}$, not an order of magnitude larger) is modest for the deuteron (factors of 2-3) compared to the $X(3872)$ (factor of $\approx 300)$. Moreover, MC studies with PYTHIA have found considerably better agreement, within a factor of 2-3, between the estimated and observed cross sections of the deuteron. Discrepancies of this size in $e^{+} e^{-}$collisions at LEP were considered reasonably close to the measurements and do not justify rejecting the MC altogether [336]. Finally, contends [335], the deuteron is a system qualitatively different from a $D^{* 0} \bar{D}^{0}$ molecule because the $D^{0}$ is spinless and cannot participate in spin interactions. Conversely, spin interactions play an important role in the determination of the deuteron binding: the spin-singlet deuteron and its isospin partner, the dineutron, are not bound.

Much of the motivation for the $X(3872)$ molecular interpretation, which assumes $S$-wave binding, no longer applies if its quantum numbers are found to be $2^{-+}$, as preferred by a recent $B A B A R[93]$ analysis. A $D^{* 0} \bar{D}^{0} 2^{-+}$ state would require a relative $P$-wave. It is unlikely [335], that $\pi$-exchange could bind such a state, given that, even in an $S$-wave configuration, it is not clear that the attraction is sufficiently strong. Even if such a state exists, there remains the further problem that unless spindependent forces prevent the binding, one should expect partner states with $0^{-+}$and $\left(J^{P C}\right.$-exotic) $1^{-+}$, for which there is no experimental evidence. A $P$-wave $2^{-+}$ molecule would also imply the existence of an extremely narrow, more deeply bound $S$-wave $1^{++}$molecule. Alternatively, forming a $2^{-+} S$-wave bound state would require a different molecule type such as $D_{2} D$ or $D_{1} D^{*}$, which would require not only an immense binding energy of some $500 \mathrm{MeV}$, but also loss of the appealing connection between the mass of the $X(3872)$ and $D^{* 0} \bar{D}^{0}$ threshold.

On the basis of a study by Cho and Wise [337], it is difficult to reconcile the observed $X(3872)$ prompt production cross section with the expectations for $1^{1} D_{2}$ stan- 
dard charmonium. The integrated prompt cross section found using the Cho and Wise gluon fragmentation function in a $1^{1} D_{2}$ state is

$$
\sigma\left(p \bar{p} \rightarrow 1^{1} D_{2}+\text { all }\right)=0.6 \mathrm{nb}
$$

some 50 and 120 times smaller than the estimated experimental cross section [335]. As for the mass of the ${ }^{1} D_{2}$ charmonium, there are a number of studies available in the literature [335]. A hadron-string calculation, with results that agree very well with previous determinations of charmonium and bottomonium levels, is also proposed in [335]. Most of these calculations indicate that the $X(3872)$ has the most difficulty matching quarkonium levels. In this model, while all charmonium and bottomonium levels agree with data within $\sim 10 \mathrm{MeV}$ (excluding the $1^{3} D_{1}$, which departs from the experimental mass by $40 \mathrm{MeV}$ ), the predicted $1^{1} D_{2}$, the would-be $X(3872)$, falls short of the measured value by $80 \mathrm{MeV}$. The mass mismatch and production cross section jeopardize a $2^{-+}$charmonium interpretation of the $X(3872)$. Clarification of the $X(3872)$ decay modes and relative branching fractions would help disentangle the possible explanations. The prominent radiative decay mode for a $D$-wave charmonium $X(3872)$ is expected to be $X(3872) \rightarrow h_{c} \gamma \rightarrow J / \psi \pi^{0} \gamma$ while the $\eta_{c}(1 S) \pi \pi$ channel should have the highest rate among hadronic modes.

The reader is also directed to Sects. 2.9.4 and 4.2.2 for more information on the $X(3872)$.

\subsubsection{Tetraquark states}

The nonstandard decay patterns of $X(3872)$ suggested other theoretical interpretations as well. Could the $X(3872)$ be a pointlike hadron resulting from the binding of a diquark and an antidiquark? This idea was discussed in Maiani et al. [285], following one interpretation of pentaquark baryons (antidiquark-antidiquark-quark states) proposed by Jaffe and Wilczek [338] and the recent discussion of light scalar mesons in terms of tetraquarks by 't Hooft et al. [339, 340] (see also the review [341] and references therein). Some considerations by 't Hooft on an open-string description of baryons [342] were also sources of inspiration.

\section{Diquarks}

A spin-zero diquark operator in the attractive antitriplet color (greek subscripts $\alpha, \beta, \gamma$ ) channel, antisymmetric in flavor (latin subscripts $i, j, k$ ) can be written as:

$$
[q q]_{i \alpha}=\epsilon_{i j k} \epsilon_{\alpha \beta \gamma} \bar{q}_{c}^{j \beta} \gamma_{5} q^{k \gamma}
$$

where the subscript $c$ denotes charge conjugation. The Fermi statistics of light quarks is respected in Eq. (58). Spin-one diquarks (the so called 'bad' ones) can be conceived but they are believed to have a smaller binding energy with respect to spin-zero candidates (see, e.g., [343]). A 'bad' diquark (spin-one) operator can be written as:

$$
[q q]_{\alpha}^{i j r}=\epsilon_{\alpha \beta \gamma}\left(\bar{q}_{c}^{i \beta} \gamma^{r} q^{j \gamma}+\bar{q}_{c}^{j \beta} \gamma^{r} q^{i \gamma}\right)
$$

which is a $\mathbf{6}$ in flavor space (and has three spin components) as required by Fermi statistics $(r=1,2,3)$. Both represent positive parity states, $0^{+}$and $1^{+}$, respectively. Similarly one can construct $0^{-}$and $1^{-}$operators, $\bar{q}_{c} q$ and $\bar{q}_{c} \gamma^{r} \gamma_{5} q$. The latter are identically zero in the 'single mode configuration': quarks that are unexcited with respect to one another. In fact, the most solid tetraquark candidates are scalar mesons made up of 'good' diquarks. The spin-zero light diquarks are very effective at reducing the number of expected four-quark states. A $q q \bar{q} \bar{q}$ multiplet should contain $81=\mathbf{3} \otimes \mathbf{3} \otimes \overline{\mathbf{3}} \otimes \overline{\mathbf{3}}$ particles for three quark flavors. But if the diquark degrees of freedom are the relevant ones, the number of states is reduced to $9=\mathbf{3} \otimes \overline{\mathbf{3}}$ for a diquark that behaves as an antiquark and an antidiquark as a quark (see Eq. (58)). In the case of light scalar mesons this represents a way of encrypting the exoticity (we would have 9 light scalar mesons even if their structure were $q \bar{q}[339,340])$. Spin-one diquarks of Eq. (59) would enlarge the flavor structure as they are $\mathbf{6}_{f}$ operators.

\section{Tetraquarks}

The tetraquark model provides fertile ground for investigations of heavy-flavored states. One of the features of the diquark-antidiquark model proposed in [285], which could also be considered a drawback, is the proliferation of predicted states. Another is the paucity of insight from selection rules that could explain why many of these states are not observed (for a recent account, see, e.g., [344]). It is quite possible that those states are waiting to be discovered.

A tetraquark in the diquark-antidiquark incarnation is a state like $[q q]_{i \alpha}[\bar{q} \bar{q}]^{j \alpha}$, if spin-zero diquarks are concerned, as is the case for the tetraquark interpretation of light scalar mesons. To use a notation making flavor explicit one can write $\left[q_{1} q_{2}\right]\left[\bar{q}_{3} \bar{q}_{4}\right]$. There is no real distinction between 'good' and 'bad' diquarks once one of the quarks in the bound state is heavy: spin-spin interactions between quarks are $1 / m$ suppressed and $m \rightarrow \infty$ with respect to the light quark mass scale. In other words, one can expect that tetraquarks like $[c q]\left[\bar{c} \bar{q}^{\prime}\right]$ have the same chances to be formed by spin-one or spin-zero diquarks. Moreover, the considerations of Fermi statistics made above are no longer valid here. This enlarges the spectrum of predicted states.

There is also the question discussed in Sect. 2.9.3 of whether an $X(3872)$ tetraquark state can better match observed cross sections at the Tevatron. If only spin-zero diquarks were allowed, a $J^{P C}=1^{++} X(3872)$ could not be described as a tetraquark. For a $2^{-+} X(3872)$, however, the tetraquark interpretation is still viable. Known problems with the proliferation of states are shifted to lower mass scales [335]. 
The $[c q]\left[\bar{c} \bar{q}^{\prime}\right]$ states should also appear in charged combinations. Although there is unconfirmed evidence of a $Z(4430)^{-}$state (see Sect. 2.3.4) decaying into charmonium plus charged pion, there is yet no evidence of charged, almost degenerate partners, of the $X(3872)$. Another example of a possible tetraquark meson is the $Y(4260)$, a $1^{--}$resonance decaying into $\pi \pi J / \psi$, as described in Sect. 2.3.2. The dipion mass distribution is consistent with there being a substantial $f_{0}(980)$ component in the $Y(4260)$ decay, suggesting an exotic tetraquark structure $[c s][\bar{c} \bar{s}]$. The tetraquark model suggests a $Y(4260) \rightarrow D_{s}^{+} D_{s}^{-}$decay mode [345], a mode for which the experimental upper limit at $90 \% \mathrm{CL}$ on branching fraction, $<1.3$ relative to $\pi^{+} \pi^{-} J / \psi$ (see Table 16 ), is not a particularly stringent one.

As stated above, the main drawback of the tetraquark model is the proliferation of predicted particles. For example, using a naive constituent diquark model, in the hidden-strange and hidden-charm sector one can predict a quite complex pattern of states [344]. But since no such states near thresholds, such as $\phi J / \psi$ or $f_{0}(980) J / \psi$, are predicted, this model cannot account for the unconfirmed evidence ( $3.8 \sigma$ significance) for a new resonance, $Y(4140)$, decaying to $\phi J / \psi$ near threshold, as reported by CDF [106] (see Table 9 and Sect. 2.3.3). The naive constituent diquark model [344] does predict, however, a $0^{-+}$state decaying to $\phi J / \psi$ at about $4277 \mathrm{MeV}$. The measured $\phi J / \psi$ mass spectrum reported by CDF [106] does show an intriguing enhancement near this mass, but the statistical significance reported by CDF for this structure, $<3 \sigma$, leaves the possibility that it is an artifact or a fluctuation. If this peak becomes more significant with more data, it could bolster the constituent diquark approach.

Constituent quark models can only give rough estimates of the expected mass values. In contrast, the most striking prediction of the model are particles decaying to charmonia plus charged pions or $\rho$-mesons. One or more of the unconfirmed $Z(4430)^{+}, Z_{1}(4050)^{-}$, and $Z_{2}(4250)^{-}$ (see Table 9 and Sect. 2.3.4) could be examples of such particles. If any of these were confirmed by CDF (or by LHC experiments), there would be a much stronger argument in favor of the tetraquark model than any mass spectrum determination of neutral candidates.

\section{Baryonia}

Assuming an open-string hadron picture of diquarkantidiquark tetraquarks, compelling evidence for their existence should be found in experimental searches for narrow structures coupled preferentially to a baryon and an antibaryon. It has been proposed [321] that there is compelling experimental evidence for a single vector baryonium candidate, $Y_{B}$, that explains two unconfirmed states reported by Belle [25, 114]. These states, the $X(4630)$, observed in the decay to $\Lambda_{c}^{+} \Lambda_{c}^{-}$, and $Y(4660)$, which decays to $\pi^{+} \pi^{-} \psi(2 S)$ (see Table 9 and Sect. 2.3.2), have reported masses that differ by only two standard deviations and compatible widths. They can also be fit well by a single resonance [321]

$$
\begin{aligned}
m\left(Y_{B}\right) & =4661 \pm 9 \mathrm{MeV} \\
\Gamma\left(Y_{B}\right) & =63 \pm 23 \mathrm{MeV} .
\end{aligned}
$$

The mass of the $Y_{B}$ is significantly above the decay threshold and a straightforward four-quark interpretation explains its decay modes. The ratio of branching ratios found,

$$
\frac{\mathcal{B}\left(Y_{B} \rightarrow \Lambda_{c}^{+} \Lambda_{c}^{-}\right)}{\mathcal{B}\left(Y_{B} \rightarrow \psi(2 S) \pi^{+} \pi^{-}\right)}=25 \pm 7
$$

highlights a strong affinity of $Y_{B}$ to the baryonantibaryon decay mode. The phase space involved in the decays in Eq. (61) are rather similar because the $\pi^{+} \pi^{-}$pair results from an $f_{0}(980)$ decay (see Fig. 21 in Sect. 2.3.2). As the $f_{0}(980)$ can be identified as a diquark-antidiquark particle (see 't Hooft et al. [339, 340]), the $Y_{B}$ can also be interpreted [321] as such an exotic state. A $[c d][\bar{c} \bar{d}]$ assignment for $Y_{B}$ naturally explains [321] the ratio found in Eq. (61); in the baryonantibaryon mode, a string of two heavy quarks with angular momentum excitation $\ell \neq 0$ would break:

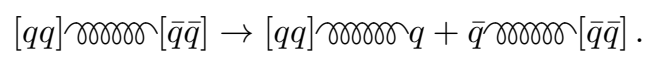

While baryonic decays of tetraquarks should be the most favorable according to the string-color picture, but these are typically phase-space forbidden.

The $Y(4360)$ (see Sect. 2.3.2) could be the radial ground state $(1 P)$ of the $Y_{B}(4660)(2 P)$. These two states both decay into $\pi^{+} \pi^{-} \psi(2 S)$ rather than $\pi^{+} \pi^{-} J / \psi$, a puzzling characteristic that awaits explanation [321]. An alternative molecular interpretation of the $Y(4660)$ can be found in [306].

The $Y(2175)$, observed by BABAR [346], BES [347], and Belle [348], is another interesting baryonium-like candidate, albeit in the light-quark sector. Assuming that $Y(2175)$ is a four-quark meson [349], it should preferentially decay into $\Lambda \bar{\Lambda}$. Because the $\Lambda \bar{\Lambda}$ threshold is $\approx 2231 \mathrm{MeV}$, this decay proceeds through the high-mass tail of the $Y(2175)$. The BABAR data are consistent with this hypothesis [349].

What about doubly charged particles? A diquarkantidiquark open-charm $[c u][\bar{d} \bar{s}]$ composition, denoted here by $A^{++}$, could exist [350] and decay, e.g., into $D^{+} K^{+}$. It is very unlikely that a loosely bound molecule of this kind could be produced. There has not been a search for a doubly charged particle like $A^{++}$close to the $D^{+} K^{+}$mass. Theory is still not able to reliably predict the $A^{++}$rate within the tetraquark model.

\section{Counting quarks in heavy-ion collisions}

Heavy-ion collisions also provide a means for definitively determining the quark nature of, e.g., the $X(3872)$. The nuclear modification ratios $R_{A A}$ and $R_{C P}$ (see Sect. 5.5 and Eqs. (184) and (185), respectively) of the $X(3872)$ and its anisotropy coefficient, $v_{2}$ (see Sect. 5.5), 
TABLE 18: For significant tetraquark candidates, their spinparity $\left(J^{P C}\right)$, decay modes, and quark content

\begin{tabular}{cccc}
\hline \hline $4 q$ candidate & $J^{P C}$ & Decay Modes & $([q q][\bar{q} \bar{q}])_{n J}$ \\
\hline$Y_{B}(4660)$ & $1^{--}$ & $\Lambda_{c}^{+} \Lambda_{c}^{-}, \pi \pi \psi(2 S)$ & $([c d][\bar{c} \bar{d}])_{2 P}$ \\
$Z(4430)^{+}$ & $1^{+-}$ & $\pi^{+} \psi(2 S)$ & $([c u][\bar{c} \bar{d}])_{2 S}$ \\
$Y(4260)$ & $1^{--}$ & $\pi \pi J / \psi, D_{s}^{+} D_{s}^{-}(?)$ & $([c s][\bar{c} \bar{s}])_{1 P}$ \\
$X(3872)$ & $1^{++}$ & $\rho^{0} J / \psi, \omega J / \psi, D \bar{D} \pi$ & $([c u][\bar{c} \bar{u}])_{1 S}$ \\
\hline \hline
\end{tabular}

which can be measured by ALICE, could be useful tools in this task. In the recombination picture, the $X(3872)$ is expected to be produced with rates similar to charm mesons and baryons. Thus the soft part of the spectrum, where recombination is more effective, can be highly populated with $X$ 's. On the other hand, the fragmentation functions of a tetraquark- $X$ are different from those of a $D^{* 0} \bar{D}^{0}$ molecule since the $D$ fragmentation functions are the standard ones. This effect could be studied in a manner similar to those described for light scalar mesons in [351].

To summarize, we refer to Table 18, which lists the most significant tetraquark candidates. The $Y_{B}$ and $Z(4430)^{+}$are the most likely. Experimental study of the $Y(4260) \rightarrow D_{s}^{+} D_{s}^{-}$decay, responsible for most of the width in the tetraquark model, would be an important discriminant to assess its nature. More work on the tetraquark picture has been done recently by Ali et al. [352, 353] and Ebert et al. [354].

\subsubsection{Hadrocharmonium}

The decay pattern for six of the new states ( $Y(4260), Y(4360), Y(4660), Z_{1}(4050)^{-}, Z_{2}(4250)^{-}$, and $Z(4430)^{+}$) could be interpreted [355] as an indication of an intact charmonium state within a more complex hadronic structure. These states have only been observed decaying to a single preferred charmonium state accompanied by one or more light mesons (see Table 9). Decays into a different, apparently nonpreferred, charmonium resonance with the same quantum numbers $(e . g ., J / \psi$ instead of $\psi(2 S)$ for $Y(4660)$ ) or open-charm hadrons have not been observed. In some cases there are meaningful experimental upper bounds on such decays, as shown in Table 16. One explanation [355] is that each such state consists of its preferred charmonium embedded in a shell of light-quark and gluon matter, i.e., a compact charmonium is bound inside a spatially large region of excited light matter. The observed decays can then be viewed as the de-excitation of the light hadronic matter into light mesons and liberation of the compact charmonium. This structure is referred to as hadrocharmonium [355], or more generally, hadroquarkonium.

The picture of a hadroquarkonium mesonic resonance is quite similar to the much-discussed nuclear-bound quarkonium. The primary difference is that, instead of a nucleus, an excited mesonic resonance provides the large spatial configuration of light-quark matter. For the quarkonium to remain intact inside hadroquarkonium, the binding has to be relatively weak. In complete analogy with the treatment of charmonium binding in nuclei (in terms of the QCD multipole expansion) [356-359], the interaction between a compact, colorless quarkonium and the soft, light matter can be described [282] by an effective Hamiltonian proportional to the quarkonium chromopolarizability $\alpha$,

$$
H_{e f f}=-\frac{1}{2} \alpha \vec{E}^{a} \cdot \vec{E}^{a},
$$

where $\vec{E}^{a}$ is the operator of the chromoelectric field. The chromopolarizability can be estimated from the transition $\psi(2 S) \rightarrow \pi \pi J / \psi$, giving [360] $\alpha^{(12)} \approx 2 \mathrm{GeV}^{-3}$. The average of the gluonic operator over light hadron matter $(h)$ with mass $m_{h}$ can be found using the conformal anomaly relation in QCD:

$$
\left\langle h\left|\frac{1}{2} \vec{E}^{a} \cdot \vec{E}^{a}\right| h\right\rangle \geq \frac{8 \pi^{2}}{9} m_{h},
$$

which provides an estimate of the strength of the van der Waals-type quarkonium-light hadrons interaction. The likelihood of binding charmonium in light hadronic matter depends on the relation between the mass $m_{h}$ and the spatial extent of $h$ [282]. In a particular model [361, 362] of mesonic resonances based on an AdS/QCD correspondence, it can be proven [363] that a heavy quarkonium does form a bound state inside a sufficiently excited light-quark resonance. The decay of such a bound state into open heavy-flavor hadrons is suppressed in the heavy-quark limit as $\exp \left(-\sqrt{\Lambda_{\mathrm{QCD}} / m}\right)$, where $m$ is the heavy-quark mass. This is consistent with the nonobservation of $Y$ and $Z$ decays into charm meson pairs. However, it is not clear whether the charm quark is heavy enough for the heavy-quark limit to be applicable.

If the $Y$ and $Z$ resonances are hadrocharmonia, it is expected [282] that:

- Bound states of $J / \psi$ and $\psi(2 S)$ with light nuclei and with baryonic resonances should exist, e.g., baryocharmonium decaying into $p J / \psi(+$ pions $)$.

- Resonances containing $\chi_{c J}$ that decay into $\chi_{c J}+$ pion(s) should also exist. The as-yet-unconfirmed $Z_{1}(4050)^{-}$and $Z_{2}(4250)^{-}$states reported by Belle [105] are candidates (see Table 9 and Sect. 2.3.4).

- Decays of hadrocharmonia candidates to nonpreferred charmonium states, e.g., $Y(4260) \rightarrow$ $\pi^{+} \pi^{-} \psi(2 S)$, or $Y(4360) \rightarrow \pi^{+} \pi^{-} J / \psi$, should be suppressed relative to preferred charmonia. 
- Resonances containing excited bottomonia such as $\Upsilon(3 S), \chi_{b}(2 P)$, and/or $\Upsilon(1 D)$ should exist in the mass range $11-11.5 \mathrm{GeV}$.

\subsubsection{QCD sum rules}

QCD sum rules (QCDSR) [238, 364-366] provide a method to perform QCD calculations of hadron masses, form factors and decay widths. The method is based on identities between two- or three-point correlation functions, which connect hadronic observables with QCD fundamental parameters, such as quark masses, the strong coupling constant, and the quantities which characterize the QCD vacuum, i.e., the condensates. In these identities (sum rules), the phenomenological side (which contains information about hadrons) is related to the QCD or OPE (operator product expansion) side, where the information about the quark content is introduced. Since the correlation functions are written in terms of well-defined quark currents, the method is effective in establishing the nature of the exotic states (molecule, tetraquark, hybrid, etc.).

In principle, QCDSR allows first-principle calculations. In practice, however, in order to extract the result, it is necessary to make expansions, truncations, and other approximations that may reduce the power of the formalism and introduce large errors. In addition, the convergence of the method often critically depends upon the decay channel.

\section{Formalism}

QCD sum rule calculations of hadron masses are based on the correlator of two hadronic currents:

$$
\Pi(q) \equiv i \int d^{4} x e^{i q \cdot x}\left\langle 0\left|T\left[j(x) j^{\dagger}(0)\right]\right| 0\right\rangle,
$$

where $j(x)$ is a current with the appropriate quantum numbers. The phenomenological and OPE correlation functions must then be identified. The Borel transformation [238, 364-366], which converts the Euclidean fourmomentum squared, $Q^{2}$, into the variable $M^{2}$ (where $M$ is the Borel mass), is applied to improve the overlap between the two sides of this identity. Working in Euclidean space is necessary to avoid singularities in the propagators in Eq. (65). More precisely, it is necessary to be in the deep Euclidean region, i.e., $Q^{2}=-q^{2} \gg \Lambda_{\mathrm{QCD}}$. The Borel transform is well-defined in this region and a good OPE convergence is obtained, dominated by the perturbative term.

After equating the two sides of the sum rule, assuming quark-hadron duality [238, 364-366] and making a Borel transform, the sum rule can be written as:

$$
\lambda e^{-m^{2} / M^{2}}=\int_{s_{\min }}^{s_{0}} d s \rho^{\mathrm{OPE}}(s),
$$

where $m$ is the mass of the particle, $M$ is the Borel mass, $\rho$ is the spectral density obtained from the OPE side (tak- ing the imaginary part of the correlation function), $s_{0}$ is the parameter which separates the pole (particle) from the continuum (tower of excitations with the same quantum numbers), and $s_{\min }$ is determined by kinematical considerations. The parameter $\lambda$ represents the coupling of the current to the hadron. Solving Eq. (66) for the mass, a function which is approximately independent of $M$ should be obtained. In practice, the result depends on the Borel mass and a value of $M$ must be chosen within a domain called the Borel window. In order to determine the Borel window, the OPE convergence and the pole contribution are examined: the minimum value of $M$ is fixed by considering the convergence of the OPE, while the maximum value of $M$ is determined by requiring that the pole contribution be larger than the continuum contribution. As pointed out in [367], it becomes more difficult for tetraquarks to simultaneously satisfy pole dominance and OPE convergence criteria. Reasonably wide Borel windows in which these two conditions are satisfied can exist only for heavy systems. Increasing the number of quark lines in a given system, the OPE convergence becomes gradually more problematic. For example, when a change is made from a meson (two quark lines) to a baryon (three quark lines), the perturbative term goes from a single loop to a double loop, suppressed by a factor of $\pi^{2}$ with respect to the single loop. At the same time, higher-order quark condensates become possible, while the nonperturbative corrections grow larger. As a consequence, larger Borel masses must be used to obtain convergence. However, at higher Borel masses the correlation function is dominated by the continuum contribution. With more quark lines, it becomes difficult to find a Borel window where both OPE convergence and pole dominance are satisfied. When a heavy quark is present, its mass provides a hard scale, which helps to make the OPE convergent at lower Borel masses. In summary: the calculations seem to indicate that it is more difficult to keep a larger number of quarks together with small spatial separation.

\section{$Q C D S R$ and $X(3872)$}

The $X(3872)$ (see Sect. 2.3.1 and Tables 9, 10, 11, 12, and 13) has quantum numbers $J^{P C}=1^{++}$or $2^{-+}$. It decays with equal strength into $\pi^{+} \pi^{-} J / \psi$ and $\pi^{+} \pi^{-} \pi^{0} J / \psi$, indicating strong isospin-violation, which is incompatible with a $c \bar{c}$ state. Its mass and the isospin-violation could be understood in several four-quark approaches. However, this state has a decay width of less than $2.2 \mathrm{MeV}$, which is sometimes difficult to accommodate. In order to discuss four-quark configurations in more detail, a distinction between a tetraquark and a molecule will be made: the former is simply a combination of four quarks with the correct quantum numbers, whereas the latter is a combination of two meson-like color-neutral objects. This separation can easily be made at the start of a calculation when the current is chosen. However, performing a Fierz transformation on the currents will mix tetraquarks with molecules. Having this ambiguity in mind, this no- 
tation will be used to clearly refer to the employed currents. The treatment given below applies only for the $J^{P C}=1^{++}$assignment for $X(3872)$.

A current can be constructed for the $X$ based on diquarks in the color-triplet configuration with symmetric spin distribution: $[c q]_{S=1}[\bar{c} \bar{q}]_{S=0}+[c q]_{S=0}[\bar{c} \bar{q}]_{S=1}$ (see Eq. (46)). Therefore the corresponding lowest-dimension interpolating operator for describing $X_{q}$ as a tetraquark state is given by

$$
\begin{aligned}
j_{\mu}^{(q, \mathrm{di})} & =\frac{i \epsilon_{a b c} \epsilon_{d e c}}{\sqrt{2}}\left[\left(q_{a}^{T} C \gamma_{5} c_{b}\right)\left(\bar{q}_{d} \gamma_{\mu} C \bar{c}_{e}^{T}\right)\right. \\
& \left.+\left(q_{a}^{T} C \gamma_{\mu} c_{b}\right)\left(\bar{q}_{d} \gamma_{5} C \bar{c}_{e}^{T}\right)\right],
\end{aligned}
$$

where $q$ denotes a $u$ or $d$ quark and $c$ is the charm quark. We can also construct a current describing $X_{q}$ as a molecular $D^{* 0} \overline{D^{0}}$ state:

$$
\begin{aligned}
j_{\mu}^{(q, \mathrm{~mol})}(x) & =\frac{1}{\sqrt{2}}\left[\left(\bar{q}_{a}(x) \gamma_{5} c_{a}(x) \bar{c}_{b}(x) \gamma_{\mu} q_{b}(x)\right)\right. \\
& \left.-\left(\bar{q}_{a}(x) \gamma_{\mu} c_{a}(x) \bar{c}_{b}(x) \gamma_{5} q_{b}(x)\right) .\right]
\end{aligned}
$$

The currents in Eqs. (67) [368] and (68) [369] have both been used. In each case it was possible to find a Borel window where the pole contribution is bigger than the continuum contribution and with a reasonable OPE convergence. On the OPE side, the calculations were done to leading order in $\alpha_{\mathrm{s}}$ including condensates up to dimension eight. The mass obtained in [368] considering the allowed Borel window and the uncertainties in the parameters was $m_{X}=(3.92 \pm 0.13) \mathrm{GeV}$, compatible with the measured value. For the current in Eq. (68), the OPE convergence and pole contribution yield a similar Borel window, resulting in a predicted mass [369] of $m_{X}=(3.87 \pm 0.07) \mathrm{GeV}$, also consistent with the measured value and more precise than that obtained with the tetraquark current.

In principle, we might expect a large partial decay width for the decay $X \rightarrow \rho^{0} J / \psi$. The initial state already contains the four necessary quarks and no rules prohibit the decay. Therefore this decay is allowed, similar to the case of the light scalars $\sigma$ and $\kappa$ studied in [370], with widths of order $400 \mathrm{MeV}$. The decay width is essentially determined by the $X J / \psi V(V=\rho, \omega)$ coupling constant. The decay width was computed using QCDSR [371] with $g_{X J / \psi V}$ evaluated assuming that the $X(3872)$ is described by the tetraquark current, Eq. (67). The QCDSR calculation for the vertex $X(3872) J / \psi V$ is based on the evaluation of the three-point correlation function, which is a straightforward extension of Eq. (65) to the case of three currents representing the three particles in the decay. In [372] the current representing the $X$ was given by:

$$
j_{\alpha}^{X}=\cos \theta j_{\alpha}^{(u, \mathrm{di})}+\sin \theta j_{\alpha}^{(d, \mathrm{di})},
$$

with $j_{\alpha}^{(q, \mathrm{di})}$ given in Eq. (67). This mixing between diquarks with different light flavors was first introduced [285] to explain the decay properties of the
$X(3872)$. Using $\theta \approx 20^{\circ}$ it is possible to reproduce the measured ratio $r_{\omega}$ given in Table 12. With the same angle, the $X J / \psi \omega$ coupling constant was calculated with QCDSR [371] and found to be $g_{X \psi \omega}=13.8 \pm 2.0$. This value is much bigger than the estimate of [285] and leads to a large partial decay width of $\Gamma(X \rightarrow J / \psi(n \pi))=$ $(50 \pm 15) \mathrm{MeV}$. A similar value was also obtained [372] using a molecular current similar to Eq. (68). Therefore it is not possible to explain the small width of the $X(3872)$ from a QCDSR calculation if the $X$ is a pure four-quark state. In [372], the $X(3872)$ was treated as a mixture of a $c \bar{c}$ current with a molecular current, similar to the mixing considered in [373] to study the light scalar mesons:

$$
J_{\mu}^{q}(x)=\sin \alpha j_{\mu}^{(q, \mathrm{~mol})}(x)+\cos \alpha j_{\mu}^{(q, 2)}(x),
$$

with $j_{\mu}^{(q, \mathrm{~mol})}(x)$ given in Eq. (68) and

$$
j_{\mu}^{(q, 2)}(x)=\frac{1}{6 \sqrt{2}}\langle\bar{q} q\rangle\left[\bar{c}_{a}(x) \gamma_{\mu} \gamma_{5} c_{a}(x)\right] .
$$

The introduction of the quark condensate, $\langle\bar{q} q\rangle$, ensures that $j_{\mu}^{(q, 2)}(x)$ and $j_{\mu}^{(q, \mathrm{~mol})}(x)$ have the same dimension.

It is not difficult to reproduce the experimental mass of the $X(3872)$ [368-370, 372]. This is also true for the current in Eq. (70) for a wide range of mixing angles, $\alpha$, but, as observed in [372], it is not possible to match the measured value of $r_{\omega}$. In order to reproduce the ratio $r_{\omega}$ given in Table 12, it is also necessary to consider a mixture of $D^{+} D^{*-}$ and $D^{-} D^{*+}$ components [285]. In this case the current is given by

$$
j_{\mu}^{X}(x)=\cos \theta J_{\mu}^{u}(x)+\sin \theta J_{\mu}^{d}(x),
$$

where $J_{\mu}^{u}(x)$ and $J_{\mu}^{d}(x)$ are given by Eq. (70). With this particular combination one obtains:

$$
\frac{\Gamma\left(X \rightarrow J / \psi \pi^{+} \pi^{-} \pi^{0}\right)}{\Gamma\left(X \rightarrow J / \psi \pi^{+} \pi^{-}\right)} \simeq 0.15\left(\frac{\cos \theta+\sin \theta}{\cos \theta-\sin \theta}\right)^{2},
$$

exactly the same relation $[285,371]$ that imposes $\theta \sim 20^{\circ}$ in Eq. (69) and compatible with the measured value of $r_{\omega}$ given in Table 12.

It was shown [372] that, with Eq. (72) and a mixing angle $\alpha=(9 \pm 4)^{\circ}$ in Eq. (70), it is possible to describe the measured $X(3872)$ mass with a decay width of $\Gamma(X \rightarrow$ $J / \psi(n \pi))=(9.3 \pm 6.9) \mathrm{MeV}$, which is compatible with the experimental upper limit. The same mixing angle was used to evaluate the ratio

$$
r_{\gamma 1} \equiv \frac{\Gamma(X \rightarrow \gamma J / \psi)}{\Gamma\left(X \rightarrow \pi^{+} \pi^{-} J / \psi\right)},
$$

obtaining $r_{\gamma 1}=0.19 \pm 0.13$ [374], in excellent agreement with the measured value given in Table 12 . Hence QCDSR calculations strongly suggest that the $X(3872)$ can be well described by a $c \bar{c}$ current with a small, but fundamental, admixture of molecular $\left(D \bar{D}^{*}\right)$ or 
tetraquark $[c q][\bar{c} \bar{q}]$ currents. In connection with the discussion in Sect. 2.9.3, a possible $D_{s}^{+} D_{s}^{*-}$ molecular state, such an $X_{s}$ state, was also considered. The $X_{s}$ mass obtained, $3900 \mathrm{MeV}$, was practically degenerate with the $X(3872)$. Therefore QCDSR indicate a larger binding energy for $X_{s}$ than for the $X(3872)$, leading to a smaller mass than predicted in [344].

It is straightforward to extend the analysis done for the $X(3872)$ to the case of the bottom quark. Using the same interpolating field of Eq. (67) with the charm quark replaced by the bottom quark, the analysis done for $X(3872)$ was repeated [368] for an analogous $X_{b}$. Here there is also a good Borel window. The prediction for the mass of the state that couples to a tetraquark $[b q][\bar{b} \bar{q}]$ with $J^{P C}=1^{++}$current is $m_{X_{b}}=(10.27 \pm 0.23) \mathrm{GeV}$. The central value is close to the mass of $\Upsilon(3 S)$ and appreciably below the $B^{*} \bar{B}$ threshold at about $10.6 \mathrm{GeV}$. For comparison, the molecular model predicts a mass for $X_{b}$ which is about $50-60 \mathrm{MeV}$ below this threshold [33], while a relativistic quark model without explicit $(b \bar{b})$ clustering predicts a value about $133 \mathrm{MeV}$ below threshold [286].

Summarizing, in QCDSR it is possible to satisfactorily explain all the $X(3872)$ properties with a mixture of $\mathrm{a} \approx 97 \% c \bar{c}$ component and $\mathrm{a} \approx 3 \%$ meson molecule component. This molecular component must be a mixture of $88 \% D^{0} D^{* 0}$ and $12 \% D^{+} D^{*-}$ [372]. These conclusions hold only for the quantum number assignment $J^{P C}=1^{++}$.

\section{QCDSR and the $Y$ states}

The states $Y(4260), Y(4360)$ and $Y(4660)$ do not easily fit in the predictions of the standard quark model. The $Y(4260)$ has a $\pi^{+} \pi^{-} J / \psi$ decay width of $\simeq 100 \mathrm{MeV}$, and no isospin-violating decay such as $Y \rightarrow J / \psi \pi^{+} \pi^{-} \pi^{0}$ has been observed. With these features, the $Y$ is likely to be a meson molecule or a hybrid state. QCDSR calculations of the mass strongly suggest the $D^{*} \bar{D}_{0}-\bar{D}^{*} D_{0}$ and $D \bar{D}_{1}-\bar{D} D_{1}$ as favorite molecular combinations, where the symbols $D, D^{*}, D_{0}$ and $D_{1}$ represent the lowest-lying pseudoscalar, vector, scalar, and axial-vector charm messons, respectively. From now on we shall omit the combinations arising from symmetrization and use the short forms for these states, e.g., $D_{0} \bar{D}$.

The vector $Y$ states can be described by molecular or tetraquark currents, with or without an $s \bar{s}$ pair. QCD sum rule calculations have been performed [375, 376] using these currents. A possible interpolating operator representing a $J^{P C}=1^{--}$tetraquark state with the symmetric spin distribution

$$
[c s]_{S=0}[\bar{c} \bar{s}]_{S=1}+[c s]_{S=1}[\bar{c} \bar{s}]_{S=0}
$$

is given by:

$$
\begin{aligned}
j_{\mu} & =\frac{\epsilon_{a b c} \epsilon_{d e c}}{\sqrt{2}}\left[\left(s_{a}^{T} C \gamma_{5} c_{b}\right)\left(\bar{s}_{d} \gamma_{\mu} \gamma_{5} C \bar{c}_{e}^{T}\right)\right. \\
& \left.+\left(s_{a}^{T} C \gamma_{5} \gamma_{\mu} c_{b}\right)\left(\bar{s}_{d} \gamma_{5} C \bar{c}_{e}^{T}\right)\right] .
\end{aligned}
$$

This current has good OPE convergence and pole dominance in a given Borel window. The result for the mass of the state described by the current in Eq. (76) is $m_{Y}=(4.65 \pm 0.10) \mathrm{GeV}$ [375], in excellent agreement with the mass of the $Y(4660)$ meson, lending credence to the conclusion [375] that the $Y(4660)$ meson can be described with a diquark-antidiquark tetraquark current with a spin configuration given by scalar and vector diquarks. The quark content of the current in Eq. (76)) is also consistent with the experimental dipion invariant mass spectra, which give some indication that the $Y(4660)$ has a well-defined dipion intermediate state consistent with $f_{0}(980)$.

Replacing the strange quarks in Eq. (76) by a generic light quark $q$, the mass obtained for a $1^{--}$state described with the symmetric spin distribution

$$
[c q]_{S=0}[\bar{c} \bar{q}]_{S=1}+[c q]_{S=1}[\bar{c} \bar{q}]_{S=0}
$$

is $m_{Y}=(4.49 \pm 0.11) \mathrm{GeV}$ [375], which is slightly larger than but consistent with the measured $Y(4360)$ mass.

The $Y$ mesons can also be described by molecular-type currents. A $D_{s 0}(2317) \bar{D}_{s}^{*}(2110)$ molecule with $J^{P C}=$ $1^{--}$could also decay into $\psi(2 S) \pi^{+} \pi^{-}$with a dipion mass spectrum consistent with $f_{0}(980)$. A current with $J^{P C}=$ $1^{--}$and a symmetric combination of scalar and vector mesons is

$$
j_{\mu}=\frac{1}{\sqrt{2}}\left[\left(\bar{s}_{a} \gamma_{\mu} c_{a}\right)\left(\bar{c}_{b} s_{b}\right)+\left(\bar{c}_{a} \gamma_{\mu} s_{a}\right)\left(\bar{s}_{b} c_{b}\right)\right] .
$$

The mass obtained in [375] for this current is $m_{D_{s 0} \bar{D}_{s}^{*}}=$ $(4.42 \pm 0.10) \mathrm{GeV}$, which is in better agreement with $Y(4360)$ than $Y(4660)$.

To consider a molecular $D_{0} \bar{D}^{*}$ current with $J^{P C}=$ $1^{--}$, the strange quarks in Eq. (78) must be replaced with a generic light quark $q$. The mass obtained with such current is [375] $m_{D_{0} \bar{D}^{*}}=(4.27 \pm 0.10) \mathrm{GeV}$, in excellent agreement with the $Y(4260)$ mass. In order to associate this molecular state with $Y(4260)$, a better understanding of the dipion invariant mass spectra in $Y(4260) \rightarrow \pi^{+} \pi^{-} J / \psi$ is needed. From the measured spectra, it seems that the $Y(4260)$ is consistent with a nonstrange molecular state $D_{0} \bar{D}^{*}$. Using a $D_{0}$ mass of $m_{D_{0}}=2352 \pm 50 \mathrm{MeV}$, the $D_{0} \bar{D}^{*}$ threshold is $\approx 4360 \mathrm{MeV}, 100 \mathrm{MeV}$ above the $4.27 \pm 0.10 \mathrm{GeV}$ quoted above, indicating the possibility of a bound state.

A $J^{P C}=1^{--}$molecular current can also be constructed with pseudoscalar and axial-vector mesons. A molecular $D \bar{D}_{1}$ current was used in [376]. The mass obtained with this current is $m_{D \bar{D}_{1}}=(4.19 \pm 0.22) \mathrm{GeV}$. Thus, taking the mass uncertainty into account, the molecular $D \bar{D}_{1}$ assignment for the $Y(4260)$ is also viable, in agreement with a meson-exchange model [377]. The $D \bar{D}_{1}$ threshold is $\approx 4285 \mathrm{MeV}$, close to the $Y(4260)$ mass, indicating the possibility of a loosely bound molecular state.

Summarizing, the $Y$ states can be understood as charmonium hybrids, tetraquark states, and a $D_{0} \bar{D}^{*}$ or $D \bar{D}_{1}$ molecular state for $Y(4260)$. Also possible are a tetraquark state with two axial $[c s] P$-wave diquarks, or two scalar $[c s] P$-wave diquarks for $Y(4360)$. 
$\underline{Z(4430)^{+}}$

A current describing the $Z(4430)^{+}$as a $D^{*} D_{1}$ molecule with $J^{P}=0^{-}$is [378]

$$
\begin{aligned}
j=\frac{1}{\sqrt{2}}[ & \left(\bar{d}_{a} \gamma_{\mu} c_{a}\right)\left(\bar{c}_{b} \gamma^{\mu} \gamma_{5} u_{b}\right)+ \\
& \left.\left(\bar{d}_{a} \gamma_{\mu} \gamma_{5} c_{a}\right)\left(\bar{c}_{b} \gamma^{\mu} u_{b}\right)\right] .
\end{aligned}
$$

This current corresponds to a symmetric $D^{*+} \bar{D}_{1}^{0}+$ $\bar{D}^{* 0} D_{1}^{+}$state with positive $G$-parity, consistent with the observed decay $Z(4430)^{+} \rightarrow \pi^{+} \psi(2 S)$. The mass obtained in a QCDSR calculation using such a current is [378] $m_{D^{*} D_{1}}=(4.40 \pm 0.10) \mathrm{GeV}$, in an excellent agreement with the measured mass.

To check if the $Z(4430)^{+}$could also be described as a diquark-antidiquark state with $J^{P}=0^{-}$, the current [379]

$$
\begin{aligned}
& j_{0^{-}}=\frac{i \epsilon_{a b c} \epsilon_{d e c}}{\sqrt{2}}\left[\left(u_{a}^{T} C \gamma_{5} c_{b}\right)\left(\bar{d}_{d} C \bar{c}_{e}^{T}\right)-\right. \\
& \left.\left(u_{a}^{T} C c_{b}\right)\left(\bar{d}_{d} \gamma_{5} C \bar{c}_{e}^{T}\right)\right]
\end{aligned}
$$

was used to obtain the mass $m_{Z_{\left(0^{-}\right)}}=(4.52 \pm$ 0.09) GeV [379], somewhat larger than, but consistent with, the experimental value. The result using a molecular-type current is in slightly better agreement with the experimental value. However, since there is no one-to-one correspondence between the structure of the current and the state, this result cannot be used to conclude that the $Z(4430)^{+}$is favored as a molecular state over a diquark-antidiquark state. To get a measure of the coupling between the state and the current, the parameter $\lambda$, defined in Eq. (66), is evaluated as $\lambda_{D^{*} D_{1}} \simeq 1.5 \lambda_{Z_{\left(0^{-}\right)}}$. This suggests that a physical particle with $J^{P}=0^{-}$and quark content $c \bar{c} u \bar{d}$ has a stronger coupling to the molecular $D^{*} D_{1}$-type current than with that of Eq. (80).

A diquark-antidiquark interpolating operator with $J^{P}=1^{-}$and positive $G$-parity was also considered [379]:

$$
\begin{aligned}
j_{\mu}^{1^{-}} & =\frac{\epsilon_{a b c} \epsilon_{d e c}}{\sqrt{2}}\left[\left(u_{a}^{T} C \gamma_{5} c_{b}\right)\left(\bar{d}_{d} \gamma_{\mu} \gamma_{5} C \bar{c}_{e}^{T}\right)\right. \\
& \left.+\left(u_{a}^{T} C \gamma_{5} \gamma_{\mu} c_{b}\right)\left(\bar{d}_{d} \gamma_{5} C \bar{c}_{e}^{T}\right)\right] .
\end{aligned}
$$

In this case the Borel stability obtained is worse than for the $Z^{+}$with $J^{P}=0^{-}$[379]. The mass obtained is $m_{Z_{\left(1^{-}\right)}}=(4.84 \pm 0.14) \mathrm{GeV}$, much larger than both the measured value and that obtained using the $J^{P}=0^{-}$ current. Thus, it is possible to describe the $Z(4430)^{+}$as a diquark-antidiquark or molecular state with $J^{P}=0^{-}$, and $J^{P}=1^{-}$configuration is disfavored.

It is straightforward to extend the $D^{*} D_{1}$-molecule analysis to the bottom quark. Using the same interpolating field of Eq. (79) but replacing the charm quark with bottom, an investigation of the hypothetical $Z_{b}$ is performed [378]. The OPE convergence is even better than for $Z(4430)^{+}$. The predicted mass is $m_{Z_{B^{*} B_{1}}}=$
TABLE 19: Summary of QCDSR results [381]. The labels 1,3 , and $\overline{3}$ refer to singlet, triplet and anti-triplet color configurations, respectively. The symbols $\mathrm{S}, \mathrm{P}, \mathrm{V}$, and A refer to scalar, pseudoscalar, vector and axial-vector $q \bar{q}, q q$ or $\bar{q} \bar{q}$ combinations, respectively

\begin{tabular}{ccc}
\hline \hline State & Configuration & Mass $(\mathrm{GeV})$ \\
\hline$X(3872)$ & {$[c q]_{3}[\bar{c} \bar{q}]_{3}(\mathrm{~S}+\mathrm{A})$} & $3.92 \pm 0.13$ \\
& {$[c \bar{q}]_{1}[\bar{c}]_{1}(\mathrm{P}+\mathrm{V})$} & $3.87 \pm 0.07$ \\
& {$[c \bar{c}]_{1}(A)+$} & \\
$Y(4140)$ & {$[c \bar{q}]_{1}[\bar{c} q]_{1}(\mathrm{P}+\mathrm{V})$} & $3.77 \pm 0.18$ \\
& {$[c \bar{q}]_{1}[\bar{c} q]_{1}(\mathrm{~V}+\mathrm{V})$} & $4.13 \pm 0.11$ \\
$Y(4260)$ & {$[c \bar{s}]_{1}[\bar{c} s]_{1}(\mathrm{~V}+\mathrm{V})$} & $4.14 \pm 0.09$ \\
& {$[c \bar{q}]_{1}[\bar{c} q]_{1}(\mathrm{~S}+\mathrm{V})$} & $4.27 \pm 0.10$ \\
$Y(4360)$ & {$[c \bar{c}]_{1}[\bar{c} q]_{1}(\mathrm{P}+\mathrm{A})$} & $4.19 \pm 0.22$ \\
& {$[c q]_{\overline{3}}[\bar{c} \bar{q}]_{3}(\mathrm{~S}+\mathrm{V})$} & $4.49 \pm 0.11$ \\
$Y(4660)$ & {$[c \bar{s}]_{1}[\bar{c} s]_{1}(\mathrm{~S}+\mathrm{V})$} & $4.42 \pm 0.10$ \\
$Z(4430)$ & {$[c s]_{3}[\bar{c}]_{3}(\mathrm{~S}+\mathrm{V})$} & $4.65 \pm 0.10$ \\
& {$[c \bar{d}]_{1}[\bar{c} u]_{1}(\mathrm{~V}+\mathrm{A})$} & $4.40 \pm 0.10$ \\
& {$[c u]_{3}[\bar{c} \bar{c}]_{3}(\mathrm{~S}+\mathrm{P})$} & $4.52 \pm 0.09$ \\
\hline \hline
\end{tabular}

$(10.74 \pm 0.12) \mathrm{GeV}$, in agreement with that of [380]. For the analogous strange meson $Z_{s}^{+}$, considered as a pseudoscalar $D_{s}^{*} D_{1}$ molecule, the current is obtained by replacing the $d$ quark in Eq. (79) with an $s$ quark. The predicted mass is [378] $m_{D_{s}^{*} D_{1}}=(4.70 \pm 0.06) \mathrm{GeV}$, larger than the $D_{s}^{*} D_{1}$ threshold of $\sim 4.5 \mathrm{GeV}$, indicating that this state is probably very broad and therefore might be difficult to observe.

Summarizing, the $Z(4430)^{+}$has been successfully described by both a $D^{*} D_{1}$ molecular current and a diquarkantidiquark current with $J^{P}=0^{-}$.

Table 19 summarizes the QCDSR results for the masses and corresponding quark configurations of the new states [381]. Masses obtained with QCDSR in the molecular approach can be found in [382] (they agree with those in Table 19 from [381] apart from small discrepancies, which should be addressed). The principal input parameters used to obtain the values in Table 19 are

$$
\begin{aligned}
m_{c}\left(m_{c}\right) & =(1.23 \pm 0.05) \mathrm{GeV} \\
\langle\bar{q} q\rangle & =-(0.23 \pm 0.03)^{3} \mathrm{GeV}^{3} \\
\langle\bar{q} g \sigma . G q\rangle & =m_{0}^{2}\langle\bar{q} q\rangle \\
m_{0}^{2} & =0.8 \mathrm{GeV}^{2} \\
\left\langle g^{2} G^{2}\right\rangle & =0.88 \mathrm{GeV}^{4}
\end{aligned}
$$

Uncertainties in Table 19 come from several sources: quark masses and $\alpha_{\mathrm{s}}$ are varied by their errors around their central values in Eq. (82); the condensates are taken from previous QCDSR analyses and their uncertainties propagated; particle masses are extracted for a range of Borel masses throughout the Borel window; and in the case of mixing, the masses and widths are computed for 
several values of the mixing angle centered on its optimal value.

Some of the states discussed above can be understood as both tetraquark and molecular structures. This freedom will be reduced once a comprehensive study of the decay width is performed. At this point, it appears that the only decay width calculated with QCDSR is in [372]. The next challenge for the QCDSR community is to understand the existing data on decays. Explaining these decays will impose severe constraints in the present picture of the new states [372].

\subsubsection{Theoretical explanations for new states}

Table 20 lists the new states with their properties and proposed theoretical explanations. The theoretical hypothesis list is far from exhaustive.

\subsection{Beyond the Standard Model}

\subsubsection{Mixing of a light CP-odd Higgs and $\eta_{b}(n S)$ resonances}

This section explores the possibility that the measured $\eta_{b}(1 S)$ mass (Table 7 and Eq. (5)) is smaller than the predictions (see Sects. 2.5.3, 2.6.2, 2.7, and 2.8.1) due to mixing with a CP-odd Higgs scalar $A$, and predictions for the spectrum of the $\eta_{b}(n S)$ - $A$ system and the branching fractions into $\tau^{+} \tau^{-}$as functions of $m_{A}$ are made. Such mixing can cause masses of the $\eta_{b}$-like eigenstates of the full mass matrix to differ considerably from their values in pure QCD [389-391]. Thus the mass of the state interpreted as the $\eta_{b}(1 S)$ can be smaller than expected if $m_{A}$ is slightly above $9.4 \mathrm{GeV}$. The masses of the states interpreted as $\eta_{b}(2 S)$ and $\eta_{b}(3 S)$ can also be affected. Furthermore, all $\eta_{b}(n S)$ states can acquire non-negligible branching ratios into $\tau^{+} \tau^{-}$due to their mixing with $A$.

A relatively light, CP-odd Higgs scalar can appear, e.g., in nonminimal supersymmetric extensions of the Standard Model (SM) as the NMSSM (Next-to-Minimal Supersymmetric Standard Model); see [392] and references therein. Its mass must satisfy constraints from LEP, where it could have been produced in $e^{+} e^{-} \rightarrow$ $Z^{*} \rightarrow Z H$ and $H \rightarrow A A$ (where $H$ is a CP-even Higgs scalar). For $m_{A}>10.5 \mathrm{GeV}$, where $A$ would decay dominantly into $b \bar{b}$, and $m_{H}<110 \mathrm{GeV}$, corresponding LEP constraints are quite strong [393]. If $2 m_{\tau}<m_{A}<10.5 \mathrm{GeV}, A$ would decay dominantly into $\tau^{+} \tau^{-}$and values for $m_{H}$ down to $\sim 86 \mathrm{GeV}$ are allowed [393] even if $H$ couples to the $Z$ boson with the strength of a SM Higgs boson. A possible explanation of an excess of $b \bar{b}$ events found at LEP [394, 395] provides additional motivation for a CP-odd Higgs scalar with a mass below 10.5 GeV. However, a recent (but preliminary) analysis from ALEPH has found no further evidence of such an excess.
The masses of all 4 physical states (denoted by $\eta_{i}$, $i=1 \ldots 4$ ) as functions of $m_{A}$ are shown together with the uncertainty bands in Fig. 35 [396]. By construction, $m_{\eta_{1}} \equiv m_{\text {obs }}\left[\eta_{b}(1 S)\right]$ is constrained to the measured value. For clarity the assumed values for $m_{\eta_{b}^{0}(n S)}$ are indicated as horizontal dashed lines. For $m_{A}$ not far above $9.4 \mathrm{GeV}$, the effects of the mixing on the states $\eta_{b}^{0}(2 S)$ and $\eta_{b}^{0}(3 S)$ are negligible, but for larger $m_{A}$ the spectrum can differ considerably from the standard one.

Now turning to the tauonic branching ratios of the $\eta_{i}$ states induced by their $A$-components, assuming $\Gamma_{\eta_{b}^{0}(1 S)} \sim 5-20 \mathrm{MeV}$, the predicted branching ratio of $\eta_{b}(1 S) \rightarrow \tau^{+} \tau^{-}$is compatible with the BABAR upper limit of $8 \%$ at $90 \%$ CL [397]. For the heavier $\eta_{i}$ states, the corresponding branching fractions vary with $m_{A}$ as shown in Fig. 36, where $\Gamma_{\eta_{b}^{0}(1 S)} \sim 10 \mathrm{MeV}$ and $\Gamma_{\eta_{b}^{0}(2 S)} \sim \Gamma_{\eta_{b}^{0}(3 S)} \sim 5 \mathrm{MeV}$. With larger (smaller) total widths, these branching fractions would be smaller (larger).

The predicted masses and branching fractions in Figs. 35 and 36, respectively, together with an accurate test of lepton-universality-breaking in $\Upsilon$ decays [396, 398], can play an important role both in the experimental search for excited $\eta_{b}(n S)$ states and subsequent interpretation of the observed spectrum. Such comparisons would test the hypothesis of $\eta_{b}$ mixing with a light CPodd Higgs boson.

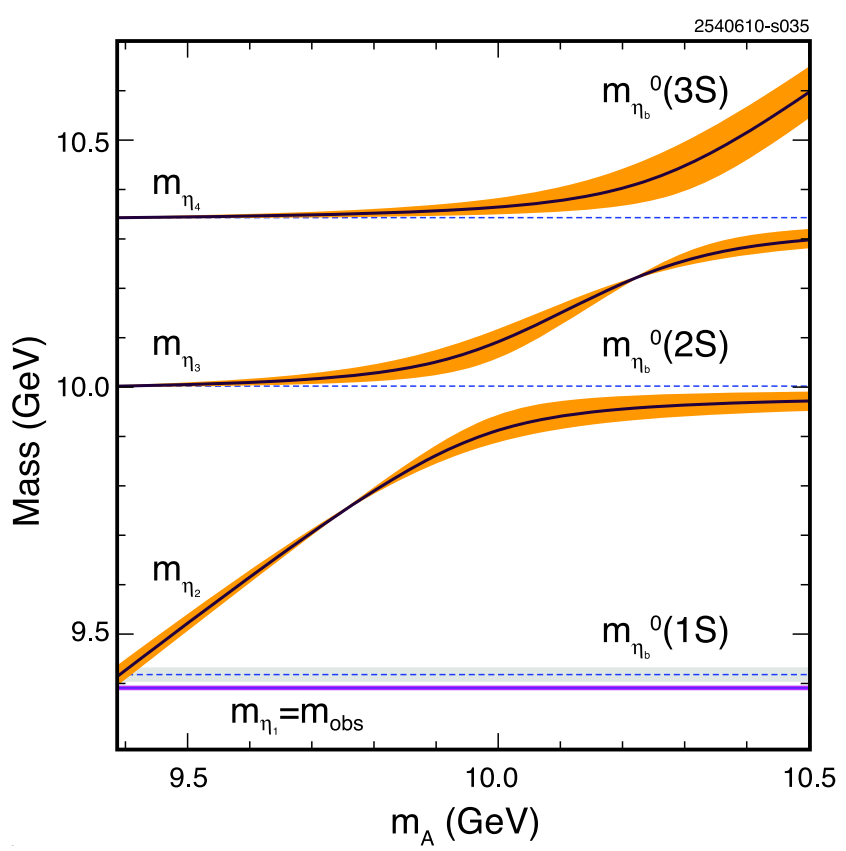

FIG. 35: The masses of all eigenstates as function of $m_{A}$ 
TABLE 20: As in Table 9, new unconventional states in the $c \bar{c}, b \bar{c}$, and $b \bar{b}$ regions, ordered by mass, with possible interpretations (which do not apply solely to the decay modes listed alongside). References are representative, not necessarily exhaustive. The QCDSR notation is explained in the caption to Table 19

\begin{tabular}{|c|c|c|c|c|c|c|}
\hline State & $m(\mathrm{MeV})$ & $\Gamma(\mathrm{MeV})$ & $J^{P C}$ & Modes & Interpretation & Reference(s) \\
\hline$X(3872)$ & $3871.52 \pm 0.20$ & $1.3 \pm 0.6$ & $1^{++} / 2^{-+}$ & $\begin{array}{l}\pi^{+} \pi^{-} J / \psi \\
D^{* 0} \bar{D}^{0} \\
\gamma J / \psi, \gamma \psi(2 S) \\
\omega J / \psi\end{array}$ & $\begin{array}{c}D^{* 0} \bar{D}^{0} \text { molecule (bound) } \\
D^{* 0} \bar{D}^{0} \text { unbound } \\
\text { if } 1^{++}, \chi_{c 2}(2 P) \\
\text { if } 2^{-+}, \eta_{c 2}(1 D) \\
\text { charmonium + mesonic-molecule mixture } \\
\text { QCDSR: }[c q]_{\overline{3}}[\bar{c} \bar{q}]_{3}(\mathrm{~S}+\mathrm{A}) \\
\text { QCDSR: }[c \bar{q}]_{1}[\bar{c} q]_{1}(\mathrm{P}+\mathrm{V}) \\
\text { QCDSR: }[c \bar{c}]_{1}(\mathrm{~A})+[c \bar{q}]_{1}[\bar{c} q]_{1}(\mathrm{P}+\mathrm{V})\end{array}$ & $\begin{array}{c}{[121,122]} \\
{[383-385]} \\
\\
{[71]} \\
{[81,93,126]} \\
{[381]} \\
{[381]} \\
{[381]} \\
{[381]}\end{array}$ \\
\hline$X(3915)$ & $3915.6 \pm 3.1$ & $28 \pm 10$ & $0,2^{?+}$ & $\omega J / \psi$ & $D^{*+} D^{*-}+D^{* 0} \bar{D}^{* 0}$ & {$[386]$} \\
\hline$Z(3930)$ & $3927.2 \pm 2.6$ & $24.1 \pm 6.1$ & $2^{++}$ & $D \bar{D}$ & $\begin{array}{c}\chi_{c 2}(2 P)\left(i . e ., 2^{3} P_{2} c \bar{c}\right) \\
1^{3} F_{2} c \bar{c}\end{array}$ & {$[71]$} \\
\hline$X(3940)$ & $3942_{-8}^{+9}$ & $37_{-17}^{+27}$ & $?^{?+}$ & $D \bar{D}^{*}$ & & \\
\hline$G(3900)$ & $3943 \pm 21$ & $52 \pm 11$ & $1^{--}$ & $D \bar{D}$ & Coupled-channel effect & {$[34]$} \\
\hline$Y(4008)$ & $4008_{-49}^{+121}$ & $226 \pm 97$ & $1^{--}$ & $\pi^{+} \pi^{-} J / \psi$ & & \\
\hline$Z_{1}(4050)^{+}$ & $4051_{-43}^{+24}$ & $82_{-55}^{+51}$ & $?$ & $\pi^{+} \chi_{c 1}(1 P)$ & hadrocharmonium & {$[282,355]$} \\
\hline$Y(4140)$ & $4143.0 \pm 3.1$ & $11.7_{-6.2}^{+9.1}$ & $?^{?+}$ & $\phi J / \psi$ & $\begin{array}{c}\text { QCDSR: }[c \bar{q}]_{1}[\bar{c} q]_{1}(\mathrm{~V}+\mathrm{V}) \\
\text { QCDSR: }[c \bar{s}]_{1}[\bar{c} s]_{1}(\mathrm{~V}+\mathrm{V}) \\
D_{s}^{*+} D_{s}^{*-}\end{array}$ & $\begin{array}{l}{[381]} \\
{[381]} \\
{[386]}\end{array}$ \\
\hline$X(4160)$ & $4156_{-25}^{+29}$ & $139_{-65}^{+113}$ & $?^{?+}$ & $D \bar{D}^{*}$ & & \\
\hline$Z_{2}(4250)^{+}$ & $4248_{-45}^{+185}$ & $177_{-72}^{+321}$ & $?$ & $\pi^{+} \chi_{c 1}(1 P)$ & hadrocharmonium & {$[282,355]$} \\
\hline$Y(4260)$ & $4263 \pm 5$ & $108 \pm 14$ & $1^{--}$ & $\begin{array}{l}\pi^{+} \pi^{-} J / \psi \\
\pi^{0} \pi^{0} J / \psi\end{array}$ & $\begin{array}{c}\text { charmonium hybrid } \\
J / \psi f_{0}(980) \text { bound state } \\
D_{0} \bar{D}^{*} \text { molecular state } \\
{[c s][\bar{c} \bar{s}] \text { tetraquark state }} \\
\text { hadrocharmonium } \\
\text { QCDSR: }[c \bar{q}]_{1}[\bar{c} q]_{1}(\mathrm{~S}+\mathrm{V}) \\
\text { QCDSR: }[c \bar{q}]_{1}[\bar{c} q]_{1}(\mathrm{P}+\mathrm{A})\end{array}$ & $\begin{array}{c}{[276-278]} \\
{[313]} \\
{[375]} \\
{[345,381]} \\
{[282,355]} \\
{[381]} \\
{[381]}\end{array}$ \\
\hline$Y(4274)$ & $4274.4_{-6.7}^{+8.4}$ & $32_{-15}^{+22}$ & $?^{?+}$ & $B \rightarrow K(\phi J / \psi)$ & $(\operatorname{see} Y(4140))$ & \\
\hline$X(4350)$ & $4350.6_{-5.1}^{+4.6}$ & $13.3_{-10.0}^{+18.4}$ & $0,2^{++}$ & $\phi J / \psi$ & & \\
\hline$Y(4360)$ & $4353 \pm 11$ & $96 \pm 42$ & $1^{--}$ & $\pi^{+} \pi^{-} \psi(2 S)$ & $\begin{array}{c}\text { hadrocharmonium } \\
\text { crypto-exotic hybrid } \\
Y_{B}(4360)=[c d][\bar{c} \bar{d}](1 P), \text { baryonium } \\
\text { QCDSR: }[c q]_{\overline{3}}[\bar{c} \bar{q}]_{3}(\mathrm{~S}+\mathrm{V}) \\
\text { QCDSR: }[c \bar{s}]_{1}[\bar{c} s]_{1}(\mathrm{~S}+\mathrm{V})\end{array}$ & $\begin{array}{c}{[282,355]} \\
{[71]} \\
{[321]} \\
{[381]} \\
{[381]}\end{array}$ \\
\hline$Z(4430)^{+}$ & $4443_{-18}^{+24}$ & $107_{-71}^{+113}$ & $?$ & $\pi^{+} \psi(2 S)$ & $\begin{array}{c}D^{*+} \bar{D}_{1}^{0} \text { molecular state } \\
{[c u][\bar{c} \bar{d}] \text { tetraquark state }} \\
\text { hadrocharmonium } \\
\text { QCDSR: }[c \bar{d}]_{1}[\bar{c} u]_{1}(\mathrm{~V}+\mathrm{A}) \\
\text { QCDSR: }[c u]_{3}[\bar{c} \bar{d}]_{3}(\mathrm{~S}+\mathrm{P})\end{array}$ & $\begin{array}{c}{[378,387]} \\
{[379]} \\
{[282,355]} \\
{[381]} \\
{[381]}\end{array}$ \\
\hline$X(4630)$ & $4634_{-11}^{+9}$ & $92_{-32}^{+41}$ & $1^{--}$ & $\Lambda_{c}^{+} \Lambda_{c}^{-}$ & $\begin{array}{c}Y_{B}(4660)=[c d][\bar{c} \bar{d}](2 P), \text { baryonium } \\
\psi(2 S) f_{0}(980) \text { molecule }\end{array}$ & $\begin{array}{l}{[321]} \\
{[324]}\end{array}$ \\
\hline$Y(4660)$ & $4664 \pm 12$ & $48 \pm 15$ & $1^{--}$ & $\pi^{+} \pi^{-} \psi(2 S)$ & $\begin{array}{c}\psi(2 S) f_{0}(980) \text { molecule } \\
{[c s][\bar{c} \bar{s}] \text { tetraquark state }} \\
\text { hadrocharmonium } \\
Y_{B}(4660)=[c d][\bar{c} \bar{d}](2 P), \text { baryonium } \\
\text { QCDSR: }[c s]_{\overline{3}}[\bar{c} \bar{s}]_{3}(\mathrm{~S}+\mathrm{V})\end{array}$ & $\begin{array}{c}{[306]} \\
{[375]} \\
{[282,355]} \\
{[321]} \\
{[381]}\end{array}$ \\
\hline$Y_{b}(10888)$ & $10888.4 \pm 3.0$ & $30.7_{-7.7}^{+8.9}$ & $1^{--}$ & $\pi^{+} \pi^{-} \Upsilon(n S)$ & $\begin{array}{c}\Upsilon(5 S) \\
b \text {-flavored } Y(4260)\end{array}$ & $\begin{array}{c}{[388]} \\
{[37,117]}\end{array}$ \\
\hline
\end{tabular}




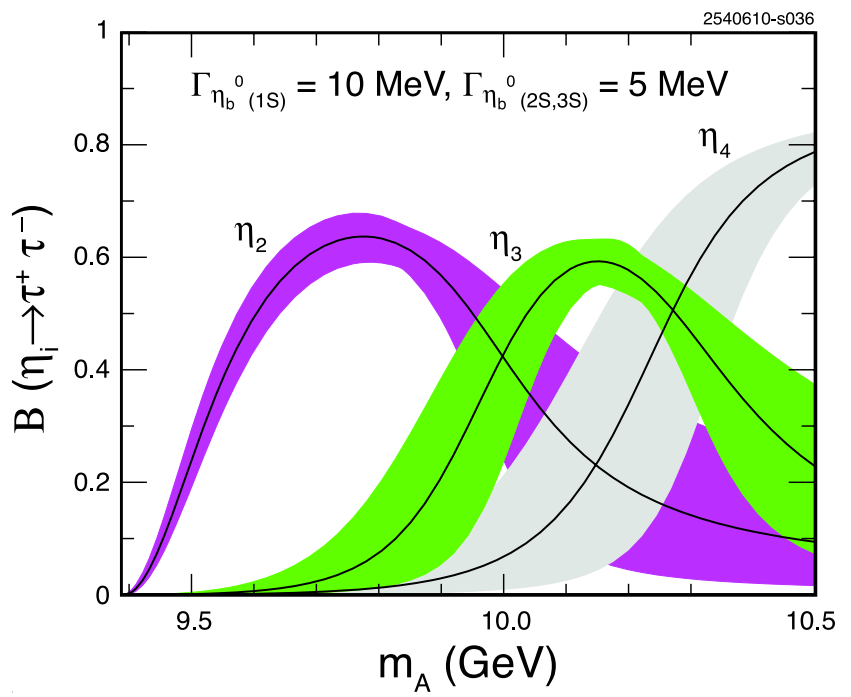

FIG. 36: The branching ratios into $\tau^{+} \tau^{-}$for the eigenstates $\eta_{2}, \eta_{3}$ and $\eta_{4}$ as functions of $m_{A}$

\subsubsection{Supersymmetric quarkonia}

As the top quark is too heavy to form QCD bound states (it decays via the electroweak transition $t \rightarrow b W$ ), the heaviest quarkonia that can be formed in the Standard Model are restricted to the bottom-quark sector. This, however, does not preclude the existence of the heavier quarkonium-like structures in theories beyond the Standard Model.

In particular, there exists some interest in detection of bound states of a top squark, $\widetilde{t}$, i.e., stoponium. From a practical point of view, observation of such a state would allow precise determination of squark masses, since stoponium decays via annihilation into SM particles do not have missing energy signatures with weakly-interacting lightest supersymmetric particles (LSPs). From a theoretical point of view such a scenario could be interesting because it could generate correct relic abundance for neutralino Dark Matter and baryon number asymmetry of the universe [399].

As it turns out, some supersymmetric models allow for a relatively light top squark that can form bound states. One condition for such an occurrence involves forbidding two-body decay channels

$$
\tilde{t}_{1} \rightarrow b \widetilde{C}_{1}, \quad \tilde{t}_{1} \rightarrow t \widetilde{N}_{1},
$$

where $\widetilde{t}_{1}$ is the lighter mass eigenstate of a top squark, and $\widetilde{C}_{1}$ and $\widetilde{N}_{1}$ are the lightest chargino and neutralino states, respectively. These decay channels can be kinematically forbidden if $m_{\widetilde{t}_{1}}-m_{\widetilde{C}_{1}}<m_{b}$ and $m_{\widetilde{t}_{1}}-m_{\widetilde{N}_{1}}<$ $m_{t}$, which can be arranged in SUSY models (although not in mSUGRA). Other decay channels are either flavorviolating or involve more than two particles in the final state and are therefore suppressed enough to allow formation of stoponia. Since top squarks are scalars, the lowest-energy bound state $\eta_{\widetilde{t}}$ has spin zero and mass predicted in the range of $200-800 \mathrm{GeV}$ [400-402].

The $\eta_{\tilde{t}}$ spin-zero state can be most effectively produced at the LHC in the $g g \rightarrow \eta_{\tilde{t}}$ channel. Possible decay channels of $\eta_{\tilde{t}}$ include $\gamma \gamma, g g, Z Z, W W$, as well as hadronic and leptonic final states. It is the $\gamma \gamma$ decay channel that has recently received most attention.

The production cross section for a stoponium $\eta_{\widetilde{t}}$ detected in the $\gamma \gamma$ channel can be written as [402]

$$
\begin{aligned}
\sigma\left(p p \rightarrow \eta_{\widetilde{t}} \rightarrow \gamma \gamma\right) & =\frac{\pi^{2}}{8 m_{\eta_{\tilde{t}}^{3}}^{3}} \mathcal{B}\left(\eta_{\widetilde{t}} \rightarrow g g\right) \Gamma\left(\eta_{\widetilde{t}} \rightarrow \gamma \gamma\right) \\
& \times \int_{\tau}^{1} d x \frac{t}{x} g\left(x, Q^{2}\right) g\left(\tau / x, Q^{2}\right),
\end{aligned}
$$

where $g\left(x, Q^{2}\right)$ is the gluon parton distribution function, and $\mathcal{B}\left(\eta_{\tilde{t}} \rightarrow g g\right)=\Gamma\left(\eta_{\tilde{t}} \rightarrow g g\right) / \Gamma_{\text {tot }}$ is the branching fraction for $\eta_{\tilde{t}} \rightarrow g g$ decay. Note that Eq. (84) contains $\eta_{\tilde{t}}$ decay widths into $\gamma \gamma$ and $g g$ channels that depend on the value of the $\eta_{\widetilde{t}}$ wave function at the origin,

$$
\begin{aligned}
\Gamma\left(\eta_{\tilde{t}} \rightarrow \gamma \gamma\right) & =\frac{4}{3} \frac{8}{9} \frac{4 \pi \alpha^{2}}{m_{\eta_{\tilde{t}}}^{2}}\left|\psi_{\eta_{\tilde{t}}}(0)\right|^{2}, \\
\Gamma\left(\eta_{\widetilde{t}} \rightarrow g g\right) & =\frac{9 \alpha_{\mathrm{s}}}{8 \alpha} \Gamma\left(\eta_{\tilde{t}} \rightarrow \gamma \gamma\right) .
\end{aligned}
$$

These decay widths have recently been evaluated taking into account one-loop QCD corrections [403, 404]. These next-to-leading order QCD corrections to $\Gamma\left(\eta_{\tilde{t}} \rightarrow \gamma \gamma\right)$ significantly decrease [403] the rate while NLO corrections to the production cross section tend to increase it [404], leaving the product in Eq. (84) largely unchanged from the leading-order prediction.

It appears that, if possible, observation of stoponium would require significant statistics at the LHC. For example, for stoponium masses on the order of or less than $300 \mathrm{GeV}$, an integrated luminosity of $10 \mathrm{fb}^{-1}$ [402] is required. Larger mass values would require even greater statistics. Thus discovery of stoponium decays could only improve determinations of the squark mass, which would probably be already available using other methods. The possibility of learning about bound-state QCD dynamics in a regime where calculations are under better theoretical control makes this study worthwhile.

\subsubsection{Invisible decays of $\psi$ and $\Upsilon$}

Measurements of invisible, meaning undetected, decay rates of $\psi$ and $\Upsilon$ resonances can discover or place strong constraints on dark matter scenarios where candidate dark matter constituents are lighter than the $b$ quark [405].

According to the SM, invisible decays of the $\Upsilon(1 S)$ can proceed via $b \bar{b}$ annihilation into a $\nu \bar{\nu}$ pair with a branching fraction $\mathcal{B}(\Upsilon(1 S) \rightarrow \nu \bar{\nu}) \simeq 10^{-5}$ [406], well below current experimental sensitivity. However, models containing low-mass dark matter components might enhance 
such invisible decay modes up to observable rates [407410]. Interestingly, from the astroparticle and cosmological side, DAMA and CoGeNT experiments have reported the observation of events compatible with a Light Dark-Matter (LDM) candidate with a mass inside the interval $[5,10] \mathrm{GeV}$ [411]. Such a mass range for LDM constituents is especially attractive in certain cosmological scenarios, e.g., the Asymmetric Dark Matter model where the cosmological dark matter density arises from the baryon asymmetry of the universe and is expected to have an LDM mass of order [1-10] GeV (see [412, 413] and references therein).

Searches for the $\Upsilon(1 S) \rightarrow$ invisible mode have been carried out by Argus [414], CLEO [415], Belle [416], and BABAR [417] using the cascade decay, $\Upsilon(2,3 S) \rightarrow$ $\pi^{+} \pi^{-} \Upsilon(1 S)$, in which the dipion tags the invisiblydecaying $\Upsilon(1 S)$. The most recent experimental data from BABAR [417] yield an upper limit at the 90\% CL of

$$
\mathcal{B}(\Upsilon(1 S) \rightarrow \text { invisible })<3 \times 10^{-4} \text { at } 90 \% \mathrm{CL},
$$

which is only one order of magnitude above the SM expectation. This bound only applies to LDM candidates with masses less than half the $\Upsilon(1 S)$ mass. The corresponding limit from BES [418] is

$$
\mathcal{B}(J / \psi \rightarrow \text { invisible })<7.2 \times 10^{-4} \text { at } 90 \% \mathrm{CL} .
$$

However, the invisible decay mode induced by scalar or pseudoscalar mediators (like CP-odd or CP-even Higgs bosons) actually vanishes, independent of the character of the dark matter candidate (either scalar, Dirac or Majorana fermion), if the decaying resonance has $J^{P C}=1^{--}[419]$. In order to get a non-vanishing decay rate, a new vector $U$-boson associated with gauging an extra $U(1)$ symmetry (i.e., a new kind of interaction) would be required [419]. Hence a different type of search has been performed by looking at the decay $\Upsilon(1 S) \rightarrow \gamma+$ invisible, which can proceed via a light scalar or pseudoscalar Higgs mediator decaying into a LDM pair. As discussed in Sect. 2.10.1, a light CP-odd Higgs boson $A_{1}$ mixing with $\eta_{b}$ resonances could naturally become the mediator of the decay into undetected dark matter particles: $\Upsilon \rightarrow \gamma A_{1}(\rightarrow$ invisible). The result for $\Upsilon(3 S)$ decays from BABAR [420] is

$$
\begin{array}{r}
\mathcal{B}(\Upsilon(3 S) \rightarrow \gamma+\text { invisible })<(0.7-30) \times 10^{-6} \\
\text { at } 90 \% \mathrm{CL}, \text { for } s_{\mathrm{inv}}^{1 / 2}<7.8 \mathrm{GeV}
\end{array}
$$

where $s_{\text {inv }}$ denotes the invariant-mass squared of the hypothetical LDM pair. The corresponding limit from CLEO [421] for $J / \psi$ decays is

$$
\begin{array}{r}
\mathcal{B}(J / \psi \rightarrow \gamma+\text { invisible })<(2.5-6.5) \times 10^{-6} \\
\text { at } 90 \% \mathrm{CL}, \text { for } s_{\mathrm{inv}}^{1 / 2}<960 \mathrm{MeV} .
\end{array}
$$

Experimental systematic effects make improving limits from searches of this type at higher mass difficult. The energy of the final state photon gets progressively smaller for larger invisible mass; at low energy, the energy resolution and number of fake photon candidates are all typically less favorable than at higher photon energy. Nevertheless, such improvements, if achieved, would provide important constraints on theoretical possibilities by reaching higher LDM masses.

\section{DECAY $^{8}$}

\subsection{Radiative transitions}

An electromagnetic transition between quarkonium states, which occurs via emission of a photon, offers the distinctive experimental signature of a monochromatic photon, a useful production mechanism for discovery and study of the lower-lying state, and a unique window on the dynamics of such systems. Below we first review the status and open questions regarding the relevant theoretical frameworks and tools, and then describe important measurements of charmonium and bottomonium electromagnetic transitions.

\subsubsection{Theoretical status}

The nonrelativistic nature of heavy quarkonium may be exploited to calculate electromagnetic transitions. Nonrelativistic effective field theories provide a way to systematically implement the expansion in the relative heavy-quark velocity, $v$. Particularly useful are nonrelativistic QCD (NRQCD) coupled to electromagnetism [137, 138], which follows from QCD (and QED) by integrating out the heavy quark mass scale $m$, and potential NRQCD coupled to electromagnetism [134, 139, 140, 422], which follows from NRQCD (and NRQED) by integrating out the momentum transfer scale $m v$.

Electromagnetic transitions may be classified in terms of electric and magnetic transitions between eigenstates of the leading-order pNRQCD Hamiltonian. The states are classified in terms of the radial quantum number, $n$, the orbital angular momentum, $l$, the total spin, $s$, and the total angular momentum, $J$. In the nonrelativistic limit, the spin dependence of the quarkonium wave function decouples from the spatial dependence. The spatial part of the wave function, $\psi(x)$, can be expressed in terms of a radial wave function, $u_{n l}(r)$, and the spherical harmonics, $Y_{l m}$, as $\psi(x)=Y_{l m}(\theta, \phi) u_{n l}(r) / r$. The spatial dependence of the electromagnetic transition amplitudes reduces to expectation values of various functions

\footnotetext{
${ }^{8}$ Contributing authors: E. Eichten ${ }^{\dagger}$, R. E. Mitchell ${ }^{\dagger}$, A. Vairo ${ }^{\dagger}$, A. Drutskoy, S. Eidelman, C. Hanhart, B. Heltsley, G. Rong, and C.-Z. Yuan
} 
of quark position and momentum between the initial- and final-state wave functions [1].

Magnetic transitions flip the quark spin. Transitions that do not change the orbital angular momentum are called magnetic dipole, or M1, transitions. In the nonrelativistic limit, the spin-flip transition decay rate between an initial state $i=n^{2 s+1} l_{J}$ and a final state $f=n^{\prime 2 s^{\prime}+1} l_{J^{\prime}}$ is:

$$
\begin{aligned}
\Gamma(i \stackrel{\mathrm{M} 1}{\longrightarrow} \gamma+f) & = \\
& \frac{16}{3} \alpha e_{Q}^{2} \frac{E_{\gamma}^{3}}{m_{i}^{2}}\left(2 J^{\prime}+1\right) \mathrm{S}_{i f}^{\mathrm{M}}\left|\mathcal{M}_{i f}\right|^{2},
\end{aligned}
$$

where $e_{Q}$ is the electrical charge of the heavy quark $Q$ $\left(e_{b}=-1 / 3, e_{c}=2 / 3\right), \alpha$ the fine structure constant, $E_{\gamma}=\left(m_{i}^{2}-m_{f}^{2}\right) /\left(2 m_{i}\right)$ is the photon energy, and $m_{i}, m_{f}$ are the masses of the initial- and final-state quarkonia, respectively. The statistical factor $\mathrm{S}_{i f}^{\mathrm{M}}=\mathrm{S}_{f i}^{\mathrm{M}}$ reads

$$
\begin{aligned}
\mathrm{S}_{i f}^{\mathrm{M}}=6(2 s+1)\left(2 s^{\prime}+1\right) \times & \left\{\begin{array}{ccc}
J & 1 & J^{\prime} \\
s^{\prime} & l & s
\end{array}\right\}^{2}\left\{\begin{array}{ccc}
1 & \frac{1}{2} & \frac{1}{2} \\
\frac{1}{2} & s^{\prime} & s
\end{array}\right\}^{2} .
\end{aligned}
$$

For $l=0$ transitions, $S_{i f}^{\mathrm{M}}=1$. For equal quark masses $m$, the overlap integral $\mathcal{M}_{i f}$ is given by

$$
\begin{aligned}
& \mathcal{M}_{i f}=\left(1+\kappa_{Q}\right) \times \\
& \int_{0}^{\infty} d r u_{n l}(r) u_{n^{\prime} l}^{\prime}(r) j_{0}\left(\frac{E_{\gamma} r}{2}\right),
\end{aligned}
$$

where $j_{n}$ are spherical Bessel functions and $\kappa_{Q}$ is the anomalous magnetic moment of a heavy quarkonium $Q \bar{Q}$. In pNRQCD, the quantity $1+\kappa_{Q}$ is the Wilson coefficient of the operator $S^{\dagger} \sigma \cdot e_{Q} \mathbf{B}^{\mathrm{em}} /(2 m) S$, where $\mathbf{B}^{\mathrm{em}}$ is the magnetic field and $S$ is a $Q \bar{Q}$ color-singlet field.

Electric transitions do not change the quark spin. Transitions that change the orbital angular momentum by one unit are called electric dipole, or E1, transitions. In the nonrelativistic limit, the spin-averaged electric transition rate between an initial state $i=n^{2 s+1} l_{J}$ and a final state $f=n^{\prime 2 s^{\prime}+1} l_{J^{\prime}}^{\prime}\left(l=l^{\prime} \pm 1\right)$ is

$$
\Gamma(i \stackrel{\mathrm{E} 1}{\longrightarrow} \gamma+f)=\frac{4}{3} \alpha e_{Q}^{2} E_{\gamma}^{3}\left(2 J^{\prime}+1\right) \mathrm{S}_{i f}^{\mathrm{E}}\left|\mathcal{E}_{i f}\right|^{2},
$$

where the statistical factor $\mathrm{S}_{i f}^{\mathrm{E}}=\mathrm{S}_{f i}^{\mathrm{E}}$ is

$$
\mathrm{S}_{i f}^{\mathrm{E}}=\max \left(l, l^{\prime}\right)\left\{\begin{array}{ccc}
J & 1 & J^{\prime} \\
l^{\prime} & s & l
\end{array}\right\}^{2} .
$$

The overlap integral $\mathcal{E}_{\text {if }}$ for equal quark masses $m$ is given by

$$
\begin{aligned}
\mathcal{E}_{i f}= & \frac{3}{E_{\gamma}} \int_{0}^{\infty} d r u_{n l}(r) u_{n^{\prime} l^{\prime}}(r) \times \\
& {\left[\frac{E_{\gamma} r}{2} j_{0}\left(\frac{E_{\gamma} r}{2}\right)-j_{1}\left(\frac{E_{\gamma} r}{2}\right)\right] . }
\end{aligned}
$$

Since the leading-order operator responsible for the electric transition does not undergo renormalization, the electric transition rate does not depend on a Wilson coefficient, analogous to the case of the quarkonium magnetic moment appearing in the magnetic transitions.

If the photon energy is smaller than the typical inverse radius of the quarkonium, we may expand the overlap integrals in $E_{\gamma} r$, generating electric and magnetic multipole moments. At leading order in the multipole expansion, the magnetic overlap integral reduces to $\mathcal{M}_{i f}=\delta_{n n^{\prime}}$. Transitions for which $n=n^{\prime}$ are called allowed M1 transitions, transitions for which $n \neq n^{\prime}$ are called hindered transitions. Hindered transitions happen only because of higher-order corrections and are suppressed by at least $v^{2}$ with respect to the allowed ones. At leading order in the multipole expansion the electric overlap integral reduces to

$$
\mathcal{E}_{i f}=\int_{0}^{\infty} d r u_{n l}(r) r u_{n^{\prime} l^{\prime}}(r) .
$$

Note that E1 transitions are more copiously observed than allowed M1 transitions, because the rates of the electric transitions are enhanced by $1 / v^{2}$ with respect to the magnetic ones. Clearly, the multipole expansion is always allowed for transitions between states with the same principal quantum numbers $\left(E_{\gamma} \sim m v^{4}\right.$ or $\left.m v^{3} \ll m v\right)$ or with contiguous principal quantum numbers $\left(E_{\gamma} \sim\right.$ $\left.m v^{2} \ll m v\right)$. For transitions that involve widely separated states, the hierarchy $E_{\gamma} \ll m v$ may not be realized. For example, in $\Upsilon(3 S) \rightarrow \gamma \eta_{b}(1 S)$, we have $E_{\gamma} \approx 921 \mathrm{MeV}$, which is smaller than the typical momentum transfer in the $\eta_{b}(1 S)$, about $1.5 \mathrm{GeV}$ [175], but may be comparable to or larger than the typical momentum transfer in the $\Upsilon(3 S)$. On the other hand, in $\psi(2 S) \rightarrow \gamma \chi_{c 1}$, we have $E_{\gamma} \approx 171 \mathrm{MeV}$, which is smaller than the typical momentum transfer in both the $\psi(2 S)$ and the $\chi_{c 1}$.

Beyond the nonrelativistic limit, Eqs. (90) and (93) get corrections. These are radiative corrections counted in powers of $\alpha_{\mathrm{s}}(m)$ and relativistic corrections counted in powers of $v$. These last ones include proper relativistic corrections of the type $(m v)^{2} / m^{2}$, recoil corrections and, for weakly-coupled quarkonia, also corrections of the type $\Lambda_{\mathrm{QCD}} /(m v)$. Finally, we also have corrections of the type $E_{\gamma} /(m v)$ that involve the photon energy. In the charmoniun system, $v^{2} \approx 0.3$, and corrections may be as large as $30 \%$. Indeed, a negative correction of about $30 \%$ is required to bring the nonrelativistic prediction of $\mathcal{B}\left(J / \psi \rightarrow \gamma \eta_{c}(1 S)\right)$, which is about $3 \%$, close to the experimental value, which is about $2 \%$. We will see that this is actually the case. In the bottomonium system, $v^{2} \approx 0.1$ and corrections may be as large as $10 \%$.

For a long time, corrections to the electromagnetic transitions have been studied almost entirely within phenomenological models [31, 423-435] (a sum rule analysis appears in [436]). We refer to reviews in [1, 81]; a textbook presentation can be found in [437]. In contrast to models, the effective field theory approach allows 
a systematic and rigorous treatment of the higher-order corrections. The use of EFTs for electromagnetic transitions was initiated in [422], in which a study of magnetic transitions was performed. The results of that analysis may be summarized in the following way.

- The quarkonium anomalous magnetic moment $\kappa_{Q}$ does not get contributions from the scale $m v$ : it is entirely determined by the quark anomalous magnetic moment. Since the quark magnetic moment appears at the scale $m$, it is accessible by perturbation theory: $\kappa_{Q}=2 \alpha_{\mathrm{s}}(m) /(3 \pi)+\mathcal{O}\left(\alpha_{\mathrm{s}}{ }^{2}\right)$. As a consequence, $\kappa_{Q}$ is a small positive quantity, about 0.05 in the bottomonium case and about 0.08 in the charmonium one. This is confirmed by lattice calculations [438] and by the analysis of higher-order multipole amplitudes (see Sect. 3.1.6).

- QCD does not allow for a scalar-type contribution to the magnetic transition rate. A scalar interaction is often postulated in phenomenological models.

The above conclusions were shown to be valid at any order of perturbation theory as well as nonperturbatively. They apply to magnetic transitions from any quarkonium state. For ground state magnetic transitions, we expect that perturbation theory may be used at the scale $m v$. Under this assumption, the following results were found at relative order $v^{2}$.

- The magnetic transition rate between the vector and pseudoscalar quarkonium ground state, including the leading relativistic correction (parametrized by $\alpha_{\mathrm{s}}$ at the typical momentum-transfer scale $\left.m_{i} \alpha_{\mathrm{s}} / 2\right)$ and the leading anomalous magnetic moment (parametrized by $\alpha_{\mathrm{s}}$ at the mass scale $m_{i} / 2$ ), reads

$$
\begin{gathered}
\Gamma(i \rightarrow \gamma+f)=\frac{16}{3} \alpha e_{Q}^{2} \frac{E_{\gamma}^{3}}{m_{i}^{2}} \times \\
{\left[1+\frac{4}{3} \frac{\alpha_{\mathrm{s}}\left(m_{i} / 2\right)}{\pi}-\frac{32}{27} \alpha_{\mathrm{s}}^{2}\left(m_{i} \alpha_{\mathrm{s}} / 2\right)\right]}
\end{gathered}
$$

in which $i=1^{3} 0_{1}$ and $f=1^{1} 0_{1}$. This expression is not affected by nonperturbative contributions. Applied to the charmonium and bottomonium case it gives: $\mathcal{B}\left(J / \psi \rightarrow \gamma \eta_{c}(1 S)\right)=(1.6 \pm 1.1) \%$ (see Sect. 3.1.2 for the experimental situation) and $\mathcal{B}\left(\Upsilon(1 S) \rightarrow \gamma \eta_{b}(1 S)\right)=(2.85 \pm 0.30) \times 10^{-4}$ (see Sect. 3.1.8 for some experimental perspectives).

- A similar perturbative analysis, performed for hindered magnetic transitions, mischaracterizes the experimental data by an order of magnitude, pointing either to a breakdown of the perturbative approach for quarkonium states with principal quantum number $n>1$, or to large higher-order relativistic corrections.
The above approach is well suited to studying the lineshapes of the $\eta_{c}(1 S)$ and $\eta_{b}(1 S)$ in the photon spectra of $J / \psi \rightarrow \gamma \eta_{c}(1 S)$ and $\Upsilon(1 S) \rightarrow \gamma \eta_{b}(1 S)$, respectively. In the region of $E_{\gamma} \ll m \alpha_{\mathrm{s}}$, at leading order, the lineshape is given by [439]

$$
\begin{aligned}
\frac{d \Gamma}{d E_{\gamma}}(i \rightarrow \gamma+f)= & \frac{16}{3} \frac{\alpha e_{Q}^{2}}{\pi} \frac{E_{\gamma}^{3}}{m_{i}^{2}} \times \\
& \frac{\Gamma_{f} / 2}{\left(m_{i}-m_{f}-E_{\gamma}\right)^{2}+\Gamma_{f}^{2} / 4}
\end{aligned}
$$

which has the characteristic asymmetric behavior around the peak seen in the data (compare with the discussion in Sect. 3.1.2).

No systematic analysis is yet available for relativistic corrections to electromagnetic transitions involving higher quarkonium states, i.e., states for which $\Lambda_{\mathrm{QCD}}$ is larger than the typical binding energy of the quarkonium. These states are not described in terms of a Coulombic potential. Transitions of this kind include magnetic transitions between states with $n>1$ and all electric transitions, $n=2$ bottomonium states being on the boundary. Theoretical determinations rely on phenomenological models, which we know do not agree with QCD in the perturbative regime and miss some of the terms at relative order $v^{2}$ [422]. A systematic analysis is, in principle, possible in the same EFT framework developed for magnetic transitions. Relativistic corrections would turn out to be factorized in some high-energy coefficients, which may be calculated in perturbation theory, and in Wilson-loop amplitudes similar to those that encode the relativistic corrections of the heavy quarkonium potential [179]. At large spatial distances, Wilson-loop amplitudes cannot be calculated in perturbation theory but are well-suited for lattice measurements. Realizing the program of systematically factorizing relativistic corrections in Wilson-loop amplitudes and evaluating them on the lattice, would, for the first time, produce modelindependent determinations of quarkonium electromagnetic transitions between states with $n>1$. These are the vast majority of transitions observed in nature. Finally, we note that, for near-threshold states such as $\psi(2 S)$, intermediate meson loops may provide important contributions [440], which should be systematically accounted for.

Higher-order multipole transitions have been observed in experiments (see Sect. 3.1.6), Again, a systematic treatment is possible in the EFT framework outlined above, but has not yet been realized.

$$
\text { 3.1.2. Study of } \psi(1 S, 2 S) \rightarrow \gamma \eta_{c}(1 S)
$$

Using a combination of inclusive and exclusive techniques, CLEO [69] has recently measured

$$
\begin{aligned}
\mathcal{B}\left(J / \psi \rightarrow \gamma \eta_{c}(1 S)\right) & =(1.98 \pm 0.09 \pm 0.30) \% \\
\mathcal{B}\left(\psi(2 S) \rightarrow \gamma \eta_{c}(1 S)\right) & =(0.432 \pm 0.016 \pm 0.060) \% .(99)
\end{aligned}
$$


The lineshape of the $\eta_{c}(1 S)$ in these M1 transitions was found to play a crucial role. Because the width of the $\eta_{c}(1 S)$ is relatively large, the energy dependence of the phase space term and the matrix element distort the lineshape (see Eq. (98)). Indeed, the photon spectrum measured by CLEO shows a characteristic asymmetric behaviour (see Fig. 37). The theoretical uncertainty in this lineshape represents the largest systematic error in the branching ratios. Both M1 transitions are found to be larger than previous measurements due to a combination of a larger $\eta_{c}(1 S)$ width and the first accounting for the pronounced asymmetry in the lineshape. This process has also been recently measured by KEDR [441].

The distortion of the $\eta_{c}(1 S)$ lineshape also has implications for the mass of the $\eta_{c}(1 S)$. As of 2006, there was a $3.3 \sigma$ discrepancy between $\eta_{c}(1 S)$ mass measurements made from $\psi(1 S, 2 S) \rightarrow \gamma \eta_{c}(1 S)$ (with a weighted average of $2977.3 \pm 1.3 \mathrm{MeV}$ ) and from $\gamma \gamma$ or $p \bar{p}$ production (averaging 2982.6 $\pm 1.0 \mathrm{MeV}$ ). The CLEO [69] analysis suggests that the solution to this problem may lie in the lineshape of the $\eta_{c}(1 S)$ in the M1 radiative transitions. When no distortion in the lineshape is used in the CLEO fit to $J / \psi \rightarrow \gamma \eta_{c}(1 S)$, the resulting $\eta_{c}(1 S)$ mass is consistent with other measurements from M1 transitions (2976.6 $\pm 0.6 \mathrm{MeV}$, statistical error only); however, when a distorted lineshape is taken into account, the mass is consistent with those from $\gamma \gamma$ or $p \bar{p}$ production $(2982.2 \pm 0.6 \mathrm{MeV}$, statistical error only). Recent

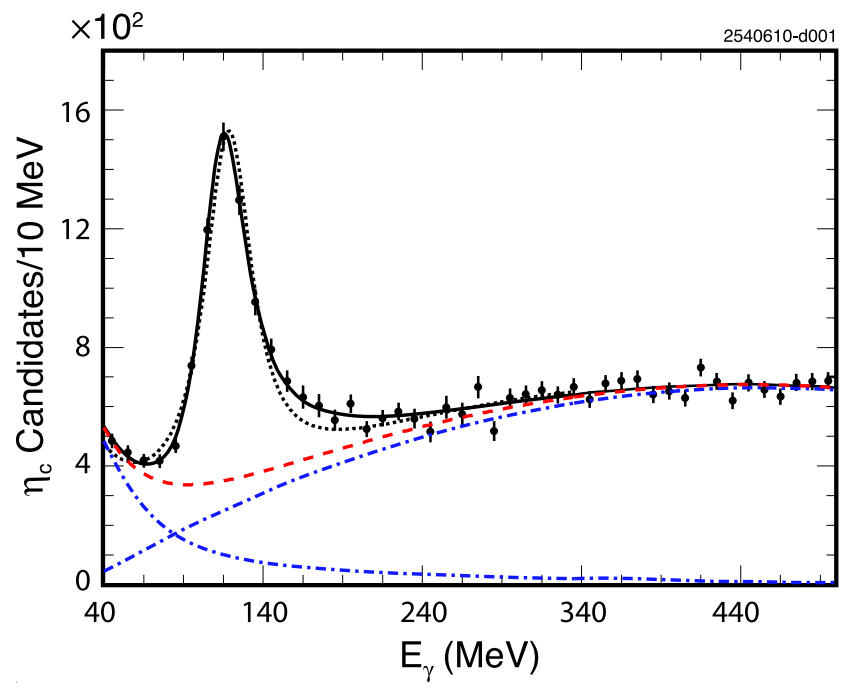

FIG. 37: From CLEO [69], the photon energy spectrum from $J / \psi \rightarrow \gamma \eta_{c}(1 S)$. The $\eta_{c}(1 S)$ is reconstructed in 12 different exclusive decay modes. The dotted curve represents a fit with a relativistic Breit-Wigner function; the solid curve uses a relativistic Breit-Wigner function distorted by the energy dependence of the phase-space term and the matrix element. The dash-dotted curves show two components of the background, which when summed become the dashed curve. Adapted from [69] with kind permission, copyright (2009) The American Physical Society measurements of the $\eta_{c}(1 S)$ mass in $\gamma \gamma$ production are consistent with this general picture. An $\eta_{c}(1 S)$ mass of $2986.1 \pm 1.0 \pm 2.5 \mathrm{MeV}$ is reported in a Belle analysis of $\gamma \gamma \rightarrow h^{+} h^{-} h^{+} h^{-}$, where $h=\pi, K$ [442], consistent with the higher $\eta_{c}(1 S)$ mass. Also, BABAR measures a mass of $2982.2 \pm 0.4 \pm 1.6 \mathrm{MeV}$ in $\gamma \gamma \rightarrow K_{S} K^{ \pm} \pi^{\mp}[443]$.

\subsubsection{Observation of $h_{c}(1 P) \rightarrow \gamma \eta_{c}(1 S)$}

The decay chain $\psi(2 S) \rightarrow \pi^{0} h_{c}(1 P), h_{c}(1 P) \rightarrow$ $\gamma \eta_{c}(1 S)$ was first observed by CLEO [45, 46] using 24.5 million $\psi(2 S)$ events, and later confirmed with higher statistics by BESIII [47] using 106 million $\psi(2 S)$. While the mass difference of the $h_{c}(1 P)$ and $\chi_{c J}(1 P)$ states is a measure of the hyperfine splitting in the $1 P$ $c \bar{c}$ system, the product branching fraction can be used to glean information about the size of the E1 transition $h_{c}(1 P) \rightarrow \gamma \eta_{c}(1 S)$. The product branching fraction $\mathcal{B}\left(\psi(2 S) \rightarrow \pi^{0} h_{c}(1 P)\right) \times \mathcal{B}\left(h_{c}(1 P) \rightarrow \gamma \eta_{c}(1 S)\right)$ was measured to be

$$
\begin{array}{ll}
(4.19 \pm 0.32 \pm 0.45) \times 10^{-4} & \text { CLEO [46] } \\
(4.58 \pm 0.40 \pm 0.50) \times 10^{-4} & \text { BESIII [47] }
\end{array}
$$

where both CLEO and BESIII used an inclusive technique (requiring reconstruction of just the $\pi^{0}$ and the transition photon and imposing appropriate kinematic constraints), but CLEO also utilized a fully exclusive technique (in addition to the $\pi^{0}$ and $\gamma$, reconstructing the $\eta_{c}(1 S)$ in multiple exclusive decay channels). BESIII has also measured $\mathcal{B}\left(\psi(2 S) \rightarrow \pi^{0} h_{c}(1 P)\right.$ ) (see Sect. 3.3.3), allowing extraction of

$$
\mathcal{B}\left(h_{c}(1 P) \rightarrow \gamma \eta_{c}(1 S)\right)=(54.3 \pm 6.7 \pm 5.2) \% .
$$

As part of the same study, CLEO has measured the angular distribution of the transition photon from $h_{c}(1 P) \rightarrow \gamma \eta_{c}(1 S)$ (Fig. 38). Fitting to a curve of the form $N\left(1+\alpha \cos ^{2} \theta\right)$, and combining the results from the inclusive and exclusive analyses, it was found that $\alpha=1.20 \pm 0.53$, consistent with $\alpha=1$, the expectation for E1 transitions.

\subsubsection{Nonobservation of $\psi(2 S) \rightarrow \gamma \eta_{c}(2 S)$}

After years of false alarms, the $\eta_{c}(2 S)$ was finally observed in $B$-decays and two-photon fusion (see Sect. 2.2.2). In an attempt to both discover new decay modes and to observe it in a radiative transition, CLEO [68] modeled an $\eta_{c}(2 S)$ analysis after its effort on the $\eta_{c}(1 S)$, wherein a systematic study of many exclusive hadronic decay modes aided in measuring the lineshapes and branching fractions in $\psi(1 S, 2 S) \rightarrow \gamma \eta_{c}(1 S)$ transitions [69]. Eleven modes, which were chosen based in part upon success in finding similar $\eta_{c}(1 S)$ decays, were sought in the exclusive decay chain $\psi(2 S) \rightarrow \gamma \eta_{c}(2 S)$, 


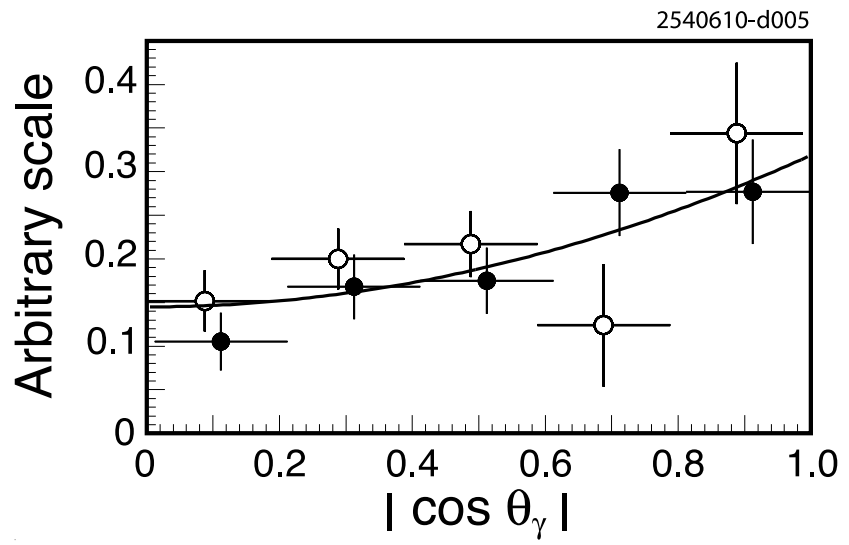

FIG. 38: From CLEO [46], the distribution of the photon polar angle in the $e^{+} e^{-}$center-of-mass frame from the transition sequence $\psi(2 S) \rightarrow \pi^{0} h_{c}(1 P), h_{c}(1 P) \rightarrow \gamma \eta_{c}(1 S)$. Open circles represent data from an inclusive $\eta_{c}(1 S)$ decays and solid circles data from exclusive $\eta_{c}(1 S)$ decays (see text). The solid curve represents a fit of both inclusive and exclusive data to $N\left(1+\alpha \cos ^{2} \theta\right)$, from which $\alpha$ was found to be $1.20 \pm 0.53$. Adapted from [46] with kind permission, copyright (2008) The American Physical Society

$\eta_{c}(2 S) \rightarrow$ hadrons in CLEO's 26 million $\psi(2 S)$ sample. One of the modes sought was the dipion transition $\eta_{c}(2 S) \rightarrow \pi^{+} \pi^{-} \eta_{c}(1 S)$, which used proven hadronic decay modes of the $\eta_{c}(1 S)$. No $\eta_{c}(2 S)$ signals were found, and eleven product branching fraction upper limits were set. None but one of these products can be used to directly set a limit on the transition because none but one have a measured $\eta_{c}(2 S)$ branching fraction. Using the $B A B A R$ [444] branching fraction for $\eta_{c}(2 S) \rightarrow K K \pi$ allows CLEO to set an upper limit

$$
\mathcal{B}\left(\psi(2 S) \rightarrow \gamma \eta_{c}(2 S)\right)<7.6 \times 10^{-4} .
$$

This value is greater than a phenomenological limit obtained [68] by assuming the matrix element is the same as for $J / \psi \rightarrow \gamma \eta_{c}(1 S)$ and correcting the measured $J / \psi$ branching fraction by the ratio of total widths and phasespace factors, $(3.9 \pm 1.1) \times 10^{-4}$.

\subsection{5. $\psi(2 S) \rightarrow \gamma \gamma J / \psi$ through $\chi_{c J}$}

One component of the CLEO [445, 446] $\psi(2 S) \rightarrow$ $X J / \psi$ branching fraction analysis described in Sect. 3.3.2 addresses the $\gamma \gamma J / \psi$ final states that proceed through the doubly-radiative decays $\psi(2 S) \rightarrow \gamma \chi_{c J}, \chi_{c J} \rightarrow \gamma J / \psi$. The resulting product branching fractions, measured using CLEO's dataset of $27 \mathrm{M} \psi(2 S)$ decays, are shown in Table 21. These inputs are used to determine $\mathcal{B}\left(\chi_{c J} \rightarrow\right.$ $\gamma J / \psi)$ branching fractions, which are calculated by the Particle Data Group [447] from world averages of the quantities in Table 21 and those for $\psi(2 S) \rightarrow \gamma \chi_{c J}$.

A substantial difference between the original [445] and final [446] CLEO analyses, aside from an eightfold in-
TABLE 21: Results from CLEO [445, 446] for the $\psi(2 S) \rightarrow$ $\gamma \gamma J / \psi$ branching fractions through either $\chi_{c J}$ intermediate states or a nonresonant (nr) channel

\begin{tabular}{lc}
\hline \hline Final state & $\mathcal{B}(\%)$ \\
\hline$\gamma(\gamma J / \psi)_{\chi_{c 0}}$ & $0.125 \pm 0.007 \pm 0.013$ \\
$\gamma(\gamma J / \psi)_{\chi_{c 1}}$ & $3.56 \pm 0.03 \pm 0.12$ \\
$\gamma(\gamma J / \psi)_{\chi_{c 2}}$ & $1.95 \pm 0.02 \pm 0.07$ \\
$\gamma(\gamma J / \psi)_{\mathrm{nr}}$ & $\leq 0.1$ \\
\hline \hline
\end{tabular}

crease in statistics, is the treatment of the transition through $\chi_{c 0}$, which has the smallest rate of the three. The primary systematic challenge for this mode is dealing with its small $\gamma J / \psi$ branching fraction relative to that of $\chi_{c 1}$. In the energy spectrum of the lower energy photon in such decays, there are peaks near 128, 172, and $258 \mathrm{MeV}$, corresponding to the intermediate $\chi_{c 2}, \chi_{c 1}$, and $\chi_{c 0}$ states, respectively (see Fig. 4 of [446]). However, due to nonzero photon energy-measurement resolution and nonzero natural widths of the $\chi_{c J}$, the three peaks overlap one another. In particular, the high-side tail of the lower-energy photon's spectrum for the transition through $\chi_{c 1}$ has a significant contribution in the $\chi_{c 0}$ energy region (relative to the small $\chi_{c 0}$ signal), a fact which introduces subtleties to the analysis. Because of the proximity of the photon lines to one another and the large disparity of rates, the measured product branching fraction for the $\chi_{c 0}$ transition is sensitive to $\Gamma\left(\chi_{c 1}\right)$ and to the detailed lineshape in these decays, i.e., some of the apparent $\chi_{c 0}$ signal is actually feedacross from $\chi_{c 1}$. The second CLEO analysis [446] implemented the $E_{\gamma}^{3}$-weighting in its MC simulation of the transition, as required from phase-space considerations for E1 decays given in Eq. (93). The inclusion of the $E_{\gamma}^{3}$ factor significantly increases the contribution of $\chi_{c 1}$ near the $\chi_{c 0}$ peak (relative to not using this factor). However, even if the MC simulation of these decays allows for a $\chi_{c 1}$ natural width of up to $1 \mathrm{MeV}$ (about $2.5 \sigma$ higher than the nominal value [18]) and uses the expected E1-transition energy dependence of the lineshape, the region in the lower-energy photon spectrum in the valley between the $\chi_{c 1}$ and $\chi_{c 0}$ peaks showed an excess of events in the CLEO data over the number expected. One hypothesis for filling that deficit suggested by CLEO is the nonresonant (nr) decay $\psi(2 S) \rightarrow \gamma \gamma J / \psi$. CLEO estimated that a branching fraction of $\approx 0.1 \%$ or smaller could be accommodated by the data. However, CLEO did not claim observation of this nonresonant decay mode due to a combination of limited statistics, uncertainties about $\chi_{c J}$ widths, and possible dynamical distortion of the lineshape, all matters deserving further attention. In fact, such a distortion was later observed in radiative transitions from $\psi(2 S)$ to $\eta_{c}(1 S)$ (see Sect. 3.1.2), raising the importance of verifying the assumed photon lineshapes with data for any 
future analysis.

A preliminary analysis of nonresonant $\psi(2 S) \rightarrow \gamma \gamma J / \psi$ decays from BESIII has been released [448, 449], which uses a dataset of $106 \mathrm{M} \psi(2 S)$ decays, nearly four times that of CLEO. A statistically significant signal is claimed, and a branching fraction of $\approx 0.1 \%$ is measured, compatible with the CLEO upper bound. BESIII also raised the issue of how interference between the resonant $\gamma \gamma J / \psi_{\chi_{c J}}$ final states might affect the lower-energy photon spectrum, and what the effect might be from interference between the nonresonant and resonant channels.

\subsubsection{Higher-order multipole amplitudes}

The radiative decays $\psi(2 S) \rightarrow \gamma \chi_{c 1,2}$ and $\chi_{c 1,2} \rightarrow$ $\gamma J / \psi$ are dominated by electric dipole (E1) amplitudes. However, they are expected to have a small additional contribution from the higher-order magnetic quadrupole (M2) amplitudes. Previous measurements of the relative sizes of the M2 amplitudes have disagreed with theoretical expectations. CLEO [450] has recently revisited this issue with a high-statistics analysis of the decay chains $\psi(2 S) \rightarrow \gamma \chi_{c J} ; \chi_{c J} \rightarrow \gamma J / \psi ; J / \psi \rightarrow$ $l^{+} l^{-}(l=e, \mu)$ for $J=1,2$. Starting with $24 \times 10^{6} \psi(2 S)$ decays, CLEO observes approximately 40000 events for $J=1$ and approximately 20000 events for $J=2$, significantly larger event samples than previous measurements. Using an unbinned maximum-likelihood fit to angular distributions, CLEO finds the normalized M2 admixtures in Table 22. For the quoted $J=2$ measurements, the electric octupole (E3) moments were fixed to zero. As shown in Fig. 39, these new measurements agree well with theoretical expectations when the anomalous magnetic moment of the charm quark is assumed to be zero and the mass of the charm quark is assumed to be $1.5 \mathrm{GeV}$.

\subsubsection{Observation of $\psi(3770) \rightarrow \gamma \chi_{c J}(1 P)$}

The existence of the $\psi(3770)$ has long been established, and it has generally been assumed to be the $1^{3} D_{1}$ char-

TABLE 22: From CLEO [450], normalized magnetic dipole (M2) amplitudes from an analysis of $\psi(2 S) \rightarrow \gamma \chi_{c J}$, $\chi_{c J} \rightarrow \gamma J / \psi$ decays. For the $J=2$ values, the electric octupole (E3) moments were fixed to zero

\begin{tabular}{lcc}
\hline \hline Decay & Quantity & Value $\left(10^{-2}\right)$ \\
\hline$\psi(2 S) \rightarrow \gamma \chi_{c 1}$ & $b_{2}^{J=1}$ & $2.76 \pm 0.73 \pm 0.23$ \\
$\psi(2 S) \rightarrow \gamma \chi_{c 2}$ & $b_{2}^{J=2}$ & $1.0 \pm 1.3 \pm 0.3$ \\
$\chi_{c 1} \rightarrow \gamma J / \psi$ & $a_{2}^{J=1}$ & $-6.26 \pm 0.63 \pm 0.24$ \\
$\chi_{c 2} \rightarrow \gamma J / \psi$ & $a_{2}^{J=2}$ & $-9.3 \pm 1.6 \pm 0.3$ \\
\hline \hline
\end{tabular}

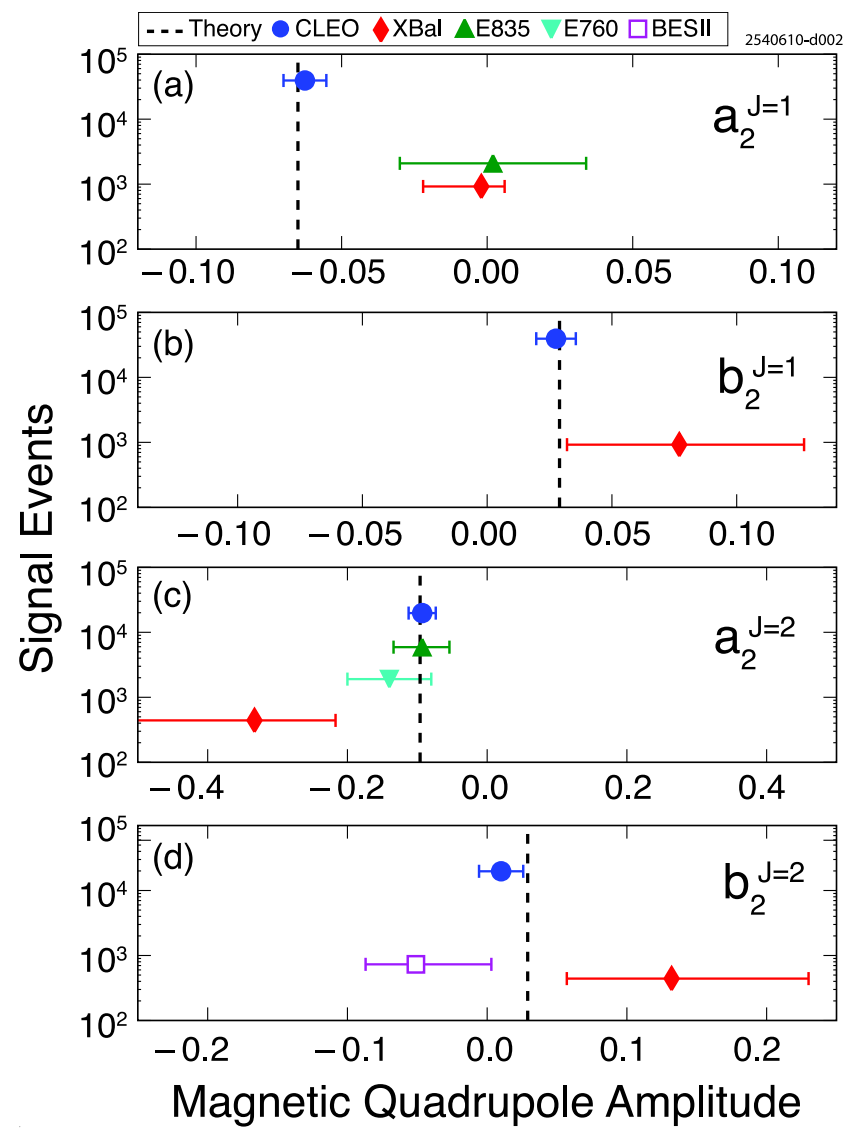

FIG. 39: A compilation of measurements of normalized magnetic dipole amplitudes from $\chi_{c 1} \rightarrow \gamma J / \psi\left(a_{2}^{J=1}\right), \psi(2 S) \rightarrow$ $\gamma \chi_{c 1}\left(b_{2}^{J=1}\right), \chi_{c 2} \rightarrow \gamma J / \psi\left(a_{2}^{J=2}\right)$, and $\psi(2 S) \rightarrow \gamma \chi_{c 2}\left(b_{2}^{J=2}\right)$. The solid circles represent data from CLEO [450], which show consistency with predictions (dashed vertical lines), unlike some earlier measurements. The nonrelativistic theoretical expectations are calculated with an anomalous magnetic moment of the charm quark of zero and an assumed $1.5 \mathrm{GeV}$ charm quark mass. Adapted from [450] with kind permission, copyright (2009) The American Physical Society

monium state with a small admixture of $2^{3} S_{1}$. However, because it predominantly decays to $D \bar{D}$, its behavior as a state of charmonium has gone relatively unexplored in comparison to its lighter partners. The charmonium nature of the $\psi(3770)$ is especially interesting given the unexpected discoveries of the $X, Y$, and $Z$ states, opening up the possibility that the $\psi(3770)$ could include more exotic admixtures. The electromagnetic transitions, $\psi(3770) \rightarrow \gamma \chi_{c J}$, because they are straightforward to calculate assuming the $\psi(3770)$ is the ${ }^{3} D_{1}$ state of charmonium, provide a natural testing ground for the nature of the $\psi(3770)$ [31, 451, 452].

CLEO has observed these transitions in two independent analyses. In the first [453], the $\chi_{c J}$ were reconstructed exclusively in the decay chain $\psi(3770) \rightarrow \gamma \chi_{c J}$, $\chi_{c J} \rightarrow \gamma J / \psi, J / \psi \rightarrow \ell^{+} \ell^{-}$, with results depicted in Fig. 40. In the second [454], the $\chi_{c J}$ were reconstructed 
in several exclusive hadronic modes and then normalized to $\psi(2 S) \rightarrow \gamma \chi_{c J}$ using the same exclusive modes, with results shown in Fig. 41. Due to differing relative rates of the $\chi_{c J}$ decay modes employed, the first method has more sensitivity to the transitions to $\chi_{c 1,2}$ whereas the second is more suited to $\chi_{c 0}$. Combining the results of the two analyses, the partial widths of $\psi(3770) \rightarrow \gamma \chi_{c} J$ were found to be $172 \pm 30 \mathrm{keV}$ for $J=0,70 \pm 17 \mathrm{keV}$ for $J=1$, and $<21 \mathrm{keV}$ at $90 \% \mathrm{CL}$ for $J=2$. These measurements are consistent with relativistic calculations assuming the $\psi(3770)$ is the ${ }^{3} D_{1}$ state of charmonium.

\subsubsection{Observation of $\Upsilon(2 S, 3 S) \rightarrow \gamma \eta_{b}(1 S)$}

The recent discovery of the $\eta_{b}(1 S)$ state by BABAR [59] has, through a measurement of the $\eta_{b}(1 S)$ mass, given us our first measurement of the $1 S$ hyperfine splitting in the bottomonium system. This is obviously an important accomplishment for spectroscopy as it provides a window into the spin-spin interactions within the $b \bar{b}$ system. However, in addition to its contributions to

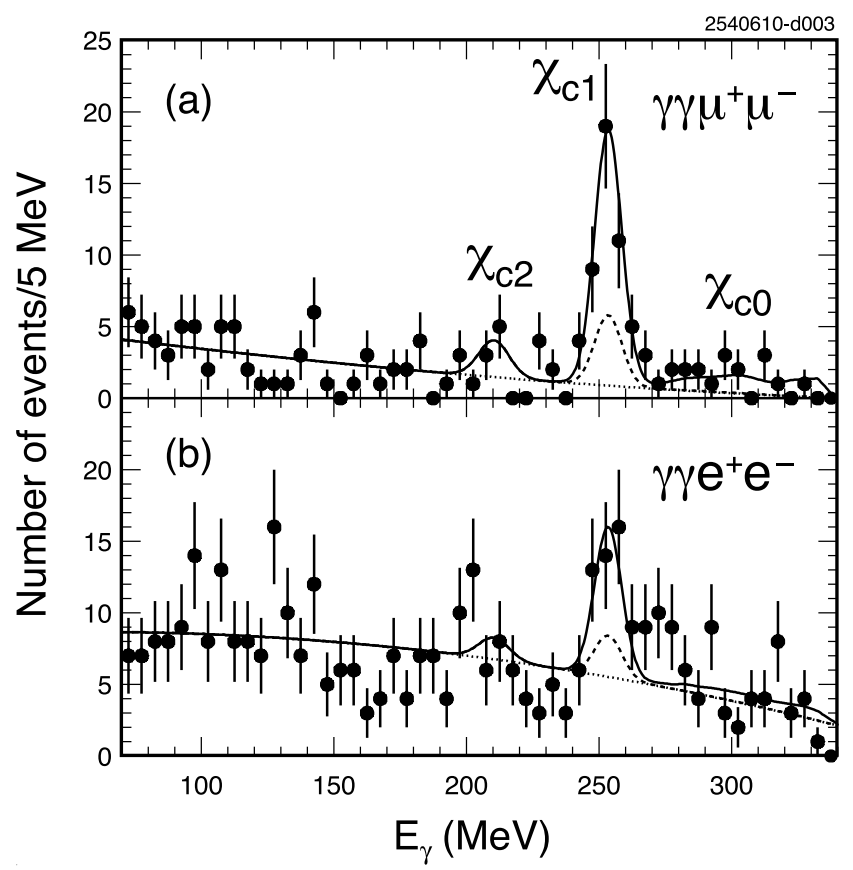

FIG. 40: From CLEO [453], the energy of the transition (lower energy) photon from $\psi(3770) \rightarrow \gamma \chi_{c J}$ found when reconstructing $\chi_{c J} \rightarrow \gamma J / \psi$ and requiring the $J / \psi$ decay to (a) $\mu^{+} \mu^{-}$or (b) $e^{+} e^{-}$. Solid circles represent data, the dotted curve shows the smooth fitted background, the dashed curve shows the sum of the smooth background fit and an estimated contribution from the tail of the $\psi(2 S)$ (events individually indistinguishable from signal), and the solid curve is a result of a fit of the data to all background and signal components. Background saturates the data at the $\chi_{c 2}$ and $\chi_{c 0}$, but a significant $\chi_{c 1}$ signal is obtained. Adapted from [453] with kind permission, copyright (2006) The American Physical Society spectroscopy, the observation of the decays $\Upsilon(2 S, 3 S) \rightarrow$ $\gamma \eta_{b}(1 S)$ has resulted in our first measurements of M1 radiative transition rates in the bottomonium system. A large range of theoretical predictions have been made for these rates [455], especially for the hindered transitions, to which the experimental measurement brings much-needed constraints.

Using 109 million $\Upsilon(3 S)$ decays and 92 million $\Upsilon(2 S)$ decays, BABAR [59, 61] has measured

$$
\begin{aligned}
& \mathcal{B}\left(\Upsilon(3 S) \rightarrow \gamma \eta_{b}(1 S)\right)=(4.8 \pm 0.5 \pm 0.6) \times 10^{-4} \\
& \mathcal{B}\left(\Upsilon(2 S) \rightarrow \gamma \eta_{b}(1 S)\right)=\left(4.2_{-1.0}^{+1.1} \pm 0.9\right) \times 10^{-4}
\end{aligned}
$$

Both measurements assume an $\eta_{b}(1 S)$ width of $10 \mathrm{MeV}$. The large systematic errors in the branching fractions are due to the difficulty in isolating the small $\eta_{b}(1 S)$ signal from other nearby photon lines $\left(\chi_{b J}(2 P, 1 P) \rightarrow \gamma \Upsilon(1 S)\right.$ and $\Upsilon(3,2 S) \rightarrow \gamma \Upsilon(1 S))$ and from the large background in the energy spectrum of inclusive photons.

In addition to the M1 transition rates, the energy dependence of the matrix elements is also of interest. In the case of charmonium (see below), this energy dependence can introduce a nontrivial distortion of the $\eta_{c}(1 S)$ lineshape which can artificially pull the mass measurement several $\mathrm{MeV}$ from its true value. It is expected that the same distortion mechanism will hold in the bot-

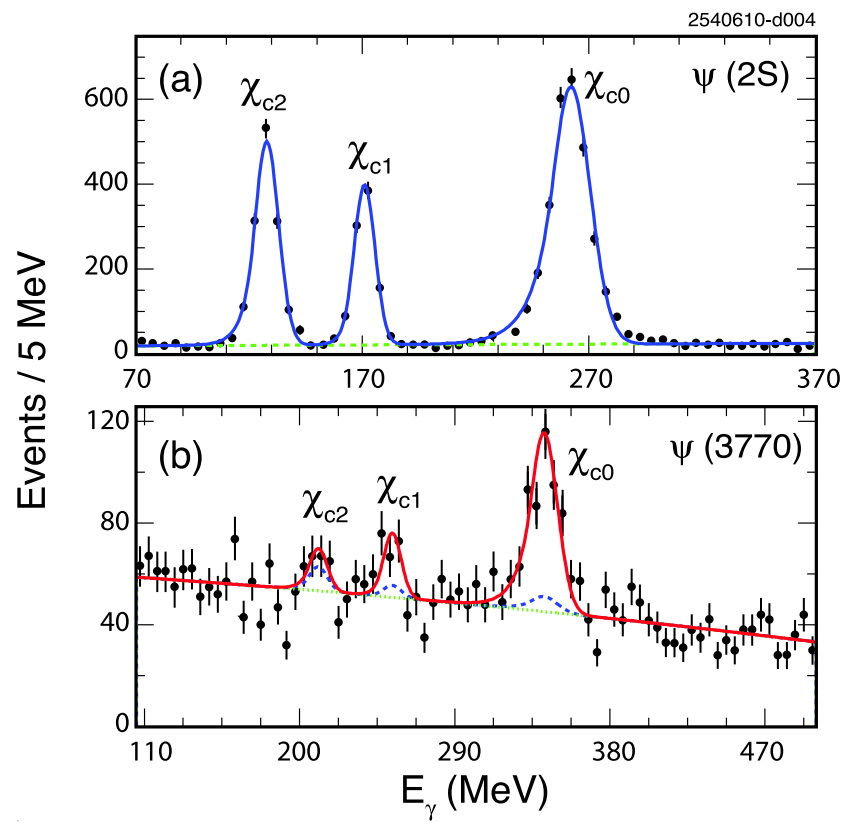

FIG. 41: From CLEO [454], the energy of the transition photon from (a) $\psi(2 S)$, or (b) $\psi(3770)$ decaying to $\gamma \chi_{c J}$ when the $\chi_{c J}$ are reconstructed in exclusive hadronic modes. Short-dashed curves represent fits to the smooth background. The long-dashed curve in (b) represents estimated background from the tail of the $\psi(2 S)$, for which events are individually indistinguishable from signal. A significant $\psi(3770) \rightarrow \gamma \chi_{c 0}$ signal is obtained. Adapted from [454] with kind permission, copyright (2006) The American Physical Society 
tomonium system. This effect must then be understood if M1 transitions are to be used for precision $\eta_{b}(1 S)$ mass measurements.

Studying the $\eta_{b}(1 S)$ lineshape in M1 transitions will require a large reduction in background levels. One possibility would be to study exclusive $\eta_{b}(1 S)$ decays. Using exclusive $\eta_{b}(1 S)$ decays could also allow a measurement of $\mathcal{B}\left(\Upsilon(1 S) \rightarrow \gamma \eta_{b}(1 S)\right)$, the allowed M1 transition, since background levels in the $\Upsilon(1 S)$ inclusive photon energy spectrum are likely prohibitively large.

\subsection{Radiative and dileptonic decays}

Here we review theoretical status and experimental results for radiative and dileptonic decays of heavy quarkonia. The simplest parton-level decay of any heavy quarkonium vector state occurs through annihilation into a virtual photon and thence into dilepton or quarkantiquark pairs. The latter can be difficult to isolate from ggg decay at the charmonium and bottomonium mass scales, but fortunately is known to have a rate proportional to $R \equiv \sigma\left(e^{+} e^{-} \rightarrow\right.$ hadrons $) / \sigma\left(e^{+} e^{-} \rightarrow \mu^{+} \mu^{-}\right)$, a quantity that is well-measured with off-resonance data and which has a well-understood energy dependence. Conversely, dilepton pairs have distinctive experimental signatures for which most modern detectors are optimized, offering the prospect of high precision. This high precision is quite useful in studies of both production and decays of vector charmonium and bottomonium states. Dileptonic widths also offer relative and absolute measures of wave function overlap at the origin. For all these reasons, decays to $\ell^{+} \ell^{-}$are heavily studied and used to characterize the most basic features of each vector state.

The simplest three-body decays of vector quarkonia are to $\gamma \gamma \gamma, \gamma g g$, and $g g g$, and their relative rates should reflect directly upon the value of $\alpha_{\mathrm{s}}$ at the relevant mass scale: naively, $R_{\gamma} \equiv \mathcal{B}(\gamma g g) / \mathcal{B}(g g g) \simeq \alpha / \alpha_{\mathrm{s}}(m)$. Although the $\gamma \gamma \gamma$ final state is experimentally straightforward to isolate, its rate is exceedingly small. Conversely, while $\gamma g g$ and $g g g$ decays are abundant, distinguishing them from each other and from transitions involving final state hadrons is quite challenging. Indeed, $g g g$ decays cannot be effectively differentiated on an event-to-event basis from quarkonium annihilation into light quark-antiquark pairs nor from $\gamma g g$ final states with soft photons. Experimental study of $\gamma \gamma \gamma, \gamma g g$, and ggg quarkonium decays has progressed substantially, but has not yet entered the realm of precision.

The simplest decay or production mechanism of any scalar or tensor quarkonium state is to and from a pair of photons. Two-photon decay offers clean experimental signatures and a measure of the frequently small twophoton branching fraction, whereas production via twophoton fusion in $e^{+} e^{-}$collisions offers the prospect of determination of the diphotonic width. As with vector states and three photons, the two-photon coupling provides quite basic information about scalar and tensor quarkonia.

For completeness, we mention that radiative decays offer convenient production mechanisms for scalar and tensor states such as light-quark or hybrid mesons, glueball candidates, and non-Standard-Model Higgs or axion searches. Treatment of such decays is beyond the scope of this review, but some examples can be found in Sect. 3.2.4.

\subsubsection{Theoretical status}

Quarkonium annihilation happens at the heavy-quark mass scale $m$. Processes that happen at the scale $m$ are best described by NRQCD. The NRQCD factorization formula for the quarkonium annihilation width into light hadrons or photons or lepton pairs reads [138, 456]:

$$
\Gamma_{H \text {-annih. }}=\sum_{n} \frac{2 \operatorname{Im} c_{n}}{m^{\left(d_{O_{n}}-4\right)}}\left\langle H\left|O_{n}^{4 \text {-fermion }}\right| H\right\rangle,
$$

where $O_{n}^{4 \text {-fermion }}$ are four-fermion operators, $c_{n}$ are their Wilson coefficients, $d_{O_{n}}$ their dimensions, and $|H\rangle$ is the state that describes the quarkonium in NRQCD. The Wilson coefficients $c_{n}$ are series in powers of the strong coupling constant $\alpha_{\mathrm{s}}$, evaluated at the heavy-quark mass, and the matrix elements $\left\langle H\left|O_{n}^{4-\text { fermion }}\right| H\right\rangle$ are counted in powers of the heavy quark velocity $v$. The matrix elements live at the scale $m v$ : they are nonperturbative if $m v \gtrsim \Lambda_{\mathrm{QCD}}$, while they may be evaluated in perturbation theory if $m v^{2} \gtrsim \Lambda_{\mathrm{QCD}}$.

Substantial progress has been made in the evaluation of the factorization formula at order $v^{7}$ [457, 458], in the lattice evaluation of the NRQCD matrix elements $\left\langle H\left|O_{n}^{4 \text {-fermion }}\right| H\right\rangle$ [233], and in the data of many hadronic and electromagnetic decays (see [1] and subsequent sections). As discussed in [1], the data are clearly sensitive to NLO corrections in the Wilson coefficients $c_{n}$ (and presumably also to relativistic corrections). For an updated list of ratios of $P$-wave charmonium decay widths, see Table 23.

In [232], the high precision of data and matrix elements has been exploited to provide a new determination of $\alpha_{\mathrm{s}}$ from

$$
\begin{gathered}
\frac{\Gamma(\Upsilon(1 S) \rightarrow \gamma \mathrm{LH})}{\Gamma(\Upsilon(1 S) \rightarrow \mathrm{LH})}: \\
\alpha_{\mathrm{S}}\left(m_{\Upsilon(1 S)}\right)=0.184_{-0.014}^{+0.015},
\end{gathered}
$$

implying

$$
\alpha_{\mathrm{s}}\left(m_{Z^{0}}\right)=0.119_{-0.005}^{+0.006} .
$$

The NRQCD factorization formulas for electromagnetic and inclusive hadronic decay widths lose their predictive power as soon as we go to higher orders in $v$, due to the rapid increase in the number of nonperturbative 
TABLE 23: Comparison of measured $\chi_{c J}$ decay-width ratios (using PDG10 [447]) with LO and NLO determinations [1], assuming $m_{c}=1.5 \mathrm{GeV}$ and $\alpha_{\mathrm{s}}\left(2 m_{c}\right)=0.245$, but without corrections of relative order $v^{2} . \mathrm{LH} \equiv$ light hadrons

\begin{tabular}{cccc}
\hline \hline Ratio & PDG & LO & NLO \\
\hline$\frac{\Gamma\left(\chi_{c 0} \rightarrow \gamma \gamma\right)}{\Gamma\left(\chi_{c 2} \rightarrow \gamma \gamma\right)}$ & 4.5 & 3.75 & 5.43 \\
$\frac{\Gamma\left(\chi_{c 2} \rightarrow \mathrm{LH}\right)-\Gamma\left(\chi_{c 1} \rightarrow \mathrm{LH}\right)}{\Gamma\left(\chi_{c 0} \rightarrow \gamma \gamma\right)}$ & 450 & 347 & 383 \\
$\frac{\Gamma\left(\chi_{c 0} \rightarrow \mathrm{LH}\right)-\Gamma\left(\chi_{c 1} \rightarrow \mathrm{LH}\right)}{\Gamma\left(\chi_{c 0} \rightarrow \gamma \gamma\right)}$ & 4200 & 1300 & 2781 \\
$\frac{\Gamma\left(\chi_{c 0} \rightarrow \mathrm{LH}\right)-\Gamma\left(\chi_{c 2} \rightarrow \mathrm{LH}\right)}{\Gamma\left(\chi_{c 2} \rightarrow \mathrm{LH}\right)-\Gamma\left(\chi_{c 1} \rightarrow \mathrm{LH}\right)}$ & 8.4 & 2.75 & 6.63 \\
$\frac{\Gamma\left(\chi_{c 0} \rightarrow \mathrm{LH}\right)-\Gamma\left(\chi_{c 1} \rightarrow \mathrm{LH}\right)}{\Gamma\left(\chi_{c 2} \rightarrow \mathrm{LH}\right)-\Gamma\left(\chi_{c 1} \rightarrow \mathrm{LH}\right)}$ & 9.4 & 3.75 & 7.63 \\
\hline \hline
\end{tabular}

matrix elements [1, 457, 458]. Quarkonia, with typical binding energies much smaller than $\Lambda_{\mathrm{QCD}}$, conservatively include all quarkonia above the ground state. Matrix elements of these states are inherently nonperturbative and may be evaluated on the lattice [233]; few, however, are known. A way to reduce the number of these unknown matrix elements is to go to the lower-energy EFT, pNRQCD, and to exploit the hierarchy $m v \gg m v^{2}$. In pNRQCD, NRQCD matrix elements factorize into two parts: one, the quarkonium wave-function or its derivative at the origin, and the second, gluon-field correlators that are universal, i.e., independent of the quarkonium state. The pNRQCD factorization has been exploited for $\mathrm{P}$-wave and S-wave decays in [181].

Quarkonium ground states have typical binding energy larger than or of the same order as $\Lambda_{\mathrm{QCD}}$. Matrix elements of these states may be evaluated in perturbation theory with the nonperturbative contributions being small corrections encoded in local or nonlocal condensates. Many higher-order corrections to spectra, masses, and wave functions have been calculated in this manner [157], all of them relevant to the quarkonium ground state annihilation into light hadrons and its electromagnetic decays. For some recent reviews about applications, see [270, 459]. In particular, $\Upsilon(1 S), \eta_{b}(1 S), J / \psi$, and $\eta_{c}(1 S)$ electromagnetic decay widths at NNLL have been evaluated [260, 460]. The ratios of electromagnetic decay widths were calculated for the ground state of charmonium and bottomonium at NNLL order [460], finding, e.g.,

$$
\frac{\Gamma\left(\eta_{b}(1 S) \rightarrow \gamma \gamma\right)}{\Gamma\left(\Upsilon(1 S) \rightarrow e^{+} e^{-}\right)}=0.502 \pm 0.068 \pm 0.014
$$

A partial NNLL-order analysis of the absolute widths of $\Upsilon(1 S) \rightarrow e^{+} e^{-}$and $\eta_{b}(1 S) \rightarrow \gamma \gamma$ can be found in [260].
As the analysis of $\Gamma\left(\Upsilon(1 S) \rightarrow e^{+} e^{-}\right)$of [260] illustrates, for this fundamental quantity there may be problems of convergence of the perturbative series. Problems of convergence are common and severe for all the annihilation observables of ground state quarkonia and may be traced back to large logarithmic contributions, to be resummed by solving suitable renormalization group equations, and to large $\beta_{0} \alpha_{\mathrm{s}}$ contributions of either resummable or nonresummable nature (these last ones are known as renormalons). Some large $\beta_{0} \alpha_{\mathrm{s}}$ contributions were successfully treated [461] to provide a more reliable estimate for

$$
\frac{\Gamma\left(\eta_{c}(1 S) \rightarrow \mathrm{LH}\right)}{\Gamma\left(\eta_{c}(1 S) \rightarrow \gamma \gamma\right)}=(3.26 \pm 0.6) \times 10^{3},
$$

or $(3.01 \pm 0.5) \times 10^{3}$ in a different resummation scheme. A similar analysis could be performed for the $\eta_{b}(1 S)$, which combined with a determination of $\Gamma\left(\eta_{b}(1 S) \rightarrow \gamma \gamma\right)$ would then provide a theoretical determination of the $\eta_{b}(1 S)$ width. At the moment, without any resummation or renormalon subtraction performed,

$$
\frac{\Gamma\left(\eta_{b}(1 S) \rightarrow \mathrm{LH}\right)}{\Gamma\left(\eta_{b}(1 S) \rightarrow \gamma \gamma\right)} \simeq(1.8-2.3) \times 10^{4} .
$$

Recently a new resummation scheme has been suggested for electromagnetic decay ratios of heavy quarkonium and applied to determine the $\eta_{b}(1 S)$ decay width into two photons [462]:

$$
\Gamma\left(\eta_{b}(1 S) \rightarrow \gamma \gamma\right)=0.54 \pm 0.15 \mathrm{keV}
$$

Substituting Eq. (111) into Eq. (110) gives $\Gamma\left(\eta_{b}(1 S) \rightarrow\right.$ $\mathrm{LH})=7-16 \mathrm{MeV}$.

\subsubsection{Measurement of $\psi, \Upsilon \rightarrow \gamma g g$}

In measurements of the $\gamma g g$ rate from $J / \psi$ [235], $\psi(2 S)$ [236], and $\Upsilon(1 S, 2 S, 3 S)$ [230], CLEO finds that the most effective experimental strategy to search for $\gamma g g$ events is to focus solely upon those with energetic photons (which are less prone to many backgrounds), then to make the inevitable large subtractions of $g g g$, $q \bar{q}$, and transition backgrounds on a statistical basis, and finally to extrapolate the radiative photon energy spectrum to zero with the guidance of both theory and the measured high energy spectrum. The most troublesome background remaining is from events with energetic $\pi^{0} \rightarrow \gamma \gamma$ decays which result in a high-energy photon in the final state. One of several methods used to estimate this background uses the measured charged pion spectra and the assumption of isospin invariance to simulate the resulting photon spectrum with Monte Carlo techniques; another measures the exponential shape of the photonfrom- $\pi^{0}$ distribution at low photon energy, where $\gamma g g$ decays are few, and extrapolates to the full energy range. Backgrounds to $\gamma g g$ from transitions require the input of 
the relevant branching fractions and their uncertainties. The rate for $g g g$ decays is then estimated as that fraction of decays that remains after all dileptonic, transition, and $q \bar{q}$ branching fractions are subtracted, again requiring input of many external measurements and their respective uncertainties. Not surprisingly, the relative errors on the results of $10-30 \%$ are dominated by the systematic uncertainties incurred from background subtraction methods, photon-spectrum model-dependence, and external branching fractions.

The CLEO measurements of the observable $R_{\gamma}$ defined in Eq. (22) are shown in Tables 24 and 25. It should be noted that the uncertainties in $R_{\gamma}$ for $V=\Upsilon(1 S, 2 S, 3 S)$ are partially correlated with one another because of shared model dependence and analysis systematics. In addition, the shape of the measured direct photon spectrum from $J / \psi$ is quite similar to that of the $\Upsilon$ 's, in contrast to the $\psi(2 S)$, for which the spectrum appears to be softer. Absolute values for the branching fractions have been calculated with input of other world-average branching fractions in PDG10 [447], and are reproduced in Table 25. See Sect. 2.8.1 for discussion of extraction of $\alpha_{\mathrm{s}}$ from $R_{\gamma}$ measurements.

\subsubsection{Observation of $J / \psi \rightarrow \gamma \gamma \gamma$}

Orthopositronium, the ${ }^{3} S_{1} e^{+} e^{-}$bound state, decays to $3 \gamma$ almost exclusively and has long been used for precision tests of QED [464]. The rate of its analog for QCD, three-photon decay of vector charmonium, in particular that of the $J / \psi$, acts as a probe of the strong interaction [355]. Due to similarities at the parton level, relative rate measurements of the branching fractions for $J / \psi \rightarrow$ $3 \gamma, J / \psi \rightarrow \gamma g g, J / \psi \rightarrow 3 g$, and $J / \psi \rightarrow l^{+} l^{-}$provide crucial grounding for QCD predictions [355, 465, 466]. Previous searches for the quarkonium decay to $3 \gamma$ have yielded only upper limits: $\mathcal{B}(\omega \rightarrow 3 \gamma)<1.9 \times 10^{-4}$ at $95 \% \mathrm{CL}$ [467] and $\mathcal{B}(J / \psi \rightarrow 3 \gamma)<5.5 \times 10^{-5}$ at 90\% CL [468].

CLEO [469] performed a search for the all-photon decays of the $J / \psi$ using tagged $J / \psi$ mesons from $9.6 \times 10^{6}$ $\psi(2 S) \rightarrow J / \psi \pi^{+} \pi^{-}$decays. After excluding backgrounds from $J / \psi \rightarrow \gamma \eta / \eta^{\prime} / \eta_{c}(1 S) \rightarrow 3 \gamma$ with restrictions on photon-pair masses, and minimizing $J / \psi \rightarrow \gamma \pi^{0} \pi^{0}$ backgrounds in which two of the photons are very soft by imposing stringent energy-momentum conservation via a kinematic fit, CLEO reported the first observation of the decay $J / \psi \rightarrow 3 \gamma$ by finding 38 events, 12.8 of which were estimated to be background, mostly from various sources of $J / \psi \rightarrow \gamma \pi^{0} \pi^{0} \rightarrow 5 \gamma$ decays. The branching fraction is measured to be

$$
\mathcal{B}(J / \psi \rightarrow 3 \gamma)=(1.2 \pm 0.3 \pm 0.2) \times 10^{-5}
$$

with statistical significance for the signal of $6.3 \sigma$. Diphoton mass plots are shown in Fig. 42 for data, signal MC, and two possible sources of background. The measured
TABLE 24: Measured values of $R_{\gamma}$, as defined in Eq. (22), assuming the Garcia-Soto (GS) [231] or Field [463] models of the direct photon spectrum from $\Upsilon$ decays, and their averages, from CLEO [230]. The uncertainty on each average includes a component to account for model dependence (which dominates the other uncertainties); statistical errors are negligible

\begin{tabular}{cccc}
\hline \hline State & & \multicolumn{3}{c}{$R_{\gamma}(\%)$} \\
& GS & Field & Average \\
\hline$\Upsilon(1 S)$ & $2.46 \pm 0.13$ & $2.90 \pm 0.13$ & $2.70 \pm 0.27$ \\
$\Upsilon(2 S)$ & $3.06 \pm 0.22$ & $3.57 \pm 0.22$ & $3.18 \pm 0.47$ \\
$\Upsilon(3 S)$ & $2.58 \pm 0.32$ & $3.04 \pm 0.32$ & $2.72 \pm 0.49$ \\
\hline \hline
\end{tabular}

TABLE 25: Measured values of $R_{\gamma}$ from CLEO, as defined in Eq. (22), and the respective absolute branching fractions from PDG10 [447]

\begin{tabular}{ccccc}
\hline \hline State & $R_{\gamma}(\%)$ & $\mathcal{B}(\gamma g g)(\%)$ & $\mathcal{B}(g g g)(\%)$ & Source \\
\hline$J / \psi$ & $13.7 \pm 1.7$ & $8.8 \pm 0.5$ & $64.1 \pm 1.0$ & {$[235,447]$} \\
$\psi(2 S)$ & $9.7 \pm 3.1$ & $1.02 \pm 0.29$ & $10.6 \pm 1.6$ & {$[236,447]$} \\
$\Upsilon(1 S)$ & $2.70 \pm 0.27$ & $2.21 \pm 0.22$ & $81.7 \pm 0.7$ & {$[230,447]$} \\
$\Upsilon(2 S)$ & $3.18 \pm 0.47$ & $1.87 \pm 0.28$ & $58.8 \pm 1.2$ & {$[230,447]$} \\
$\Upsilon(3 S)$ & $2.72 \pm 0.49$ & $0.97 \pm 0.18$ & $35.7 \pm 2.6$ & {$[230,447]$} \\
\hline \hline
\end{tabular}

three-photon branching fraction lies between the zerothorder predictions [465] for $\mathcal{B}_{3 \gamma} / \mathcal{B}_{\gamma g g} \approx\left(\alpha / \alpha_{\mathrm{s}}\right)^{2} / 3$ and $\mathcal{B}_{3 \gamma} / \mathcal{B}_{3 g} \approx\left(\alpha / \alpha_{\mathrm{s}}\right)^{3}$ and is consistent with both, but is a factor of $\approx 2.5$ below that for $\mathcal{B}_{3 \gamma} / \mathcal{B}_{l l} \approx \alpha / 14$, all assuming $\alpha_{\mathrm{s}}\left(m_{J / \psi}\right) \approx 0.3$. Upper limits on the branching fractions for $J / \psi \rightarrow \gamma \gamma, 4 \gamma$, and $5 \gamma$ were also determined, setting more stringent restrictions than previous experiments.

\subsubsection{Nonobservation of $\psi(2 S), \Upsilon(1 S) \rightarrow \gamma \eta$}

Both BESII [470] and CLEO [471] report studies on the exclusive final states $\gamma\left(\pi^{0} / \eta / \eta^{\prime}\right)$ from charmonium, BESII from $J / \psi$ decays alone, and CLEO from decays of $J / \psi, \psi(2 S)$, and $\psi(3770)$. Resulting branching fractions appear in Table 26, where it can be seen that the results from the two experiments are consistent with each other, and that precision has steadily improved. From the perspective of charmonium physics, the most striking feature of these numbers is the nonobservation of $\psi(2 S) \rightarrow \gamma \eta$.

CLEO and BESII have consistent values of the ratio

$$
r_{n} \equiv \frac{\mathcal{B}(\psi(n S) \rightarrow \gamma \eta)}{\mathcal{B}\left(\psi(n S) \rightarrow \gamma \eta^{\prime}\right)}
$$

for $J / \psi$ of $r_{1} \approx 0.2$ within a few percent; the naive expectation would be that, for $\psi(2 S), r_{2} \approx r_{1}$. Yet the CLEO result implies that $r_{2}<1.8 \%$, or $r_{2} / r_{1}<8 \%$, both at 


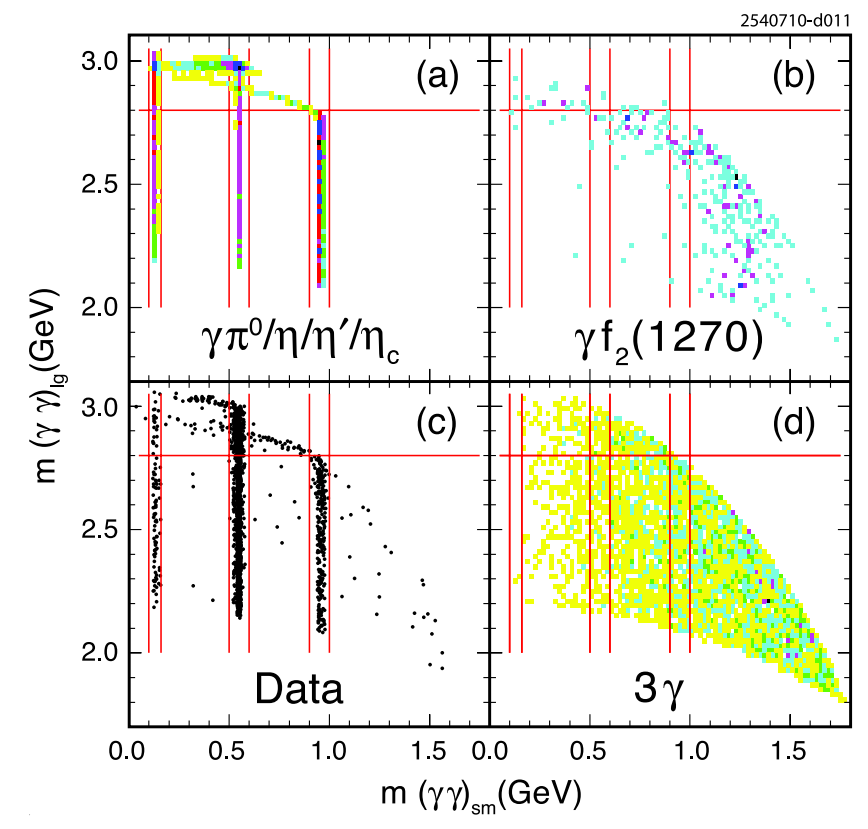

FIG. 42: From CLEO [469], distributions of the largest vs. the smallest diphoton mass combination per event in $\psi(2 S) \rightarrow$ $\pi^{+} \pi^{-} J / \psi, J / \psi \rightarrow 3 \gamma$ candidates. Parts (a), (c), and (d) are from MC simulations of $J / \psi \rightarrow \gamma \eta / \eta^{\prime} / \eta_{c}(1 S) \rightarrow 3 \gamma$, $\gamma f_{2}(1270) \rightarrow \gamma \pi^{0} \pi^{0} \rightarrow 5 \gamma$, and the signal process, $J / \psi \rightarrow 3 \gamma$, respectively. In these three MC plots darker shading of bins signifies relatively larger event density than lighter shades. Part (b) shows the CLEO data, where each solid circle indicates a single event. Solid lines demarcate regions excluded from the $J / \psi \rightarrow 3 \gamma$ event selection. 38 data events populate the signal region, of which $24.2_{-6.0}^{+7.2}$ were estimated to be from $J / \psi \rightarrow 3 \gamma$, with the remainder due to background, mostly various sources of $J / \psi \rightarrow \gamma \pi^{0} \pi^{0}$. Adapted from [469] with kind permission, copyright (2008) The American Physical Society

$90 \%$ CL. Specifically, the rate of $\gamma \eta$ relative to $\gamma \eta^{\prime}$ from $\psi(2 S)$ is at least an order of magnitude smaller than that from $J / \psi$. If instead we characterize the effect in terms of "the $12 \%$ rule" (Sect. 3.4.1), we note that, relative to its dileptonic width, $\psi(2 S) \rightarrow \gamma \eta^{\prime}$ is suppressed by a factor of five with respect to $J / \psi \rightarrow \gamma \eta^{\prime}$, but $\psi(2 S) \rightarrow \gamma \eta$ is suppressed by at least two orders of magnitude with respect to $J / \psi \rightarrow \gamma \eta$.

Do we see such transitions at expected rates in $\Upsilon$ decays? The CLEO [472] search for $\Upsilon(1 S) \rightarrow \gamma \eta^{(\prime)}$ failed to find evidence for either pseudoscalar meson in radiative decays, setting the limits

$$
\begin{aligned}
& \mathcal{B}(\Upsilon(1 S) \rightarrow \gamma \eta)<1.0 \times 10^{-6} \\
& \mathcal{B}\left(\Upsilon(1 S) \rightarrow \gamma \eta^{\prime}\right)<1.9 \times 10^{-6} .
\end{aligned}
$$

These values rule out the predictions of Chao [473], which are based on mixing of $\eta, \eta^{\prime}$, and $\eta_{b}(1 S)$, but not sensitive enough to probe those of Ma [474], which uses a QCDfactorization approach, nor those of $\mathrm{Li}$ [475], which posits a substantial two-gluon component in $\eta$ and $\eta^{\prime}$ within a perturbative QCD framework.

What dynamical effect is present in $\psi(2 S)$ decays that is absent in $J / \psi$ decays that can explain this large of a suppression? Is it related to $\rho \pi$ suppression in $\psi(2 S)$ decays relative to $J / \psi$ (Sect. 3.4.1), another vectorpseudoscalar final state? Is there a connection between the suppression of $\gamma \eta$ and $\gamma \eta^{\prime}$ in $\Upsilon(1 S)$ decay and that of $\gamma \eta$ in $\psi(2 S)$ decay? These questions remain unanswered.

Events selected from this charmonium analysis have been used to address physics questions other than those directly associated with $c \bar{c}$ bound states:

- The flavor content of $\eta$ and $\eta^{\prime}$ mesons, which are commonly thought to be mixtures of the pure $\mathrm{SU}(3)$-flavor octet and singlet states, with a possible admixture of gluonium [476-478]; if $J / \psi \rightarrow$ $\gamma \eta^{(\prime)}$ occurs through $c \bar{c} \rightarrow \gamma g g$, which is expected to fragment in a flavor-blind manner, the mixing angle can be extracted from $r_{1}$ as defined in Eq. (113). The measured value [470] from charmonium is consistent with that obtained from other sources [479481].

- The high-statistics sample of $J / \psi \rightarrow \gamma \eta^{\prime}$ decays was also used by CLEO to perform the first simultaneous measurement of the largest five $\eta^{\prime}$ branching fractions [471], attaining improved precisions, and to improve the measurement precision of the $\eta^{\prime}$ mass [482].

- Although CLEO [471] only set limits for $\psi(3770)$ decays to these final states, clean signals for both $\gamma \eta$ and $\gamma \eta^{\prime}$ final states were observed. However, these rates were seen to be consistent with that

TABLE 26: Branching fractions (in units of $10^{-4}$ ) for charmonium decays to $\gamma\left(\pi^{0} / \eta / \eta^{\prime}\right)$ from CLEO [471] and PDG08 [18], the latter of which is dominated by BESII [470]. The rightmost column shows the difference between the two in units of standard error $(\sigma)$. Upper limits are quoted at $90 \%$ CL. Entries in the last two rows include the effects of estimated continuum background and ignore (include) maximal destructive interference between $\psi(3770)$ and continuum sources

\begin{tabular}{cccc}
\hline \hline Mode & CLEO & PDG08 & $\# \sigma$ \\
\hline$J / \psi \rightarrow \gamma \pi^{0}$ & $0.363 \pm 0.036 \pm 0.013$ & $0.33_{-0.04}^{+0.06}$ & 0.4 \\
$\rightarrow \gamma \eta$ & $11.01 \pm 0.29 \pm 0.22$ & $9.8 \pm 1.0$ & 1.2 \\
$\rightarrow \gamma \eta^{\prime}$ & $52.4 \pm 1.2 \pm 1.1$ & $47.1 \pm 2.7$ & 1.7 \\
$\psi(2 S) \rightarrow \gamma \pi^{0}$ & $<0.07$ & $<54$ & - \\
$\rightarrow \gamma \eta$ & $<0.02$ & $<0.9$ & - \\
$\rightarrow \gamma \eta^{\prime}$ & $1.19 \pm 0.08 \pm 0.03$ & $1.36 \pm 0.24$ & -0.7 \\
$\psi(3770) \rightarrow \gamma \pi^{0}$ & $<3$ & - & - \\
$\rightarrow \gamma \eta$ & $<0.2(1.5)$ & - & - \\
$\rightarrow \gamma \eta^{\prime}$ & $<0.2(1.8)$ & - & - \\
\hline \hline
\end{tabular}


expected from continuum production as extrapolated from the bottomonium energy region using the only other measurement of $e^{+} e^{-} \rightarrow \gamma \eta^{(\prime)}$, by BABAR [483].

- Rosner [484] explores the implications of these measurements on production mechanisms for $\gamma P$ $(P=$ pseudoscalar) final states, in particular the contribution of the vector dominance model (through $\rho \pi$ ) to $J / \psi \rightarrow \gamma \pi^{0}$.

\subsubsection{Two-photon widths of charmonia}

Considerable progress has been made with measurements of the two-photon width of the $\eta_{c}(1 S)$ and $\chi_{c}(1 P)$ states. Two different approaches have been used. The first one uses the formation of a charmonium state in twophoton collisions followed by the observation of its decay products. In this case the directly measured quantity is the product of the charmonium two-photon width and the branching ratio of its decay to a specific final state. A two-photon width can then be computed from the product if the corresponding branching fraction has been measured. Such measurements were performed at Belle for the following decays: $\chi_{c 0(2)} \rightarrow \pi^{+} \pi^{-}, K^{+} K^{-}$[485], $\eta_{c}(1 S) \rightarrow p \bar{p}$ [486], $\chi_{c 0(2)} \rightarrow K_{S}^{0} K_{S}^{0}$ [487], $\chi_{c 0(2)} \rightarrow$ $\pi^{0} \pi^{0}[488,489]$ and at CLEO for the $\chi_{c 2} \rightarrow J / \psi \gamma$ decay [490]. Belle also applied this method to study the two-photon formation of the $\eta_{c}(1 S), \chi_{c 0}(1 P)$ and $\chi_{c 2}(1 P)$ via various final states with four charged particles $\left(2 \pi^{+} 2 \pi^{-}, \pi^{+} \pi^{-} K^{+} K^{-}, 2 K^{+} 2 K^{-}\right)$and quasi-twobody final states $(\rho \rho, \phi \phi, \eta \eta, \ldots)[442,491]$. In the latter study [442], Belle also sought the two-photon production of the $\eta_{c}(2 S)$ and did not find a significant signal in any four-body final state

The second method is based on the charmonium decay into two photons. CLEO performed [492] such measurements using the reactions $\psi(2 S) \rightarrow \gamma_{1} \chi_{c J}, \quad \chi_{c J} \rightarrow \gamma_{2} \gamma_{3}$, where $\gamma_{1}$ is the least-energetic final-state photon in the $\psi(2 S)$ center-of-mass frame. Clear signals were observed for the $\chi_{c 0}$ and $\chi_{c 2}$, as shown in Fig. 43. (Two-photon decay of spin-one states is forbidden by the Landau-Yang theorem [493, 494]). Using the measured signal yield and the previously-determined number of $\psi(2 S)$ produced (24.6 million), the product of the branching fractions is determined as:

$$
\begin{aligned}
\mathcal{B}\left(\psi(2 S) \rightarrow \gamma \chi_{c 0}\right) \times & \mathcal{B}\left(\chi_{c 0} \rightarrow \gamma \gamma\right)= \\
& (2.17 \pm 0.32 \pm 0.10) \times 10^{-5} \\
\mathcal{B}\left(\psi(2 S) \rightarrow \gamma \chi_{c 2}\right) \times & \mathcal{B}\left(\chi_{c 2} \rightarrow \gamma \gamma\right)= \\
( & 2.68 \pm 0.28 \pm 0.15) \times 10^{-5}
\end{aligned}
$$

World-average values for the $\psi(2 S) \rightarrow \gamma \chi_{c J}$ branching fractions and total widths of the $\chi_{c 0(2)}$ states can then used to calculate the two-photon widths for the corresponding charmonia. In Table 27 we list these twophoton widths, which result from a constrained fit to

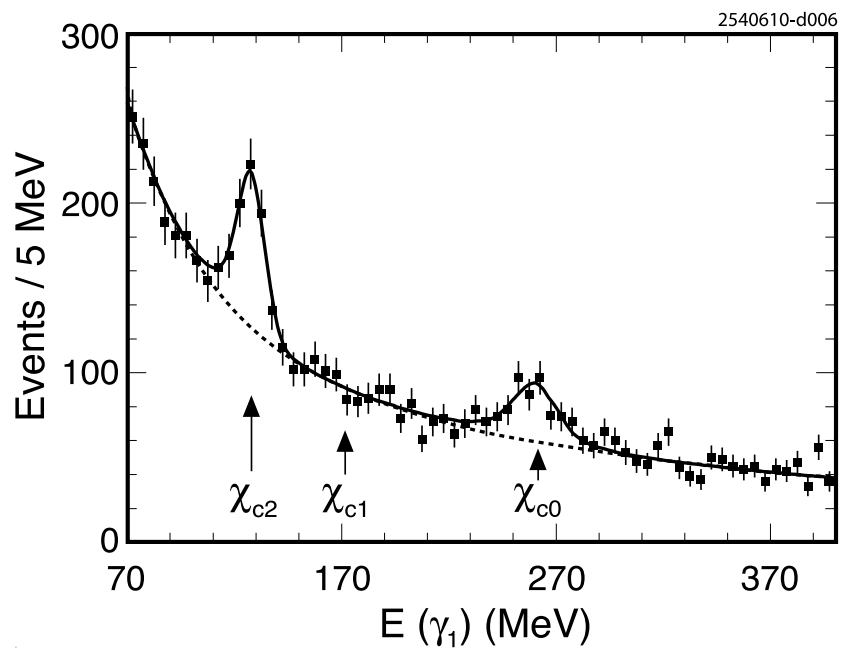

FIG. 43: From CLEO [492], the fitted spectrum for $E\left(\gamma_{1}\right)$ from the reaction $\psi(2 S) \rightarrow \gamma_{1} \chi_{c J}, \quad \chi_{c J} \rightarrow \gamma_{2} \gamma_{3}$, where $\gamma_{1}$ is the least energetic photon in the $\psi(2 S)$ center-of-mass frame. The expected positions of $E\left(\gamma_{1}\right)$ from $\chi_{c 0}, \chi_{c 1}, \chi_{c 2}$ are marked with arrows. The dashed curve represents a fit of the nonpeaking background to a polynomial. Adapted from [492] with kind permission, copyright (2008) The American Physical Society

TABLE 27: World-average values of the two-photon width for various charmonium states from PDG08 [18]

\begin{tabular}{lccc}
\hline \hline State & $\chi_{c 0}(1 P)$ & $\chi_{c 2}(1 P)$ & $\eta_{c}(1 S)$ \\
\hline$\Gamma_{\gamma \gamma}(\mathrm{keV})$ & $2.36 \pm 0.25$ & $0.515 \pm 0.043$ & $7.2 \pm 0.7 \pm 2.0$ \\
\hline \hline
\end{tabular}

all relevant experimental information from PDG08 [18]. Using those values, the experimental ratio becomes $\Gamma_{\gamma \gamma}\left(\chi_{c 2}\right) / \Gamma_{\gamma \gamma}\left(\chi_{c 0}\right)=0.22 \pm 0.03$. The LO and a NLO determination are shown in the first row of Table 23; some relevant theoretical issues are discussed in Sect. 3.2.1.

As part of the $J / \psi \rightarrow 3 \gamma$ analysis described in Sect. 3.2.3, CLEO [469] failed to find evidence for the decay $\eta_{c}(1 S) \rightarrow \gamma \gamma$. The product branching fraction for the decay chain $J / \psi \rightarrow \gamma \eta_{c}(1 S), \eta_{c}(1 S) \rightarrow \gamma \gamma$ was measured to be $\left(1.2_{-1.1}^{+2.7} \pm 0.3\right) \times 10^{-6}\left(<6 \times 10^{-6}\right.$ at $90 \% \mathrm{CL})$, which, using the CLEO [69] measurement of $\mathcal{B}\left(J / \psi \rightarrow \gamma \eta_{c}(1 S)\right)=(1.98 \pm 0.31) \%$ (discussed in Sect. 3.1.2), can be rewritten as

$$
\begin{aligned}
\mathcal{B}\left(\eta_{c}(1 S) \rightarrow \gamma \gamma\right) & =\left(6.1_{-5.6}^{+13.7}\right) \times 10^{-5}(\mathrm{CLEO}) \\
& <2.4 \times 10^{-4} \text { at } 90 \% \mathrm{CL}
\end{aligned}
$$

This central value is smaller than but consistent with the only other direct measurement of $\eta_{c}(1 S) \rightarrow \gamma \gamma$ measured in $B^{ \pm} \rightarrow K^{ \pm} \eta_{c}(1 S)$ decays by Belle [496], which obtains

$$
\mathcal{B}\left(\eta_{c}(1 S) \rightarrow \gamma \gamma\right)=\left(2.4_{-0.9}^{+1.2}\right) \times 10^{-4} \text { (Belle) }
$$

for which the Belle product branching fraction was un- 
folded by PDG08 [18].

\subsubsection{Dileptonic widths in the $\psi$ family}

BABAR [497], CLEO [498-500], and KEDR [501] have all performed measurements of dileptonic decays from the narrow members of the $\psi$ resonance family with muchimproved precisions. BABAR [497] pioneered the use of vector charmonia produced through initial-state radiation from $e^{+} e^{-}$collisions collected for other purposes at energies higher than the state being studied, in particular for the $J / \psi$ from $\sqrt{s} \approx 10.58 \mathrm{GeV}$. CLEO followed BABAR's lead, studying $J / \psi[498]$ and $\psi(2 S)$ [500] mesons from $\sqrt{s}=3770 \mathrm{MeV}$. KEDR [501] followed the more straightforward route, which requires a dedicated scan of the resonance. In both kinds of analyses the directly measured quantity is $\Gamma\left(J / \psi \rightarrow e^{+} e^{-}\right) \times \mathcal{B}\left(J / \psi \rightarrow \ell^{+} \ell^{-}\right)$. The dileptonic branching fraction can then be divided out from the result once to obtain $\Gamma_{e e}$ and twice for $\Gamma_{\text {tot }}$. CLEO and BABAR used only $J / \psi \rightarrow \mu^{+} \mu^{-}$decays because the $e^{+} e^{-}$final state has larger backgrounds from radiative Bhabha events, whereas KEDR used dimuons and dielectrons, obtaining the best precision to date. In all three cases $e^{+} e^{-} \rightarrow \ell^{+} \ell^{-}$and its interference with the resonant signal must be addressed. The results are listed in Table 28, where it can be seen that the four measurements are consistent with one another and have combined precision of $1.4 \%$.

CLEO [499] also provided an independent measurement of $J / \psi$ dileptonic branching fractions using $\psi(2 S) \rightarrow J / \psi \pi^{+} \pi^{-}, J / \psi \rightarrow \ell^{+} \ell^{-}$decay chains, and normalizing to all $J / \psi$ decays produced via the $\pi^{+} \pi^{-}$ transition by fitting the dipion recoil mass distribution.

TABLE 28: Measurements of $\Gamma\left(J / \psi \rightarrow e^{+} e^{-}\right) \times \mathcal{B}(J / \psi \rightarrow$ $\left.\ell^{+} \ell^{-}\right)$and their relative accuracies $\delta$; the weighted average and its $\left(\chi^{2} /\right.$ d.o.f. $)$ are shown assuming lepton universality. The two values from KEDR have some systematic uncertainties in common and are shown separately below the average

\begin{tabular}{lccc}
\hline \hline Experiment & $\ell^{+} \ell^{-}$ & $\begin{array}{c}\Gamma\left(J / \psi \rightarrow e^{+} e^{-}\right) \times \\
\mathcal{B}\left(J / \psi \rightarrow \ell^{+} \ell^{-}\right)(\mathrm{keV})\end{array}$ & $\delta(\%)$ \\
\hline BABAR [497] & $\mu^{+} \mu^{-}$ & $0.3301 \pm 0.0077 \pm 0.0073$ & 3.2 \\
CLEO [498] & $\mu^{+} \mu^{-}$ & $0.3384 \pm 0.0058 \pm 0.0071$ & 2.7 \\
KEDR [501] & $\ell^{+} \ell^{-}$ & $0.3321 \pm 0.0041 \pm 0.0050$ & 1.9 \\
Avg (0.43/2) & $\ell^{+} \ell^{-}$ & $0.3334 \pm 0.0047$ & 1.4 \\
$\operatorname{KEDR~[501]~}$ & $\mu^{+} \mu^{-}$ & $0.3318 \pm 0.0052 \pm 0.0063$ & 2.5 \\
$\operatorname{KEDR~[501]~}$ & $e^{+} e^{-}$ & $0.3323 \pm 0.0064 \pm 0.0048$ & 2.4 \\
\hline \hline
\end{tabular}

CLEO obtained

$$
\begin{aligned}
\mathcal{B}\left(J / \psi \rightarrow \mu^{+} \mu^{-}\right) & =(5.960 \pm 0.065 \pm 0.050) \% \\
\mathcal{B}\left(J / \psi \rightarrow e^{+} e^{-}\right) & =(5.945 \pm 0.067 \pm 0.042) \% \\
\frac{\mathcal{B}\left(J / \psi \rightarrow e^{+} e^{-}\right)}{\mathcal{B}\left(J / \psi \rightarrow \mu^{+} \mu^{-}\right)} & =(99.7 \pm 1.2 \pm 0.6) \% \\
\mathcal{B}\left(J / \psi \rightarrow \ell^{+} \ell^{-}\right) & =(5.953 \pm 0.070) \%,
\end{aligned}
$$

consistent with and having uncertainties at least factor of two smaller than previous determinations. With this measurement, the assumption of lepton universality, and the weighted average from Table 28, we obtain $\Gamma_{e e}(J / \psi)=5.60 \pm 0.10 \mathrm{keV}(1.8 \%)$ and $\Gamma_{\text {tot }}(J / \psi)=$ $94.1 \pm 2.6 \mathrm{keV}(2.7 \%)$, compared to relative uncertainties on these quantities before any of these measurements (PDG04 [17]) of $3.1 \%$ and $3.5 \%$, respectively.

For $\psi(2 S)$, CLEO [500] used the decays $\psi(2 S) \rightarrow$ $X_{i} J / \psi, J / \psi \rightarrow \ell^{+} \ell^{-}$, where $X_{i}=\pi^{+} \pi^{-}, \pi^{0} \pi^{0}$, and $\eta$, directly measuring the products $\mathcal{B}(\psi(2 S) \rightarrow$ $\left.X_{i} J / \psi\right) \times \Gamma_{e e}[\psi(2 S)]$. CLEO initially used $\mathcal{B}(\psi(2 S) \rightarrow$ $\left.X_{i} J / \psi\right)$ values from [445] to extract $\Gamma_{e e}[\psi(2 S)]=2.54 \pm$ $0.11 \mathrm{keV}(4.3 \%)$, but if instead we use CLEO's updated values [446], described below in Sect. 3.3.2, we obtain $\Gamma_{e e}[\psi(2 S)]=2.407 \pm 0.083 \mathrm{keV}(3.4 \%)$. We then obtain the ratio $\Gamma_{e e}[\psi(2 S)] / \Gamma_{e e}(J / \psi)=0.43 \pm 0.02$, a quantity which might be more precisely predicted in lattice QCD than either $\Gamma_{e e}$ alone [211], in which we have used our updated $\Gamma_{e e}[\psi(2 S)]$ and our world-average $\Gamma_{e e}[J / \psi]$ from above.

BES [502, 503] studied the energy range $\sqrt{s}=3.660$ $3.872 \mathrm{GeV}$ to determine the resonance parameters of $\psi(2 S)$ and $\psi(3770)$. From the fit of the cross sections for $D^{0} \bar{D}^{0}, D^{+} D^{-}$and non- $D \bar{D}$ production the branching fractions and partial widths for $\psi(2 S) \rightarrow e^{+} e^{-}$and $\psi(3770) \rightarrow e^{+} e^{-}$decays were determined. The results of these measurements of $\Gamma_{e e}[\psi(2 S)]$ are shown in Table 29, where the improvement since 2004 can be observed.

Decays of the $\psi(2 S)$ into $\tau$-lepton pairs are less probable and therefore less studied. BES [504] used a sample of 14 million produced $\psi(2 S)$ to measure the corresponding branching fraction. The result, $\mathcal{B}\left(\psi(2 S) \rightarrow \tau^{+} \tau^{-}\right)=$ $(3.08 \pm 0.21 \pm 0.38) \times 10^{-3}$, has better relative preci-

TABLE 29: Measurements of $\Gamma_{e e}(\psi(2 S))$ and their relative accuracies $\delta$. The CLEO value quoted has been updated with branching fractions from [446] and the weighted-average with $\left(\chi^{2} /\right.$ d.o.f. $)$ of the top three measurements is given

\begin{tabular}{lcc}
\hline \hline Experiment & $\Gamma\left(\psi(2 S) \rightarrow e^{+} e^{-}\right)(\mathrm{keV})$ & $\delta(\%)$ \\
\hline CLEO [446, 500] & $2.407 \pm 0.083$ & 3.4 \\
BES [502] & $2.330 \pm 0.036 \pm 0.110$ & 5.0 \\
BES [503] & $2.388 \pm 0.037 \pm 0.096$ & 4.3 \\
Avg $(0.29 / 2)$ & $2.383 \pm 0,056$ & 2.3 \\
PDG04 [17] & $2.12 \pm 0.12$ & 5.7 \\
\hline \hline
\end{tabular}


sion $(14 \%)$ than previous measurements $[505,506]$. Using part of their statistics KEDR measured $\Gamma(\psi(2 S) \rightarrow$ $\left.e^{+} e^{-}\right) \times \mathcal{B}\left(\psi(2 S) \rightarrow \tau^{+} \tau^{-}\right)$to be $9.0 \pm 2.6 \mathrm{eV}$ [507], which, using the average from Table 29 , implies a value $\mathcal{B}\left(\psi(2 S) \rightarrow \tau^{+} \tau^{-}\right)=(3.8 \pm 1.1) \times 10^{-3}$, consistent with but considerably less precise than the result from BES.

CLEO [508] measured the hadronic cross section at a single energy point near the peak of the $\psi(3770)$, $\sqrt{s}=3773 \mathrm{MeV}$, taking interference between the final states of resonance decays and nonresonant $e^{+} e^{-}$annihilation into account. From the observed cross section, which is significantly smaller than some of the previous measurements $[8,11], \Gamma_{e e}[\psi(3770)]$ is also obtained. In a scan over the $\psi(3770)$ energy region, 68 energy points in the range $3.650-3.872 \mathrm{GeV}$, BES [509] measured $R \equiv \sigma\left(e^{+} e^{-} \rightarrow\right.$ hadrons $) / \sigma\left(e^{+} e^{-} \rightarrow \mu^{+} \mu^{-}\right)$, determining the parameters of the $\psi(3770)$ resonance, including the leptonic width. The results of the described measurements of $\Gamma_{e e}[\psi(3770)]$ are shown in Table 30, where it can be seen that world-average uncertainty improved by more than a factor of two between 2004 and 2010 .

Finally, BES [13] performed a global fit of $R$ in the energy range 3.7-5.0 GeV, covering the four resonances, $\psi(3770), \psi(4040), \psi(4160)$, and $\psi(4415)$ [15]. Interference between the four $\psi$ states was accounted for (which was not the case for the $\Gamma_{e e}[\psi(3770)]$ measurements in Table 30) and an energy-dependent width based on all accessible two-body decay channels was used. The results are shown in Table 31. It can be seen that the new results have larger uncertainties than previous ones, which ignored interference with higher-mass $\psi$ states.

\subsubsection{Dileptonic widths in the $\Upsilon$ family}

CLEO [510-512] has made a systematic study of dileptonic decays of the narrow states in the $\Upsilon$ family. To determine dimuonic branching fractions, the quantities $\tilde{\mathcal{B}}_{\mu \mu} \equiv \Gamma_{\mu \mu} / \Gamma_{\text {had }}$ are measured for each $\Upsilon(n S)$, where $\Gamma_{\mu \mu}\left(\Gamma_{\text {had }}\right)$ is the rate for $\Upsilon$ decay to $\mu^{+} \mu^{-}$(hadrons).

TABLE 30: Measurements of $\Gamma_{e e}(\psi(3770))$ and their relative accuracies $\delta$. The BES and CLEO results listed do not include the potential effect of interference with higher-mass $\psi$-states

\begin{tabular}{lcc}
\hline \hline Experiment & $\Gamma\left(\psi(3770) \rightarrow e^{+} e^{-}\right)(\mathrm{keV})$ & $\delta(\%)$ \\
\hline PDG04 [17] & $0.26 \pm 0.04$ & 15.4 \\
CLEO [508] & $0.203 \pm 0.003_{-0.027}^{+0.041}$ & ${ }_{-13.3}^{+20.0}$ \\
BES [502] & $0.251 \pm 0.026 \pm 0.011$ & 11.2 \\
BES [509] & $0.277 \pm 0.011 \pm 0.013$ & 6.1 \\
PDG10 [447] & $0.259 \pm 0.016$ & 6.2 \\
\hline \hline
\end{tabular}

TABLE 31: $\Gamma_{e e}$ of the higher $\psi$ states from PDG04 [17] and the BES [15] global fit

\begin{tabular}{ccc}
\hline \hline Resonance & $\begin{array}{c}\Gamma_{e e}(\mathrm{keV}) \\
\text { from PDG04 }\end{array}$ & $\begin{array}{c}\Gamma_{e e}(\mathrm{keV}) \\
\text { from BES }\end{array}$ \\
\hline$\psi(3770)$ & $0.26 \pm 0.04$ & $0.22 \pm 0.05$ \\
$\psi(4040)$ & $0.75 \pm 0.15$ & $0.83 \pm 0.20$ \\
$\psi(4160)$ & $0.77 \pm 0.23$ & $0.48 \pm 0.22$ \\
$\psi(4415)$ & $0.47 \pm 0.10$ & $0.35 \pm 0.12$ \\
\hline \hline
\end{tabular}

Assuming lepton universality,

$$
\mathcal{B}_{\mu \mu}=\frac{\Gamma_{\mu \mu}}{\Gamma_{\text {tot }}}=\frac{\tilde{\mathcal{B}}_{\mu \mu}}{1+3 \tilde{\mathcal{B}}_{\mu \mu}} .
$$

The results of this analysis, based on much larger data samples than available to previous experiments, are summarized in Table 32. While the result for the $\Upsilon(1 S)$ is in good agreement with the world average, the CLEO results for the $\Upsilon(2 S)$ and $\Upsilon(3 S)$ are about $3 \sigma$ larger than previous world averages. However, the CLEO values are confirmed by their proximity to $\tau^{+} \tau^{-}$branching fraction measurements, which are discussed below.

In order to measure a quantity very close to $\Gamma_{\mathrm{ee}}$, CLEO [511] performed dedicated scans to measure the integral of the $\Upsilon$ production cross section over incident $e^{+} e^{-}$energies to determine

$$
\begin{aligned}
\tilde{\Gamma}_{e e} & \equiv \frac{\Gamma_{e e} \Gamma_{\text {had }}}{\Gamma_{\text {tot }}} \\
\tilde{\Gamma}_{e e} & =\frac{m_{\Upsilon}^{2}}{6 \pi^{2}} \int \sigma\left(e^{+} e^{-} \rightarrow \Upsilon \rightarrow \text { hadrons }\right) d E .
\end{aligned}
$$

The resulting values, listed in Table 33, are consistent with, but more precise than, the PDG world averages.

CLEO [512] also addressed the third dileptonic width, $\Gamma_{\tau \tau}$ (making the first observation of the decay $\Upsilon(3 S) \rightarrow$ $\tau^{+} \tau^{-}$) and measured precise values of $\mathcal{B}_{\tau \tau} / \mathcal{B}_{\mu \mu}$ for $\Upsilon(n S), n=1,2,3$, as shown in Table 34. Using the CLEO values of $\mathcal{B}_{\mu \mu}$ [510] (discussed above) allowed reporting of the absolute $\mathcal{B}_{\tau \tau}$ values as well. The results obtained are consistent with the expectations from

TABLE 32: Dimuonic branching fractions of the narrow $\Upsilon$ states from PDG04 [17] and CLEO [510]

\begin{tabular}{ccc}
\hline \hline Resonance & $\begin{array}{c}\mathcal{B}_{\mu \mu}(\%) \\
\text { from PDG04 }\end{array}$ & $\begin{array}{c}\mathcal{B}_{\mu \mu}(\%) \\
\text { from CLEO }\end{array}$ \\
\hline$\Upsilon(1 S)$ & $2.48 \pm 0.06$ & $2.49 \pm 0.02 \pm 0.07$ \\
$\Upsilon(2 S)$ & $1.31 \pm 0.21$ & $2.03 \pm 0.03 \pm 0.08$ \\
$\Upsilon(3 S)$ & $1.81 \pm 0.17$ & $2.39 \pm 0.07 \pm 0.10$ \\
\hline \hline
\end{tabular}


TABLE 33: From CLEO [511], measured values of $\Gamma_{e e} \Gamma_{\text {had }} / \Gamma_{\text {tot }}$ for the narrow $\Upsilon$ states

\begin{tabular}{lc}
\hline \hline Resonance & $\Gamma_{e e} \Gamma_{\text {had }} / \Gamma_{\text {tot }}(\mathrm{keV})$ \\
\hline$\Upsilon(1 S)$ & $1.252 \pm 0.004 \pm 0.019$ \\
$\Upsilon(2 S)$ & $0.581 \pm 0.004 \pm 0.009$ \\
$\Upsilon(3 S)$ & $0.413 \pm 0.004 \pm 0.006$ \\
\hline \hline
\end{tabular}

TABLE 34: From CLEO [512], measured $\tau$-pair branching fractions of the narrow $\Upsilon$ states and ratios to corresponding dimuonic rates

\begin{tabular}{lcc}
\hline \hline Resonance & $\mathcal{B}_{\tau \tau} / \mathcal{B}_{\mu \mu}$ & $\mathcal{B}_{\tau \tau}(\%)$ \\
\hline$\Upsilon(1 S)$ & $1.02 \pm 0.02 \pm 0.05$ & $2.54 \pm 0.04 \pm 0.12$ \\
$\Upsilon(2 S)$ & $1.04 \pm 0.04 \pm 0.05$ & $2.11 \pm 0.07 \pm 0.13$ \\
$\Upsilon(3 S)$ & $1.05 \pm 0.08 \pm 0.05$ & $2.52 \pm 0.19 \pm 0.15$ \\
\hline \hline
\end{tabular}

the Standard Model, and $\mathcal{B}_{\tau \tau}$ values for $\Upsilon(1 S, 2 S)$ have much-improved precision over previous measurements.

The total width of the resonances can be expressed as

$$
\Gamma_{\text {tot }}=\frac{\tilde{\Gamma}_{e e}}{\mathcal{B}_{\mu \mu}\left(1-3 \mathcal{B}_{\mu \mu}\right)}
$$

where $\tilde{\Gamma}_{e e}$ is measured as in Eq. (120), and which, when combined with the more precise $\mathcal{B}_{\mu \mu}$ also described above, yields improved measurements of the total widths $\Gamma_{\text {tot }}$. The larger $\mathcal{B}_{\mu \mu}$ for $\Upsilon(2 S, 3 S)$ as determined by CLEO (validated by consistent values of $\mathcal{B}_{\tau \tau}$ ) leads to smaller and more precise $\Gamma_{\text {tot }}(2,3 S)$, as seen in Table 35 .

Improved measurements of the $\Upsilon(4 S)$ parameters were reported by $B A B A R$ [513]. Three scans of the energy range $\sqrt{s}=10.518-10.604 \mathrm{GeV}$ were performed with 11,7 , and 5 energy points, respectively, with integrated luminosity of typically $0.01 \mathrm{fb}^{-1}$ per point. This information was complemented by a large data sample of $76 \mathrm{fb}^{-1}$ collected at the peak of the $\Upsilon(4 S)$, from which the cross section at the peak was determined. The nominal $\sqrt{s}$ values of the scans were corrected using an energy calibration based on the dedicated run at the $\Upsilon(3 S)$. A fit of the energy dependence allows a determination of the $\Upsilon(4 S)$ parameters, among them mass, total, and electronic width, obtaining

$$
\begin{aligned}
m(\Upsilon(4 S)) & =10579.3 \pm 0.4 \pm 1.2 \mathrm{MeV} \\
\Gamma_{\text {tot }}(\Upsilon(4 S)) & =20.7 \pm 1.6 \pm 2.5 \mathrm{MeV} \\
\Gamma_{e e}(\Upsilon(4 S)) & =321 \pm 17 \pm 29 \mathrm{eV}
\end{aligned}
$$

all of which dominate the PDG08 [18] world averages.
TABLE 35: Values of $\Gamma_{\text {tot }}$ for the $\Upsilon(1 S, 2 S, 3 S)$ from PDG04 [17], CLEO [510], and PDG08 [18]

\begin{tabular}{cccc}
\hline \hline$\Upsilon(n S)$ & & $\Gamma_{\text {tot }}(\mathrm{keV})$ & \\
& PDG04 & CLEO & PDG08 \\
\hline$\Upsilon(1 S)$ & $53.0 \pm 1.5$ & $52.8 \pm 1.8$ & $54.02 \pm 1.25$ \\
$\Upsilon(2 S)$ & $43 \pm 6$ & $29.0 \pm 1.6$ & $31.98 \pm 2.63$ \\
$\Upsilon(3 S)$ & $26.3 \pm 3.4$ & $20.3 \pm 2.1$ & $20.32 \pm 1.85$ \\
\hline \hline
\end{tabular}

\subsection{Hadronic transitions}

\subsubsection{Theoretical status}

The general form for a hadronic transition is

$$
\Phi_{i} \rightarrow \Phi_{f}+h
$$

where $\Phi_{i}, \Phi_{f}$ and $h$ stand for the initial state, final state quarkonia, and the emitted light hadron(s). In the $c \bar{c}$ and $b \bar{b}$ systems, the mass $m_{\Phi_{i}}-m_{\Phi_{f}}$ varies from a few hundred $\mathrm{MeV}$ to slightly over a $\mathrm{GeV}$, so the kinematically allowed final light hadron(s) $h$ are dominated by singleparticle $\left(\pi^{0}, \eta, \omega, \ldots\right)$ or two-particle $(2 \pi$ or $2 K)$ states. The low momenta of the light hadrons in these transitions allow the application of chiral lagrangian methods. To date, over twenty hadronic transitions have been observed experimentally.

Hadronic transitions are important decay modes for low-lying heavy quarkonium states. In fact, the first observed hadronic transition, $\psi(2 S) \rightarrow \pi \pi J / \psi$, has a branching fraction recently measured by CLEO [446] to be $(52.7 \pm 1.3) \%$ (see Sect. 3.3.2). Calculating such transitions requires nonperturbative QCD. The standard approach is a QCD Multipole Expansion (QCDME) for gluon emission, which is modeled after the multipole expansion used for electromagnetic transitions (see Sect. 3.1.1).

Many contributed to the early development of the QCDME approach [158, 514, 515], but Yan [516] was the first to present a gauge-invariant formulation within QCD. For a heavy $Q \bar{Q}$ bound state, a dressed (constituent) quark is defined as

$$
\tilde{\psi}(\mathbf{x}, t) \equiv U^{-1}(\mathbf{x}, t) \psi(x)
$$

where $\psi(x)$ is the usual quark field and $U$ is defined as a path-ordered exponential along a straight line from $\mathbf{X} \equiv$ $\left(\mathbf{x}_{1}+\mathbf{x}_{2}\right) / 2$ (the center-of-mass coordinate of $Q$ and $\bar{Q}$ ) to $\mathbf{x}$,

$$
U(\mathbf{x}, t)=P \exp \left[i g_{s} \int_{\mathbf{x}}^{\mathbf{x}} \mathbf{A}\left(\mathbf{x}^{\prime}, t\right) \cdot d \mathbf{x}^{\prime}\right] .
$$

Gluon-field color indices have been suppressed. The 
dressed gluon field is defined by

$$
\begin{aligned}
\tilde{A}_{\mu}(\mathbf{x}, t) \equiv & U^{-1}(\mathbf{x}, t) A_{\mu}(x) U(\mathbf{x}, t) \\
& -\frac{i}{g_{s}} U^{-1}(\mathbf{x}, t) \partial_{\mu} U(\mathbf{x}, t) .
\end{aligned}
$$

Now we can make the QCD multipole expansion, in powers of $(\mathbf{x}-\mathbf{X}) \cdot \nabla$ operating on the gluon field in exact analogy with QED:

$$
\begin{aligned}
\tilde{A}_{0}(\mathbf{x}, t) & =A_{0}(\mathbf{X}, t)-(\mathbf{x}-\mathbf{X}) \cdot \mathbf{E}(\mathbf{X}, t)+\cdots \\
\tilde{\mathbf{A}}(\mathbf{X}, t) & =-\frac{1}{2}(\mathbf{x}-\mathbf{X}) \times \mathbf{B}(\mathbf{X}, t)+\cdots
\end{aligned}
$$

where $\mathbf{E}$ and $\mathbf{B}$ are color-electric and color-magnetic fields, respectively. The resulting Hamiltonian for a heavy $Q \bar{Q}$ system is then [516]

$$
H_{\mathrm{QCD}}^{\mathrm{eff}}=H_{\mathrm{QCD}}^{(0)}+H_{\mathrm{QCD}}^{(1)}+H_{\mathrm{QCD}}^{(2)},
$$

with $H_{\mathrm{QCD}}^{(0)}$ taken as the zeroth-order Hamiltonian, even though it does not represent free fields but instead the sum of the kinetic and potential energies of the heavy quarks. We also define

$$
H_{\mathrm{QCD}}^{(1)} \equiv Q_{a} A_{0}^{a}(\mathbf{X}, t),
$$

in which $Q_{a}$ is the color charge of the $Q \bar{Q}$ system (zero for color-singlets), and

$$
H_{\mathrm{QCD}}^{(2)} \equiv-\mathbf{d}_{a} \cdot \mathbf{E}^{a}(\mathbf{X}, \mathbf{t})-\mathbf{m}_{\mathbf{a}} \cdot \mathbf{B}^{\mathbf{a}}(\mathbf{X}, \mathbf{t})+\cdots,
$$

which is treated perturbatively. The quantities

$$
\mathbf{d}_{a}^{i}=g_{E} \int d^{3} x \tilde{\psi}^{\dagger}(x-X)^{i} t_{a} \tilde{\psi}
$$

and

$$
\mathbf{m}_{a}^{i}=g_{M} / 2 \int d^{3} x \tilde{\psi}^{\dagger} \epsilon^{i j k}(x-X)_{j} \gamma_{k} t_{a} \tilde{\psi}
$$

are the color-electric dipole moment (E1) and the colormagnetic dipole moment (M1) of the $Q \bar{Q}$ system, respectively. Higher-order terms (not shown) give rise to higher-order electric $(\mathrm{E} 2, \mathrm{E} 3, \ldots)$ and magnetic moments $(\mathrm{M} 2, \ldots)$. Because $H_{\mathrm{QCD}}^{(2)}$ in Eq. (130) couples colorsinglet to octet $Q \bar{Q}$ states, the transitions between eigenstates $|i\rangle$ and $|f\rangle$ of $H_{\mathrm{QCD}}^{(0)}$ are at least second-order in $H_{\mathrm{QCD}}^{(2)}$. The leading-order term is given by:

$$
\begin{gathered}
\left\langle f h\left|H_{2} \frac{1}{E_{i}-H_{\mathrm{QCD}}^{(0)}+i \partial_{0}-H_{\mathrm{QCD}}^{(1)}}\right| i\right\rangle= \\
\sum_{K L}\left\langle f h\left|H_{2}\right| K L\right\rangle \frac{1}{E_{i}-E_{K L}}\left\langle K L\left|H_{2}\right| i\right\rangle,
\end{gathered}
$$

where the sum $K L$ is over a complete set of color-octet $Q \bar{Q}$ states $|K L\rangle$ with associated energy $E_{K L}$. Finally, a connection is made to the physical hadronic transitions in Eq. (123) by assuming factorization of the heavy-quark interactions and the production of light hadrons. For example, the leading order E1-E1 transition amplitude is:

$$
\begin{aligned}
& \mathcal{M}\left(\Phi_{i} \rightarrow \Phi_{f}+h\right)= \\
& \quad \frac{1}{24} \sum_{K L} \frac{\left\langle f\left|d_{m}^{i a}\right| K L\right\rangle\left\langle K L\left|d_{m a}^{j}\right| i\right\rangle}{E_{i}-E_{K L}}\left\langle h\left|\mathbf{E}^{a i} \mathbf{E}_{a}^{j}\right| 0\right\rangle .(13)
\end{aligned}
$$

The allowed light hadronic final state $h$ is determined by the quantum numbers of the gluonic operator. The leading order term E1-E1 in Eq. (133) has $C P=++$ and $L=0,2$ and hence couples to $2 \pi$ and $2 K$ in $I=0$ states. Higher-order terms (in powers of $v$ ) couple as follows: E1-M1 in $O(v)$ with $(C P=--)$ couples to $\omega$; E1-M1, E1-E2 in $O(v)$ and M1-M1, E1-M2 in $O\left(v^{2}\right)$ with $(C P=+-)$ couples to both $\pi^{0}$ (isospin-breaking) and $\eta$ (SU(3)-breaking); and M1-M1, E1-E3, E2-E2 ( $C P=$ $++)$ are higher-order corrections to the E1-E1 terms.

Applying this formulation to observed hadronic transitions requires additional phenomenological assumptions. Following Kuang and Yan [516, 517], the heavy $Q \bar{Q}$ bound states spectrum of $H_{\mathrm{QCD}}^{(0)}$ is calculated by solving the state equation with a given potential model. The intermediate octet $Q \bar{Q}$ states are modeled by the Buchmueller-Tye quark-confining string (QCS) model [518]. Then chiral symmetry relations can be employed to parametrize the light hadronic matrix element. The remaining unknown coefficients in the light hadron matrix elements are set by experiment or calculated using a duality argument between the physical light hadron final state and associated two-gluon final state. A detailed discussion of all these assumptions can be found in the QWG review [1].

For the most common transitions, $h=\pi_{1}+\pi_{2}$, the effective chiral lagrangian form is [519]

$$
\begin{array}{r}
\frac{g_{E}^{2}}{6}\left\langle\pi_{1} \pi_{2}\left|\mathbf{E}_{i}^{a} \mathbf{E}_{a j}\right| 0\right\rangle=\frac{1}{\sqrt{\left(2 \omega_{1}\right)\left(2 \omega_{2}\right)}}\left[C_{1} \delta_{i j} q_{1}^{\mu} q_{2 \mu}+\right. \\
\left.C_{2}\left(q_{1 k} q_{2 l}+q_{1 l} q_{2 k}-\frac{2}{3} \delta_{i j} q_{1}^{\mu} q_{2 \mu}\right)\right]
\end{array}
$$

If the polarization of the heavy $Q \bar{Q}$ initial and final states is measured, more information can be extracted from these transitions and a more general form of Eq. (135) is appropriate [520].

Important single light-hadron transitions include those involving the $\eta, \pi^{0}$, or $\omega$ mesons. The general form of the light-hadronic factor for the $\eta$ transition, which is dominantly E1-M2, is [521]

$$
\frac{g_{e} g_{M}}{6}\left\langle\eta\left|\mathbf{E}_{i}^{a} \partial_{i} \mathbf{B}_{j}^{a}\right| 0\right\rangle=i(2 \pi)^{\frac{3}{2}} C_{3} q_{j} .
$$

The $\pi^{0}$ and $\eta$ transitions are related by the structure of chiral symmetry-breaking [522]. Many more details 
for these and other transitions within the context of the Kuang-Yan model can be found in the review of Kuang [521].

A summary of all experimentally observed hadronic transitions and their corresponding theoretical expectations within the Kuang-Yan (KY) model is presented in Table 36. The experimental partial widths are determined from the measured branching fractions and total width of the initial state. If the total width is not wellmeasured, the theoretically-expected width is used, as indicated. The theory expectations are adjusted using the current experimental inputs to rescale the model parameters $\left|C_{1}\right|$ and $\left|C_{2}\right|$ in Eq. (135) and $\left|C_{3}\right|$ in Eq. (136).

The multipole expansion works well for transitions of heavy $Q \bar{Q}$ states below threshold [81]. Within the specific KY model a fairly good description of the rates for the two-pion transitions is observed. The partial width $\Gamma\left(\Upsilon(3 S) \rightarrow \Upsilon(1 S) \pi^{+} \pi^{-}\right)$was predicted to be suppressed due to cancellations between the various QCS intermediate states [517], allowing nonleading terms, $O\left(v^{2}\right)$, to contribute significantly. The non- $S$-wave behavior of the $m_{\pi^{+} \pi^{-}}$dependence in $\Upsilon(3 S)$ decays, also observed in the $\Upsilon(4 S) \rightarrow \Upsilon(2 S) \pi^{+} \pi^{-}$transitions, may well reflect this influence of higher-order terms. Other possibilities are discussed in Sect. 3.3.11. For single light-hadron transitions some puzzles remain. For example, the ratio

$$
\frac{\Gamma(\Upsilon(2 S) \rightarrow \eta \Upsilon(1 S))}{\Gamma(\psi(2 S) \rightarrow \eta J / \psi(1 S))}
$$

is much smaller than expected from theory (see Sect. 3.3.6).

The situation is more complicated for above-threshold, strong open-flavor decays. The issues are manifest for $\Upsilon(5 S)$ two-pion transitions to $\Upsilon(n S)(n=1,2,3)$. First, states above threshold do not have sizes that are small compared to the QCD scale $\left(e . g ., \sqrt{\left\langle r^{2}\right\rangle_{\Upsilon(5 S)}}=1.2 \mathrm{fm}\right)$, making the whole QCDME approach less reliable. Second, even within the KY model, the QCS intermediate states are no longer far away from the initial-state mass. Thus the energy denominator, $E_{i}-E_{K L}$ in Eq. (134), can be small, leading to large enhancements in the transition rates that are sensitive to the exact position of the intermediate states [528]. This is the reason for the large theory widths seen in Table 36 . Third, a number of new states (see Sects. 2.3) that do not fit into the conventional $Q \bar{Q}$ spectra have been observed, implying additional degrees of freedom appearing in the QCD spectrum beyond naive-quark-model counting. Hence the physical quarkonium states have open-flavor meson-pair contributions and possible hybrid $(Q \bar{Q} g)$ or tetraquark contributions. The effect of such terms on hadronic transitions is not yet understood [531]. A possibly-related puzzle is the strikingly-large ratio

$$
R_{\eta}[\Upsilon(4 S)] \equiv \frac{\Gamma(\Upsilon(4 S) \rightarrow \Upsilon(1 S) \eta)}{\Gamma\left(\Upsilon(4 S) \rightarrow \Upsilon(1 S) \pi^{+} \pi^{-}\right)} \approx 2.5
$$

This ratio is over a hundred times larger than one would expect within the KY model, which is particularly sur-
TABLE 36: Partial widths for observed hadronic transitions. Experimental results are from PDG08 [18] unless otherwise noted. Partial widths determined from known branching fractions and total widths. Quoted values assume total widths of $\Gamma_{\text {tot }}\left(\chi_{b 2}(2 P)\right)=138 \pm 19 \mathrm{keV}$ [523], $\Gamma_{\text {tot }}\left(\chi_{b 1}(2 P)\right)=$ $96 \pm 16 \mathrm{keV}$ [523], $\Gamma_{\text {tot }}\left(\Upsilon\left(1^{3} D_{2}\right)\right)=28.5 \mathrm{keV}[524,525]$ and $\Gamma_{\text {tot }}(\Upsilon(5 S))=43 \pm 4 \mathrm{MeV}$ [36]. Only the charged dipion transitions are shown here, but the corresponding measured $\pi^{0} \pi^{0}$ rates, where they exist, are consistent with a parent state of $I=0$. Theoretical results are given using the Kuang and Yan (KY) model [517, 521, 526]. Current experimental inputs were used to rescale the parameters in the theory partial rates. $\left(\left|C_{1}\right|=10.2 \pm 0.2 \times 10^{-3}, C_{2} / C_{1}=1.75 \pm 0.14\right.$,

\begin{tabular}{|c|c|c|}
\hline Transition & $\begin{array}{l}\Gamma_{\text {partial }}(\mathrm{keV}) \\
(\text { Experiment })\end{array}$ & $\begin{array}{c}\Gamma_{\text {partial }}(\mathrm{keV}) \\
(\mathrm{KY} \text { Model }) \\
\end{array}$ \\
\hline \multicolumn{3}{|l|}{$\psi(2 S)$} \\
\hline $\begin{array}{l}\rightarrow J / \psi+\pi^{+} \pi^{-} \\
\rightarrow J / \psi+\eta \\
\rightarrow J / \psi+\pi^{0} \\
\rightarrow h_{c}(1 P)+\pi^{0}\end{array}$ & $\begin{array}{c}102.3 \pm 3.4 \\
10.0 \pm 0.4 \\
0.411 \pm 0.030[446] \\
0.26 \pm 0.05[47]\end{array}$ & $\begin{array}{c}\text { input }\left(\left|C_{1}\right|\right) \\
\text { input }\left(C_{3} / C_{1}\right) \\
0.64[522] \\
0.12-0.40[527]\end{array}$ \\
\hline \multicolumn{3}{|l|}{$\psi(3770)$} \\
\hline $\begin{array}{l}\quad \rightarrow J / \psi+\pi^{+} \pi^{-} \\
\quad \rightarrow J / \psi+\eta \\
\psi(3 S)\end{array}$ & $\begin{array}{c}52.7 \pm 7.9 \\
24 \pm 11\end{array}$ & input $\left(C_{2} / C_{1}\right)$ \\
\hline$\rightarrow J / \psi+\pi^{+} \pi^{-}$ & $<320(90 \% \mathrm{CL})$ & \\
\hline \multicolumn{3}{|l|}{$\Upsilon(2 S)$} \\
\hline $\begin{array}{l}\rightarrow \Upsilon(1 S)+\pi^{+} \pi^{-} \\
\rightarrow \Upsilon(1 S)+\eta\end{array}$ & $\begin{array}{c}5.79 \pm 0.49 \\
(6.7 \pm 2.4) \times 10^{-3}\end{array}$ & $\begin{array}{c}8.7[528] \\
0.025[521]\end{array}$ \\
\hline \multicolumn{3}{|l|}{$\Upsilon\left(1^{3} D_{2}\right)$} \\
\hline$\rightarrow \Upsilon(1 S)+\pi^{+} \pi^{-}$ & $0.188 \pm 0.046[63]$ & $0.07[529]$ \\
\hline \multicolumn{3}{|l|}{$\chi_{b 1}(2 P)$} \\
\hline $\begin{array}{l}\rightarrow \chi_{b 1}(1 P)+\pi^{+} \pi^{-} \\
\rightarrow \Upsilon(1 S)+\omega\end{array}$ & $\begin{array}{c}0.83 \pm 0.33[523] \\
1.56 \pm 0.46\end{array}$ & $0.54[530]$ \\
\hline \multicolumn{3}{|l|}{$\chi_{b 2}(2 P)$} \\
\hline $\begin{array}{l}\rightarrow \chi_{b 2}(1 P)+\pi^{+} \pi^{-} \\
\rightarrow \Upsilon(1 S)+\omega\end{array}$ & $\begin{array}{l}0.83 \pm 0.31[523] \\
1.52 \pm 0.49\end{array}$ & $0.54[530]$ \\
\hline \multicolumn{3}{|l|}{$\Upsilon(3 S)$} \\
\hline $\begin{array}{l}\rightarrow \Upsilon(1 S)+\pi^{+} \pi^{-} \\
\rightarrow \Upsilon(1 S)+\eta \\
\rightarrow \Upsilon(2 S)+\pi^{+} \pi^{-}\end{array}$ & $\begin{array}{l}0.894 \pm 0.084 \\
<3.7 \times 10^{-3} \\
0.498 \pm 0.065\end{array}$ & $\begin{array}{c}1.85[528] \\
0.012[521] \\
0.86[528]\end{array}$ \\
\hline \multicolumn{3}{|l|}{$\Upsilon(4 S)$} \\
\hline $\begin{array}{l}\rightarrow \Upsilon(1 S)+\pi^{+} \pi^{-} \\
\rightarrow \Upsilon(1 S)+\eta \\
\rightarrow \Upsilon(2 S)+\pi^{+} \pi^{-}\end{array}$ & $\begin{array}{l}1.64 \pm 0.25 \\
4.02 \pm 0.54 \\
1.76 \pm 0.34\end{array}$ & $\begin{array}{l}4.1[528] \\
1.4[528]\end{array}$ \\
\hline \multicolumn{3}{|l|}{$\Upsilon(5 S)$} \\
\hline $\begin{array}{l}\rightarrow \Upsilon(1 S)+\pi^{+} \pi^{-} \\
\rightarrow \Upsilon(1 S)+K^{+} K^{-} \\
\rightarrow \Upsilon(2 S)+\pi^{+} \pi^{-} \\
\rightarrow \Upsilon(3 S)+\pi^{+} \pi^{-}\end{array}$ & $\begin{aligned} 228 & \pm 33 \\
26.2 & \pm 8.1 \\
335 & \pm 64 \\
206 & \pm 80\end{aligned}$ & \\
\hline
\end{tabular}
$C_{3} / C_{1}=0.78 \pm 0.02$ for the Cornell case) 
prising, since the similarly-defined ratio, $R_{\eta}[\Upsilon(2 S)] \approx$ $10^{-3}$, is actually less than half of the KY model expectations (see Table 36) and the experimental upper bound on $R_{\eta}[\Upsilon(3 S)]$ is already slightly below KY-model expectations. Much theoretical work remains in order to understand the hadronic transitions of the heavy $Q \bar{Q}$ systems above threshold.

Many of the new $X Y Z$ states (see Sects. 2.3) are candidates for so-called hadronic molecules. If this were the case, and they indeed owe their existence to nonperturbative interactions among heavy mesons, the QCDME needs to be extended by heavy-meson loops. These loops provide nonmultipole, long-ranged contributions, so as to allow for the inclusion of their influence. However, if hadron loops play a significant, sometimes even nonperturbative, role above $\bar{D} D$ threshold, one should expect them to be at least of some importance below the lowest inelastic threshold. Correspondingly, one should expect to find some systematic deviations between quark-model predictions and data. By including intermediate heavy-meson effects within the framework of QCDME [532, 533], improved agreement with the experimental data on dipion transitions in the $\psi$ and $\Upsilon$ systems was obtained.

Alternatively, a nonrelativistic effective field theory (NREFT) was introduced [534] that allows one to study the effect of heavy-meson loops on charmonium transitions with controlled uncertainty. In this work, it was argued that the presence of meson loops resolves the long-standing discrepancy between, on the one hand, the values of the light-quark mass-differences extracted from the masses of the Goldstone bosons, and on the other, the ratio of selected charmonium transitions, namely $\psi(2 S) \rightarrow J / \psi \pi^{0} / \psi(2 S) \rightarrow J / \psi \eta$. NREFT uses the velocity of the heavy mesons in the intermediate state, $v \sim \sqrt{\left|m-2 m_{D}\right| / m_{D}}$, as expansion parameter. Thus, for transitions of states below $D \bar{D}$ threshold, the analytic continuation of the standard expression is to be used. For low-lying charmonium transitions, $v$ is found to be of order 0.5. A typical transition via a $D$-meson loop may then be counted as

$$
v^{3} /\left(v^{2}\right)^{2} \times \text { vertex factors }
$$

For the transition between two $S$-wave charmonia, which decay into $D^{(*)} \bar{D}^{(*)}$ via a $P$-wave vertex, the vertex factors scale as $v^{2}$. Thus the loop contributions appear to scale as order $v$, and, for values of the velocity small relative to those that can be captured by QCDME, are typically suppressed. However, in certain cases enhancements may occur. For example, for $\psi(2 S) \rightarrow J / \psi \pi^{0}$ and $\psi(2 S) \rightarrow J / \psi \eta$, flavor symmetry is broken, and therefore the transition matrix element needs to scale as $\delta$, the energy scale that quantifies the degree of flavor-symmetry violation in the loop and which originates from the mass differences of charged and neutral $D$-mesons. However, if an energy scale is pulled out of the integral, this needs to be balanced by removing the energy scale $v^{2}$ from the power counting. Thus the estimate for the loops con- tribution scales as $\delta / v$ compared to the piece of order $\delta$ that emerges from QCDME. Hence, for certain transitions, meson loops are expected to significantly influence the rates.

The contributions of heavy-meson loops to charmonium decays follow a very special pattern. They are expected to be more important for charmonia pairs close to the lowest two-meson threshold. In addition, loops appear to be suppressed for transitions between $S$ and $P$ wave charmonia [535]. These conjectures can be tested experimentally by systematic, high-precision measurements of as many transitions as possible. Once the role of meson loops is established for transitions between conventional heavy quarkonia below or very close to openflavor threshold, an extension from perturbative to a nonperturbative treatment might lead to a combined analysis of excited charmonia and at least a few of the $X Y Z$ states.

\subsubsection{Branching fractions for $\psi(2 S) \rightarrow X J / \psi$}

Precision measurements of the hadronic transitions $\psi(2 S) \rightarrow X J / \psi$ are important for a number of reasons beyond the obvious one of providing an accurate normalization and accounting for $\psi(2 S)$ decays. First, they allow experimental comparisons with increasingly sophisticated theoretical calculations (see Sect. 3.3.1). Second, they can be used in comparisons with the analogous $\Upsilon$ transitions in the bottomonium system. Finally, the transitions provide a convenient way to access the $J / \psi$, where the transition pions in $\psi(2 S) \rightarrow \pi^{+} \pi^{-} J / \psi$, for example, can be used in tagging a clean and wellnormalized $J / \psi$ sample. Using its full sample of 27 million $\psi(2 S)$ decays, CLEO [446] measured these rates with substantially improved precision over previous measurements. The analysis measures $\mathcal{B}\left(\psi(2 S) \rightarrow \pi^{+} \pi^{-} J / \psi\right)$ inclusively, selecting events based upon the mass recoiling against the transition dipion and placing no restriction on the $J / \psi$ decay. Its uncertainty is dominated by a $2 \%$ systematic error on the produced number of $\psi(2 S)$. Other transitions to $J / \psi$, the exclusive modes through $\pi^{0} \pi^{0}, \eta$, $\pi^{0}$, and $\psi(2 S) \rightarrow \gamma \chi_{c J} \rightarrow \gamma \gamma J / \psi$, as well as the inclusive rate for $\psi(2 S) \rightarrow J / \psi+$ any, are all measured relative to the $\pi^{+} \pi^{-}$transition and use $J / \psi \rightarrow \ell^{+} \ell^{-}$, thereby reducing systematic error from the number of $\psi(2 S)$, which cancels in the ratios. These relative rates are measured with precision from $2-6 \%$. The absolute branching fractions determined from this analysis are shown in Table 37 which are higher values than most previous measurements. The ratio of $\pi^{0} \pi^{0}$ to $\pi^{+} \pi^{-}$transitions is consistent with one-half, the expectation from isospin invariance, which was not the case in some earlier analyses. See Sect. 3.1.5 for a discussion of the $\gamma \gamma J / \psi$ portion of this analysis, which addresses the product branching fractions $\mathcal{B}\left(\psi(2 S) \rightarrow \gamma \chi_{c J}\right) \times \mathcal{B}\left(\chi_{c J} \rightarrow \gamma J / \psi\right)$ and whether any nonresonant such final states are present. 
TABLE 37: Results from the branching fraction analyses for $\psi(2 S)$ and $\psi(3770)$ decays to $X J / \psi$ from CLEO $[446,500]$

\begin{tabular}{lcc}
\hline \hline Quantity & $\psi(2 S)(\%)$ & $\psi(3770)(\%)$ \\
\hline $\mathcal{B}\left(\pi^{+} \pi^{-} J / \psi\right)$ & $35.04 \pm 0.07 \pm 0.77$ & $0.189 \pm 0.020 \pm 0.020$ \\
$\mathcal{B}\left(\pi^{0} \pi^{0} J / \psi\right)$ & $17.69 \pm 0.08 \pm 0.53$ & $0.080 \pm 0.025 \pm 0.016$ \\
$\mathcal{B}(\eta J / \psi)$ & $3.43 \pm 0.04 \pm 0.09$ & $0.087 \pm 0.033 \pm 0.022$ \\
$\mathcal{B}\left(\pi^{0} J / \psi\right)$ & $0.133 \pm 0.008 \pm 0.003$ & $<0.028$ at $90 \% \mathrm{CL}$ \\
$\mathcal{B}(J / \psi+$ any $)$ & $62.54 \pm 0.16 \pm 1.55$ & - \\
$\frac{\mathcal{B}\left(\pi^{0} \pi^{0} J / \psi\right)}{\mathcal{B}\left(\pi^{+} \pi^{-} J / \psi\right)}$ & $50.47 \pm 1.04$ & $42 \pm 17$ \\
\hline \hline
\end{tabular}

\subsubsection{Observation of $\psi(2 S) \rightarrow \pi^{0} h_{c}(1 P)$}

The hadronic transition $\psi(2 S) \rightarrow \pi^{0} h_{c}(1 P)$ was first observed by CLEO for $h_{c}(1 P) \rightarrow \gamma \eta_{c}(1 S)$ [45, 46] and later seen for $h_{c}(1 P) \rightarrow 2\left(\pi^{+} \pi^{-}\right) \pi^{0}$ [536] (see Sect. 3.4.2). However, those analyses could only measure product branching fractions. BESIII [47] has used its much larger 106 million $\psi(2 S)$ sample to inclusively measure $\mathcal{B}\left(\psi(2 S) \rightarrow \pi^{0} h_{c}(1 P)\right)$ by observing a significant enhancement at the $h_{c}(1 P)$ in the mass recoiling against a reconstructed $\pi^{0}$, with none of the $h_{c}(1 P)$ decay products reconstructed. The result is $\mathcal{B}(\psi(2 S) \rightarrow$ $\left.\pi^{0} h_{c}(1 P)\right)=(8.4 \pm 1.3 \pm 1.0) \times 10^{-4}$, in agreement with the range predicted in [527] (see Table 36).

\subsubsection{Nonobservation of $\eta_{c}(2 S) \rightarrow \pi^{+} \pi^{-} \eta_{c}(1 S)$}

The historically uncooperative nature of the $\eta_{c}(2 S)$ has continued into the modern era, despite its discovery (Sect. 2.2.2). CLEO [68] failed not only to see $\eta_{c}(2 S)$ in radiative transitions (Sect. 3.1.4), but also failed to observe the expected dipion transition to $\eta_{c}(1 S)$, setting the upper limit

$$
\begin{aligned}
\mathcal{B}(\psi(2 S) \rightarrow & \left.\gamma \eta_{c}(2 S)\right) \times \mathcal{B}\left(\eta_{c}(2 S) \rightarrow \pi^{+} \pi^{-} \eta_{c}(1 S)\right) \\
& <1.7 \times 10^{-4} \text { at } 90 \% \mathrm{CL}
\end{aligned}
$$

\subsubsection{Observation of $\psi(3770) \rightarrow X J / \psi$}

Hadronic transitions of the $\psi(3770)$ to $J / \psi$ are sensitive to the relative sizes of the $2^{3} S_{1}$ and $1^{3} D_{1}$ admixture contained in the $\psi(3770)$, and thus, at least in principle, contain information about the nature of the $\psi(3770)$. BESII [537] first reported evidence for the transition $\psi(3770) \rightarrow \pi^{+} \pi^{-} J / \psi$ at $\sim 3 \sigma$ significance using approximately $2 \times 10^{5} \psi(3770)$ decays. CLEO [500], with roughly $2 \times 10^{6} \psi(3770)$ decays, later observed the $\pi^{+} \pi^{-}$transition at $11.6 \sigma$, and also found evidence for the $\pi^{0} \pi^{0} J / \psi(3.4 \sigma)$ and $\eta J / \psi(3.5 \sigma)$ transitions. The
CLEO results appear in Table 37. With more data, these hadronic transitions could be used to shed light on the $c \bar{c}$ purity of the $\psi(2 S)$ and $\psi(3770)$ states [538].

$$
\text { 3.3.6. Observation of } \Upsilon(2 S) \rightarrow \eta \Upsilon(1 S)
$$

The observation of $\Upsilon(2 S) \rightarrow \eta \Upsilon(1 S)$ using 9 million $\Upsilon(2 S)$ decays collected with the CLEO III detector represents the first observation of a quarkonium transition involving the spin flip of a bottom quark. The transition rate is sensitive to the chromomagnetic moment of the $b$ quark. Using three decay modes, $\eta \rightarrow \gamma \gamma$, $\pi^{+} \pi^{-} \pi^{0}$, and $3 \pi^{0}$, CLEO [539] observes $13.9_{-3.8}^{+4.5}$ events of the form $\Upsilon(2 S) \rightarrow \eta \Upsilon(1 S)$ (Fig. 44). Using the difference in log-likelihood for fits with and without signal, it was determined that this corresponds to a $5.3 \sigma$ observation. Correcting for acceptance and incorporating systematic errors, the observed number of events translates to a branching fraction of

$$
\begin{aligned}
& \mathcal{B}(\Upsilon(2 S) \rightarrow \eta \Upsilon(1 S))=\left(2.1_{-0.6}^{+0.7} \pm 0.3\right) \times 10^{-4} \\
& \mathcal{B}(\Upsilon(3 S) \rightarrow \eta \Upsilon(1 S))<1.8 \times 10^{-4} \text { at } 90 \% \mathrm{CL}
\end{aligned}
$$

The $2 S$ rate is a factor of four smaller than one would expect scaling from the analogous charmonium transition rate, $\psi(2 S) \rightarrow \eta J / \psi$. Similarly, the $3 S$ limit is already two times smaller than what one would expect from the same scaling. The interpretation of this pattern is still unclear.

\subsubsection{Observation of $\chi_{b 1,2}(2 P) \rightarrow \omega \Upsilon(1 S)$}

In 2003, CLEO [540] reported the observation of the decay chain $\Upsilon(3 S) \rightarrow \gamma \chi b J(2 P), \chi b J(2 P) \rightarrow \omega \Upsilon(1 S)$, for $J=1,2$, with $\omega \rightarrow \pi^{+} \pi^{-} \pi^{0}$ and $\Upsilon(1 S) \rightarrow \ell^{+} \ell^{-}$, for which there is marginal phase space available, and measured

$$
\begin{aligned}
& \mathcal{B}\left(\chi_{b 1} \rightarrow \omega \Upsilon(1 S)\right)=\left(1.63_{-0.31-0.15}^{+0.35+0.16}\right) \% \\
& \mathcal{B}\left(\chi_{b 2} \rightarrow \omega \Upsilon(1 S)\right)=\left(1.10_{-0.28}^{+0.32+0.11}\right) \%
\end{aligned}
$$

The relative rates are comparable, in agreement with Voloshin's prediction [541] on the basis of $S$-wave phase space factors and the $E 1 * E 1 * E 1$ gluon configurations expected by the multipole expansion.

\subsubsection{Dipion transitions from $\Upsilon(2 S, 3 S)$}

The double-hump dipion invariant mass distribution in $\Upsilon(3 S) \rightarrow \Upsilon(1 S) \pi \pi$ transitions has been thought to be at least puzzling, and frequently thought to be anomalous, indicative of either new physics or an intermediate scalar dipion resonance. However, a CLEO [542] analysis has offered an alternative to these characterizations; simply, that dipion-quarkonium dynamics expected to occur within QCD are responsible. Brown and 


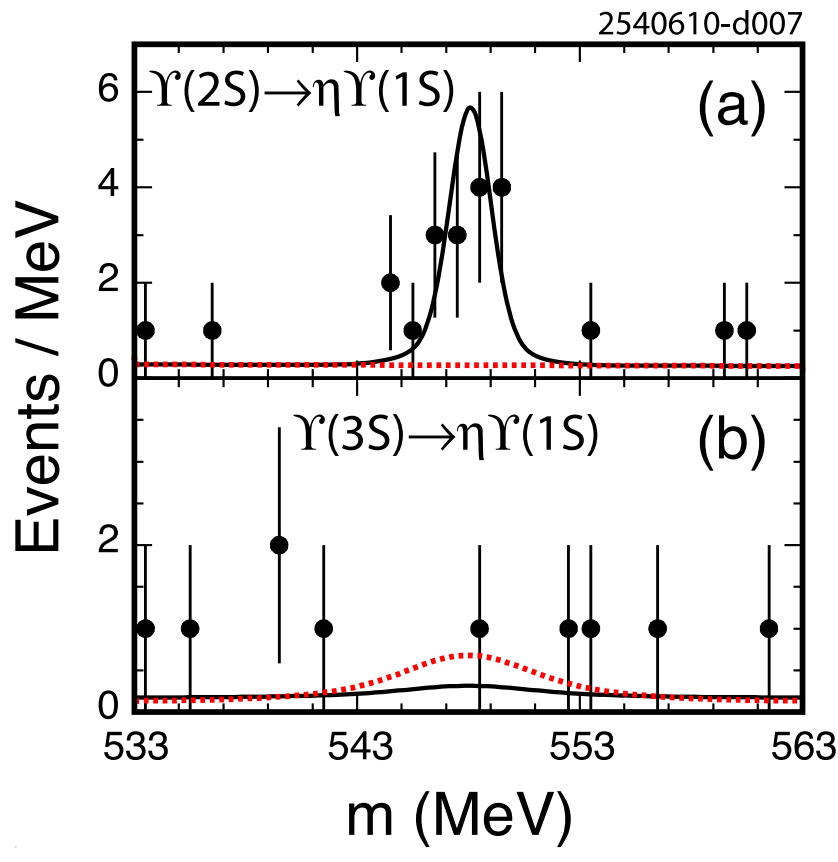

FIG. 44: From CLEO [539], the invariant mass of the $\eta$ candidate for (a) $\Upsilon(2 S) \rightarrow \eta \Upsilon(1 S)$ and (b) $\Upsilon(3 S) \rightarrow \eta \Upsilon(1 S)$ for $\eta$-mesons exclusively reconstructed in the three decay modes $\eta \rightarrow \gamma \gamma, \pi^{+} \pi^{-} \pi^{0}$, and $3 \pi^{0}$. In each case, the solid curve corresponds to the best fit to a flat background and signal. The dotted curve represents (a) the best-fit background, and (b) the 90\% CL upper limit for a signal. Adapted from [539] with kind permission, copyright (2008) The American Physical Society

Cahn [519] derived the general matrix element for dipion transitions from heavy vector quarkonia; it is constrained by PCAC and simplified by treating it as a multipole expansion $[514,516,543]$. This general matrix element has three terms: one proportional to $\left(m_{\pi \pi}^{2}-2 m_{\pi}^{2}\right)$, one proportional to the product $E_{1} E_{2}$ of the two pion energies in the parent rest frame, and a third which characterizes the transition requiring a $b$-quark spin-flip. Although the third chromomagnetic term is thought to be highly suppressed, and therefore ignorable, even the second term had generally been neglected prior to the CLEO analysis. Sensitivity to the second term, which is also proportional to $\cos \theta_{X}, \theta_{X}$ being the dipion helicity angle, is greatest at low $m_{\pi \pi}$, where experiments using only charged pions can only reconstruct very few events: soft charged particles curl up in the detector magnetic field before reaching any tracking chambers. The notable aspect of the CLEO analysis is not only that it fits for all three terms with complex form factors $\mathcal{A}, \mathcal{B}$, and $\mathcal{C}$, respectively, but also that it performs a simultaneous fit to dipion transitions through both charged and neutral pion pairs. The latter subset of the data enhances sensitivity to the $\cos \theta_{X}$ dependence because even when neutral pions are slow, frequently both $\pi^{0}$-decay photons can be reconstructed in the calorimeter.
Results are shown in Fig. 45 and Table 38, from which the following conclusions are drawn:

- The CLEO data in all three $\Upsilon$ dipion transitions can be represented well by a two-term form of the matrix element, provided the form factors are allowed to be different for all three decays.

- Sensitivity of the CLEO data to the chromomagnetic term, i.e., to a nonzero value of $\mathcal{C} / \mathcal{A}$, is small because the functional dependencies of the $\mathcal{B}$ and $\mathcal{C}$ terms are quite similar; a much larger dataset of $\Upsilon(3 S) \rightarrow \Upsilon(1 S) \pi \pi$ is required to probe this component. None of the three $\Upsilon$ dipion transitions require a nonzero $\mathcal{C} / \mathcal{A}$; i.e., there is no evidence yet that spin-flips play a significant role in these de-
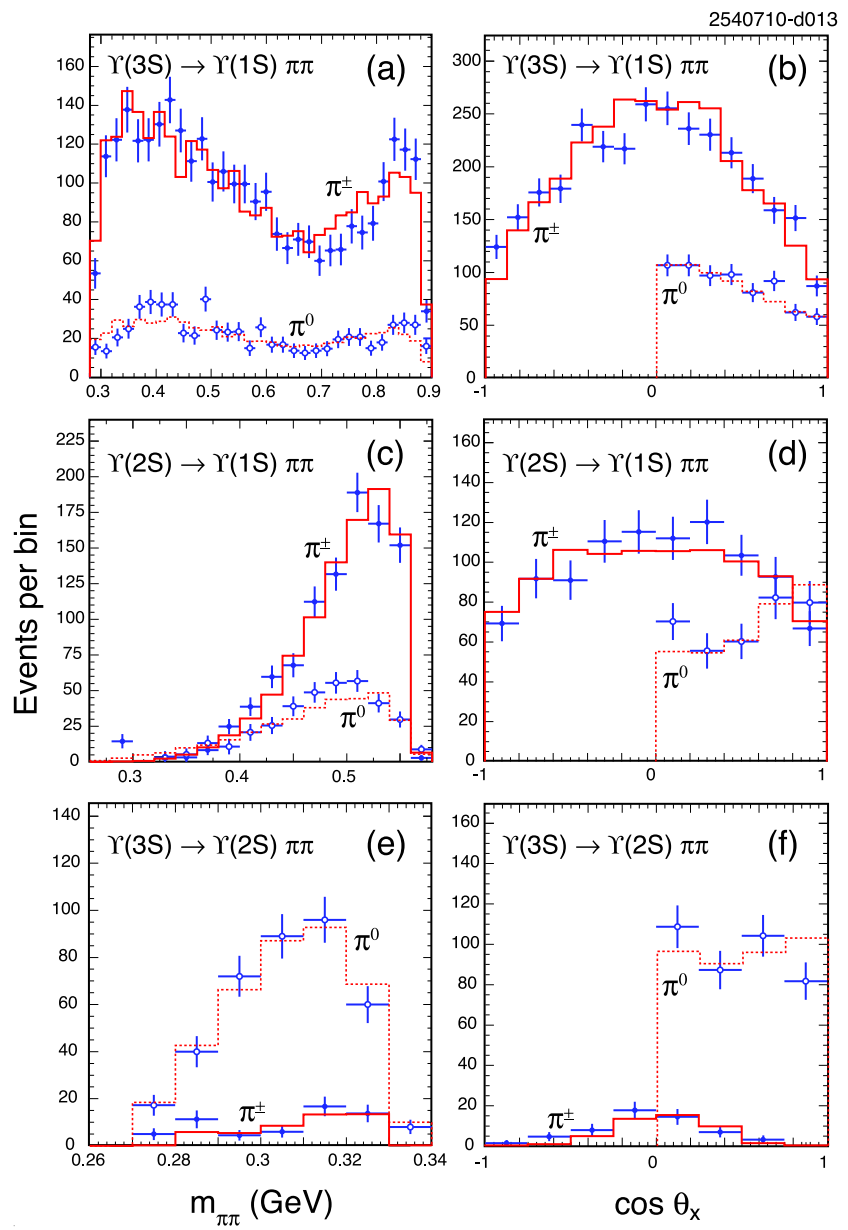

FIG. 45: From CLEO [542], (a), (c), (e) the dipion mass distributions and (b), (d), (f) dipion helicity angle $\cos \theta_{X}$ distributions for the transitions (a), (b) $\Upsilon(3 S) \rightarrow \Upsilon(1 S) \pi \pi$, (c), (d) $\Upsilon(2 S) \rightarrow \Upsilon(1 S) \pi \pi$, and (e), (f) $\Upsilon(3 S) \rightarrow \Upsilon(2 S) \pi \pi$, in which closed (open) circles represent the $\pi^{+} \pi^{-}\left(\pi^{0} \pi^{0}\right)$ data, and solid (dotted) line histogram the MC simulation for $\pi^{+} \pi^{-}\left(\pi^{0} \pi^{0}\right)$ transitions generated with the best-fit parameters in Table 38. Adapted from [542] with kind permission, copyright (2007) The American Physical Society 
TABLE 38: Results from fits to the CLEO [542] $\Upsilon(m S) \rightarrow$ $\pi \pi \Upsilon(n S),(m, n)=(3,2),(3,1),(2,1)$ transitions data for the complex form factors $(\mathcal{A}, \mathcal{B}, \mathcal{C})$ of the three terms, and the associated phase angle, $\delta_{\mathcal{B} \mathcal{A}}$. The phase angles are quoted in degrees, and have a two-fold ambiguity of reflection in the real axis. The first three fits constrain the chromomagnetic coefficient to be zero $(\mathcal{C} \equiv 0)$, but $\mathcal{C}$ floats in the fourth fit. The operators $\mathcal{R}$ and $\mathcal{I}$ denote real and imaginary parts, respectively

\begin{tabular}{clc}
\hline \hline Transition & Quantity & Value \\
\hline$\Upsilon(3 S) \rightarrow \Upsilon(1 S) \pi \pi$ & $\Re(\mathcal{B} / \mathcal{A})$ & $-2.52 \pm 0.04$ \\
& $\Im(\mathcal{B} / \mathcal{A})$ & $\pm 1.19 \pm 0.06$ \\
$(\mathcal{C} \equiv 0)$ & $|\mathcal{B} / \mathcal{A}|$ & $2.79 \pm 0.05$ \\
& $\delta_{\mathcal{B} \mathcal{A}\left(^{\circ}\right)}$ & $155(205) \pm 2$ \\
$\Upsilon(2 S) \rightarrow \Upsilon(1 S) \pi \pi$ & $\Re(\mathcal{B} / \mathcal{A})$ & $-0.75 \pm 0.15$ \\
& $\Im(\mathcal{B} / \mathcal{A})$ & $0.00 \pm 0.11$ \\
$(\mathcal{C} \equiv 0)$ & $|\mathcal{B} / \mathcal{A}|$ & $0.75 \pm 0.15$ \\
& $\delta_{\mathcal{B} \mathcal{A}\left(^{\circ}\right)}$ & $180 \pm 9$ \\
$\Upsilon(3 S) \rightarrow \Upsilon(2 S) \pi \pi$ & $\Re(\mathcal{B} / \mathcal{A})$ & $-0.40 \pm 0.32$ \\
$(\mathcal{C} \equiv 0)$ & $\Im(\mathcal{B} / \mathcal{A})$ & $0.00 \pm 1.1$ \\
$\Upsilon(3 S) \rightarrow \Upsilon(1 S) \pi \pi$ & $|\mathcal{B} / \mathcal{A}|$ & $2.89 \pm 0.25$ \\
$(\mathcal{C}$ floats $)$ & $|\mathcal{C} / \mathcal{A}|$ & $0.45 \pm 0.40$ \\
\hline \hline
\end{tabular}

cays. In the case of $\Upsilon(3 S) \rightarrow \Upsilon(1 S) \pi \pi$, where a slight sensitivity to this term exists, an upper limit of $|\mathcal{C} / \mathcal{A}|<1.09$ at $90 \% \mathrm{CL}$ is given.

- The dynamics in $\Upsilon(2 S) \rightarrow \Upsilon(1 S) \pi \pi$ are reproduced by the fits, but only if nonzero values of $\mathcal{B} / \mathcal{A}$ are allowed. The data in this transition are described well without any complex component in $\mathcal{B} / \mathcal{A}$.

- The two-peak structure in $\Upsilon(3 S) \rightarrow \Upsilon(1 S) \pi \pi$ is reproduced without any new physics or any intermediate dipion resonances, $\mathcal{B} / \mathcal{A}$ is found to have a significant complex component, and the dipionhelicity form factor is nearly three times larger than that of the dipion-mass-dependent term.

Dubynskiy and Voloshin [520] comment further, on both the formalism and prospects for learning more from such decays. They cast doubt upon the possibility that $\mathcal{B}$ can have have a complex component when $\mathcal{C} \equiv 0$, on general principles; conclude that $\mathcal{B}$ and $\mathcal{C}$ terms are degenerate unless $\mathcal{C}$ is set to zero; and suggest resolving the $\mathcal{B}-\mathcal{C}$ terms' degeneracy by including initial- and final-state polarization information in fits to experimental data.

CLEO [544] used the matrix elements determined above to obtain correct efficiencies in the first new measurement of these transition branching ratios in a decade. $B A B A R$ [545] followed suit soon after, using radiative returns from $e^{+} e^{-}$collisions at $10.58 \mathrm{GeV}$ and the nearby continuum to the narrow $\Upsilon$ states as a source. Both groups used $\Upsilon \rightarrow \ell^{+} \ell^{-}$decays; BABAR used only charged, fully-reconstructed dipions, whereas CLEO used both charged and neutral, except for $\Upsilon(3 S) \rightarrow \Upsilon(2 S) \pi \pi$, where the low efficiency for charged track reconstruction made the charged mode too difficult to normalize precisely. CLEO gained some statistical power by performing both exclusive $\left(\Upsilon \rightarrow \ell^{+} \ell^{-}\right)$and inclusive $(\Upsilon \rightarrow$ any) versions for each transition; in the latter case the signal was obtained by a fit to the invariant mass recoiling against the dipion for a smooth background and signal term peaking at the appropriate $\Upsilon$ mass. $B A B A R$ extracted signals by fitting distributions of $\Delta m \equiv m\left(\pi^{+} \pi^{-} \ell^{+} \ell^{-}\right)-m\left(\ell^{+} \ell^{-}\right)$for a smooth background and signal at the mass difference appropriate for the desired parent $\Upsilon$, after cutting on $m\left(\ell^{+} \ell^{-}\right)$around the mass appropriate for the desired daughter $\Upsilon$. The combination of CLEO and BABAR branching fractions, as shown in Table 39, which are consistent with each other and previous measurements, made significant improvements to the branching fraction precisions and dominate the PDG10 [447] world averages shown.

TABLE 39: Branching fractions for bottomonium dipion transitions $\Upsilon(n S) \rightarrow \pi \pi \Upsilon(m S)$ for $n=2,3$ and $m<n$, as compiled by the Particle Data Group as indicated and as measured by CLEO and BABAR; the PDG10 [447] (PDG08 [18]) numbers include (do not include) the CLEO [544] and BABAR [545] results

\begin{tabular}{lcc}
\hline \hline Transition & $\mathcal{B}(\%)$ & Source \\
\hline$\Upsilon(2 S) \rightarrow \pi^{+} \pi^{-} \Upsilon(1 S)$ & $18.8 \pm 0.6$ & PDG08 [18] \\
& $18.02 \pm 0.02 \pm 0.61$ & CLEO [544] \\
& $17.22 \pm 0.17 \pm 0.75$ & BABAR [545] \\
$\Upsilon(2 S) \rightarrow \pi^{0} \pi^{0} \Upsilon(1 S)$ & $9.0 \pm 0.8$ & PDG08 [18] \\
& $8.43 \pm 0.16 \pm 0.42$ & CLEO [544] \\
$\Upsilon(3 S) \rightarrow \pi^{+} \pi^{-} \Upsilon(1 S)$ & $8.6 \pm 0.4$ & PDG10 [447] \\
& $4.46 \pm 0.01 \pm 0.13$ & CLEO [544] \\
& $4.17 \pm 0.06 \pm 0.19$ & BABAR [545] \\
& $4.40 \pm 0.10$ & PDG10 [447] \\
$\Upsilon(3 S) \rightarrow \pi^{0} \pi^{0} \Upsilon(1 S)$ & $2.06 \pm 0.28$ & PDG08 [18] \\
& $2.24 \pm 0.09 \pm 0.11$ & CLEO [544] \\
& $2.20 \pm 0.13$ & PDG10 [447] \\
$\Upsilon(3 S) \rightarrow \pi^{+} \pi^{-} \Upsilon(2 S)$ & $2.8 \pm 0.6$ & PDG08 [18] \\
& $2.40 \pm 0.10 \pm 0.26$ & BABAR [545] \\
& $2.45 \pm 0.23$ & PDG10 [447] \\
& $2.00 \pm 0.32$ & PDG08 [18] \\
& $1.82 \pm 0.09 \pm 0.12$ & CLEO [544] \\
& $1.85 \pm 0.14$ & PDG10 [447] \\
\hline \hline
\end{tabular}




\subsubsection{Observation of $\Upsilon\left(1^{3} D_{J}\right) \rightarrow \pi^{+} \pi^{-} \Upsilon(1 S)$}

The $\Upsilon\left(1^{3} D_{J}\right) \rightarrow \pi^{+} \pi^{-} \Upsilon(1 S)$ measurements from BABAR [63] (see Sect. 2.2.7) represent the only available data on hadronic transitions of the $\Upsilon\left(1^{3} D_{J}\right)$ states. Partial rates are expected to be independent of $J$ [526]. Using predicted [524] branching fractions for $\Upsilon(3 S) \rightarrow$ $\gamma_{\chi_{b J^{\prime}}}(2 P)$ and $\chi_{b J^{\prime}}(2 P) \rightarrow \gamma \Upsilon\left(1^{3} D_{J}\right)$, BABAR quotes

$$
\begin{aligned}
\mathcal{B}\left(\Upsilon\left(1^{3} D_{1}\right) \rightarrow\right. & \left.\pi^{+} \pi^{-} \Upsilon(1 S)\right)= \\
& \left(0.42_{-0.23}^{+0.27} \pm 0.10\right) \%(<0.82 \%), \\
\mathcal{B}\left(\Upsilon\left(1^{3} D_{2}\right) \rightarrow\right. & \left.\pi^{+} \pi^{-} \Upsilon(1 S)\right)= \\
& \left(0.66_{-0.14}^{+0.15} \pm 0.06\right) \%, \text { and } \\
\mathcal{B}\left(\Upsilon\left(1^{3} D_{3}\right) \rightarrow\right. & \left.\pi^{+} \pi^{-} \Upsilon(1 S)\right)= \\
& \left(0.29_{-0.18}^{+0.22} \pm 0.06\right) \%(<0.62 \%),
\end{aligned}
$$

where upper limits are given at 90\% CL and include systematic uncertainties. This hadronic $\Upsilon\left(1^{3} D_{2}\right)$ transition provides an important benchmark for comparing various theoretical predictions for partial rates [524, 526, 529]. Furthermore, comparing with the observed $\psi(3770) \rightarrow$ $\pi^{+} \pi^{-} J / \psi$ transition may give insight into threshold effects in the $\psi(3770)$ state.

\subsubsection{Dipion and $\eta$ transitions from $\Upsilon(4 S)$}

Even above open-bottom threshold, the $b \bar{b}$ vector bound state undergoes hadronic transitions, as first measured by BABAR [546], which was later updated [545]. The latter analysis is done in a manner similar to that described in Sect. 3.3.8, using mass-difference windows around $\Delta m=m[\Upsilon(4 S)]-m[\Upsilon(j S)]$, with $j=1,2$. Belle [547] reported a similar analysis, but only on $\Upsilon(4 S) \rightarrow \pi^{+} \pi^{-} \Upsilon(1 S)$. The two resulting branching fractions for the latter transition are consistent with one other; PDG10 reports (averaged for $\Upsilon(4 S) \rightarrow$ $\pi^{+} \pi^{-} \Upsilon(1 S)$ ) branching fractions

$$
\begin{aligned}
& \mathcal{B}\left(\Upsilon(4 S) \rightarrow \pi^{+} \pi^{-} \Upsilon(1 S)\right)= \\
& (0.810 \pm 0.06) \times 10^{-4} \\
& \mathcal{B}\left(\Upsilon(4 S) \rightarrow \pi^{+} \pi^{-} \Upsilon(2 S)\right)= \\
& \quad(0.86 \pm 0.11 \pm 0.07) \times 10^{-4} \\
& \frac{\mathcal{B}\left(\Upsilon(4 S) \rightarrow \pi^{+} \pi^{-} \Upsilon(2 S)\right)}{\mathcal{B}\left(\Upsilon(4 S) \rightarrow \pi^{+} \pi^{-} \Upsilon(1 S)\right)}=1.16 \pm 0.16 \pm 0.14
\end{aligned}
$$

Of particular note is that while the dipion mass spectrum for $\Upsilon(4 S) \rightarrow \pi^{+} \pi^{-} \Upsilon(1 S)$ has a typical spectrum with a single peak, that of $\Upsilon(4 S) \rightarrow \pi^{+} \pi^{-} \Upsilon(2 S)$ appears to have a double-peak structure like $\Upsilon(3 S) \rightarrow \pi \pi \Upsilon(1 S)$, as seen in Fig. 46.

In the same analysis, BABAR [546] sought $\eta$ transitions in the $\Upsilon$ system as well, via $\eta \rightarrow \pi^{+} \pi^{-} \pi^{0}$. While no evidence is found for such transitions from $\Upsilon(2 S)$ or $\Upsilon(3 S)$ (consistent with the CLEO results discussed in Sect. 3.3.6), a quite significant signal was observed for $\eta \Upsilon(1 S)$ final states in the $\Upsilon(4 S)$ data sample, which, if attributed fully to resonant production, corresponds to

$$
\begin{gathered}
\mathcal{B}(\Upsilon(4 S) \rightarrow \eta \Upsilon(1 S))=(1.96 \pm 0.06 \pm 0.09) \times 10^{-4} \\
\frac{\mathcal{B}(\Upsilon(4 S) \rightarrow \eta \Upsilon(1 S))}{\mathcal{B}\left(\Upsilon(4 S) \rightarrow \pi^{+} \pi^{-} \Upsilon(1 S)\right)}=2.41 \pm 0.40 \pm 0.12
\end{gathered}
$$

The BABAR continuum sample taken just below $\Upsilon(4 S)$ is only a small fraction of that taken on- $\Upsilon(4 S)$, so even though no $\eta \Upsilon(1 S)$ are observed in that sample, the possibility that the observed events are attributable to $e^{+} e^{-} \rightarrow \eta \Upsilon(1 S)$ can only be excluded at the $2.7 \sigma$ level.

\subsubsection{Dipion transitions near $\Upsilon(5 S)$}

As described in Sect. 2.3.2, Belle [117] reported first observation of apparent dipion transitions from the $\Upsilon(5 S)$ to the lower mass narrow $\Upsilon$ states. The caveat "apparent" is applied because a later analysis, also by Belle [37], and also described in Sect. 2.3.2, suggests that these transitions may originate not from the enhancement in the hadronic cross section from $e^{+} e^{-}$collisions near $\sqrt{s} \approx 10.87 \mathrm{GeV}$ known as " $(5 S)$ ", but from a separate new state $Y_{b}$ close by in mass, much as $Y(4260)$ was observed via dipion transitions to lower-mass charmonium.

If interpreted as $\Upsilon(5 S)$ transitions, the measured dipion decay widths are more than two orders of magnitude larger than those in the similar $\Upsilon(4 S) \rightarrow \Upsilon(1 S) \pi^{+} \pi^{-}[545,547]$ (see Sect. 3.3.10), and $\Upsilon(2 S, 3 S) \rightarrow \Upsilon(1 S) \pi^{+} \pi^{-}$(see Sect. 3.3.8) transitions. The reason for such large differences is not clear. Two theoretical ideas have been proposed. The first approach [548] assumes that the bottomonium transitions

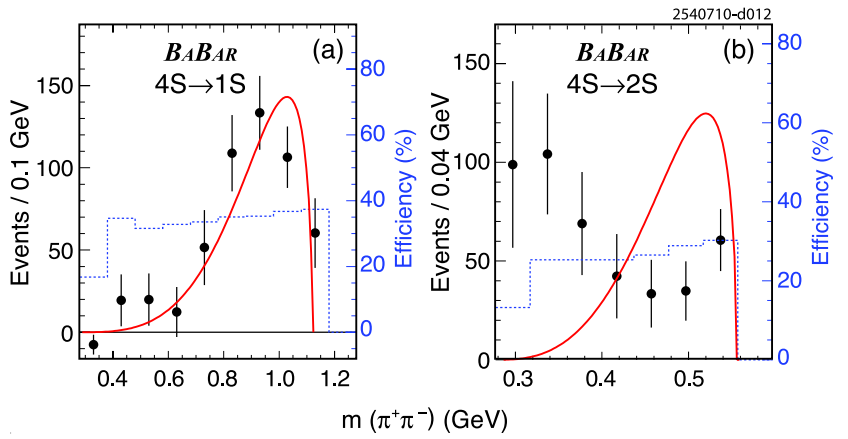

FIG. 46: From BABAR [546], efficiency-corrected dipion mass distributions for (a) $\Upsilon(4 S) \rightarrow \pi^{+} \pi^{-} \Upsilon(1 S)$, and (b) $\Upsilon(4 S) \rightarrow$ $\pi^{+} \pi^{-} \Upsilon(2 S)$, in which solid circles denote data, dotted $h i-$ tograms denote reconstruction and event selection efficiencies, which follow the scales on the right-side axes, and the solid curves represent theoretical predictions [546]. Experimental resolution on $m_{\pi^{+} \pi^{-}}$is less than $5 \mathrm{MeV}$. Adapted from [546] with kind permission, copyright (2006) The American Physical Society 
with two-pion emission come through intermediate virtual $B \bar{B} \pi(\pi)$ formation, followed by the recombination of the $B$ pair into the $\Upsilon$. The difference between the $\Upsilon(5 S)$ and $\Upsilon(4 S)$ decays results from a large imaginary part in the $\Upsilon(5 S)$ decay amplitude appearing due to the positive difference between the $\Upsilon(5 S)$ mass and the sum of the masses of the $B \bar{B} \pi \pi$ system. A similar approach was also used [549, 550], in which specific decay parameters were predicted with good accuracy. An alternative theoretical idea was proposed [388], in which the large $\Upsilon(5 S) \rightarrow \Upsilon(1 S) \pi^{+} \pi^{-}$decay width was explained as a possible indication of the production of a $b \bar{b} g$ hybrid state $Y_{b}$ with a mass close to the mass of the $\Upsilon(5 S)$ resonance.

\subsubsection{Observation of $\chi_{b J}(2 P) \rightarrow \pi \pi \chi_{b J}(1 P)$}

The decay $\chi_{b J}(2 P) \rightarrow \pi \pi \chi_{b J}(1 P)(J=1,2)$ was observed by CLEO [523] using 6 million decays of the $\Upsilon(3 S)$. This is the only observed hadronic transition from the $\chi_{b J}(n P)$ states aside from the surprisingly large $\chi_{b J}(2 P) \rightarrow \omega \Upsilon(1 S)$; as such, it provides an important benchmark. The following decay chain was used in the search: $\Upsilon(3 S) \rightarrow \gamma \chi_{b J^{\prime}}(2 P) ; \chi_{b J^{\prime}}(2 P) \rightarrow \pi \pi \chi_{b J}(1 P)$; $\chi_{b J}(1 P) \rightarrow \gamma \Upsilon(1 S) ; \Upsilon(1 S) \rightarrow \ell^{+} \ell^{-}$. Both charged and neutral pion pairs were sought, with results combined assuming isospin invariance. The data were not adequate to distinguish between allowed values of $J^{\prime}$ and $J$; hence it was assumed that $J^{\prime}=J$. The $J=0$ transition was inaccessible due to the smallness of the branching fractions in the decay chain. Assuming the $J=2$ and $J=1$ transitions have the same partial width, it was found that

$$
\Gamma\left(\chi_{b}(2 P) \rightarrow \pi \pi \chi_{b}(1 P)=0.83 \pm 0.22 \pm 0.21 \mathrm{keV} .\right.
$$

This rate is consistent with theoretical expectations [517].

\subsection{Hadronic decays}

In general, the nonrelativistic quark model does a remarkable job also for decays of the lowest (below $\bar{D} D$ threshold) charmonia - see, e.g., [31]. However, there are some striking discrepancies where additional experimental as well as theoretical work is necessary, as will be outlined below - see, e.g., Sect. 3.4.1. Improved data, e.g., sensitive to lineshapes due to improved resolution and statistics, as well as data on additional transitions, should shed important light on the structure of the light charmonia.

As described at the end of Sect. 3.3.1, for some transitions heavy meson loops might play a significant role. The natural question that arises asks what their influence on decays could be. Here, unfortunately, the NREFT described above is not applicable anymore, for the momenta of the final state particles as well as the intermediate heavy meson velocities get too large for a controlled expansion. However, those studies can be performed within phenomenological models [440, 551-553].

\subsubsection{The $12 \%$ rule and $\rho \pi$ puzzle}

From perturbative QCD (pQCD), it is expected that both $J / \psi$ and $\psi(2 S)$ decay into any exclusive lighthadron final state with a width proportional to the square of the wave function at the origin $[554,555]$. This yields the pQCD " $12 \%$ rule",

$$
\begin{aligned}
Q_{h} & \equiv \frac{\mathcal{B}(\psi(2 S) \rightarrow h)}{\mathcal{B}(J / \psi \rightarrow h)} \\
& =\frac{\mathcal{B}\left(\psi(2 S) \rightarrow e^{+} e^{-}\right)}{\mathcal{B}\left(J / \psi \rightarrow e^{+} e^{-}\right)} \approx 12 \%
\end{aligned}
$$

A large violation of this rule was first observed in decays to $\rho \pi$ and $K^{*+} K^{-}+$c.c. by Mark II [556], and became known as the $\rho \pi$ puzzle. Since then, many two-body decay modes of the $\psi(2 S)$ (and some multibody ones) have been measured by BES [557-563] and CLEO [564]; some decays obey the rule while others violate it to varying degrees.

The $\rho \pi$ mode is essential for this study - the recent measurements, together with the old information, show us a new picture of the charmonium decay dynamics [565]. With a weighted average of $\mathcal{B}(J / \psi \rightarrow$ $\left.\pi^{+} \pi^{-} \pi^{0}\right)=(2.00 \pm 0.09) \%$ from the existing measurements, and an estimation of

$$
\frac{\mathcal{B}(J / \psi \rightarrow \rho \pi)}{\mathcal{B}\left(J / \psi \rightarrow \pi^{+} \pi^{-} \pi^{0}\right)}=1.17 \times(1 \pm 10 \%)
$$

using the information given in [566], one gets $\mathcal{B}(J / \psi \rightarrow$ $\rho \pi)=(2.34 \pm 0.26) \%$. This is substantially larger than the world average listed by PDG08 [18], which is (1.69 \pm $0.15) \%$, from a simple average of many measurements. The branching fraction of $\psi(2 S) \rightarrow \pi^{+} \pi^{-} \pi^{0}$ is measured to be $(18.1 \pm 1.8 \pm 1.9) \times 10^{-5}$ and $\left(18.8_{-1.5}^{+1.6} \pm 1.9\right) \times 10^{-5}$ at BESII [567] and CLEO [564], respectively. To extract the $\rho \pi$ component, however, the experiments make different choices, which in turn lead to different answers. BESII uses a partial wave analysis (PWA), while CLEO counts the number of events by applying a $\rho$ mass cut. The branching fraction from BESII is $(5.1 \pm 0.7 \pm 1.1) \times 10^{-5}$, while that from CLEO is $\left(2.4_{-0.7}^{+0.8} \pm 0.2\right) \times 10^{-5}$. If we take a weighted average and inflate the resulting uncertainty with a PDG-like scale factor accounting for the disagreement, we obtain $\mathcal{B}(\psi(2 S) \rightarrow \rho \pi)=(3.1 \pm 1.2) \times 10^{-5}$. With the results from above, one gets

$$
Q_{\rho \pi}=\frac{\mathcal{B}(\psi(2 S) \rightarrow \rho \pi)}{\mathcal{B}(J / \psi \rightarrow \rho \pi)}=(0.13 \pm 0.05) \%
$$

The suppression compared to the $12 \%$ rule is about two orders of magnitude.

There are enough measurements of $\psi(2 S)$ and $J / \psi$ decays for an extensive study of the " $12 \%$ rule" [18, 557564, 568-570], among which the Vector-Pseudoscalar (VP) modes, like the $\rho \pi$, have been measured with the highest priority. The ratios of the branching fractions are generally suppressed relative to the $12 \%$ rule for 
the non-isospin-violating VP and Vector-Tensor (VT) modes (i.e., excluding modes like $\omega \pi^{0}$ and $\rho^{0} \eta$ ), while Pseudoscalar-Pseudoscalar (PP) modes are enhanced. The multihadron modes and the baryon-antibaryon modes cannot be simply characterized, as some are enhanced, some are suppressed, and some match the expectation of the rule. Theoretical models, developed for interpreting specific modes, have not yet provided a solution for all of the measured channels. For a recent review, see $[1,571]$.

\subsubsection{Observation of $h_{c}(1 P) \rightarrow 2\left(\pi^{+} \pi^{-}\right) \pi^{0}$}

CLEO reported the first evidence for an exclusive hadronic decay mode of the $h_{c}(1 P)$ [536], previously only seen through its radiative transition to the $\eta_{c}(1 S)$. Using $25.7 \times 10^{6} \psi(2 S)$ decays, CLEO performed a search for hadronic decays of the $h_{c}(1 P)$ in the channels $\psi(2 S) \rightarrow$ $\pi^{0} h_{c}(1 P) ; h_{c}(1 P) \rightarrow n\left(\pi^{+} \pi^{-}\right) \pi^{0}$, where $n=1,2,3$. Upper limits were set for the $3 \pi$ and $7 \pi$ decay modes, but evidence for a signal was found in the $5 \pi$ channel with a significance of $4.4 \sigma$. The $5 \pi$ mass distribution from data is shown in Fig. 47(a); the corresponding spectrum from background Monte Carlo is shown in Fig. 47(b). The measured mass is consistent with previous measurements. The measured product branching fraction is

$$
\begin{gathered}
\mathcal{B}\left(\psi(2 S) \rightarrow \pi^{0} h_{c}(1 P)\right) \times \mathcal{B}\left(h_{c}(1 P) \rightarrow 2\left(\pi^{+} \pi^{-}\right) \pi^{0}\right)= \\
\left(1.88_{-0.45-0.30}^{+0.48+0.47}\right) \times 10^{-5}
\end{gathered}
$$

This value is approximately $5 \%$ of that given in Eq. (100), indicating the total hadronic decay width of the $h_{c}(1 P)$ is likely of the same order as its radiative transition width to the $\eta_{c}(1 S)$.

\subsection{3. $\chi_{c J}(1 P)$ hadronic decays}

Precision in study of hadronic $\chi_{c J}(1 P)$ decays continues to improve with larger datasets, and is beginning to approach that achieved for $J / \psi$ and $\psi(2 S)$. Decays of the $P$-wave states provide information complementary to that from the $S$-wave states, which probe short-range processes. At CLEO and BESII the $P$-wave states are accessed through the predominant E1 radiative transitions $\psi(2 S) \rightarrow \gamma \chi_{c J}(1 P)$. Recent measurements have also come from Belle through the process $\gamma \gamma \rightarrow \chi_{c 0,2}(1 P)$.

A large number of different hadronic decay modes of the $\chi_{c J}(1 P)$ states have recently been measured, many for the first time. These include two-meson decays at BESII [572], CLEO [573], and Belle [485, 487-489, 491]; two-baryon decays at CLEO [574]; three-meson decays at CLEO [575] and BESII [576]; four-meson decays at BESII [577-580], CLEO [581], and BELLE [442]; as well as others like $4 \pi p \bar{p}$ and $p \bar{n} \pi^{0}$ at BESII [563, 582].

More work is required on the theoretical front to understand the wide variety of hadronic $\chi_{c J}(1 P)$ decays now experimentally observed. There are indications that the pattern of decays may require the introduction of a color octet mechanism that includes contributions from the subprocess $c \bar{c} g \rightarrow q \bar{q}$ [456]. Hadronic decays of the $\chi_{c J}(1 P)$ may also provide insight into the relative contributions of singly and doubly-OZI violating processes [583].

\subsubsection{Non-D $\bar{D} \psi(3770)$ hadronic decays}

Experimental measurements of the branching fractions for $\psi(3770) \rightarrow$ non- $D \bar{D}$ provide important information about the nature of the $\psi(3770)$ resonance. While the $\psi(3770)$ is thought to be dominantly the $1^{3} D_{1}$ state of charmonium, its dilepton width indicates it should also contain a substantial $n^{3} S_{1}$ component. The size of this component is related to the non- $D \bar{D}$-width of the $\psi(3770)[425,452]$. Several measurements of the cross

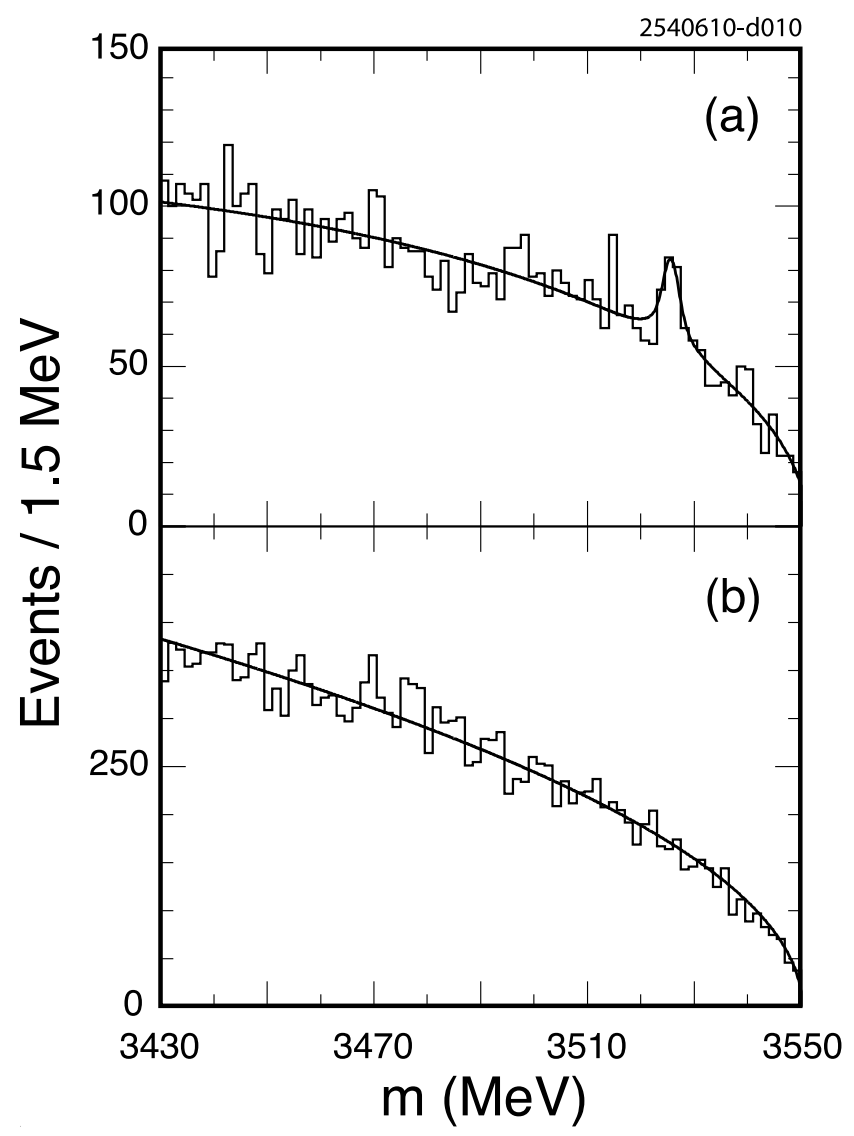

FIG. 47: From CLEO [536], the $2\left(\pi^{+} \pi^{-}\right) \pi^{0}$ mass distribution showing evidence for the $\psi(2 S) \rightarrow \pi^{0} h_{c}(1 P)$ followed by $h_{c}(1 P) \rightarrow 2\left(\pi^{+} \pi^{-}\right) \pi^{0}$. Solid line histograms show (a) data and (b) Monte Carlo simulation of backgrounds. Solid curves show the results of fits to the respective histograms for a smooth background and $h_{c}(1 P)$ signal. Adapted from [536] with kind permission, copyright (2009) The American Physical Society 
sections for $\psi(3770) \rightarrow$ non- $D \bar{D}$ final states have recently been made, but there is a disagreement between those from BES [502, 503, 584, 585] and that of CLEO [508].

The most recent and precise result from BES [503] is $\mathcal{B}(\psi(3770) \rightarrow$ non- $D \bar{D})=(15.1 \pm 5.6 \pm 1.8) \%$. This was obtained by counting inclusively selected hadronic events with a charged kaon of energy 1.15-2.00 GeV, a range which excludes $D \bar{D}$ events, in the range of centerof-mass energies 3.650-3.872 GeV, and correcting the observed number for efficiency and integrated luminosity at each energy point. These cross sections were then fit for a smooth background and the expected lineshape of $\psi(3770)$ with floating normalization. Figure 48 shows these measured cross sections versus the center-of-mass energy together with the best fit, which indicates evidence for non- $D \bar{D}$ decays. Combining this result with another one, also by BES [585], which measured cross sections at just two points $(3.773 \mathrm{GeV}$ and $3.650 \mathrm{GeV})$ gives a $4.8 \sigma$ signal significance for $\psi(3770) \rightarrow$ non- $D \bar{D}$ decays. Other measurements from BES - which include variations on the above-described techniques - are listed in Table 40.

CLEO [508] measured the cross section for $\psi(3770) \rightarrow$ non- $D \bar{D}$ by comparing the cross sections for $\psi(3770) \rightarrow$ hadrons to that measured for $\psi(3770) \rightarrow D \bar{D}$ [30]. The

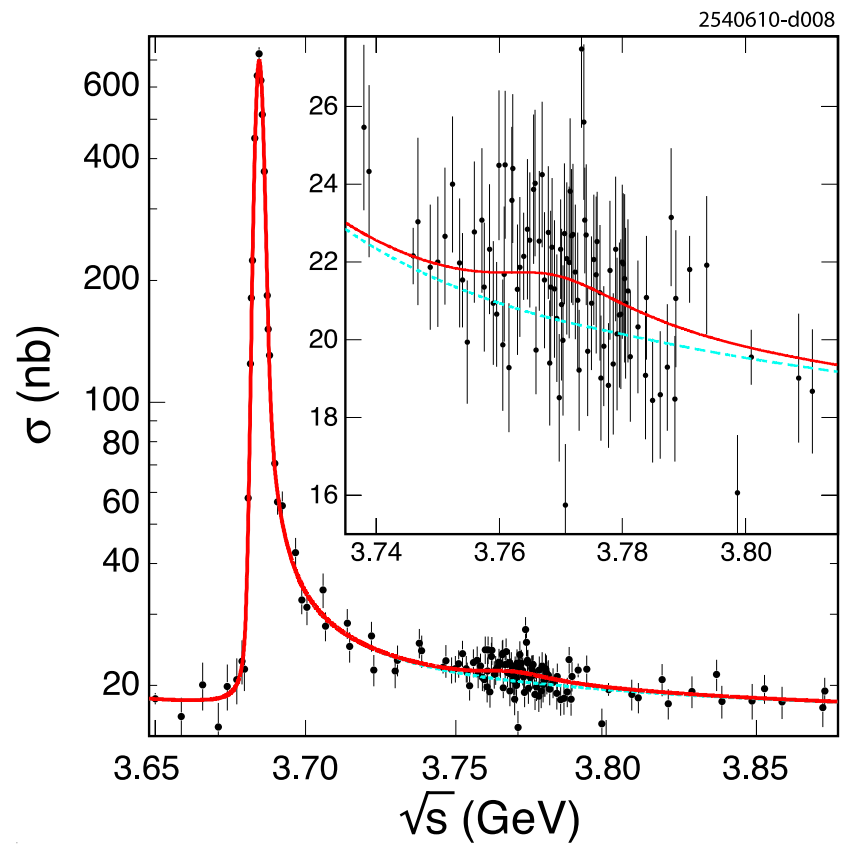

FIG. 48: From BES [503], the hadronic cross section versus $\sqrt{s}$, extracted from counting inclusively selected hadronic events with a charged kaon of energy 1.15-2.00 GeV, a range which excludes $D \bar{D}$ events. Solid circles represent data, the dashed curve represents the contributions from $J / \psi, \psi(2 S)$, and continuum hadron production, and the solid curve the best fit to the data of expected background plus a floating $\psi(3770) \rightarrow$ non- $D \bar{D}$ component (see text). Adapted from [503] with kind permission, copyright (2008) Elsevier
TABLE 40: Measurements of branching fractions for $\psi(3770) \rightarrow D \bar{D}$ and non- $D \bar{D}$ at BES, CLEO, and as averaged by PDG10. Rates for $D^{0} \bar{D}^{0}$ and $D^{+} D^{-}$are constrained to sum to that of $D \bar{D}$, and the rates for $D \bar{D}$ and non- $D \bar{D}$ are constrained to sum to unity. Hence within each set of measurements, uncertainties are highly correlated

\begin{tabular}{lcc}
\hline \hline Final state & $\mathcal{B}(\%)$ & Source \\
\hline$D^{0} \bar{D}^{0}$ & $49.9 \pm 1.3 \pm 3.8$ & BES [502] \\
$D^{+} D^{-}$ & $35.7 \pm 1.1 \pm 3.4$ & BES [502] \\
$D \bar{D}$ & $85.5 \pm 1.7 \pm 5.8$ & BES [502] \\
non- $D \bar{D}$ & $14.5 \pm 1.7 \pm 5.8$ & BES [502] \\
$D^{0} \overline{D^{0}}$ & $46.7 \pm 4.7 \pm 2.3$ & BES [584] \\
$D^{+} D^{-}$ & $36.9 \pm 3.7 \pm 2.8$ & BES [584] \\
$D \bar{D}$ & $83.6 \pm 7.3 \pm 4.2$ & BES [584] \\
non- $D \bar{D}$ & $16.4 \pm 7.3 \pm 4.2$ & BES [584] \\
$D \bar{D}$ & $86.6 \pm 5.0 \pm 3.6$ & BES [503] \\
non- $D \bar{D}$ & $13.4 \pm 5.0 \pm 3.6$ & BES [503] \\
$D \bar{D}$ & $103.3 \pm 1.4_{-6.6}^{+4.8}$ & CLEO [508] \\
non- $D \bar{D}$ & $-3.3 \pm 1.4_{-4.8}^{+6.6}$ & CLEO [508] \\
$D \bar{D}$ & $93_{-9}^{+8}$ & PDG10 [447] \\
non- $D \bar{D}$ & $7_{-8}^{+9}$ & PDG10 [447] \\
\hline \hline
\end{tabular}

TABLE 41: Measurements of non- $D \bar{D}$ cross sections for $\psi(3770)$ decays and the experimentally observed cross section for $\psi(3770)$ production at $3.773 \mathrm{GeV}$

\begin{tabular}{lcc}
\hline \hline Experiment & $\sigma_{\text {non- }}^{\text {obs }} D \bar{D}(\mathrm{nb})$ & $\sigma_{\psi(3770)}^{\text {obs }}(\mathrm{nb})$ \\
\hline CLEO [508] & $-0.01 \pm 0.08_{-0.30}^{+0.41}$ & $6.38 \pm 0.08_{-0.30}^{+0.41}$ \\
BESII [502] & $1.14 \pm 0.08 \pm 0.59$ & $7.18 \pm 0.20 \pm 0.63$ \\
BESII [584] & $1.04 \pm 0.23 \pm 0.13$ & $6.94 \pm 0.48 \pm 0.28$ \\
BESII [503] & $0.95 \pm 0.35 \pm 0.29$ & $7.07 \pm 0.36 \pm 0.45$ \\
BESII [585] & $1.08 \pm 0.40 \pm 0.15$ & - \\
MARKII [11] & - & $9.1 \pm 1.4$ \\
\hline \hline
\end{tabular}

former quantity is obtained by subtracting the hadronic cross section measured on the "continuum" at $\sqrt{s}=$ $3671 \mathrm{MeV}$, as extrapolated to $\sqrt{s}=3773 \mathrm{MeV}$ and corrected for interference with $\psi(2 S)$ decays, from the hadronic cross section measured near the peak of the $\psi(3770), \sqrt{s}=3773 \mathrm{MeV}$. The net non- $D \bar{D}$ cross section obtained by CLEO is smaller than those of BES and is consistent with zero. These non- $D \bar{D}$ cross sections are summarized in Table 41, and resulting branching fractions in Table 40. As the CLEO and BES results are in conflict, the PDG10 average in Table 40 averages between the two and inflates the combined uncertainty so as to be consistent with both. 
To search for light hadron decays of $\psi(3770)$, both BES [586-592] and CLEO [593-595] extensively studied various exclusive light hadron decay modes for $\psi(3770) \rightarrow \mathrm{LH}(\mathrm{LH} \equiv$ light hadron), but for only one channel was found to have a significant signal: CLEO [595] measured the branching fraction $\mathcal{B}(\psi(3770) \rightarrow \phi \eta)=(3.1 \pm 0.6 \pm 0.3) \times 10^{-4}$. This branching fraction is obtained by subtracting the extrapolated continuum cross section for $e^{+} e^{-} \rightarrow \phi \eta$ measured at $\sqrt{s}=3671 \mathrm{MeV}$ from that measured at $3.773 \mathrm{GeV}$. CLEO's measurement explicitly ignored the possible interference among amplitudes for this final state from $\psi(3770)$, continuum, and $\psi(2 S)$.

Although CLEO did not claim observations for other light hadron decay modes, some evidence for such decays can be found in the CLEO cross sections. Table 42 lists some cross sections for $e^{+} e^{-} \rightarrow \mathrm{LH}$ measured at 3.773 and $3.671 \mathrm{GeV}$ by CLEO [595]. Curiously, the final states $\pi^{+} \pi^{-} \pi^{0}, \rho \pi$, and $\omega \eta$ have smaller cross sections at $\sqrt{s}=3773 \mathrm{MeV}$ than at $3671 \mathrm{MeV}$, suggesting that an interference effect has come into play to reduce the observed cross section. If this is the cause, this would imply $\psi(3770)$ branching fractions for these modes of order $10^{-4}-10^{-3}$. It is also noteworthy that $K^{* 0} \bar{K}^{0}$ is produced more copiously than $K^{*+} K^{-}$(by a factor of at least 20) at both energies. Whether these two phenomena are related remains an open question.

BES [19] has observed a $\psi(3770)$-lineshape anomaly in measurement of inclusive cross sections measured in the range $\sqrt{s}=3.70-3.87 \mathrm{GeV}$ (see also Sect. 2.1.1). It was suggested by BES and by Dubynskiy and Voloshin [596] that a second structure near $3765 \mathrm{MeV}$ could be responsible. If such a structure exists, and it decays into some of the low multiplicity LH states discussed above, yet another amplitude comes into play which could interfere and cause observed cross sections to be smaller near the $\psi(3770)$ than on the continuum. A very recent preliminary analysis by KEDR [20] of its $e^{+} e^{-}$scan data near

TABLE 42: Measurements from CLEO [595] of cross sections for $e^{+} e^{-} \rightarrow \pi^{+} \pi^{-} \pi^{0}$ and $e^{+} e^{-} \rightarrow \mathrm{VP}$ channels on the continuum just below the $\psi(2 S)$ at $\sqrt{s}=3.671 \mathrm{GeV}$ and on the $\psi(3770)$ resonance at $\sqrt{s}=3.773 \mathrm{GeV}$

\begin{tabular}{lcc}
\hline \hline Final state & $\sigma(\mathrm{pb})$ & $\sigma(\mathrm{pb})$ \\
& $\sqrt{s}=3.671 \mathrm{GeV}$ & $\sqrt{s}=3.773 \mathrm{GeV}$ \\
\hline$\pi^{+} \pi^{-} \pi^{0}$ & $13.1_{-1.7}^{+1.9} \pm 2.1$ & $7.4 \pm 0.4 \pm 2.1$ \\
$\rho \pi$ & $8.0_{-1.4}^{+1.7} \pm 0.9$ & $4.4 \pm 0.3 \pm 0.5$ \\
$\rho^{0} \pi^{0}$ & $3.1_{-0.8}^{+1.0} \pm 0.4$ & $1.3 \pm 0.2 \pm 0.2$ \\
$\rho^{+} \pi^{-}$ & $4.8_{-1.2}^{+1.5} \pm 0.5$ & $3.2 \pm 0.3 \pm 0.2$ \\
$\omega \eta$ & $2.3_{-1.0}^{+1.8} \pm 0.5$ & $0.4 \pm 0.2 \pm 0.1$ \\
$\phi \eta$ & $2.1_{-1.2}^{+1.9} \pm 0.2$ & $4.5 \pm 0.5 \pm 0.5$ \\
$K^{* 0} \bar{K}^{0}$ & $23.5_{-3.9}^{+4.6} \pm 3.1$ & $23.5 \pm 1.1 \pm 3.1$ \\
$K^{*+} K^{-}$ & $1.0_{-0.7}^{+1.1} \pm 0.5$ & $<0.6$ \\
\hline \hline
\end{tabular}

$\psi(3770)$ finds inconsistency with this lineshape anomaly. The conflict about the fraction of non- $D \bar{D}$ decays from $\psi(3770)$ is not restricted to being between the BESII and CLEO experiments; an inclusive-exclusive rift also remains to be bridged. Inclusive-hadronic measurements alone have difficulty supporting a $\psi(3770) \rightarrow$ non- $D \bar{D}$ branching fraction of more than several percent without some confirmation in exclusive mode measurements, which currently show a large number of modes, including the leading candidate low-multiplicity transitions and decays, to be quite small. The large datasets expected at BESIII offer an opportunity for such a multifaceted approach to $\psi(3770) \rightarrow$ non- $D \bar{D}$.

\subsubsection{Observation of $\Upsilon(1 S) \rightarrow$ antideuteron $+X$}

The appearance of deuterons in fragmentation has been addressed theoretically in the framework of a coalescence model [597, 598], via the binding of a nearby neutron and proton. Experimental constraints on the process from measured deuteron production are limited. ARGUS [599] found evidence in $\Upsilon(1 S, 2 S)$ decays for such production but with very low statistics. These results have been accommodated in a string-model calculation [600]. Further experimental information is essential.

CLEO [334] addressed this issue with a measurement of antideuteron production in samples of 22, 3.7, and 0.45 million $\Upsilon(1 S, 2 S, 4 S)$ decays, respectively. Only antideuterons were sought due to the presence of a large deuteron background. This background arises from nuclear interactions with matter (such as gas, vacuum chambers, and beam collimators) initiated by either particles created in the $e^{+} e^{-}$annihilation or errant $e^{ \pm}$from the colliding beams. These interactions result in the appearance of neutrons, protons, and deuterons in the detector. Antideuterons are identified primarily by their distinctive energy loss $(d E / d x)$ as a function of momentum, as measured in tracking chambers, but residual pion and proton backgrounds are additionally suppressed by requiring deuteron-appropriate response in the RICH detectors. This identification is relatively background-free over the momentum range of $0.45-1.45 \mathrm{GeV} / c$, and is found to correspond to a branching fraction

$$
\mathcal{B}(\Upsilon(1 S) \rightarrow \overline{\mathrm{d}} X)=(2.86 \pm 0.19 \pm 0.21) \times 10^{-5} \text {. }
$$

How often is the baryon-number conservation for the $\overline{\mathrm{d}}$ accomplished with a deuteron? Deuteron background becomes tolerable in $\overline{\mathrm{d}}$-tagged events, and three d $\overline{\mathrm{d}}$ candidate events are found in the CLEO $\Upsilon(1 S)$ data sample, meaning that baryon-number compensation occurs with a deuteron about $1 \%$ of the time. By counting events with zero, one, or two protons accompanying an identified $\overline{\mathrm{d}}$, CLEO finds that compensation by each of $p n$, $n p, n n$, or $p p$ occurs at roughly the same rate, about a quarter of the time.

CLEO also measures antideuteron fractions in the data samples of $\Upsilon(2 S)$ and $\Upsilon(4 S)$. The $\Upsilon(2 S)$ result can be 
used to calculate the rate of $\chi_{b J} \rightarrow \overline{\mathrm{d}} X$ by subtracting a scaled $\Upsilon(1 S) \overline{\mathrm{d}}$-fraction to account for $\Upsilon(2 S) \rightarrow X \Upsilon(1 S)$ transitions, and again, with a different scaling factor, to account for $\Upsilon(2 S) \rightarrow g g g$, $\gamma g g$ decays, assuming they have the same $\overline{\mathrm{d}}$-fraction as $\Upsilon(1 S)$; the balance are attributed to appearance through $\Upsilon(2 S) \rightarrow \gamma \chi_{b J}$, $\chi_{b J} \rightarrow \overline{\mathrm{d}} X$. No significant excess is observed, either here nor in $\Upsilon(4 S)$ decays, so CLEO reports the upper limits $\mathcal{B}\left(\chi_{b J} \rightarrow \overline{\mathrm{d}} X\right)<1.1 \times 10^{-4}$, averaged over $J=0,1,2$, and $\mathcal{B}(\Upsilon(4 S) \rightarrow \overline{\mathrm{d}} X)<1.3 \times 10^{-5}$, both at $90 \% \mathrm{CL}$.

Artoisenet and Braaten [333] find this CLEO result useful in tuning parameters of event generators in a study of production of loosely bound hadronic molecules (see Sect. 2.9.3 for a discussion of the relevance of this measurement to the nature of the $X(3872)$ ). Brodsky [601] comments on how measurements such as these should be extended in order to probe the hidden-color structure of the antideuteron wave function.

\subsubsection{Observation of $\Upsilon, \chi_{b J} \rightarrow$ open charm}

Very little is known about the heavy-flavor (i.e., open charm) content of bottomonium hadronic decays, which can be used as a tool to probe of the post- $b \bar{b}$-annihilation fragmentation processes. $\Upsilon(n S)$ hadronic decays are dominated by those materializing through three gluons $(g g g), \chi_{b 0,2}$ through $g g$, and $\chi_{b 1}$ through $q \bar{q} g$ [495]; each of these processes is expected to have its own characteristic open-charm content.

CLEO [602] first selected events from its $\Upsilon(2 S, 3 S)$ data samples to have a $D^{0}$ (or $\bar{D}^{0}$ ) meson exclusively reconstructed, and with momentum $>2.5 \mathrm{GeV} / c$; the momentum cut is required to suppress backgrounds. The single-photon energy spectra for these events were then fit for the presence of narrow peaks corresponding to the radiative transitions $\Upsilon(m S) \rightarrow \chi_{b J}(n P)$. Significant signals for $D^{0}$ production only from $\chi_{b 1}(1 P, 2 P)$ are observed with branching fractions (not correcting for the $D^{0}$ momentum cut) of about $10 \%$, with upper limits at $90 \%$ CL for the others ranging from 2-10\%. CLEO then combines these measured fractions with some assumptions and theoretical input to simultaneously extract $\rho_{8}$, an NRQCD non-perturbative parameter, and branching fractions for $D^{0}$ mesons produced with any momenta; the assumptions and $\rho_{8}$ directly affect the spectrum of $D^{0}$ mesons. With these assumptions, CLEO obtains $\rho_{8} \approx 0.09$ and

$$
\mathcal{B}\left(\chi_{b 1}(1 P, 2 P) \rightarrow D^{0} X\right) \approx 25 \%
$$

both in agreement with predictions [603, 604]. Upper limits at $90 \%$ CL are set for the remaining four $\chi_{b J}$ that are also consistent with the predictions, with the exception of $\chi_{b 2}(2 P)$, which has an upper limit that is half the predicted value of $12 \%$. The largest branching fractions occur for $\chi_{b 1}(1 P, 2 P)$, as expected, which are the states expected to decay via $q \bar{q} g$.
$B A B A R$ [605] searched its $\Upsilon(1 S)$ data sample for the presence of $D^{*+}$ (and $D^{*-}$ ) mesons. BABAR measures

$$
\mathcal{B}\left(\Upsilon(1 S) \rightarrow D^{*+} X\right)=(2.52 \pm 0.13 \pm 0.15) \%,
$$

the first such observation of charm in $\Upsilon(1 S)$ decays, and extracts the resulting $D^{*+}$ momentum spectrum. $B A B A R$ also finds this rate to be considerably in excess (i.e., roughly double) of that expected from $b \bar{b}$ annihilation into a single photon. The excess is seen to be in agreement with a prediction [606] based on splitting a virtual gluon, but appears to be too small to accommodate an octet-state contribution [607].

\subsubsection{Observation of $\chi_{b J}(1 P, 2 P) \rightarrow$ hadrons}

Using its full sample of 9 million $\Upsilon(2 S)$ and 6 million $\Upsilon(3 S)$ decays collected with the CLEO III detector, CLEO made the first measurements of branching fractions of exclusive decays of the $\chi_{b J}(1 P, 2 P)$ states [608], which were accessed through the allowed E1 radiative transitions $\Upsilon(3 S) \rightarrow \gamma \chi_{b J}(2 P)$ and $\Upsilon(2 S) \rightarrow \gamma \chi_{b J}(1 P)$.

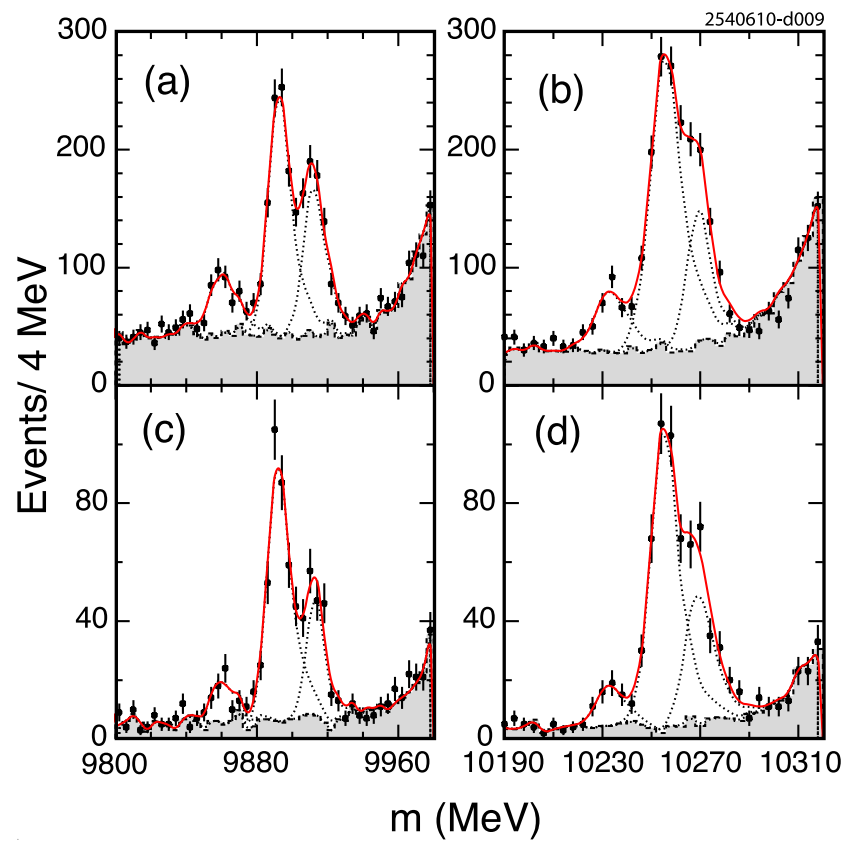

FIG. 49: From CLEO [608], the invariant mass of the sum of exclusive decay modes of the $\chi_{b J}(1 P)$ from (a), (c) $\Upsilon(2 S)$ and (b), (d) $\Upsilon(3 S$ ) radiative decays. (a) and (b) show the sum of 659 exclusive decay modes, while (c) and (d) show the sum of the 14 decay modes with significant branching fractions, the values of which were measured. Solid circles represent data, shaded histograms the backgrounds determined from $\Upsilon(1 S)$ data, dotted curves the contributions of the individual $\chi_{b J}$ signals as determined from fits to the data, and solid curves the sum of background and fitted signals. Adapted from [608] with kind permission, copyright (2008) The American Physical Society 
A comprehensive set of 659 decay exclusive modes was included in the search, where each decay mode was a combination of $\pi^{ \pm}, \pi^{0}, \eta, K^{ \pm}, K_{S}$, and $p^{ \pm}$. Of these 659,14 were found that have significances of greater than $5 \sigma$ for both $\chi_{b J}(1 P)$ and $\chi_{b J}(2 P)$ decays (combined for $J=0,1,2)$. Figure 49 shows the invariant mass of the exclusive decay modes for all 659 and the selected 14 decay modes of the $\chi_{b J}(1 P)$ and $\chi_{b J}(2 P)$. Branching fractions were measured for the 14 decay modes from both the $\chi_{b J}(1 P)$ and $\chi_{b J}(2 P)$ for $J=0,1,2$. The branching fractions ranged from approximately $(1-20) \times 10^{-4}$. The largest branching fractions measured were to $6 \pi 2 \pi^{0}$ and $8 \pi 2 \pi^{0}$. This survey of branching fractions, besides being useful for testing models of bottomonium decays, also gives some indication of which exclusive channels might be most fruitful in searches for new states in the bottomonium region.

\section{PRODUCTION ${ }^{9}$}

\subsection{Introduction to theoretical concepts}

In this subsection, we provide an introduction to some of the theoretical ideas that will appear in subsequent subsections, many of which are based on various factorization formulas for quarkonium production and decay. This subsection also serves to establish notation and nomenclature that is used in subsequent subsections.

\subsubsection{Momentum scales and factorization}

A heavy quarkonium has at least three intrinsic momentum scales: the heavy-quark mass $m_{Q}$; the momentum of the heavy quark or antiquark in the quarkonium rest frame, which is of order $m_{Q} v$; and the binding energy of the heavy quark-antiquark $(Q \bar{Q})$ pair, which is of order $m_{Q} v^{2}$. Here $v$ is the typical velocity of the heavy quark or antiquark in the quarkonium rest frame. $\left(v^{2} \approx 0.3\right.$ for the $J / \psi$ and $v^{2} \approx 0.1$ for the $\Upsilon$.)

If a heavy quarkonium is produced in a hard-scattering process, then, in addition to the intrinsic scales of the quarkonium, the hard-scattering scale $p$ enters into the description of the production process. The hardscattering scale $p$ is usually set by a large momentum transfer in the production process. In quarkonium production in hadron-hadron collisions (hadroproduction) and hadron-lepton collisions, $p$ is usually taken to be of order $p_{T}$, the transverse momentum of the quarkonium, while in quarkonium production in $e^{+} e^{-}$collisions, $p$ is

9 P. Artoisenet, A. Bertolin, G. T. Bodwin ${ }^{\dagger}$, C.-H. Chang, K.-T. Chao, J.-P. Lansberg, F. Maltoni, A. Meyer ${ }^{\dagger}$, V. Papadimitriou ${ }^{\dagger}$, and J.-W. Qiu usually taken to be of order $p^{*}$, the quarkonium momentum in the $e^{+} e^{-}$center-of-mass frame.

One might expect intuitively that the production process could be understood in terms of two distinct steps: the production of the $Q \bar{Q}$ pair, which would occur at the scale $p$, and the subsequent evolution of the $Q \bar{Q}$ pair into the quarkonium, which would involve the smaller dynamical scales $m_{Q} v$ and $m_{Q} v^{2}$. The first step would be calculable in an expansion in powers of $\alpha_{s}(p)$, while the second step would typically involve inherently nonperturbative physics. The term "short distance" is often used to refer to the momentum scale $p$ (distance scale $1 / p$ ), while the term "long distance" is often used to refer to typical hadronic momentum scales, such as $m_{Q} v, m_{Q} v^{2}$, or $\Lambda_{\mathrm{QCD}}$. The term "short distance" is also sometimes used to refer to the scale $m_{Q}$ in the context of NRQCD.

In order to establish that this intuitive picture of quarkonium production is actually a property of QCD, one must demonstrate that the short-distance, perturbative effects at the scale $p$ can be separated from the longdistance, nonperturbative dynamics. Such a separation is known as "factorization." In proving a "factorization theorem," one must show that an amplitude or cross section can be expressed as a sum of products of infraredsafe, short-distance coefficients with well defined operator matrix elements. Such short-distance coefficients are perturbatively calculable. The operator matrix elements would contain all of the long-distance, nonperturbative physics. They might be determined phenomenologically or, possibly, through lattice simulations. If it can be further demonstrated that the long-distance matrix elements are universal, i.e., process independent, then factorization formulas yield much greater predictive power.

The nonperturbative evolution of the $Q \bar{Q}$ pair into a quarkonium has been discussed extensively in terms of models and in terms of the language of effective theories of QCD [1, 134, 138]. Different treatments of this evolution have led to various theoretical models for inclusive quarkonium production. Most notable among these are the color-singlet model (CSM), the color-evaporation model (CEM), the nonrelativistic QCD (NRQCD) factorization approach, and the fragmentation-function approach.

\subsubsection{The color-singlet model}

The CSM was first proposed shortly after the discovery of the $J / \psi[609-616]$. In this model, it is assumed that the $Q \bar{Q}$ pair that evolves into the quarkonium is in a color-singlet state and that it has the same spin and angular-momentum quantum numbers as the quarkonium. In the CSM, the production rate for each quarkonium state is related to the absolute values of the color-singlet $Q \bar{Q}$ wave function and its derivatives, evaluated at zero $Q \bar{Q}$ separation. These quantities can be extracted by comparing theoretical expressions for quarkonium decay rates in the CSM with experimental mea- 
surements. Once this extraction has been carried out, the CSM has no free parameters. The CSM was successful in predicting quarkonium production rates at relatively low energy [617]. Recently, it has been found that, at high energies, very large corrections to the CSM appear at next-to-leading order (NLO) and next-to-next-to-leading order (NNLO) in $\alpha_{s}$ [618-620]. (See Sect. 4.2.1). Consequently, the possibility that the CSM might embody an important production mechanism at high energies has re-emerged. However, given the very large corrections at NLO and NNLO, it is not clear that the perturbative expansion in $\alpha_{s}$ is convergent. Furthermore, in the production and decay of $P$-wave and higher-orbital-angularmomentum quarkonium states, the CSM is known to be inconsistent because it leads to uncanceled infrared divergences. (See Ref. [1] and references therein.) As we will describe below, the NRQCD factorization approach encompasses the color-singlet model, but goes beyond it.

\subsubsection{The color-evaporation model}

The CEM [621-626] is motivated by the principle of quark-hadron duality. In the CEM, it is assumed that every produced $Q \bar{Q}$ pair evolves into a quarkonium if it has an invariant mass that is less than the threshold for producing a pair of open-flavor heavy mesons. It is further assumed that the nonperturbative probability for the $Q \bar{Q}$ pair to evolve into a quarkonium state $H$ is given by a constant $F_{H}$ that is energy-momentum and process independent. Once $F_{H}$ has been fixed by comparison with the measured total cross section for the production of the quarkonium $H$, the CEM can predict, with no additional free parameters, the momentum distribution of the quarkonium production rate. The CEM predictions provide rough descriptions of the CDF data for $J / \psi, \psi(2 S)$, and $\chi_{c J}$ production at $\sqrt{s}=1.8 \mathrm{TeV}$ [626]. In Ref. [627], the CEM predictions are fit to the CDF data for $J / \psi$, $\psi(2 S)$, and $\chi_{c J}$ production at $\sqrt{s}=1.8 \mathrm{TeV}$ [628]. The quality of these fits is generally poor, with $\chi^{2} /$ d.o.f. for the $J / \psi$ fits of about $7-8$ without initial-state $k_{T}$ smearing and 2-4.5 with initial-state $k_{T}$ smearing. In contrast, the NRQCD factorization approach, which we are about to describe, yields fits to the $\mathrm{CDF} J / \psi$ data with $\chi^{2} /$ d.o.f. of about 1 .

\subsubsection{The NRQCD factorization approach}

The NRQCD factorization approach [138] to heavyquarkonium production is by far the most sound theoretically and most successful phenomenologically. NRQCD is an effective theory of QCD and reproduces full QCD dynamics accurately at momentum scales of order $m_{Q} v$ and smaller. Dynamics involving momentum scales of order $m_{Q}$ or larger are taken into account through the short-distance coefficients of the operators that appear in the NRQCD action. The NRQCD factorization approach expresses the probability for a $Q \bar{Q}$ pair to evolve into a quarkonium in terms of matrix elements of NRQCD operators. These matrix elements can be characterized in terms of their scaling with the heavy-quark velocity $v$ [138]. In the NRQCD factorization approach, the inclusive cross section for the direct production of a quarkonium state $H$ is written as a sum of products of these NRQCD matrix elements with the corresponding $Q \bar{Q}$ production cross sections:

$$
\sigma(H)=\sum_{n} \sigma_{n}(\Lambda)\left\langle\mathcal{O}_{n}^{H}(\Lambda)\right\rangle .
$$

Here $\Lambda$ is the ultraviolet cutoff of the effective theory, the $\sigma_{n}$ are expansions in powers of $v$ of the cross sections to produce a $Q \bar{Q}$ pair in the color, spin, and orbital-angular momentum state $n$. The $\sigma_{n}$ are convolutions of partonlevel cross sections at the scale $p$ with parton distribution functions. (The former are short-distance quantities, while the latter are long-distance quantities that depend on the nonperturbative dynamics of the initial hadrons.) The matrix elements $\left\langle\mathcal{O}_{n}^{H}(\Lambda)\right\rangle$ are vacuum-expectation values of four-fermion operators in NRQCD. We emphasize that Eq. (154) represents both processes in which the $Q \bar{Q}$ pair is produced in a color-singlet state and processes in which the $Q \bar{Q}$ pair is produced in a color-octet state. It is conjectured that the NRQCD factorization expression in Eq. (154) holds when the momentum transfer $p$ in the hard-scattering production process is of order $m_{Q}$ or larger.

Unlike the CSM and the CEM expressions for the production cross section, the NRQCD factorization formula for heavy-quarkonium production depends on an infinite number of unknown matrix elements. However, the sum in Eq. (154) can be organized as an expansion in powers of $v$. Hence, the NRQCD factorization formula is a double expansion in powers of $v$ and powers of $\alpha_{s}$. In phenomenological applications, the sum in Eq. (154) is truncated at a fixed order in $v$, and only a few matrix elements typically enter into the phenomenology. The predictive power of the NRQCD factorization approach is based on the validity of such a truncation, as well as on perturbative calculability of the $Q \bar{Q}$ cross sections and the universality of the long-distance matrix elements.

If one retains in Eq. (154) only the color-singlet contributions of leading order in $v$ for each quarkonium state, then one obtains the CSM. As we have mentioned, such a truncation leads to inconsistencies because the omission of color-octet contributions results in uncanceled infrared divergences in the production rates of $P$-wave and higher-orbital-angular-momentum quarkonium states.

The CEM implies that certain relationships must hold between the NRQCD long-distance matrix elements [627]. These relationships are generally inconsistent with the scaling of the matrix elements with $v$ that is predicted by NRQCD. The shortcomings of the CEM in describing the Fermilab Tevatron data can be traced, at least in part, to these inconsistencies [627].

As we will explain in more detail below, in the case 
of inclusive quarkonium production, a compelling proof of NRQCD factorization is still lacking. A further difficulty with the NRQCD factorization formula in Eq. (154) is that a straightforward perturbative expansion of the short-distance coefficients may not yield an optimal organization of the expression for the cross section. The difficulty with such a straightforward expansion is that it ignores the fact that different orders in $\alpha_{s}$ in the perturbative expansion may have different dependences on $m_{Q} / p$. Consequently, at large $p / m_{Q}$, higher orders in the perturbation expansion may be more important than lower orders. Therefore, it may be useful to organize the production cross section in powers of $p / m_{Q}$ before expanding the short-distance coefficients in powers of $\alpha_{s}$ [629-631].

Although the application of NRQCD factorization to heavy-quarkonium production processes has had many successes, there remain a number of discrepancies between its predictions and experimental measurements. The most important of these successes and discrepancies are discussed in the remainder of Sect. 4.

\subsubsection{The fragmentation-function approach}

In the fragmentation-function approach to factorization for inclusive quarkonium production [629-631], one writes the production cross section in terms of convolutions of parton production cross sections with light-cone fragmentation functions. This procedure provides a convenient way to organize the contributions to the cross section in terms of powers of $m_{Q} / p$. As we will explain below, it might also represent the first step in proving NRQCD factorization [629-631]. In the second step, one would establish that the light-cone fragmentation functions could be expanded in terms of NRQCD matrix elements.

We now describe the fragmentation-function approach for the specific case of single inclusive heavy-quarkonium production at transverse momentum $p_{T} \gg m_{Q}$. The contribution to the cross section at the leading power in $m_{Q} / p_{T}$ is given by the production of a single parton $(e . g$. , a gluon), at a distance scale of order $1 / p_{T}$, which subsequently fragments into a heavy quarkonium [632]. The contribution to the cross section at the first subleading power in $m_{Q} / p_{T}$ is given by the production of a $Q \bar{Q}$ pair in a vector- or axial-vector state, at a distance scale of order $1 / p_{T}$, which then fragments into a heavy quarkonium [631]. It was shown in the perturbative-QCD factorization approach $[629,631]$ that the production cross section can be factorized as

$$
\begin{aligned}
& d \sigma_{A+B \rightarrow H+X}\left(p_{T}\right)= \\
& \sum_{i} d \hat{\sigma}_{A+B \rightarrow i+X}\left(p_{T} / z, \mu\right) \otimes D_{i \rightarrow H}\left(z, m_{Q}, \mu\right) \\
& +\sum_{[Q \bar{Q}(\kappa)]} d \hat{\sigma}_{A+B \rightarrow[Q \bar{Q}(\kappa)]+X}\left(P_{[Q \bar{Q}(\kappa)]}=p_{T} / z, \mu\right) \\
& \quad \otimes D_{[Q \bar{Q}(\kappa)] \rightarrow H}\left(z, m_{Q}, \mu\right) \\
& +\mathcal{O}\left(m_{Q}^{4} / p_{T}^{4}\right)
\end{aligned}
$$

where the first term in Eq. (155) gives the contribution of leading power in $m_{Q} / p$, and the second term gives the first contribution of subleading power in $m_{Q} / p$. $A$ and $B$ are the initial particles in the hard-scattering process and $\otimes$ represents a convolution in the momentum fraction $z$. In the first term in Eq. (155), the cross section for the inclusive production of a single particle $i, d \hat{\sigma}_{A+B \rightarrow i+X}$, contains all of the information about the incoming state and includes convolutions with parton distributions in the cases in which $\mathrm{A}$ or $\mathrm{B}$ is a hadron. The cross section $d \hat{\sigma}_{A+B \rightarrow i+X}$ is evaluated at the factorization scale $\mu \sim p_{T}$. The quantity $D_{i \rightarrow H}$ is the fragmentation function for an off-shell parton of flavor $i$ to fragment into a quarkonium state $H$ [633]. The argument $m_{Q}$ indicates explicitly the dependence of $D_{i \rightarrow H}$ on the heavyquark mass. Similarly, in the second term in Eq. (155), $d \hat{\sigma}_{A+B \rightarrow[Q \bar{Q}(\kappa)]+X}$ is the inclusive cross section to produce an on-shell $Q \bar{Q}$ pair with spin and color quantum numbers $\kappa$. The cross section $d \hat{\sigma}_{A+B \rightarrow[Q \bar{Q}(\kappa)]+X}$ is also evaluated at the factorization scale $\mu \sim p_{T}$, but it is suppressed by a factor $m_{Q}^{2} / p_{T}^{2}$ relative to $d \hat{\sigma}_{A+B \rightarrow i+X}$. The quantity $D_{[Q \bar{Q}(\kappa)] \rightarrow H}$ is the fragmentation function for an off-shell $Q \bar{Q}$ pair with quantum numbers $\kappa$ to fragment into a quarkonium state $H$ [631]. The predictive power of the factorization formula in Eq. (155) relies on the perturbative calculability of the single-particle inclusive and $Q \bar{Q}$ inclusive cross sections and the universality of the fragmentation functions.

The dependences of the single-parton and the $Q \bar{Q}$-pair fragmentation functions on the factorization scale $\mu$ are given by their respective evolution equations. These evolution equations can be used to express the fragmentation functions at the scale $\mu \sim p$ in terms of the fragmentation functions at the scale $\mu_{0} \sim 2 m_{Q}$, thereby resumming the logarithms of $\mu / m_{Q}$ that are contained in the fragmentation functions.

\subsubsection{Relationship of the fragmentation-function approach to the NRQCD factorization approach}

If the NRQCD factorization formula in Eq. (154) is valid for the leading and first subleading power of $m_{Q}^{2} / p_{T}^{2}$, then it implies that the fragmentation functions in Eq. (155) can be expanded in terms of NRQCD matrix 
elements [629-631]:

$$
\begin{aligned}
& D_{i \rightarrow H}\left(z, m_{Q}, \mu_{0}\right)=\sum_{n} d_{i \rightarrow n}\left(z, m_{Q}, \mu_{0}\right)\left\langle\mathcal{O}_{n}^{H}\right\rangle \\
& D_{[Q \bar{Q}(\kappa)] \rightarrow H}\left(z, m_{Q}, \mu_{0}\right)= \\
& \quad \sum_{n} d_{[Q \bar{Q}(\kappa)] \rightarrow n}\left(z, m_{Q}, \mu_{0}\right)\left\langle\mathcal{O}_{n}^{H}\right\rangle
\end{aligned}
$$

Here, the short-distance coefficients $d_{i \rightarrow n}\left(z, m_{Q}, \mu_{0}\right)$ and $d_{[Q \bar{Q}(\kappa)] \rightarrow n}\left(z, m_{Q}, \mu_{0}\right)$ describe, respectively, the perturbative evolution at the scale $\mu_{0}$ of an off-shell parton of flavor $i$ and a $Q \bar{Q}$ pair with quantum numbers $\kappa$ into a $Q \bar{Q}$ pair in the nonrelativistic state $n$. Viewed in this way, the factorization formula in Eq. (155) is simply a reorganization of the sum over $n$ in Eq. (154). (The contributions denoted by $\mathcal{O}\left(m_{Q}^{4} / p_{T}^{4}\right)$ in Eq. (155) are the difference between the NRQCD expression in Eq. (154) and the first two terms in Eq. (155) expanded as a series in $\alpha_{s}$ [634].) Although Eqs. (154) and (155) are equivalent if the NRQCD factorization formalism is valid for heavy-quarkonium production, the formula in Eq. (155) provides a systematic reorganization of the cross section in term of powers of $m_{Q} / p_{T}$ and a systematic method for resumming potentially large logarithms of $p_{T} / m_{Q}$. That reorganization and resummation may make the $\alpha_{s}$ expansion more convergent.

\subsubsection{Difficulties in establishing NRQCD factorization}

The fragmentation functions $D_{i \rightarrow H}$ and $D_{[Q \bar{Q}(\kappa)] \rightarrow H}$ include certain contributions whose compatibility with NRQCD factorization is not obvious. These contributions arise from processes which, when viewed in the quarkonium rest frame, involve the emission of a gluon with momentum of order $m_{Q}$ from the fragmenting parton. That relatively hard gluon can exchange soft gluons with the color-octet $Q \bar{Q}$ pair that evolves into the quarkonium. Such soft interactions can produce logarithmic infrared divergences, which must be absorbed into the NRQCD matrix elements in Eq. (156) in order to obtain short-distance coefficients $d_{i \rightarrow n}$ and $d_{[Q \bar{Q}(\kappa)] \rightarrow n}$ that are infrared safe. The interactions of soft gluons with the gluon that has momentum of order $m_{Q}$ can be represented by interactions of the soft gluons with a lightlike eikonal line (gauge-field link) [630]. Similar lightlike eikonal lines are required in order to render the color-octet NRQCD long-distance matrix elements gauge invariant [629-631]. If it can be shown that the coloroctet NRQCD matrix elements are independent of the directions of these eikonal lines, then it follows that the infrared-divergent soft interactions with gluons that have momenta of order $m_{Q}$ can be absorbed into universal (i.e., process independent) NRQCD long-distance matrix elements. It has been shown that this is the case through two-loop order and, therefore, that the NRQCD factorization in Eq. (156) is valid through two-loop order [629-631]. However, the NRQCD factorization in
Eq. (156) has not been verified at higher orders and, therefore, it is not known if the NRQCD factorization formula in Eq. (154) is valid [629-631]. Note that, because the potential violations of NRQCD factorization at higher loop orders involve gluons with arbitrarily soft momenta, such violations are not suppressed by powers of $\alpha_{s}$ and, consequently, they could completely invalidate the NRQCD factorization formula in Eq. (154).

It is clear that the NRQCD factorization formula cannot apply directly to reactions in which an additional heavy $Q$ or $\bar{Q}$ is produced nearly co-moving with the $Q \bar{Q}$ pair that evolves into the heavy quarkonium. That is because the NRQCD factorization formula is designed to take into account only a heavy quark and a heavy antiquark at small relative velocity $[635,636]$. If an additional heavy $Q$ or $\bar{Q}$ is nearly co-moving with a $Q \bar{Q}$ pair, a color-octet $Q \bar{Q}$ pair could evolve into a color-singlet $Q \bar{Q}$ pair by exchanging soft gluons with the additional $Q$ or $\bar{Q}$. Such nonperturbative color-transfer processes could be taken into account by generalizing the existing NRQCD factorization formalism to include long-distance matrix elements that involve additional heavy quarks or antiquarks. Such color-transfer processes might be identified experimentally by looking for an excess of heavyflavored mesons near the direction of the quarkonium.

\subsection{8. $k_{T}$ factorization}

The $k_{T}$-factorization approach is an alternative to standard collinear factorization that has been applied to analyses of inclusive hard-scattering processes. In the case of quarkonium production, the $k_{T}$-factorization approach has usually been applied within the CSM [637642]. In $k_{T}$-factorization formulas, the parton distributions for the initial-state hadrons depend on the parton transverse momentum, as well as on the parton longitudinal momentum fraction. The leading-order $k_{T^{-}}$ factorization expressions for hard-scattering rates contain some contributions that appear in the standard collinear-factorization formulas in higher orders in $\alpha_{s}$ and $\Lambda_{\mathrm{QCD}} / p$. In some kinematic situations, these higher-order corrections might be important numerically, and the $k_{T}$-factorization predictions in leading order might, in principle, be more accurate than the collinearfactorization predictions in leading order. (An example of such a kinematic situation is the high-energy limit $s \gg \hat{s}$, where $s(\hat{s})$ denotes the square of the total fourmomentum of the colliding hadrons (partons).) On the other hand, the $k_{T}$-dependent parton distributions are less constrained by phenomenology than are the standard parton distributions, and the uncertainties in the $k_{T}$-dependent parton distributions are not yet well quantified in comparison with the uncertainties in the standard parton distributions. Consequently, in practice, the $k_{T}$-factorization predictions may be more uncertain than the corresponding collinear-factorization predictions. 


\subsubsection{Factorization in exclusive quarkonium production}

NRQCD factorization has been proven for the amplitudes for two exclusive quarkonium production processes [643, 644]: exclusive production of a quarkonium and a light meson in $B$-meson decays and exclusive production of two-quarkonium states in $e^{+} e^{-}$annihilation. The proofs begin by factoring nonperturbative processes that involve virtualities of order $\Lambda_{\mathrm{QCD}}$ or smaller from hard processes that involve virtualities of order $p$. (Here, $p$ is the $e^{+} e^{-}$center-of-mass energy in $e^{+} e^{-}$annihilation, and $p$ is the $B$-meson mass in $B$-meson decays.) At this stage, the quarkonia enter through gauge-invariant quarkonium distribution amplitudes. It is then argued that each quarkonium distribution amplitude can be written as a sum of products of perturbatively calculable shortdistance coefficients with NRQCD long-distance matrix elements. The difficulties that occur in establishing this step for inclusive quarkonium production do not appear in the case of exclusive quarkonium production because only color-singlet $Q \bar{Q}$ pairs evolve into quarkonia in exclusive production. The proofs of factorization for exclusive quarkonium production reveal that the violations of factorization are generally suppressed by a factor $m_{Q} v / p$ for each final-state quarkonium and, therefore, vanish in calculations of quarkonium production at order $v^{0}$ in the velocity expansion.

The factorization proofs in Refs. [643, 644] also establish factorized forms in which the light-cone distribution amplitudes, rather than NRQCD long-distance matrix elements, account for the nonperturbative properties of the quarkonia. In contrast with an NRQCD long-distance matrix element, which is a single number, a light-cone distribution amplitude is a function of the heavy-quark longitudinal momentum fraction. Hence, the light-cone distribution amplitudes are incompletely determined by phenomenology, and predictions that are based on lightcone factorization formulas [645-649] must rely on constrained models for the light-cone distribution amplitudes. Generally, the quantitative effects of the model assumptions on the light-cone factorization predictions are not yet known.

\subsubsection{Factorization in quarkonium decays}

There are NRQCD factorization formulas for exclusive quarkonium decay amplitudes and for inclusive quarkonium decay rates [138]. As in the NRQCD factorization formula for inclusive quarkonium production in Eq. (154), these decay formulas consist of sums of products of NRQCD matrix elements with short-distance coefficients. In the cases of decays, the short-distance coefficients are evaluated at a scale $\mu$ of order $m_{Q}$ and are thought to be calculable as power series in $\alpha_{s}(\mu)$. It is generally believed that the NRQCD factorization formula for quarkonium decays can be proven by making use of standard methods for establishing perturbative factoriza- tion. The color-singlet NRQCD long-distance production matrix elements are proportional, up to corrections of relative order $v^{4}$, to the color-singlet NRQCD long-distance decay matrix elements. However, there is no known relationship between the color-octet production and decay matrix elements.

\subsubsection{Future opportunities}

One of the crucial theoretical issues in quarkonium physics is the validity of the NRQCD factorization formula for inclusive quarkonium production. It is very important either to establish that the NRQCD factorization formula is valid to all orders in perturbation theory or to demonstrate that it breaks down at some fixed order in perturbation theory.

The NRQCD factorization formula is known to break down when an additional heavy quark or antiquark is produced in close proximity to a $Q \bar{Q}$ pair that evolves into a quarkonium. It would help in assessing the numerical importance of such processes if experimental measurements could determine the rate at which heavy-flavored mesons are produced nearby in phase space to a heavy quarkonium. If such processes prove to be important numerically, then it would be useful to extend the NRQCD factorization formalism to include them.

\subsection{Production at the Tevatron, RHIC and the LHC}

The first measurements by the CDF collaboration of the direct production ${ }^{10}$ of the $J / \psi$ and the $\psi(2 S)$ at $\sqrt{s}=1.8 \mathrm{TeV}[628,650]$ revealed a striking discrepancy with the existing theoretical calculations: The observed rates were more than an order of magnitude greater than the calculated rates at leading order ( $\mathrm{LO})$ in $\alpha_{s}$ in the CSM. (See Sect. 4.1.2 for a discussion of the CSM.) This discrepancy has triggered many theoretical studies of quarkonium hadroproduction, especially in the framework of NRQCD factorization. (See Sect. 4.1.4 for a discussion of NRQCD factorization.) In the NRQCD factorization approach, mechanisms beyond those in the CSM arise, in which the production of charmonium states proceeds through the creation of a $c \bar{c}$ pair in a color-octet state. For the specific case of the production of the $J / \psi$ or the $\psi(2 S)$ (henceforth denoted collectively as " $\psi$ "), these color-octet transitions take place at higher orders in $v$. Depending on the convergence of the expansions

10 "Prompt production" excludes quarkonium production from weak decays of more massive states, such as the $B$ meson. "Direct production" further excludes quarkonium production from feeddown, via the electromagnetic and strong interactions, from more massive states, such as higher-mass quarkonium states. 
in $\alpha_{s}$ and $v$ and the validity of the NRQCD factorization formula, the NRQCD factorization approach may provide systematically improvable approximations to the inclusive quarkonium production rates. For some recent reviews, see Refs. [1, 651-653]. For some perspectives on quarkonium production at the LHC, see Ref. [654].

Despite recent theoretical advances, which we shall detail below, we are still lacking a clear picture of the mechanisms at work in quarkonium hadroproduction. These mechanisms would have to explain, in a consistent way, both the cross section measurements and the polarization measurements for charmonium production at the Tevatron [329, 628, 650, 655-658] and at RHIC [659-664]. For example, the observed $p_{T}$ spectra in prompt $\psi$ production seem to suggest that a dominant contribution at large $p_{T}$ arises from a color-octet process in which a gluon fragments into a $Q \bar{Q}$ pair, which then evolves nonrelativistically into a quarkonium. Because of the approximate heavy-quark spin symmetry of NRQCD, the dominance of such a process would lead to a substantial transverse component for the polarization of $\psi$ 's produced at large $p_{T}$ [665-667]. This prediction is clearly challenged by the experimental measurements [658].

A possible interpretation of such a failure of NRQCD factorization is that the charmonium system is too light for relativistic effects to be small and that, in phenomenological analyses, the velocity expansion of NRQCD [138] may have been truncated at too low an order. However, such an explanation would seem to be at odds with other successful predictions of the NRQCD approach to charmonium physics. If the convergence of the velocity expansion of NRQCD is indeed an issue, then one would expect better agreement between theory and the available experimental data on hadroproduction in the case of the bottomonium states and, in particular, in the case of the $\Upsilon$. Better convergence of the velocity expansion might explain, for example, why a computation that retains only color-singlet contributions [620] (that is, only contributions of leading order in $v$ ) seems to be in better agreement with the data for $\Upsilon$ production [668-671] than with the data for $\psi$ production [329]. We will discuss this comparison between theory and experiment in greater detail later.

In efforts to identify the mechanisms that are at work in inclusive $\psi$ or $\Upsilon$ production, it is important to have control of the higher-order perturbative corrections to the short-distance coefficients that appear in the NRQCD factorization formula. Several works have been dedicated to the study of the corrections of higher-order in $\alpha_{s}$ and their phenomenological implications for the differential production rates. We summarize these results in Sects. 4.2.1 and 4.2.2. An important observable that has been reanalyzed in the context of higher-order perturbative corrections is the polarization of the quarkonium. We review the analyses of the quarkonium polarization in Sect. 4.2.3. In addition to the rates and polarizations in inclusive quarkonium production, other observables have been shown to yield valuable informa- tion about the production mechanisms. We discuss them in Sect. 4.2.4. Finally, we summarize the future opportunities for theory and experiment in inclusive quarkonium hadroproduction in Sect. 4.2.5.

\subsubsection{Channels at higher-order in $\alpha_{s}$}

At the LHC, the Tevatron, and RHIC, quarkonium production proceeds predominantly via gluon-fusion processes. The production cross sections differential in $p_{T}$ for $S$-wave quarkonium states have been calculated only recently at NLO in $\alpha_{s}$ (Refs. [618, 619, 672-674]). These NLO calculations also provide predictions for the quarkonium polarization differential in $p_{T}$. In the case of the production of spin-triplet $P$-wave quarkonium states, the NLO corrections to the production rate differential in $p_{T}$ have been calculated even more recently [675].

One common outcome of these calculations is that the total cross sections are not much affected by corrections of higher order in $\alpha_{s}[619,676]$. That is, the perturbation series for the total cross sections seem to exhibit normal convergence. However in the case of $\psi, \Upsilon$, or $\chi_{c J}$ production via color-singlet channels, very large corrections appear in the cross sections differential in $p_{T}^{2}$ at large $p_{T}$. This behavior, which has also been seen in photoproduction [677], is well understood. QCD corrections to the color-singlet parton cross section open new production channels, whose contributions fall more slowly with $p_{T}$ than do the LO contributions. Hence, the contributions from the new channels increase substantially the cross section in the large- $p_{T}$ region.

We now discuss this phenomenon briefly. If only the LO (order- $\alpha_{s}^{3}$ ) contribution to the production of a colorsinglet ${ }^{3} S_{1} Q \bar{Q}$ state is taken into account, then the partonic cross section differential in $p_{T}^{2}$ scales as $p_{T}^{-8}$ (Refs. [612-614, 616, 678]). This behavior comes from the contributions that are associated with "box" graphs, such as the one in Fig. 50(a). At NLO in $\alpha_{s}$ (order $\alpha_{s}^{4}$ ), several contributions with distinct kinematic properties arise. The loop corrections, illustrated in Fig. 50(b), are not expected to have substantially different $p_{T}$ scaling than the Born contributions. However, the t-channel gluon-exchange diagrams, such as the one depicted in Fig. 50(c), yield contributions that scale as $p_{T}^{-6}$ and are, therefore, kinematically enhanced in comparison with the Born contribution. At sufficiently large $p_{T}$, this contribution has a kinematic enhancement that compensates for its $\alpha_{s}$ suppression, and it is expected to dominate over the Born contribution. At NLO, there is also a contribution that arises from the process in which a second $Q \bar{Q}$ pair is produced, in addition to the $Q \bar{Q}$ pair that evolves into a quarkonium. In this "associated production" process, both $Q \bar{Q}$ pairs have the same flavor. In the limit $p_{T} \gg m_{Q}$, the associated-production mechanism reduces to heavy-quark fragmentation. Therefore, in this limit, the corresponding partonic cross section differential in $p_{T}^{2}$ scales as $p_{T}^{-4}$. The associated-production contribu- 


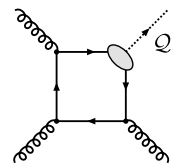

(a)

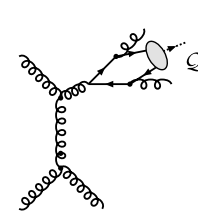

(e)

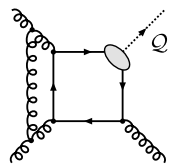

(b)

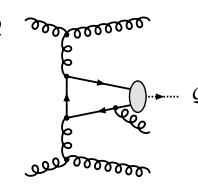

(f)

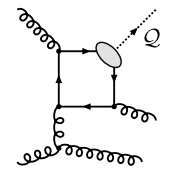

(c)

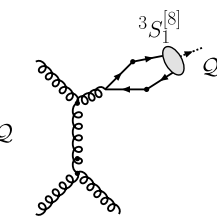

(g)

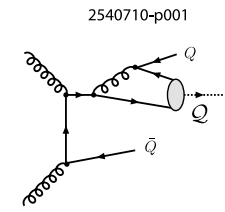

(d)
FIG. 50: Representative diagrams that contribute to the hadroproduction of ${ }^{3} S_{1}$ quarkonium states via color-singlet channels at orders $\alpha_{s}^{3}(\mathrm{a}), \alpha_{s}^{4}(\mathrm{~b}, \mathrm{c}, \mathrm{d})$, and $\alpha_{s}^{5}(\mathrm{e}, \mathrm{f})$, and via color-octet channels at order $\alpha_{s}^{3}(\mathrm{~g}, \mathrm{~h})$. The quark and antiquark that are attached to the ellipses are taken to be on shell, and their relative velocity is set to zero. (a) $-(\mathrm{f}),(\mathrm{g})-(\mathrm{h})$ from [620, 652] with kind permission, copyrights (2008, 2009), The American Physical Society and Springer-Verlag, respectively

tion eventually provides the bulk of the color-singlet parton cross section at sufficiently large $p_{T}$. In the $p_{T}$ region that is covered by the current experiments, though, this contribution is relatively small, owing to phase-space suppression [618].

Similar arguments can be used to understand the impact of QCD corrections on the rates differential in $p_{T}^{2}$ for the production of a $Q \bar{Q}$ pair in a color-octet state. At LO in $\alpha_{s}$, the production of a color-octet ${ }^{3} S_{1} Q \bar{Q}$ pair proceeds, at large $p_{T}$, predominantly through gluon fragmentation [Fig. 50(g)]. Thus, the rate differential in $p_{T}^{2}$ scales as $p_{T}^{-4}$. This is the smallest power of $1 / p_{T}$ that is possible in partonic cross sections. Hence, in this case, the NLO correction cannot contain a kinematically enhanced channel and does not affect substantially the shape of the differential rate [674]. The situation is different for the production of a $C$-even color-octet $Q \bar{Q}$ pair because, in this case, there is no fragmentation process at LO in $\alpha_{s}$. [See Fig. 50(h).] At LO in $\alpha_{s}$, the rates for these channels, differential in $p_{T}^{2}$, scale as $p_{T}^{-6}$. The fragmentation channels appear at NLO in $\alpha_{s}$. Consequently the NLO correction to the differential rate is expected to yield a substantial enhancement at large $p_{T}$. This feature has been checked explicitly in Ref. [674] in the specific case of the production of a color-octet ${ }^{1} S_{0} Q \bar{Q}$ state.

In view of the strong impact of the correction at NLO in $\alpha_{s}$ on the color-singlet differential rate at large transverse momentum, it is natural to examine the QCD corrections that appear at even higher orders in the $\alpha_{s}$ expansion. At NNLO (order $\alpha_{s}^{5}$ ), new, important channels with specific kinematic properties continue to appear. Some of these channels have actually been studied for some time in specific kinematic limits in which one can take advantage of large separations between different perturbative energy scales. The color-singlet gluonfragmentation channel [Fig. 50(e)] has been investigated in the framework of the fragmentation approximation [679], which is relevant in the limit $p_{T} \gg m_{Q}$. The processes in which the $Q \bar{Q}$ pair is produced by the exchange of two gluons in the $t$ channel [Fig. 50(f)] were investigated in the $k_{T}$-factorization approach [639-642]. This approach is relevant in the high-energy limit $s \gg \hat{s}$, where $s(\hat{s})$ denotes the square of the total four-momentum of the colliding hadrons (partons). In that kinematic limit, other enhanced processes, which are initiated by a symmetric two-gluon color-octet state and an additional gluon, have been investigated more recently in Ref. [680]. These processes correspond to higher-order contributions in the framework of the (standard) collinear approximation of perturbative QCD. The advantage in considering either of these kinematic limits is that the perturbation expansion can be reorganized in such a way as to simplify the evaluation of the dominant contribution. Furthermore, the convergence of the perturbation expansion is improved because large logarithms of the ratio of the disparate energy scales are resummed. Away from the asymptotic regime, the corrections to each of these approaches may be important. The impacts of these corrections in the kinematic region that is covered at the current hadron colliders is not known accurately. There is an alternative method that has been proposed for estimating the NNLO corrections to the color-singlet differential rate that is known as the $\mathrm{NNLO}^{\star}$ method [620]. The $\mathrm{NNLO}^{\star}$ method does not attempt to separate the various energy scales. Instead, it considers only NNLO corrections involving real gluon emission and imposes an infrared cutoff to control soft and collinear divergences. The $\mathrm{NNLO}^{\star}$ estimates suffer from large uncertainties, which arise primarily from the sensitivities of these estimates to the infrared cutoff and to the choice of renormalization scale.

A specific higher-order process that has been investigated is the so-called " $s$-channel $Q \bar{Q}$-cut" process $[681,682]$. In this process, an on-shell $Q \bar{Q}$ pair is produced. That pair then rescatters into a quarkonium state. The contribution of $\mathrm{LO}$ in $\alpha_{s}$ to the amplitude for this process is given by the imaginary part of a specific set of one-loop diagrams [683], and the square of this amplitude contributes to the cross section at order $\alpha_{s}^{5}$.

In addition to these new results for the QCD corrections to inclusive quarkonium production, there have also been new results for the QED and relativistic corrections to inclusive quarkonium production. The QED correction to the inclusive $J / \psi$ production rate in hadronhadron collisions has been computed $[684,685]$ and turns out to be small in the region of $p_{T}$ that is covered by the current experiments. Relativistic corrections to the color-singlet rate for inclusive $J / \psi$ hadroproduction have been shown to be negligible over the entire $p_{T}$ range that is currently accessible [686]. 


\subsubsection{Phenomenology, including QCD corrections}

\section{$\psi, \Upsilon$, and $\chi_{c}$ production at the Tevatron}

In the case of $\Upsilon$ production, the contributions of the NLO corrections to the color-singlet channels reduce the discrepancy between the color-singlet contribution to the inclusive cross section and the data collected by the CDF collaboration, as is illustrated in Fig. 51. ${ }^{11}$ However, the predicted NLO rate drops too rapidly at large $p_{T}$, indicating that another production mechanism is at work in that phase-space region. A recent study [620] has shown that contributions from channels that open at NNLO (or-

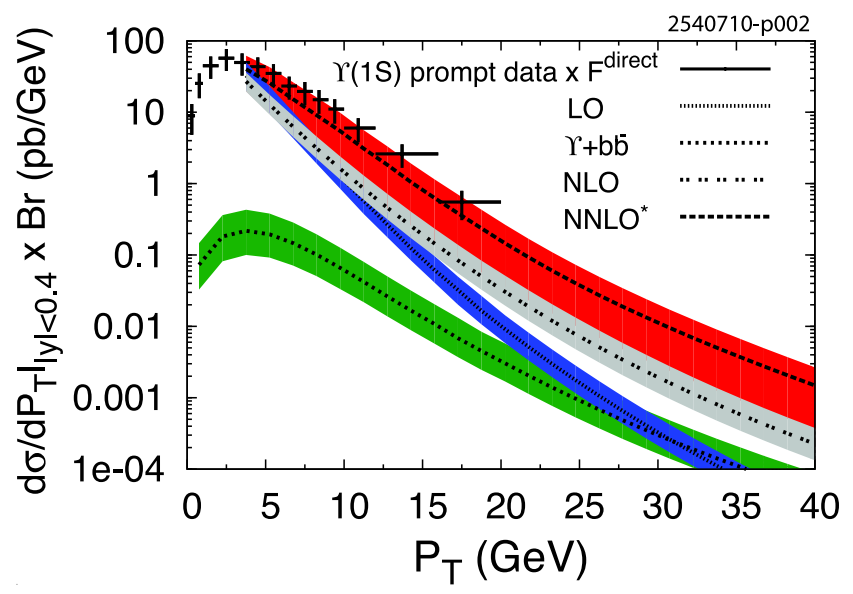

FIG. 51: Comparison between the CSM predictions at NLO and $\mathrm{NNLO}^{\star}$ accuracy for the $\Upsilon$ cross section as a function of the $\Upsilon$ transverse momentum at the Tevatron at $\sqrt{s}=$ $1.80 \mathrm{TeV}$ (Ref. [620]) and the CDF data for $\Upsilon(1 S)$ production [669]. The crosses are the CDF data for prompt $\Upsilon(1 S)$ production, multiplied by $F^{\text {direct }}$, the fraction of direct $\Upsilon(1 S)$ 's in prompt $\Upsilon(1 S)$ events, as measured by the CDF collaboration using an older event sample [668]. The lines show the central values of the theoretical predictions, and the bands depict the theoretical uncertainties. The theoretical uncertainty bands for the LO, NLO, and $\Upsilon+b \bar{b}$ contributions were obtained by combining the uncertainty from $m_{b}$ with the uncertainties that are obtained by varying the renormalization scale $\mu_{f}$ and the factorization scale $\mu_{r}$ between $2 m_{T}$ and $m_{T} / 2$, where $m_{T}=\sqrt{4 m_{b}^{2}+p_{T}^{2}}$. The error band for the NNLO* contribution is obtained by varying the infrared cutoff $s_{i j}^{\min }$ between $2 m_{b}^{2}$ and $m_{b}^{2} / 2$ and by varying $\mu_{f}$ and $\mu_{r}$ between $2 m_{T}$ and $m_{T} / 2$. From [620] with kind permission, copyright (2008) The American Physical Society

11 We note that no phenomenological analysis of $\chi_{b J}$ production is available yet at NLO accuracy. Such an analysis would be necessary in order to predict the prompt $\Upsilon$ cross section to NLO accuracy. As a makeshift, one could multiply the available data by the measurement of the fraction of direct $\Upsilon$ 's in the total rate (integrated over $p_{T}$ ) [668]. Note, however, that the NLO calculation of the $\chi_{c J}$ production rate [675] indicates that the fraction of direct $J / \psi$ 's may depend rather strongly on $p_{T}$. $\left.\operatorname{der} \alpha_{s}^{5}\right)$ may fill the remaining gap between the colorsinglet contribution at NLO and the data. The estimate of the NNLO contribution from this study, called the "NNLO* contribution", is shown in the (red) band labeled $\mathrm{NNLO}^{\star}$ in Fig. 51. Owing to the large theoretical uncertainties, this improved prediction for the colorsinglet contribution does not imply any severe constraint on other possible contributions, such as a color-octet contribution. However, in contrast with previous LO analyses, in which color-octet contributions were required in order to describe the data, the $\mathrm{NNLO}^{\star}$ estimate of the color-singlet contribution shows that color-octet contributions are now merely allowed by the rather large theoretical uncertainties in the $\mathrm{NNLO}^{\star}$ estimate.

The impact of the QCD corrections on the colorsinglet contribution has also been studied in the case of $\psi$ hadroproduction [652]. The comparison with the data is simpler in the case of the $\psi(2 S)$ than in the case of the $J / \psi$, owing to the absence of significant feeddown from excited charmonium states to the $\psi(2 S)$. In a recent paper, the CDF collaboration has reported a new measurement of the inclusive $\psi(2 S)$ cross section [329]. The rates for the prompt production of the $\psi(2 S)$ and for the production of the $\psi(2 S)$ in $B$-meson decays were also extracted in that analysis. The reconstructed differential rate for the prompt component is compared to the prediction for the color-singlet rate at LO, NLO and NNLO* accuracy in Fig. 52. At medium values of $p_{T}$, the upper limit of the $\mathrm{NNLO}^{\star}$ rate is compatible with the CDF results. At larger values of $p_{T}$, a gap appears between the color-singlet rate and the data [652]. The $J / \psi$ differential production rate has the same qualitative features as the $\psi(2 S)$ differential production rate [687]. It is worth emphasizing that the current discrepancy between the color-singlet rate and the Tevatron data has been dramatically reduced by the inclusion of higher-order QCD corrections.

NLO QCD corrections to the color-octet production channels ${ }^{3} S_{1}$ and ${ }^{1} S_{0}$ have been analyzed for $J / \psi$ production [674] and for $\Upsilon$ production [688]. In both cases, these corrections proved to be small when the $p_{T}$ of the produced quarkonium is less than $20 \mathrm{GeV}$. In Ref. [674], values of the NRQCD long-distance matrix elements $\left\langle O^{J / \psi}\left({ }^{3} S_{1}^{[8]}\right)\right\rangle$ and $\left\langle O^{J / \psi}\left({ }^{1} S_{0}^{[8]}\right)\right\rangle$ were obtained by fitting the theoretical prediction to the prompt production rate that was measured by the CDF collaboration [657]. The values of the NRQCD matrix elements that were extracted in this analysis are compatible with the values that were extracted in LO analyses. In the analysis of Ref. [674], feeddown contributions were ignored and the $P$-wave color-octet long-distance matrix elements were set to zero. A satisfactory fit could not be obtained for the experimental data points that have $p_{T}<6 \mathrm{GeV}$, and so these points were not included in the fit. In this regard, it should be kept in mind that resummation of large logarithms may be needed at small $p_{T}$ and that NRQCD factorization may break down at small $p_{T}$.

The $s$-channel $c \bar{c}$-cut $(s \mathrm{CC})$ contributions to $\psi$ 


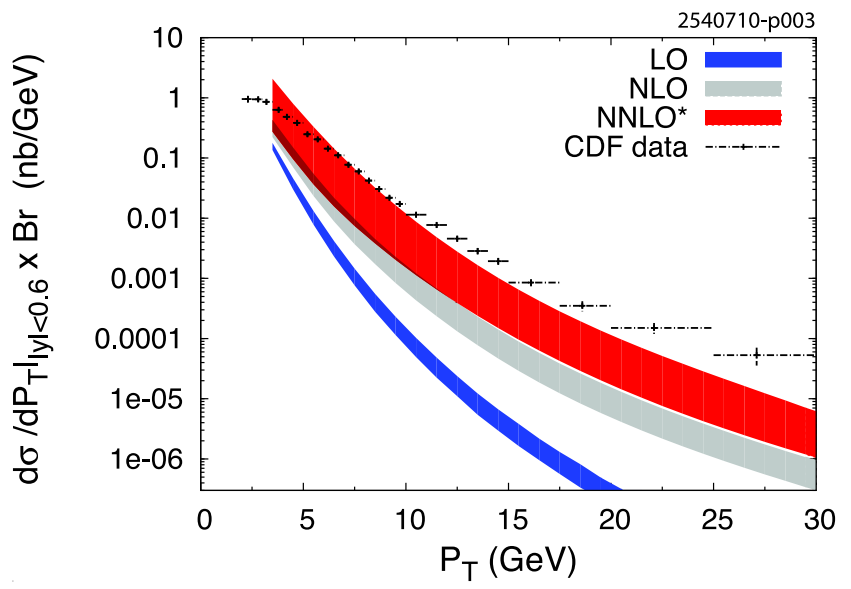

FIG. 52: Comparison between the CSM predictions for the $\psi(2 S)$ cross sections at LO, NLO, and NNLO ${ }^{\star}$ accuracy as a function of the $\psi(2 S) p_{T}$ at the Tevatron at $\sqrt{s}=1.96 \mathrm{TeV}$ (Ref. [652]) and the CDF prompt $\psi(2 S)$ data [329]. The theoretical uncertainty bands for the LO and NLO contributions were obtained by combining the uncertainty from $m_{c}$ with the uncertainties that are obtained by varying the renormalization scale $\mu_{f}$ and the factorization scale $\mu_{r}$ between $2 m_{T}$ and $m_{T} / 2$, where $m_{T}=\sqrt{4 m_{c}^{2}+p_{T}^{2}}$. The theoretical uncertainty band for the $\mathrm{NNLO}^{\star}$ contribution was obtained by varying the infrared cut-off $s_{i j}^{\min }$ between $4 m_{c}^{2}$ and $m_{c}^{2}$ and by varying $\mu_{f}$ and $\mu_{r}$ between $2 m_{T}$ and $m_{T} / 2$. From [652] with kind permission, copyright (2009) Springer-Verlag

hadroproduction have been investigated in Ref. [681, 682] in the framework of a phenomenological model. A first analysis of this model [682], which was based on the data of Refs. [628,657] and incorporated constraints for the small- and large- $p_{T}$ regions, supported rates that are significantly larger than those of the CSM prediction. This analysis, which did not include resummation of initialgluon contributions, yielded a good fit to the $p_{T}$ dependence of the RHIC data. However, it has been shown recently, by evaluating the leading-order contribution of the $s \mathrm{CC}$ amplitude in the framework of NRQCD, that the $s \mathrm{CC}$ contributions can account only for a negligible fraction of the $J / \psi$ production rate that is measured by the CDF collaboration [683].

Some specific NNLO contributions in the CSM were considered in Ref. [680]. These contributions are referred to in the literature as the "gluon-tower model." They can be viewed as LO BFKL contributions, and, thus, they are expected to be enhanced in comparison to other NNLO contributions by a factor $\log s / \hat{s}$. At large $s$, this logarithm may compensate for the $\alpha_{s}^{2}$ suppression of these contributions. At $\sqrt{s}=1.96 \mathrm{TeV}$ and $|y|<0.6$, the gluon-tower model predicts the $J / \psi$ cross section, integrated over $p_{T}$, to be $\sigma(|y|<0.6)=2.7 \mu \mathrm{b}$ (Ref. [680]), in near agreement with the CDF measure- ment $\sigma(|y|<0.6)=4.1_{-0.5}^{+0.6} \mu \mathrm{b}$ (Ref. [689]). ${ }^{12}$ However, the theoretical prediction is somewhat sensitive to an effective gluon mass that is introduced as an infrared cutoff in the model. The comparison does not take into account feeddown from $P$-wave states in the measured cross section, and the model cannot, at present, predict the $p_{T}$ dependence of the cross section.

At LO in $\alpha_{s}$, NRQCD factorization predicts that the ratio of production cross sections, $R_{\chi_{c}}=\sigma_{\chi_{c 2}} / \sigma_{\chi_{c 1}}$, is dominated by the color-octet contribution at large $p_{T}$ and approaches the value $R_{\chi_{c}}=5 / 3$ as $p_{T}$ increases. This LO prediction is in sharp disagreement with the CDF measurement [691], which finds that $R_{\chi_{c}} \approx 0.75$ at large $p_{T}$. Recently, NLO corrections to $\chi_{c J}$ production have been calculated in Ref. [675]. It is found that the NLO corrections are large at large $p_{T}$. They make the contributions of the color-singlet ${ }^{3} P_{J}$ channels negative and comparable to the color-octet contribution for large $p_{T}$. They also cause the ${ }^{3} P_{1}$ color-singlet contribution to fall at a slower rate than the ${ }^{3} P_{2}$ color-singlet contribution as $p_{T}$ increases. Taking into account the large NLO correction, the authors of Ref. [675] were able to fit the measured $p_{T}$ distribution of $R_{\chi_{c}}$, using a plausible value for the ratio of the relevant NRQCD long-distance matrix elements. Hence, there may now be a resolution of this outstanding conflict between theory and experiment. One interesting prediction of the fit to $R_{\chi_{c}}$ in Ref. [675] is that the feeddown from the $\chi_{c J}$ states to the $J / \psi$ state may be quite large - perhaps $30 \%$ of the prompt $J / \psi$ rate at $p_{T}=20 \mathrm{GeV}$. Such a large proportion of feeddown events in the prompt $J / \psi$ rate could have an important effect on the prompt $J / \psi$ polarization.

\section{New NLO hadroproduction results}

As this article was nearing completion, two papers [692, 693] appeared that give complete calculations of the corrections of NLO in $\alpha_{\mathrm{s}}$ for the color-octet production channels through relative order $v^{4}$, that is, for the color-octet ${ }^{3} S_{1},{ }^{1} S_{0}$, and ${ }^{3} P_{J}$ channels We now give a brief account of these results.

The calculations in Refs. [692, 693] are in numerical agreement for the short-distance coefficients for $J / \psi$ production at the Tevatron. The calculations confirm that the corrections to the color-octet ${ }^{3} S_{1}$ and ${ }^{1} S_{0}$ channels are small, but also show that there is a large, negative $K$ factor in the color-octet ${ }^{3} P_{J}$ channel.

In Ref. [692], the NLO calculation was fit to the CDF data for the prompt production of the $J / \psi$ and the $\psi(2 S)$ $[329,657]$, and values were obtained for two linear combinations of NRQCD long-distance matrix elements. In the case of the $J / \psi$, these fits took into account feeddown from the $\psi(2 S)$ state and the $\chi_{c J}$ states, where the

\footnotetext{
12 We note that the LO CSM result for the $p_{T}$-integrated direct $J / \psi$ cross section $d \sigma / d y$, evaluated at $y=0$ (Ref. [690]), is compatible with the CDF [657] and PHENIX [659,663] measurements.
} 
latter was obtained from the NLO calculation of $\chi_{c}$ production [675] that was described above. Satisfactory fits could not be obtained to the experimental data points for the $J / \psi$ and the $\psi(2 S)$ with $p_{T}<7 \mathrm{GeV}$, and so these points were excluded from the fits. The fitted values of the linear combinations of matrix elements were used to predict the cross section for $J / \psi$ production at CMS, and good agreement with the CMS data [694] was obtained. This analysis suggests the possibility that that the cross section is dominated by the color-octet ${ }^{1} S_{0}$ contribution, rather than by the color-octet ${ }^{3} S_{1}$ contribution, in contrast with conclusions that had been drawn on the basis of LO fits to the Tevatron data.

In Ref. [693], values of the NRQCD long-distance matrix elements were extracted by using the NLO calculation of $J / \psi$ hadroproduction of Ref. [693] and an NLO calculation of $J / \psi$ photoproduction from Ref. [695] to make a combined fit to the CDF Run II data for prompt $J / \psi$ production [657] and to the HERA I and HERA II H1 data for prompt $J / \psi$ photoproduction [696, 697]. In this fit, only CDF data with $p_{T}>3 \mathrm{GeV}$ were used, as the flattening of the cross section at smaller values of $p_{T}$ cannot be described by fixed-order perturbation theory. Feeddown of the $\psi(2 S)$ and $\chi_{c J}$ states to the $J / \psi$ was not taken into account in the fits. This is the first multiprocess fit of NRQCD long-distance matrix elements for quarkonium production. The values of the NRQCD long-distance matrix elements that were obtained in this fit do not differ greatly from those that were obtained in LO fits. They were used to predict the cross sections for prompt $J / \psi$ production at PHENIX [662] and CMS [694], and good agreement with the data was achieved in both cases.

The values of the linear combinations of $J / \psi$ NRQCD long-distance matrix elements that were obtained in Ref. [692] are not consistent with the values of the NRQCD long-distance matrix elements that were obtained in Ref. [693]. Since the calculations of Refs. [692, 693] are in agreement on the short-distance cross sections, any discrepancies in the extracted NRQCD long-distance matrix elements must be due to differences in the fitting procedures. Clearly, it is necessary to understand the significance of the various choices that have been made in the fitting procedures before any definite conclusions can be drawn about the sizes of the NRQCD long-distance matrix elements.

\section{$J / \psi$ production at $R H I C$}

Recently, the STAR collaboration at RHIC has reported an analysis of prompt $J / \psi$ production for values of $p_{T}$ up to $12 \mathrm{GeV}$ (Ref. [664]). In Ref. [664], the measured production rate as a function of $p_{T}$ is compared with predictions based on NRQCD factorization at LO [698] and the CSM up to $\mathrm{NNLO}^{\star}$ accuracy [620]. The calculations do not include feeddown from the $\psi(2 S)$ and the $\chi_{c}$ states. The data clearly favor the NRQCD factorization prediction over the CSM prediction. However, no definite conclusions can be drawn because the effects of feeddown have not been taken into account.

A calculation of prompt $J / \psi$ production at RHIC, including feeddown from the $\psi(2 S)$ and $\chi_{c}$ states, has been carried out in Ref. [699] in the CSM and the NRQCD factorization formalism at LO. In Fig. 53, we show a comparison between the predictions of Ref. [699] for the prompt $J / \psi$ cross section as a function of $p_{T}$ and data from the PHENIX collaboration [662, 663]. Again, the NRQCD predictions are favored over the CSM predictions. However, in this case, the small values of $p_{T}$ involved may call into question the validity of perturbation theory, and the omission of higher-order corrections to the CSM, which are known to be large, also undermines the comparison.

Higher-order corrections to the color-singlet contribution to $J / \psi$ production at RHIC have been considered in Ref. [700] and were found to be large. A comparison between the predictions of Ref. [700] for the cross section differential in $p_{T}$ and the PHENIX and STAR prompt $J / \psi$ data is shown in Fig. 54. The color-singlet contributions through NLO agree with the PHENIX prompt $J / \psi$ data for $p_{T}$ in the range $1-2 \mathrm{GeV}$, but fall substantially below the PHENIX and STAR prompt $J / \psi$ data for larger values of $p_{T}$. The $\mathrm{NNLO}^{\star}$ color-singlet contribution can be computed reliably only for $p_{T}>5 \mathrm{GeV}$. The upper limit of the theoretical uncertainty band for the $\mathrm{NNLO}^{\star}$ contribution is compatible with the PHENIX

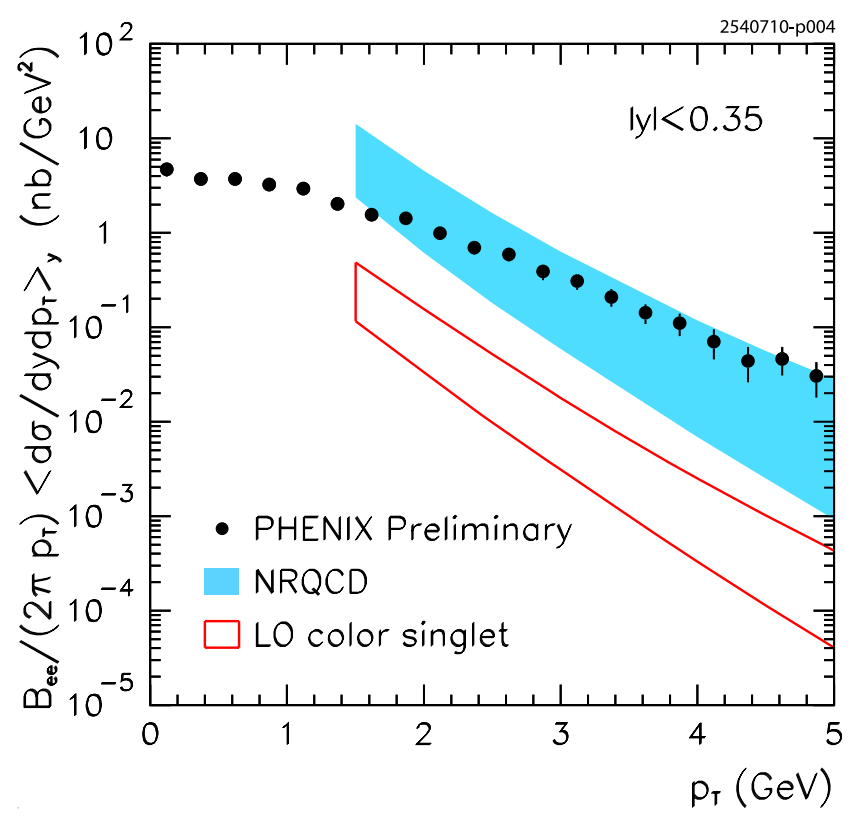

FIG. 53: Comparison of the LO NRQCD and the LO CSM predictions for the $J / \psi$ cross section as a function of the $J / \psi$ transverse momentum [699] with the data from the PHENIX collaboration [662, 663]. The theoretical uncertainty bands were obtained by combining the uncertainties from $m_{c}$ and the NRQCD long-distance matrix elements with the uncertainties that are obtained by varying the renormalization scale $\mu_{r}$ and the factorization scale $\mu_{f}$ between $2 m_{T}$ and $m_{T} / 2$. Here $m_{T}=\sqrt{4 m_{c}^{2}+p_{T}^{2}}$. From [699] with kind permission, copyright (2010) The American Physical Society 
and STAR data, although the theoretical uncertainties are very large.

As we have mentioned above, the NLO analysis of Ref. [693], which includes the ${ }^{3} S_{1},{ }^{1} S_{0}$, and ${ }^{3} P_{J}$ coloroctet channels, uses matrix elements that are extracted from a combined fit to CDF data [657] and $\mathrm{H} 1$ data $[696,697]$ to predict the cross sections for $J / \psi$ production at HERA. This prediction agrees well with PHENIX data [662].

\section{Exclusive production of charmonia}

The exclusive production of charmonium states (plus beam particles) has also been observed at hadron-hadron colliders. In most current theoretical models, the exclusive production of states with charge parity -1 , such as the $J / \psi$ or the $\psi(2 S)$, is dominated by the process of photon-Pomeron fusion (photoproduction), while the exclusive production of states with charge parity +1 , such as the $\chi_{c 0}$, is dominated by the process of PomeronPomeron fusion. In perturbative model calculations, the Pomeron is represented as an exchange of two or more

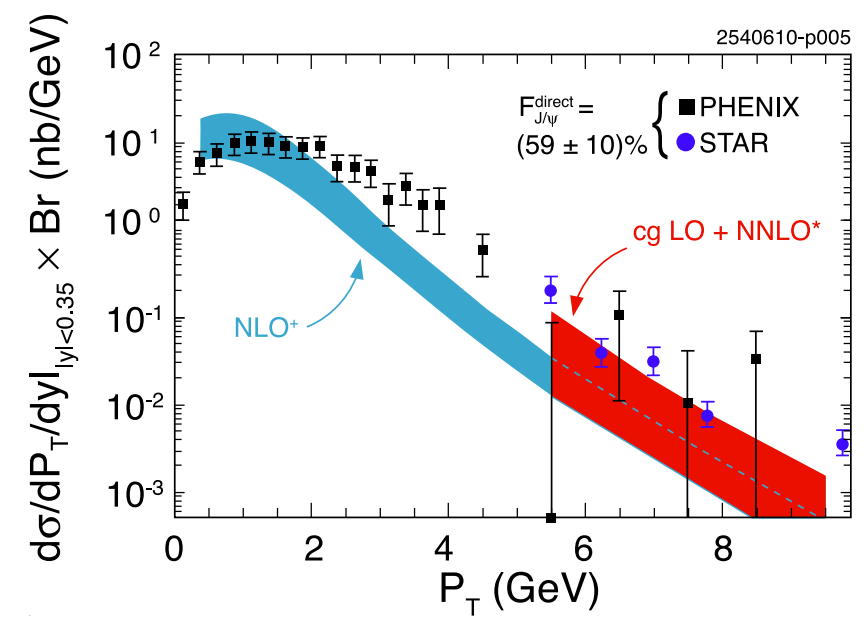

FIG. 54: Comparison between CSM prediction for the $J / \psi$ cross section at NLO accuracy as a function of the $J / \psi$ transverse momentum in $p p$ collisions at RHIC at $\sqrt{s_{N N}}=$ $200 \mathrm{GeV}$ and $|y|<0.35$ (Ref. [700]) and the PHENIX [659] and STAR [664] prompt $J / \psi$ data. The $\mathrm{NLO}^{+}$contribution contains the contributions from $g g$ and $g q$ fusion at NLO accuracy, where $q$ is a light quark, plus the contribution from $c g$ fusion at LO accuracy. The theoretical uncertainty band for the $\mathrm{NLO}^{+}$contribution was obtained by combining the uncertainties that are obtained by varying $m_{c}$ in the range $1.4 \mathrm{GeV}<m_{c}<1.6 \mathrm{GeV}$ and by varying the factorization scale $\mu_{f}$ and the renormalization scale $\mu_{r}$ through the values $((0.75,0.75) ;(1,1) ;(1,2) ;(2,1) ;(2,2)) \times m_{T}$. Here $m_{T}=\sqrt{4 m_{c}^{2}+p_{T}^{2}}$. The theoretical uncertainty band for the $\mathrm{NNLO}^{\star}$ contribution was obtained by combining the uncertainties that are obtained by varying $m_{c}$ in the range $1.4 \mathrm{GeV}<m_{c}<1.6 \mathrm{GeV}$, by varying $\mu_{f}$ and $\mu_{r}$ in the range $0.5 m_{T}<\mu_{f}=\mu_{r}<2 m_{T}$, and by varying the infrared cutoff $s_{i j}^{\text {min }}$ in the range $2.25 \mathrm{GeV}^{2}<s_{i j}^{\text {min }}<9.00 \mathrm{GeV}^{2}$. From Ref. [700] gluons in a color-singlet state. Exclusive quarkonium production could provide an important tool with which to probe these mechanisms.

The CDF Collaboration has measured exclusive $J / \psi$, $\psi(2 S)$, and $\chi_{c 0}$ production in $p \bar{p}$ collisions at $\sqrt{s}=$ $1.96 \mathrm{TeV}$ (Ref. [701]). The CDF measurements are in agreement with theoretical predictions that are based on models for the Pomeron [702-710]. The PHENIX collaboration has measured exclusive $J / \psi$ production in $\mathrm{Au}+\mathrm{Au}$ collisions at $\sqrt{s_{N N}}=200 \mathrm{GeV}$ (Ref. [711]) and also finds agreement with theoretical predictions [712719]. See Sect. 5.7 for more on photoproduction in nuclear collisions.

X(3872) production at the Tevatron and the LHC

Since the discovery of the $X(3872)$ by the Belle collaboration in 2003 (Ref. [85]), this state has attracted a large interest in the particle-physics community. The $X(3872)$ is the exotic state for which the largest experimental data set is available. The production of the $X(3872)$ at the Tevatron has been analyzed by both the CDF collaboration [88-90] and the $\mathrm{D} \varnothing$ collaboration [91] in the $J / \psi \pi^{+} \pi^{-}$decay channel. The CDF collaboration has shown that most of the $X(3872)$ 's at the Tevatron are produced promptly, rather than through $b$-hadron decays [720].

As is discussed in Sects. 2.3.1, 2.9.3, and 2.9.4 of this article, the exact nature of the $X(3872)$ is still subject to debate. Nevertheless, it is plausible that the prompt production rate of the $X(3872)$ can be predicted correctly in the factorization framework of NRQCD. The reason for this is that, in all the viable hypotheses as to the nature of the $X(3872)$, the particle content of the state includes a charm-quark pair with a relative momentum $q \ll m_{c}$. The small size of $q$ suggests that one can make use of the NRQCD expansion of the production rate in powers of $q / m_{c}$. It follows that the expression for the cross section is given by the NRQCD factorization formula in Eq. (154). It was argued in Refs. [332, 721] that it is reasonable to truncate the NRQCD series so that it includes only contributions to the $X(3872)$ production rate from $c \bar{c}$ pairs that are created in an $S$-wave configuration. Moreover, in the case of hadroproduction, it was argued that a truncation that retains only the coloroctet ${ }^{3} S_{1}$ channel would provide a reliable prediction at large transverse momentum. It follows that the corresponding NRQCD long-distance matrix element can be extracted from the measured production rate at the Tevatron in the $J / \psi \pi^{+} \pi^{-}$decay channel. In Ref. [332], the aforementioned simplifying assumptions are used in the NRQCD factorization framework to predict the prompt production rate for $X(3872) \rightarrow J / \psi \pi^{+} \pi^{-}$as a function of $p_{T}$ for various LHC experiments. In the same work, the production of the $X(3872)$ from $b$-hadron decays is discussed. The data samples at the LHC are predicted to be large, suggesting that the $X(3872)$ can be studied very effectively at the LHC. Measurements of the prompt production rate at the $\mathrm{LHC}$ as a function of $p_{T}$ would 
provide a key test of the NRQCD factorization approach to $X(3872)$ hadroproduction.

\subsubsection{Quarkonium polarization: a key observable}

Measurements of quarkonium polarization observables may yield information about quarkonium production mechanisms that is not available from the study of unpolarized cross sections alone.

The three polarization states of a $J=1$ quarkonium can be specified in terms of a particular coordinate system in the rest frame of the quarkonium. This coordinate system is often called the "spin-quantization frame." In a hadron collider, the $J / \psi, \psi(2 S)$ and $\Upsilon$ resonances are reconstructed through their electromagnetic decays into a lepton pair. The information about the polarization of the quarkonium state is encoded in the angular distribution of the leptons. This angular distribution is usually described in the quarkonium rest frame with respect to a particular spin-quantization frame. In that case, the angular distribution of the quarkonium can be expressed in terms of three real parameters that are related to the spin-polarization amplitudes of the $J=1$ quarkonium state.

In hadron-hadron collisions, polarization analyses are often restricted to the measurement of the distribution as a function of the polar angle with respect to the chosen spin-quantization axis. This distribution is parametrized as

$$
1+\alpha \cos ^{2} \theta_{\ell \ell}
$$

The parameter $\alpha$ in Eq. (157) is directly related to the fraction of the cross section that is longitudinal (or transverse) with respect to the chosen spin-quantization axis: $\alpha=1$ corresponds to $100 \%$ transverse polarization; $\alpha=-1$ corresponds to $100 \%$ longitudinal polarization.

In experimental analyses, knowledge of the angular distribution of dileptons from quarkonium decay is important because, typically, detector acceptances fall as dileptons are emitted more along the direction of the quarkonium momentum - especially at small $p_{T}$. This effect is included in the corrections to the experimental acceptance. However, it induces systematic experimental uncertainties.

In theoretical calculations, polarization parameters, such as the polar asymmetry $\alpha$, can be expressed in terms of ratios of polarized quarkonium cross sections. In some cases, these ratios are less sensitive than the production cross sections to the theoretical uncertainties from quantities such as the factorization scale, the renormalization scale, the heavy-quark mass, and the NRQCD long-distance matrix elements. For example, in the cases of the production of the $J / \psi$ or the $\psi(2 S)$ in the NRQCD factorization formalism, the polarization parameter $\alpha$ depends, to good approximation, on ratios of color-octet long-distance matrix elements, but not on their magnitudes.
One should keep in mind that a measurement of the polar asymmetry parameter $\alpha$ alone does not give complete information about the polarization state of the produced quarkonium. The importance of measuring all of the parameters of the dilepton angular distribution for a variety of choices of the spin-quantization frame has been emphasized in Refs. [722, 723]. The significance of the information that is obtained in measuring $\alpha$ alone depends very much on the orientation of the spin-quantization axis. So far, most of the theoretical studies of polarization in quarkonium production have been carried out for the case in which the spin-quantization axis is taken to be along the direction of the quarkonium momentum in the laboratory frame $[618,620,639-642,652,665-$ $667,672,674,680-682,724,725]$. That choice of spinquantization axis [665] is often referred to as the "helicity frame." In Ref. [726], it is shown that one can make more sophisticated choices of the spin-quantization axis, which involve not only the kinematics of the quarkonium state, but also the kinematics of other produced particles. These alternative choices of spin-quantization axis can increase the significance of the measurement of $\alpha$. However, their optimization requires knowledge of the dominant quarkonium production mechanism.

Experimental measurements of quarkonium polarization have been made for a variety of spin-quantization frames. Measurements by the CDF [656, 658, 669], DØ [671], and PHENIX [661-663] collaborations were carried out in the helicity frame, while some measurements at fixed-target experiments [727, 728] were carried out in the Collins-Soper frame [729]. Recently, the Hera-B collaboration has analyzed quarkonium polarizations [730] not only in the helicity and Collins-Soper frames, but also in the Gottfried-Jackson frame [731], in which the spin-quantization axis is along the direction of the incident beam. In Ref. [722], a global analysis was made of polarization measurements that were carried out in the Collins-Soper and helicity frames. That analysis shows that the results that were obtained in these two spin-quantization frames are plausibly compatible when the experimental rapidity ranges are taken into account. However, it is clear that additional analyses in different spin-quantization frames would be very informative.

According to the CDF Run II measurement of the $\psi$ polarization in the helicity frame [658], the prompt $\psi$ yield becomes increasingly longitudinal as $p_{T}$ increases. The disagreement of this result with a previous CDF polarization measurement that was based on Run I data [656] has not been resolved. In Fig. 55 the CDF measurement of the polarization parameter $\alpha$ for the prompt $J / \psi$ production at the Tevatron in Run II is compared with the NRQCD factorization prediction at LO in $\alpha_{s}$ (Ref. [667]). This prediction ignores possible violations of the heavy-quark spin symmetry, which appear at relative order $v^{3}$. The effects of feeddown from the $\psi(2 S)$ and the $\chi_{c J}$ states are taken into account in the NRQCD factorization prediction. However, it should be kept in mind that the corrections at NLO in $\alpha_{s}$ to 
the $\chi_{c}$ production rate are large [675] and are not taken into account in the NRQCD prediction in Fig. 55. The solid line in Fig. 55 is the prediction from the $k_{T}$ factorization approach [640], which includes only color-singlet contributions.

At LO accuracy in $\alpha_{\mathrm{s}}$, the NRQCD factorization prediction for the $J / \psi$ polarization clearly disagrees with the observation of a very small polar asymmetry in the helicity frame. One obvious issue is the effect of corrections of higher order in $\alpha_{\mathrm{s}}$ on the NRQCD factorization prediction.

Corrections of higher order in $\alpha_{\mathrm{s}}$ to $J / \psi$ production via the color-singlet channel dramatically affect the polarization in that channel. While the prediction at LO in $\alpha_{\mathrm{s}}$ for the helicity of the $J / \psi$ in the color-singlet channel is mainly transverse at medium and large $p_{T}$, calculations at NLO or $\mathrm{NNLO}^{\star}$ accuracy for the color-singlet channel reveal a polarization that is increasingly longitudinal as $p_{T}$ increases, as can be seen in Fig. 56. A similar trend for the polarization as a function of $p_{T}$ is found in some other analyses of the color-singlet channel, such those in the $k_{T}$ factorization approach [639-642] (see Fig. 55), the gluon-tower approach [680], and the $s$-channel- $Q \bar{Q}$ cut approach [681, 682].

In the case of the color-octet ${ }^{3} S_{1}$ channel, the NLO correction to the helicity of the $J / \psi$ is very small [674].

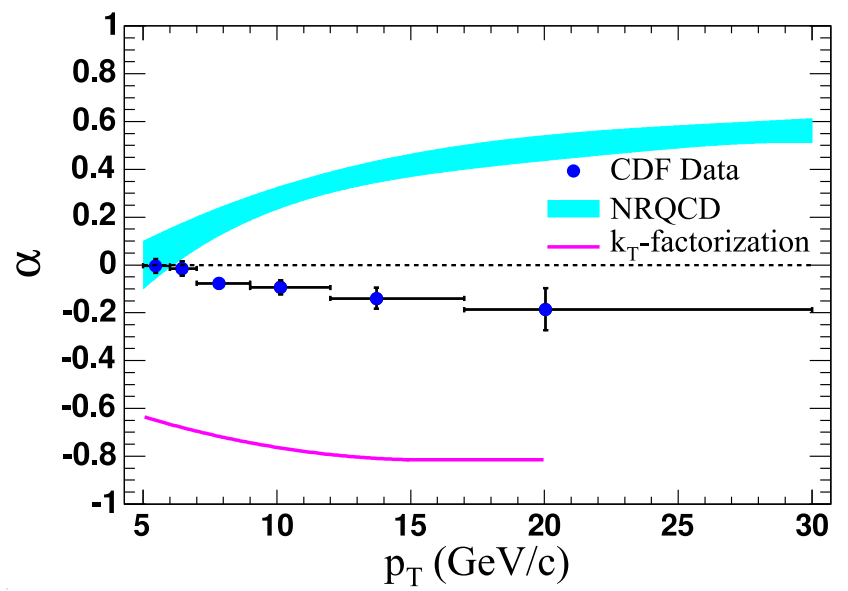

FIG. 55: The polarization parameter $\alpha$ for prompt $J / \psi$ production in $p \bar{p}$ collisions at $\sqrt{s}=1.96 \mathrm{TeV}$ as a function of $p_{T}$. The points are the CDF data [658], the band is the prediction from LO NRQCD factorization [667], and the line is the prediction from $k_{T}$ factorization [640]. The theoretical uncertainty in the LO NRQCD factorization prediction was obtained by combining the uncertainties from the parton distributions (estimated by comparing the MRST98LO (Ref. [732]) and the CTEQ5L (Ref. [733]) distributions), the uncertainties from the color-octet NRQCD long-distance matrix elements, the uncertainties that are obtained by varying $m_{c}$ in the range $1.45 \mathrm{GeV}<m_{c}<1.55 \mathrm{GeV}$, and the uncertainties that are obtained by varying the factorization and renormalization scales in the range $0.5 m_{T}<\mu_{f}=\mu_{r}<2 m_{T}$. Here $m_{T}=\sqrt{4 m_{c}^{2}+p_{T}^{2}}$. From [658] with kind permission, copyright (2007) The American Physical Society
This NLO correction would not change substantially the comparison between the NRQCD factorization prediction and the experimental data that is shown in Fig. 55. As we have explained in Sect. 4.2.2, the NLO analysis of Ref. [692] suggests the possibility that the $J / \psi$ directproduction cross section is dominated by the color-octet ${ }^{1} S_{0}$ contribution, rather than by the color-octet ${ }^{3} S_{1}$ contribution, even at the largest values of $p_{T}$ that are accessed in the Tevatron measurements. However, the NLO analysis in Ref. [693] concludes that the color-octet ${ }^{3} S_{1}$ contribution at NLO is not very different from that at LO. A complete NLO analysis of the direct $J / \psi$ polarization, including the contribution of the color-octet ${ }^{3} P_{J}$ channel is still lacking and is an important theoretical goal. Further progress in determining the relevant production mechanisms would be aided significantly by highstatistics measurements of the polarizations of the $J / \psi$, the $\chi_{c J}$, and the $\psi(2 S)$ in direct production.

The polarization of the $J / \psi$ has also been measured in hadronic collisions at $\sqrt{s}=200 \mathrm{GeV}$. Data from the PHENIX collaboration for prompt $J / \psi$ polarization as a function of $p_{T}$ (Refs. [662, 663]) exist in the range $0<p_{T}<3 \mathrm{GeV}$ and indicate a polarization that is compatible with zero, with a trend toward longitudinal polarization as $p_{T}$ increases. Comparisons of the data with LO calculations in the CSM and the NRQCD factorization formalism [699] are given in Refs. [662, 699]. As can be seen from Fig. 57, the data favor the NRQCD factorization prediction and are in agreement with it. However, the small values of $p_{T}$ involved may call into question the validity of the NRQCD factorization formula. The NLO color-singlet contribution to the $J / \psi$ polarization at RHIC has been computed in Ref. [700]. A comparison of this prediction with the PHENIX prompt $J / \psi$ data differential in $p_{T}[661,662]$ is shown in Fig. 58. As can be seen, the CSM contributions to the polarization through NLO are in agreement with the PHENIX prompt $J / \psi$ data.

The polarization of the $\Upsilon(1 S)$ in prompt produc-

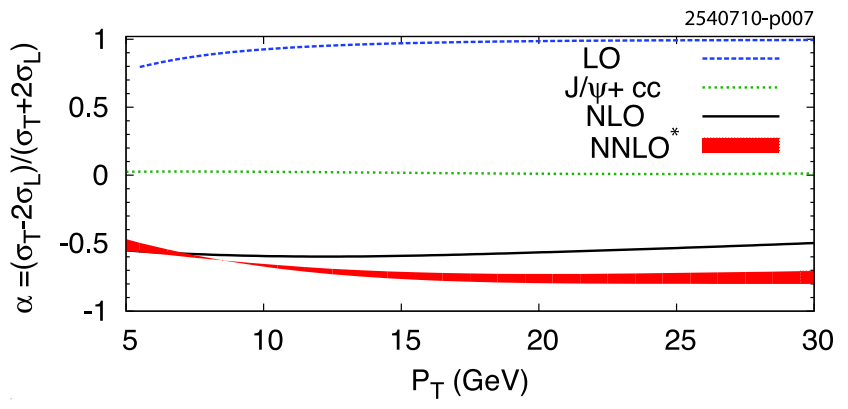

FIG. 56: Predictions for the polarization parameter $\alpha$ for direct $J / \psi$ production in the color-singlet channel in $p \bar{p}$ collisions at the Tevatron at $\sqrt{s}=1.96 \mathrm{TeV}$ at LO, NLO and $\mathrm{NNLO}^{\star}$ accuracy [652]. Most of the uncertainties in $\alpha$ for the $\mathrm{LO}, J / \psi+c \bar{c}$, and NLO cases cancel. The theoretical uncertainty band for the $\mathrm{NNLO}^{\star}$ case was obtained by varying the infrared cutoff $s_{i j}^{\min }$ between $2 m_{c}^{2}$ and $m_{c}^{2} / 2$ 


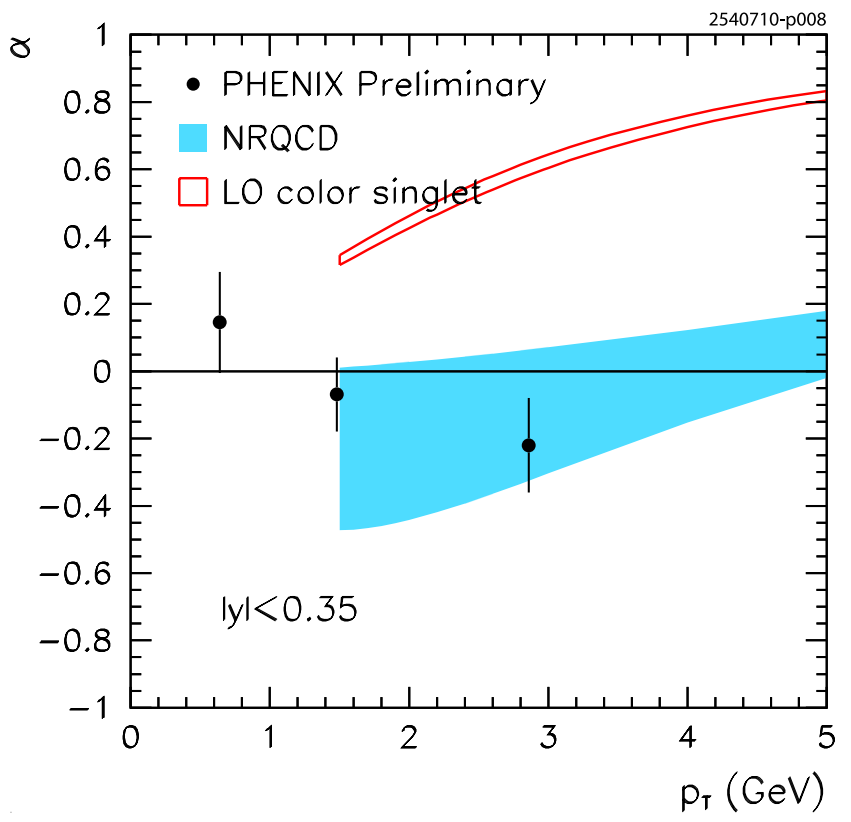

FIG. 57: Comparison of the LO NRQCD and LO CSM predictions for the prompt $J / \psi$ polarization in $p p$ collisions at RHIC at $\sqrt{S_{n n}}=200 \mathrm{GeV}$ and $|y|<0.35$ (Ref. [699]) with the data from the PHENIX collaboration [662, 663]. The theoretical uncertainty bands were obtained by combining the uncertainties from $m_{c}$ and the NRQCD long-distance matrix elements with the uncertainties that are obtained by varying the renormalization scale $\mu_{f}$ and the factorization scale $\mu_{r}$ between $2 m_{T}$ and $m_{T} / 2$. Here $m_{T}=\sqrt{4 m_{c}^{2}+p_{T}^{2}}$. From [699] with kind permission, copyright (2010) The American Physical Society

tion has been measured by both the CDF collaboration (Run I) [669] and by the DØ collaboration (Run II) [671]. The DØ measurement has substantially larger experimental uncertainties than the CDF measurement. The CDF collaboration has recently reported a new preliminary measurement of the polarization of prompt $\Upsilon$ 's that is based on a larger data set from Run II [734]. This Run II measurement is consistent with the CDF Run I measurement. The results of the CDF Run II and $\mathrm{D} \varnothing$ measurements are shown in Fig. 59, along with the NRQCD factorization prediction at LO in $\alpha_{s}$ (Ref. [735]). The origin of the large discrepancy between the CDF and $\mathrm{D} \varnothing$ data is unclear. However, we note that the CDF measurement was made over the rapidity interval $|y|<0.6$, while the $\mathrm{D} \varnothing$ measurement was made over the rapidity interval $|y|<1.8$. The NRQCD factorization prediction in Ref. [735] was integrated over the range $|y|<0.4$ (Ref. [736]). The LO NRQCD factorization prediction is marginally compatible with the CDF data at medium $p_{T}$ and incompatible with the CDF data at large $p_{T}$, while the LO NRQCD factorization prediction is incompatible with the $\mathrm{D} \varnothing$ data at medium $p_{T}$ and compatible with the $\mathrm{D} \emptyset$ data at large $p_{T}$. The effects of higher-order QCD corrections on the polarization of direct $\Upsilon$ 's produced via the color-singlet channel are shown in Fig. 60. The

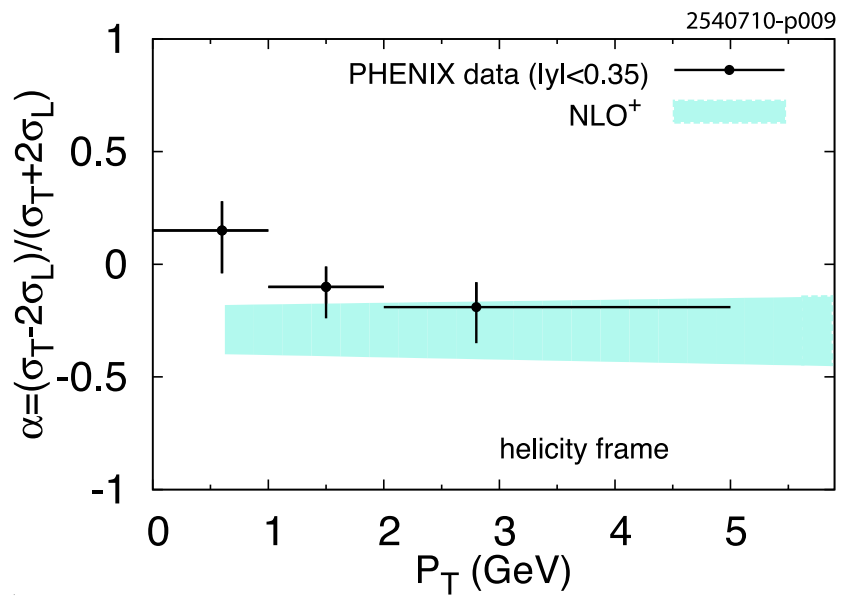

FIG. 58: Comparison of the NLO CSM calculation of $J / \psi$ polarization in $p p$ collisions at RHIC at $\sqrt{S_{N N}}=200 \mathrm{GeV}$ and $|y|<0.35$ (Ref. [700]) with the prompt $J / \psi$ polarization data from the PHENIX collaboration [661, 662]. The theoretical uncertainty band was obtained by combining the uncertainties that are obtained by varying $m_{c}$ in the range $1.4 \mathrm{GeV}<m_{c}<1.6 \mathrm{GeV}$, by varying the factorization scale $\mu_{f}$ and the renormalization scale $\mu_{r}$ over the values $((0.75,0.75) ;(1,1) ;(1,2) ;(2,1) ;(2,2)) \times m_{T}$. Here $m_{T}=$ $\sqrt{4 m_{c}^{2}+p_{T}^{2}}$. From Ref. [700]

higher-order corrections in this case have the same qualitative features as in the case of $\psi$ : The higher-order corrections change the polarization from nearly $100 \%$ transverse to substantially longitudinal. A complete computation of the prompt $\Upsilon$ polarization at NLO in the NRQCD factorization, including feeddown from $\chi_{b J}$ states, is not yet available.

\subsubsection{New observables in hadroproduction}

It may be useful, in order to progress in the understanding of the mechanisms that are responsible for heavy-quarkonium production, to identify, to compute, and to measure new observables.

As we have seen from previous discussions, the $\psi$ and $\Upsilon$ production rates in hadron-hadron collisions, differential in $p_{T}$, are complicated to calculate because the dominant color-singlet channels at large $p_{T}$ arise beyond LO in $\alpha_{s}$. In the case of the $\Upsilon$, higher-order corrections bring the color-singlet contribution close to the Tevatron data. However, in the case of $\psi$, there is a gap, which increases with increasing $p_{T}$, between the higher-order color-singlet contributions and the data. In both cases, the uncertainties are very large and, in the $\Upsilon$ case, they are too large to make a definite statement about the relative proportions of color-singlet and color-octet contributions. However, it is worth noting that the uncertainties in the higher-order color-singlet contributions affect the normalization of the differential rates much more than the shape. This suggests the use of observables that do 


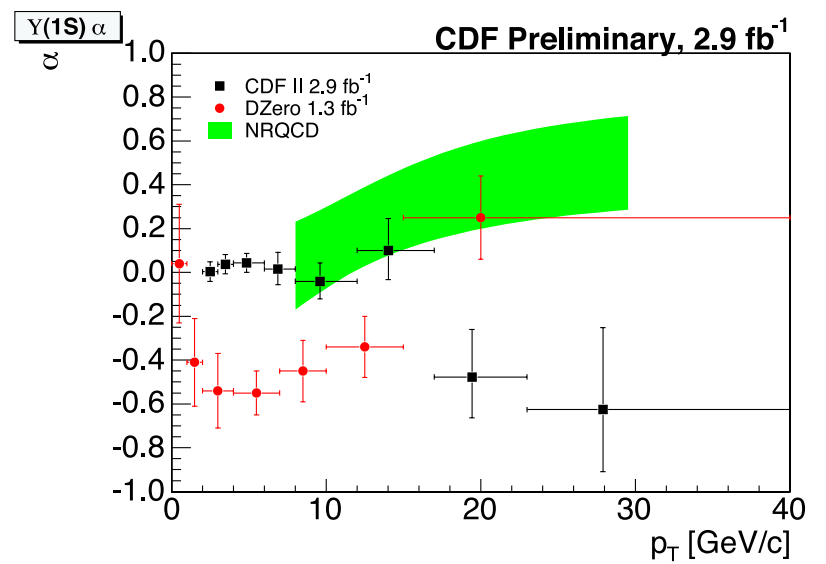

FIG. 59: The polarization parameter $\alpha$ in the helicity frame for prompt $\Upsilon(1 S)$ production in $p \bar{p}$ collisions at $\sqrt{s}=$ $1.96 \mathrm{TeV}$. The NRQCD factorization prediction at LO in $\alpha_{s}$ (Ref. [735]) is compared with the data of the CDF collaboration [734] and the $\mathrm{D} \varnothing$ collaboration [671]. The CDF measurement was made over the rapidity interval $|y|<0.6$, while the $\mathrm{D} \varnothing$ measurement was made over the rapidity interval $|y|<1.8$. The NRQCD factorization prediction in Ref. [735] was integrated over the range $|y|<0.4$ (Ref. [736]) and includes feeddown from the $\Upsilon(2 S), \Upsilon(3 S), \chi_{b}(1 P)$, and $\chi_{b}(2 P)$ states. The theoretical uncertainty band was obtained by combining the uncertainties from the NRQCD long-distance color-singlet and color-octet matrix elements, $m_{b}$, the parton distributions, and the quarkonium branching fractions with uncertainties that are obtained by varying the renormalization and factorization scales from $\mu_{T} / 2$ to $2 \mu_{T}$. Here $\mu_{T}=\sqrt{m_{b}^{2}+p_{T}^{2}}$. Figure provided by Hee Sok Chung, using Ref. [737], which is based on the analysis of Ref. [734]

not depend on the total rate. One interesting proposal is to compare ratios of differential cross sections at different values of $\sqrt{s}$, the center-of-mass energy of the colliding hadrons [738]. For a given color channel, this ratio can be predicted quite accurately. If the ratios that are associated with the color-singlet and color-octet channels are sufficiently well separated, then the relative sizes of the color-singlet and color-octet contributions could be extracted by comparing the measured production rate at the Tevatron with the measured production rate at the LHC.

One interesting observable is the hadronic activity near the quarkonium direction [739] or, more generally, the $J / \psi$-hadron azimuthal correlation. The UA1 collaboration compared their charged-track distributions with Monte Carlo simulations for a $J / \psi$ produced in the decay of a $b$ hadron and a $J / \psi$ produced in feeddown from a $\chi_{c J}$ state $[740,741]$. At the time of the UA1 study, $\chi_{c}$ feeddown was still expected to be the dominant source of prompt $J / \psi$ 's. In either the NRQCD factorization formalism or the CSM at higher orders in $\alpha_{s}$, it is expected that the production process for prompt $J / \psi$ 's is more complex than the $\chi_{c J}$-feeddown process alone. Recently, the STAR collaboration reported the first measurement of the $J / \psi$-hadron azimuthal correlation at

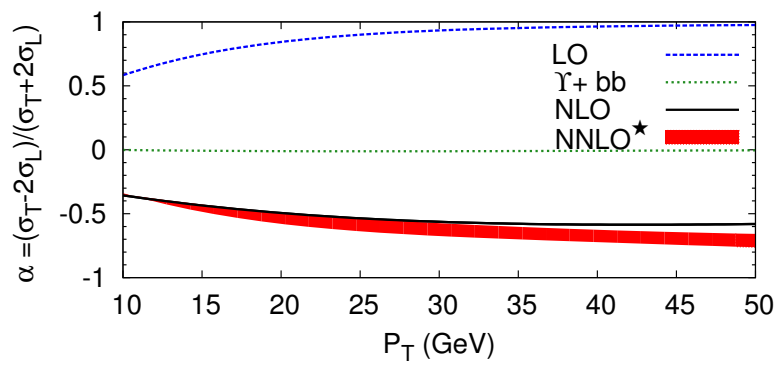

FIG. 60: Polarization parameter $\alpha$ for direct $\Upsilon$ production in the color-singlet channel in $p \bar{p}$ collisions at $\sqrt{s}=1.96 \mathrm{TeV}$ at LO, NLO and $\mathrm{NNLO}^{\star}$ accuracy. Most of the uncertainties in $\alpha$ for the LO, $\Upsilon+b \bar{b}$, and NLO cases cancel. The theoretical uncertainty band for the $\mathrm{NNLO}^{\star}$ case was obtained by varying the infrared cutoff $s_{i j}^{\min }$ between $2 m_{b}^{2}$ and $m_{b}^{2} / 2$ and by varying $\mu_{f}$ and $\mu_{r}$ between $m_{T} / 2$ and $2 m_{T}$. Here $m_{T}=\sqrt{4 m_{c}^{2}+p_{T}^{2}}$. The variations with respect to $\mu_{f}$ and $\mu_{r}$ are negligible in comparison with the variation with respect to $s_{i j}^{\min }$. From [620] with kind permission, copyright (2008) The American Physical Society

RHIC [664, 742]. The STAR collaboration compared its measurement with up-to-date LO PYTHIA predictions ${ }^{13}$ and found no significant hadronic yield in the direction of the $J / \psi$ beyond that which is expected from the LO PYTHIA predictions. Observation of the hadronic activity around the quarkonium might help to disentangle color-octet contributions from color-singlet contributions to the inclusive production process. One expects additional hadronic activity around the quarkonium in color-octet production. However, in practice, it may be difficult to identify this hadronic activity [739] because there are competing effects, such as the suppression of collinear gluon radiation from massive particles (deadcone effect) and the suppression of soft gluon radiation from color-singlet objects, both of which would be difficult to compute reliably.

It would be useful to identify additional observables that can be computed reliably in order to test the many production models that are available [1, 651]. One such observable could be the rate of production of heavy-flavor mesons in association with a quarkonium. Final states that could be studied include $\psi+c \bar{c}$ and $\Upsilon+b \bar{b}$. Associated production could be investigated first in $p p$ collisions and subsequently in $p A$ and $A A$ collisions. The study of associated production in hadron collisions is motivated by measurements that were carried out at the $B$ factories that show that, in a surprisingly large fraction of $J / \psi$ events, a second $c \bar{c}$ pair is produced. (See Sect. 4.6.) It is not yet known whether such a large fraction of $J / \psi+c \bar{c}$ events occurs in hadroproduction. Analyses

13 Note that one expects such predictions to be affected by channels that appear beyond LO in $\alpha_{s}$. 
at the Tevatron and at RHIC are already possible. The LO prediction for associated production at the Tevatron at $\sqrt{s}=1.96 \mathrm{TeV}$ has been computed in Ref. [618] and shows that the integrated cross sections are significant:

$$
\begin{aligned}
\sigma(J / \psi+c \bar{c}) \times \mathcal{B}\left(J / \psi \rightarrow \mu^{+} \mu^{-}\right) & \approx 1 \mathrm{nb} \\
\sigma(\Upsilon+b \bar{b}) \times \mathcal{B}\left(\Upsilon \rightarrow \mu^{+} \mu^{-}\right) & \approx 1 \mathrm{pb}
\end{aligned}
$$

In order to illustrate the measurement potential at RHIC, the author of Ref. [652] computed the differential cross sections as a function of $p_{T}$ and found them to be on the order of $1 \mathrm{pb} / \mathrm{GeV}$ at $p_{T}=5 \mathrm{GeV}$ for the STAR kinematics. Measurements of such processes would provide tests of the NRQCD factorization formalism. They would also provide information about the color-transfer mechanism [635, 636] (see Sect. 4.1.7), which involves soft-gluon exchanges between comoving heavy particles and is known to violate standard NRQCD factorization. In this case, it would be useful to compare heavy-flavor activity near the quarkonium direction and away from the quarkonium direction.

A new observable that could be measured in existing and future experiments is the rate of production of a photon in association with a $J / \psi$ or an $\Upsilon$. The QCD corrections to the rates for these processes have been computed recently at NLO [724] and $\mathrm{NNLO}^{\star}$ [725] accuracy. As is argued in Ref. [725], a measurement of such processes would provide information on the quarkonium production mechanisms that is complementary to that which is provided by measurements of inclusive quarkonium production.

In order to facilitate phenomenological studies, an automated tree-level amplitude generator MadOnia [743] has been developed for processes involving quarkonium production or decay. It is now embedded in the online version of MadGraph/MadEvent [744] and, thus, is publicly available. A number of studies [618, 620, 676, 725, $745,746]$ have already taken advantage of the flexibility of MadOnia and of the possibility to interface it with showering and hadronization programs.

\subsubsection{Future opportunities}

The results of the past few years, on both the theoretical and experimental fronts, have yielded important clues as to the mechanisms that are at work in inclusive quarkonium hadroproduction. In general, however, theoretical uncertainties remain too large to draw any definite conclusions about the production mechanisms.

Regarding these uncertainties, one of the key issues is that, in color-singlet production channels, the mechanisms that are dominant at large $p_{T}$ appear only at higher orders in $\alpha_{s}$. (See Sects. 4.2.1 and 4.2.2.) In addition to the new complete calculations of NLO contributions, estimates have been made of the NNLO contributions to $\psi$ and $\Upsilon$ production in the color-singlet channels in several different frameworks (the fragmentation approximation, the $k_{T}$ factorization approach, the gluon-tower model, and the $\mathrm{NNLO}^{\star}$ approach). A more accurate treatment of higher-order corrections to the color-singlet contributions at the Tevatron and the LHC is urgently needed. Here, the re-organization of the perturbation series that is provided by the fragmentation-function approach (Sect. 4.1.5) may be an important tool.

Furthermore, the current theoretical predictions suffer from uncertainties that are related to the long-distance dynamics that is involved in quarkonium production. For example, the prediction of the $\psi$ or $\Upsilon$ polarization relies on the approximate heavy-quark spin symmetry of NRQCD. This approximate symmetry is based on the application of the velocity-scaling rules of NRQCD to evaluate the order of suppression of the spin-flip contribution. In the case of inclusive quarkonium decays, calculations of the NRQCD long-distance matrix elements on the lattice [233] have constrained the size of the spinflip contribution. A similar constraint in case of inclusive quarkonium production would obviously be very valuable.

More generally, lattice determinations of the NRQCD long-distance production matrix elements would provide very useful constraints on the theoretical predictions and would also serve to check the phenomenological determinations of the long-distance matrix elements. An outstanding theoretical challenge is the development of methods for carrying out such lattice calculations, which are, at present, stymied by fundamental issues regarding the correct lattice formulation of single-particle inclusive rates in Euclidean space.

Further light could be shed on the NRQCD velocity expansion and its implications for low-energy dynamics by comparing charmonium and bottomonium production. The heavy-quark velocity $v$ is much smaller in bottomonium systems than in charmonium systems. Hence, the velocity expansion is expected to converge more rapidly for bottomonium systems than for charmonium systems. In particular, spin-flip effects and color-octet contributions are expected to be smaller in bottomonium systems than in charmonium systems. The NRQCD factorization formula for inclusive quarkonium production, if it is correct, becomes more accurate as $p_{T}$ increases and probably holds only for values of $p_{T}$ that are greater than the heavy-quark mass. Therefore, the high- $p_{T}$ reach of the LHC may be crucial in studying bottomonium production.

There are many unresolved theoretical issues at present that bear on the reliability of predictions for prompt $J / \psi$ and $\Upsilon$ production. These issues may affect predictions for both the direct production of the $J / \psi$ and the $\Upsilon$ and the production of the higher-mass quarkonium states that feed down into the $J / \psi$ and the $\Upsilon$. Therefore, it would be of considerable help in disentangling the theoretical issues in $J / \psi$ and $\Upsilon$ production if experimental measurements could separate direct production of the $J / \psi$ and the $\Upsilon$ from production via feeddown from higher-mass charmonium and bottomonium states. Ideally, the direct production cross sections and polarizations would 
both be measured differentially in $p_{T}$. Measurement of the direct $J / \psi$ cross section and polarization might be particularly important at large $p_{T}$, given the large proportion of $\chi_{c J}$ feeddown events in the prompt $J / \psi$ rate at large $p_{T}$ that is predicted in Ref. [675].

Although it would be ideal to have measurements of direct quarkonium production rates and polarizations, it is, of course, very important to resolve the existing discrepancy between the CDF and D $\varnothing$ measurements of the prompt $\Upsilon$ polarization. The CDF measurement of the $\Upsilon(1 S)$ polarization is for the rapidity range $|y|<0.6$, while the $\mathrm{D} \varnothing$ measurement is for the rapidity range $|y|<1.8$. It would be very useful for the two experiments to provide polarization measurements that cover the same rapidity range.

It might also be useful to formulate new measurements and observables that would provide information that is complementary to that which is provided by the differential rates and polarization observables. The large rates for $J / \psi$ and $\Upsilon$ production that are expected at the LHC open the door to new analyses. As we have mentioned in Sect. 4.2.4, the possibilities include studies of quarkonium production at different values of $\sqrt{s}$, studies of hadronic energy near and away from the quarkonium direction, and studies of the production of heavy-flavor mesons in association with a quarkonium. It is important in all of these studies to identify observables that are accessible under realistic experimental conditions and that can be calculated accurately enough to allow meaningful comparisons with experimental measurements. In this endeavor, communication between the experimental and theoretical experts in these areas will be crucial.

\section{3. $e p$ collisions}

Inelastic production of charmonia in ep collisions at HERA proceeds via photon-gluon fusion: A photon emitted from the incoming electron or positron interacts with a gluon from the proton to produce a $c \bar{c}$ pair that evolves into a color-neutral charmonium state by the radiation of soft and/or hard gluons.

The elasticity observable $z$ is defined as the fraction of energy of the incoming photon, in the proton rest frame, that is carried by the final-state charmonium. The kinematic region of inelastic charmonium production is $0.05 \lesssim z<0.9$. In the so-called "photoproduction regime," at low photon virtuality $Q^{2}$, the incoming electron is scattered through a small angle, and the incoming photon is quasi real. The invariant mass of the $\gamma p$ system $W_{\gamma p}$ depends on the energy of the incoming photon. In photoproduction, photons can interact directly with the charm quark (direct processes), or via their hadronic component (resolved processes). Resolved processes are relevant at low elasticities $(z \lesssim 0.3)$. HERA has been a unique laboratory for the observation of photoproduction in the photon-proton center-of-mass range $20<W_{\gamma p}<320 \mathrm{GeV}$.
In $e p$ scattering at HERA, toward high values of elasticity $(z>0.95)$, another production mechanism that is distinctly different from boson-gluon fusion becomes dominant. In this mechanism, which applies both to exclusive and diffractive charmonium production, the incoming photon fluctuates into a $c \bar{c}$ QCD dipole state which, subsequently interacts with the proton by the exchange of two or more gluons in a colorless state. This colorless interaction transfers momentum that allows the $c \bar{c}$ pair to form a bound quarkonium state. Experiments distinguish between two categories of diffractive processes. In elastic processes, the proton stays intact, i.e., $\gamma p \rightarrow J / \psi p(z \approx 1)$. In proton-dissociative processes, the proton breaks up into a low-mass final state, i.e., $\gamma p \rightarrow J / \psi Y$. In proton-dissociative processes, $m_{Y}$, mass of the state $Y$, is less than about $2 \mathrm{GeV}$ or $z$ lies in the $0.95 \lesssim z \lesssim 1$. Many measurements of the diffractive production of the $\rho^{0}, \omega, \phi, J / \psi, \psi(2 S)$ and $\Upsilon$ states have been performed at HERA [747-758]. ${ }^{14}$ These measurements were crucial in reaching a new understanding of the partonic structure of hard diffraction, the distributions of partons in the proton, the validity of evolution equations in the low- $x$ limit, and the interplay between soft and hard QCD scales. For a detailed report on diffractive quarkonium production at HERA, we refer the reader to Ref. [759]. Possible future opportunities involving measurements of diffractive quarkonium production are discussed in Sect. 6.11.

The ZEUS and H1 collaborations have published several measurements of inelastic $J / \psi$ and $\psi(2 S)$ production that are based on data from HERA Run I [697, 760]. A new measurement, making use of the full Run II data sample, was published recently by the $\mathrm{H} 1$ collaboration [696]. The ZEUS collaboration has published a new measurement of the $J / \psi$ decay angular distributions in inelastic photoproduction, making use of the collaboration's full data sample [761].

The data samples of $J / \psi$ events that result from the experimental selection cuts are dominated by inelastic production processes in which the $J / \psi$ 's do not originate from the decay of a heavier resonance. Sub-dominant diffractive backgrounds, as well as feeddown contributions from the $\psi(2 S)$, the $\chi_{c J}$, and $b$-flavored hadrons are estimated to contribute between $15 \%$ and $25 \%$ of the total $J / \psi$ events, depending on the kinematic region. These backgrounds are usually neglected in theoretical predictions of direct $J / \psi$ production rates.

The measurements of $J / \psi$ cross sections and polarization parameters reported by the ZEUS and H1 collaborations have been compared extensively to NRQCD factorization predictions at LO in $\alpha_{s}$. In these studies, a truncation of the NRQCD velocity expansion is used, in which the independent long-distance matrix elements are $\left\langle\mathcal{O}_{1}^{J / \psi}\left({ }^{3} S_{1}\right)\right\rangle,\left\langle\mathcal{O}_{8}^{J / \psi}\left({ }^{3} S_{8}\right)\right\rangle,\left\langle\mathcal{O}_{8}^{J / \psi}\left({ }^{1} S_{0}\right)\right\rangle$ and

\footnotetext{
14 Here, only the most recent measurements are cited.
} 
$\left\langle\mathcal{O}_{8}^{J / \psi}\left({ }^{3} P_{0}\right)\right\rangle$. In the CSM, all of these matrix elements, except for the first one, are, in effect, set to zero. Usually the values of the long-distance color-octet matrix elements are extracted from the Tevatron data, in which case the comparisons of the resulting predictions for $J / \psi$ production at HERA with the data offer the opportunity to assess the universality of the long-distance matrix elements. The comparisons between the predictions at LO in $\alpha_{s}$ and the data are summarized in Ref. [1].

In calculations in the NRQCD factorization formalism, large uncertainties arise from the sensitivity to the input parameters: the mass of the charm quark, the factorization and renormalization scales, and the values of the color-octet matrix elements, which are obtained from fits to the Tevatron data. One also expects sizable uncertainties owing to the omission of corrections of higher-order in both $\alpha_{s}$ and $v$. As we shall see, because of these theoretical uncertainties, the relative sizes of the color-singlet and color-octet contributions in charmonium photoproduction are still unclear.

In fixed-order, tree-level predictions of the color-octet contribution to photoproduction, a large peak appears in the $z$ distribution near the kinematic endpoint $z=1$. This feature, which was first interpreted as a failure of the universality of the long-distance matrix elements, has since been attributed to the breakdown of the NRQCD velocity expansion and the perturbation expansion in $\alpha_{s}$ near $z=1$. In this region, in order to obtain a reliable theoretical prediction, one must resum large perturbative corrections to all orders in $\alpha_{s}$ and large nonperturbative corrections to all orders in $v$. It is known that the resummation of the color-octet contribution leads to a significant broadening of the peak at large $z$ [762]. However, the effects of the nonperturbative resummation of the velocity expansion cannot be determined precisely without further information about the so-called "shape function." In principle, that information could be extracted from data on charmonium production in $e^{+} e^{-}$collisions.

$J / \psi$ photoproduction at HERA has also been studied in the $k_{T}$-factorization scheme [640, 763]. In this framework, it has been argued that the color-singlet contribution alone can explain the HERA data. A prediction for the color-singlet yield at LO in $\alpha_{s}$ in the $k_{T^{-}}$ factorization approach reproduces the measured shapes of the $p_{T}$ and $z$ distributions reasonably well. However, it should be kept in mind that these predictions rely on unintegrated parton distributions, which are not well known at present. This uncertainty might be reduced as more accurate unintegrated parton distributions sets become available [764].

The pioneering calculation of the correction of NLO in $\alpha_{s}$ to the color-singlet contribution to the direct $J / \psi$ cross section was presented in Ref. [765]. The NLO correction affects not only the normalization of the photoproduction rate, but also the shape of the $p_{T}$ distribution. Both of these effects bring the color-singlet contribution into better agreement with the data. The large impact of the NLO correction at large transverse momentum can be understood in terms of the kinematic enhancement of NLO color-singlet production processes relative to the LO color-singlet production processes. This effect is similar to the one that appears in hadroproduction in the color-singlet channel. (See Sect. 4.2.1.)

The NLO calculation of Ref. [765] suggests that production in the color-singlet channel might be the main mechanism at work in $J / \psi$ photoproduction. However, in more recent work [695, 766-769], which confirms the calculation of Ref. [765], it has been emphasized that the factorization and renormalization scales in Ref. [765] have been set a value $\left(m_{c} / \sqrt{2}\right)$ that is generally considered to be too low in the region of large $p_{T}$. As we shall see, a more physical choice of scale, such as $\sqrt{4 m_{c}^{2}+p_{T}^{2}}$, leads to predictions for cross sections differential in $p_{T}^{2}$ or in $z$ that lie considerably below the $\mathrm{H} 1$ and ZEUS measurements. Hence, the size of the NLO color-singlet contribution does not exclude the possibility that other contributions, such as those from the color-octet channel, are at least as large as the color-singlet contribution. In the region of low transverse momentum, which is the dominant region for $J / \psi$ production at HERA, the sensitivity to the factorization and renormalization scales is very large and complicates the identification of the dominant production mechanism at HERA.

\subsubsection{Phenomenology of the cross section, including NLO corrections}

In Fig. 61 we show a comparison between data from the H1 collaboration [696] for the $J / \psi$ photoproduction cross sections differential in $p_{T}^{2}$ and in $z$ and calculations in the NRQCD factorization formalism from Refs. $[695,768,769] .{ }^{15}$ The dashed line depicts the central values of the complete NRQCD factorization prediction (including color-singlet and color-octet contributions) at NLO in $\alpha_{s}$, and the band shows the uncertainty in that prediction that arises from the uncertainties in the color-octet long-distance NRQCD matrix elements. Note that the contributions from resolved photoproduction, which are important in the low- $z$ region, and the contributions from diffractive production, which are important near $z=1$, are not included in the NRQCD factorization prediction. The uncertainties that are shown arise from the uncertainties in the NRQCD color-octet longdistance matrix elements. These matrix elements were obtained through a fit to the Tevatron hadroproduction data that used the NRQCD prediction at LO in $\alpha_{s}$, augmented by an approximate calculation of some higherorder corrections from multiple-gluon radiation [770].

The NRQCD factorization prediction at NLO accuracy in $\alpha_{s}$ is in better agreement with the $\mathrm{H} 1$ data than the

15 A more detailed comparison of the $\mathrm{H} 1$ data with theory predictions can be found in Ref. [696]. 

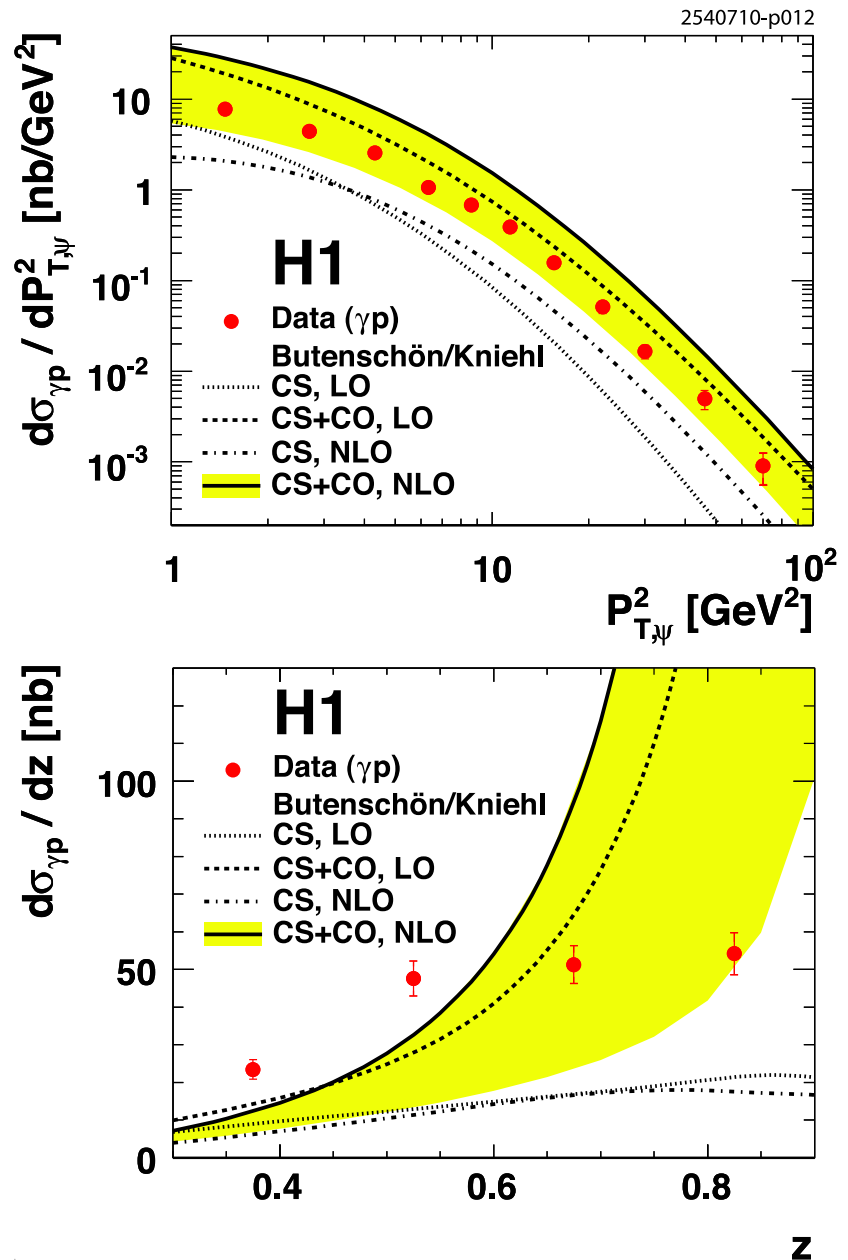

FIG. 61: Cross sections differential in $p_{T}^{2}$ and in $z$ for $J / \psi$ photoproduction at HERA. The measurement by the $\mathrm{H} 1 \mathrm{col}-$ laboration [696] is compared to the CSM and NRQCD predictions at LO and NLO in $\alpha_{s}$ from Refs. [695, 768]. "CS" and "CO" denote the color-singlet and color-octet contributions, respectively. The dashed line depicts the central values of the complete NRQCD factorization prediction (including colorsinglet and color-octet contributions) at NLO in $\alpha_{s}$, and the band shows the uncertainty in that prediction that arises from the uncertainties in the color-octet long-distance NRQCD matrix elements. From Ref. [696]

color-singlet contribution alone. However, it should be kept in mind that the mass and scale uncertainties of the color-singlet contribution have not been displayed here. As we have already mentioned, these uncertainties are large, even at NLO in $\alpha_{s}$.

The NRQCD factorization prediction for the cross section differential in $z$ shows a rise near $z=1$ that is characteristic of the color-octet contributions. As we have mentioned, resummations of the series in $\alpha_{s}$ and in $v$ are needed in order to obtain a reliable theoretical prediction in this region. In the low- $z$ region, the NRQCD factorization prediction undershoots the data. In this region, the corrections to resolved photoproduction through NLO in $\alpha_{s}$ may be needed in order to bring the theory into agree- ment with the data.

\subsubsection{Polarization}

In addition to the studies of $J / \psi$ differential cross sections that we have mentioned, there have also been recent analyses of polarization in $J / \psi$ photoproduction. The polarization observables may provide additional information about the production mechanisms.

Experimentally, the $J / \psi$ polarization is extracted from the angular distribution of the leptons that originate in $J / \psi$ decays. In the $J / \psi$ rest frame, the distribution takes the general form

$$
\begin{aligned}
& \frac{d \Gamma\left(J / \psi \rightarrow l^{+} l^{-}\right)}{d \Omega} \propto 1+\lambda \cos ^{2} \theta+\mu \sin 2 \theta \cos \phi \\
& +\frac{\nu}{2} \sin ^{2} \theta \cos 2 \phi,
\end{aligned}
$$

where $\theta$ and $\phi$ are the polar and azimuthal angles of the $l^{+}$three-momentum with respect to a particular spinquantization frame. (See Sect. 4.2.3.) The ZEUS collaboration has published a new measurement of the parameters $\lambda$ and $\nu$ in the target spin-quantization frame [771] that is based on an integrated luminosity of $468 \mathrm{pb}^{-1}$ [761]. The H1 collaboration has published new measurements of the parameters $\lambda$ and $\nu$ in both the helicity and the Collins-Soper spin-quantization frames that are based on an integrated luminosity of $165 \mathrm{pb}^{-1}$ [696]. The H1 collaboration uses a more restricted range in the energy fraction $z$, namely, $0.3<z<0.9$, in order to suppress possible contributions from diffractive or feeddown processes. In both experiments, the polarization parameters in each bin are extracted by comparing the data with Monte Carlo distributions for different values of the polarization parameters, using a $\chi^{2}$ criterion to assess the probability of each distribution.

We show comparisons of several theoretical predictions with the ZEUS data in Fig. 62 and with the H1 data in Fig. 63.

The curves in Fig. 62 that are labeled "LO CS+CO" are the complete NRQCD factorization predictions (including color-singlet and color-octet contributions) at LO in $\alpha_{s}$ [771]. These agree reasonably well with the ZEUS data in the target frame, except for the value of $\nu$ in the lowest- $p_{T}$ bin. However, at such a low value of $p_{T}$, the NRQCD factorization formula is not expected to be valid.

The curves that are labeled "LO $k_{T}$ " and "CSM $k_{T}$ " are predictions in the $k_{T}$-factorization scheme [772]. The set of unintegrated parton distribution functions that is used is indicated in parentheses. The $k_{T}$-factorization predictions bracket the ZEUS data in the target frame for both $\lambda$ and $\nu$ and are in reasonable agreement with the H1 data for $\lambda$ and $\nu$ in the helicity frame, if one takes the difference between the two $k_{T}$-factorization predictions to be a measure of the theoretical uncertainty. 

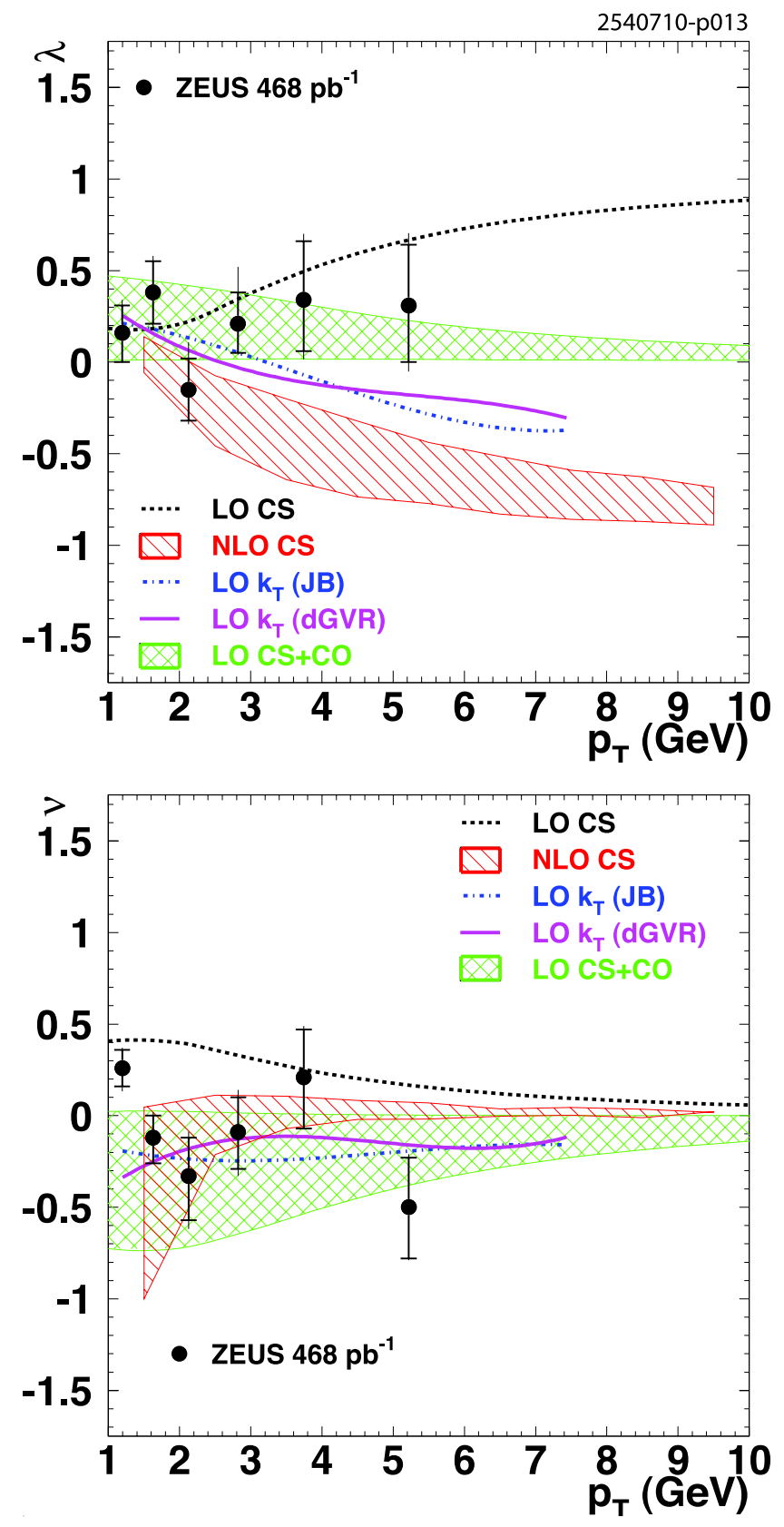

FIG. 62: Polarization parameters $\lambda$ and $\nu$ in the target frame as a function of $p_{T}$ for $J / \psi$ photoproduction at HERA. The measurement by the ZEUS collaboration [761] of the polarization parameters in the target frame is compared with the color-singlet contribution at LO in $\alpha_{s}$ (labeled LO CS) [771] and at NLO in $\alpha_{s}$ (labeled NLO CS) [766], with predictions in the $k_{T}$-factorization approach for two different sets of unintegrated parton-distributions functions (labeled LO $k_{T}$ (JB) and LO $k_{T}$ (dGRV)) [772], and with the complete NRQCD factorization predictions (including color-singlet and coloroctet contributions) at LO in $\alpha_{s}$ (labeled LO CS+CO) [771]. The theoretical uncertainty bands labeled NLO CS were obtained by varying the factorization scale $\mu_{f}$ and the renormalization scale $\mu_{r}$ in the range defined by $0.5 \mu_{0}<\mu_{f}, \mu_{r}<2 \mu_{0}$, and $0.5<\mu_{r} / \mu_{f}<2$, where $\mu_{0}=4 m_{c}$. The theoretical uncertainty bands labeled LO CS+CO were obtained by considering uncertainties in the values of the color-octet NRQCD long-distance matrix elements. From [761] with kind permission, copyright (2009) Springer-Verlag
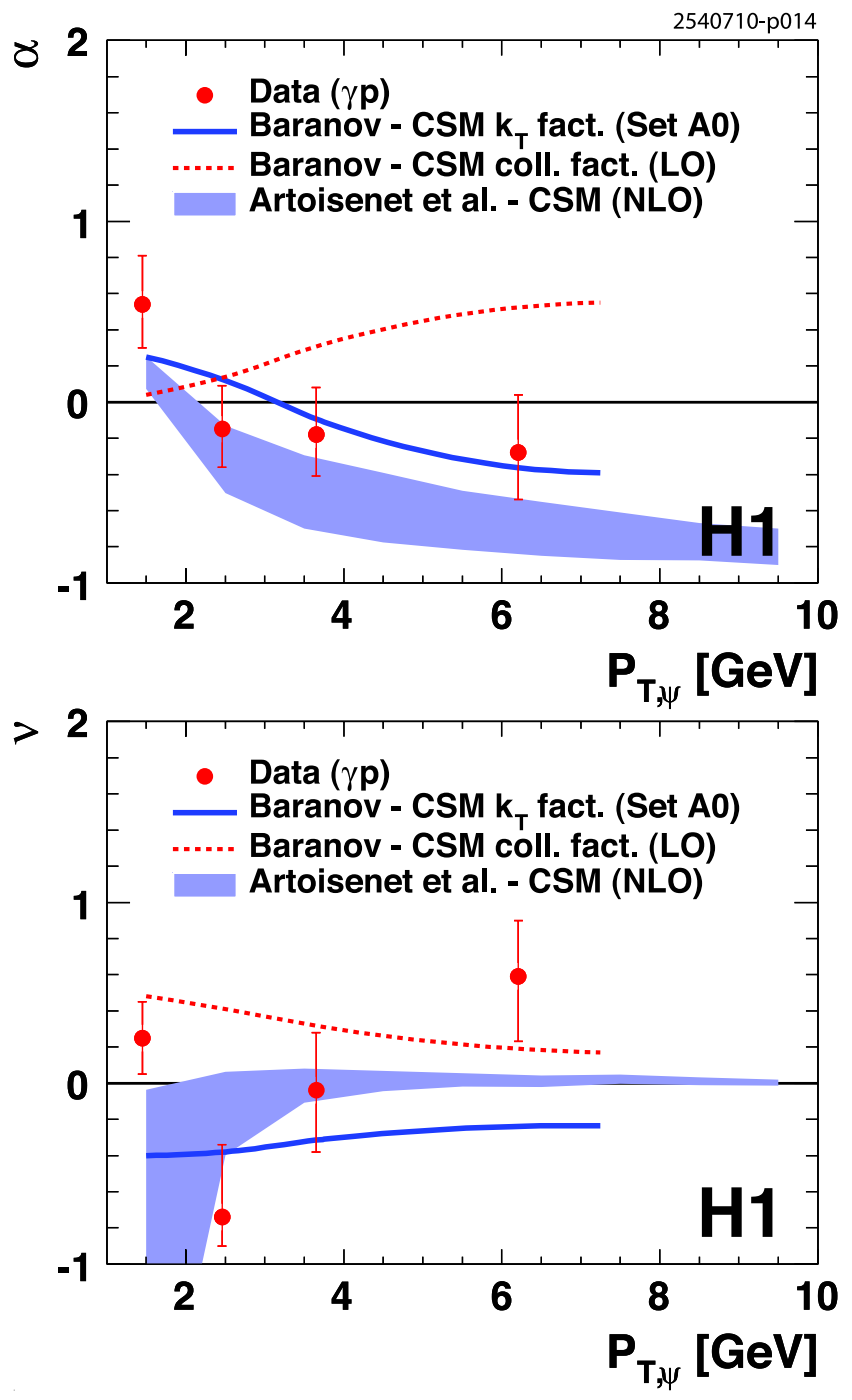

FIG. 63: Polarization parameters $\alpha=\lambda$ and $\nu$ in the helicity frame as a function of $p_{T}$ for $J / \psi$ photoproduction at HERA. The measurement by the H1 collaboration [696] of the polarization parameters in the helicity frame is compared with the predictions in the $k_{T}$-factorization approach (solid line) [772] and with the predictions in color-singlet model (CSM) at LO in $\alpha_{s}$ (dashed line) [772] and at NLO $\alpha_{s}$ (filled band) $[766,773]$. The theoretical uncertainty bands were obtained by varying the factorization scale $\mu_{f}$ and the renormalization scale $\mu_{r}$ in the range defined by $0.5 \mu_{0}<\mu_{f}, \mu_{r}<2 \mu_{0}$, and $0.5<\mu_{r} / \mu_{f}<2$, where $\mu_{0}=4 m_{c}$. From Ref. [696]

The bands that are labeled "NLO CS" and "CSM NLO" correspond to predictions in the color-singlet model at NLO in $\alpha_{s}$ (Ref. [766]). (Similar results for the polarization at NLO in $\alpha_{s}$ in the color-singlet model were obtained in Ref. [767].) The uncertainties that are shown in these bands arise from the sensitivity of the polarization to the factorization and renormalization scales and are much larger than the uncertainties that arise from the uncertainty in the value of $m_{c}$. A comparison of the LO and NLO predictions of the CSM shows the 
large impact of the NLO correction on the polarization parameters. At NLO, the parameter $\lambda$ is predicted to decrease with increasing $p_{T}$, in both the target and the helicity frames. (This trend is also observed in the LO prediction of the $k_{T}$ factorization formalism, which effectively accounts for some topologies that occur at higher orders in $\alpha_{s}$ in the collinear factorization scheme.) The NLO color-singlet predictions for $\lambda$ are compatible with the $\mathrm{H} 1$ data in the helicity frame, but differ significantly from the ZEUS data in the target frame. In contrast, the NLO color-singlet prediction for the parameter $\nu$ is in reasonable agreement with the ZEUS data in the target frame, as well as with the $\mathrm{H} 1$ data in the helicity frame.

A more complete presentation of the comparison between theory and experiment for the polarization parameters $\lambda$ and $\nu$, including the distributions of these parameters as functions of $z$, can be found in Refs. [696, 761].

\subsubsection{Future opportunities}

In spite of the recent advances that we have described, it is still unclear which mechanisms are at work in $J / \psi$ photoproduction at HERA. The new computations of NLO corrections to the differential cross-sections and the polarization parameters in the collinear-factorization scheme show that there is room for a color-octet contribution. Indeed, the NLO NRQCD factorization prediction for the cross section fits the data reasonably, with central values that are closer to the data points than are the central values of the NLO color-singlet contribution. Recent analyses of the color-singlet contribution in the $k_{T}$ factorization scheme also show reasonable agreement with the data.

In both $k_{T}$ factorization and in collinear factorization, theoretical uncertainties remain substantial. Improvement of the situation for $k_{T}$ factorization will require better knowledge of the $k_{T}$-dependent parton distributions. In collinear factorization, the large sensitivity of the NLO color-singlet rates to the renormalization scale signals that QCD corrections beyond NLO might be relevant, especially for the description of the polarization parameters. Here, an analysis in the fragmentation-function approach (see Sect. 4.1.5) might help to bring the perturbation series under better control. Theoretical uncertainties in the NRQCD factorization prediction for the $J / \psi$ polarization could be reduced by computing the color-octet contributions to the polarization parameters at NLO accuracy in $\alpha_{s}$. The large uncertainties in the color-octet long-distance matrix elements dominate the uncertainties in the NRQCD factorization prediction for the cross section. Since these matrix elements are obtained by fitting NRQCD factorization predictions to the Tevatron data, improvements in the theoretical uncertainties in the Tevatron (or LHC) predictions are necessary in order to reduce the uncertainties in the matrix elements. Finally, theoretical uncertainties in the region near the kinematic endpoint $z=1$ might be reduced through a systematic study of resummations of the perturbative and velocity expansions in both $e p$ and $e^{+} e^{-}$quarkonium production.

\subsection{Fixed-target production}

\subsubsection{Phenomenology of fixed-target production}

The NRQCD factorization approach has also been tested against the charmonium production data that have been obtained from fixed-target experiments and $p p$ experiments at low energies. Owing to the limited statistics, quarkonium observables measured in these experiments have, in general, been restricted to total cross sections for $J / \psi$ and $\psi(2 S)$ production. Also, in the case of $J / \psi$ production, feeddown contributions have not been subtracted.

In the most recent analysis of the fixed-target and lowenergy $p p$ quarkonium production data [774], experimental results for the inclusive production rates of the $J / \psi$ and the $\psi(2 S)$ have been examined, along with the experimental results for the ratios of these cross sections. A total of 29 experimental results have been analyzed in order to extract the color-octet contribution to the observed rates. By comparing the values of the color-octet matrix elements that are extracted from the fixed-target data with the values that are extracted from the Tevatron data, one can test the universality of the NRQCD matrix elements.

The analysis of Ref. [774] made use of the NRQCD short-distance coefficients through order $\alpha_{s}^{3}$ for the $P$ wave channels and for all of the color-octet channels that are of leading order in $v$ (Ref. [466]). The LO shortdistance coefficients for the color-singlet ${ }^{3} S_{1}$ channel were employed, as the computation of the QCD correction to these short-distance coefficients in $p p$ collisions had not yet been completed at the time of the analysis of Ref. [774].

In extracting the color-octet contribution from the production rates that are measured in fixed-target experiments, the authors of Ref. [774] treated the NRQCD long-distance matrix elements as follows: Heavy-quark spin symmetry was employed; the color-singlet matrix elements were taken from the potential-model calculation of Ref. [775]; the color-octet matrix element for $P$-wave charmonium states was set equal to a value that was extracted in Ref. [776] from the CDF data [650]. Regarding the color-octet matrix elements for the $J / \psi$ and $\psi(2 S)$, it was assumed that $\left\langle\mathcal{O}_{8}^{H}\left({ }^{1} S_{0}\right)\right\rangle=\left\langle\mathcal{O}_{8}^{H}\left({ }^{3} P_{0}\right)\right\rangle / m_{c}^{2}$ and that the ratios of these matrix elements to $\left\langle\mathcal{O}_{8}^{H}\left({ }^{3} S_{1}\right)\right\rangle$ are given by the values that were extracted from the Tevatron data. The color-octet contributions to the direct production of the $J / \psi$ and the $\psi(2 S)$ were multiplied by rescaling parameters $\lambda_{J / \psi}$ and $\lambda_{\psi^{\prime}}$, respectively, which were varied in order to fit the fixed-target data. In addition to these two parameters, the factorization and renormalization scales were varied in the fit, while the mass of 
the charm quark was held fixed at $1.5 \mathrm{GeV}$.

The $\chi^{2}$ of the fit favors a nonzero value for the coloroctet contribution to direct $J / \psi$ and $\psi(2 S)$ production. This can be seen in Fig. 64 for the case of the $J / \psi$ : The color-singlet contribution alone (dotted-dashed line) systematically undershoots the data, while the fitted complete NRQCD contribution (solid line) is in good agreement with the data. However, the fit also indicates that the values of the color-octet matrix elements that are needed to explain the fixed-target data are only about $10 \%$ of the values that were extracted from fits to the Tevatron data. A similar result was obtained in the case of the color-octet matrix elements for the $\psi(2 S)$. In both cases, the stability of the fitting procedure was checked by changing the selection of measurements used in the fits and by changing the set of parton distribution functions that was used in computing the cross sections. In any of these scenarios, the values of the color-octet matrix elements that were extracted from the fixed-target data are smaller than the values that were extracted from the Tevatron data.

The analysis of fixed-target data that we have described could be updated by making use of the recent results for the NLO corrections to the hadroproduction of $S$-wave states [619, 674]. Furthermore, a more accurate value for the $J / \psi$ color-singlet matrix element has been derived recently in Ref. [777]. However, it is expected, even with these improvements, that the qualitative conclusions of Ref. [774] would still hold.

It is interesting to consider this fixed-target result in light of two recent developments: (1) the possible impact of higher-order corrections to the color-singlet yield on the phenomenology of $J / \psi$ production at the Tevatron

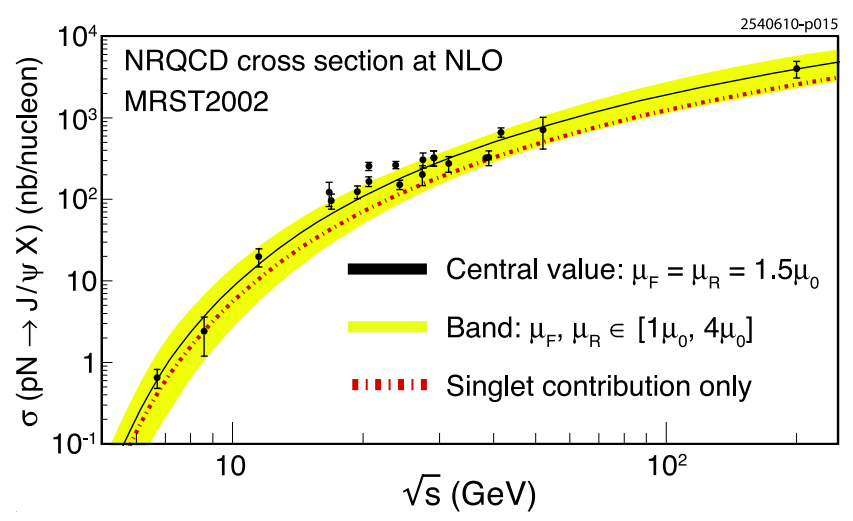

FIG. 64: Fit of the NRQCD factorization cross section for the production of the $J / \psi$ as a function of the center-of-mass energy $\sqrt{s}$ to data from fixed-target experiments and $p p$ experiments at low energies. The curves are the result of the fitting procedure that is described in the text. The color-singlet component is shown as a dotted-dashed line. The theoretical uncertainty band was obtained by varying the factorization scale $\mu_{F}$ and the renormalization scale $\mu_{R}$ in the ranges $\mu_{0}<\mu_{F}<4 \mu_{0}$ and $\mu_{0}<\mu_{R}<4 \mu_{0}$, where $\mu_{0}=2 m_{c}$. From [774] with kind permission, copyright (2006) Elsevier
(Sect. 4.2.2) and (2) the latest $J / \psi$ polarization measurement by the CDF collaboration [658] (Sect. 4.2.3). Both of these developments suggest that the values of the color-octet NRQCD matrix elements may be smaller than had previously been supposed. However, in assessing the significance of the fixed-target result, it should be remembered that the fixed-target data, integrated over $p_{T}$, are dominated by data from the lowest values of $p_{T}$. At low $p_{T}$, one would not expect NRQCD factorization to hold.

\subsubsection{Future opportunities}

Fixed-target experiments provide another means by which to test the various theories of inclusive quarkonium production. In order to test definitively theoretical hypotheses, such as NRQCD factorization, that are based on hard-scattering factorization, it is necessary to make measurements at values of $p_{T}$ that are much greater than the heavy-quark mass. If fixed-target experiments with such a high reach in $p_{T}$ could be devised, then it would be very useful to measure both the cross section and the quarkonium polarization as functions of $p_{T}$.

\subsection{Exclusive production in $e^{+} e^{-}$collisions}

\subsubsection{Theory vs. experiment}

As recently as three years ago, large discrepancies existed between experimental measurements and theoretical predictions for the process $e^{+} e^{-} \rightarrow J / \psi+\eta_{c}$ at the $B$-factory energy of $\sqrt{s}=10.58 \mathrm{GeV}$. In its initial measurement of this process, the Belle collaboration obtained $\sigma\left(e^{+} e^{-} \rightarrow J / \psi+\eta_{c}\right) \times \mathcal{B}_{\geq 4}=33_{-6}^{+7} \pm 9 \mathrm{fb}$ (Ref. [778]), where $\sigma\left(e^{+} e^{-} \rightarrow J / \psi+\eta_{c}\right)$ is the cross section and $\mathcal{B}_{\geq 4}$ is the branching fraction of the $\eta_{c}$ into four or more charged tracks. The first theoretical predictions for $\sigma\left(e^{+} e^{-} \rightarrow J / \psi+\eta_{c}\right)$ were based on calculations in the NRQCD factorization approach [138]. These initial calculations were carried out at LO in $\alpha_{s}$ and $v$. They gave the following predictions for $\sigma\left(e^{+} e^{-} \rightarrow J / \psi+\eta_{c}\right)$ : $5.5 \mathrm{fb}$ (Ref. [779]), $3.78 \pm 1.26 \mathrm{fb}$ (Ref. [780]), and $2.3 \mathrm{fb}$ (Ref. [781]). ${ }^{16}$ The differences between the calculations of Refs. [779-781] arise from QED effects, which are included only in Ref. [780]; from contributions from an intermediate $Z$ boson, which are included only in Ref. [781]; and from different choices of $m_{c}$, the NRQCD longdistance matrix elements, and $\alpha_{s}$. The sensitivities of the calculations to the values of these parameters are important sources of theoretical uncertainties, which we will

${ }^{16}$ In Ref. [780] a cross section of $2.31 \pm 1.09 \mathrm{fb}$ was reported initially. Later, a sign error in the QED interference term was corrected and the value cited above was obtained. 
discuss later.

The Belle collaboration has, more recently, measured the quantity $\sigma\left(e^{+} e^{-} \rightarrow J / \psi+\eta_{c}\right) \times \mathcal{B}_{>2}=25.6 \pm 2.8 \pm$ $3.4 \mathrm{fb}$ (Ref. [782]), where $\mathcal{B}_{>2}$ is the branching fraction of the charmonium state that is recoiling against the $J / \psi$ (in this case the $\eta_{c}$ ) into more than two charged tracks. This cross section times branching fraction has also been measured by the $B A B A R$ collaboration, which obtains $\sigma\left(e^{+} e^{-} \rightarrow J / \psi+\eta_{c}\right) \times \mathcal{B}_{>2}=17.6 \pm 2.8 \pm 2.1 \mathrm{fb}$ (Ref. [53]). These more recent experimental results reduced the discrepancy between theory and experiment, but did not eliminate it. In assessing the size of the discrepancy, it is important to recognize that the experimental results are cross sections times branching fractions. Hence, they are lower bounds on the cross sections, which are the quantities that appear in the theoretical predictions.

Table 43 contains a summary of experimental measurements and NRQCD predictions for the process $e^{+} e^{-} \rightarrow$ $J / \psi+H$, where $H$ is $\eta_{c}, \chi_{c 0}$, or $\eta_{c}(2 S)$. As can be seen from Table 43, significant discrepancies exist between LO NRQCD predictions and experiment, not only for exclusive production of $J / \psi+\eta_{c}$, but also for exclusive production of $J / \psi+\chi_{c 0}$ and $J / \psi+\eta_{c}(2 S)$. An important step toward resolving the discrepancy between theory and experiment for $\sigma\left(e^{+} e^{-} \rightarrow J / \psi+\eta_{c}\right)$ was the calculation in Ref. [786] of the corrections of NLO in $\alpha_{s}$. These corrections yield a $K$ factor of about 1.96. This result has been confirmed in Ref. [787]. While this $K$ factor is substantial, it does not, by itself, eliminate the discrepancy between theory and experiment.

In the NRQCD factorization formalism there are, in addition to corrections of higher order in $\alpha_{s}$, corrections of higher order in $v$, i.e., relativistic corrections. In $\sigma\left(e^{+} e^{-} \rightarrow J / \psi+\eta_{c}\right)$, relativistic corrections can arise in two ways. First, they can appear directly as corrections to the process $e^{+} e^{-} \rightarrow J / \psi+\eta_{c}$ itself. Second, they can arise indirectly through the NRQCD longdistance matrix elements that appear in the expression for $\sigma\left(e^{+} e^{-} \rightarrow J / \psi+\eta_{c}\right)$. For example, the matrix element of leading order in $v$ that appears in $J / \psi$ production can be determined phenomenologically from the experimental value for the width for $J / \psi \rightarrow e^{+} e^{-}$and the theoretical expression for that width. There are relativistic corrections to the theoretical expression for the width, which affect the value of the long-distance matrix element that one obtains.

The first relativistic correction appears at relative order $v^{2}$, where $v^{2} \approx 0.3$ for charmonium. It has been known for some time that this correction is potentially large: In Ref. [780], it was found that the order- $v^{2}$ correction is $1.95\left\langle v^{2}\right\rangle_{J / \psi}+2.37\left\langle v^{2}\right\rangle_{\eta_{c}}$. Here, $\left\langle v^{2}\right\rangle_{H}$ is the ratio of an order- $v^{2}$ NRQCD long-distance matrix element to the LO matrix element in the quarkonium state $H$. The authors of Ref. [780] estimated the matrix elements of order $v^{2}$ by making use of the Gremm-Kapustin relation [788], which follows from the NRQCD equations of motion. On the basis of these estimates, they found that the
$K$ factor for the relativistic corrections is about $2.0_{-1.1}^{+10.9}$. The very large uncertainties in this $K$ factor reflect the large uncertainties in the Gremm-Kapustin-relation estimates of the matrix elements of order $v^{2}$.

In Ref. [789], significant progress was made in reducing the uncertainties in the order- $v^{2}$ NRQCD matrix elements. The approach in this work was to make use of a static-potential model to calculate the quarkonium wave functions and, from those wave functions, to compute the dimensionally regulated NRQCD matrix elements. If the static potential is known accurately, for example, from lattice calculations, then the corrections to the static potential model are of relative order $v^{2}$ (Ref. [140, 179]). Hence, one can regard the potential-model calculation as a first-principles calculation with controlled uncertainties.

Making use of the results of Ref. [789], the authors of Ref. [783] computed the relativistic corrections to $\sigma\left(e^{+} e^{-} \rightarrow J / \psi+\eta_{c}\right)$. Taking into account the corrections of NLO in $\alpha_{s}$ from Ref. [786], they obtained $\sigma\left(e^{+} e^{-} \rightarrow J / \psi+\eta_{c}\right)=17.5 \pm 5.7 \mathrm{fb}$, where the quoted uncertainty reflects only the uncertainties in $m_{c}$ and the order- $v^{2}$ NRQCD long-distance matrix elements. This result includes the effects of a resummation of a class of relativistic corrections that arise from the quarkonium wave function. One might worry that the large relativistic corrections that appear in this calculation, which result in a $K$ factor of about 2.6, are an indication that the $v$ expansion of NRQCD is out of control. However, this large correction is the result of several corrections of a more modest size: a direct correction of about $40 \%$ and two indirect corrections (for the $J / \psi$ and the $\eta_{c}$ ) of about $37 \%$ each. Furthermore, higher-order terms in the resummation of wave-function corrections change the direct correction by only about $13 \%$, suggesting that the $v$ expansion is indeed converging well.

The authors of Ref. [784] took a different approach to calculating relativistic corrections, determining the NRQCD long-distance matrix elements of LO in $v$ and of relative order $v^{2}$ by using $\Gamma\left(J / \psi \rightarrow e^{+} e^{-}\right), \Gamma\left(\eta_{c} \rightarrow \gamma \gamma\right)$, and $\Gamma(J / \psi \rightarrow$ light hadrons $)$ as inputs. Their result, $\sigma\left(e^{+} e^{-} \rightarrow J / \psi+\eta_{c}\right)=20.04 \mathrm{fb}$, is in agreement with the result of Ref. [783]. However, the values of the NRQCD matrix elements that are given in Ref. [784] differ significantly from those that were used in Ref. [783]. This difference probably arises mainly because of the very large relativistic corrections to $\Gamma(J / \psi \rightarrow$ light hadrons $)$, which may not be under good control.

The results of Refs. [783, 784] greatly reduced the difference between the experimental and theoretical central values for $\sigma\left(e^{+} e^{-} \rightarrow J / \psi+\eta_{c}\right)$. However, in order to assess the significance of these results, it is essential to have a reliable estimate of the theoretical uncertainties. Such an estimate was provided in Ref. [785]. In this work, which was based on the method of Ref. [783], uncertainties from various input parameters, such as $m_{c}$ and the electromagnetic widths of the $J / \psi$ and the $\eta_{c}$, were taken into account, as well as uncertainties from the truncations 
TABLE 43: Experimental measurements and NRQCD predictions for $e^{+} e^{-} \rightarrow J / \psi+H$, where $H$ is $\eta_{c}, \chi_{c 0}$, or $\eta_{c}(2 S)$. Cross sections are in units of $\mathrm{fb}$. The quantity $\mathcal{B}_{>2}$ is the branching fraction of the charmonium state that is recoiling against the $J / \psi$ into more than two charged tracks

\begin{tabular}{lccc}
\hline \hline Quantity & $\eta_{c}(1 S)$ & $\chi_{c 0}(1 P)$ & $\eta_{c}(2 S)$ \\
\hline$\sigma \times \mathcal{B}_{>2}$ (Belle [782]) & $25.6 \pm 2.8 \pm 3.4$ & $6.4 \pm 1.7 \pm 1.0$ & $16.5 \pm 3.0 \pm 2.4$ \\
$\sigma \times \mathcal{B}_{>2}($ BABAR [53]) & $17.6 \pm 2.8_{-2.1}^{+1.5}$ & $10.3 \pm 2.5_{-1.8}^{+1.4}$ & $16.4 \pm 3.7_{-3.0}^{+2.4}$ \\
\hline$\sigma$ (Liu, He, Chao [779]) & 5.5 & 6.9 & 3.7 \\
$\sigma$ (Braaten, Lee [780]) & $3.78 \pm 1.26$ & $2.40 \pm 1.02$ & $1.57 \pm 0.52$ \\
$\sigma$ (Hagiwara, Kou, Qiao [781]) & 2.3 & & \\
$\sigma$ (Bodwin et al. $[783])$ & $17.5 \pm 5.7$ & \\
$\sigma$ (He, Fan, Chao [784]) & 20.4 & \\
$\sigma$ (Bodwin, Lee, Yu [785]) & $17.6_{-6.7}^{+8.1}$ & & \\
\hline \hline
\end{tabular}

of the $\alpha_{s}$ and $v$ expansions. Correlations between uncertainties in various components of the calculation were also taken into account. In addition, various refinements were included in the calculation, such as the use of the vector-meson-dominance method to reduce uncertainties in the QED contribution and the inclusion of the effects of interference between relativistic corrections and corrections of NLO in $\alpha_{s}$. The conclusion of Ref. [785] is that $\sigma\left(e^{+} e^{-} \rightarrow J / \psi+\eta_{c}\right)=17.6_{-6.7}^{+8.1} \mathrm{fb}$. This result is in agreement, within uncertainties, with the BABAR result, even if one allows for the fact that the branching fraction $\mathcal{B}_{>2}$ could be as small as $0.5-0.6$.

An alternative approach to theoretical calculations of exclusive quarkonium production in $e^{+} e^{-}$annihilation is the light-cone method [645-649]. Generally, the lightcone-method predictions for exclusive quarkonium production cross sections are in agreement with the experimental results. The light-cone approach to quarkonium production can be derived from QCD. ${ }^{17}$ In principle, the light-cone approach is as valid as the NRQCD factorization approach. In practice, it is, at present, necessary to model the light-cone wave functions of the quarkonia, possibly making use of constraints from QCD sum rules [790, 791]. Consequently, the existing light-cone calculations are not first-principles calculations, and it is not known how to estimate their uncertainties reliably. The light-cone approach automatically includes relativistic corrections that arise from the quarkonium wave function. As has been pointed out in Ref. [792], the light-cone calculations contain contributions from regions in which the quarkonia wave-function momenta are of order $m_{c}$ or greater. In NRQCD, such contributions are contained in corrections to the short-distance coefficients of higher order in $\alpha_{s}$. Therefore, in order to avoid double counting, one should refrain from combining light-cone results

17 Light-cone factorization formulas are derived in Ref. [643] in the course of proving NRQCD factorization formulas. with NRQCD corrections of higher order in $\alpha_{s}$. Finally, we note that, in Ref. [792], it was suggested that resummations of logarithms of $\sqrt{s} / m_{c}$ have not been carried out correctly in some light-cone calculations.

\subsubsection{Future opportunities}

Clearly, it would be desirable to reduce both the theoretical and experimental uncertainties in the rates for the exclusive production of quarkonia in $e^{+} e^{-}$annihilation and to extend theory and experiment to processes involving additional quarkonium states.

On the experimental side, the central values of the Belle and BABAR measurements of $\sigma\left(e^{+} e^{-} \rightarrow J / \psi+\right.$ $\left.\eta_{c}\right) \times \mathcal{B}_{>2}$ differ by about twice the uncertainty of either measurement. Although those uncertainties are small in comparison with the theoretical uncertainties, the rather large difference in central values suggests that further experimental work would be useful. Furthermore, it would be very useful, for comparisons with theory, to eliminate the uncertainty in the cross section that arises from the unmeasured branching fraction $\mathcal{B}_{>2}$.

On the theoretical side, the largest uncertainty in $\sigma\left(e^{+} e^{-} \rightarrow J / \psi+\eta_{c}\right)$ arises from the uncertainty in $m_{c}$. One could take advantage of the recent reductions in the uncertainty in $m_{c}[228,248]$ to reduce the theoretical uncertainty in $\sigma\left(e^{+} e^{-} \rightarrow J / \psi+\eta_{c}\right)$ from this source. The next largest source of theoretical uncertainty arises from the omission of the correction to the $J / \psi$ electromagnetic width at NNLO in $\alpha_{s}$ (Ref. [793, 794]). Unfortunately, the large scale dependence of this correction is a serious impediment to progress on this issue. The uncalculated correction to $\sigma\left(e^{+} e^{-} \rightarrow J / \psi+\eta_{c}\right)$ of relative order $\alpha_{s} v^{2}$ is potentially large, as is the uncalculated correction of relative order $\alpha_{s}^{4}$. While the calculation of the former correction may be feasible, the calculation of the latter correction is probably beyond the current state of the art. However, one might be able to identify large contributions that could be resummed to all orders in $\alpha_{s}$. 
Finally, it would be desirable to extend the theoretical calculations to include $P$-wave and higher $S$-wave states. In the NRQCD approach, a serious obstacle to such calculations is the fact that the relativistic corrections become much larger for excited states, possibly spoiling the convergence of the NRQCD velocity expansion.

\subsection{Inclusive production in $e^{+} e^{-}$collisions}

\subsubsection{Experiments and LO theoretical expectations}

In 2001, the prompt $J / \psi$ inclusive production cross section $\sigma\left(e^{+} e^{-} \rightarrow J / \psi+X\right)$ was measured to be $\sigma_{\text {tot }}=$ $2.52 \pm 0.21 \pm 0.21 \mathrm{pb}$ by the BABAR collaboration [795]. A smaller value, $\sigma_{\text {tot }}=1.47 \pm 0.10 \pm 0.13 \mathrm{pb}$ was found by the Belle collaboration [796]. The color-singlet contribution to the prompt $J / \psi$ inclusive production cross section at $\mathrm{LO}$ in $\alpha_{s}$, including contributions from the processes $e^{+} e^{-} \rightarrow J / \psi+c \bar{c}, e^{+} e^{-} \rightarrow J / \psi+g g$, and $e^{+} e^{-} \rightarrow J / \psi+q \bar{q}+g g(q=u, d, s)$, was estimated to be only about $0.3-0.5 \mathrm{pb}$ (Ref. [797-802]), which is much smaller than the measured value. This would suggest that the color-octet contribution might play an important role in inclusive $J / \psi$ production [797-802]. However, it was found by the Belle collaboration [778] that the associated production cross section

$$
\sigma\left(e^{+} e^{-} \rightarrow J / \psi+c \bar{c}\right)=\left(0.87_{-0.19}^{+0.21} \pm 0.17\right) \mathrm{pb}
$$

is larger, by at least a factor of 5 , than the LO NRQCD factorization prediction, which includes both the colorsinglet contribution [798-803] and the color-octet contribution [803]. The ratio of the $J / \psi+c \bar{c}$ cross section to the $J / \psi$ inclusive cross section was found by the Belle collaboration [778] to be

$$
R_{c \bar{c}}=\frac{\sigma\left(e^{+} e^{-} \rightarrow J / \psi+c \bar{c}\right)}{\sigma\left(e^{+} e^{-} \rightarrow J / \psi+X\right)}=0.59_{-0.13}^{+0.15} \pm 0.12
$$

which is also much larger than LO NRQCD factorization prediction. If one includes only the color-singlet contribution at LO in $\alpha_{s}$, then the ratio is predicted to be $R_{c \bar{c}}=0.1-0.3$ (Ref. [797-802, 804]), depending on the values of input parameters, such as $\alpha_{s}, m_{c}$, and, especially, the color-singlet matrix elements. A large color-octet contribution could enhance substantially the $J / \psi$ inclusive cross section (the denominator) but could enhance only slightly the $J / \psi+c \bar{c}$ cross section (the numerator)[803] and, therefore, would have the effect of decreasing the prediction for $R_{c \bar{c}}$. Thus, the LO theoretical results and the experimental results in Eqs. (160) and (161) presented a serious challenge to the NRQCD factorization picture. ${ }^{18}$

18 The light-cone perturbative-QCD approach [805] gives a prediction that $R_{c \bar{c}}=0.1-0.3$, while the CEM gives a prediction that
Several theoretical studies were made with the aim of resolving this puzzle in $J / \psi$ production. The authors of Ref. [807] used soft-collinear effective theory (SCET) to resum the color-octet contribution to the $J / \psi$ inclusive cross section, the authors of Ref. [808] used SCET to analyze the color-singlet contribution to $e^{+} e^{-} \rightarrow J / \psi+g g$, and the authors of Ref. [809] resummed the LO and NLO logarithms in the color-singlet contribution to the $J / \psi$ inclusive cross section. These resummation calculations, while potentially useful, did not resolve the puzzle.

Very recently, the Belle collaboration reported new measurements [810]:

$$
\begin{aligned}
\sigma(J / \psi+X) & =(1.17 \pm 0.02 \pm 0.07) \mathrm{pb} \\
\sigma(J / \psi+c \bar{c}) & =\left(0.74 \pm 0.08_{-0.08}^{+0.09}\right) \mathrm{pb} \\
\sigma\left(J / \psi+X_{\text {non } c \bar{c}}\right) & =(0.43 \pm 0.09 \pm 0.09) \mathrm{pb}
\end{aligned}
$$

the value of the inclusive $J / \psi$ cross section in Eq. (162) is significantly smaller than the values that were obtained previously by the $B A B A R$ collaboration [795] and by the Belle collaboration [796], but it is still much larger than the LO NRQCD prediction. The cross section $\sigma\left(e^{+} e^{-} \rightarrow\right.$ $J / \psi+c \bar{c})$ in Eq. (163) is also much larger than the LO color-singlet and color-octet predictions.

$$
\text { 4.6.2. } e^{+} e^{-} \rightarrow J / \psi+c \bar{c} \text { at } N L O
$$

An important step toward resolving the puzzle of the $J / \psi$ production cross section was taken in Ref. [811], where it was found that the correction of NLO in $\alpha_{s}$ to $e^{+} e^{-} \rightarrow J / \psi+c \bar{c}$ gives a large enhancement. In this work, a value for the square of the $J / \psi$ wave function at the origin was obtained by comparing the observed $J / \psi$ leptonic decay width $(5.55 \pm 0.14 \pm 0.02 \mathrm{keV})$ with the theoretical expression for that width, including corrections of NLO in $\alpha_{s}$. (The square of the wave function at the origin is proportional to the color-singlet NRQCD long-distance matrix element of leading order in $v$.) The value $\left|R_{S}(0)\right|^{2}=1.01 \mathrm{GeV}^{3}$ that was obtained in this work is a factor of 1.25 larger than the value $\left|R_{S}(0)\right|^{2}=0.810 \mathrm{GeV}^{3}$ that was obtained in potential-model calculations and used in Ref. [803] to calculate the $e^{+} e^{-} \rightarrow J / \psi+c \bar{c}$ cross section. Taking $m_{c}=1.5 \mathrm{GeV}$, the renormalization scale $\mu_{R}=2 m_{c}$, and $\Lambda_{\overline{\mathrm{MS}}}^{(4)}=0.338 \mathrm{GeV}$, the authors of Ref. [811] found the direct production cross section at NLO in $\alpha_{s}$ to be

$$
\sigma^{\mathrm{NLO}}\left(e^{+} e^{-} \rightarrow J / \psi+c \bar{c}+X\right)=0.33 \mathrm{pb},
$$

which is a factor of 1.8 larger than the LO result $(0.18$ $\mathrm{pb})$ that is obtained with the same set of input parameters. Results for the NLO cross section for other values of the input parameters can be found in Table 44 .

$R_{c \bar{c}}=0.06$ (Ref. [806]), both of which are far below the measured value of $R_{c \bar{c}}$. 
TABLE 44: Color-singlet contributions to cross sections for $J / \psi$ production in $e^{+} e^{-}$annihilation at the $B$-factory energy, $\sqrt{s}=10.58 \mathrm{GeV}$. The table shows cross sections for the production of $J / \psi+c \bar{c}$ (Ref. [811]) and $J / \psi+g g$ (Ref. [812]), as well as the ratio $R_{c \bar{c}}$, which is computed from the expression in Eq. (161) by summing, in the denominator, over only the $J / \psi+c \bar{c}$ and $J / \psi+g g$ cross sections. The cross sections, in units of $\mathrm{pb}$, are shown for different values of $m_{c}(1.4$ and $1.5 \mathrm{GeV})$ and $\mu_{R}\left(2 m_{c}\right.$ and $\left.\sqrt{s} / 2\right)$, along with the corresponding value of $\alpha_{s}\left(\mu_{R}\right)$. The prompt $J / \psi+c \bar{c}$ cross sections include feeddown from the $\psi(2 S)$ and the $\chi_{c J}$ states, while the prompt $J / \psi+g g$ cross sections include feeddown from the $\psi(2 S)$ state

\begin{tabular}{lcccc}
\hline \hline$\mu_{R}(\mathrm{GeV})$ & 2.8 & 3.0 & 5.3 & 5.3 \\
$\alpha_{s}\left(\mu_{R}\right)$ & 0.267 & 0.259 & 0.211 & 0.211 \\
$m_{c}(\mathrm{GeV})$ & 1.4 & 1.5 & 1.4 & 1.5 \\
\hline$\sigma^{\mathrm{LO}}(g g)$ & 0.42 & 0.32 & 0.26 & 0.22 \\
$\sigma^{\mathrm{NLO}}(g g)$ & 0.50 & 0.40 & 0.39 & 0.32 \\
$\sigma_{\text {prompt }}^{\mathrm{NLO}}(g g)$ & 0.67 & 0.54 & 0.53 & 0.44 \\
\hline$\sigma^{\mathrm{LO}}(c \bar{c})$ & 0.27 & 0.18 & 0.17 & 0.12 \\
$\sigma^{\mathrm{NLO}}(c \bar{c})$ & 0.47 & 0.33 & 0.34 & 0.24 \\
$\sigma_{\mathrm{prompt}}^{\mathrm{NLO}}(c \bar{c})$ & 0.71 & 0.51 & 0.53 & 0.39 \\
\hline$R_{c \bar{c}}^{\mathrm{LO}}$ & 0.39 & 0.36 & 0.40 & 0.35 \\
$R_{c \bar{c}}^{\mathrm{NLO}}$ & 0.51 & 0.49 & 0.50 & 0.47 \\
\hline \hline
\end{tabular}

From Table 44 and Fig. 65, it can be seen that the renormalization-scale dependence of the NLO cross section for $e^{+} e^{-} \rightarrow J / \psi+c \bar{c}$ is quite strong. This strong $\mu_{R}$ dependence is related to the large size of the NLO contribution relative to the $\mathrm{LO}$ contribution. The cross section is also sensitive to the value of the charm-quark mass. It is larger for smaller values of $m_{c}$.

In Ref. [811] the QED contribution at order $\alpha_{s} \alpha^{3}$ and the contribution from $e^{+} e^{-} \rightarrow 2 \gamma^{*} \rightarrow J / \psi+c \bar{c}$ were found to increase the $J / \psi+c \bar{c}$ cross section by only a small amount. The feeddown contributions $e^{+} e^{-} \rightarrow$ $\psi(2 S)+c \bar{c} \rightarrow J / \psi+c \bar{c}+X$ and $e^{+} e^{-} \rightarrow \chi_{c J}+c \bar{c} \rightarrow$ $J / \psi+c \bar{c}+X$ were also estimated in Ref. [811]. The primary feeddown contribution comes from the $\psi(2 S)$ and produces an enhancement factor of 1.355 for the prompt $J / \psi c \bar{c}$ cross section.

Taking into account all of the aforementioned contributions, we obtain the following estimate for the prompt cross section:

$$
\sigma_{\text {prompt }}^{\mathrm{NLO}}\left(e^{+}+e^{-} \rightarrow J / \psi+c \bar{c}+X\right)=0.51 \mathrm{pb},
$$

where the input values $m_{c}=1.5 \mathrm{GeV}$ and $\mu_{R}=2 m_{c}$ have been used. As is shown in Table 44, despite the uncertainties in the input parameters, the NLO correction to the color-singlet contribution to $e^{+} e^{-} \rightarrow J / \psi+c \bar{c}$ substantially increases the cross section and largely reduces the discrepancy between experiment and theory. Furthermore, the NLO relativistic correction to this process is found to be negligible [784], in contrast with the NLO

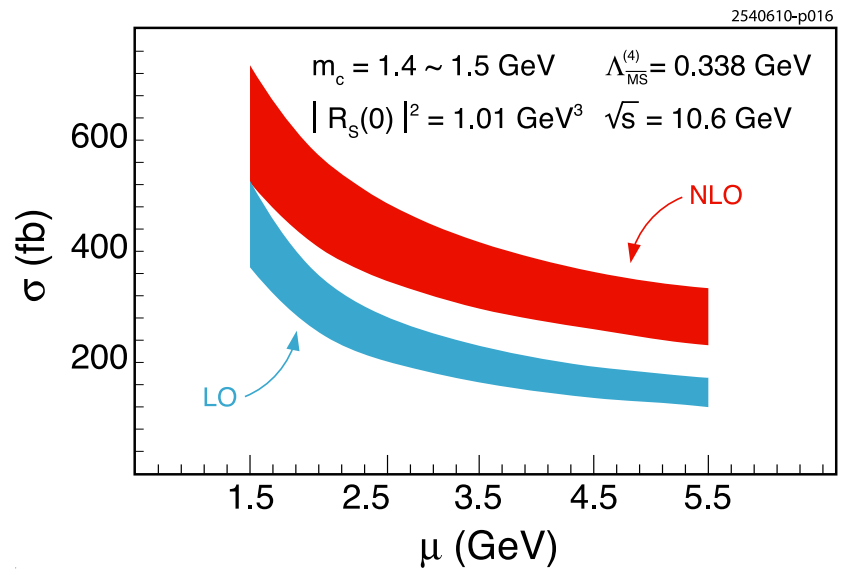

FIG. 65: Color-singlet contributions to the direct production cross sections for $e^{+} e^{-} \rightarrow J / \psi+c \bar{c}$ as functions of the renormalization scale $\mu_{R}$. The following values of the input parameters were taken: $\left|R_{S}(0)\right|^{2}=1.01 \mathrm{GeV}^{3}, \Lambda_{\overline{\mathrm{MS}}}^{(4)}=0.338 \mathrm{GeV}$, $\sqrt{s}=10.6 \mathrm{GeV}$. Results at NLO in $\alpha_{s}$ are represented by the upper band, and results at LO in $\alpha_{s}$ are represented by the lower band. In each case, the upper border corresponds to the input value $m_{c}=1.4 \mathrm{GeV}$ and the lower border corresponds to the input value $m_{c}=1.5 \mathrm{GeV}$. From [811] with kind permission, copyright (2007) The American Physical Society

relativistic correction to the process $e^{+}+e^{-} \rightarrow J / \psi+\eta_{c}$ (Sect. 4.5).

Recently, the authors of Ref. [813] confirmed the results of Ref. [811] and presented a more detailed analysis of the $J / \psi$ angular distributions and polarization parameters, using slightly different input parameters than those in Ref. [811].

$$
\text { 4.6.3. } e^{+} e^{-} \rightarrow J / \psi+g g \text { at } N L O
$$

In NRQCD factorization, the production cross section for the $J / \psi$ in association with light hadrons, $\sigma\left(e^{+} e^{-} \rightarrow J / \psi+X_{\text {non- }-c \bar{c}}\right)$, includes the color-singlet contribution $\sigma(J / \psi+g g)$, and the color-octet contributions $\sigma\left(J / \psi\left({ }^{3} P_{J}^{[8]},{ }^{1} S_{0}^{[8]}\right)+g\right)$. Contributions from other Fock states are suppressed by powers of $\alpha_{s}$ or $v$. The corrections of NLO in $\alpha_{s}$ to $\sigma(J / \psi+g g)$ were calculated in Refs. [812, 814] and found to enhance the LO cross section by about $20-30 \%$. The prompt production cross section $\sigma\left(e^{+} e^{-} \rightarrow J / \psi+g g\right)$ at NLO in $\alpha_{s}$, including the feeddown contribution from the $\psi(2 S)$, can be found in Table 44. From Fig. 66, it can be seen that the renormalization-scale dependence at NLO is moderate and much improved in comparison with the renormalization-scale dependence at LO. It can also be seen that the NLO result is consistent with the latest Belle measurement of $\sigma\left(e^{+} e^{-} \rightarrow J / \psi+X_{\text {non- }-c \bar{c}}\right)$ (Ref. [810]), given the experimental uncertainties. Resummation of the leading logarithms near the kinematic endpoint of the $J / \psi$ momentum distribution is found to 
change the endpoint momentum distribution, but to have only a small effect on the total $J / \psi+g g$ cross section [812].

Results for the color-singlet contributions to $\sigma\left(e^{+} e^{-} \rightarrow\right.$ $J / \psi+c \bar{c})(\operatorname{Ref} .[811]), \sigma(J / \psi+g g)$ (Ref. [812]), and the corresponding ratio $R_{c \bar{c}}$ are summarized in Table 44. In Table 44, the color-octet contribution $\sigma\left(J / \psi\left({ }^{3} P_{J}^{[8]},{ }^{1} S_{0}^{[8]}\right)+g\right)$ is ignored. It can be seen that the NLO results significantly reduce the discrepancies between theory and experiment.

In Fig. 67, the measured distributions in the $J / \psi$ momentum $p^{*}$ are shown for the processes $e^{+} e^{-} \rightarrow J / \psi+c \bar{c}$ and $e^{+} e^{-} \rightarrow J / \psi+X_{\text {non }-c \bar{c}}$ (Ref. [810]). The calculated $J / \psi$ momentum distributions for the process $e^{+} e^{-} \rightarrow J / \psi+g g$ at $\mathrm{NLO}$ in $\alpha_{s}$ (Refs. [812, 814]) are roughly compatible with the $e^{+} e^{-} \rightarrow J / \psi+X_{\text {non-ci }}$ data. We note that the NLO $J / \psi$ momentum distribution is much softer than the LO $J / \psi$ momentum distribution (see Fig. 6 of Ref. [812]), resulting in better agreement with data.

Finally, it was found in Ref. [816] that the relativeorder- $v^{2}$ relativistic correction can enhance the $e^{+} e^{-} \rightarrow$ $J / \psi+g g$ cross section by $20-30 \%$, which is comparable to the enhancement that arises from the corrections of NLO in $\alpha_{s}$ (Ref. [812, 814]). This relativistic correction has been confirmed in Ref. [817]. If one includes both the correction of NLO in $\alpha_{s}$ and the relative-order- $v^{2}$ relativistic correction, then the color-singlet contribution to $e^{+} e^{-} \rightarrow J / \psi+g g$ saturates the latest observed cross section for $e^{+} e^{-} \rightarrow J / \psi+X_{\text {non }-c \bar{c}}$ in Eq.(164), even if a significantly smaller color-singlet matrix element is chosen. This leaves little room for the color-octet contribution $\sigma\left(J / \psi\left({ }^{3} P_{J}^{[8]},{ }^{1} S_{0}^{[8]}\right)+g\right)$ and may imply that the

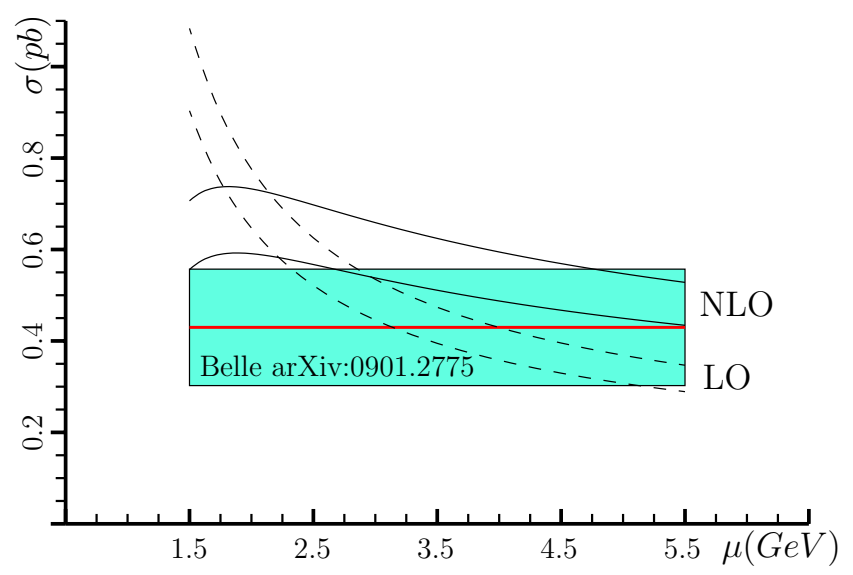

FIG. 66: Prompt cross sections for the process $e^{+} e^{-} \rightarrow$ $J / \psi+g g$. The curves are the predictions of Ref. [812], plotted as functions of the renormalization scale $\mu_{R}$ at LO (dashed curves) and NLO (solid curves) in $\alpha_{s}$. In each case, the upper curves correspond to $m_{c}=1.4 \mathrm{GeV}$, and the lower curves correspond to $m_{c}=1.5 \mathrm{GeV}$. The experimental datum (shaded band) is for the process $e^{+} e^{-} \rightarrow J / \psi+X_{\text {non }-c \bar{c}}$ (Ref. [810]). From [812] with kind permission, copyright (2009) The American Physical Society true values of the color-octet matrix elements are much smaller than those that have been extracted in LO fits to the Tevatron data or those that would be expected from a naive application of the NRQCD velocity-scaling rules.

\subsubsection{Color-octet process $e^{+} e^{-} \rightarrow J / \psi\left({ }^{3} P_{J}^{[8]},{ }^{1} S_{0}^{[8]}\right)+g$}

The color-octet contribution to the cross section at LO in $\alpha_{s}$ is given at LO in $v$ by $\sigma\left(J / \psi\left({ }^{3} P_{J}^{[8]},{ }^{1} S_{0}^{[8]}\right)+g\right)$. This contribution was calculated in Ref. [818], and an enhancement near the kinematic endpoint, $z=1$, was predicted. Here, $z=E_{c \bar{c}} / E_{c \bar{c}}^{\max }$ is the energy of the $c \bar{c}$ pair divided by the maximum possible energy of the $c \bar{c}$ pair. As can be seen from Fig. 67, measurements of the $J / \psi$ momentum distribution do not show any enhancement near the kinematic endpoint. In Ref. [807], resummations of the NRQCD velocity expansion near the endpoint and resummations of logarithms of $1-z$ were considered. These resummations smear out the peak near $z=1$ and shift it to smaller values of $z$, making the theory more compatible with the data. However, the resummation results rely heavily on a nonperturbative shape function that is not well known, and so it is not clear if they can reconcile the theoretical and experimental results. The non-

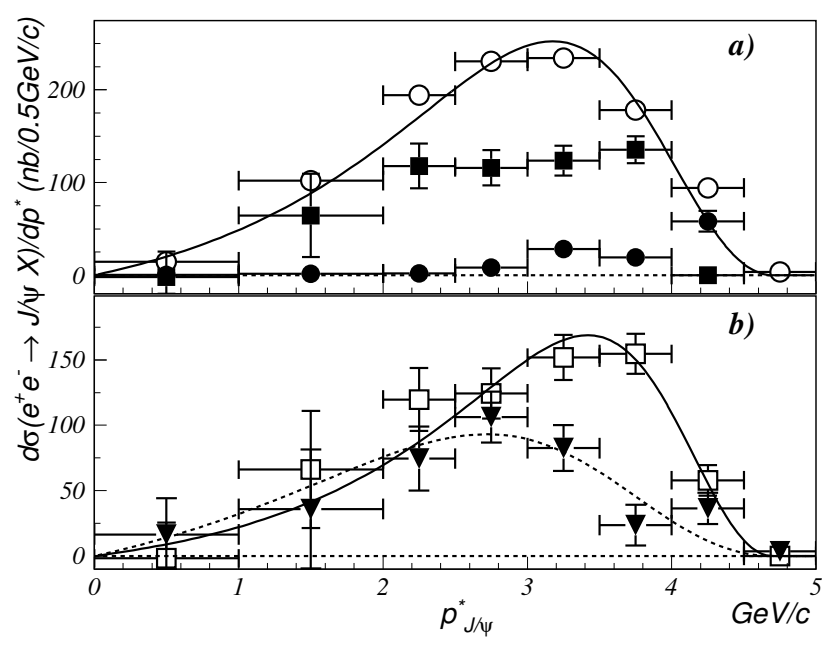

FIG. 67: $J / \psi$ momentum distributions: (a) the Belle measurements [810] for the inclusive distribution (open circles), the distribution from $e^{+} e^{-} \rightarrow J / \psi+H_{c}+X$ (filled squares), where $H_{c}$ is a charmed hadron, and the distribution from double-charmonium production (filled circles); (b) the Belle measurements [810] for the distribution for the sum of all $e^{+} e^{-} \rightarrow J / \psi+c \bar{c}$ processes (open squares) and the distribution from the $e^{+} e^{-} \rightarrow J / \psi+X_{\text {non-c亠 }}$ processes (filled triangles). The curves are the results of using the Peterson function [815] to fit the inclusive distribution [solid curve in (a)], the $e^{+} e^{-} \rightarrow J / \psi+c \bar{c}$ distribution [solid curve in (b)], and the $e^{+} e^{-} \rightarrow J / \psi+X_{\text {non-co }}$ distribution [dashed curve in (b)]. From [810] with kind permission, copyright (2009) The American Physical Society 
observation of an enhancement near $z=1$ might also point to a possibility that we mentioned in Sect. 4.6.3, namely, that the ${ }^{3} P_{J}$ and ${ }^{1} S_{0}$ color-octet matrix elements are much smaller than would be expected from the LO fits to the Tevatron data or the NRQCD velocity-scaling rules.

Very recently, the corrections at NLO in $\alpha_{s}$ to the color-octet contribution to inclusive $J / \psi$ production have been calculated [819]. In comparison with the LO result, the NLO contributions are found to enhance the short-distance coefficients in the color-octet contributions $\sigma\left(e^{+} e^{-} \rightarrow c \bar{c}\left({ }^{1} S_{0}^{(8)}\right)+g\right)$ and $\sigma\left(e^{+} e^{-} \rightarrow c \bar{c}\left({ }^{3} P_{J}^{(8)}\right)+g\right)$ (with $J=0,1,2$ ) by a factor of about 1.9. Moreover, the NLO corrections smear the peak at the endpoint in the $J / \psi$ energy distribution, although the bulk of the color-octet contribution still comes from the region of large $J / \psi$ energy. One can obtain an upper bound on the sizes of the color-octet matrix elements by setting the color-singlet contribution to be zero in $\sigma\left(e^{+} e^{-} \rightarrow J / \psi+X_{\text {non }-c \bar{c}}\right)$. The result, at NLO in $\alpha_{s}$, is

$$
\begin{gathered}
\left\langle 0\left|\mathcal{O}^{J / \psi}\left({ }^{1} S_{0}^{(8)}\right)\right| 0\right\rangle+4.0\left\langle 0\left|\mathcal{O}^{J / \psi}\left({ }^{3} P_{0}^{(8)}\right)\right| 0\right\rangle / m_{c}^{2} \\
<(2.0 \pm 0.6) \times 10^{-2} \mathrm{GeV}^{3} .
\end{gathered}
$$

This bound is smaller by about a factor of 2 than the values that were extracted in $\mathrm{LO}$ fits to the Tevatron data for the combination of matrix elements in Eq. (167).

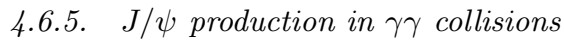

Photon-photon $(\gamma \gamma)$ collisions can be can be studied at $e^{+} e^{-}$colliders by observing processes in which the incoming $e^{+}$and $e^{-}$each emit a virtual $\gamma$ that is very close to its mass shell. The inclusive cross section differential in $p_{T}$ for the production of $J / \psi$ in $\gamma \gamma$ collisions at LEP has been measured by the DELPHI collaboration [820, 821].

The cross section differential in $p_{T}$ has been calculated in the CSM and the NRQCD factorization approach at LO in $\alpha_{s}$ (Refs. [822-826]). The computations include three processes: the direct- $\gamma$ process $\gamma \gamma \rightarrow(c \bar{c})+g$, which is of order $\alpha^{2} \alpha_{s}$; the single-resolved- $\gamma$ process $i \gamma \rightarrow(c \bar{c})+i$, which is of order $\alpha \alpha_{s}^{2}$; and the doubleresolved- $\gamma$ process $i j \rightarrow(c \bar{c})+k$, which is of order $\alpha_{s}^{3}$. Here, $i j=g g, g q, g \bar{q}$, or $q \bar{q}$, where $q$ is a light quark. Because the leading contribution to the distribution of a parton in a $\gamma$ is of order $\alpha / \alpha_{s}$, all of the processes that we have mentioned contribute to the $\gamma \gamma$ production rate in order $\alpha^{2} \alpha_{s}$. The corrections of NLO in $\alpha_{s}$ to the direct- $\gamma$ process have been computed in Ref. [827].

A comparison of the LO CSM and NRQCD factorization predictions with the DELPHI data is shown in Fig. 68. The data clearly favor the NRQCD factorization prediction. However, it should be kept in mind that there may be large NLO corrections to the color-singlet contribution, as is the case in $p p$ and $e p$ charmonium production. Therefore, no firm conclusions can be drawn until a complete NLO calculation of the production cross section is available.

\subsubsection{Future opportunities}

The central values for the prompt $J / \psi$ inclusive production cross section that were obtained by the BABAR collaboration and the Belle collaboration differ more than a factor of 2 . It would certainly be desirable to clear up this discrepancy. Furthermore, the BABAR collaboration has not presented results for $\sigma\left(e^{+} e^{-} \rightarrow J / \psi+c \bar{c}\right)$ and $\sigma\left(e^{+} e^{-} \rightarrow J / \psi+X_{\text {non- }-c \bar{c}}\right)$. It is very important that the BABAR collaboration check the Belle results for these cross sections, which play a central role in efforts to understand the mechanisms of inclusive quarkonium production. Additionally, measurements of greater accuracy of the charmonium angular distributions and polarization parameters would be useful in understanding the mechanisms of quarkonium production. Measurements of in-

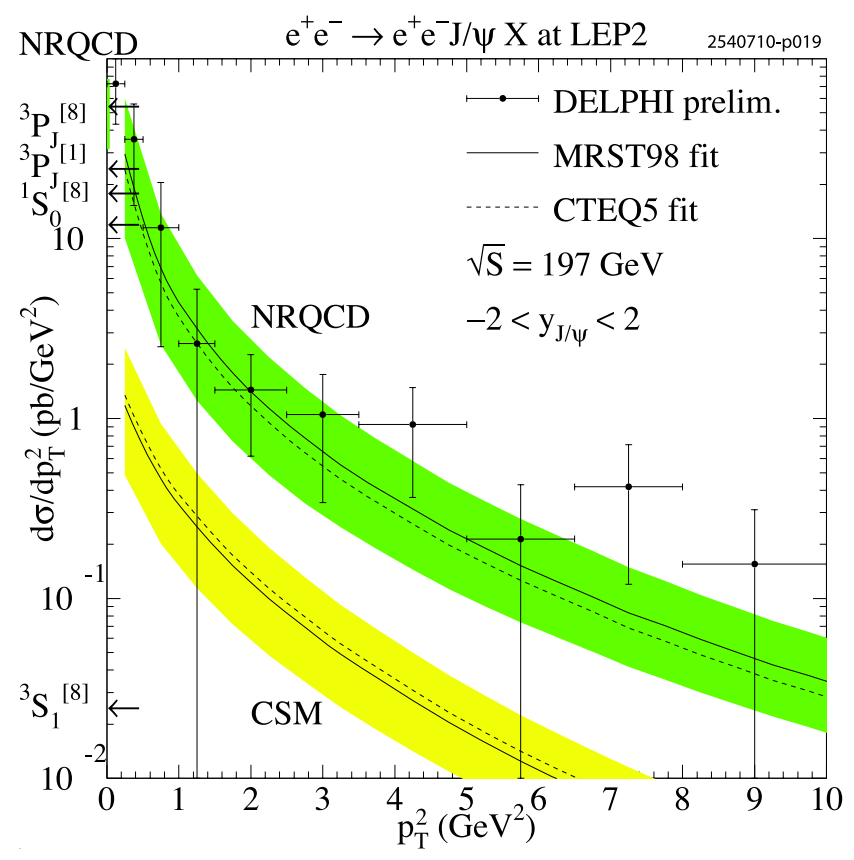

FIG. 68: Comparison of the inclusive cross section differential in $p_{T}$ for $\gamma \gamma$ production of the $J / \psi$ (Refs. [820, 821]) with the predictions at LO in $\alpha_{s}$ of the CSM and the NRQCD factorization approach. The upper two curves are the NRQCD factorization predictions, and the lower two curves are the CSM predictions. The solid and dashed curves correspond to the MRST98LO (Ref. [732]) and the CTEQ5L (Ref. [733]) parton distributions, respectively. The arrows indicate the NRQCD factorization predictions at $p_{T}=0$ for the ${ }^{3} P_{J}^{[1]}$, ${ }^{1} S_{0}^{[8]},{ }^{3} S_{1}^{[8]}$, and ${ }^{3} P_{J}^{[8]}$ contributions. The theoretical uncertainty bands were obtained by combining the uncertainties from $m_{c}=1.5 \pm 0.1 \mathrm{GeV}$, the decay branching fractions of the $\psi(2 S)$ and the $\chi_{c J}$ states, the parton distributions, and the NRQCD long-distance matrix elements with the uncertainties that are obtained by varying the renormalization factorization scales between $2 m_{T}$ and $m_{T} / 2$. Here $m_{T}=\sqrt{4 m_{c}^{2}+p_{T}^{2}}$. From [826] with kind permission, copyright (2002) The American Physical Society 
clusive cross sections for the production of charmonium states other than the $J / \psi$ might also yield important clues regarding the production mechanisms.

In the theoretical prediction for the color-singlet contribution to $\sigma\left(e^{+} e^{-} \rightarrow J / \psi+c \bar{c}\right)$, large uncertainties arise from the uncertainties in the renormalization scale $\mu_{R}$ and $m_{c}$. (The uncertainties in the theoretical prediction for the color-singlet contribution to $\sigma\left(e^{+} e^{-} \rightarrow J / \psi+g g\right)$ seem to be under rather good control.) The uncertainties in the color-singlet contribution to $\sigma\left(e^{+} e^{-} \rightarrow J / \psi+c \bar{c}\right)$ might be reduced by understanding the origins of the large corrections at NLO in $\alpha_{s}$ and by taking advantage of recent progress in determining $m_{c}$ (Ref. [227]). It is important to examine further whether the observed value of $\sigma\left(e^{+} e^{-} \rightarrow J / \psi+X_{\text {non }-c \bar{c}}\right)$ is actually saturated by $\sigma\left(e^{+} e^{-} \rightarrow J / \psi+g g\right)$, which would imply that the color-octet $\left({ }^{3} P_{J}^{[8]},{ }^{1} S_{0}^{[8]}\right)$ contributions are negligible. In this regard, one might obtain additional information by examining the $J / \psi$ angular distribution and polarization parameters, in addition to the total cross section and momentum distribution. Finally, we mention, as we did in Sect. 4.3.3, that theoretical uncertainties in the region near the kinematic endpoint $z=1$ might be reduced through a systematic study of resummations of the perturbative and velocity expansions in both $e p$ and $e^{+} e^{-}$ quarkonium production.

Quarkonium production in $\gamma \gamma$ collisions provides yet another opportunity to understand quarkonium production mechanisms. In order to make a definitive comparison of the DELPHI data for the $J / \psi$ cross section differential in $p_{T}$ with the NRQCD factorization prediction, it is necessary to have a complete calculation of all of the direct and resolved contributions at least through NLO in $\alpha_{s}$. Further measurements of quarkonium production in $\gamma \gamma$ collisions should be carried out at the next opportunity at an $e^{+} e^{-}$or $e p$ collider. It may also be possible to measure quarkonium production in $\gamma \gamma$ and $\gamma p$ collisions at the LHC [828].

\section{7. $B_{c}$ production}

\subsubsection{Experimental progress}

The first observation of the $B_{c}$ was reported by the CDF collaboration in Ref. [73]. Subsequently, this unique double-heavy-flavored meson has been observed by both the $\mathrm{CDF}$ and $\mathrm{D} \varnothing$ collaborations at the Tevatron via two decay channels: $B_{c} \rightarrow J / \psi+\bar{l} \nu_{l}$ and $B_{c} \rightarrow J / \psi+\pi^{+}$ (Refs. [57, 58, 75, 77, 173]). Using an event sample corresponding to an integrated luminosity of $360 \mathrm{pb}^{-1}$ at $\sqrt{s}=1.96 \mathrm{TeV}$, the CDF collaboration has measured the $B_{c}$ lifetime in the decay $B_{c}^{+} \rightarrow J / \psi e^{+} \nu_{e}$ (Ref. [75]) and obtained

$$
\tau_{B_{c}}=0.463_{-0.065}^{+0.073} \pm 0.036 \mathrm{ps} .
$$

The $B_{c}$ lifetime has also been measured by the CDF collaboration in the decay $B_{c} \rightarrow J / \psi+l^{ \pm}+X$ (Ref. [829]).
The result of this measurement, which is based on an event sample corresponding to an integrated luminosity of $1 \mathrm{fb}^{-1}$ at $\sqrt{s}=1.96 \mathrm{TeV}$, is

$$
\tau_{B_{c}}=0.475_{-0.049}^{+0.053} \pm 0.018 \mathrm{ps} .
$$

The CDF collaboration has measured the $B_{c}$ mass in the decay $B_{c}^{ \pm} \rightarrow J / \psi \pi^{ \pm}$(Refs. [57, 173]), obtaining in its most recent measurement [57], which is based on an integrated luminosity of $2.4 \mathrm{fb}^{-1}$ at $\sqrt{s}=1.96 \mathrm{TeV}$, the value

$$
m_{B_{c}}=6275.6 \pm 2.9 \pm 2.5 \mathrm{MeV} .
$$

The DØ collaboration, making use of an event sample based on an integrated luminosity of $1.3 \mathrm{fb}^{-1}$ at $\sqrt{s}=1.96 \mathrm{TeV}$, has also provided measurements of the $B_{c}$ lifetime [58],

$$
\tau_{B_{c}}=0.448_{-0.036}^{+0.038} \pm 0.032 \mathrm{ps},
$$

and the $B_{c}$ mass [77],

$$
m_{B_{c}}=6300 \pm 14 \pm 5 \mathrm{MeV} .
$$

The results obtained by the two collaborations are consistent with each other. (See Sect. 2.2.4 of this article for a further discussion of the $B_{c}$ mass and lifetime.)

Recently, the CDF collaboration has updated a previous measurement [830] of the ratio

$$
R_{B_{c}}=\frac{\sigma\left(B_{c}^{+}\right) \mathcal{B}\left(B_{c} \rightarrow J / \psi+\mu^{+}+\nu_{\mu}\right)}{\sigma\left(B^{+}\right) \mathcal{B}\left(B^{+} \rightarrow J / \psi+K^{+}\right)} .
$$

The new analysis [831], which used a data set that corresponds an integrated luminosity of $1 \mathrm{fb}^{-1}$, yielded the results

$$
R_{B_{c}}=0.295 \pm 0.040(\text { stat })_{-0.026}^{+0.033}(\text { sys }) \pm 0.036\left(p_{T}\right),
$$

for $p_{T}>4 \mathrm{GeV}$, and

$$
R_{B_{c}}=0.227 \pm 0.040(\text { stat })_{-0.017}^{+0.024}(\text { sys }) \pm 0.014\left(p_{T}\right),
$$

for $p_{T}>6 \mathrm{GeV}$, where $p_{T}$ is the $B_{c}$ transverse momentum. The measurements in Refs. [830, 831] provided the first, indirect, experimental information on the production cross section.

\subsubsection{Calculational schemes}

Experimental studies of $B_{c}$ production could help to further theoretical progress in understanding the production mechanisms for heavy-quark bound states. On the other hand, experimental observations of the $B_{c}$ are very challenging and might benefit from theoretical predictions of $B_{c}$ production rates, which could be of use in devising efficient observational strategies, for example, in selecting decay channels to study. 
So far, two theoretical approaches have been used to obtain predictions for $B_{c}$ hadroproduction. Both of them are based on the NRQCD factorization approach.

The simplest approach conceptually is to calculate the contributions from all of the hard subprocesses, through a fixed order in $\alpha_{s}$, that produce a $c \bar{c}$ pair and a $b \bar{b}$ pair. The $c$ quark (antiquark) is required to be nearly co-moving with the $b$ antiquark (quark) in order to produce a $B_{c}$. We call this approach the "fixed-order approach." A typical Feynman diagram that appears in this approach is shown in Fig. 69. If the initial-state partons are light (i.e., gluons, or $u, d$, or $s$ quarks or antiquarks), then the leading order for $B_{c}$ production in the fixed-order approach is $\alpha_{s}^{4}$. LO computations in this approach can be found in Refs. [832-840].

An alternative approach is the fragmentation approximation, in which the production process is factorized into convolutions of fragmentation functions with a simple perturbative-QCD hard-scattering sub-process [841, 842]. An example of such a factorized contribution is one in which $p p \rightarrow b \bar{b}$, with the final-state $b$ or $\bar{b}$ fragmenting to $B_{c}+\bar{c}$. This process is illustrated in Fig. 70 . The fragmentation approximation drastically simplifies the calculation and also provides a formalism with which to resum large final-state logarithms of $p_{T} / m_{b}$. However, it has been shown in Ref. [843] that the fragmentation diagrams are not dominant at the Tevatron unless the $B_{c}$ is produced at very large (experimentally inaccessible) values of $p_{T}$. A similar behavior has been found in Ref. [618] in the case of charmonium or bottomonium production at the Tevatron and the LHC in association with a heavyquark pair. Therefore, we can conclude that there is no need to resum logarithms of $p_{T} / m_{b}$ at the values of $p_{T}$ that are accessible at the current hadron colliders. That is, a fixed-order calculation is sufficient, and the fragmentation approach is not relevant.

Within either the fixed-order approach or the fragmentation approach, various choices of factorization scheme are possible. In the simplest scheme, which is known as the fixed-flavor-number (FFN) scheme, the flavor content of the proton is held fixed and includes only the light flavors. Specifically, it is assumed that only the light quarks are "active" flavors, which means that there are contribu-

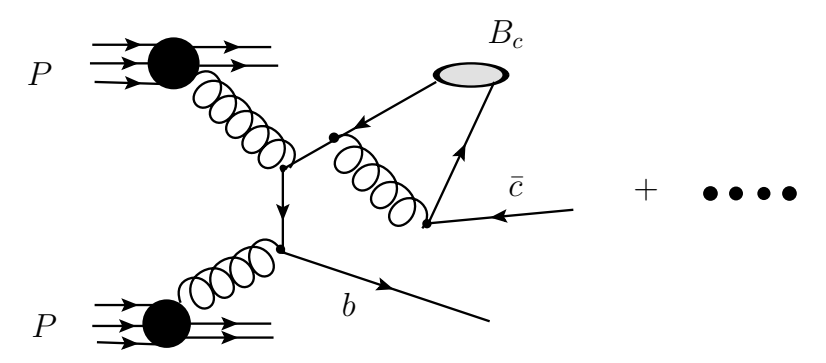

FIG. 69: Typical Feynman diagram for $B_{c}$ production via gluon-gluon fusion in the fixed-order approach at order $\alpha_{s}^{4}$ tions involving light-quark (and antiquark) parton distributions, but there are no contributions involving $c$ - and $b$-quark (and antiquark) parton distributions.

Alternatives to the FFN scheme have been proposed with the aim of improving upon FFN calculations by resumming large initial-state collinear logarithms of $Q / m_{c}$ and $Q / m_{b}$, where $Q$ is a kinematic scale in the production process $\left(e . g ., p_{T}\right)$. Such logarithms arise, for example, in a process in which an initial-state gluon splits into a quark-antiquark pair. The quark or antiquark then participates in a hard scattering, giving rise to a $B_{c}$. (An example of such a process is $g \rightarrow c \bar{c}$, followed by $g \bar{c} \rightarrow B_{c} \bar{b}$.) It has been suggested that one can resum these initialstate collinear logarithms by making use of heavy-flavor parton distributions. The basic idea is that one can absorb logarithms of $Q / m_{c}$ or $Q / m_{b}$ into massless $c$-quark (or antiquark) and $b$-quark (or antiquark) parton distributions, in which they can be computed through DGLAP evolution. In such methods, there are contributions in which the $B_{c}$ is produced in a $2 \rightarrow 2$ scattering that involves a $c$ or $b$ initial-state parton. Because the contributions involving massless $c$ and $b$ parton distributions are good approximations to the physical process only at large $Q$, the formalism must suppress these contributions when $Q$ is of the order of the heavy-quark mass or less. A factorization scheme in which the number of active heavy quarks varies with $Q$ in this way is called a general-mass variable-flavor-number (GM-VFN) scheme. Typical Feynman diagrams that enter into the calculation of $B_{c}$ production in a GM-VFN scheme are shown in Fig. 71.

The GM-VFN scheme was first applied to $B_{c}$ production in Refs. [844, 845]. (Earlier calculations [846-849] in the GM-VFN scheme did not address $B_{c}$ production.) Comparisons [845] of results from both FFN and GMVFN schemes for $B_{c}\left({ }^{1} S_{0}\right)$ and $B_{c}\left({ }^{3} S_{1}\right)$ production at the Tevatron are shown in Figs. 72 and 73 , respectively. The FFN and GM-VFN approaches yield different results at small $p_{T}$, possibly because, in the implementation of the GM-VFN scheme in Ref. [845], the factorization scale

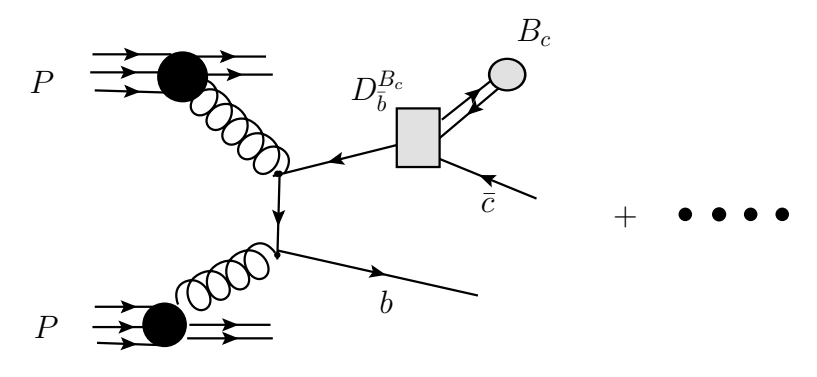

FIG. 70: Typical Feynman diagram for $B_{c}$ production via gluon-gluon fusion in the fragmentation approach at order $\alpha_{s}^{2} . D_{\bar{b}}^{B_{c}}$ is the fragmentation function for a $b$ antiquark into a $B_{c}$ 


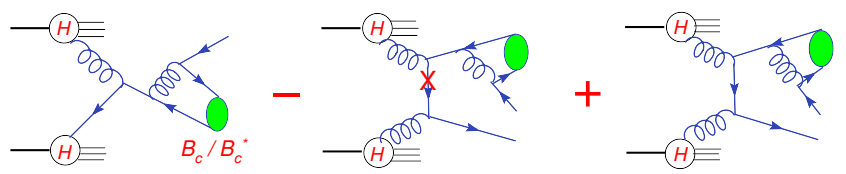

FIG. 71: Typical Feynman diagrams for $B_{c}$ production via gluon-gluon fusion in a GM-VFN scheme. The middle diagram represents a subtraction that removes double-counting of the contribution in the right-hand diagram in which the lower initial-state gluon produces a heavy quark-antiquark pair that is collinear to that gluon. From [844] with kind permission, copyright (2006) The American Physical Society

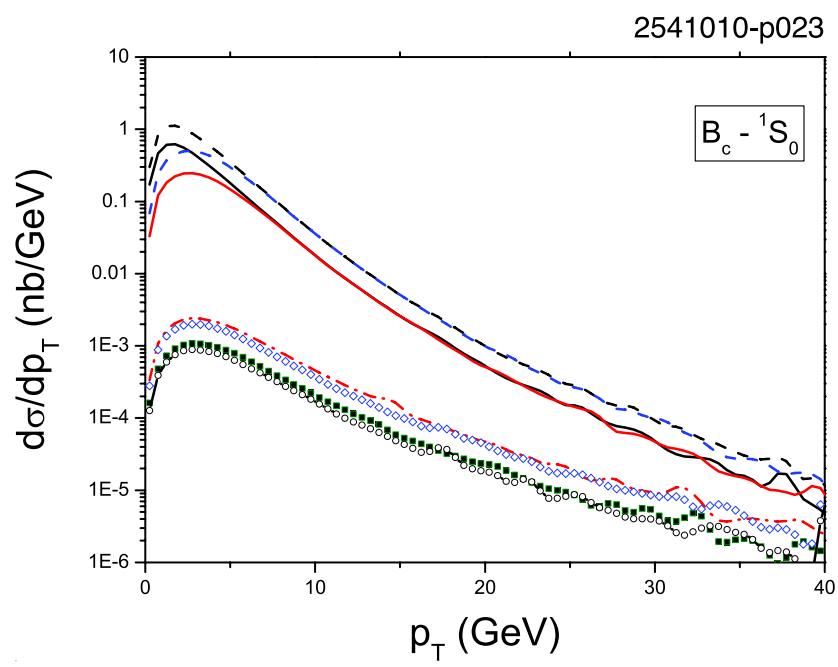

FIG. 72: Predictions for $p_{T}$ distributions of the $B_{c}\left({ }^{1} S_{0}\right)$ in production at the Tevatron [845]. Four pairs of curves are shown, corresponding, from top to bottom, to the following contributions: $g g$-fusion with the cut $|y|<1.3, g g$-fusion with the cut $|y|<0.6, q \bar{q}$-annihilation with the cut $|y|<1.3$, and $q \bar{q}$-annihilation with the cut $|y|<0.6$. Here, $q$ is a light quark. In each pair of curves, the upper curve is the GMVFN prediction, and the lower curve is the FFN prediction

$\mu_{F}=\sqrt{p_{T}^{2}+m_{B_{c}}^{2}}$ was chosen, which implies that there are contributions from initial $c$ and $b$ quarks down to $p_{T}=0$. Outside the small- $p_{T}$ region, the FFN and GMVFN approaches give very similar results. This implies that the resummations that are contained in the GMVFN scheme have only a small effect and that there is no compelling need to resum initial-state logarithms.

In conclusion, there is no evidence of a need to improve the FFN order- $\alpha_{s}^{4}$ computation of $B_{c}$ production by resumming large final-state or initial-state logarithms. On the other hand, a complete computation of the NLO corrections in the FFN approach would be very welcome in order to allow one to improve the dependence of the predictions on the renormalization scale and reduce the theoretical uncertainties.

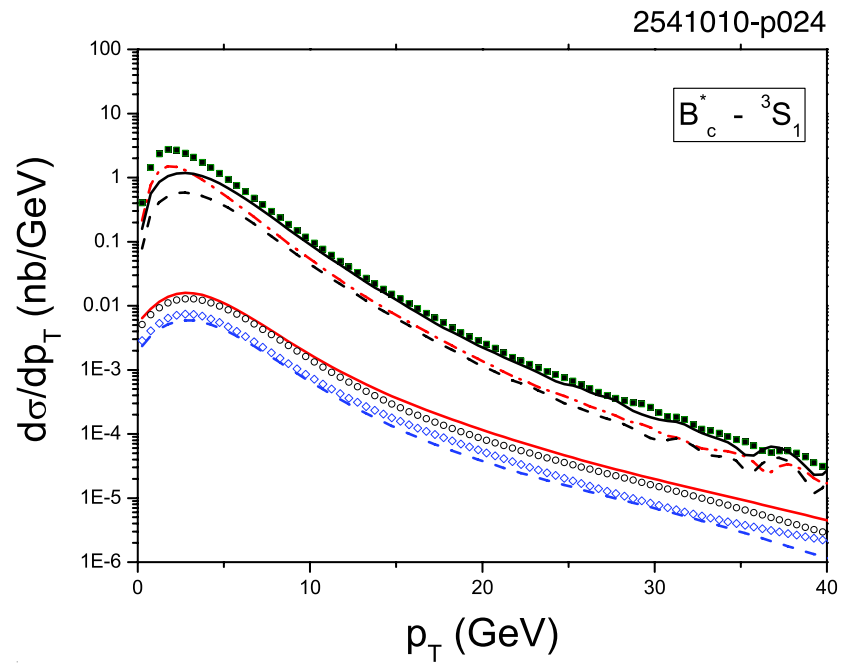

FIG. 73: Predictions for $p_{T}$ distributions of the $B_{c}^{*}\left({ }^{3} S_{1}\right)$ in production at the Tevatron [845]. Four pairs of curves are shown, corresponding, from top to bottom, to the following contributions: $g g$-fusion with the cut $|y|<1.3, g g$-fusion with the cut $|y|<0.6, q \bar{q}$-annihilation with the cut $|y|<1.3$, and $q \bar{q}$-annihilation with the cut $|y|<0.6$. Here, $q$ is a light quark. In each pair of curves, the upper curve is the GMVFN prediction, and the lower curve is the FFN prediction

\subsubsection{Phenomenology}

The study of $B_{c}$ production and decays will be possible at the $\mathrm{LHC}$ as well as at the Tevatron. The $\mathrm{LHCb}$ experiment, in particular, has been designed especially for the study of $b$ hadrons (e.g., $B^{ \pm}, B^{0}, \bar{B}^{0}, B_{s}, B_{c}$, $\left.\Lambda_{b}\right)$. Thus, many new results for the $B_{c}$ meson are expected. New data will be useful in understanding the production mechanism itself and also in determining the decay branching ratios.

Because the excitations of the $B_{c}$ carry both $b$ and $c$ flavor quantum numbers, they must decay into the $B_{c}$ ground state with almost $100 \%$ probability, either directly or via strong and/or electromagnetic cascades. No experimental distinction among direct or indirect production is possible at the moment. It is therefore important that predictions for $B_{c}$ production include feeddown from the excitations of the $B_{c}$, such as the $B_{c}^{*}\left({ }^{3} S_{1}\right)$, $B_{c J, L=1}^{*}, B_{c J, L=2}^{* *}$, etc., and the radially excited states. Predictions in LO perturbative QCD for the production cross sections of the $B_{c}^{*}$ and the low-lying $P$-wave excited states $B_{c J, L=1}^{*}$ are available in analytic and numerical form in Refs. [850, 851] and in numerical form in Ref. [743]. These predictions show that the contributions of the color-octet channels, $\left[(c \bar{b})_{\mathbf{8}},{ }^{1} S_{0}\right]$ and $\left[(c \bar{b})_{\mathbf{8}},{ }^{3} S_{1}\right]$, to the production of low-lying $P$-wave excited states, such as the $B_{c J, L=1}^{*}$, are comparable to the contributions of the leading color-singlet channels, provided that the values of the color-octet matrix elements are consistent with NRQCD velocity scaling.

Simulations of the production of $c \bar{b}$ and $\bar{c} b$ mesons 
are now implemented in the programs BCVEGPY [852] and MadOnia [743]. Both programs are interfaced to PYTHIA [853].

In Figs. 74 and 75 , we show typical distributions in $y$ and $p_{T}$ for the production of excited $(c \bar{b})$ mesons at the LHC and the Tevatron [851]. The contributions from the various parton-level production channels are shown separately.

\subsubsection{Future opportunities}

The observation and study of the $B_{c}$ mesons and their excitations are new and exciting components of the quarkonium-physics plans for both the Tevatron and the LHC. Much has yet to be learned about the production
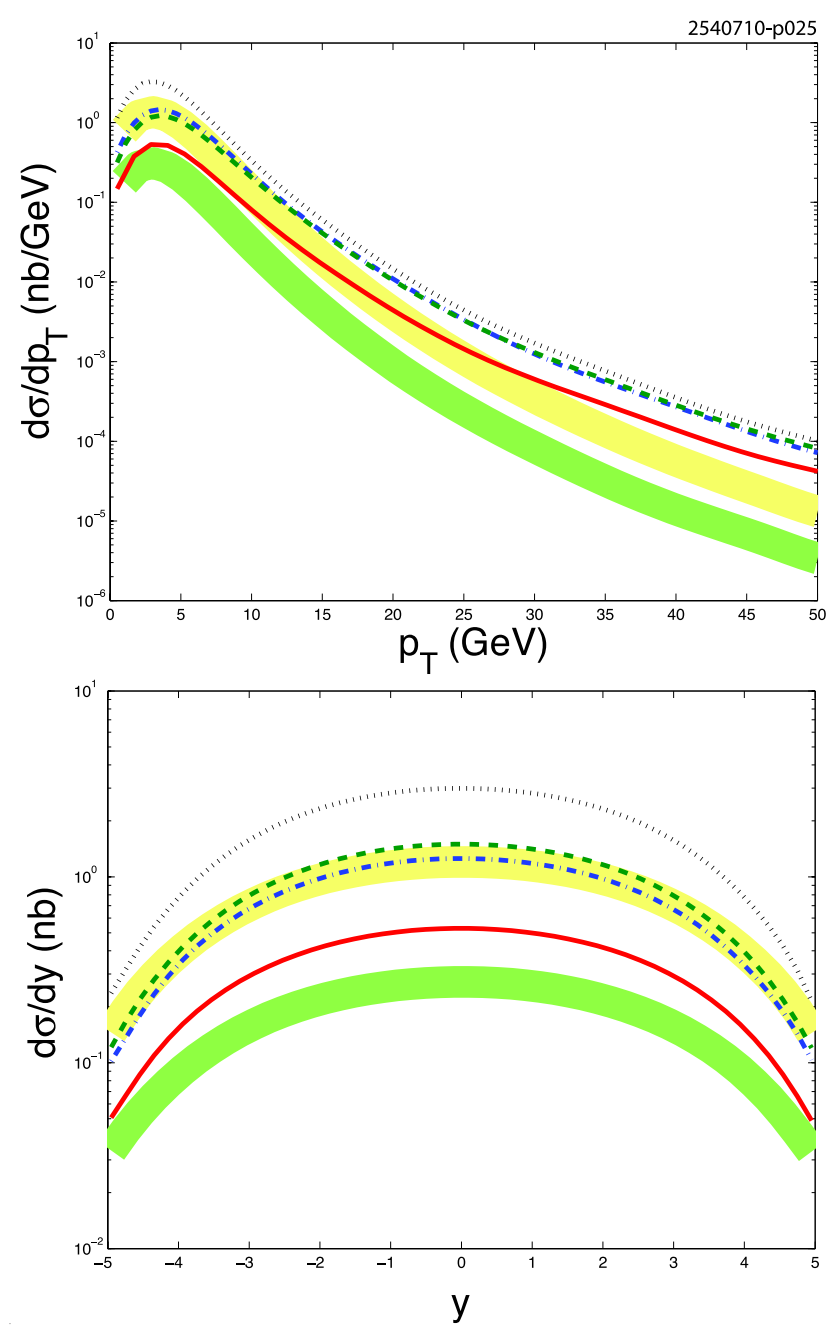

FIG. 74: Distributions in $p_{T}$ and $y$ for production of $c \bar{b}$ mesons at the LHC. The dashed, solid, dash-dotted, and dotted lines represent the color-singlet ${ }^{1} P_{1},{ }^{3} P_{0},{ }^{3} P_{1}$, and ${ }^{3} P_{2}$ contributions, respectively. The lower and upper shaded bands represent the color-octet ${ }^{1} S_{0}$ and ${ }^{3} S_{1}$ contributions, respectively. From [851] with kind permission, copyright (2005) The American Physical Society and decay of these states.

A number of improvements in the theoretical predictions for $B_{c}$ mesons are needed, such as computations of the NLO corrections to the production cross sections. In addition, new mechanisms for $B_{c}$ production at high $p_{T}$ should be explored. These include production via $Z^{0}$ or top-quark decays [854].
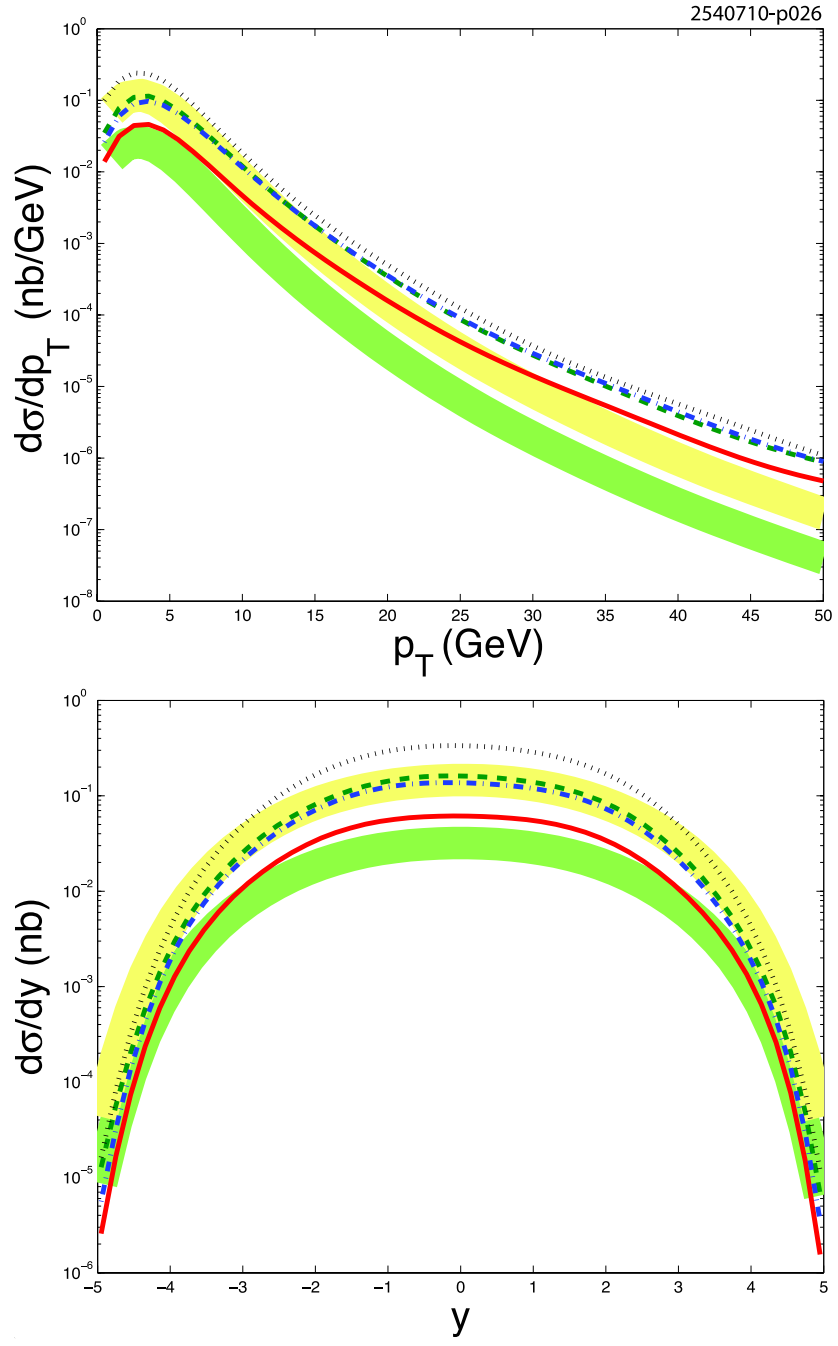

FIG. 75: Distributions in $p_{T}$ and $y$ for production of $c \bar{b}$ mesons at the Tevatron. The dashed, solid, dash-dotted, and dotted lines represent the color-singlet ${ }^{1} P_{1},{ }^{3} P_{0},{ }^{3} P_{1}$, and ${ }^{3} P_{2}$ contributions, respectively. The lower and upper shaded bands represent the color-octet ${ }^{1} S_{0}$ and ${ }^{3} S_{1}$ contributions, respectively. From [851] with kind permission, copyright (2005) The American Physical Society 


\section{IN MEDIUM ${ }^{19}$}

\subsection{Quarkonia as a probe of hot and dense matter}

It is expected that strongly-interacting matter shows qualitatively new behavior at temperatures and/or densities which are comparable to or larger than the typical hadronic scale. It has been argued that under such extreme conditions deconfinement of quarks and gluons should set in and the thermodynamics of stronglyinteracting matter could then be understood in terms of these elementary degrees of freedom. This new form of matter is called quark-gluon plasma [855], or QGP. The existence of such a transition has indeed been demonstrated from first principles using Monte Carlo simulations of lattice QCD. The properties of this new state of matter have also been studied [856-859].

In addition to theoretical efforts, the deconfinement transition and the properties of hot, strongly-interacting matter are also studied experimentally in heavy-ion collisions [860, 861]. A significant part of the extensive experimental heavy-ion program is dedicated to measuring quarkonium yields since Matsui and Satz suggested that quarkonium suppression could be a signature of deconfinement [862]. In fact, the observation of anomalous suppression was considered to be a key signature of deconfinement at SPS energies [863].

However, not all of the observed quarkonium suppression in nucleus-nucleus $(A B)$ collisions relative to scaled proton-proton $(p p)$ collisions is due to quark-gluon plasma formation. In fact, quarkonium suppression was also observed in proton-nucleus $(p A)$ collisions, so that part of the nucleus-nucleus suppression is due to coldnuclear-matter effects. Therefore it is necessary to disentangle hot- and cold-medium effects. We first discuss cold-nuclear-matter effects at different center-of-mass energies. Then we discuss what is known about the properties of heavy $Q \bar{Q}$ states in hot, deconfined media. Finally, we review recent experimental results on quarkonium production from $p A$ collisions at the SPS and from $p p, \mathrm{~d}+\mathrm{Au}$, and $A A$ collisions at RHIC.

\subsection{Cold-nuclear-matter effects}

The baseline for quarkonium production and suppression in heavy-ion collisions should be determined from studies of cold-nuclear-matter (CNM) effects. The name cold matter arises because these effects are observed in hadron-nucleus interactions where no hot, dense matter effects are expected. There are several CNM effects. Modifications of the parton distribution functions in the

19 Contributing authors: A. D. Frawley ${ }^{\dagger}$, P. Petreczky $^{\dagger}$, R. Vogt $^{\dagger}$, R. Arnaldi, N. Brambilla, P. Cortese, S. R. Klein, C. Lourenço, A. Mocsy, E. Scomparin, and H. K. Wöhri nucleus, relative to the nucleon, (i.e., shadowing) and energy loss of the parton traversing the nucleus before the hard scattering are both assumed to be initial-state effects, intrinsic to the nuclear target. Another CNM effect is absorption (i.e., destruction) of the quarkonium state as it passes through the nucleus. Since the latter occurs after the $Q \bar{Q}$ pair has been produced and while it is traversing the nuclear medium, this absorption is typically referred to as a final-state effect. In order to disentangle the mechanisms affecting the produced $Q \bar{Q}$, data from a variety of center-of-mass energies and different phase-space windows need to be studied. In addition, the inclusive $J / \psi$ yield includes contributions from $\chi_{c}$ and $\psi(2 S)$ decays to $J / \psi$ at the $30-35 \%$ level [864]. While there is some information on the $A$ dependence of $\psi(2 S)$ production, that on $\chi_{c}$ is largely unknown [865].

Even though the contributions to CNM effects may seem rather straightforward, there are a number of associated uncertainties. First, while nuclear modifications of the quark densities are relatively well-measured in nuclear deep-inelastic scattering (nDIS), the modifications of the gluon density are not directly measured. The nDIS measurements probe only the quark and antiquark distributions directly. The scaling violations in nDIS can be used to constrain the nuclear gluon density. Overall momentum conservation provides another constraint. However, more direct probes of the gluon density are needed. Current shadowing parametrizations are derived from global fits to the nuclear parton densities and give wide variations in the nuclear gluon density, from almost no effect to very large shadowing at low- $x$, compensated by strong antishadowing around $x \sim 0.1$. The range of the possible shadowing effects is illustrated in Fig. 76 by the new EPS09 [866] parametrization and its associated uncertainties, employing the scale values used to fix the $J / \psi$ and $\Upsilon$ cross sections below the open-heavy-flavor threshold [867].

The color glass condensate (CGC) is expected to play an important role in quarkonium production at $\mathrm{RHIC}$ and the LHC since the saturation scale $Q_{S, A}(x)$ is com-

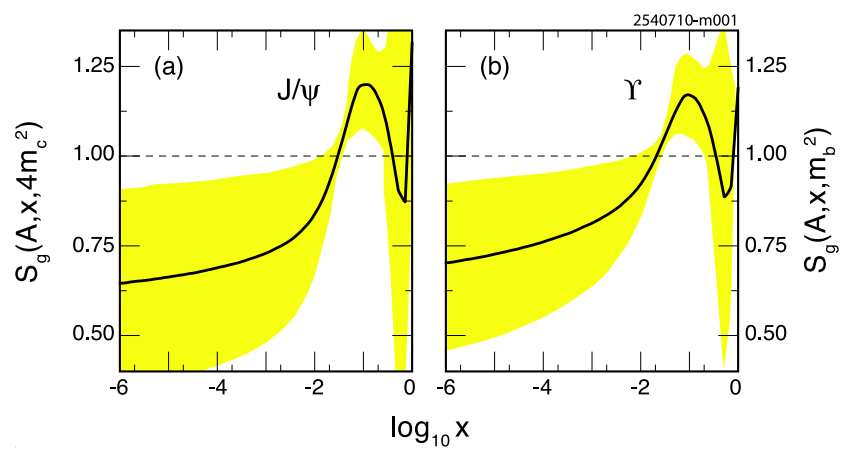

FIG. 76: The EPS09 gluon-shadowing parametrization [866] at $Q=2 m_{c}$ and $m_{b}$. The central value (solid curves) and the associated uncertainty (shaded band) are shown 
parable to the charm quark mass [868]. In this picture, collinear factorization of $J / \psi$ production is assumed to break down and forward $J / \psi$ production is suppressed. Indeed, CGC suppression of $J / \psi$ formation may mask some QGP effects [869].

The nuclear absorption survival probability depends on the quarkonium absorption cross section. There are more inherent uncertainties in absorption than in the shadowing parametrization, which is obtained from data on other processes and is independent of the final state. Typically an absorption cross section is fit to the $A$ dependence of $J / \psi$ and/or $\psi(2 S)$ production at a given energy. This is rather simplistic since it is unknown whether the object traversing the nucleus is a precursor color-octet state or a fully-formed color-singlet quarkonium state. If it is an octet state, it is assumed to immediately interact with a large, finite cross section since it is a colored object [870]. In this case, it has often been assumed that all precursor quarkonium states will interact with the same cross section. If it is produced as a small color-singlet, the absorption cross section immediately after the production of the $Q \bar{Q}$ pair should be small and increasing with proper time until, at the formation time, it reaches its final-state size [871]. High-momentum color-singlet quarkonium states will experience negligible nuclear absorption effects since they will be formed well outside the target. See [865] for a discussion of the $A$ dependence of absorption for all the quarkonium states.

Fixed-target data taken in the range $400 \leq E_{\text {lab }} \leq$ $800 \mathrm{GeV}$ have shown that the $J / \psi$ and $\psi(2 S)$ absorption cross sections are not identical, as the basic color-octet absorption mechanism would suggest [872-874]. The difference between the effective $A$ dependence of $J / \psi$ and $\psi(2 S)$ seems to decrease with beam energy. The $J / \psi$ absorption cross section at $y \sim 0$ is seen to decrease with energy, regardless of the chosen shadowing parametrization [875], as shown in Fig. 77.

Recent analyses of $J / \psi$ production in fixed-target interactions [875] show that the effective absorption cross section depends on the energy of the initial beam and the rapidity or $x_{F}$ of the observed $J / \psi$. One possible interpretation is that low-momentum color-singlet states can hadronize in the target, resulting in larger effective absorption cross sections at lower center-of-mass energies and backward $x_{F}$ (or center-of-mass rapidity). At higher energies, the states traverse the target more rapidly so that the $x_{F}$ values at which they can hadronize in the target move back from midrapidity toward more negative $x_{F}$. Finally, at sufficiently high energies, the quarkonium states pass through the target before hadronizing, resulting in negligible absorption effects. Thus the effective absorption cross section decreases with increasing center-of-mass energy because faster states are less likely to hadronize inside the target.

At higher $x_{F}$, away from midrapidity, the effective absorption cross section becomes very large, as shown in the top panel of Fig. 78. The increase in $\sigma_{\text {abs }}^{J / \psi}$ begins closer to midrapidity for lower incident energies.

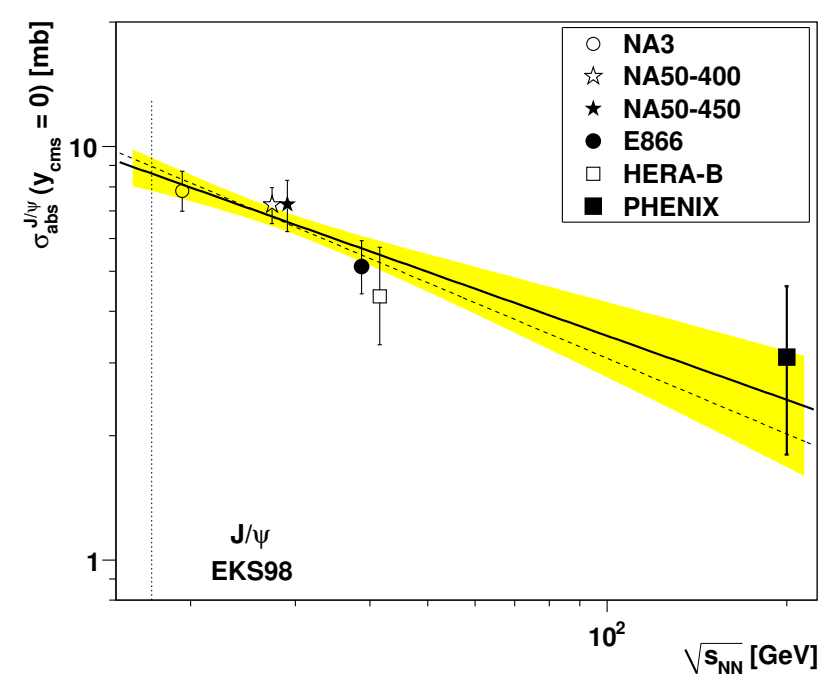

FIG. 77: The extracted energy dependence of $\sigma_{\text {abs }}^{J / \psi}$ at midrapidity. The solid line is a power law approximation to $\sigma_{\text {abs }}^{J / \psi}\left(y=0, \sqrt{s_{N N}}\right)$ using the EKS98 [876, 877] shadowing parametrization with the CTEQ61L parton densities $[878,879]$. The band indicates the uncertainty in the extracted cross sections. The dashed curve shows an exponential fit for comparison. The data at $y_{\mathrm{cms}} \sim 0$ from NA3 [880], NA50 at $400 \mathrm{GeV}$ [872] and $450 \mathrm{GeV}$ [873], E866 [874], HERA-B [881], and PHENIX [663] are also shown. The vertical dotted line indicates the energy of the $\mathrm{Pb}+\mathrm{Pb}$ and $\mathrm{In}+\mathrm{In}$ collisions at the CERN SPS. Adapted from [875] with kind permission, copyright (2009) Springer-Verlag

There appears to be some saturation of the effect since the $800 \mathrm{GeV}$ fixed-target data exhibit the same trend as the most recent (preliminary) PHENIX data [884] as a function of center-of-mass rapidity, $y_{\mathrm{cms}}$, as seen in the bottom panel of Fig. 78. Model calculations including CGC effects can reproduce the general trend of the high$x_{F}$ behavior of $J / \psi$ production at $800 \mathrm{GeV}$ without invoking energy loss [869]. However, the fact that the NA3 data at $\sqrt{s_{N N}}=19 \mathrm{GeV}$ exhibit the same trend in $x_{F}$ as E866 calls the CGC explanation into question.

As previously discussed, such an increase in the apparent absorption cannot be due to interactions with nucleons. In addition, since the large- $x_{F}$ dependence seems to be independent of the quarkonium state (i.e., the same for $J / \psi$ and $\psi(2 S)$ [874], and also for $\Upsilon(1 S)$ and $\Upsilon(2 S)+\Upsilon(3 S))$ [885], it likely cannot be attributed to the size of the final state and should thus be an initial-state effect, possibly energy loss. (See [886] for a discussion of several types of energy-loss models and their effect on $J / \psi$ production.) Work is in progress to incorporate this effect using a new approach, based on the number of soft collisions the projectile parton undergoes before the hard scattering to produce the $Q \bar{Q}$ pair.

It is also well known that feeddown from $P$ and higher$S$ states through radiative and hadronic transitions, respectively, accounts for almost half of the observed $J / \psi$ 

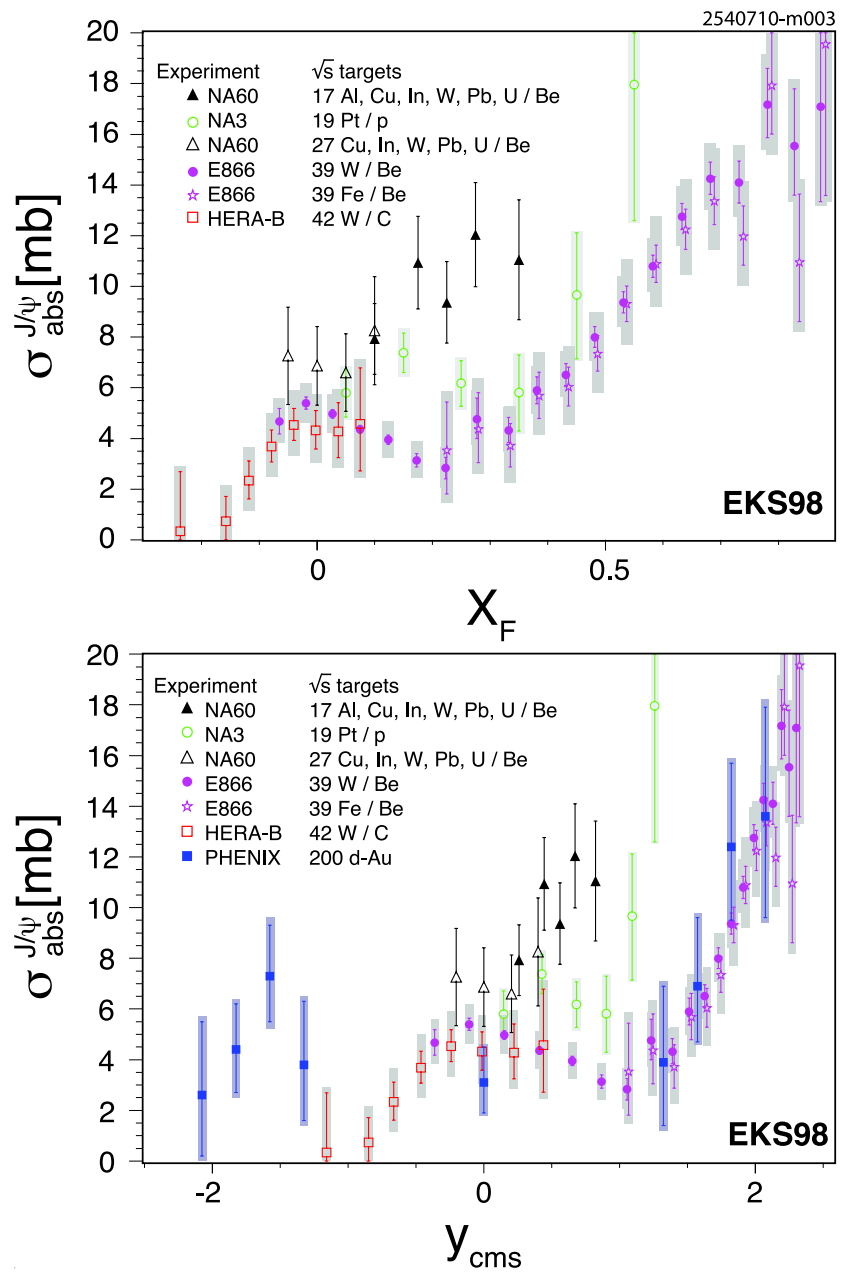

FIG. 78: Top: The $x_{F}$ dependence of $\sigma_{\text {abs }}^{J / \psi}$ for incident fixed-target energies from $158 \mathrm{GeV}[882], 200 \mathrm{GeV}[880]$, $400 \mathrm{GeV}$ [872], $\quad 450 \mathrm{GeV}$ [873], $\quad 800 \mathrm{GeV}$ [874], and $920 \mathrm{GeV}[881]$ obtained using the EKS98 shadowing parametrization [876, 877]. The E866 [874] and HERA$\mathrm{B}$ [881] results were previously shown in [875]. Bottom: The same results as above but as a function of center-of-mass rapidity $y_{\mathrm{CMS}}$. The absorption cross sections extracted from the preliminary PHENIX [663] results at $\left|y_{\mathrm{CMS}}\right|>0$ and the central rapidity result [883] are also included. The boxes surrounding the data points represent the statistical and total systematic uncertainties added in quadrature, except for PHENIX where the global term ${ }_{-4.1}^{+5.0} \mathrm{mb}$, common to all rapidity bins) is missing

and $\Upsilon(1 S)$ yields. The excited quarkonium states have very different sizes and formation times and should thus have different absorption cross sections. For example, the absorption cross section of quarkonium state $C$ may be proportional to its area, $\sigma_{C} \propto r_{C}^{2}$ [887].

It should be noted, however, that the fitted absorption cross sections used for extracting the "normal absorption" baseline for $\mathrm{Pb}+\mathrm{Pb}$ collisions at the SPS have treated $J / \psi$ and $\psi(2 S)$ absorption independently, ignoring feeddown and formation times, and have not taken initial-state shadowing into account [872, 873]. As discussed above, more detailed analyses show that the quarkonium absorption cross section decreases with increasing energy [875, 888]. More recent fixed-target analyses [882, 889], comparing measurements at 158 and $400 \mathrm{GeV}$, have begun to address these issues (see Sect. 5.4). Indeed, the extracted absorption cross section is found to be larger at $158 \mathrm{GeV}$ than at $400 \mathrm{GeV}$, contrary to previous analyses, which assumed a universal, constant absorption cross section [872, 873]. When these latest results are extrapolated to nucleus-nucleus collisions at the same energy, the anomalous suppression is significantly decreased relative to the new baseline [882].

The cold-nuclear-matter effects suggested (initial-state energy loss, shadowing, final-state breakup, etc.) depend differently on the quarkonium kinematic variables and the collision energy. It is clearly unsatisfactory to combine all these mechanisms into an effective absorption cross section, as employed in the Glauber formalism, that only evaluates final-state absorption. Simply taking the $\sigma_{\text {abs }}$ obtained from the analysis of the $p A$ data and using it to define the $\mathrm{Pb}+\mathrm{Pb}$ baseline may not be sufficient.

A better understanding of absorption requires more detailed knowledge of the production mechanism. Most calculations of the $A$ dependence use the color evaporation model (CEM), in which all quarkonia are assumed to be produced with the same underlying kinematic distributions [890]. This model works well for fixed-target energies and for RHIC [867], as does the LO color-singlet model (CSM) [676]. In the latter case, but contrary to the CEM at LO, $J / \psi$ production is necessarily accompanied by the emission of a perturbative final-state gluon which can be seen as an extrinsic source of transverse momentum. This induces modifications in the relations between the initial-state gluon momentum fractions and the momentum of the $J / \psi$. In turn, this modifies [891] the gluon-shadowing corrections relative to those expected from the LO CEM where the transverse momentum of the $J / \psi$ is intrinsic to the initial-state gluons. Further studies are being carried out, including the impact of feeddown, the extraction of absorption cross sections for each of the charmonium states, and the dependence on the partonic $J / \psi$ production mechanism. A high precision measurement of the $\psi(2 S)$ and $\Upsilon(3 S)$ production ratios in $p A$ interactions as a function of rapidity would be desirable since they are not affected by feeddown contributions. In addition, measurements of the feeddown contributions to $J / \psi$ and $\Upsilon(1 S)$ as a function of rapidity and $p_{T}$ would be very useful.

On the other hand, the higher- $p_{T}$ Tevatron predictions have been calculated within the nonrelativistic QCD (NRQCD) approach [138], which includes both singlet and octet matrix elements. These high $p_{T}$ calculations can be tuned to agree with the high $p_{T}$ data but cannot reproduce the measured quarkonium polarization at the same energy [1]. If some fraction of the final-state quarkonium yields can be attributed to color-singlet production, then absorption need not be solely due to either 
singlet or octet states but rather some mixture of the two, as dictated by NRQCD [865, 892, 893]. A measurement of the $A$ dependence of $\chi_{c}$ production would be particularly helpful to ensure significant progress toward understanding the production mechanism.

\subsection{Quarkonium in hot medium}

\subsubsection{Spectral properties at high temperature}

There has been considerable interest in studying quarkonia in hot media since publication of the famous Matsui and Satz paper [862]. It has been argued that color screening in a deconfined QCD medium will destroy all $Q \bar{Q}$ bound states at sufficiently high temperatures. Although this idea was proposed long ago, first principle QCD calculations, which go beyond qualitative arguments, have been performed only recently. Such calculations include lattice QCD determinations of quarkonium correlators [894-898], potential model calculations of the quarkonium spectral functions with potentials based on lattice QCD [899-906], as well as effective field theory approaches that justify potential models and reveal new medium effects [907-910]. Furthermore, better modeling of quarkonium production in the medium created by heavy-ion collisions has been achieved. These advancements make it possible to disentangle the cold- and hotmedium effects on the quarkonium states, crucial for the interpretation of heavy-ion data.

\subsubsection{Color screening and deconfinement}

At high temperatures, strongly-interacting matter undergoes a deconfining phase transition to a quark-gluon plasma. This transition is triggered by a rapid increase of the energy and entropy densities as well as the disappearance of hadronic states. (For a recent review, see [858].) According to current lattice calculations [911920] at zero net-baryon density, deconfinement occurs at $T \sim 165-195 \mathrm{MeV}$. The QGP is characterized by color screening: the range of interaction between heavy quarks becomes inversely proportional to the temperature. Thus at sufficiently high temperatures, it is impossible to produce a bound state between a heavy quark ( $c$ or $b)$ and its antiquark.

Color screening is studied on the lattice by calculating the spatial correlation function of a static quark and antiquark in a color-singlet state which propagates in Euclidean time from $\tau=0$ to $\tau=1 / T$, where $T$ is the temperature (see [921, 922] for reviews). Lattice calculations of this quantity with dynamical quarks have been reported [923-928]. The logarithm of the singlet correlation function, also called the singlet free energy, is shown in Fig. 79. As expected, in the zero-temperature limit the singlet free energy coincides with the zero-temperature potential. Figure 79 also illustrates that, at sufficiently short distances, the singlet free energy is temperature independent and equal to the zero-temperature potential. The range of interaction decreases with increasing temperature. For temperatures above the transition temperature, $T_{c}$, the heavy-quark interaction range becomes comparable to the charmonium radius. Based on this general observation, one would expect that the charmonium states, as well as the excited bottomonium states, do not remain bound at temperatures just above the deconfinement transition, often referred to as dissociation or melting.

\subsubsection{Quarkonium spectral functions and quarkonium potential}

In-medium quarkonium properties are encoded in the corresponding spectral functions, as is quarkonium dissolution at high temperatures. Spectral functions are defined as the imaginary part of the retarded correlation function of quarkonium operators. Bound states appear as peaks in the spectral functions. The peaks broaden and eventually disappear with increasing temperature. The disappearance of a peak signals the melting of the given quarkonium state.

In lattice QCD, the meson correlation functions, $G(\tau, T)$, are calculated in Euclidean time. These correlation functions are related to the spectral functions $\sigma(\omega, T)$ by

$$
G(\tau, T)=\int_{0}^{\infty} d \omega \sigma(\omega, T) \frac{\cosh (\omega(\tau-1 /(2 T)))}{\sinh (\omega /(2 T))} .
$$

Detailed information on $G(\tau, T)$ would allow reconstruction of the spectral function from the lattice data. In practice, however, this turns out to be a very difficult

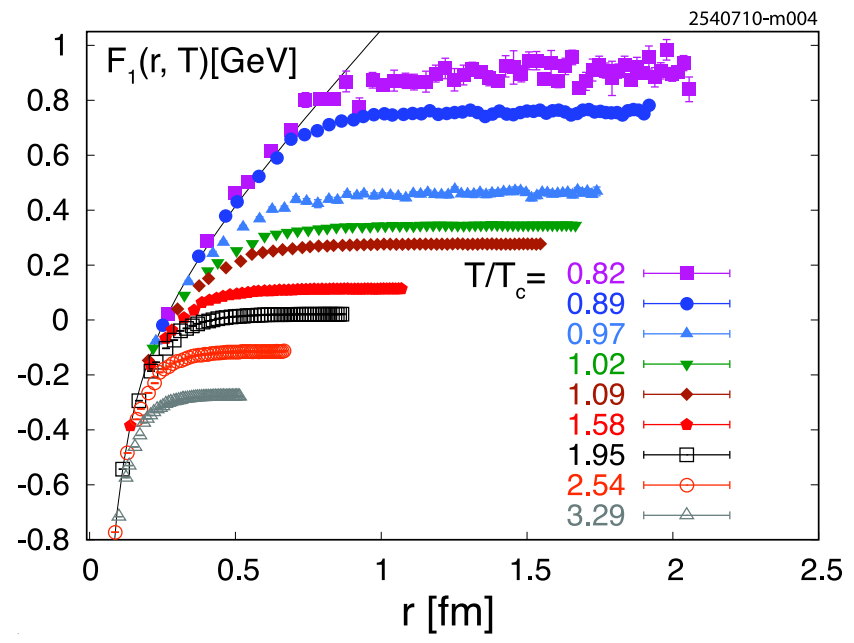

FIG. 79: Heavy-quark-singlet free energy versus quark separation calculated in $2+1$ flavor QCD on $16^{3} \times 4$ lattices at different temperatures [928, 929] 
task because the time extent is limited to $1 / T$ (see the discussion in [897] and references therein). Lattice artifacts in the spectral functions at high energies $\omega$ are also a problem when analyzing the correlation functions calculated on the lattice [930].

The quarkonium spectral functions can be calculated in potential models using the singlet free energy from Fig. 79 or with different lattice-based potentials obtained using the singlet free energy as an input [905, 906] (see also [931] for a review). The results for quenched QCD calculations are shown in Fig. 80 for $S$-wave charmonium (top) and bottomonium (bottom) spectral functions [905]. All charmonium states are dissolved in the deconfined phase while the bottomonium $1 S$ state may persist up to $T \sim 2 T_{c}$. The temperature dependence of the Euclidean correlators can be predicted using Eq. (176). Somewhat surprisingly, the Euclidean correlation functions in the pseudoscalar channel show very little temperature dependence, irrespective of whether a state remains bound $\left(\eta_{b}\right)$ or not $\left(\eta_{c}\right)$. Note also that correlators from potential models are in accord with the lattice calculations (see insets in Fig. 80). Initially, the weak temperature dependence of the pseudoscalar correlators was considered to be evidence for the survival of $1 S$ quarkonium states [896]. It is now clear that this conclusion was premature. In other channels one sees significant temperature dependence of the Euclidean correlation functions, especially in the scalar and axial-vector channels where it has been interpreted as evidence for dissolution of the quarkonium $1 P$ states. However, this temperature dependence is due to the zero-mode contribution, i.e., a peak in the finite temperature spectral functions at $\omega \simeq 0[932,933]$. After subtracting the zeromode contribution, the Euclidean correlation functions show no temperature dependence within the uncertainties [934]. Thus melting of the quarkonium states is not visible in the Euclidean correlation functions.

There is a large enhancement in the threshold region of the spectral functions relative to the free spectral function, as shown in Fig. 80. This threshold enhancement compensates for the absence of bound states and leads to Euclidean correlation functions with very weak temperature dependencies [905]. It further indicates strong residual correlations between the quark and antiquark, even in the absence of bound states. Similar analyses were done for the $P$-wave charmonium and bottomonium spectral functions [905, 906]. An upper bound on the dissociation temperature (the temperatures above which no bound states peaks can be seen in the spectral function and bound state formation is suppressed) can be obtained from the analysis of the spectral functions. Conservative upper limits on the dissociation temperatures for the different quarkonium states obtained from a full QCD calculation [906] are given in Table 45.

The problems with the potential model approach described above are how to relate it to first principles QCD and how to define the in-medium quark-antiquark potential. These problems can be addressed using an EFT

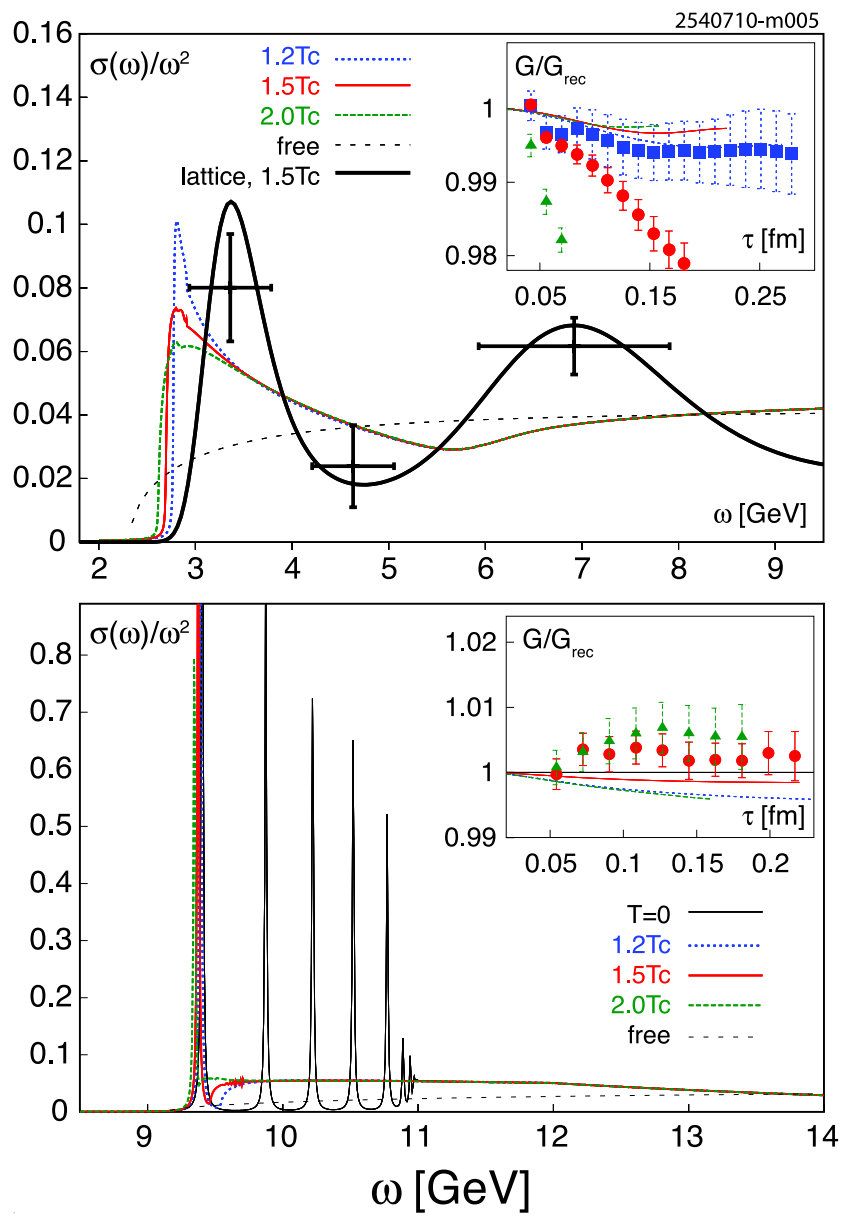

FIG. 80: The $S$-wave charmonium (upper) and bottomonium (lower) spectral functions calculated in potential models. Insets: correlators compared to lattice data. The dotted curves are the free spectral functions. Adapted from [905] with kind permission, copyright (2008) The American Physical Society

TABLE 45: Upper bounds on the dissociation temperatures. Adapted from [906] with kind permission, copyright (2007) The American Physical Society

\begin{tabular}{ccccccc}
\hline \hline State & $\chi_{c J}(1 P)$ & $\psi(2 S)$ & $J / \psi$ & $\Upsilon(2 S)$ & $\chi_{b J}(1 P)$ & $\Upsilon$ \\
\hline$T_{\text {diss }}$ & $\leq T_{c}$ & $\leq T_{c}$ & $1.2 T_{c}$ & $1.2 T_{c}$ & $1.3 T_{c}$ & $2 T_{c}$ \\
\hline \hline
\end{tabular}

approach to heavy-quark bound states in a real time formalism. The first steps in this direction were taken in $[907,935]$. The static potential (for infinitely heavy $Q \bar{Q}$ pairs) was calculated in the regime $T \gg 1 / r \gtrsim m_{D}$, where $m_{D}$ is the Debye mass and $r$ the $Q \bar{Q}$ separation, by analytically continuing the Euclidean Wilson loop to real time. The calculation was done in weak-coupling resummed perturbation theory. The imaginary part of the gluon self-energy gives an imaginary part to the static potential and thus introduces a thermal width in the 
$Q \bar{Q}$ bound state. The heavy quarkonium contribution to the spectral function of the electromagnetic current was calculated in the same framework [908, 936]. Static $Q \bar{Q}$ pairs in a real-time formalism are considered in [937], where the potential for distances $1 / r \sim m_{D}$ in a hot QED plasma is derived. The real part of the static potential was in agreement with the singlet free energy while the damping factor (imaginary part of the potential) at large distances agreed with the one found in [935]. This damping can be thought of as quarkonia scattering with light partons in a thermal bath where the collisional width increases with temperature. The real part of the static potential was found to agree with the singlet free energy and the damping factor with the one found in [935]. In [938], a study of bound states in a hot QED plasma was performed in a non-relativistic EFT framework. In particular, the hydrogen atom was studied for temperatures ranging from $T \ll m \alpha^{2}$ to $T \sim m$, where the imaginary part of the potential becomes larger than the real part and the hydrogen ceases to exist. The same study has been extended to muonic hydrogen in [939].

The EFT framework quarkonium at finite temperature for different distance regimes was developed in [910] using the real time formalism and weak coupling techniques. In a zero-temperature medium, the behavior of quarkonia is characterized by different energy and momentum scales related to non-relativistic bound states with heavy quark velocity $v$ and mass $m$. The scale related to the inverse distance between the $Q$ and $\bar{Q}$ is $m v$ while $m v^{2}$ is the scale related to the binding energy of the state. Finally, $\Lambda_{\mathrm{QCD}}$ is related to the nonperturbative features of the QCD vacuum. In the weakcoupling regime, $v \sim \alpha_{\mathrm{S}}$ and the hierarchy of scales follows $m \gg m v \gg m v^{2} \gg \Lambda_{\mathrm{QCD}}{ }^{20}$. In addition, there are thermodynamical scales: the temperature $T$; the inverse of the screening length of chromo-electric interactions, the Debye mass $\left(m_{D} \sim g T\right)$; and the static magnetic scale, $g^{2} T$. In the weak-coupling regime at finite temperature, the ordering of these scales follows $T \gg g T \gg g^{2} T$.

If there exists such a hierarchy of scales, any quantity of interest maybe expanded in some ratio of the scales. If the contributions from the different scales are separated explicitly at the Lagrangian level, this amounts to replacing QCD with a hierarchy of EFTs equivalent to QCD order by order in the expansion parameters. As described in 2.5, at $T=0$ the EFTs that follow from QCD by integrating over the scales $m$ and $m v$ are called nonrelativistic QCD (NRQCD) and potential NRQCD (pNRQCD) respectively. This procedure for constructing EFTs can be generalized to finite temperatures. The construction of EFTs for heavy-quark bound states and their resulting

20 A hierarchy of bound state scales may exist beyond the weakcoupling regime, e.g., $m v \simeq \Lambda_{\mathrm{QCD}}$, but has not yet been considered at finite temperature. forms depend on how the bound-state scales are related to the thermal scales. If all the bound state scales are larger than $T$, the relevant EFT is zero temperature $\mathrm{pN}$ RQCD. In this case, there are no corrections to the heavy $Q \bar{Q}$ potential even though there may be thermal corrections to the quarkonium binding energies and widths. If $m v>T>m v^{2}$ the relevant EFT can be constructed similarly to the $T=0$ case but now the temperature scale is integrated over. Now there are thermal corrections to the potential which have both real and imaginary parts $[910,940]$. Finally, when $T>m v$ the temperature scale must be integrated out after the scale $m$ is integrated over but before the scale $m v$. This procedure leads to an EFT very similar to NRQCD but a modification of the Lagrangian corresponding to gluon and light quark fields is necessary. This part of the Lagrangian is replaced by the hard thermal loop (HTL) Lagrangian [941-944]. The resulting effective theory is referred to as NRQCD $_{\text {HTL }}[910,940]$. Subsequent integration over the scale $m v$ leads to a new EFT called pNRQCD ${ }_{\text {HTL }}$ similar to pNRQCD. Now the heavy quark potential receives both real and imaginary thermal corrections. Furthermore, for $r \sim 1 / m_{D}$, the real part of the potential is exponentially screened and the imaginary part is much larger than the real part [910, 940]. Below we summarize what has been learned from the EFT approach to heavy quark bound states at finite temperature.

The thermal part of the potential has both a real and an imaginary part. The imaginary part of the potential smears out the bound state peaks of the quarkonium spectral function, leading to their dissolution prior to the onset of Debye screening in the real part of the potential (see, e.g., the discussion in [909]). Therefore, quarkonium dissociation appears to be a consequence of the thermal decay width rather than color screening of the real part of the potential. This conclusion follows from the observation that the thermal decay width becomes comparable to the binding energy at temperatures below that required for the onset of color screening.

Two mechanisms contribute to the thermal decay width: the imaginary part of the gluon self-energy induced by Landau damping, as also observed in QED, see [935] and the quark-antiquark color singlet to color octet thermal break-up (a new effect, specific to QCD) [910]. Parametrically, the first mechanism dominates at temperatures where the Debye mass $m_{D}$ is larger than the binding energy, while the latter effect dominates for temperatures where $m_{D}$ is smaller than the binding energy. The dissociation temperature is related to the coupling by $\pi T_{\text {dissoc }} \sim m g^{\frac{4}{3}}[909,938]$.

The derived color-singlet thermal potential, $V$, is neither the color-singlet $Q \bar{Q}$ free energy nor its internal energy. Instead it has an imaginary part and may contain divergences that eventually cancel in physical observables [910].

Finally, there may be other finite-temperature effects other than screening. These typically may take the form of power-law corrections or have a logarithmic tempera- 
ture dependence [910, 938].

The EFT framework thus provides a clear definition of the potential and a coherent and systematic approach for calculating quarkonium masses and widths at finite temperature. In [945], heavy quarkonium energy levels and decay widths in a quark-gluon plasma, below the quarkonium dissociation temperature where the temperature and screening mass satisfy the hierarchy $m \alpha_{\mathrm{s}} \gg$ $\pi T \gg m \alpha_{\mathrm{s}}{ }^{2} \gg m_{D}$, have been calculated to order $m \alpha_{\mathrm{s}}{ }^{5}$, relevant for bottomonium $1 S$ states $\left(\Upsilon(1 S), \eta_{b}\right)$ at the LHC. At leading order the quarkonium masses increase quadratically with $T$, the same functional increase with energy as dileptons produced in electromagnetic decays [945]. A thermal correction proportional to $T^{2}$ appears in the quarkonium electromagnetic decay rates. The leading-order decay width grows linearly with temperature, implying that quarkonium dissociates by decaying to the color-octet continuum.

This EFT approach was derived assuming weak coupling and neglecting nonperturbative effects. In particular, the role of the color-octet degrees of freedom in pNRQCD beyond perturbation theory needs to be better understood (see e.g., [946]). Comparison of certain static $Q \bar{Q}$ correlators calculated in the EFT framework with results from lattice QCD, partly discussed in subsection 5.3.2, could prove useful in this respect. The correlation function of two Polyakov loops, which is gauge invariant and corresponds to the free energy of a static $Q \bar{Q}$ pair could be particularly suitable for this purpose. Therefore, in [947] the Polyakov loop and the correlator of two Polyakov loops have been calculated to next-tonext-to-leading order at finite temperature in the weakcoupling regime and at $Q \bar{Q}$ separations shorter than the inverse of the temperature and for Debye masses larger than the Coulomb potential. The relationship between the Polyakov loop correlator and the singlet and octet $Q \bar{Q}$ correlator has been established in the EFT framework. A related study of cyclic Wilson loops at finite temperature in perturbation theory was reported in [948]. A further attempt to relate static $Q \bar{Q}$ correlation functions to the real-time potential was discussed in [949]. Very recently the first lattice NRQCD calculations of bottomoniun at finite temperature have appeared [950]. The initial discussion on the possibility of calculating quarkonium properties at finite temperature using NRQCD goes back to the first meeting of the QWG at CERN in 2002.

In addition, the effects of medium anisotropies on the quarkonium states have been considered, both on the real [951] and imaginary [952-954] parts of the potential, as well as on bound-state production. Polarization of the $P$ states has been predicted to arise from the medium anisotropies, resulting in a significant $(\sim 30 \%)$ effect on the $\chi_{b}$ states [955]. A weak medium anisotropy may also be related to the shear viscosity [956]. Thus the polarization can directly probe the properties of the medium produced in heavy-ion collisions.

\subsubsection{Dynamical production models}

While it is necessary to understand the quarkonium spectral functions in equilibrium QCD, this knowledge is insufficient for predicting effects on quarkonium production in heavy-ion collisions because, unlike the light degrees of freedom, heavy quarks are not fully thermalized in heavy-ion collisions. Therefore it is nontrivial to relate the finite temperature quarkonium spectral functions to quarkonium production rates in heavy-ion collisions without further model assumptions. The bridge between the two is provided by dynamical models of the matter produced in heavy-ion collisions. Some of the simple models currently available are based on statistical recombination [957], statistical recombination and dissociation rates [958], or sequential melting [959]. Here we highlight a more recent model, which makes closer contact with both QCD and experimental observations [960].

The bulk evolution of the matter produced in heavyion collisions is well described by hydrodynamics (see [961] for a recent review). The large heavy quark mass makes it possible to model its interaction with the medium by Langevin dynamics [962]. Such an approach successfully describes the anisotropic flow of charm quarks observed at RHIC [962, 963] (see also the review [964] and references therein). Potential models have shown that, in the absence of bound states, the $Q \bar{Q}$ pairs are correlated in space $[905,906]$. This correlation can be modeled classically using Langevin dynamics, including a drag force and a random force between the $Q$ (or $\bar{Q}$ ) and the medium as well as the forces between the $Q$ and $\bar{Q}$ described by the potential. It was recently shown that a model combining an ideal hydrodynamic expansion of the medium with a description of the correlated $Q \bar{Q}$ pair dynamics by the Langevin equation can describe charmonium suppression at RHIC quite well [960]. In particular, this model can explain why, despite the fact that a deconfined medium is created at RHIC, there is only a $40-50 \%$ suppression in the charmonium yield. The attractive potential and the finite lifetime of the system prevents the complete decorrelation of some of the $Q \bar{Q}$ pairs [960]. Once the matter has cooled sufficiently, these residual correlations make it possible for the $Q$ and $\bar{Q}$ to form a bound state. Charmonium production by recombination can also be calculated in this approach [965]. Although recombination was found to be significant for the most central collisions, it is still subdominant [965].

The above approach, which neglects quantum effects, is applicable only if there are no bound states, as is likely to be the case for the $J / \psi$. If heavy quark bound states are present, as is probable for the $\Upsilon(1 S)$, the thermal dissociation rate will be most relevant for understanding the quarkonium yield. It is expected that the interaction of a color-singlet quarkonium state with the medium is much smaller than that of heavy quarks. Thus, to first approximation, medium effects will only lead to quarkonium dissociation. 


\subsubsection{Summary of hot-medium effects}

Potential model calculations based on lattice QCD, as well as resummed perturbative QCD calculations, indicate that all charmonium states and the excited bottomonium states dissolve in the deconfined medium. This leads to the reduction of the quarkonium yields in heavyion collisions compared to the binary scaling of $p p$ collisions. Recombination and edge effects, however, guarantee a nonzero yield.

One of the great opportunities of the LHC and RHIC-II heavy-ion programs is the ability to study bottomonium yields. From a theoretical perspective, bottomonium is an important and clean probe for at least two reasons. First, the effective field theory approach, which provides a link to first principles QCD, is more applicable for bottomonium due to better separation of scales and higher dissociation temperatures. Second, the heavier bottom quark mass reduces the importance of statistical recombination effects, making bottomonium a good probe of dynamical models.

\subsection{Recent results at SPS energies}

Studies of charmonium production and suppression in cold and hot nuclear matter have been carried out by the NA60 collaboration [882, 966, 967]. In particular, data have been taken for In+In collisions at $158 \mathrm{GeV} /$ nucleon and for $p A$ collisions at 158 and $400 \mathrm{GeV}$. In the following, the primary NA60 results and their impact on the understanding of the anomalous $J / \psi$ suppression, first observed by the NA50 collaboration in $\mathrm{Pb}+\mathrm{Pb}$ collisions [968], are summarized. A preliminary comparison between the suppression patterns observed at the SPS and RHIC is discussed in Sect. 5.6.

\subsection{1. $J / \psi$ production in $p A$ collisions}

One of the main results of the SPS heavy-ion program was the observation of anomalous $J / \psi$ suppression. Results obtained in $\mathrm{Pb}+\mathrm{Pb}$ collisions at $158 \mathrm{GeV} /$ nucleon by the NA50 collaboration showed that the $J / \psi$ yield was suppressed with respect to estimates that include only cold-nuclear-matter effects [968]. The magnitude of the cold-nuclear-matter effects has typically been extracted by extrapolating the $J / \psi$ production data obtained in $p A$ collisions. Until recently the reference SPS $p A$ data were based on samples collected at $400 / 450 \mathrm{GeV}$ by the NA50 collaboration, at higher energy than the nuclear collisions and in a slightly different rapidity domain [872, 873, 969].

The need for reference $p A$ data taken under the same conditions as the $A A$ data was a major motivation for the NA60 run with an SPS primary proton beam at $158 \mathrm{GeV}$ in 2004. Seven nuclear targets (Be, Al, Cu, In, W, Pb, and $\mathrm{U}$ ) were simultaneously exposed to the beam. The sophisticated NA60 experimental setup [970], based on a high-resolution vertex spectrometer coupled to the muon spectrometer inherited from NA50, made it possible to unambiguously identify the target in which the $J / \psi$ was produced as well as measure muon pairs from its decay with $\mathrm{a} \sim 70 \mathrm{MeV}$ invariant mass resolution. During the same period, a $400 \mathrm{GeV}$ pA data sample was taken with the same experimental setup.

Cold-nuclear-matter effects were evaluated comparing the cross section ratio

$$
\frac{\sigma_{p A}^{J / \psi}}{\sigma_{p \mathrm{Be}}^{J / \psi}}
$$

for each nucleus with mass number $A$, relative to the lightest target $(\mathrm{Be})$. The beam luminosity factors cancel out in the ratio, apart from a small beam-attenuation factor. However, since the sub-targets see the vertex telescope from slightly different angles, the track reconstruction efficiencies do not completely cancel out. Therefore an accurate evaluation of the time evolution of such quantities was performed target-by-target, with high granularity and on a run-by-run basis. The results [882, 967], shown in Fig. 81, are integrated over $p_{T}$ and are given in the rapidity region covered by all the sub-targets, $0.28<y_{\mathrm{CMS}}<0.78$ for the $158 \mathrm{GeV}$ sample and $-0.17<y_{\mathrm{CMS}}<0.33$ for the $400 \mathrm{GeV}$ sample. Systematic errors include uncertainties in the target thickness, the rapidity distribution used in the acceptance calculation, and the reconstruction efficiency. Only the fraction of systematic errors not common to all the points is shown since it affects the evaluation of nuclear effects.

Nuclear effects have usually been parametrized by fitting the $A$ dependence of the $J / \psi$ production cross section using the expression

$$
\sigma_{p A}^{J / \psi}=\sigma_{p p}^{J / \psi} A^{\alpha}
$$

Alternatively, the effective absorption cross section, $\sigma_{\text {abs }}^{J / \psi}$, can be extracted from the data using the Glauber model. Both $\alpha$ and $\sigma_{\text {abs }}^{J / \psi}$ are effective quantities since they represent the strength of the cold-nuclear-matter effects that reduce the $J / \psi$ yield. However, they cannot distinguish among the different effects, e.g., shadowing and nuclear absorption, contributing to this reduction. The results in Fig. 81 were used to extract

$$
\begin{aligned}
\sigma_{\mathrm{abs}}^{J / \psi} & =7.6 \pm 0.7 \text { (stat.) } \pm 0.6 \text { (syst.) } \mathrm{mb} ; \\
\alpha & =0.882 \pm 0.009 \pm 0.008
\end{aligned}
$$

at $158 \mathrm{GeV}$ and

$$
\begin{aligned}
\sigma_{\mathrm{abs}}^{J / \psi} & =4.3 \pm 0.8 \text { (stat.) } \pm 0.6 \text { (syst.) } \mathrm{mb} ; \\
\alpha & =0.927 \pm 0.013 \pm 0.009
\end{aligned}
$$

at $400 \mathrm{GeV}$. Thus $\sigma_{\text {abs }}^{J / \psi}$ is larger at $158 \mathrm{GeV}$ than at $400 \mathrm{GeV}$ by three standard deviations. The $400 \mathrm{GeV}$ result is, on the other hand, in excellent agreement with the previous NA50 result obtained at the same energy [872]. 


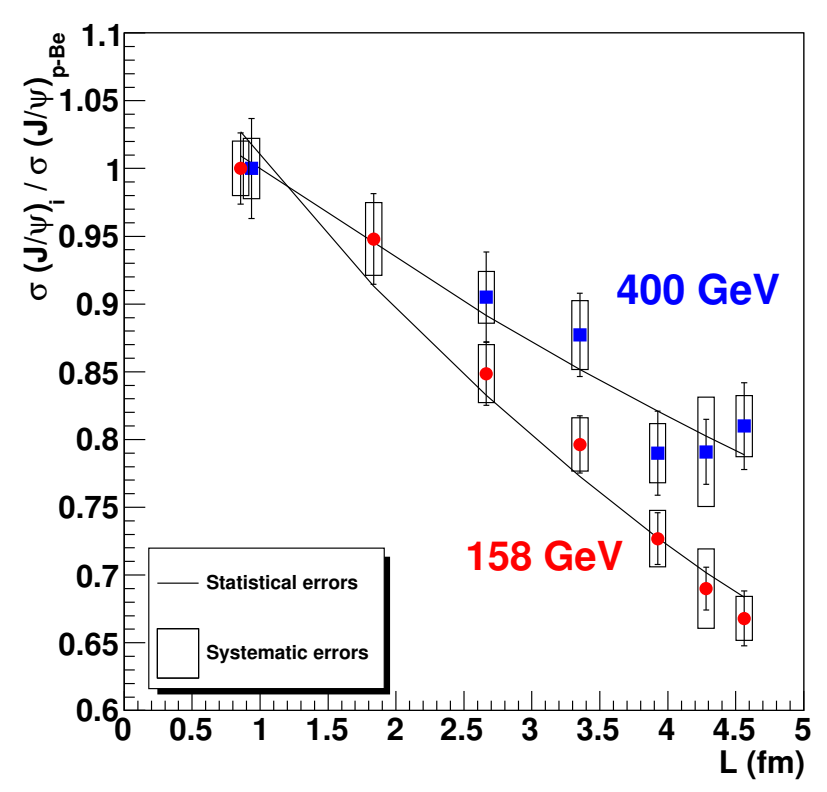

FIG. 81: The $J / \psi$ cross section ratios for $p A$ collisions at $158 \mathrm{GeV}$ (circles) and $400 \mathrm{GeV}$ (squares), as a function of $L$, the mean thickness of nuclear matter traversed by the $J / \psi$. From [882] with kind permission, copyright (2009) Elsevier

The study of cold-nuclear-matter effects at fixed-target energies is a subject which has attracted considerable interest. In Fig. 82, a compilation of previous results for $\sigma_{\text {abs }}^{J / \psi}$ as a function of $x_{F}[873,874,880,881]$ is presented, together with the new NA60 results [967]. Contrary to Fig. 78 , the values of $\sigma_{\text {abs }}^{J / \psi}$ in Fig. 82 do not include any shadowing contribution, only absorption. There is a systematic increase in the nuclear effects going from low to high $x_{F}$ as well as when from high to low incident proton energies. As shown in Fig. 82, the new NA60 results at $400 \mathrm{GeV}$ confirm the NA50 values obtained at a similar energy. On the other hand, the NA60 $158 \mathrm{GeV}$ data suggest higher values of $\sigma_{\text {abs }}^{J / \psi}$ and hint at increased absorption over the $x_{F}$ range. Note also that the older NA3 $J / \psi$ results are in partial contradiction with these observations, giving lower values of $\sigma_{\text {abs }}^{J / \psi}$, similar to those obtained from the higher energy data samples. Such a complex pattern of nuclear effects results from a delicate interplay of various nuclear effects (final-state absorption, shadowing, initial-state energy loss, etc.) and has so far not been satisfactorily explained by theoretical models [886]. A first attempt to disentangle the contribution of shadowing from $\sigma_{\text {abs }}^{J / \psi}$ (as extracted from the NA60 results) has been carried out using the EKS98 [877] parametrization of the nuclear PDFs. It was found that a larger $\sigma_{\text {abs }}^{J / \psi}$ is needed to describe the measured data:

$$
\begin{aligned}
\sigma_{\mathrm{abs}}^{J / \psi}(158 \mathrm{GeV}) & =9.3 \pm 0.7 \pm 0.7 \mathrm{mb} \\
\sigma_{\mathrm{abs}}^{J / \psi}(400 \mathrm{GeV}) & =6.0 \pm 0.9 \pm 0.7 \mathrm{mb}
\end{aligned}
$$

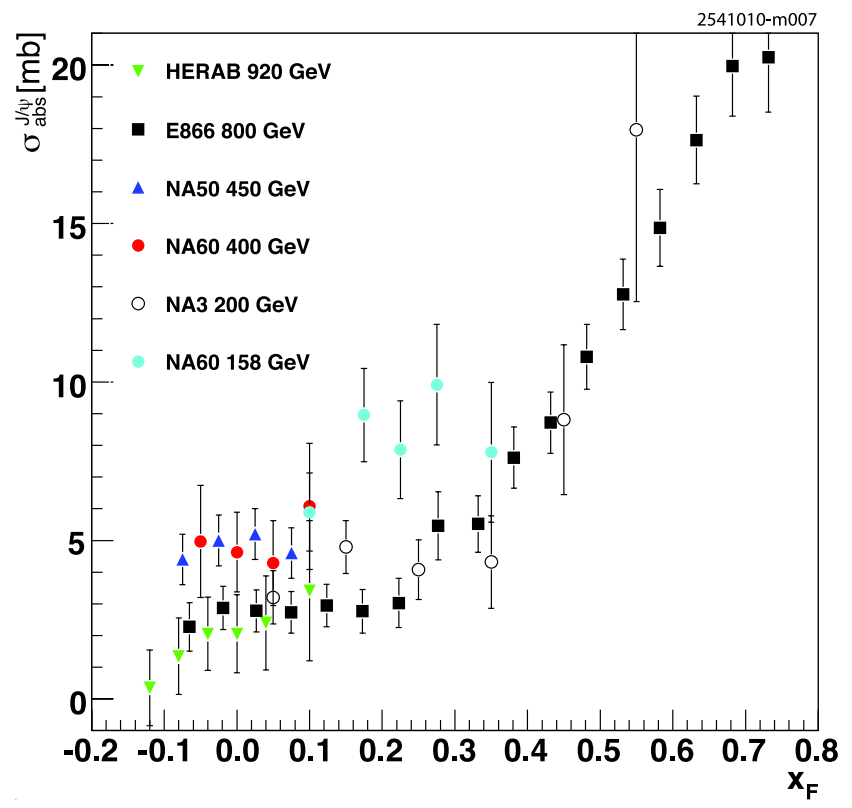

FIG. 82: Compilation of $\sigma_{\text {abs }}^{J / \psi}$ as a function of $x_{F}$ with no additional cold-matter effects included. From [967] with kind permission, copyright (2009) Elsevier

The results thus depend on the parametrization of the nuclear modifications of the PDFs. For example, slightly higher (5-10\%) values of $\sigma_{\text {abs }}^{J / \psi}$ are obtained if the EPS08 [971] parametrization is used.

\subsubsection{Anomalous $J / \psi$ suppression}

The $p A$ results at $158 \mathrm{GeV}$ shown in the previous section have been collected at the same energy and in the same $x_{F}$ range as the SPS $A A$ data. It is therefore natural to use these results to calculate the expected magnitude of cold-nuclear-matter effects on $J / \psi$ production in nuclear collisions. In order to do so, the expected shape of the $J / \psi$ distribution as a function of the forward energy in the zero degree calorimeter, $d N_{J / \psi}^{\text {expect }} / d E_{\mathrm{ZDC}}$, has been determined using the Glauber model. The $J / \psi$ yield is assumed to scale with the number of $N N$ collisions. The effective $J / \psi$ absorption cross section in nuclear matter is assumed to be the same as the value at $158 \mathrm{GeV}$ deduced in the previous section.

The measured $J / \psi$ yield, $d N_{J / \psi} / d E_{\mathrm{ZDC}}$, is normalized to $d N_{J / \psi}^{\text {expect }} / d E_{\mathrm{ZDC}}$ using the procedure detailed in [966]. This procedure previously did not take shadowing effects into account when extrapolating from $p A$ to $A A$ interactions. In $p A$ collisions, only the target partons are affected by shadowing, while in $A A$ collisions, effects on both the projectile and target must be taken into account. If shadowing is neglected in the $p A$ to $A A$ extrapolation, a small bias is introduced, resulting in an artificial $\sim 5 \%$ suppression of the $J / \psi$ yield with the 
EKS98 parametrization [889]. Therefore, if shadowing is properly accounted for in the $p A$ to $A A$ extrapolation, the amount of the anomalous $J / \psi$ suppression is reduced. Figure 83 presents the new results for the anomalous $J / \psi$ suppression in $\mathrm{In}+\mathrm{In}$ and $\mathrm{Pb}+\mathrm{Pb}$ collisions $[882,967]$ as a function of $N_{\text {part }}$, the number of participant nucleons. Up to $N_{\text {part }} \sim 200$ the $\mathrm{J} / \psi$ yield is, within errors, compatible with the extrapolation of cold-nuclear-matter effects. When $N_{\text {part }}>200$, there is an anomalous suppression of up to $\sim 20-30 \%$ in the most central $\mathrm{Pb}+\mathrm{Pb}$ collisions. This new, smaller anomalous suppression is primarily due to the larger $\sigma_{\text {abs }}^{J / \psi}$ extracted from the evaluation of coldnuclear-matter effects.

\subsection{Recent hadroproduction results from RHIC}

The strategy of the RHIC $J / \psi$ program has been to measure production cross sections in $\sqrt{s_{N N}}=200 \mathrm{GeV}$ collisions for $p p, \mathrm{~d}+\mathrm{Au}, \mathrm{Au}+\mathrm{Au}$ and $\mathrm{Cu}+\mathrm{Cu}$ collisions. RHIC has also studied $J / \psi$ production in $\mathrm{Cu}+\mathrm{Cu}$ collisions at $\sqrt{s_{N N}}=62 \mathrm{GeV}$ and will also study $J / \psi$ production in $p p$ collisions at $\sqrt{s}=500 \mathrm{GeV}$. The $p p$ collisions are studied both to learn about the $J / \psi$ production mechanism and to provide baseline production cross sections needed for understanding the $\mathrm{d}+\mathrm{Au}$ and $A A$ data.

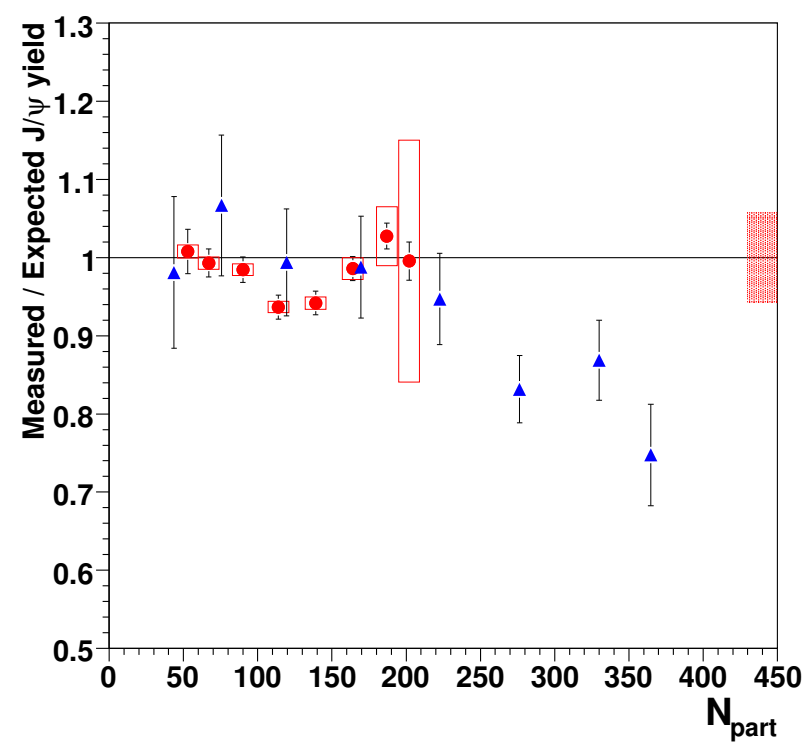

FIG. 83: Anomalous $J / \psi$ suppression in In+In (circles) and $\mathrm{Pb}+\mathrm{Pb}$ collisions (triangles) as a function of $N_{\text {part }}$. The boxes around the In+In points represent correlated systematic errors. The filled box on the right corresponds to the uncertainty in the absolute normalization of the In+In points. A $12 \%$ global error, due to the uncertainty on $\sigma_{\text {abs }}^{J / \psi}$ at $158 \mathrm{GeV}$ is not shown. From [967] with kind permission, copyright (2009) Elsevier
Similarly, the $\mathrm{d}+\mathrm{Au}$ measurements are inherently interesting because they study the physical processes that modify $J / \psi$ production cross sections in nuclear targets and also provide the crucial cold-nuclear-matter baseline for understanding $J / \psi$ production in $A A$ collisions. Note that $\mathrm{d}+\mathrm{Au}$ collisions are studied at RHIC instead of $p+\mathrm{Au}$ collisions for convenience $-p+\mathrm{Au}$ collisions are possible at $\mathrm{RHIC}$, but would require a dedicated $p+\mathrm{Au}$ run.

The last few years of the RHIC program have produced $J / \psi$ data from PHENIX for $p p, \mathrm{~d}+\mathrm{Au}$ and $\mathrm{Au}+\mathrm{Au}$ collisions with sufficient statistical precision to establish the centrality dependence of both hot and cold-nuclearmatter effects at $\sqrt{s_{N N}}=200 \mathrm{GeV}$. The data cover the rapidity range $|y|<2.4$.

We introduce some quantities that have been applied to $\mathrm{d}+\mathrm{Au}$ and $A A$ collisions at RHIC to describe the impact parameter, $b$ (also called centrality), dependence of the quarkonium results. While most of the data taken are at large impact parameter (peripheral collisions), the small impact parameter (central) collisions are more likely to produce a quark-gluon plasma. Therefore it is important to study quantities over a range of centralities, using impact-parameter dependent variables such as the number of participant nucleons, $N_{\text {part }}$, and the number of collisions, $N_{\text {coll }}$. The number of participants depends on $b$ as

$$
\begin{aligned}
& N_{\text {part }}(b)=\int d^{2} s \\
& \quad\left[T_{A}(s)\left(1-\exp \left[-\sigma_{\text {inel }}\left(s_{N N}\right) T_{B}(|\vec{b}-\vec{s}|)\right]\right)+\right. \\
& \left.T_{B}(|\vec{b}-\vec{s}|)\left(1-\exp \left[-\sigma_{\text {inel }}\left(s_{N N}\right) T_{A}(s)\right]\right)\right]
\end{aligned}
$$

Here $\sigma_{\text {inel }}$ is the inelastic nucleon-nucleon cross section, $42 \mathrm{mb}$ at $\mathrm{RHIC}$, and $T_{A / B}(s)=\int d z \rho_{A / B}(s, z)$, the line integral of the nuclear density, $\rho$, in the beam direction, is the nuclear profile function. Large values of $N_{\text {part }}$ are obtained for small impact parameters with $N_{\text {part }}(b=0)=2 A$ for spherical nuclei. Small values of $N_{\text {part }}$ occur in very peripheral collisions. The number of collisions, $N_{\text {coll }}\left(s_{N N} ; b\right)=\sigma_{\text {inel }}\left(s_{N N}\right) T_{A B}(b)$, depends on the nuclear overlap integral,

$$
T_{A B}(b)=\int d^{2} s d z d z^{\prime} \rho_{A}(s, z) \rho_{B}\left(|\vec{b}-\vec{s}|, z^{\prime}\right) .
$$

In $p A$ collisions, we assume that the proton has a negligible size, $\rho_{A}(s, z)=\delta(s) \delta(z)$ so that $T_{A B}(b)$ collapses to the nuclear profile function. The deuteron cannot be treated as a point particle since it is large and diffuse. Thus the Húlthen wave function [972, 973] is used to calculate the deuteron density distribution. No shadowing effects are included on the deuteron.

The nuclear suppression factor, $R_{A B}$, for $\mathrm{d} A$, and $A A$ collisions is defined as the ratio

$$
R_{A B}\left(N_{\text {part }} ; b\right)=\frac{d \sigma_{A B} / d y}{T_{A B}(b) d \sigma_{p p} / d y}
$$


where $d \sigma_{A B} / d y$ and $d \sigma_{p p} / d y$ are the quarkonium rapidity distributions in $A B$ and $p p$ collisions and $T_{A B}$ is the nuclear overlap function, defined in Eq. (183). In $A A$ collisions, $R_{A A}$ is sometimes shown relative to the extracted cold-nuclear-matter baseline, $R_{A A}^{\mathrm{CNM}}$. PHENIX has also shown both $\mathrm{d}+\mathrm{Au}$ and $A A$ data as a function of $R_{C P}$, the ratio of $A B$ cross sections in central relative to peripheral collisions,

$$
R_{C P}(y)=\frac{T_{A B}\left(b_{P}\right)}{T_{A B}\left(b_{C}\right)} \frac{d \sigma_{A B}\left(b_{C}\right) / d y}{d \sigma_{A B}\left(b_{P}\right) / d y},
$$

where $b_{C}$ and $b_{P}$ correspond to the central and peripheral values of the impact parameter since systematic uncertainties cancel in the ratio. Another quantity of interest is $v_{2}$, the second harmonic of the azimuthal Fourier decomposition of the momentum distribution, $d N / d p_{T} \propto 1+2 v_{2} \cos \left(2\left(\phi-\phi_{r}\right)\right)$ where $\phi$ is the particle emission angle and $\phi_{r}$ is the reaction plane angle, known as the elliptic flow. It gives some indication of the particle response to the thermalization of the medium. A finite $J / \psi \quad v_{2}$ would give some indication of whether the $J / \psi$ distribution becomes thermal. The strength of $v_{2}$ depends on the proportion of $J / \psi$ produced by coalescence.

In the next few years the increased RHIC luminosity and the commissioning of upgraded detectors and triggers for PHENIX and STAR will enable a next generation of RHIC measurements, extending the program to the $\Upsilon$ family, excited charmonium states, and $J / \psi v_{2}$ and high- $p_{\mathrm{T}}$ suppression measurements. There have already been low-precision, essentially proof-of-principle, measurements of most of those signals. Very importantly, upgraded silicon vertex detectors for both PHENIX and STAR are expected to produce qualitatively better open charm measurements that will provide important inputs to models of $J / \psi$ production in heavy-ion collisions.

In addition to the results discussed here, there have been PHENIX results on $J / \psi$ photoproduction in peripheral $\mathrm{Au}+\mathrm{Au}$ collisions [974] and a proof-of-principle measurement of the $J / \psi \quad v_{2}$ in $\mathrm{Au}+\mathrm{Au}$ collisions by PHENIX [975] with insufficient precision for physics conclusions.

\subsubsection{Charmonium from $p p$ collisions}

PHENIX [662] has reported measurements of the inclusive $J / \psi$ polarization in $200 \mathrm{GeV} p p$ collisions at midrapidity. Results for the polarization parameter $\lambda$, defined in the Helicity frame, are shown in Fig. 84 and compared to COM [699] and s-channel-cut CSM [682] predictions. The latter has been shown to describe the rapidity and $p_{\mathrm{T}}$ dependence of the PHENIX $200 \mathrm{GeV} p p J / \psi$ data [976] using a two-parameter fit to CDF data at $\sqrt{s}=1.8 \mathrm{TeV}$.

At Quark Matter 2009, PHENIX [663] showed preliminary measurements of the $p_{\mathrm{T}}$ dependence of the $\psi(2 S)$ cross section at $200 \mathrm{GeV}$. This is the first measurement of the $p_{\mathrm{T}}$-dependence of an excited charmonium state at
RHIC. PHENIX measured the feeddown contribution of the $\psi(2 S)$ to the $J / \psi$ to be $(8.6 \pm 2.3) \%$, in good agreement with the world average.

STAR [664] has published measurements of the $J / \psi$ cross section in $200 \mathrm{GeV} p p$ collisions for $5<p_{\mathrm{T}}<$ $13 \mathrm{GeV} / c$. This greatly extends the $p_{\mathrm{T}}$ range over which $J / \psi$ data are available at RHIC. Although PHENIX can trigger at all $p_{\mathrm{T}}$, it has so far been limited to $p_{\mathrm{T}}$ below about $9 \mathrm{GeV} / c$ [663] because of its much smaller acceptance.

\subsubsection{Charmonium from $\mathrm{Cu}+\mathrm{Cu}$ collisions}

Measured quarkonium production rates from heavyion collisions are commonly presented in terms of a nuclear modification factor, $R_{A A}$, defined in Eq. (184). PHENIX [977] results on the rapidity and $p_{\mathrm{T}}$ dependence of $R_{A A}$ values for $J / \psi$ from $200 \mathrm{GeV} \mathrm{Cu}+\mathrm{Cu}$ collisions were published some time ago. However, those results were limited to $p_{T}<5 \mathrm{GeV} / c$, and do not address the high- $p_{\mathrm{T}}$ behavior of the measurements very well. STAR [664] has now published $\mathrm{Cu}+\mathrm{Cu} R_{A A}$ data for $J / \psi$ at 5.5 and $7 \mathrm{GeV} / c$ that yield an average $\left\langle R_{A A}\right\rangle=1.4 \pm 0.4$ (stat) \pm 0.2 (sys) above $5 \mathrm{GeV} / c$ for the $0-20 \%$ most central collisions. The $R_{A A}$ data for the $0-60 \%$ most central collisions have very similar values, in contrast to the PHENIX data below $5 \mathrm{GeV} / c$ that yield $\left\langle R_{A A}\right\rangle \approx 0.52$ for central $\mathrm{Cu}+\mathrm{Cu}$ collisions.

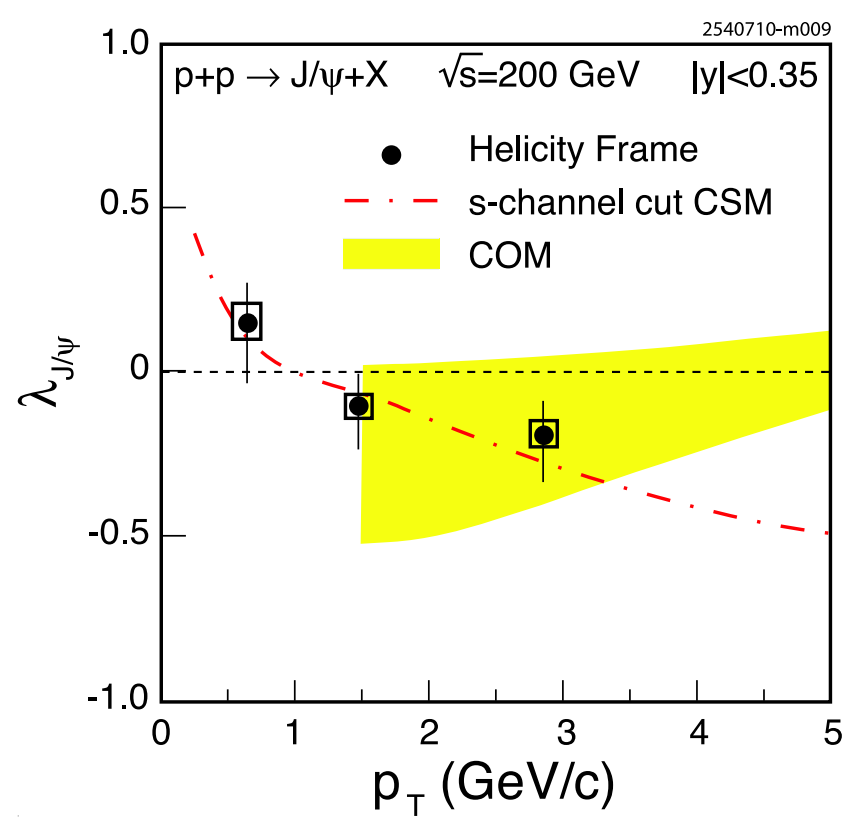

FIG. 84: The polarization extracted from $200 \mathrm{GeV}$ PHENIX $p p$ data at midrapidity as a function of $p_{T}$. The data are compared with the $s$-channel-cut CSM [682] and a COM [699] prediction. Adapted from [662] with kind permission, copyright (2010) The American Physical Society 
PHENIX [975] has also released preliminary data on the $R_{A A}$ for $J / \psi$ from minimum bias $(0-94 \%$ centrality) $\mathrm{Cu}+\mathrm{Cu}$ data at 7 and $9 \mathrm{GeV} / c$. The minimum bias PHENIX data should be comparable to the STAR 0-60\% data, but the PHENIX results are more consistent with a nearly $p_{\mathrm{T}}$-independent $R_{A A}$. However, both measurements have large statistical uncertainties and a direct comparison [978] of the STAR and PHENIX $\mathrm{Cu}+\mathrm{Cu} R_{A A}$ data at high $p_{\mathrm{T}}$ suggests that more data will be required to definitively determine the high- $p_{\mathrm{T}}$ behavior of $R_{A A}$ in central collisions.

\subsubsection{Bottomonium production}

PHENIX [979] showed a preliminary result for the $\Upsilon(1 S)+\Upsilon(2 S)+\Upsilon(3 S)$ cross section at forward and backward rapidity $(1.2<|y|<2.4)$ at Quark Matter 2006. More recently, PHENIX [663] showed a preliminary result at Quark Matter 2009 for $\Upsilon(1 S)+\Upsilon(2 S)+$ $\Upsilon(3 S)$ production in $200 \mathrm{GeV} p p$ collisions at midrapidity $(|y|<0.35)$. The measured cross section is $\mathcal{B} d \sigma / d y=114_{-45}^{+46} \mathrm{pb}$ at $y=0$, where the presence of the $\mathcal{B}$ reflects that the results have not been separated by individual $\Upsilon(n S)$ resonance nor corrected for the dilepton branching fractions $\mathcal{B}\left(\Upsilon(n S) \rightarrow e^{+} e^{-}\right)$.

STAR [980] published a measurement of the $\Upsilon(1 S)+$ $\Upsilon(2 S)+\Upsilon(3 S) \rightarrow e^{+} e^{-}$cross section at $|y|<0.5$ for $200 \mathrm{GeV} p p$ collisions. The measured value is $\mathcal{B} d \sigma / d y=$ $114 \pm 38$ (stat) ${ }_{-24}^{+23}$ (syst) pb at $y=0$. STAR [981] also has a preliminary result for the $\Upsilon(1 S)+\Upsilon(2 S)+\Upsilon(3 S) \rightarrow$ $e^{+} e^{-}$cross section at midrapidity in $\mathrm{d}+\mathrm{Au}$ collisions at $200 \mathrm{GeV} / c$. The cross section was found to be $\mathcal{B} d \sigma / d y=$ $35 \pm 4$ (stat) \pm 5 (syst) nb. The midrapidity value of $R_{\mathrm{dAu}}$ was found to be $0.98 \pm 0.32$ (stat) \pm 0.28 (syst), consistent with binary scaling.

PHENIX has made a preliminary measurement of the dielectron yield in the $\Upsilon(1 S)+\Upsilon(2 S)+\Upsilon(3 S)$ mass range at midrapidity in $\mathrm{Au}+\mathrm{Au}$ collisions [975]. In combination with the PHENIX $\Upsilon(1 S)+\Upsilon(2 S)+\Upsilon(3 S) p p$ result at midrapity, a $90 \%$ CL upper limit on $R_{\mathrm{dAu}}$ of 0.64 was found for the $\Upsilon(1 S)+\Upsilon(2 S)+\Upsilon(3 S)$ mass region. The significance of this result is not yet very clear since the measurement is for all three $\Upsilon$ states combined.

\subsection{4. $J / \psi$ production from $d+A u$ collisions}

As discussed previously, modification of the $J / \psi$ production cross section due to the presence of a nuclear target is expected to be caused by shadowing, breakup of the precursor $J / \psi$ state by collisions with nucleons, initialstate energy loss, and other possible effects. Parametrizing these effects by employing a Glauber model with a fitted effective $J / \psi$-absorption cross section, $\sigma_{\text {abs }}^{J / \psi}$, results in an effective cross section with strong rapidity and $\sqrt{s_{N N}}$ dependencies [875] that are not well understood. A large increase in the effective absorption cross section is observed by E866/NuSea [874] at forward rapidity. This increase cannot be explained by shadowing models alone, suggesting that there are important physics effects omitted from the Glauber absorption-plus-shadowing model.

The extraction of hot-matter effects in the $\mathrm{Au}+\mathrm{Au} J / \psi$ data at RHIC has been seriously hampered by the poor understanding of $J / \psi$ production in nuclear targets, including the underlying $J / \psi$ production mechanism. Thus the cold-nuclear-matter baseline has to be obtained experimentally.

The PHENIX $J / \psi$ data obtained in the 2003 RHIC $\mathrm{d}+\mathrm{Au}$ run did not have sufficient statistical precision either for studies of cold-nuclear-matter effects or for setting a cold-nuclear-matter baseline for the $\mathrm{Au}+\mathrm{Au}$ data [883]. This low-statistics measurement has been augmented by the large $J / \psi$ data set obtained in the 2008 $\mathrm{d}+\mathrm{Au}$ run. PHENIX [663] has released $\mathrm{d}+\mathrm{Au} R_{C P}$ data for $J / \psi$ production in nine rapidity bins over $|y|<2.4$. Systematic uncertainties associated with the beam luminosity, detector acceptance, trigger efficiency, and tracking efficiency cancel in $R_{C P}$, defined in Eq. (185). There is a remaining systematic uncertainty due to the centrality dependence of the tracking and particle identification efficiencies.

The use of a Glauber model also gives rise to significant systematic uncertainties in the centrality dependence of $R_{C P}$. The model is used to calculate the average number of nucleon-nucleon collisions as a means of estimating the relative normalization between different centrality bins. The systematic uncertainty due to this effect is independent of rapidity.

The PHENIX $\mathrm{d}+\mathrm{Au} R_{C P}$ data have been independently fitted at each of the nine rapidities [884] employing a model including shadowing and $J / \psi$ absorption. The model calculations [888] use the EKS98 and nDSg shadowing parametrizations with $0 \leq \sigma_{\text {abs }} \leq 15 \mathrm{mb}$. The best fit absorption cross section was determined at each rapidity, along with the $\pm 1 \sigma$ uncertainties associated with both rapidity-dependent and rapidity-independent systematic effects. The results are shown in Fig. 85. The most notable feature is the stronger effective absorption cross section at forward rapidity, similar to the behavior observed at lower energies [874]. In fact, it is striking that the extracted cross sections at forward rapidity are very similar for PHENIX $\left(\sqrt{s_{N N}}=200 \mathrm{GeV}\right)$ and E866 [875] $\left(\sqrt{s_{N N}}=38.8 \mathrm{GeV}\right)$ (see the lower panel of Fig. 78), despite the large difference in center-of-mass energies.

Note the large global systematic uncertainty in $\sigma_{\text {abs }}$ extracted from the PHENIX $R_{C P}$ data, dominated by the uncertainty in the Glauber estimate of the average number of collisions at each centrality. Although it does not affect the shape of the rapidity dependence of $\sigma_{\text {abs }}^{J / \psi}$, it results in considerable uncertainty in the magnitude of the effective absorption cross section.

It has been suggested [891] that the large increase in effective absorption cross section at forward rapidity obtained from a CEM calculation [884] may be moderated significantly if the $2 \rightarrow 2$ kinematics of the leading-order 


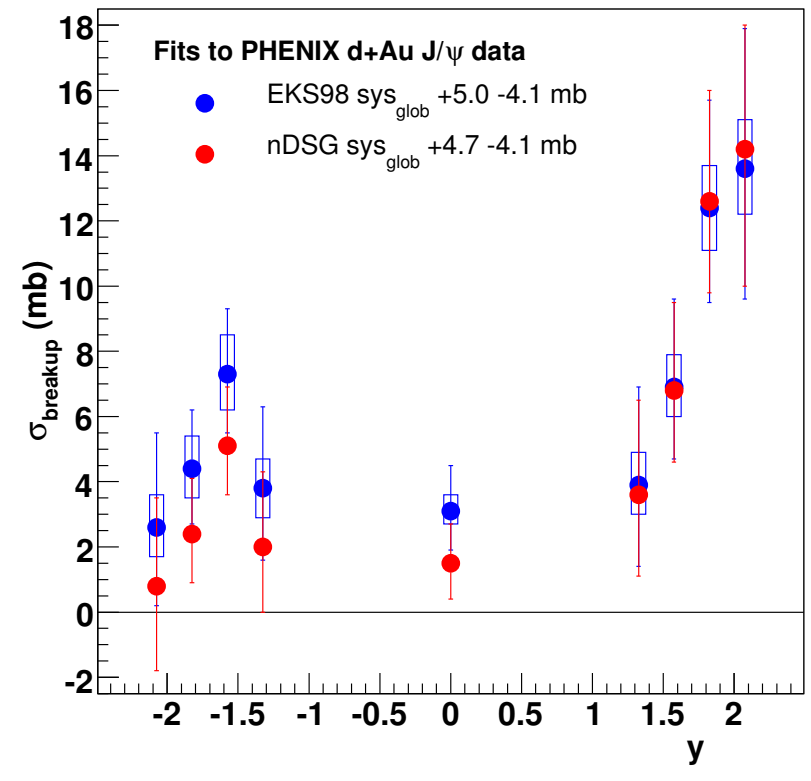

FIG. 85: The effective absorption cross section as a function of rapidity extracted from PHENIX $\mathrm{d}+\mathrm{Au} R_{C P}$ data using the EKS98 and $\mathrm{nDSg}$ shadowing parametrizations. The vertical bars show uncorrelated point-to-point uncertainties, the boxes show correlated uncertainties, and the global uncertainties are given in the legend

CSM is used. This difference emphasizes the importance of understanding the underlying production mechanism.

PHENIX has very recently released [982] final $R_{\mathrm{dAu}}$ and $R_{C P}$ data from the $2008 \mathrm{~d}+\mathrm{Au}$ RHIC run. The final $R_{C P}$ data are in good agreement with the preliminary data, discussed earlier, as well as in the next section. A comparison [982] of the $R_{\mathrm{dAu}}$ data, which has not been shown before, with the $R_{C P}$ data shows that a simultaneous description of the two observables will require a stronger than linear dependence of the $J / \psi$ suppression on the nuclear thickness function at forward rapidity. The dependence of the suppression on nuclear thickness is at least quadratic, and is likely higher. The result has important implications for the understanding of forwardrapidity $\mathrm{d}+\mathrm{Au}$ physics. Since the calculations of the cold matter contributions to $R_{A A}$ assumed that shadowing depends linearly on the nuclear thickness, the calculations of $R_{A A}$ shown in the next section should be be revisited.

\subsection{5. $J / \psi$ production from $A u+A u$ collisions}

PHENIX [983] has published the centrality dependence of $R_{A A}$ for $\mathrm{Au}+\mathrm{Au}$ collisions using $\mathrm{Au}+\mathrm{Au}$ data from the 2004 RHIC run and $p p$ data from the 2005 run. The data are shown in Fig. 86. The suppression is considerably stronger at forward rapidity than at midrapidity. The significance of this difference with respect to hot-

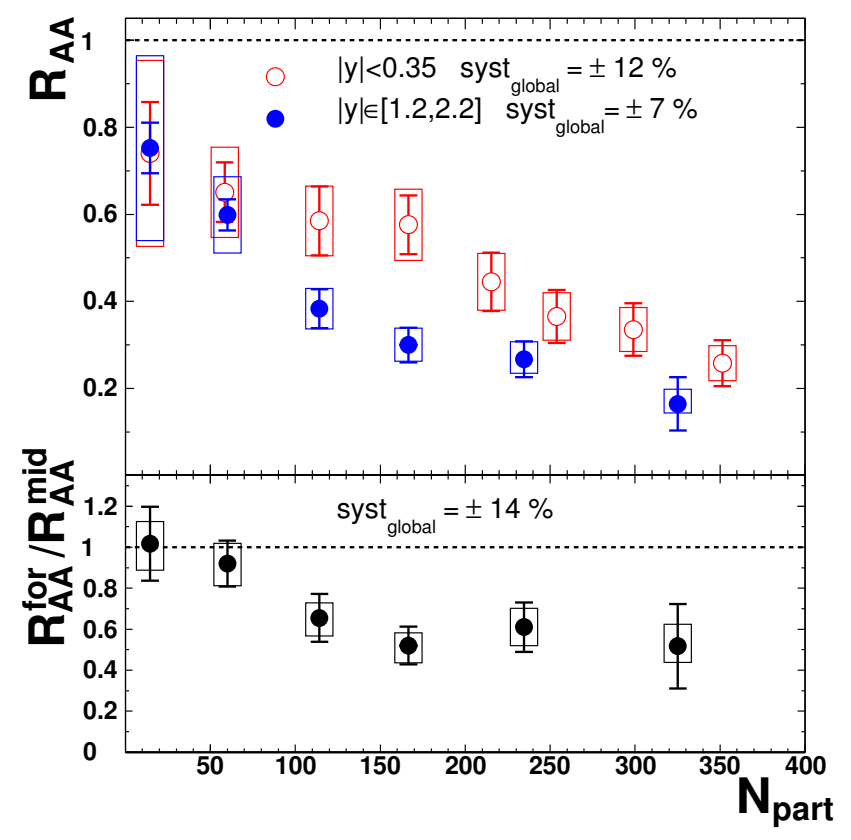

FIG. 86: The PHENIX $\mathrm{Au}+\mathrm{Au} R_{A A}$ as a function of centrality for $|y|<0.35$ and $1.2<|y|<2.2$

matter effects will not be clear, however, until the suppression due to cold-nuclear-matter effects is more accurately known.

To estimate the cold-nuclear-matter contribution to the $\mathrm{Au}+\mathrm{Au} \quad J / \psi \quad R_{A A}$ the $\mathrm{d}+\mathrm{Au} \quad J / \psi \quad R_{C P}$ data were extracted using the EKS98 and nDSg shadowing parametrizations, as described earlier, except that, in this case, the $\sigma_{\text {abs }}^{J / \psi}$ values in $\mathrm{d}+\mathrm{Au}$ collisions were fitted independently in three rapidity intervals: $-2.2<$ $y<-1.2,|y|<0.35$, and $1.2<y<2.2$. In effect, this tunes the calculations to reproduce the $\mathrm{d}+\mathrm{Au} R_{C P}$ independently in each of the three rapidity windows in which the $\mathrm{Au}+\mathrm{Au} R_{A A}$ data were measured. The cold-nuclearmatter $R_{A A}$ for $\mathrm{Au}+\mathrm{Au}$ collisions was then estimated in a Glauber calculation using the fitted absorption cross sections and the centrality-dependent $R_{p \mathrm{Au}}$ values calculated using EKS98 and nDSg shadowing parametrizations [984]. Each nucleon-nucleon collision contributes differently to the $R_{A A}$ in each rapidity window. To more directly simulate nucleon-nucleus interactions, the analysis assumes that $R_{A A}$ can be treated as a convolution of $p+\mathrm{Au}$ and $\mathrm{Au}+p$ collisions in the three rapidity windows. The impact-parameter dependence of $R_{p \mathrm{Au}}$ is determined separately to infer the $R_{A A}$ centrality dependence for a rapidity-dependent absorption cross section. Thus the value of $R_{p \mathrm{Au}}$ at the impact parameter of nucleon 1 in the projectile is convoluted with the value of $R_{\text {Aup }}$ at the impact parameter of nucleon 2 in the target. Effectively, this means that to obtain $R_{A A}$ for $1.2<|y|<2.2$, $R_{p \mathrm{Au}}$ for the forward-moving nucleon $(1.2<y<2.2)$ is multiplied by $R_{p \mathrm{Au}}$ for the backward-moving nucleon $(-2.2<y<-1.2)$. When $|y|<0.35$, the $R_{p \mathrm{Au}}$ calcu- 
lations at midrapidity are used. The number of participants, obtained from a Glauber calculation, is used to bin the collisions in centrality with a cut on peripheral events to mimic the effect of the PHENIX trigger efficiency at large impact parameter. The uncertainty in the calculated CNM $R_{A A}$ was estimated by repeating the calculation with $\sigma_{\text {abs }}^{J / \psi}$ varied away from best-fit values. This variation ranged over the rapidity-dependent systematic uncertainty determined when fitting the $\mathrm{d}+\mathrm{Au} R_{C P}$.

The global systematic uncertainty in $\sigma_{\text {abs }}^{J / \psi}$ was neglected in the calculation of the CNM $R_{A A}$. This was done because the same Glauber model was used to obtain both the number of nucleon-nucleon collisions, $N_{\text {coll }}$, in $\mathrm{d}+\mathrm{Au}$ and $\mathrm{Au}+\mathrm{Au}$ interactions and the fitted $\sigma_{\text {abs }}^{J / \psi}$ values. Therefore, if, for example, $N_{\text {coll }}$ is underestimated for the $\mathrm{d}+\mathrm{Au} R_{C P}$, the fitted absorption cross section will be overestimated. However, this would be compensated in the calculated CNM $R_{A A}$ by the underestimated $N_{\text {coll }}$ value. Any possible differences in the details of the $\mathrm{d}+\mathrm{Au}$ and $\mathrm{Au}+\mathrm{Au}$ Glauber calculations would result in an imprecise cancellation of the uncertainties. This effect has not yet been studied.

Note that there is a significant difference between the impact-parameter dependence of the $R_{p \mathrm{Au}}$ and $R_{\mathrm{dAu}}$ calculations [884], primarily for peripheral collisions, due to the smearing caused by the finite size of the deuteron. Since $R_{\mathrm{dAu}}$ and $R_{p \mathrm{Au}}$ are calculated using the same basic model, this smearing does not present a problem in the present analysis. However, if the measured $R_{\mathrm{dAu}}$ was used directly in a Glauber model, as was done with the RHIC 2003 data [883], a correction would be necessary.

The resulting Glauber calculations of the cold-nuclearmatter $R_{A A}$ using the EKS98 shadowing parametrization are shown in Fig. 87. The values obtained with $\mathrm{nDSg}$ are almost identical, as they should be since both methods parametrize the same data.

We emphasize that the kinematic-dependent differences in the effective absorption cross sections noted in the previous section do not affect the cold-nuclear-matter $R_{A A}$ derived from the data. As long as the method of fitting the $\mathrm{d}+\mathrm{Au}$ data is consistent with the estimate of the cold nuclear matter $R_{A A}$, the result should be model independent.

The $J / \psi$ suppression beyond CNM effects in $\mathrm{Au}+\mathrm{Au}$ collisions can be estimated by dividing the measured $R_{A A}$ by the estimates of the CNM $R_{A A}$. The result for EKS98 is shown in Fig. 88. The result for $\mathrm{nDSg}$ is nearly identical.

Assuming that the final PHENIX $R_{\mathrm{dAu}}$ confirms the strong suppression at forward rapidity seen in $R_{C P}$, it would suggest that the stronger suppression seen at forward/backward rapidity in the PHENIX $\mathrm{Au}+\mathrm{Au} R_{A A}$ data is primarily due to cold-nuclear-matter effects. The suppression due to hot-matter effects seems to be comparable at midrapidity and at forward/backward rapidity.

Finally, it is possible to use the effective absorption cross sections obtained from the $\mathrm{d}+\mathrm{Au} J / \psi R_{C P}$ data in a similar Glauber calculation of $R_{p \mathrm{Cu}}$ to estimate the

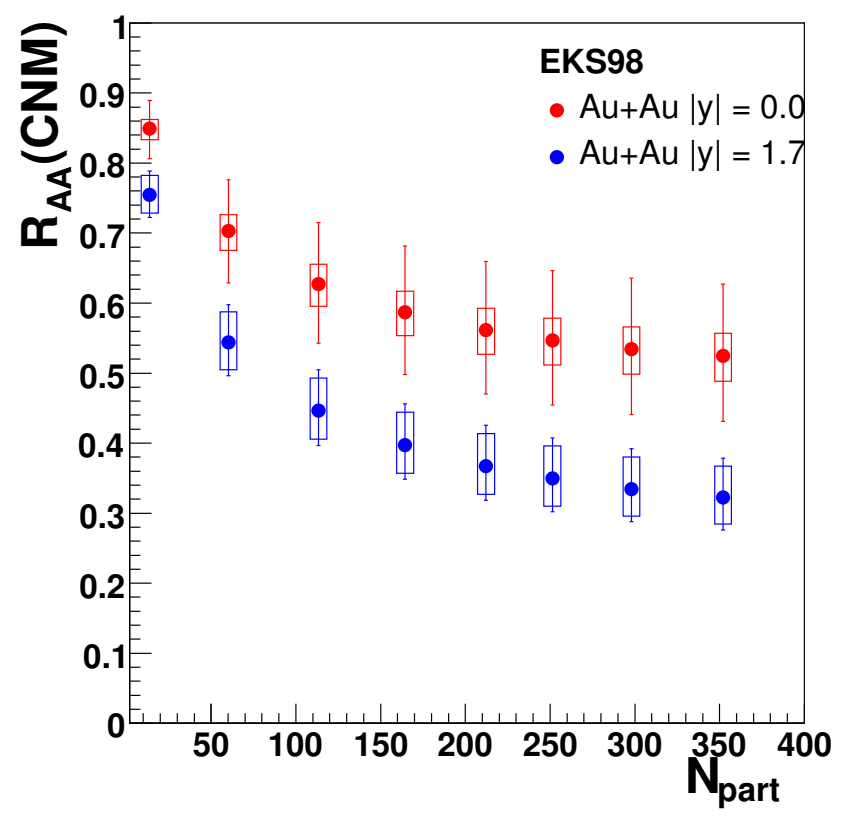

FIG. 87: The estimated $\mathrm{Au}+\mathrm{Au}$ cold-nuclear-matter $R_{A A}$ as a function of centrality for $|y|<0.35$ and $1.2<|y|<2.2$. The vertical bar represents the rapidity-dependent systematic uncertainty in the fitted $\sigma_{\text {abs }}^{J / \psi}$

cold-nuclear-matter $R_{A A}$ for $\mathrm{Cu}+\mathrm{Cu}$ collisions. However, the resulting CNM $R_{A A}$ for $\mathrm{Cu}+\mathrm{Cu}$ is significantly different for EKS98 and nDSg [884], most likely due to the different $A$ dependences of EKS98 and nDSg. Measurements of $J / \psi$ production in $p+\mathrm{Cu}$ or $\mathrm{d}+\mathrm{Cu}$ collisions would be needed to reduce the model dependence of the estimated $\mathrm{CNM} R_{A A}$ for $\mathrm{Cu}+\mathrm{Cu}$ collisions.

\subsection{Anomalous suppression: SPS vs RHIC}

The preliminary PHENIX $\mathrm{d}+\mathrm{Au}$ results at $\sqrt{s}=$ $200 \mathrm{GeV}$ are, for the first time, based on a high-statistics sample [884]. Comparing these results with the previous $\mathrm{Au}+\mathrm{Au}$ data gives an estimate of the magnitude of the anomalous $J / \psi$ suppression at RHIC. The newlyavailable NA60 $p A$ results at $158 \mathrm{GeV}$, described in Sect. 5.4, allow significant comparisons of the centrality dependence of the anomalous suppression at the SPS and that obtained at RHIC. Work is in progress to make such a comparison as a function of several variables of interest, such as the charged particle multiplicity, $d N_{\mathrm{ch}} / d \eta$, and the Bjorken energy density reached in the collision. The anomalous suppression patterns in $\mathrm{In}+\mathrm{In}$ and $\mathrm{Pb}+\mathrm{Pb}$ collisions at the SPS and the midrapidity $\mathrm{Au}+\mathrm{Au}$ results at RHIC are presented as a function of $d N_{\mathrm{ch}} / d \eta$ in Fig. 89 [985]. Note that the magnitude of the anomalous $J / \psi$ suppression is practically system- and $\sqrt{s}$-independent when expressed as a function of $d N_{\mathrm{ch}} /\left.d \eta\right|_{\eta=0}$. 


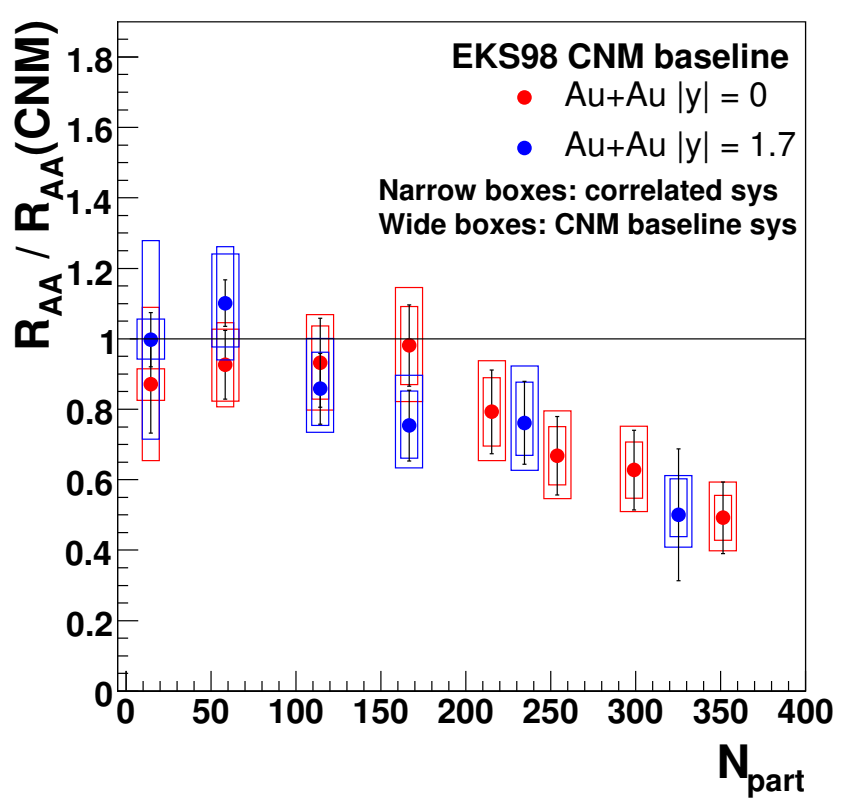

FIG. 88: The estimated $\mathrm{Au}+\mathrm{Au}$ suppression relative to the cold-nuclear-matter $R_{A A}$ as a function of centrality for $|y|<$ 0.35 and $1.2<|y|<2.2$. The systematic uncertainty of the baseline cold-nuclear-matter $R_{A A}$ is depicted by the wide box around each point. The narrow box is the systematic uncertainty in the $\mathrm{Au}+\mathrm{Au} R_{A A}$

\subsection{Photoproduction in nuclear collisions}

In addition to in-medium hadroproduction, photoproduction of quarkonium may also occur in nucleus-nucleus collisions. In this case, one nucleus acts as a photon source (the photon flux is given by the WeizsackerWilliams formalism). The photons fluctuate to virtual quark-antiquark pairs which interact with the opposite (target) nucleus [986, 987] and emerge as heavy quarkonia (e.g., $J / \psi$ and $\Upsilon$ ) or other, light, vector mesons. Such $J / \psi$ photoproduction has been observed in $\mathrm{Au}+\mathrm{Au}$ collisions with PHENIX [974] and in $\bar{p} p$ collisions at the Tevatron [701]. CDF [701] has also observed $\psi(2 S)$ photoproduction.

At the LHC, photoproduction can be studied at far higher energies than available at fixed-target facilities or at HERA. At the maximum $p p$ energy of the LHC, $\gamma p$ collisions with center-of-mass energies up to $\sqrt{s_{\gamma p}}=8.4 \mathrm{TeV}$ are accessible, forty times the energy reached at HERA. With $\mathrm{Pb}$ beams at maximum energy, the per-nucleon center-of-mass energy can reach $\sqrt{s_{\gamma N}}=950 \mathrm{GeV}$ [988], equivalent to a $480 \mathrm{TeV}$ photon beam on a fixed target.

Photoproduction is of interest because it is sensitive to the gluon distribution in the target nucleus. The cross section for $\gamma p \rightarrow V p$ scales as [989] $\left[x g\left(x, Q^{2}\right)\right]^{2}$, where $x$ is the gluon momentum fraction, $Q^{2}=m_{V}^{2} / 4$ is the photon virtuality, and $m_{V}$ is the vector meson mass. For low $p_{T}$ vector mesons, the gluon momentum fraction, $x$,

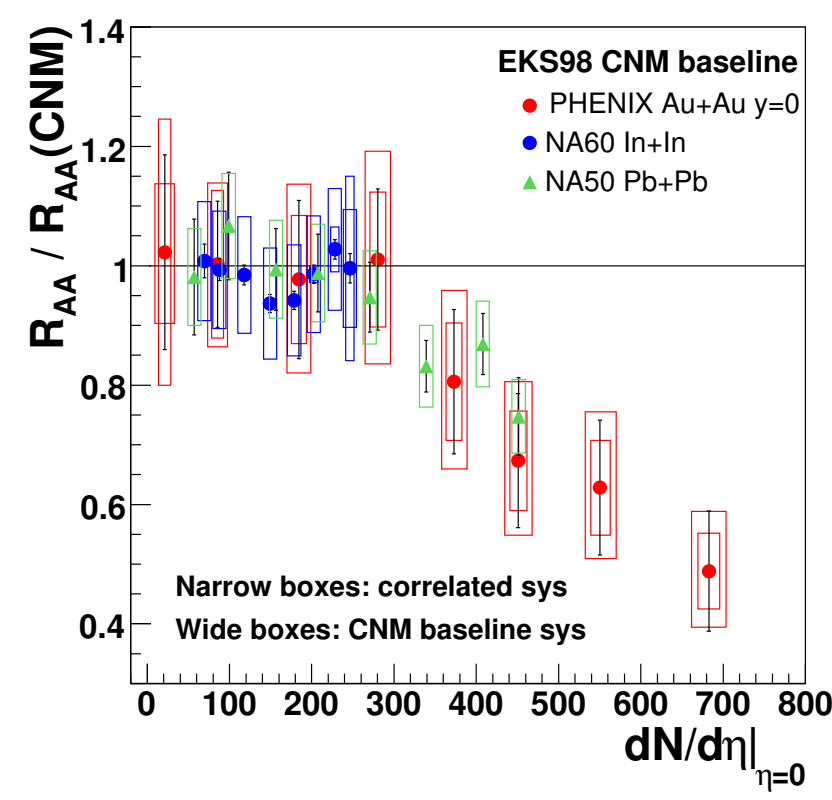

FIG. 89: Comparison of the anomalous suppression at the SPS and RHIC as a function of $d N_{\mathrm{ch}} / d \eta$ at $\eta=0$

may be related to the final state rapidity, $y$, by

$$
y=-\frac{1}{2} \ln \left(\frac{2 x \gamma m_{p}}{m_{V}}\right),
$$

where $\gamma$ is the Lorentz boost of the nuclear beam and $m_{p}$ is the proton mass. The higher energies available at the LHC allow studies at much lower $x$ than previously available, possibly down to $10^{-6}[988,990]$.

\section{Photoproduction cross sections}

The cross section for vector meson production may be calculated by integrating over photon momentum $k$ (equivalent to integrating over rapidity $y$ ):

$$
\sigma(A A \rightarrow A A V)=2 \int d k \frac{d N_{\gamma}}{d k} \sigma(\gamma A \rightarrow V A),
$$

where $d N_{\gamma} / d k$ is the photon flux, determined from the Weizsacker-Williams method, and $\sigma(\gamma A \rightarrow V A)$ is the photoproduction cross section. This cross section may be extrapolated from HERA data. A Glauber calculation is used to determine the cross sections for nuclear targets. Two Glauber calculations of $J / \psi$ and $\Upsilon$ photoproduction are available [704, 712, 713, 991, 992]; a third uses a color-glass condensate/saturation approach to describe the nuclear target [706].

The Glauber calculations successfully predict the rapidity distribution and cross section for $\rho^{0}$ photoproduction in $\mathrm{Au}+\mathrm{Au}$ collisions [993, 994], while the saturation calculation predicts a somewhat higher cross section. Calculations have also provided a reasonable estimate of the cross sections of excited meson production, such as 


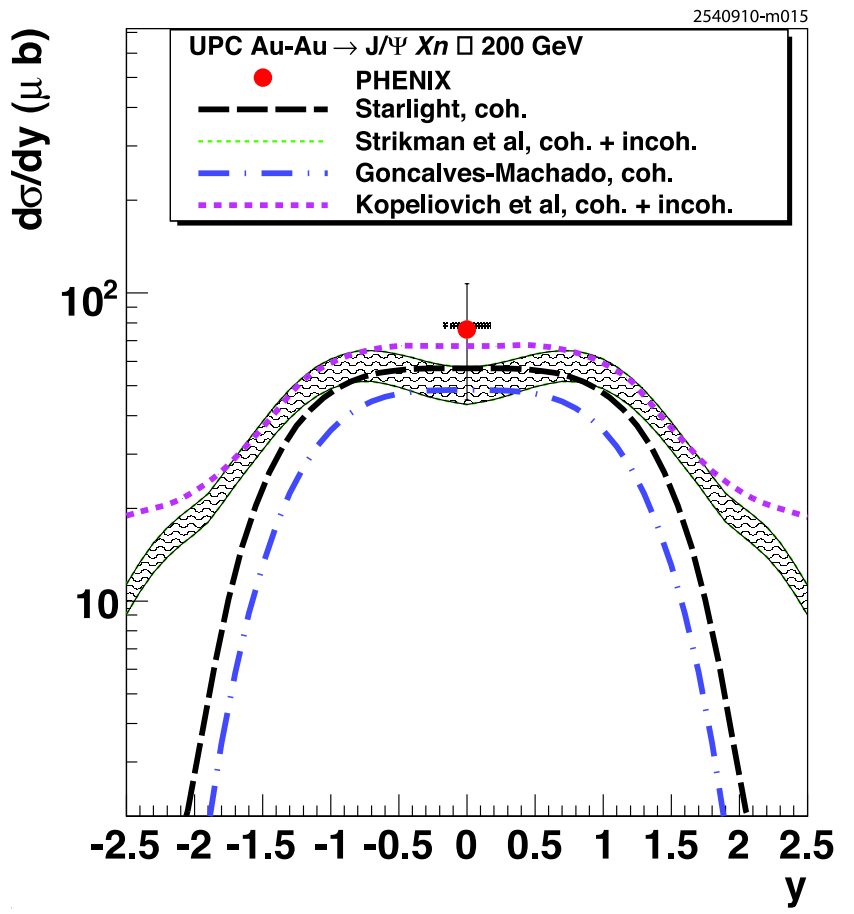

FIG. 90: The rapidity distribution, $d \sigma / d y$, of $J / \psi$ photoproduction measured by PHENIX, compared with three calculations. Coherent and incoherent predictions are summed. Coherent production dominates until $|y|>2$, where the coherent cross section is kinematically suppressed and incoherent production becomes important. From [974] with kind permission, copyright (2009) Elsevier

$\rho^{*}$ states [995-997]. The Tevatron $J / \psi$ and $\psi(2 S)$ cross sections are compatible with expectations [701]. The $J / \psi$ photoproduction cross section in $\mathrm{Au}+\mathrm{Au}$ collsions is sensitive to nuclear shadowing. The uncertainty of the PHENIX [974] measurement is still large, but, as Fig. 90 shows, the central point indicates that shadowing is not large. (At RHIC, midrapidity $J / \psi$ photoproduction corresponds to $x \approx 0.015$.)

\section{Transverse momentum spectra}

The $p_{T}$ spectrum of quarkonium photoproduction is the sum of the photon and Pomeron $p_{T}$-dependent contributions. Since the photon $p_{T}$ is small, the spectrum is dominated by the momentum transfer from the target nucleus. In $p p$ collisions, the typical momentum scale is $\sim 300 \mathrm{MeV}$, set by the size of the nucleon, while for heavy-ion collisions, the momentum scale is $\sim \hbar c / R_{A}$, where $R_{A}$ is the nuclear radius.

Photoproduction has a unique feature [998]: either nucleus can emit the photon while the other serves as the target. Because the two possibities are indistinguishable, their amplitudes add. In $p p$ and $A A$ collisions, the possibilities are related by a parity transformation. Since vector mesons have negative parity, the two amplitude subtract, leading to a net amplitude $A \approx A_{1}-A_{2} \exp \left(i p_{T} \cdot b\right)$

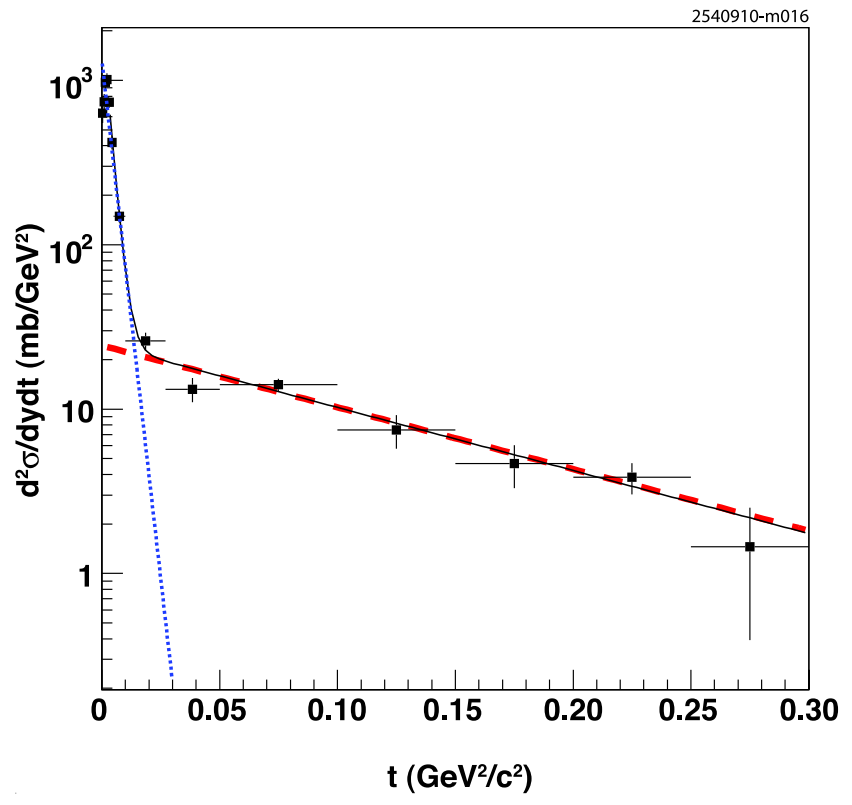

FIG. 91: The $t=p_{T}^{2}$ spectrum for $\rho^{0}$ photoproduction observed by STAR in $200 \mathrm{GeV} \mathrm{Au}+\mathrm{Au}$ collsions, averaged over $|y|<1$. The data are shown by points with error bars, and the solid curve is a fit of the data to a sum of two components, each exponential in $t$, representing that is coherent (dashed curve) at low $t$ and incoherent (dotted) at high $t$. Adapted from [993] with kind permission, copyright (2008) The American Physical Society

where $b$ is the impact parameter. The two amplitudes, $A_{1}$ and $A_{2}$, are equal at midrapidity, but may differ for $y \neq 0$ because the photon energies differ, depending on which proton or nucleus emits the photon. The exponential is a propagator from one nucleus to the other. The cross section is suppressed for $p_{T}<\langle b\rangle$ with a suppression factor proportional to $p_{T}^{2}$. Such suppression has been observed by STAR [999].

The bulk of the cross section from a nuclear target is due to coherent production since the virtual $q \bar{q}$ pair interacts in-phase with the entire nucleus. The $p_{T}$ transfer from the nucleus is small with a $p_{T}$ scale on the order of a few times $\hbar c / R_{A}$. The cross section for coherent photoproduction scales as $Z^{2}$ (from the photon flux) times $A^{\delta}$, where $4 / 3<\delta<2$. Here, $\delta=2$ corresponds to small interaction probabilities, as expected for heavy quarkonia. Larger interaction probabilities lead to smaller values of $\delta$. Studies of $\rho^{0}$ photoproduction at RHIC suggest $\delta \approx 5 / 3$.

At larger $p_{T}$, the $q \bar{q}$ pair interactions are out of phase so that the pair effectively interacts with a single nucleon. This contribution thus gives a harder slope in momentum transfer, $t$, corresponding to the size of a single nucleon, as can be seen in the STAR data in Fig. 91. At these higher $p_{T}$, the struck nucleon may be ejected from the nucleus, resulting in nuclear dissociation, making it possible to probe the dynamics of hard Pomerons [996, 997]. 
Expected at higher $p_{T}$ are additional components which probe the nucleon substructure. In this regime, the cross sections become sensitive to the quark distributions [1000]. Because of the higher momentum transfer from the target, incoherent interactions contribute at larger rapidities than coherent interactions, explaining the 'wings' at large $|y|$ in Fig. 90.

\section{Plans for the $L H C$}

ALICE, CMS, and ATLAS are all planning to study quarkonium photoproduction [988]. These events have a very clean topology: two nearly back-to-back electrons or muons, with almost nothing else in the detector. At RHIC, STAR and PHENIX found that such an analysis is relatively straightforward. The most difficult part of the study is devising a trigger to select these events. However, the LHC experiments will benefit from vastly more sophisticated triggers than are available at RHIC. Indeed CMS, ATLAS, and the ALICE forward muon spectrometer have triggers primitives that can be employed for this purpose. CMS, in particular, may be able to separately measure $\Upsilon(1 S), \Upsilon(2 S)$ and $\Upsilon(3 S)$ photoproduction.

The LHC energy and luminosity are sufficient for copious $J / \psi$ and significant $\psi(2 S)$ and $\Upsilon$ signals. The greatest physics interest may be in probing the gluon distributions, and, in particular, measuring nuclear shadowing. At midrapidity, quarkonium production probes $x$ values between $2 \times 10^{-4}(J / \psi$ in $p p$ collisions $)$ and $1.7 \times 10^{-3}$ $(\Upsilon$ in $\mathrm{Pb}+\mathrm{Pb}$ collisions). Away from midrapidity it is possible to probe $x$ values as low as $10^{-6}$ [988].

In the case of $A A$ collisions, since the photon can be emiitted from either nucleus, ambiguities arise because the photon energies, and hence the $x$ values probed, are different for the two possibilities. We mention two possible ways to resolve this two-fold ambiguity. Conceptually, the easiest is to study $p A$ collisions (or $d A$ at RHIC). Here, the ion is usually the photon emitter. In addition, it is possible to employ the difference in the $p_{T}$ spectra for photons scattering on protons and ions to separate the two possibilities. Unfortunately, $p A$ runs at the LHC are some years off. A second possiblity is to use bootstrapping, usually by comparing results at different beam energies. At each beam energy, the midrapidity cross section can be unambiguously determined, giving the cross section at a specific photon energy. At a different beam energy, the same photon energy corresponds to a different rapidity. By measuring the cross section at this rapidity and subtracting the known cross section determined previously, one obtains the cross section at the new photon energy. Unfortunately, the uncertainties add each time the cross sections are subtracted, increasing the relative error. A similar procedure may also employed by using data taken under different running conditions, such as exclusive $J / \psi$ production relative to $J / \psi$ production accompanied by mutual Coulomb excitation. At a given rapidity, these two processes contribute differently to the cross section, depending on the direction.

The LHC measurements allow for a relatively clean measurement of nuclear shadowing by taking the ratio of the $A A$ and $p p$ cross sections. In this approach, many of the theoretical and experimental uncertainties cancel in the ratio, resulting in a relatively clean determination. Additional $p A$ data would help this study by allowing cross checks between $p A$ and $p p$ interactions as well as between $A A$ and $p A$ interactions. Another possibility, for $p p$ collisions, is to use Roman pots or other small-angle detectors to tag the outgoing protons [1001]. The proton that emitted the photon will usually have lower $p_{T}$. Of course, some of these techniques are also applicable at RHIC, where the experiments are collecting large data sets with improved triggers and particle identification.

Such measurements of the nuclear gluon distributions will be important for understanding the properties of cold nuclear matter, which, in turn, clarifies the interpretation of the quarkonium signals in central heavy-ion collisions.

\section{EXPERIMENTAL OUTLOOK ${ }^{21}$}

Moving beyond the present status of heavy quarkonium physics described in the previous sections poses major challenges to the next generation of accelerators and experiments. In this section the future "players" in the field will be described with special emphasis on the potential to resolve the important open questions. Here we will present the rationale for and status of the newer facilities and experiments, from those already running (BESIII at BEPCII; ALICE, ATLAS, CMS, and $\mathrm{LHCb}$ at the LHC) to those under construction or only planned ( $\bar{P} A N D A$ and CBM at FAIR, SuperB and taucharm factories, lepton-hadron colliders, and high-energy linear $e^{+} e^{-}$colliders).

\subsection{BESIII}

For BESIII, the future is now. The Beijing ElectronPositron Collider (BEPC) and the Beijing Spectrometer (BES) operated in the tau-charm center-of-mass energy region between 2 and $5 \mathrm{GeV}$ from 1990 until 2003. Now, BEPC has been upgraded to a two-ring collider (BEPCII), and a brand new detector (BESIII) has been constructed. Commissioning of the upgraded accelerator and new detector began in spring 2008, and the first event was obtained on July 20, 2008. Approximately $13 \times 10^{6}$ $\psi(2 S)$ events were accumulated in fall 2008 , which provided data for studies of the new detector and for calibra-

21 Contributing authors: S. Eidelman ${ }^{\dagger}$, P. Robbe ${ }^{\dagger}$, A. Andronic, D. Bettoni, J. Brodzicka, G. E. Bruno, A. Caldwell, J. Catmore, E. Chudakov, P. Crochet, P. Faccioli, A. D. Frawley, C. Hanhart, F. A. Harris, D. M. Kaplan, H. Kowalski, E. Levichev, V. Lombardo, C. Lourenço, M. Negrini, K. Peters, W. Qian, E. Scomparin, P. Senger, F. Simon, S. Stracka, Y. Sumino, C. Weiss, H. K. Wöhri, and C.-Z. Yuan 
tion. In spring 2009 after running for about one month, $106 \times 10^{6} \psi(2 S)$ events were obtained, and in summer 2009 after running for six weeks, about $226 \times 10^{6} \mathrm{~J} / \psi$ events were accumulated. These are the world's largest such data sets and are approximately four times larger than the CLEO-c $\psi(2 S)$ sample and the BESII $J / \psi$ sample, respectively. The new data will allow more detailed studies of detector performance, and offers many physics opportunities.

The peak design luminosity of BEPCII is $10^{33} \mathrm{~cm}^{-2} \mathrm{~s}^{-1}$ $\left(1 \mathrm{nb}^{-1} \mathrm{~s}^{-1}\right)$ at a beam energy of $1.89 \mathrm{GeV}$, an improvement of a factor of 100 with respect to the BEPC. It will operate at a center-of-mass energy between 2 and 4.6 GeV, which allows production of almost all known charmonium and charmonium-like states. The detector performance is also greatly improved compared to BESII. BESIII [1002] is a new, general-purpose detector. It features a beryllium beam pipe; a small-cell, helium-based drift chamber (MDC); a Time-of-Flight (TOF) system; a $\mathrm{CsI}(\mathrm{Tl})$ electromagnetic calorimeter; a $1 \mathrm{~T}$ superconducting solenoidal magnet; and a muon identifier using the magnet yoke interleaved with Resistive Plate Chambers.

Running at design luminosity, BESIII will be able to accumulate $10 \times 10^{9} \mathrm{~J} / \psi$ events or $3 \times 10^{9} \psi(2 \mathrm{~S})$ events in one year's running. It will take around $20 \mathrm{fb}^{-1}$ of data each at $3.77 \mathrm{GeV}$ and $4.17 \mathrm{GeV}$ for charm physics. There is also the possibility of a high-statistics fine scan between 2 and $4.6 \mathrm{GeV}$, allowing the direct study of states with $J^{P C}=1^{--}$. States with even charge parity may be studied using radiative decays of high mass excited $\psi$ states, such as $\psi(2 S), \psi(3770), \psi(4040)$, $\psi(4160)$, and $\psi(4415)$. All these data samples allow detailed studies of charmonium physics, including the spectroscopy of conventional charmonium (see Sects. 2.1-2.2) and charmonium-like (see Sect. 2.3) states, charmonium transitions (see Sects. 3.1 and 3.3), and charmonium decays (see Sects. 3.2 and 3.4). Charmonium hadronic decay dynamics are especially interesting because of the $\rho \pi$ puzzle (see Sect. 3.4.1). The new datasets should also enable a better understanding of the physics of the strong interaction in the transition region between perturbative and nonperturbative QCD.

\subsubsection{Spin singlets: $h_{c}(1 P), \eta_{c}(1 S, 2 S)$}

Below open charm threshold there are three spinsinglet states, the $S$-wave spin-singlet, $\eta_{c}(1 S)$, its radially excited state, $\eta_{c}(2 S)$, and the $P$-wave spin-singlet, $h_{c}(1 P)$. All these may be reached from $\psi(2 S)$ transitions. The $\eta_{c}(1 S)$ can also be studied in $J / \psi$ radiative decays. Their properties are less well measured because of their low production rates in previous $e^{+} e^{-}$experiments.

BESIII will measure the $h_{c}(1 P)$ mass, width, spinparity, production rate via $\psi(2 S) \rightarrow \pi^{0} h_{c}(1 P)$, and its $E 1$ transition rate $h_{c}(1 P) \rightarrow \gamma \eta_{c}(1 S)$ (see Sects. 2.2.1 and 3.1.3). BESIII will also search for its hadronic de- cays (see Sect. 3.4.2), which are expected to be about $50 \%$ of the total decay width, and search for other transitions.

Extraction of the $\eta_{c}(1 S)$ mass and width from radiative $J / \psi$ or $\psi(2 S)$ radiative transitions is not straightforward due to the unexpected lineshape observed in such transitions (see Sect. 3.1.2). With theoretical guidance and more data, these transitions may become competitive with other $\eta_{c}(1 S)$ production sources in determination of its mass and width. In addition to increased statistics, more decay modes will be found and their branching fractions measured.

Despite the passage of eight years since the observation of the $\eta_{c}(2 S)$ (see Sect. 2.2.2), the discovery mode $\eta_{c}(2 S) \rightarrow K \bar{K} \pi$ remained the only mode observed until the summer of 2010, at which time Belle [70] reported preliminary observation of several hadronic $\eta_{c}(2 S)$ decay modes in two-photon production of $\eta_{c}(2 S)$. BESIII will search for $\eta_{c}(2 S)$ in $\psi(2 S)$ radiative decays. With much less data, CLEO-c sought 11 exclusive hadronic decay modes in radiative transitions but saw none (see Sect. 3.1.4), even in the discovery mode and in the three new modes found by Belle. With more data, BESIII will have a better chance with exclusive decay modes. However, it will be a challenge to isolate the low-energy $(\simeq 50 \mathrm{MeV})$ radiative photon due to the many background photon candidates, both genuine and fake. Observation of a signal in the inclusive photon spectrum is even more challenging, but is the only way to get the absolute $\psi(2 S) \rightarrow \gamma \eta_{c}(2 S)$ transition rate. This task will require a good understanding of both backgrounds and the electromagnetic calorimeter performance. If the $\eta_{c}(2 S)$ is found in radiative $\psi(2 S)$ decays, the photon energy lineshape can then be studied and compared to that of the $\eta_{c}(1 S)$ (see Sect. 3.1.2).

\subsubsection{Vectors above $\psi(3770): \psi$ 's and $Y$ 's}

There are many structures between $3.9 \mathrm{GeV}$ and 4.7 GeV, including the excited $\psi$ (see Sect. 2.1.1) and the $Y$ (see Sect. 2.3.2) states [33, 82, 1003]. By doing a fine scan in this energy range, BESIII may study the inclusive cross section, as well as the cross sections of many exclusive modes, such as $D \bar{D}, D^{*} \bar{D}+$ c.c., $D^{*} \bar{D}^{*}+$ c.c., $D \bar{D} \pi$, etc. (see Sect. 2.1.1). This will help in understanding the structures, for instance, whether they are really resonances, due to coupled-channel effects, finalstate interactions, or even threshold effects. BESIII will also measure the hadronic and radiative transitions of these excited $\psi$ and the $Y$ states. Other $X Y Z$ particles can also be sought in these transitions.

\subsubsection{Hadronic decays}

As discussed in Sect. 3.4.1, the $12 \%$ rule is expected to hold for exclusive and inclusive decays, but is violated 
by many such modes, including the namesake mode of the $\rho \pi$ puzzle. A plethora of experimental results exists (see Sect. 3.4.1 and references therein). With much larger datasets, a variety of theoretical explanations can be tested by BESIII at higher accuracy [571]. Moreover, studies should be made not only of ratios of $\psi(2 S)$ to $J / \psi$ decays, but also of other ratios such as those between $\eta_{c}(2 S)$ and $\eta_{c}(1 S)$ [1004], between $\psi(3770)$ and $J / \psi[565]$, and other ratios between different resonances for the same channel or between different channels from the same resonance [1005] (e.g., $\gamma \eta$ and $\gamma \eta^{\prime}$, as discussed in Sect. 3.2.4). All such studies are important to our understanding of charmonium decays.

BESIII also has an opportunity to measure the direct photon spectrum in both $J / \psi$ and $\psi(2 S)$ decays and values of $R_{\gamma}$ (see Eq. (22)) for both resonances (see discussion in Sects. 2.8.1 and 3.2.2 and in [1002]), building on the work of CLEO for $J / \psi[235]$ and $\psi(2 S)$ [236].

\subsubsection{Excited $C$-even charmonium states}

Above open charm threshold, there are still many $C$-even charmonium states not yet observed, especially the excited $P$-wave spin-triplet and the $S$-wave spinsinglet [31, 1006]. In principle, these states can be produced in the E1 or M1 transitions from excited $\psi$ states. As BESIII will accumulate much data at $4.17 \mathrm{GeV}$ for the study of charm physics, the sample can be used for such a search.

\subsubsection{Decays of $\chi_{c J}(1 P)$}

Approximately $30 \%$ of $\psi(2 S)$ events decay radiatively to $\chi_{c J}$, which decay hadronically via two or more gluons (see Sect. 3.4.3). These events and radiative $J / \psi$ decays are thought to be important processes for the production of glueball, hybrid, and other non- $q \bar{q}$ states. BESIII will study these processes and also search for charmonium rare decays. The decay $\chi_{c 1} \rightarrow \eta \pi \pi$ is a golden channel for the study of states with exotic quantum numbers $I^{G}\left(J^{P C}\right)=1^{-}\left(1^{-+}\right)$, that is, the $\pi_{1}$ states [18], since these states, can be produced in $\chi_{c 1} S$-wave decays. A detailed partial wave analysis with a large $\chi_{c 1}$ sample will shed light on these exotic states.

\subsubsection{Prospects}

The present and future large BESIII data sets and excellent new detector will allow extensive studies of charmonium states and their decays.

\subsection{ALICE}

ALICE [1007] is the experiment dedicated to the study of nucleus-nucleus collisions at the Large Hadron Collider (LHC). The study of heavy quarkonium production in nuclear collisions is one of the most important sources of information on the characteristics of the hadronic/partonic medium. (For a discussion of quarkonium physics in this medium, see Sect. 5.) ALICE will study $\mathrm{Pb}+\mathrm{Pb}$ collisions at top LHC $\mathrm{Pb}$ energy $\left(\sqrt{s_{N N}}=\right.$ $5.5 \mathrm{TeV}$ ), at a nominal luminosity $L=5 \times 10^{26} \mathrm{~cm}^{-2} \mathrm{~s}^{-1}$.

In the ALICE physics program [1008, 1009], the study of $p p$ collisions is also essential, in order to provide reference data for the interpretation of nuclear collision results. In addition, many aspects of genuine $p p$ physics can be addressed. The $p p$ luminosity in ALICE will be restricted so as to not exceed $L=3 \times 10^{30} \mathrm{~cm}^{-2} \mathrm{~s}^{-1}$. Despite this luminosity limitation, most physics topics related to charmonium and bottomonium production remain accessible.

Heavy quarkonia will be measured in the central barrel, covering the pseudorapidity range $-0.9<\eta<0.9$, and in the forward muon arm, which has a coverage $2.5<\eta<4$. In the central barrel, heavy quarkonia will be detected through the $e^{+} e^{-}$decay. ALICE can push its transverse momentum $\left(p_{\mathrm{T}}\right)$ reach for charmonium down to $p_{\mathrm{T}} \sim 0$. Electron identification is performed jointly in the TPC through the $d E / d x$ measurement and in the Transition Radiation Detector (TRD). In the forward region, quarkonia will be studied via their decay into muon pairs. Muons with momenta larger than $4 \mathrm{GeV} / c$ are detected by means of a spectrometer which includes a $3 \mathrm{Tm}$ dipole magnet, a front absorber, a muon filter, tracking (Cathode Pad Chambers, CPC) and triggering (Resistive Plate Chambers, RPC) devices.

In the following sections we will review the ALICE physics capabilities for heavy-quarkonium measurements at the top LHC energy within the running conditions specified above. A short overview of the measurements that could be performed in the first high-energy run of the LHC will also be presented. For the ALICE physics run in 2010, the forward muon spectrometer and most of the central barrel detectors have been installed and commissioned, including seven TRD supermodules (out of 18).

In the central barrel, the geometrical acceptance for $J / \psi$ produced at rapidity $|y|<0.9$ (with no $p_{\mathrm{T}}$ cut on either the $J / \psi$ or the decay electrons) is $29 \%$ for the complete TRD setup. The electron reconstruction and identification efficiency in the TRD is between 80 and $90 \%$ for $p_{\mathrm{T}}>0.5 \mathrm{GeV} / c$, while the probability of misidentifying a pion as an electron is $\sim 1 \%$. Below a few $\mathrm{GeV} / c$, particle identification in the TPC [1010] contributes substantially to hadron rejection, with an overall TPC+TRD electron reconstruction efficiency of $\sim 75 \%$.

The acceptance of the forward spectrometer, relative to the rapidity range $2.5<y<4$, is $\sim 35 \%$ for the $J / \psi$. Since most of the background is due to low transverse 
momentum muons, a $p_{\mathrm{T}}$ cut is applied to each muon at the trigger level. With a $1 \mathrm{GeV} / c p_{\mathrm{T}}$ cut, there is a $\sim 20 \%$ reduction of the $J / \psi$ acceptance. The combined efficiency for $J / \psi$ detection in the forward spectrometer acceptance, taking into account the efficiency of tracking and triggering detectors, is expected to be about $70 \%$.

\subsection{1. $\mathrm{J} / \psi$ production from $\mathrm{Pb}+\mathrm{Pb}$ collisions}

Heavy quarkonium states probe the medium created in heavy-ion collisions. Color screening in a deconfined state is expected to suppress the charmonium and bottomonium yields. In addition, at the LHC, a large multiplicity of heavy quarks (in particular, charm) may lead to significant regeneration of bound states in the dense medium during the hadronization phase. ALICE will investigate these topics through a study of the yields and differential distributions of various quarkonium states, performed as a function of the centrality of the collision.

A simulation has been performed [1011] for $J / \psi$ production in the forward muon arm, using as an input a Color Evaporation Model (CEM) calculation, based on the MRST HO set of Parton Distribution Functions (PDF), with $m_{\mathrm{c}}=1.2 \mathrm{GeV}$ and $\mu=2 m_{\mathrm{c}}$ [1012]. (For a discussion of the CEM, see Sect. 4.1.3.) With such a choice of parameters, the total $p p J / \psi$ cross section at $\sqrt{s_{N N}}=5.5 \mathrm{TeV}$, including the feeddown from higher resonances, amounts to $31 \mu \mathrm{b}$. The $p p$ cross section has been scaled to $\mathrm{Pb}+\mathrm{Pb}$ assuming binary collision scaling and taking into account nuclear shadowing through the EKS98 [877] parametrization. The differential $p_{\mathrm{T}}$ and $y$ shapes have been obtained via an extrapolation of the CDF measurements and via CEM predictions, respectively, and assuming that the $J / \psi$ are produced unpolarized. The hadronic background was simulated using a parametrized HIJING generator tuned to $d N_{\mathrm{ch}} / d y=$ 8000 for central events at midrapidity (such a high value, 3-4 times that realistically expected, represents a rather extreme evaluation of this source). Open heavy quark production was simulated using PYTHIA, tuned to reproduce the single particle results of NLO pQCD calculations.

At nominal luminosity, the expected $J / \psi \mathrm{Pb}+\mathrm{Pb}$ statistics for a $10^{6} \mathrm{~s}$ run, corresponding to the yearly running time with the $\mathrm{Pb}$ beam, are of the order of $7 \times$ $10^{5}$ events. The mass resolution will be $\sim 70 \mathrm{MeV}[1008$, 1009]. A simulation of the various background sources to the muon pair invariant mass spectrum in the $J / \psi$ region (including combinatorial $\pi$ and $K$ decays, as well as semileptonic decays of open heavy flavors) shows that the signal-to-background ratio, $S / B$, ranges from 0.13 to $\approx 7$ when moving from central to peripheral collisions. With such statistics and $S / B$ values it will be possible to study the proposed theoretical scenarios for the modification of the $J / \psi$ yield in the hot medium.

The transverse momentum distributions can be addressed with reasonable statistics even for the relatively less populated peripheral $\mathrm{Pb}+\mathrm{Pb}$ collisions. In particular, for collisions with an impact parameter $b>12 \mathrm{fm}$, we expect having more than 1000 events with $p_{\mathrm{T}}>8 \mathrm{GeV} / c$.

Finally, a study of the $J / \psi$ polarization will be performed by measuring the angular distribution of the decay products. With the expected statistics, the polarization parameter $\lambda$ extracted from the fit $d \sigma / d \cos \theta=$ $\sigma_{0}\left(1+\lambda \cos ^{2} \theta\right)$ can be measured, defining five impact parameter bins, with a statistical error $<0.05$ for each bin.

Another simulation of $J / \psi$ production at central rapidity has been carried out using as input the rates obtained from the CEM calculation described above. For high-mass electron pairs, the main background sources are misidentified pions and electrons from semileptonic $B$ and $D$ decays. The value $d N_{\mathrm{ch}} / d y=3000$ for central events at $y=0$ was used for the simulation of the hadronic background. PYTHIA was used for open heavy quark production, with the same tuning used for the forward rapidity simulations.

For $\mathrm{Pb}+\mathrm{Pb}$, the expected $J / \psi$ statistics, measured for $10^{6} \mathrm{~s}$ running time at the nominal luminosity, are about $2 \times 10^{5}$ candidates from the $10^{8}$ collisions passing the $10 \%$ most central impact parameter criteria. The mass resolution will be $\sim 30 \mathrm{MeV}$ [1013]. The background under the $J / \psi$ peak, dominated by misidentified pions, is at a rather comfortable level $(S / B=1.2)$. As for the forward region, it will therefore be possible to test the proposed theoretical models.

The $S / B$ ratio is expected to increase as a function of $p_{\mathrm{T}}$, reaching a value of $\sim 5$ at $10 \mathrm{GeV} / c$. The expected statistics at that $p_{\mathrm{T}}$ are still a few hundred counts, implying that differential $J / \psi$ spectra can also be studied.

\subsection{2. $J / \psi$ production from $p p$ collisions}

Quarkonium hadroproduction is an issue which is not yet quantitatively understood theoretically. A study of $J / \psi$ production in $p p$ collisions at ALICE aims at a comprehensive measurement of interesting observables (production cross sections, $p_{\mathrm{T}}$ spectra, polarization) useful to test theory in a still unexplored energy regime. Furthermore, the forward rapidity measurement offers a possibility to access the gluon PDFs at very low $x\left(<10^{-5}\right)$.

In the forward muon arm, $J / \psi$ production at $\sqrt{s}=$ $14 \mathrm{TeV}$ has been simulated using the CEM, with parameters identical to those listed above for $\mathrm{Pb}+\mathrm{Pb}$ collisions. The $J / \psi$ total cross section turns out to be $53.9 \mu \mathrm{b}$, including the feeddown from higher-mass resonances. A typical data taking period of one year (assuming $10^{7} \mathrm{~s}$ running time) at $L=3 \times 10^{30} \mathrm{~cm}^{-2} \mathrm{~s}^{-1}$ gives an integrated luminosity of $30 \mathrm{pb}^{-1}$. The corresponding dimuon invariant mass spectrum, for opposite-sign pairs, is shown in Fig. 92.

The expected $J / \psi$ statistics are $\sim 2.8 \times 10^{6}$ events [1014]. The background under the $J / \psi$ peak is dominated by correlated decays of heavy flavors but is 
anyway expected to be quite small $(S / B=12)$. It will be possible to study the transverse momentum distribution of the $J / \psi$ with negligible statistical errors up to at least $p_{\mathrm{T}}=20 \mathrm{GeV} / c$. By studying the shape of the $J / \psi$ rapidity distribution in the region $2.5<y<4$ it will be possible to put strong constraints on the gluon PDFs and, in particular, to discriminate between the currently available extrapolations in the region around $x=10^{-5}$. With the expected statistics it will also be possible to carry out a detailed analysis of the $p_{\mathrm{T}}$ dependence of the $J / \psi$ polarization.

In a $p p$ run, a sample of a few thousand $J / \psi$ events is expected to be acquired in minimum bias collisions. With such statistics it will be possible to measure $d \sigma / d y$ at midrapidity. In order to improve these statistics, the implementation of a Level-1 trigger for electrons is foreseen. Assuming a conservative trigger efficiency of $10 \%$, one would get about $7 \times 10^{5} \mathrm{~J} / \psi$. Such a yield would open up the possibility of measuring differential spectra up to high $p_{\mathrm{T}}$ and polarization.

\subsection{3. $J / \psi$ production from $b$-hadron decays}

When measuring $J / \psi$ production at the LHC, a significant fraction of the measured yield comes from $b$-hadron decays. This $J / \psi$ source is a very interesting physics signal for the evaluation of the open-bottom production cross section, nicely complementing measurements performed via single leptons. It is also an important component to be disentangled when one wants to identify prompt $J / \psi$ production, as it is the case for studies of yield modifications in nuclear collisions.

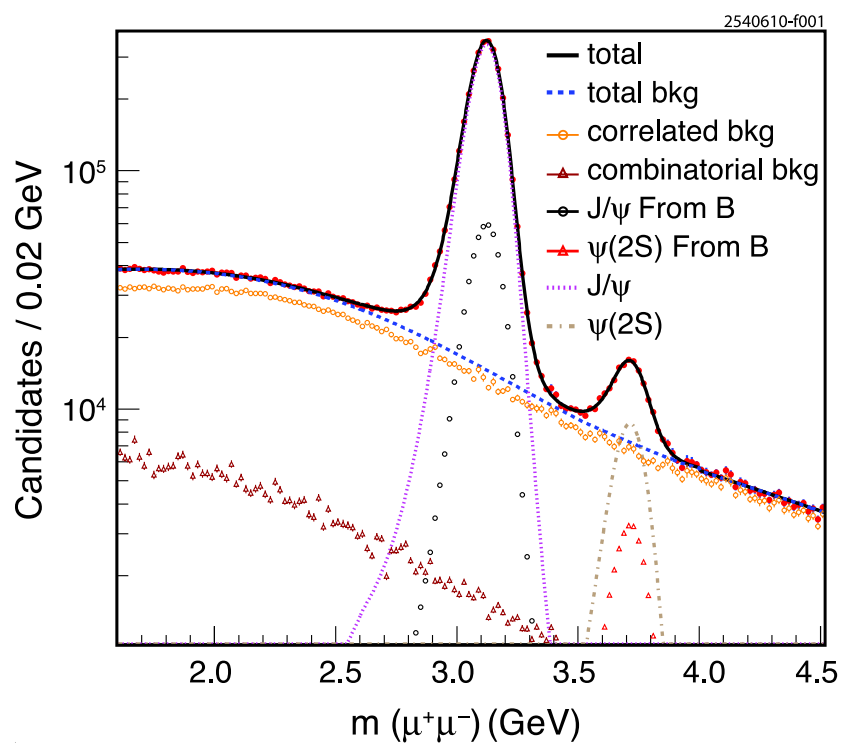

FIG. 92: Opposite-sign dimuon mass spectrum in $p p$ collisions at $\sqrt{s}=14 \mathrm{TeV}$ for a $10^{7} \mathrm{~s}$ running time at $L=$ $3 \times 10^{30} \mathrm{~cm}^{-2} \mathrm{~s}^{-1}$
At midrapidity, thanks to the excellent vertexing capabilities of ALICE, the secondary $b$-decay vertex can be separated. A good measure of the separation from the main vertex is $L_{x y}$, the signed projection of the $J / \psi$ flight distance, $\vec{L}$, on its transverse momentum, $\vec{p}_{T}$, defined as

$$
L_{x y} \equiv \frac{\vec{L} \times \vec{p}_{\mathrm{T}}}{p_{\mathrm{T}}} .
$$

To reduce the dependence on the $J / \psi$ transverse momentum distribution, the variable $x$ is used instead of $L_{x y}$,

$$
x \equiv L_{x y} \times \frac{m(J / \psi)}{p_{\mathrm{T}}},
$$

where $m(J / \psi)$ is the known $J / \psi$ mass. Studies based on Monte Carlo simulation have shown that the fractions of secondary $J / \psi$ as a function of $p_{\mathrm{T}}$ can be extracted by a likelihood fit to the dielectron invariant mass and the $x$ variable defined above with uncertainties smaller than $10 \%$. This approach will also provide a measurement of the open-bottom $p_{\mathrm{T}}$-differential cross section down to $p_{\mathrm{T}} \approx 0$.

The situation is more difficult at forward rapidity. Due to the presence of a thick hadron absorber in the path of the muons, the accuracy on the position of the $J / \psi$ production vertex is not sufficient. Work is in progress in order to evaluate the secondary $J / \psi$ yield starting from the study of events with 3 muons detected in the muon spectrometer in $p p$ collisions. Finally, the option of introducing a Si vertex tracker covering the $2.5<\eta<4$ rapidity domain is currently under study. It should be noted that open-bottom production at forward rapidity will be estimated from the study of the single- $\mu p_{\mathrm{T}}$ distributions and from the contribution to the dimuon continuum of correlated semileptonic decays of $b$-hadrons. These measurements will allow us to estimate the fraction of the $J / \psi$ yield coming from $b$ decays.

\subsubsection{Production of $\chi_{c J}(1 P)$ and $\psi(2 S)$}

It is well known that a significant fraction (up to $\approx$ $40 \%$ ) of the measured $J / \psi$ yield comes from $\chi_{c J}$ and $\psi(2 S)$ decays. An accurate measurement of the yield of these resonances is therefore an important ingredient in the interpretation of the $J / \psi$ production data. At the same time, these higher-mass resonances suffer from a much smaller feeddown contribution than the $J / \psi$ and may represent cleaner signals for theoretical calculations.

The dilepton yield from $\psi(2 S)$ is much smaller than that of $J / \psi$. At the nominal LHC energy and luminosity described above, one expects about $7.5 \times 10^{4}$ events in the forward muon arm for a standard $p p$ run, with $S / B \approx 0.6$ [1014]. In $\mathrm{Pb}+\mathrm{Pb}$ collisions, the situation is not so favorable, due to the much larger combinatorial background. The expected statistics are about $1.5 \times 10^{4}$ events, but with a $S / B$ ratio ranging from $18 \%$ to only $1 \%$ from peripheral to central collisions. The background 
levels at midrapidity are prohibitive for $\mathrm{Pb}+\mathrm{Pb}$ collisions; a measurement in $p p$ collisions also appears to be problematic.

Concerning $\chi_{c}$, a feasibility study has been performed on the detection of the radiative decay $\chi_{c} \rightarrow J / \psi \gamma$ at midrapidity in $p p$ collisions [1015]. The $J / \psi$ has been reconstructed via its $e^{+} e^{-}$decay, while the photon conversion has been reconstructed from opposite-sign tracks with opening angle $<0.1 \mathrm{rad}$ and mass $<0.175 \mathrm{GeV}$. The $\chi_{c 1}$ and $\chi_{c 2}$ states can be separated in the $\Delta m=$ $m\left(e^{+} e^{-} \gamma\right)-m\left(e^{+} e^{-}\right)$spectrum. The mean reconstruction efficiency is $0.9 \%$. As for $J / \psi$ production at midrapidity, triggering is crucial also for this signal. With a $10 \%$ trigger efficiency, several thousand events could be collected in a $p p$ run.

\subsection{5. $\Upsilon$ production}

In nucleus-nucleus collisions, the yield of $\Upsilon(1 S, 2 S, 3 S)$ states should exhibit various degree of suppression due to the screening of the color force in a Quark-Gluon Plasma. Results from $p p$ collisions will be essential as a normalization for $\mathrm{Pb}+\mathrm{Pb}$ results and extremely interesting in order to understand the related QCD topics (see Sect. 5).

In the forward rapidity region, where the muon-pair invariant-mass resolution is $\sim 100 \mathrm{MeV}$, the $\Upsilon$ states can be clearly separated. The expected yields are of the order of $7 \times 10^{3}$ events for the $\Upsilon(1 S)$ in $\mathrm{Pb}+\mathrm{Pb}$ collisions, and factors $\approx 4$ and $\approx 6.5$ smaller for the higher-mass resonances $\Upsilon(2 S)$ and $\Upsilon(3 S)$, respectively [1011]. The $S / B$ ratios will be more favorable than for the $J / \psi(\approx 1.7$ for the $\Upsilon(1 S)$ in central collisions). In $p p$ collisions, about $2.7 \times 10^{4} \Upsilon(1 S)$ events are expected for one run [1014]. These statistics will allow, in addition to the integrated cross section measurement, a study of $p_{\mathrm{T}}$ distributions and polarization.

At midrapidity, a possibility of measuring the $\Upsilon$ states is closely related to the implementation of a Level-1 trigger on electrons [1013]. Assuming a conservative 10\% trigger efficiency, about $7000 \Upsilon$ events could be collected in a $p p$ run. In a $\mathrm{Pb}+\mathrm{Pb}$ run, a significant $\Upsilon(1 S)$ sample (several thousand events) can be collected with a comfortable $S / B \approx 1$. The statistics for the higher-mass resonances depend crucially on the production mechanism. Assuming binary scaling, as for $\Upsilon(1 S)$, a measurement of $\Upsilon(2 S)$ looks very promising $(\approx 1000$ events with $S / B=0.35)$.

\subsubsection{First LHC high-energy running}

In 2010 the LHC has begun to deliver proton beams at $\sqrt{s}=7 \mathrm{TeV}$. Under the present running conditions, during 2010 it is expected that a few $10^{4} \mathrm{~J} / \psi \rightarrow \mu^{+} \mu^{-}$ will be collected in the forward spectrometer using a single muon trigger. With these statistics a measurement of the $p_{\mathrm{T}}$ distribution and a $p_{\mathrm{T}}$-integrated polarization estimate could be within reach. Several hundred $\psi(2 S)$ and $\Upsilon(1 S)$ events could be collected, enough for an estimate of the $p_{\mathrm{T}}$-integrated cross sections.

Assuming a sample of $10^{9}$ minimum bias events, the expected $J / \psi$ statistics in the central barrel are a few hundred events (due to the reduced coverage provided by the presently installed TRD supermodules). Employing the TRD trigger would enhance this sample significantly and would enable measurements of other charmonium states, as well as of the $\Upsilon$.

\subsection{ATLAS}

ATLAS $[1016,1017]$ is a general-purpose $4 \pi$ detector at the LHC. Although primarily designed for the discovery of physics beyond the Standard Model through the direct observation of new particles, indirect constraints through precise measurements of known phenomena are also an important avenue of activity. The quarkonium program of ATLAS falls into this category. Of particular importance to these studies are the tracking detector and muon spectrometer. The silicon pixels and strips close to the interaction point allow primary and secondary vertex reconstruction with good resolution. The vast muon spectrometer in the outer parts of the machine provides a flexible muon trigger scheme that can fire on pairs of lowmomentum $(4 \mathrm{GeV} / c)$ muons as well as efficient muon identification and reconstruction. Together these factors have allowed ATLAS to assemble a strong quarkonium physics program.

We review here the ATLAS capability relevant for prompt quarkonium production at the LHC, in particular the methods of separating promptly produced $J / \psi \rightarrow \mu^{+} \mu^{-}$and $\Upsilon \rightarrow \mu^{+} \mu^{-}$decays from the various backgrounds. The outlook for the first measurements at $7 \mathrm{TeV}$ is also discussed. All of the results shown here are taken from the Computing Services Commissioning [1018, p. 1083-1110] exercises on Monte Carlo carried out in 2008 .

\subsubsection{Trigger considerations}

A detailed account of the ATLAS trigger can be found in [1019], and the full details of the trigger scheme to be used in the ATLAS bottom and quarkonia program are available in [1018, p. 1044-1082]. The quarkonium program relies on two trigger methods in particular. The first requires the lowest-level trigger to fire on two overthreshold muons independently, forming two conical "Regions of Interest" (RoIs) around the muon candidate. Full track reconstruction on hits within the RoIs is then performed by higher-level trigger algorithms to confirm and refine the low-level signature. The second method requires only one muon at the lowest level; the RoI in this case encompasses a larger volume and the second 
muon is sought only in the higher-level algorithms. These methods allow thresholds in $p_{\mathrm{T}}$ down to $4 \mathrm{GeV} / c$. An alternative approach requires only one muon, with a higher $p_{\mathrm{T}}$ threshold of $10 \mathrm{GeV} / c$; in this case the other muon is sought offline.

Any determination of the quarkonia cross sections requires a detailed understanding of the trigger efficiencies. With around $10 \mathrm{pb}^{-1}$ of data it will be possible to measure the efficiency maps directly from the data, using the narrow $J / \psi$ resonance in the so-called "Tag and Probe" method [1018, p. 1069-1081]. With fewer data such maps will have to be made from Monte Carlo.

Figure 93 shows the density of the production cross section for $p p \rightarrow J / \psi \rightarrow \mu^{+} \mu^{-}$and $p p \rightarrow \Upsilon \rightarrow \mu^{+} \mu^{-}$as a function of the $p_{\mathrm{T}}$ of the two muons, with cut lines representing dimuon triggers of $(4,4)$ and $(6,4) \mathrm{GeV} / c$ and the single-muon trigger threshold of $10 \mathrm{GeV} / c$. It can be seen immediately that the situation for the two states is very different. In the case of $J / \psi$, most of the decays produce muons with $p_{\mathrm{T}}$ well below the $(4,4) \mathrm{GeV} / c$ threshold, which is as low as the ATLAS muon triggers can go. Furthermore, it is clear that increasing the thresholds to $(6,4) \mathrm{GeV} / c$ does not lose many additional events. On the other hand, the $\Upsilon$, which is three times as massive as the $J / \psi$, decays into muons with significantly higher $p_{\mathrm{T}}$. In this case the difference between thresholds of $(4,4)$ and $(6,4) \mathrm{GeV} / c$ is critical, with the lower cut capturing many more events and resulting in an order-of-magnitude increase in the accessible cross section. See Table 46 for expected cross sections from a variety of quarkonium states for different trigger configurations. Although excited $\Upsilon$ states are included in the table, it is unlikely that ATLAS will have good enough mass resolution to be able to separate them. It should also be noted that the muon trigger configuration used early-on will have a nonzero efficiency below the $(4,4) \mathrm{GeV} / c$ threshold, which will allow ATLAS to collect more events than suggested by Table 46, which assumes hard cuts. Finally, the opening angle between the muons in $\Upsilon$ decay is typically much larger than for $J / \psi$, which presents a difficulty for RoIguided triggers because the RoI is generally too narrow.

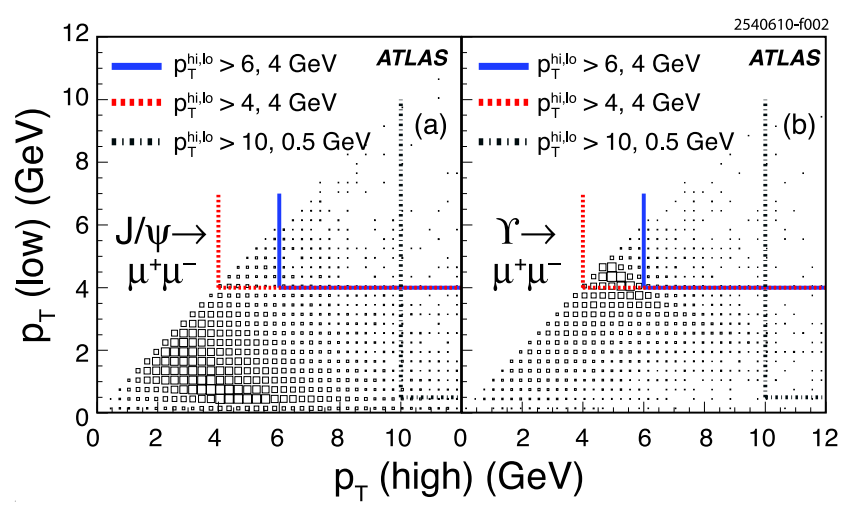

FIG. 93: Density of production cross section for $J / \psi$ and $\Upsilon$
TABLE 46: Predicted cross sections for various prompt quarkonia production and decay into dimuons for three trigger scenarios

\begin{tabular}{ccccc}
\hline \hline State & \multicolumn{4}{c}{ Cross section, nb } \\
& $\mu 4 \mu 4$ & $\mu 6 \mu 4$ & $\mu 10$ & $\mu 6 \mu 4 \cap \mu 10$ \\
\hline$J / \psi$ & 28 & 23 & 23 & 5 \\
$\psi(2 S)$ & 1.0 & 0.8 & 0.8 & 0.2 \\
$\Upsilon(1 S)$ & 48 & 5.2 & 2.8 & 0.8 \\
$\Upsilon(2 S)$ & 16 & 1.7 & 0.9 & 0.3 \\
$\Upsilon(3 S)$ & 9.0 & 1.0 & 0.6 & 0.2 \\
\hline \hline
\end{tabular}

Physics studies of $\Upsilon$ will therefore benefit from the "full scan" dimuon triggers, which allow the whole tracking volume to be accessed by the higher-level trigger algorithms rather than just hits in the RoI. Full-scan triggers are CPU-intensive and will only be available at low luminosity.

The angle $\cos \theta^{*}$, used in quarkonium spin-alignment analyses, is defined (by convention) as the angle in the quarkonium rest frame between the positive muon from the quarkonium decay and the flight direction of the quarkonium itself in the laboratory frame. The distribution of this angle may depend on the relative contributions of the different quarkonium production mechanisms that are not fully understood. Different angular distributions can have different trigger acceptances: until the spin alignment is properly understood, a proper determination of the trigger acceptance will not be possible. For quarkonium decays in which the two muons have roughly equal $p_{\mathrm{T}}, \cos \theta^{*} \approx 0$; such decays will have a high chance of being accepted by the trigger. Conversely, quarkonia decays with $\left|\cos \theta^{*}\right| \approx 1$ will have muons with very different $p_{\mathrm{T}}$, and as the lower $p_{\mathrm{T}}$ muon is likely to fall below the trigger threshold, such events have a greater chance of being rejected. Figure 94 shows the $\cos \theta^{*}$ distributions for $J / \psi$ and $\Upsilon$ after trigger cuts of $p_{\mathrm{T}}>(6,4) \mathrm{GeV}$ (solid line) and a single muon trigger cut of $p_{\mathrm{T}}>10 \mathrm{GeV}$ (dashed line). The samples were generated with zero spin alignment, so without trigger selection the $\cos \theta^{*}$ distribution would be flat across the range -1 to +1 . The figures show, first, that a narrow acceptance in $\cos \theta^{*}$ would impair the spin-alignment measurements, and second, that the single-muon trigger has much better acceptance at the extreme ends of the $\cos \theta^{*}$ distribution, since it has a much better chance of picking up events with one low- $p_{\mathrm{T}}$ and one high- $p_{\mathrm{T}}$ muon. At low luminosity such a trigger will have an acceptable rate, and, used in conjunction with the dimuon triggers, will provide excellent coverage across the whole $\cos \theta^{*}$ range. 


\subsubsection{Event selection}

Events passing the triggers are processed offline. Oppositely-charged pairs of tracks identified as muons by the offline reconstruction are fit to a common vertex, after which the invariant mass is calculated from the refitted track parameters. Candidates whose refitted mass is within $300 \mathrm{MeV}(1 \mathrm{GeV})$ of the $J / \psi(\Upsilon)$ table mass of 3097 (9460) MeV are regarded as quarkonia candidates and are accepted for further analysis. Table 47 shows the mass resolution for $J / \psi$ and $\Upsilon$ candidates for three cases: both muon tracks reconstructed in the barrel $(|\eta|<1.05)$, both in the endcaps $(|\eta|>1.05)$, and one each in the barrel and an endcap.

For prompt quarkonia candidates accepted by a dimuon trigger there are five major sources of background:

- $J / \psi \rightarrow \mu^{+} \mu^{-}$candidates from $b \bar{b}$ events

- nonresonant $\mu^{+} \mu^{-}$from $b \bar{b}$ events

- nonresonant $\mu^{+} \mu^{-}$from charm decays

- nonresonant $\mu^{+} \mu^{-}$from the Drell-Yan mechanism

- nonresonant $\mu^{+} \mu^{-}$from $\pi$ and $K$ decays-in-flight

The first two in the list are the largest: decays of the form $b \rightarrow J / \psi\left(\rightarrow \mu^{+} \mu^{-}\right) X$ and dimuons from $b \bar{b}$ events. While the charm background may be higher in aggregate, the $p_{\mathrm{T}}$ spectrum of the muons falls off sharply and the probability of a dimuon having an invariant mass close to either of the quarkonia is much lower than for the $b \bar{b}$. Monte Carlo studies indicate that the background from Drell-Yan is negligible because only a tiny fraction passes the trigger thresholds. Muons from decays-in-flight also have a very sharply falling $p_{\mathrm{T}}$ spectrum and also need to be in coincidence with another muon, such that the two form an accepted quarkonium candidate, and hence are not dominant background contributors.

Since all of the sources above (aside from Drell-Yan) produce muons which emerge from a secondary vertex, it is possible to suppress them by means of a secondaryvertex cut based on the pseudoproper time, defined as

$$
\tau=\frac{L_{x y} \times m}{p_{\mathrm{T}} \times c}
$$

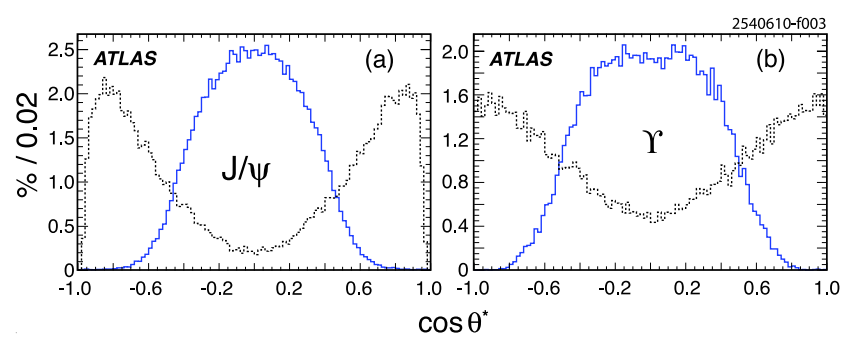

FIG. 94: Polarization angle $\cos \theta^{*}$ distributions for $J / \psi$ and $\Upsilon$ dimuon decays
TABLE 47: Mass peak positions and resolutions for prompt quarkonia production in various pseudorapidity ranges

\begin{tabular}{cccccc}
\hline \hline State & $m_{\mathrm{rec}}-m_{\mathrm{PDG}}$ & \multicolumn{4}{c}{ Resolution $\sigma(\mathrm{MeV})$} \\
& $(\mathrm{MeV})$ & Average & Barrel & Mixed & Endcap \\
\hline$J / \psi$ & $+4 \pm 1$ & 53 & 42 & 54 & 75 \\
$\Upsilon$ & $+15 \pm 1$ & 161 & 129 & 170 & 225 \\
\hline \hline
\end{tabular}

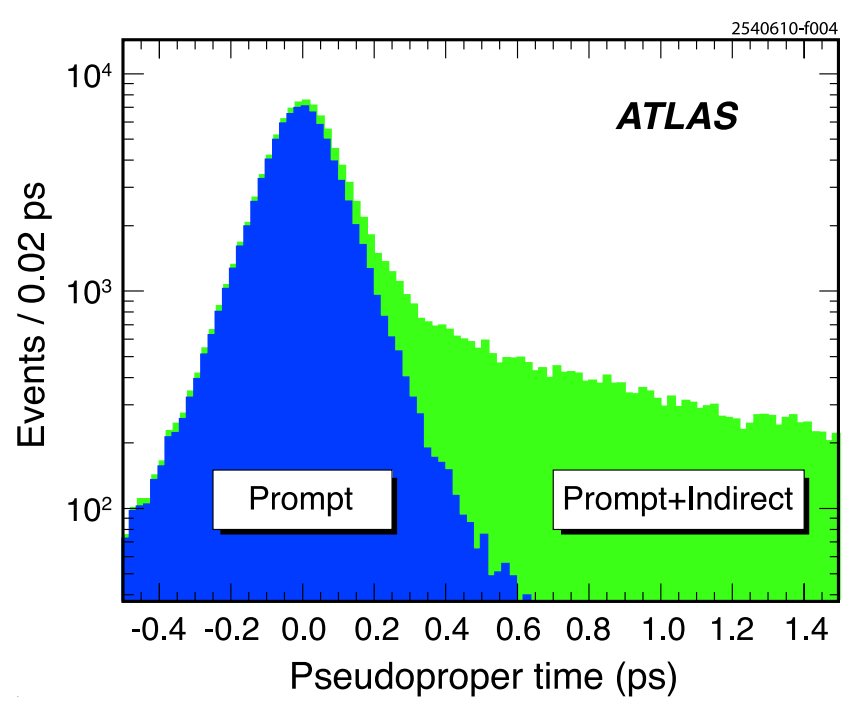

FIG. 95: Pseudoproper decay-time distribution for reconstructed prompt $J / \psi$ (dark shading) and the sum of direct and indirect contributions (lighter shading)

where $m$ and $p_{\mathrm{T}}$ are the invariant mass and transverse momentum of the quarkonium candidate, respectively, and $L_{x y}$ is the measured radial displacement of the twotrack vertex from the beamline as in Eq. (188). A collection of prompt quarkonia will have a pseudoproper time distribution around zero, while distributions for nonprompt candidates will have an exponentially decaying tail on the positive side due to the nonzero lifetime of the parent, as shown in Fig. 95. By making a cut on $\tau$, it is possible to exclude the nonprompt component by, for instance, removing all prompt $J / \psi$ candidates with $\tau>0.2 \mathrm{ps}$, thereby obtaining a sample with an efficiency of $93 \%$ and a purity of $92 \%$. In the case of $\Upsilon$ there is no background from $b \rightarrow J / \psi\left(\mu^{+} \mu^{-}\right) X$ to address. But $b \bar{b} \rightarrow \mu^{+} \mu^{-}$is more problematic in this higher-mass region: the two muons must have come from different decays, rendering the use of pseudoproper time less effective. However, it is possible, e.g., to insist that both muon tracks in the candidate are used to build the same primary vertex: in this case the $b \bar{b} \rightarrow \mu^{+} \mu^{-}$background under the $\Upsilon$ can be reduced by a factor of three or more while losing about $5 \%$ of the signal.

Figure 96 shows the quarkonia signals and the principal backgrounds for the dimuon trigger with thresholds 
TABLE 48: For prompt quarkonia with various selection and background suppression cuts, predicted and observed cross sections, and efficiencies relative to generator-level Monte Carlo

\begin{tabular}{|c|c|c|c|c|c|}
\hline & Quantity & $J / \psi$ & $J / \psi$ & $\Upsilon$ & $\Upsilon$ \\
\hline & Trigger type & $\mu 6 \mu 4$ & $\mu 10$ & $\mu 6 \mu 4$ & $\mu 10$ \\
\hline & MC cross sections & $23 \mathrm{nb}$ & $23 \mathrm{nb}$ & $5.2 \mathrm{nb}$ & $2.8 \mathrm{nb}$ \\
\hline$\epsilon_{1}$ & $\begin{array}{l}\text { Trigger, reconstruction, } \\
\text { and vertexing }\end{array}$ & $75 \%$ & $90 \%$ & $51 \%$ & $90 \%$ \\
\hline$\epsilon_{2}$ & Offline cuts & $90 \%$ & $76 \%$ & $95 \%$ & $75 \%$ \\
\hline$\epsilon$ & Overall efficiency $\epsilon_{1} \times \epsilon_{2}$ & $67 \%$ & $69 \%$ & $49 \%$ & $68 \%$ \\
\hline & Observed signal $\sigma$ & $15 \mathrm{nb}$ & $16 \mathrm{nb}$ & $2.5 \mathrm{nb}$ & $2.0 \mathrm{nb}$ \\
\hline & $N_{s}\left(10 \mathrm{pb}^{-1}\right)$ & $150 \mathrm{~K}$ & $160 \mathrm{~K}$ & $25 \mathrm{~K}$ & $20 \mathrm{~K}$ \\
\hline & $N_{b}\left(10 \mathrm{pb}^{-1}\right)$ & $7 \mathrm{~K}$ & $700 \mathrm{~K}$ & $16 \mathrm{~K}$ & $2000 \mathrm{~K}$ \\
\hline & Signal/bgd at peak & 60 & 1.2 & 10 & 0.05 \\
\hline
\end{tabular}

of $p_{\mathrm{T}}>(6,4) \mathrm{GeV}$. The higher resonances of the $\Upsilon$ were not included in the simulation; hence their absence from the plot. A pseudoproper time cut of $0.2 \mathrm{ps}$ has been applied as described above, and both muon tracks in a candidate are required to have been fitted to the same primary vertex. Table 48 summarizes the reconstruction efficiencies of all of the cuts described above for the different trigger schemes.

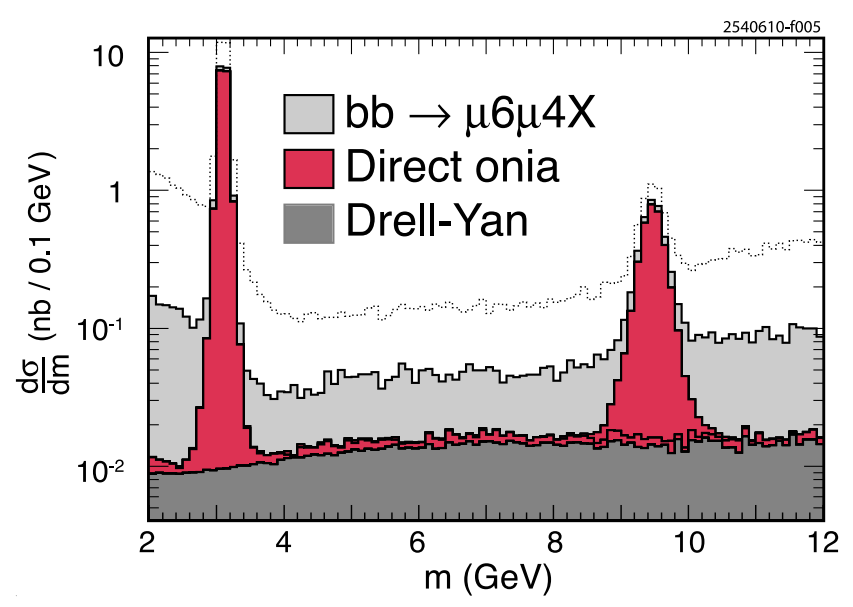

FIG. 96: Cumulative plot of the invariant mass of dimuons from various sources, reconstructed with a dimuon trigger with thresholds of $p_{\mathrm{T}}>(6,4) \mathrm{GeV}$, with the requirements that both muons are identified as coming from the primary vertex and with a pseudoproper time cut of $0.2 \mathrm{ps}$. The dotted line shows the cumulative distribution without vertex and pseudoproper time cuts

\subsubsection{Prompt quarkonium polarization}

The Color Octet Model (COM) predicts that prompt quarkonia are transversely polarized, with the degree of polarization increasing as a function of the transverse momentum of the quarkonium. Other models predict different $p_{\mathrm{T}}$-behaviors of the polarization, so this measurable quantity serves as an important discriminator of the various quarkonia production models. As discussed above, the polarization can be accessed via the distribution of the angle $\cos \theta^{*}$. This measurement is challenging due to reduced acceptance at high $\left|\cos \theta^{*}\right|$ and the difficulty of disentangling acceptance corrections from the spin alignment. Additionally, feeddown from $\chi_{c}$ and $B$ mesons may act to reduce polarization in the final quarkonia sample.

The ATLAS program, in this respect, seeks to measure the polarization of prompt quarkonia states up to transverse momenta of $\sim 50 \mathrm{GeV} / c$, with the coverage in $\cos \theta^{*}$ extended through the use of both single- and double-muon triggers. The high quarkonia rate at the LHC will allow ATLAS to obtain a high-purity prompt quarkonia sample through the use of the pseudoproper time cut, which reduces the depolarization due to contamination from nonprompt quarkonia. Taken together, these techniques will allow ATLAS to control the systematics of the polarization measurement. Of the two main quarkonium states, the $J / \psi$ is easier to deal with than the $\Upsilon$ due to a higher production cross section and much lower backgrounds with the single muon trigger. Indeed, the background to the single-muon trigger for $\Upsilon$ renders the sample available at $10 \mathrm{pb}^{-1}$ essentially unusable: the reduced acceptance in the high $\left|\cos \theta^{*}\right|$ part of the angular distribution cannot be offset with use of a single-muon trigger in the same way as for $J / \psi$. For this reason the uncertainties on the spin alignment for $\Upsilon$ are much higher than $J / \psi$.

The uncertainties from both integrated luminosity and spin alignment need to be factored into measurement errors for prompt quarkonia production cross sections. Both are expected to be high in the early running of the new machine. However, the relative magnitudes of the cross sections measured in separate $p_{\mathrm{T}}$ slices will be unaffected by both luminosity and spin-alignment uncertainties.

Summarizing the main conclusion of $[1018$, p. $1083-$ 1110]: after $10 \mathrm{pb}^{-1}$ it should be possible to measure the spin alignment, $\alpha$, of prompt $J / \psi$ with a precision of $\Delta \alpha= \pm 0.02-0.06$ for $p_{\mathrm{T}}>12 \mathrm{GeV} / c$, depending on the level of polarization. For the reasons discussed above, in the case of $\Upsilon$, the precision is about ten times worse of order 0.2 . With an integrated luminosity increased by a factor of 10 , the uncertainties on $\Upsilon$ polarization could drop by a factor of around 5 because the sample obtained with the $\mu 10$ trigger will become more useful. 


\subsubsection{Early $7 \mathrm{TeV}$ LHC running}

The quarkonium program in ATLAS has begun with the first runs of the LHC. The first task is to observe the resonances in the data, using the peaks as calibration points to assess the performance of the muon- and inner-detector track reconstruction and the muon triggers. These studies are being carried out in a rapidly changing luminosity and trigger environment as the LHC itself is commissioned.

After about $1 \mathrm{pb}^{-1}$ ATLAS should have collected some $15 \mathrm{~K} J / \psi$ and $2.5 \mathrm{~K} \Upsilon$ candidates decaying to pairs of muons passing the dimuon trigger requiring both muons to have a $p_{\mathrm{T}}$ of $4 \mathrm{GeV} / c$ and one having at $6 \mathrm{GeV} / c$. The single-muon trigger with a threshold of $10 \mathrm{GeV} / c$ will provide largely independent additional samples of $16 \mathrm{~K}$ $J / \psi$ and $2 \mathrm{~K} \Upsilon$ decays. Separately from these, some $7 \mathrm{~K}$ $J / \psi \rightarrow \mu^{+} \mu^{-}$events are expected from $b$-hadron decays. All of these decays can be used for detector performance studies. Furthermore, a measurement of the fraction of $J / \psi$ arising from $B$ decays will be possible at this level, although the muon trigger and reconstruction efficiencies will have to be estimated with Monte Carlo at this stage.

After about $10 \mathrm{pb}^{-1}$ there will be sufficient statistics to use the data-driven tag-and-probe method to calculate the efficiencies, leading to a reduction in the systematic uncertainties on the ratio measurement. The $p_{\mathrm{T}^{-}}$ dependence of the production cross section for both $J / \psi$ and $\Upsilon$ should be fairly well measured by then, over a wide range of transverse momenta $\left(10 \leq p_{\mathrm{T}} \leq 50 \mathrm{GeV} / c\right)$.

After around $100 \mathrm{pb}^{-1}$ the $J / \psi$ and $\Upsilon$ differential cross sections will be measured up to transverse momenta around $100 \mathrm{GeV} / c$. With several million $J / \psi$ and around $500 \mathrm{~K} \Upsilon$, and a good understanding of the efficiency and acceptance, polarization measurements should reach precisions of a few percent. Additional luminosity may allow the observation of resonant pairs of $J / \psi$ in the $\Upsilon$ mass region from the decays of $\eta_{b}$ and $\chi_{b}$ states.

\subsection{CMS}

The primary goal of the Compact Muon Solenoid (CMS) experiment [1020] is to explore particle physics at the $\mathrm{TeV}$ energy scale exploiting the proton-proton collisions delivered by the LHC. The central feature of the CMS apparatus is a superconducting solenoid of $6 \mathrm{~m}$ internal diameter which provides an axial magnetic field of $3.8 \mathrm{~T}$. Within the field volume are the silicon tracker, the crystal electromagnetic calorimeter and the brass/scintillator hadronic calorimeter in barrel and endcap configurations. CMS also has extensive forward calorimetry, including a steel/quartz-fibre forward calorimeter covering the $2.9<|\eta|<5.2$ region. Four stations of muon detectors are embedded in the steel return yoke, covering the $|\eta|<2.4$ window. Each station consists of several layers of drift tubes in the barrel region and cathode strip chambers in the endcap regions, both complemented by resistive plate chambers.

Having a high-quality muon measurement was one of the basic pillars in the design of CMS. Around $44 \%$ of the $J / \psi$ mesons produced in $p p$ collisions are emitted within the almost 5 units of pseudorapidity covered by the muon stations, which cover an even larger phase-space fraction for dimuons from $\Upsilon$ decays. These detectors are crucial for triggering and for muon identification purposes; CMS can easily select collisions which produced one or more muons for writing on permanent storage. The good quality of the muon measurement, however, is mostly due to the granularity of the silicon tracker (1440 silicon-pixel and 15148 silicon-strip modules) and to the very strong bending power of the magnetic field [1021]. The silicon tracker also provides the vertex position with $\sim 15 \mu \mathrm{m}$ accuracy [1022].

The performance of muon reconstruction in CMS has been evaluated using a large data sample of cosmic-ray muons recorded in 2008 [1023]. Various efficiencies, measured for a broad range of muon momenta, were found to be in good agreement with expectations from Monte Carlo simulation studies. The relative momentum resolution for muons crossing the barrel part of the detector is better than $1 \%$ at $10 \mathrm{GeV} / c$.

The CMS experiment, thanks to its good performance for the measurement of dimuons, including the capability of distinguishing prompt dimuons from dimuons produced in a displaced vertex, should be ideally placed to study the production of several quarkonia, including the $J / \psi, \psi(2 S)$ and $\Upsilon(1 S, 2 S, 3 S)$ states. Complementing the dimuon measurements with the photon information provided by the electromagnetic calorimeter should also allow reconstruction of the $\chi_{c}$ and $\chi_{b}$ states. Such measurements will lead to several studies of quarkonium production. Some will be simple analyses that will lead to the first CMS physics publications. Other rather complex ones will come later, such as the measurement of the polarization of the directly-produced $J / \psi$ mesons as a function of their $p_{\mathrm{T}}$, after subtraction of feeddown contributions from $\chi_{c}$ and $B$-meson decays.

Here we do not describe an exhaustive description of all the many interesting quarkonium physics analyses that can, in principle, be performed by CMS. Instead, we focus on only a few representative studies. We only mention measurements with dimuons in proton-proton collisions, despite the fact that similar studies could also be made with electron pairs, and/or in heavy-ion collisions, at least to some extent.

\subsubsection{Quarkonium production}

At midrapidity, the strong magnetic field imposes a minimum transverse momentum of around $3 \mathrm{GeV} / c$ for muons to reach the muon stations. At forward angles, the material thickness imposes a minimum energy on the detected muons, rather than a minimum $p_{\mathrm{T}}$. In general, for a muon to trigger it needs to cross at least two muon 
stations. This requirement rejects a significant fraction of the low $p_{\mathrm{T}} J / \psi$ dimuons which could be reconstructed from a data sample collected with a "minimum bias" trigger. In the first few months of LHC operation, while the instantaneous luminosity will be low enough, less selective triggers can be used. For instance, it is possible to combine (in the "high-level trigger" online farm) a singlemuon trigger with a silicon track, such that their pair mass is in a mass window surrounding the $J / \psi$ peak. In this way, sizeable samples of low $p_{\mathrm{T}} J / \psi$ dimuons can be collected before the trigger rates become too large.

Figure 97 shows a $J / \psi p_{\mathrm{T}}$ distribution resulting from a Monte Carlo simulation study (based on a tuned [1025] version of the PYTHIA [1026] event generator). This study [1024] was made for $p p$ collisions at $2360 \mathrm{GeV}$ and corresponds to a minimum-bias event sample, collected without any trigger selection of muon-station signals. We see that CMS should have the capability of measuring very low $p_{\mathrm{T}} J / \psi$ dimuons, especially if one of the two muons (or both) is reconstructed as a "tracker muon", meaning that it only traverses one muon station. In fact, most of the yield that could be reconstructed by CMS is contained in the muon-pair category where only one of the muons crosses two or more muon stations (the "global-tracker" pairs).

By accepting events with one of the muons measured only in one station, the signal-to-background ratio in the $J / \psi$ dimuon mass region becomes smaller than in the "global-global" category. However, it remains rather good, as illustrated in Fig. 98, where we see the $J / \psi$ peak reconstructed from $p p$ collisions at $7 \mathrm{TeV}$ after applying certain selection cuts on the muons and requiring a minimum dimuon vertex quality.

With $\sim 10 \mathrm{pb}^{-1}$ of integrated luminosity for $p p$ collisions at $7 \mathrm{TeV}$, CMS should collect a few hundred thou-

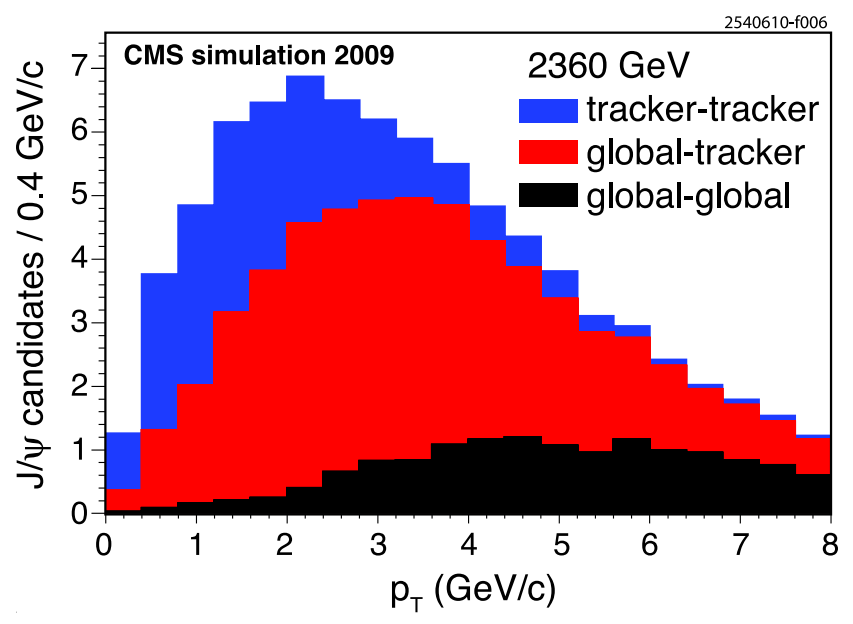

FIG. 97: Transverse momentum distribution of dimuons from $J / \psi$, reconstructed by CMS in three different muon-pair categories, which depend upon the number of stations crossed by the muons (MC study) [1024] sand $J / \psi$ dimuons and a few tens of thousands of $\Upsilon(1 S)$ dimuons. It is important to note that CMS can measure muon pairs resulting from decays of zero $p_{\mathrm{T}} \Upsilon_{\mathrm{s}}$. Indeed, the high mass of the $\Upsilon$ states $(\sim 10 \mathrm{GeV})$ gives single muons enough energy to reach the muon stations even when the $\Upsilon$ is produced at rest.

Given its very good muon momentum resolution, better than $1 \%$ (2\%) for the barrel (endcap) region for muon momenta up to $100 \mathrm{GeV} / c$ [1028], CMS will reconstruct the $J / \psi$ and $\Upsilon$ peaks with a dimuon mass resolution of around 30 and $80 \mathrm{MeV}$, respectively, better at midrapidity than at forward rapidity [1029].

The very good electromagnetic calorimeter of CMS, covering the range $|\eta|<3.0$, enables the study of $\chi_{c}$ and $\chi_{b}$ production through the measurement of their radiative decays. Such measurements are crucial to evaluate non-negligible feeddown contributions to prompt $J / \psi$ and $\Upsilon$ production, a mandatory ingredient to fully understand the physics of quarkonium production from measurements of differential cross sections and polarization. The contribution of $\psi(2 S)$ and $\chi_{c}$ decays to prompt $J / \psi$ production has recently been evaluated to be $8.1 \pm 0.3 \%$ and $25 \pm 5 \%$, respectively [864], while around half of the $\Upsilon(1 S)$ yield is due to decays of heavier bottomonium states, at least for $p_{\mathrm{T}}(\Upsilon)>8 \mathrm{GeV} / c$ [668]. The decays of $b$-hadrons also contribute to the observed $J / \psi$ yield. This further complication can be kept under control through the measurement of nonprompt $J / \psi$ production, which CMS can do efficiently thanks to very good vertexing and $b$-tagging capabilities, and profiting from the long $b$ hadron lifetimes. In the $\Upsilon$ sector there are no feeddown decays from nonprompt sources.

Given the performance capabilities of the CMS detector, which include a good dimuon mass resolution, a broad rapidity coverage, acceptance down to zero $p_{\mathrm{T}}$ for the $\Upsilon$ states (and also to relatively low $p_{\mathrm{T}}$ for the $J / \psi$ ),

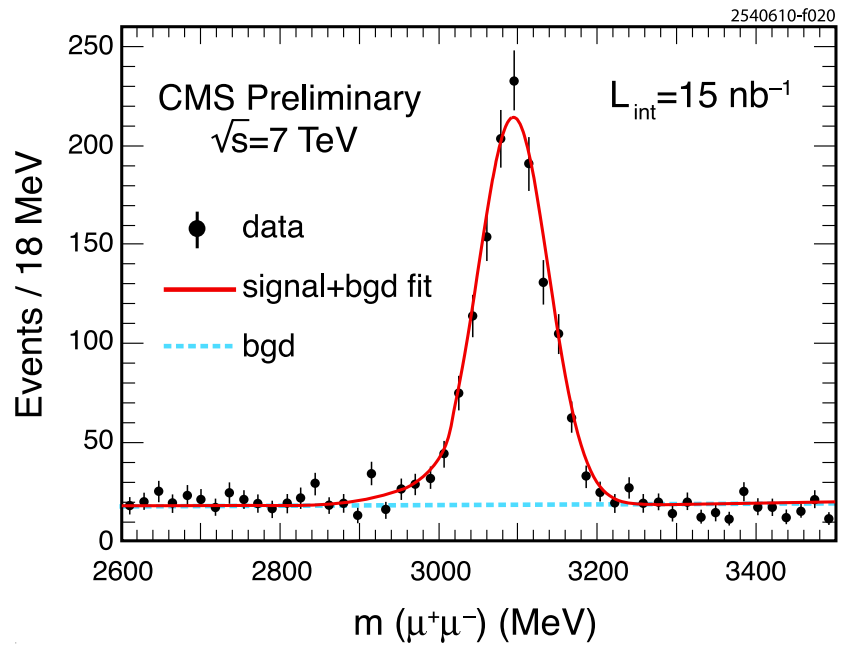

FIG. 98: Dimuon mass distribution reconstructed for $p p$ collisions at $7 \mathrm{TeV}$, for an integrated luminosity of $15 \mathrm{nb}^{-1}[1027]$ 
CMS is in a very good position to do detailed studies of the quarkonium production mechanisms, hopefully answering some of the questions left open by the lower energy experiments. Naturally, the CMS quarkonium physics program foresees measurements of the differential production cross sections, versus $p_{\mathrm{T}}$ and rapidity, of many quarkonium states. Given the large charm and bottom production cross sections at LHC energies, CMS should collect large $J / \psi$ and $\Upsilon$ event samples in only a few months of LHC operation, leading to physics publications on, in particular, their $p_{\mathrm{T}}$ distributions, very competitive with respect to the presently available Tevatron results.

\subsubsection{Quarkonium polarization}

Polarization studies, particularly challenging because of their multidimensional character, will exploit the full capabilities of the CMS detector and the ongoing optimization of dedicated trigger selections. CMS will study the complete dilepton decay distributions, including polar and azimuthal anisotropies and as functions of $p_{\mathrm{T}}$ and rapidity, in the Collins-Soper (CS) and the helicity (HX) frames. These analyses will require considerably larger event samples than the cross section measurements. The acceptance in the lepton decay angles is drastically limited by the minimum- $p_{\mathrm{T}}$ requirements on the accepted leptons (rather than reflecting geometrical detector constraints). Polarization measurements will therefore profit crucially from looser muon triggers. Moreover, such trigger-specific acceptance limitations determine a significant dependence of the global acceptances (in different degrees for different quarkonium states) on the knowledge of the polarization. The systematic contributions of the as-yet unknown polarizations to early cross section measurements will be estimated, adopting the same multidimensional approach of the polarization analyses. Plans for high-statistics runs include separate determinations of the polarizations of quarkonia produced directly and of those coming from the decays of heavier states. Current studies indicate that CMS should be able to measure the polarization of the $J / \psi$ 's that result from $\chi_{c}$ decays, together with the $p_{\mathrm{T}}$-dependent $J / \psi$ feeddown contribution from $\chi_{c}$ decays, from very low to very high $p_{\mathrm{T}}$.

All measurements will also be reported in terms of frame-invariant quantities, which will be determined, for cross-checking purposes, in more than one reference frame. These plans reflect our conviction that robust measurements of quarkonium polarization can only be provided by fully taking into account the intrinsic multidimensionality of the problem. As emphasized in $[722,1030]$, the measurements should report the full decay distribution in possibly more than one frame and avoid kinematic averages (for example, over the whole rapidity acceptance range) as much as possible.

Figure 99 shows, as a simple example, how a hypothetical Drell-Yan-like polarization (fully transverse and

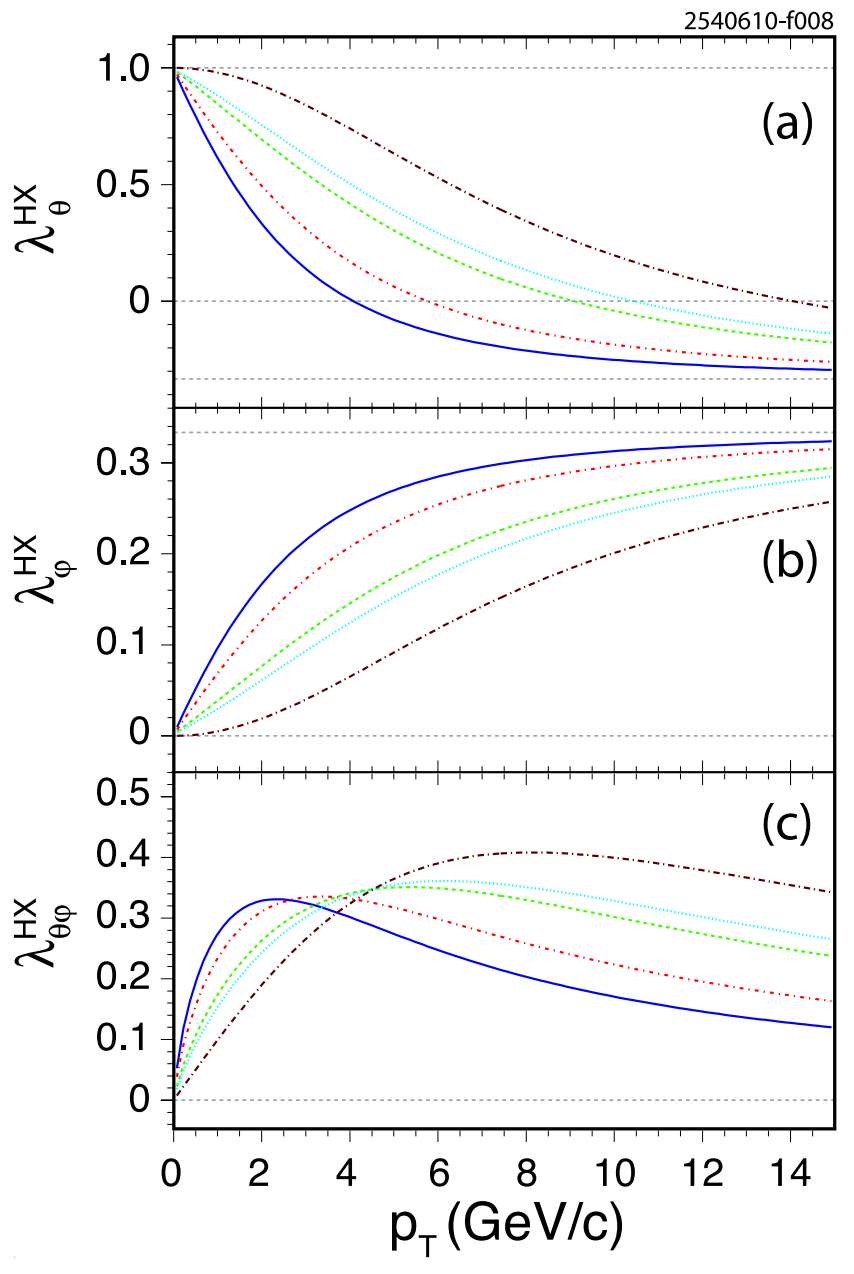

FIG. 99: Anisotropy parameters in (a) polar and (b) azimuthal angle vs. transverse momentum for $\Upsilon \rightarrow \ell^{+} \ell^{-}$decays in the HX frame for a natural polarization $\lambda_{\vartheta}=+1$ in the CS frame. The curves in each plot correspond to different rapidity intervals representative of different experiments. Starting from the solid line: $|y|<0.6$ (CDF), $|y|<0.9$ (ALICE, $e^{+} e^{-}$channel), $|y|<1.8$ (DØ), $|y|<2.5$ (ATLAS and CMS), $2<|y|<5$ (LHCb). For simplicity, the event populations have been assumed to be flat in rapidity. The vertical axis of the polarization frame is here defined as $\operatorname{sign}\left(p_{\mathrm{L}}\right)\left(\vec{P}_{1} \times \vec{P}_{2}\right) /\left|\vec{P}_{1}{ }_{1} \times \vec{P}^{\prime}{ }_{2}\right|$, where $\vec{P}^{\prime}{ }_{1}$ and $\vec{P}^{\prime}{ }_{2}$ are the momenta of the colliding protons in the quarkonium rest frame (the sign of $\lambda_{\vartheta \varphi}$ depends on this definition)

purely polar in the CS frame) in the $\Upsilon$ mass region would translate into different $p_{\mathrm{T}}$-dependent polarizations measured in the HX frame by experiments with different rapidity acceptances. The anisotropy parameters $\lambda_{\vartheta}, \lambda_{\varphi}$ and $\lambda_{\vartheta \varphi}$ are defined as in [722]. This example illustrates the following general concepts:

- The polarization depends very strongly on the reference frame. The very concepts of "transverse" and "longitudinal" are frame-dependent.

- The fundamental nature of the polarization ob- 


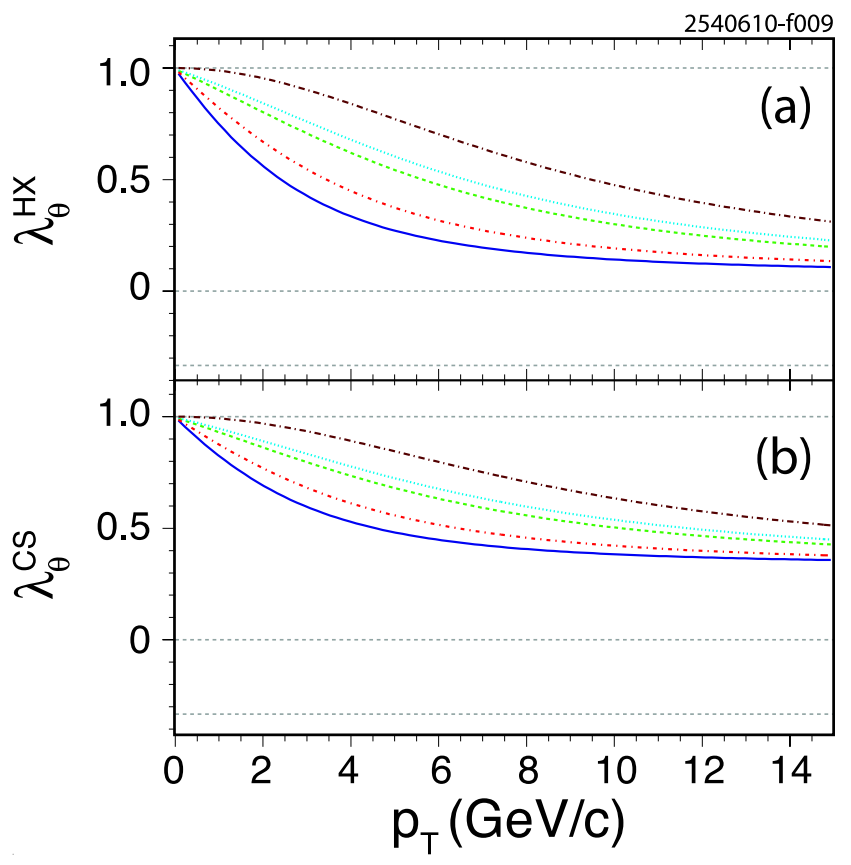

FIG. 100: Polar angle anisotropy parameter vs. transverse momentum for $\Upsilon \rightarrow \ell^{+} \ell^{-}$decay, observed in the (a) HX and (b) CS frames when $40 \%(60 \%)$ of the events have full transverse polarization in the HX (CS) frame. The curves in each plot represent measurements performed in different experimental acceptance ranges, as detailed in Fig. 99

served in one chosen frame can be correctly interpreted (without relying on assumptions) only when the azimuthal anisotropy is measured together with the polar anisotropy.

- The measured polarization may be affected by "extrinsic" kinematic dependencies due to a nonoptimal choice of the observation frame. Such extrinsic dependencies can introduce artificial differences among the results obtained by experiments performed in different acceptance windows and give a misleading view of the polarization scenario.

On the other hand, these spurious effects cannot always be eliminated by a suitable frame choice, as is shown by the further illustrative case represented in Fig. 100. Here it is assumed that $60 \%$ of the $\Upsilon$ events has natural polarization $\lambda_{\vartheta}=+1$ in the CS frame and the remaining fraction has natural polarization $\lambda_{\vartheta}=+1$ in the HX frame. While the polarizations of the two event subsamples are intrinsically independent of the production kinematics, in neither frame will measurements performed in different transverse and longitudinal momentum windows find identical results for $\lambda_{\vartheta}$ (the same is true for the other two anisotropy parameters, not shown here). This example provides a first motivation for the complementary use of a frame-invariant approach [1030], consisting of the measurement of intrinsically rotation-invariant polarization parameters like

$$
K=\frac{1+\lambda_{\theta}+2 \lambda_{\phi}}{3+\lambda_{\theta}}
$$

In the example of Fig. 100, all experiments would measure a constant, frame-independent value $K=1 / 2$. This method facilitates the comparison between different experiments, as well as between measurements and theory. Furthermore, since the acceptance distributions for the polar and azimuthal decay angles can be very different in different frames, checking whether quantities like $K$ are, as they should be, numerically independent of the reference frame provides a nontrivial systematic test of the experimental analyses.

\section{5. $\mathrm{LHCb}$}

LHCb [1031] is a dedicated experiment for $b$-physics at the LHC. Since $b$ production is peaked in the forward region at $\mathrm{LHC}$ energies, the $\mathrm{LHCb}$ detector has a forward spectrometer geometry covering an angle between $15 \mathrm{mrad}$ and $300 \mathrm{mrad}$ with respect to the beam axis. This corresponds to an $\eta$ range between 2 and 5, which will allow $\mathrm{LHCb}$ to have a unique acceptance coverage among the LHC experiments. Good vertex resolution and particle identification over a wide momentum range are key characteristics of LHCb. The trigger system retains muons with moderate $p_{\mathrm{T}}$ as well as purely hadronic final states.

\subsubsection{Charmonium physics}

The $J / \psi$ selection studies and in general all studies presented here have been performed using the full $\mathrm{LHCb}$ Monte Carlo simulation based on PYTHIA [1026], EvtGen [1032], and GEANT4 [1033]. At the generation level, color-octet $J / \psi$ production models in PYTHIA have been tuned to reproduce the cross section and $p_{T}$ spectrum observed at Tevatron energies [1025]. A fullevent reconstruction is applied to the simulated events [1034]. $J / \psi$ candidates are selected using track and vertex quality requirements, and also muon identification information. Since the first-level trigger (L0) requires at least one muon with a $p_{\mathrm{T}}$ larger than $1 \mathrm{GeV} / c$, a tighter selection is applied at reconstruction level to keep only candidates formed with at least one muon with a $p_{\mathrm{T}}$ larger than $1.5 \mathrm{GeV} / c$. The $J / \psi$ selection yields an expected number of reconstructed events equal to $3.2 \times 10^{6}$ at $\sqrt{s}=14 \mathrm{TeV}$, with $S / B=4$, for an integrated luminosity equal to $5 \mathrm{pb}^{-1}$. This number is obtained assuming a $J / \psi$ production cross section equal to $290 \mu \mathrm{b}$. This amount of data could be collected in a few days under nominal LHC running conditions. The mass resolution is $11.4 \pm 0.4 \mathrm{MeV}$.

One of the first goals of the $\mathrm{LHCb}$ experiment will be to measure the differential $J / \psi$ cross section in bins of 
$p_{\mathrm{T}}$ and $\eta$ in the range $0<p_{\mathrm{T}}<7 \mathrm{GeV} / c$ and $2<\eta<5$. Both the prompt- $J / \psi$ and the $b \rightarrow J / \psi X$ production cross sections will be accessible, thereby measuring the total $b \bar{b}$-production cross section. The two contributions will be separated using a variable which approximates the $b$-hadron proper time along the beam axis

$$
t \equiv \frac{d z \times m(J / \psi)}{p_{z}^{J / \psi} \times c}
$$

where $d z$ is the distance between the $J / \psi$ decay vertex and the primary vertex of the event projected along the beam $(z)$ axis, $p_{z}^{J / \psi}$ is the signed projection of the $J / \psi$ momentum along the $z$ axis, and $m(J / \psi)$ is the known $J / \psi$ mass. (Note that this is analogous to the ATLAS pseudoproper time definition in Eq. (190), which uses the transverse decay length and momentum instead of the longitudinal component employed here.) The expected distribution of the $t$ variable is shown in Fig. 101. The distribution can be described by

- a prompt $J / \psi$ component produced at the primary vertex of the event, represented by a Gaussian distribution to account for vertex resolution;

- an exponential $J / \psi$ component coming from $b$ hadron decays, convoluted with a Gaussian resolution function;

- a combinatorial background component due to random combinations of tracks coming from the primary vertex (the form of this component will be extracted using events in the sidebands of the dilepton mass distribution);

- a tail due to a wrong association of primary vertex when computing the $t$ variable (the shape of this component will be determined from data, associating the $J / \psi$ vertex with a different event's primary vertex).

A combined fit of the mass and $t$ distributions will extract the number of reconstructed $J / \psi$ in each $p_{\mathrm{T}}$ and $\eta$ bin [1035]. The absolute cross section in each of the bins will be obtained from this measured yield, efficiencies that will be computed from Monte Carlo simulations, and integrated luminosity. The measurement uncertainty will be dominated by systematic errors in the integrated luminosity, the resolution model, and the reconstruction and trigger efficiencies.

The unknown polarization at production of the $J / \psi$ will complicate the measurement. The acceptance of the $\mathrm{LHCb}$ detector is not uniform as a function of the $J / \psi$ polarization angle, $\theta$, defined as the angle between the $\mu^{+}$direction in the center-of-mass frame of the $J / \psi$ and the direction of the $J / \psi$ in the laboratory. Ignoring this effect adds a $25 \%$ uncertainty to the cross section measurement. Performing the measurement in bins of $\theta$ will allow determination of the $J / \psi$ production polarization.

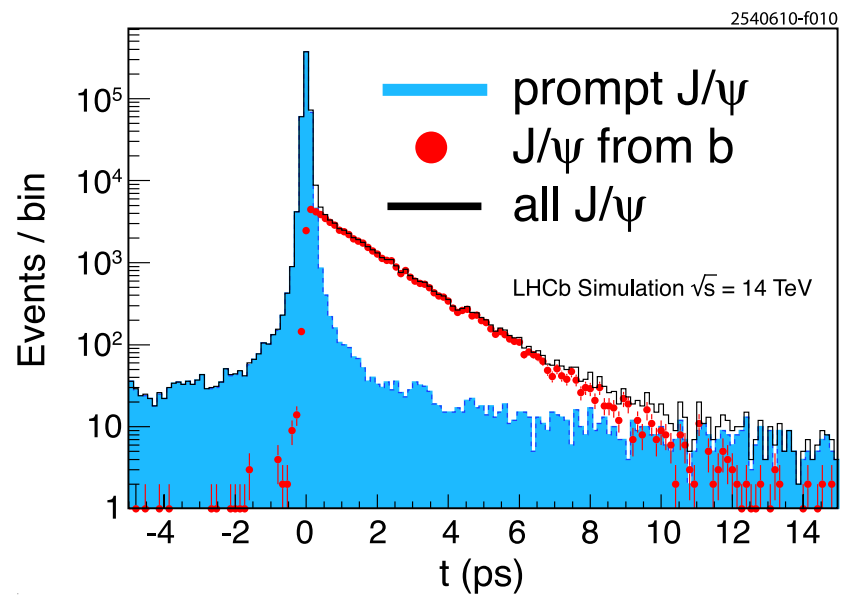

FIG. 101: Time distribution of $J / \psi$ candidates obtained with LHCb Monte Carlo simulation

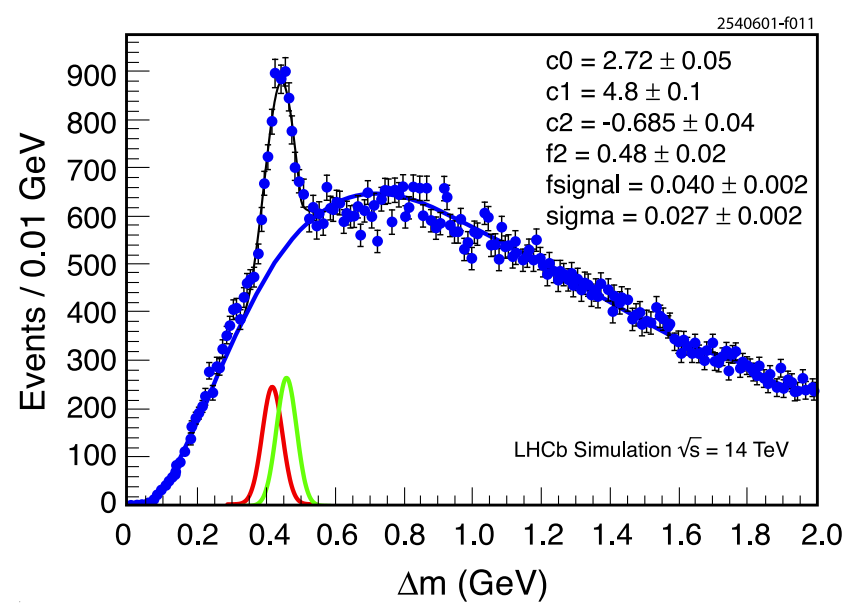

FIG. 102: $\Delta m$ distribution of $\chi_{c}$ candidates obtained with LHCb Monte Carlo simulation

Measurements of the rates and polarization of the $J / \psi$, and more generally of the charmonium and bottomonium states, will be compared to predictions of different theoretical models.

Reconstruction of $\chi_{c 1}$ and $\chi_{c 2}$ has also been studied [1034] using the decay modes $\chi_{c 1,2} \rightarrow J / \psi \gamma$. A photon candidate reconstructed in the electromagnetic calorimeter with $p_{\mathrm{T}}>500 \mathrm{MeV}$ is associated with a $J / \psi$ candidate. Figure 102 shows the $\Delta m=m\left(\mu^{+} \mu^{-} \gamma\right)-$ $m\left(\mu^{+} \mu^{-}\right)$distribution obtained from a Monte Carlo simulation. A clear signal peak can be observed. The $\Delta m$ resolution of $27 \mathrm{MeV}$ is dominated by the uncertainty in photon energy. Since the mass difference between $\chi_{c 1}$ and $\chi_{c 2}$ is known ( $\left.55 \mathrm{MeV}\right)$, imposing this constraint on the analysis should allow separation of the $\chi_{c 1}$ and $\chi_{c 2}$ contributions.

A large number of $\psi(2 S)$ mesons will also be collected at $\mathrm{LHCb}$. It is expected that the number of reconstructed 
$\psi(2 S) \rightarrow \mu^{+} \mu^{-}$decays will be equal to 2 to $4 \%$ of the number of reconstructed $J / \psi \rightarrow \mu^{+} \mu^{-}$, with $S / B$ between 1 and 2 [1036]. Because the masses of the $J / \psi$ and $\psi(2 S)$ are close, trigger and reconstruction efficiencies for the two states are similar. A measurement of

$$
\frac{\sigma(\operatorname{prompt} J / \psi)}{\sigma(\operatorname{prompt} \psi(2 S))},
$$

where a number of systematic errors cancel, will be possible.

A large sample of $X(3872) \rightarrow J / \psi \pi^{+} \pi^{-}$decays will be reconstructed at $\mathrm{LHCb}$, either prompt $X(3872)$, or $X(3872)$ from $b$-hadron decays. In particular, the decay channel $^{22} B^{+} \rightarrow X(3872)\left(\rightarrow J / \psi \rho^{0}\right) K^{+}$will be studied because an angular analysis of the decay products can lead to the determination of the now-ambiguous quantum numbers of the $X(3872)$ (see Sect. 2.3.1), allowing separation of the $1^{++}$and the $2^{-+}$hypotheses. 1850 reconstructed events are expected for $2 \mathrm{fb}^{-1}$ of data at $\sqrt{s}=14 \mathrm{TeV}$, with $B / S$ between 0.3 and 3.4 [1037]. $Z(4430)$ will be sought in the decay $B^{0} \rightarrow Z(4430)^{\mp} K^{ \pm}$, with $Z(4430)^{\mp} \rightarrow \psi(2 S)\left(\rightarrow \mu^{+} \mu^{-}\right) \pi^{\mp} .6200$ events are expected with $B / S$ between 2.7 and 5.3 , for $2 \mathrm{fb}^{-1}$ of data at $\sqrt{s}=14 \mathrm{TeV}[1037]$.

\subsection{2. $B_{c}$ physics}

The expected $B_{c}^{+}$cross section at the $\mathrm{LHC}$ is at the level of $1 \mu \mathrm{b}$, so a very large number of $B_{c}$ will be produced and recorded at $\mathrm{LHCb}$. (For the present status of $B_{c}$ measurements, see Sects. 2.2.4 and 4.7.) First studies will use the reconstruction of the mode with a large branching fraction $B_{c}^{+} \rightarrow J / \psi \mu^{+} \nu$, and most promising results are expected using the clean $B_{c}^{+} \rightarrow J / \psi \pi^{+}$decay mode. But the large number of $B_{c}^{+}$produced will allow a systematic study of the $B_{c}$ family at $\mathrm{LHCb}$.

The selection of the decay channel $B_{c}^{+} \rightarrow J / \psi \pi^{+}$, with $J / \psi \rightarrow \mu^{+} \mu^{-}$has been studied using full Monte Carlo simulation of events reconstructed by the $\mathrm{LHCb}$ detector [1038-1040]. A specific generator, BCVEGPY [1041] has been used to generate $B_{c}$ events. Since the $B_{c}$ vertex is displaced with respect to the primary vertex, impact parameter selections are imposed to the $\pi$ and $J / \psi$ candidates. Particle identification, quality of track and vertex fits, and minimum $p_{\mathrm{T}}$ requirements are applied to $B_{c}$ candidates in order to reduce the large background due to other $b$-hadron decays with a $J / \psi$ in the final state. The total reconstruction efficiency is estimated to be $(1.01 \pm 0.02) \%$, with $1<B / S<2$ at $90 \%$ CL. Assuming a $B_{c}$ production cross section of $\sigma\left(B_{c}^{+}\right)=0.4 \mu \mathrm{b}$ for $\sqrt{s}=14 \mathrm{TeV}$ and a branching fraction $\mathcal{B}\left(B_{c}^{+} \rightarrow J / \psi \pi^{+}\right)=1.3 \times 10^{-3}, 310$ signal events are expected with $1 \mathrm{fb}^{-1}$ of data.

${ }^{22}$ A charge-conjugate decay mode is implied in the rest of the text
The potential of a mass measurement has been studied using an unbinned maximum likelihood method to extract the $B_{c}^{+}$mass from the reconstructed sample of $B_{c}^{+} \rightarrow J / \psi \pi^{+}$candidates. Describing the invariant mass distribution of the signal by a single Gaussian and the combinatorial background by a first-order polynomial, the fit procedure gives a $B_{c}^{+}$mass of $m\left(B_{c}^{+}\right)=$ $6399.6 \pm 1.7 \mathrm{MeV}$, where the error is statistical only, consistent with the input value of $6400 \mathrm{MeV}$. The size of the sample used corresponds to the expected yield for $1 \mathrm{fb}^{-1}$ of data. The result of the fit and the mass distribution are shown in Fig. 103. The mass resolution is $\sigma=17.0 \pm 1.6 \mathrm{MeV}$.

The reconstructed $B_{c}^{+}$candidates will also be used to measure the lifetime of the $B_{c}^{+}$. A combined masslifetime fit is performed. The proper-time distribution is described by an exponential function convoluted with a resolution function and multiplied by an acceptance function $\epsilon(t)$ which describes the distortion of the proper-time distribution due to the trigger and offline event selections through impact parameter requirements. The form of these functions is determined from the full Monte Carlo simulation. Since the resolution of the impact parameter depends on the transverse momentum of the tracks, the proper-time acceptance function $\epsilon(t)$ depends on the $p_{\mathrm{T}}$ distribution of the $B_{c}^{+}$, and then on the generation model used for the $B_{c}^{+}$when determining $\epsilon(t)$. In order to evaluate the systematics associated with this effect, a fit was performed on a $B_{c}^{+}$sample generated with a $p_{\mathrm{T}}$ spectrum identical to the $B^{+}$spectrum observed in the simulation. A bias of $0.023 \mathrm{ps}$ is then observed in the lifetime determination. In order to reduce this bias, the lifetime fit is performed simultaneously on two samples with different $p_{\mathrm{T}}$ ranges, $5<p_{\mathrm{T}}<12 \mathrm{GeV} / c$ and $p_{\mathrm{T}}>12 \mathrm{GeV} / c$. The resulting bias is then reduced to $0.004 \mathrm{ps}$. The fit procedure applied to a sample corresponding to $1 \mathrm{fb}^{-1}$ of data gives a $B_{c}^{+}$lifetime of $\tau\left(B_{c}^{+}\right)=0.438 \pm 0.027 \mathrm{ps}$, where the error is statistical only, consistent with the input value of $0.46 \mathrm{ps}$ [1042].

$\mathrm{LHCb}$ capabilities should allow comprehensive studies of many other aspects of $B_{c}$ physics [1043]. Spectroscopy of the $B_{c}$ excited states, both below and above the $m\left(B^{0}\right)+m\left(D^{0}\right)$ threshold will be performed. For example, searches for $B_{c}^{* *} \rightarrow B_{c}^{+} \pi^{+} \pi^{-}$have been envisaged [1044]. Searches for new decay modes of the $B_{c}$ will also be made, e.g., modes with a weak decay of the $c$ quark, such as $B_{c}^{+} \rightarrow B_{s}^{0} \pi^{+}$or those with a $\bar{b} c$ annihilation [1045], such as $B_{c}^{+} \rightarrow \bar{K}^{* 0} K^{+}$.

\subsubsection{Bottomonium physics}

Analyses of bottomonium in LHCb have begun. First results show that the reconstruction of $\Upsilon(1 S) \rightarrow \mu^{+} \mu^{-}$ is possible with a mass resolution of $37 \mathrm{MeV}$. Other $\Upsilon$ states will also be observed in the detector. Using these candidates, measurements of the production cross section and of the production polarization will be performed as 


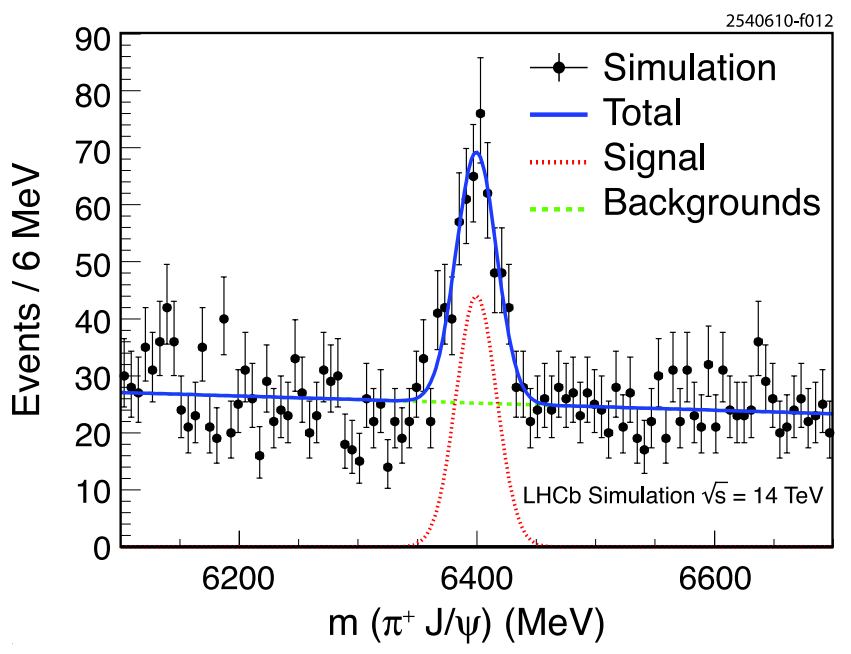

FIG. 103: $B_{c}^{+}$candidate mass distribution obtained with LHCb Monte Carlo simulation

a function of $p_{\mathrm{T}}$ and $\eta$. Similarly, the decays $\chi_{b 1,2} \rightarrow \Upsilon_{\gamma}$ are also possible to reconstruct in the $\mathrm{LHCb}$ detector, due to low- $p_{\mathrm{T}}$-photon reconstruction provided by the electromagnetic calorimeter [1034]. In addition, the equivalent for the $b$ family of the exotic $X, Y$, and $Z$ states (see Sect. 2.3) will be sought at $\mathrm{LHCb}$, for example, in the decay mode $Y_{b} \rightarrow \Upsilon(1 S) \pi^{+} \pi^{-}$(for status of the $Y_{b}$, see Sects. 2.3.2 and 3.3.11).

\subsection{RHIC}

The Relativistic Heavy-Ion Collider, RHIC, provides $\mathrm{d}+\mathrm{Au}, \mathrm{Au}+\mathrm{Au}$, and $\mathrm{Cu}+\mathrm{Cu}$ collisions at $\sqrt{s_{N N}}=$ $200 \mathrm{GeV}$, and polarized $p p$ collisions at both 200 and $500 \mathrm{GeV}$. Polarized $p p$ collisions are used for spin studies, with those from $200 \mathrm{GeV}$ serving as reference data for the heavy-ion program. The $\mathrm{d}+\mathrm{Au}$ collisions are used for studies of forward physics and to establish the cold nuclear matter baseline for heavy-ion collisions. The primary focus of the heavy-ion program is to quantify the differences between the hot, dense final state and scaled $p p$ and $\mathrm{d}+\mathrm{Au}$ reference data.

In $200 \mathrm{GeV} p p$ collisions, PHENIX [663, 979] has measured cross sections for production of the (unresolved) $\Upsilon$ states and the $\psi(2 S)$. A $\chi_{c J}$ measurement will be published soon. There is also a preliminary PHENIX [975] result showing that the $\Upsilon R_{A A}$, defined in Eq. (184)), measured in $\mathrm{Au}+\mathrm{Au}$ collisions is below 0.64 at $90 \% \mathrm{CL}$. All of these measurements involve low yields and will benefit greatly from additional luminosity. PHENIX [976] has also measured the polarization of the $J / \psi$ in $200 \mathrm{GeV} p p$ collisions and will also do so with existing $500 \mathrm{GeV}$ data.

So far, the high-statistics heavy quarkonium data available from RHIC are the $J / \psi$ data sets measured by PHENIX in $\sqrt{s_{N N}}=200 \mathrm{GeV} p p$ [976], $\mathrm{d}+\mathrm{Au}[663,883]$, $\mathrm{Cu}+\mathrm{Cu}$ [977], and $\mathrm{Au}+\mathrm{Au}$ [983] collisions. The data were measured in three rapidity ranges: $-2.2<y<-1.2$; $-0.5<y<0.5$; and $1.2<y<2.2$. There are also more recent data sets with much higher yields in $\mathrm{d}+\mathrm{Au}$ (2008) and $\mathrm{Au}+\mathrm{Au}$ (2007 and 2010) that are still being analyzed. While preliminary $J / \psi R_{C P}$ (see Eq. (185)) results were presented by PHENIX [663] at Quark Matter 2009 , the final data have not yet been released. Section 5.5 reviews the status of the $J / \psi$ program at RHIC.

STAR [1046] has measured $J / \psi$-hadron azimuthal angular correlations which have been used to infer the $B$ meson feeddown contribution to the $J / \psi$. STAR has also measured $R_{\mathrm{dAu}}$ for the combined $\Upsilon$ states using 2008 $\mathrm{d}+\mathrm{Au}$ [981] data and $2006 p p$ [980] data, and made measurements of the high $p_{T} J / \psi$ suppression factor, $R_{A A}$, from $\mathrm{Cu}+\mathrm{Cu}$ [664] collisions.

The ongoing RHIC luminosity upgrades will be completed by the 2013 run. The introduction in 2011 and 2012 of silicon vertex detectors into PHENIX at mid- and forward-rapidity, respectively, and of the STAR Heavy Flavor Tracker in 2014, will enable open charm and open bottom to be measured independently with greatly improved precision. The detector upgrades will also allow improved measurements of quarkonium states due to improved mass resolution and background rejection.

The RHIC run plan for the next 5 years or so will be centered on exploiting the capabilities of the new silicon vertex detectors and other upgrades, combined with the increased RHIC luminosity. Of greatest interest to inmedium heavy flavor physics, there will likely be long $p p, \mathrm{~d}+\mathrm{Au}$, and $\mathrm{Au}+\mathrm{Au}$ runs at $200 \mathrm{GeV}$, plus shorter runs with the same species at $62 \mathrm{GeV}$ to explore the energy dependence of open and hidden heavy flavor production. The luminosity increase and the PHENIX detector upgrades will enhance the PHENIX heavy quarkonium program in several ways: increased $p_{T}$ reach for the $J / \psi$; new studies of $J / \psi$ suppression with respect to the reaction plane; a first $J / \psi v_{2}$ measurement; better understanding of cold nuclear matter effects on $J / \psi$ production; and low-statistics measurements of the modification of the combined $\Upsilon$ states. The increased luminosity will enable the large-acceptance STAR detector to extend its $p_{T}$ reach to considerably higher values for $\Upsilon$ and $J / \psi$ measurements than previously possible.

The RHIC schedule for the period beyond about 5 years is still under development. RHIC experiments are presently engaged in preparing a decadal plan that will lay out their proposed science goals and detector upgrades for 2011 to 2020. PHENIX is considering a conceptual plan that would keep the new silicon vertex detectors but completely replace the central magnet and the outer central arm detectors. The new magnet would be a $2 \mathrm{~T}$ solenoid with an inner radius of $70 \mathrm{~cm}$. Two new silicon tracking layers would be placed inside the solenoid at 40 and $60 \mathrm{~cm}$, followed by a compact electromagnetic calorimeter of $8 \mathrm{~cm}$ depth and a preshower layer. A hadronic calorimeter would be added outside the magnet with an acceptance of $|\eta|<1$ and $2 \pi$ in azimuth. The conceptual design is still being evaluated, but 
it promises to allow powerful measurements of light quark and gluon jets; dijets and $\gamma+$ jet coincidences; charm and bottom jets; the $J / \psi$ modification factor over a range of energies; simultaneous studies of the modification of the three bound $\Upsilon$ states; and direct $\gamma^{*}$ flow. Removing the south muon spectrometer and replacing it with an electron/photon endcap spectrometer has also been discussed. This replacement would be aimed at addressing spin physics questions and possibly providing electronion capabilities in PHENIX. The north muon spectrometer would be retained to provide forward rapidity heavy flavor measurements.

In addition to the Heavy Flavor Tracker, STAR will add a new detector that is of major importance to their quarkonium program. The Muon Telescope Detector will be a large acceptance muon detector located outside the STAR magnet at midrapidity, covering $|y|<0.5$. It will provide a good signal-to-background ratio for measurements of the three $\Upsilon(n S)$ states and add the capability of measuring $J / \psi$ elliptic flow and suppression at high $p_{T}$. The upgrades being pursued by PHENIX and STAR, combined with very high RHIC luminosity, will provide the opportunity to compare, between RHIC and the LHC, in-medium quarkonium modification at energies of 62,200 , and $5500 \mathrm{GeV}$. This energy regime spans a wide range of medium temperatures and lifetimes, and also provides measurements with very different underlying heavy quark rapidity densities and thus very different contributions to quarkonia from processes involving coalescence of heavy quarks from different hard collisions.

\subsection{Super Flavor Factories}

At $e^{+} e^{-}$machines, quarkonium can be produced through several processes: directly, i.e., during energy scans for $J^{P C}=1^{--}$states in the $c \bar{c}$ and $b \bar{b}$ regions; through ISR (for $J^{P C}=1^{--}$states below the $e^{+} e^{-}$ center-of-mass energy) or two-photon (for $C=+1$ states) processes; in $B$-meson decays through color-suppressed $b \rightarrow c$ transitions. All these have been successfully employed for quarkonium studies in the CLEO, BABAR, Belle, and/or BESIII experiments, the first two of which permanently ceased taking data in early 2008. Belle acquired data until the middle of 2010 , and then shut down for a significant upgrade. CLEO-c collected $48 \mathrm{pb}^{-1}$ at the $\psi(2 S)$ (about 27 million $\psi(2 S)$ produced) and $1485 \mathrm{pb}^{-1}$ in the CM energy range $3.67-4.26 \mathrm{GeV}$; BESIII has already quadrupled the CLEO-c $\psi(2 S)$ sample, acquired $200 \mathrm{M} J / \psi$ decays, and will, in time, exceed the CLEO-c samples above open-charm threshold as well (Sect. 6.1). What will happen to $e^{+} e^{-}$quarkonium physics after BESIII and Belle programs are complete?

A new generation of Super Flavor Factories has recently been proposed in order to perform precision measurements in the flavor sector and complement New Physics (NP) searches at hadronic machines [1047, 1048]. An increase in statistics by a factor of 50-100 with respect to the current generation of still-running experiments is essential to such physics program.

Two different approaches have been devised to reach a design peak luminosity of $10^{36} \mathrm{~cm}^{-2} \mathrm{~s}^{-1}$. In the original SuperKEKB [1047] design this was to be achieved by increasing the beam currents, and introducing crab crossing to maintain large beam-beam parameters [1049]. In the SuperB design [1048] a similar luminosity goal is pursued through the reduction of the interaction point size using very small emittance beams, and with a "crab" of the focal plane to compensate for a large crossing angle and mantain optimal collisions [1050]. After KEK revised their design in favor of the nanobeam collision option [1050], the machine parameters for the SuperB factory and the KEKB upgrade are very similar [1048, 1049].

An NP-oriented program suggests that the machine be operated primarily at the $\Upsilon(4 S)$ resonance [1051], with integrated luminosity of order $25-75 \mathrm{ab}^{-1}$. However, the ability to run at other $\Upsilon$ resonances or at energies in the $c \bar{c}$ region would substantially enhance the physics potential of the machine. In one month at design luminosities it would be possible to collect about $150 \mathrm{fb}^{-1}$ at $D \bar{D}$ threshold [1048]. In about the same time, an $\Upsilon(5 S)$ run would integrate about $1 \mathrm{ab}^{-1}$, corresponding to a "short run" scenario. In a "long run" scenario at $\Upsilon(5 S)$, about $30 \mathrm{ab}^{-1}$ could be collected [1048].

The detector design will primarily address the requirements of an NP search program at the $\Upsilon(4 S)$. Other constraints are posed by the possibility to re-use components from the $B A B A R$ and Belle detectors. Improvements in the vertex resolution, to compensate a reduced beam asymmetry, as well as increased hermeticity of the detector are foreseen. Assuming the same magnetic field, a similar momentum resolution is expected. In a simplified approach, one can assume similar backgrounds and detector performances as at BABAR [1052] and Belle [1053].

A heavy-quarkonium to-do list in a Super Flavor Factory physics program can be found in the Spectroscopy (Sect. 2), Decays (Sect. 3), and Summary (Sect. 7) sections of this review. Precision measurements or simply observation of some conventional, expected processes is warranted; the as-yet-unexplained phenomena also demand attention and offer great reward. Highlights of such a program would include the following:

- Precision measurements of $\eta_{c}(1 S)$ and $\eta_{c}(2 S)$ masses and widths (probably in $\gamma \gamma$-fusion), understanding of their observed lineshapes in radiative $J / \psi$ and $\psi(2 S)$ decays, and a comprehensive inventory of radiative and hadronic decay modes and respective branching fractions (Sects. 2.2.2, 3.1.2 and Table 4).

- First observation and study of $\eta_{b}(2 S), h_{b}\left({ }^{1} P_{1}\right)$, and $\Upsilon\left(1^{3} D_{J}\right)(J=1,3)$ (Sect. 2.2.7 and Tables 4 and $8)$.

- For $X(3872)$ (see Sect. 2.3.1 and Tables 9, 10, 11, 12 , and 13 ), detailed study of the $\pi^{+} \pi^{-} J / \psi$ and 
$D^{* 0} \bar{D}^{0}$ lineshapes, high-statistics measurements of $\gamma J / \psi$ and $\gamma \psi(2 S)$ branching fractions, and a search for decay to $\pi^{0} \pi^{0} J / \psi$. The fruit of such an effort could be an answer to how much tetraquark, molecular, and/or conventional charmonium content this state contains.

- For the $Y(4260)$ and nearby states (see Sect. 2.3.2 and Tables 9, 14, 15, and 16), a comprehensive lineshape measurement accompanied by precision branching fractions could shed light on the molecular, tetraquark, or hybrid hypotheses.

- Confirmation and study of the exotic charged $Z^{+}$ states (Sect. 2.3.4 and Table 9).

- Confirmation and study of the $Y_{b}$ (Sect. 2.3.2 and Table 9). A high-statistics scan of $20 \mathrm{fb}^{-1}$ per point, necessary to reduce the relative error to the $10^{-3}$ level, might be needed [1054]. If the $Y_{b}$ is below $10800 \mathrm{MeV}$, it could also be produced by ISR with $\Upsilon(5 S)$ data collected by a Super $B$-factory. If it is above that energy, a direct scan will be necessary.

\section{8. $\overline{P A N D A}$}

$\bar{P} A N D A$ is one of the major projects at the future Facility for Antiproton and Ion Research (FAIR) [1055] at GSI in Darmstadt, which is expected to start its operations in 2014. PANDA will use the antiprotons circulating in the High Energy Storage Ring (HESR), to study their interactions with protons or nuclei on a fixed target. The antiproton momentum in the range 1.5 to $15 \mathrm{GeV} / c$ corresponds to a center-of-mass energy in $p \bar{p}$ collisions in the range $2.5-5.5 \mathrm{GeV}$. The purpose of the $\bar{P} A N D A$ experiment is to investigate QCD in the nonperturbative regime. This is achieved through the study of several topics, like QCD bound states, nonperturbative QCD dynamics, study of hadrons in nuclear matter, hypernuclear physics, electromagnetic processes, and electroweak physics. In particular, PANDA has an extensive research program in charmonium physics. Experimentally, charmonium has been studied mainly in $e^{+} e^{-}$and $p \bar{p}$ experiments. While in $e^{+} e^{-}$collisions, only the $J^{P C}=1^{--}$ states can be directly formed, in $p \bar{p}$ interactions, direct formation is possible for all the states with different quantum numbers, through coherent annihilation of the three quarks of the protons with the three antiquarks of the antiproton. An additional advantage of this technique is that the interaction energy can be precisely determined from the beam parameters and it is not limited by the detector resolution, allowing fine energy scans of narrow resonances. Historically, this experimental technique was successfully used at CERN and at Fermilab. With a higher luminosity and a better beam energy resolution with respect to previous $p \bar{p}$ experiments, $\overline{\text { PANDA will be }}$ able to obtain high-precision data on charmonia and measure the masses, widths, and excitation curves for the recently observed states with unprecedented precision.

\subsubsection{Experimental technique}

Quarkonium physics is one of the main research fields for PANDA. The precision study of resonance parameters and excitation curves is an area where the close interplay between machine and detector is fundamental. For the PANDA charmonium program, the antiproton beam collides with a fixed hydrogen target. In order to achieve the design luminosity $\left(2 \times 10^{32} \mathrm{~cm}^{-2} \mathrm{~s}^{-1}\right)$, a high-density target thickness is needed. Two solutions are under study: the cluster-jet target and the pellet target, with different implications for the beam quality and the definition of the interaction point.

Thanks to the cooling of the antiproton beam, at the HESR the energy spread of the beam will be approximately $30 \mathrm{keV}$, which is comparable with the width of the narrowest charmonia states. The knowledge of the total interaction energy from the beam parameters and the narrowness of the beam energy distribution allow the direct measurement of the mass and the width of narrow states through scans of the excitation curve.

The resonance parameters are determined from a maximum-likelihood fit to the number of observed events in a specific channel $N_{i}$, where the subscript $i$ refers to different center-of-mass energies:

$$
N_{i}=\epsilon_{i} \mathcal{L} \int \sigma_{B W}\left(E^{\prime}\right) B_{i}\left(E^{\prime}\right) d E^{\prime}
$$

where $\sigma_{B W}$ is the Breit-Wigner resonance cross section to be measured and $B_{i}$ is the center-of-mass energy distribution from beam parameters.

The energy scan of a resonance is schematically represented in Fig. 104: the energy of the interaction, shown on the horizontal axis, is obtained from the beam parameters, and is varied in the energy region to be explored. The detector is used as a simple event counter and the cross section values (vertical axis) can be obtained from the number of events observed at each energy point. Using Eq. (194), it is then possible to determine the resonance parameters. By means of fine scans it will be possible to measure masses with accuracies of the order of $100 \mathrm{keV}$ and widths at the $10 \%$ level. The entire region below and above the open charm threshold (from $2.5 \mathrm{GeV}$ to $5.5 \mathrm{GeV}$ in the center-of-mass energy) will be explored.

As described above, antiproton-proton formation will become an important tool for decisive studies of the natural widths and lineshapes of open and hidden charm states. It will also have a powerful role in measuring decay modes of very narrow states. Unfortunately, charm production cross sections in proton-antiproton annihilation in this energy regime are either unknown or, at best, poorly measured. Thus predictions for sensitivities 


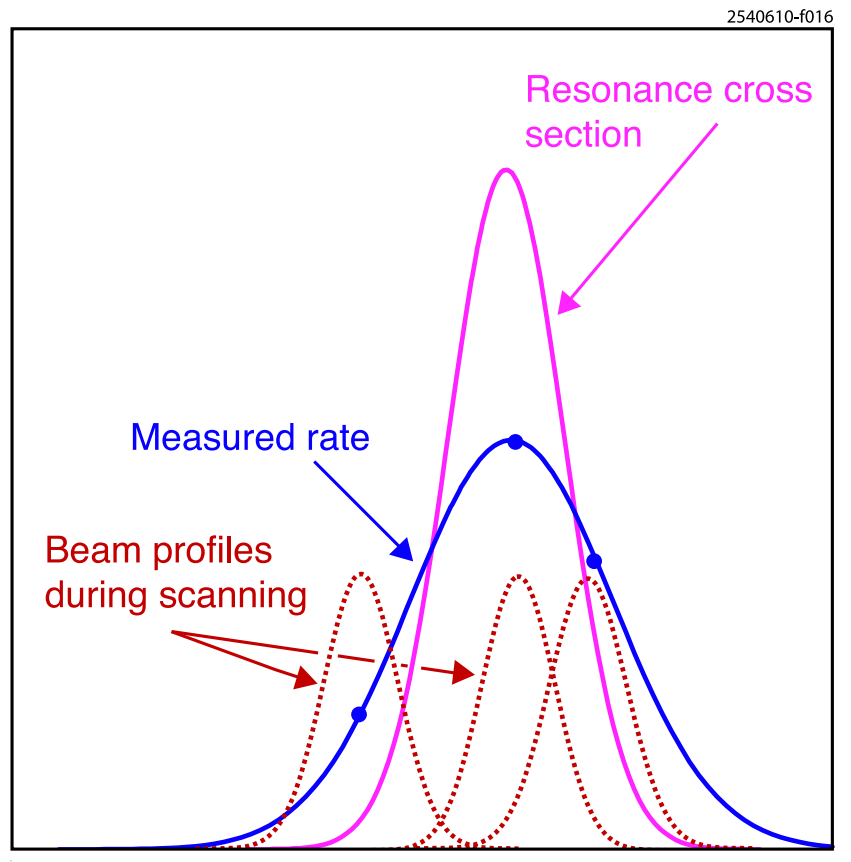

FIG. 104: Schematic representation of the scan of a resonance (lighter solid line): the center-of-mass energy is varied with the HESR to several values across the resonance (the energy distributions are shown by (dotted lines). The solid circles represent the observed cross section for each energy point, defining the measured excitation curve (darker solid line), which is the convolution of the resonance curve and the beam profile

are not reliable. In order to provide experimental input to event generators, and thereby allow credible sensivity studies, it would be worthwhile to measure inclusive open charm production at the existing antiproton facility at Fermilab with a relatively simple and small test apparatus equipped with a microvertex detector and a tracking device. Such an experiment would likely require less than a month of datataking to acquire a sufficient statistics.

\subsubsection{Detector}

The $\bar{P}$ ANDA detector is a multipurpose detector, designed to obtain the highest possible acceptance, good tracking resolution, particle identification and calorimetry. It is composed of two parts: a target spectrometer with an axial field generated by a superconducting solenoid for the measurement of the particles emitted at large angles with respect to the beam direction, and a forward spectrometer with a dipole magnet in the forward direction for the measurement of the particles leaving the interaction region at small angles with respect to the beam.

The target spectrometer is arranged in a barrel part, for angles larger than $22^{\circ}$, and an endcap part, covering the forward region down to $5^{\circ}$ in the vertical plane and $10^{\circ}$ in the horizontal plane. A silicon tracker is located close to the interaction region. It is composed of two inner layers of hybrid pixel detectors and two outer layers with silicon strip detectors, with cylindrical symmetry around the beam pipe. In addition, six silicon discs with both pixel and strip modules are located in the forward direction. The tracking system (solutions with straw tubes detector or TPC are under study) is required to provide momentum measurement with resolution $\delta p / p$ at the percent level, handling the high particle fluxes that are foreseen at the maximum luminosity. In the forward part of the target spectrometer, particles exiting the interaction region at polar angles below $22^{\circ}$ are tracked with 3 GEM detectors placed downstream. Charged particle identification is obtained by collecting complementary information from different detectors. Most charged particles with momentum exceeding $0.8 \mathrm{GeV} / c$ are identified using a Cherenkov detector. A detector of internally-reflected Cherenkov light based on the BABAR design [1056] will provide particle identification in the region $22^{\circ}$ to $140^{\circ}$. Time-of-flight will be also used in PANDA for the identification of lowmomentum particles. To obtain muon-pion separation, the yoke of the superconducting solenoid is segmented in 12 layers and the gaps are instrumented for the measurement of the interaction length in iron. Electromagnetic calorimetry is required over a wide energy range, from the $\mathrm{MeV}$ to the $\mathrm{GeV}$ scale. A lead-tungstate $\left(\mathrm{PbWO}_{4}\right)$ calorimeter, with crystal length corresponding to $22 X_{0}$, will measure energy with a resolution better than $2 \%$ at $2 \mathrm{GeV}$ and good time resolution.

A dipole magnet with $1 \mathrm{~m}$ gap and $2 \mathrm{~m}$ aperture is located between the target and the forward spectrometer. The measurement of the deflection of charged tracks is obtained by using a set of wire chambers placed before, within, and behind the dipole magnet. The expected $\delta p / p \sim 3 \%$ for $3 \mathrm{GeV} / c$ protons is limited by the scattering on gas and wires in the chambers. In the forward spectrometer, RICH or time-of-flight detectors are proposed as particle identification systems. Electromagnetic calorimetry in the forward region will be performed by a shashlyk-type calorimeter with high resolution and efficiency. An energy resolution of $4 \% / \sqrt{E}$ is foreseen. In the very forward part, a muon detector similar to the one employed in the target region will provide muon-pion separation.

\subsubsection{Charmonium and open charm physics}

The study of charmonium and open charm are among the main topics in the $\bar{P} A N D A$ physics program. Compared to Fermilab E760/E835, a multipurpose detector with magnetic field, $\bar{P}$ ANDA has better momentum resolution and higher machine luminosity, which will allow the realization of an extensive research program in these 
fields.

The existence of a large hadronic background in $p \bar{p}$ annihilation represents a challenge for the study of many final states and the capability of observing a particular final state depends on the signal-to-background ratio after the selection. A full detector simulation has been developed and used to test the separation of signal from background sources, with cross sections that are orders of magnitude larger than the channels of interest, and to prove the capability of background reduction at the level needed to perform charmonium studies in $\bar{P} A N D A$. Here we will summarize the results, which appear in more detail in the PANDA Physics Performance Report [1057], of using the full simulation on some channels of interest. In all the following cases, a simple selection is adopted for the channel of interest: particles are reconstructed starting from detected tracks, using associated PID criteria, and a kinematic constraint to the energy and momentum of the beam is imposed in order to improve on detector resolutions.

The identification of charmonium states decaying into $J / \psi$ is relatively clean due to the presence of a pair of leptons $\ell^{+} \ell^{-}$in the final state. These channels can be used to study decays from $\psi(2 S), \chi_{c J}, X(3872)$, and $Y(4260)$ that contain a $J / \psi$ in the final state. These analyses rely on the positive identification of the two leptons in the final state for the reconstruction of $J / \psi \rightarrow \ell^{+} \ell^{-}$, where the main background is represented by pairs of tracks, like $\pi^{+} \pi^{-}$, associated with large energy deposition in the electromagnetic calorimeter. The simulation shows that the resolution of the $e^{+} e^{-}$invariant mass for a reconstructed $J / \psi$ in the final state is in the range $4-8 \mathrm{MeV}$, depending on the total center-of-mass energy of the interaction.

The $J / \psi \pi^{+} \pi^{-}$final state is a key decay channel in charmonium studies. New states in the charmonia mass region, like the $X(3872)$ and the $Y(4260)$, have been discovered at the $B$-factories through this decay mode (see Sect. 2.3). The $\overline{\mathrm{P} A N D A}$ performance on $J / \psi \pi^{+} \pi^{-}$has been analyzed in detail through a complete simulation of this final state in the detector. A simple selection has been performed: adding two charged pions to a reconstructed $J / \psi$ and performing a kinematic fit with vertex constraint, the efficiency of the complete selection is approximately $30 \%$ over the energy region of interest (3.5 to $5.0 \mathrm{GeV}$ ). The main source of hadronic background for this channel comes from $p \bar{p} \rightarrow \pi^{+} \pi^{-} \pi^{+} \pi^{-}$, where two pions may be erroneously identified as electrons and contaminate the signal. At a center-of-mass energy around $4.26 \mathrm{GeV}$, the cross section for this process is a few tens of $\mu \mathrm{b}$ [1058], which is $10^{6}$ times larger than the expected signal, estimated from previous results of Fermilab E835. With the selection described, the rejection power for this background source is of the order of $10^{6}$ and the signalto-background ratio is about 2 , which should provide well-identified and relatively clean $J / \psi \pi^{+} \pi^{-}$final states in $\bar{P} A N D A$.

The discovery mode (see Sect. 2.2.1) for $h_{c}(1 P)$ is the electromagnetic transition to the ground state charmonia $h_{c}(1 P) \rightarrow \eta_{c}(1 S) \gamma$, where the $\eta_{c}(1 S)$ can then be detected through many decay modes. The decay $\eta_{c}(1 S) \rightarrow \gamma \gamma$ is characterized by a reasonably clean signature, due to the presence of two energetic photons in the detector with the $\eta_{c}(1 S)$ mass, albeit with a small branching fraction (see Sect. 3.2.5). A study has been performed to assess the $\bar{P}$ ANDA capability to detect the $h_{c}(1 P) \rightarrow \eta_{c}(1 S) \gamma \rightarrow 3 \gamma$ decay, in presence of background sources due to final states such as $p \bar{p} \rightarrow \pi^{0} \pi^{0}$, $\pi^{0} \eta$, and $\eta \eta$. Each presents hard photons in the final state and no charged tracks, a signature that could mimic the channel of interest. (See Sect. 2.2.1 for a description of a previous $p \bar{p} \rightarrow h_{c}(1 P) \rightarrow \gamma \eta_{c}(2 S) \rightarrow 3 \gamma$ analysis and Sect. 3.2.3 for a description of a $J / \psi \rightarrow 3 \gamma$ observation in $\psi(2 S) \rightarrow \pi^{+} \pi^{-} J / \psi$ decays.) In order to improve the background rejection, additional cuts are applied after the event reconstruction:

- a cut on the center-of-mass energy of the energy of the $\gamma$ coming from the radiative transition: $0.4<$ $E_{\gamma}<0.6 \mathrm{GeV}$;

- an angular cut $\left|\cos \theta_{C M}\right|<0.6$ allows rejection of a large fraction of backgrounds (like $\pi^{0} \pi^{0}$ ) that are strongly peaked in the forward direction;

- to suppress $\eta^{\prime}$ decays, the invariant mass of the radiative $\gamma$ paired with either photon coming from the decay of the $\eta_{c}(1 S)$ candidate is required to be larger than $1 \mathrm{GeV}$.

After these cuts, the efficiency on the signal is about $8 \%$ and the background suppression of the order of $10^{-6}$ or larger on many background channels. The production cross section observed by E835 [48], although with large uncertainties, can be combined with the present background suppression, to obtain an estimate of the order of 90 or more for the signal-to-background ratio.

As a benchmark channel of hadronic decays, we consider $h_{c}(1 P) \rightarrow \eta_{c}(1 S) \gamma \rightarrow \phi \phi \gamma$, with $\phi \rightarrow K^{+} K^{-}$. Three reactions are considered to be dominant contributions to the background: $p \bar{p} \rightarrow K^{+} K^{-} K^{+} K^{-} \pi^{0}$, $p \bar{p} \rightarrow \phi K^{+} K^{-} \pi^{0}$, and $p \bar{p} \rightarrow \phi \phi \pi^{0}$, with one photon from the $\pi^{0}$ undetected. To suppress such background, it is additionally required that no $\pi^{0}$ candidates (photon pair with invariant mass in the 0.115$0.150 \mathrm{GeV}$ region) be present in the event. The overall efficiency for signal events is $\sim 25 \%$. Since no experimental data is available for the three background cross sections, the only way to estimate the background contribution is to use the dual parton model (DPM); none out of $2 \times 10^{7}$ simulated events pass the selection. The three main background channels have been also simulated separately. With a total $p \bar{p}$ cross section of $60 \mathrm{mb}$, we estimate that $\sigma\left(p \bar{p} \rightarrow K^{+} K^{-} K^{+} K^{-} \pi^{0}\right)=345 \mathrm{nb}$, $\sigma\left(p \bar{p} \rightarrow \phi K^{+} K^{-} \pi^{0}\right)=60 \mathrm{nb}$, and $\sigma\left(p \bar{p} \rightarrow \phi \phi \pi^{0}\right)=3 \mathrm{nb}$. Using these values, a signal-to-background ratio of $\geq 8$ for each of the background channels is obtained. Using 
these signal-to-background values, it is possible to estimate the PANDA sensitivity in the $h_{c}(1 P)$ width measurement. A few scans of the $h_{c}(1 P)$ have been simulated for different values of $\Gamma\left(h_{c}(1 P)\right)$. The expected shape of the measured cross section is obtained from the convolution of the Breit-Wigner resonance curve with the normalised beam energy distribution plus a background term. The simulated $h_{c}(1 P)$ resonance shape for the case $\Gamma\left(h_{c}(1 P)\right)=0.5 \mathrm{MeV}$, assuming 5 days of data taking per point in high-resolution mode, is shown in Fig. 105. The accuracy on the width measurement is of the order of $0.2 \mathrm{MeV}$ for $\Gamma\left(h_{c}(1 P)\right)$ values in the range $0.5-1.0 \mathrm{MeV}$.

The ability to study charmonium states above $D \bar{D}$ threshold is important for the major part of the PANDA physics program, in topics like the study of open charm spectroscopy, the search for hybrids, and $\mathrm{CP}$ violation studies. The study of $p \bar{p} \rightarrow D \bar{D}$ as a benchmark channel will also assess the capability to separate a hadronic decay channel from a large source of hadronic background. Two benchmark channels are studied in detail:

$$
\begin{aligned}
& \text { - } p \bar{p} \rightarrow D^{+} D^{-}\left(\text {with } D^{+} \rightarrow K^{-} \pi^{+} \pi^{+}\right) \\
& \text {- } p \bar{p} \rightarrow D^{*+} D^{*-}\left(\text { with } D^{*+} \rightarrow D^{0} \pi^{+}, D^{0} \rightarrow K^{-} \pi^{+}\right)
\end{aligned}
$$

The first one is simulated at the $\psi(3770)$ and the second at the $\psi(4040)$ mass energies. We assume a conservative estimate for the charmonium production cross section above the open-charm threshold, on the order of $3 \mathrm{nb}$ for $D^{+} D^{-}$and $0.9 \mathrm{nb}$ for $D^{*+} D^{*-}$ production. The background is simulated using the DPM to produce inelastic reaction in $p \bar{p}$ annihilations. A background suppression of the order of $10^{7}$ is achieved with the previous selection. A detailed study of specific background reactions is also performed. In particular, nonresonant production of $K^{+} K^{-} 2 \pi^{+} 2 \pi^{-}$has a cross section which is $10^{6}$ times

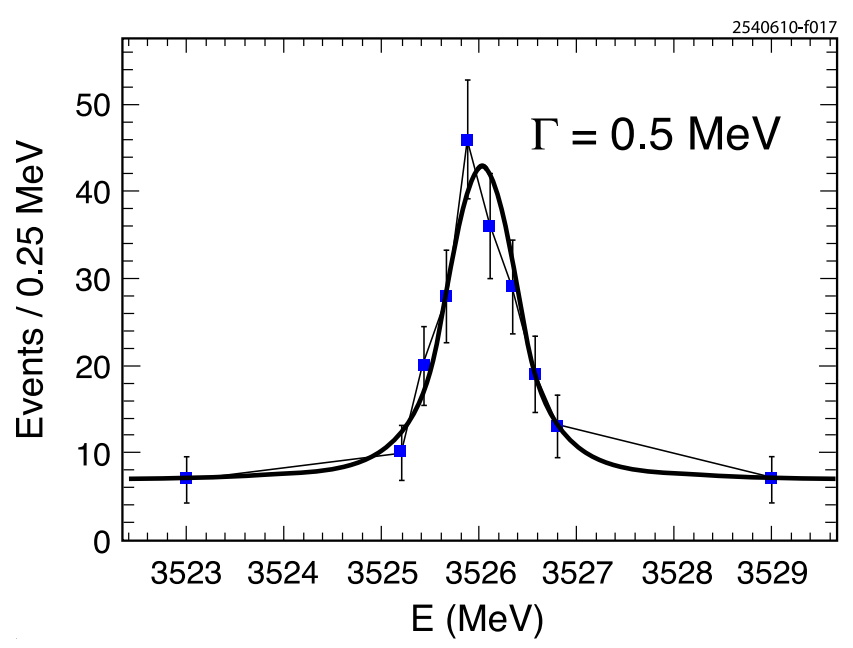

FIG. 105: Simulation of the scan of the $h_{c}(1 P)$ (with $\left.\Gamma\left(h_{c}(1 P)\right)=0.5 \mathrm{MeV}\right)$ for the measurement of the resonance width. Each point corresponds to 5 days of data-taking with PANDA larger than the $D^{+} D^{-}$signal. A cut on the longitudinal and transverse momentum of the $D^{ \pm}$can reduce the background by a factor $\sim 26$, and the remaining events leave a nonpeaking background in the loose mass region defined in the preselection. The reconstructed decay vertex location will further improve the background rejection, reaching a signal-to-background ratio near unity with an efficiency for signal events of $\approx 8 \%$. Under these assumptions, a conservative estimate of the number of reconstructed events per year of PANDA operation is of the order of $10^{4}$ and $10^{3}$ for $D^{+} D^{-}$and $D^{*+} D^{*-}$, respectively.

Performing fine energy scans, $\bar{P}$ ANDA will be able to observe the energy dependence of a cross section in proximity to a threshold. Here we report the result obtained in the simulation of $p \bar{p} \rightarrow D_{s}^{ \pm} D_{s 0}^{*}(2317)^{\mp}$, to test the sensitivity to resonance parameters measurements with this technique. The assumptions used in this study are:

- a 12-point scan in a $4 \mathrm{MeV}$-wide region;

- 14 days of data with a total integrated luminosity of $9 \mathrm{pb}^{-1} /$ day;

- a signal-to-background ratio of 1:3;

- $1 \mathrm{MeV}$ total width for the $D_{s 0}^{*}(2317)$.

The results of a fit to the scan simulation are presented in Fig. 106, where the mass and the width of the $D_{s 0}^{*}(2317)$ are free parameters. The study yields the results:

$$
\begin{aligned}
m & =2317.41 \pm 0.53 \mathrm{MeV}, \\
\Gamma & =1.16 \pm 0.30 \mathrm{MeV},
\end{aligned}
$$

to be compared with the input values of the simulation: $m=2317.30 \mathrm{MeV}$ and $\Gamma=1.00 \mathrm{MeV}$, demonstrating that such a scan would yield accurate values of mass and width with the precisions shown.

In conventional charmonia, the quantum numbers are derived directly from the excitation of the $c \bar{c}$ pair. The glue tube adds degrees of freedom that manifest themselves in unconventional quantum numbers; in the simplest $c \bar{c} g$ scenario this corresponds to the addition of a single gluon quantum number $\left(J^{P C}=1^{-}\right.$or $1^{+}$for colorelectric or color-magnetic excitation). This would result in charmonium hybrids with non-exotic and exotic quantum numbers which are expected in the $3-5 \mathrm{GeV}$ mass region. Here we will sketch out the strategy for hybrids studies in PANDA, with more details available in [1057]. Formation experiments would generate non-exotic charmonium hybrids with high cross sections while production experiments would yield a hybrid together with another particle like $\pi$ or $\eta$. In PANDA both processes are possible; the strategy would be to start searching for hybrids in production processes, fixing the $p \bar{p}$ center-ofmass energy at the highest possible value $(\sqrt{s} \simeq 5.5 \mathrm{GeV})$ and studying all the production channels. Then hybrid formation could be studied through energy scans over the regions where possible signals have been observed in production measurements. 


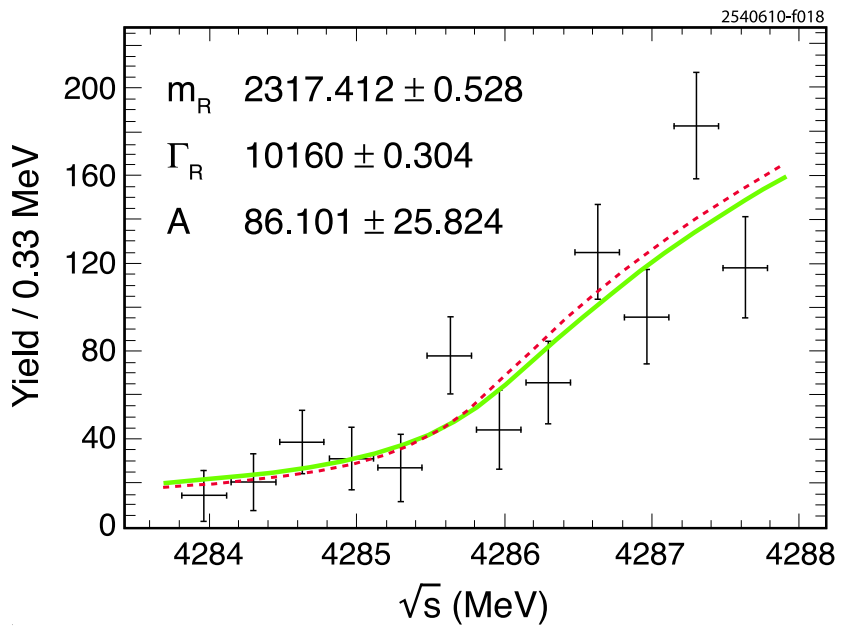

FIG. 106: Fit of the simulated excitation function for nearthreshold $p \bar{p} \rightarrow D_{s}^{ \pm} D_{s 0}^{*}(2317)^{\mp}$. The dashed line corresponds to the simulated function and the solid line is the reconstructed curve

Aside from the benchmark channels used for the detection of conventional charmonia, hybrids can be identified through reactions like:

$$
\begin{aligned}
& \text { - } p \bar{p} \rightarrow \widetilde{\eta}_{c 0,1,2} \eta \rightarrow \chi_{c 1} \pi^{0} \pi^{0} \eta \\
& \text { - } p \bar{p} \rightarrow \widetilde{h}_{c 0,1,2} \eta \rightarrow \chi_{c 1} \pi^{0} \pi^{0} \eta \\
& \text { - } p \bar{p} \rightarrow \widetilde{\psi} \eta \rightarrow J / \psi \omega \eta ; \\
& \text { - } p \bar{p} \rightarrow\left[\widetilde{\eta}_{c 0,1,2}, \widetilde{h}_{c 0,1,2}, \widetilde{\chi_{c 1}}\right] \eta \rightarrow D D^{*} \eta
\end{aligned}
$$

namely final states with charmonia accompanied by light hadrons or final states with a $D D^{*}$ pair. As a case study, we will present the results obtained for the benchmark channel $p \bar{p} \rightarrow \widetilde{\eta}_{c 1} \eta \rightarrow \chi_{c 1} \pi^{0} \pi^{0} \eta$. It can be assumed that the $p \bar{p} \rightarrow \widetilde{\eta}_{c 1} \eta$ production cross section is of the same order of $p \bar{p} \rightarrow \psi(2 S) \eta$, which is estimated to be $33 \pm$ $8 \mathrm{pb}$ [1059]. As possible sources of background, several reactions with similar topology have been considered:

$$
\begin{aligned}
& \text { - } p \bar{p} \rightarrow \chi_{c 0}(1 P) \pi^{0} \pi^{0} \eta \\
& \text { - } p \bar{p} \rightarrow \chi_{c 1}(1 P) \pi^{0} \eta \eta \\
& \text { - } p \bar{p} \rightarrow \chi_{c 0}(1 P) \pi^{0} \pi^{0} \pi^{0} \eta \\
& \text { - } p \bar{p} \rightarrow J / \psi \pi^{0} \pi^{0} \pi^{0} \eta
\end{aligned}
$$

A simple analysis is carried out. Two photons are accepted as $\pi^{0}$ or $\eta$ candidates if their invariant mass is in the range $115-150 \mathrm{MeV}$ or $470-610 \mathrm{MeV}$, respectively. The $\chi_{c 1}$ is formed adding a radiative photon to a $J / \psi$ candidate, with total invariant mass within 3.3-3.7 GeV. From these, $\chi_{c 1} \pi^{0} \pi^{0} \eta$ candidates are created and kinematically fit to the original beam energymomentum, with an additional constraint for the $\mathrm{J} / \psi$ mass. Additional cuts on the kinematic fit CL and on the invariant masses of the intermediate decay products are applied, obtaining a total efficiency around $7 \%$ for this channel. The $\widetilde{\eta}_{c 1}$ peak reconstructed in this way has a FWHM of $30 \mathrm{MeV}$. The background suppression is estimated applying the same analysis to background events. The results are summarized in Table 49.

The $J / \psi N$ dissociation cross section is as yet experimentally unknown, except for indirect information deduced from high-energy $J / \psi$ production from nuclear targets. Apart from being a quantity of its own interest, this cross section is closely related to the attempt of identifying quark-gluon plasma (QGP) formation in ultra-relativistic nucleus-nucleus collisions: the interpretation of the $J / \psi$ suppression observed at the CERN SPS [966, 968, 1060] as a signal for QGP formation relies on the knowledge of the "normal" suppression effect due to $J / \psi$ dissociation in a hadronic environment. Nuclear $J / \psi$ absorption can only be deduced from models, since the available data do not cover the kinematic regime relevant for the interpretation of the SPS results. In antiproton-nucleus collisions the $J / \psi N$ dissociation cross section can be determined for momenta around $4 \mathrm{GeV} / c$ with very little model dependence. The determination of the $J / \psi N$ dissociation cross section is in principle straightforward: the $J / \psi$ production cross section is measured for different target nuclei of mass number ranging from light $(\mathrm{d})$ to heavy (Xe or $\mathrm{Au}$ ) by scanning the $\bar{p}$ momentum across the $J / \psi$ yield profile whose width is essentially given by the known internal target-nucleon momentum distribution. The $J / \psi$ is identified by its decay to $e^{+} e^{-}$or $\mu^{+} \mu^{-}$. The attenuation of the $J / \psi$ yield per effective target proton is a direct measure of the $J / \psi N$ dissociation cross section, which can be deduced by a Glauber-type analysis. These studies may be extended to higher charmonium states like the $\psi(2 S)$, which would allow determination of the cross section for the inelastic process $\psi(2 S) N \rightarrow J / \psi N$, which is also relevant for the interpretation of the ultrarelativistic heavy-ion data. The benchmark channel studied in this context is the reaction:

$$
\bar{p}{ }^{40} \mathrm{Ca} \rightarrow J / \psi X \rightarrow e^{+} e^{-} X .
$$

The cross section for this process is estimated to be nine orders of magnitude smaller than the total antiprotonnucleus cross section. The results of the simulations show that it is possible to identify the channel of interest with good efficiency and acceptable signal-to-background ra-

TABLE 49: Background suppression $(\eta)$ for the individual background reactions

\begin{tabular}{lc}
\hline \hline Background channel & Suppression $\left(10^{3}\right)$ \\
\hline$\chi_{c 0}(1 P) \pi^{0} \pi^{0} \eta$ & 5.3 \\
$\chi_{c 1}(1 P) \pi^{0} \eta \eta$ & 26 \\
$\chi_{c 0}(1 P) \pi^{0} \pi^{0} \pi^{0} \eta$ & $>80$ \\
$J / \psi \pi^{0} \pi^{0} \pi^{0} \eta$ & 10 \\
\hline \hline
\end{tabular}


tio [1057].

\subsection{CBM at FAIR}

The Compressed Baryonic Matter (CBM) experiment will be one of the major scientific activities at FAIR [1055]. The goal of the CBM research program is to explore the QCD phase diagram in the region of high baryon densities using high-energy nucleus-nucleus collisions. This includes a study of the equation-of-state of nuclear matter at high densities, and a search for the deconfinement and chiral phase transitions. The CBM research program comprises a comprehensive scan of observables, beam energies, and collision systems. The observables include low mass dileptons, charmonia and open charm, but also collective flow of rare and bulk particles, correlations and fluctuations.

Particles with open and hidden charm are expected to provide valuable information about the conditions inside the dense fireball. For example, the excitation function of the charm particle ratios such as the $\psi(2 S) /(J / \psi)$ ratio and the $(J / \psi) / D$ ratio may vary when passing the deconfinement phase transition. In addition, the initial pressure of the partonic phase influences the elliptic flow of charmonium. The transport properties of open charm mesons in dense matter, which depend on the interaction with the medium and hence on the structure of the medium, can be studied via the yield, the elliptic flow, and the momentum distributions of charmed particles. In a baryon-dominated medium, these observables are expected to differ for $D$ and $\bar{D}$ mesons.

The experimental goal is to measure these rare probes with unprecedented precision. In order to compensate for the low yields, the measurements will be performed at exceptionally high reaction rates (up to $10 \mathrm{MHz}$ for certain observables). These conditions require the development of ultrafast and extremely radiation-hard detectors and electronics. A particular challenge for the detectors, the front-end electronics, and the data-acquisition system is the online selection of displaced vertices with the extraordinarily high speed and precision needed for open-charm measurements.

A schematic view of the proposed CBM experimental facility is shown in Fig. 107. The core of the setup is a Silicon Tracking and Vertexing System located inside a large aperture dipole magnet. The vertex detector consists of 2 stations of Monolithic Active Pixel Sensors, and the tracker comprises 8 stations of doublesided microstrip sensors. Particle identification will be performed using the momentum information from the silicon tracker and the time-of-flight measured with a large area Resistive Plate Chamber (RPC) wall. Figure 107(a) depicts the setup with the Ring Imaging Cherenkov (RICH) detector for the identification of electrons from low-mass vector-meson decays. The Transition Radiation Detector (TRD) will provide charged particle tracking and the identification of high-energy elec- trons and positrons. The Electromagnetic Calorimeter (ECAL) will be used for the identification of electrons and photons. The muon detection/hadron absorber system is shown in Fig. 107(b). It consists of 5 doubleor triple-stations of highly-granulated gaseous micropattern chambers, e.g., Gas-Electron Multiplier (GEM) detectors, sandwiched by iron plates with a total thickness equivalent to 13 absorption lengths. The status of detector $\mathrm{R} \& \mathrm{D}$ and recent results of detailed simulations are documented in [1061]. The CBM collaboration consists of more than 450 scientists from 55 institutions and 14 countries.

\subsection{Tau-charm factory in Novosibirsk}

A tau-charm factory can address various issues concerning $\tau$-leptons, charmonia, open-charm particles, and light-quark spectroscopy in a unique manner. Indeed, the $B$-factories have inadequate sensitivity for some of these physics topics, leaving a tau-charm factory as the only practical avenue for substantial progress. A nextgeneration tau-charm factory is now under consideration in Novosibirsk. A novel approach of the Crab Waist collision scheme $[1050,1062]$ allows reaching luminosity of $(1-2) \times 10^{35} \mathrm{~cm}^{-2} \mathrm{~s}^{-1}$. Suggested priorities include

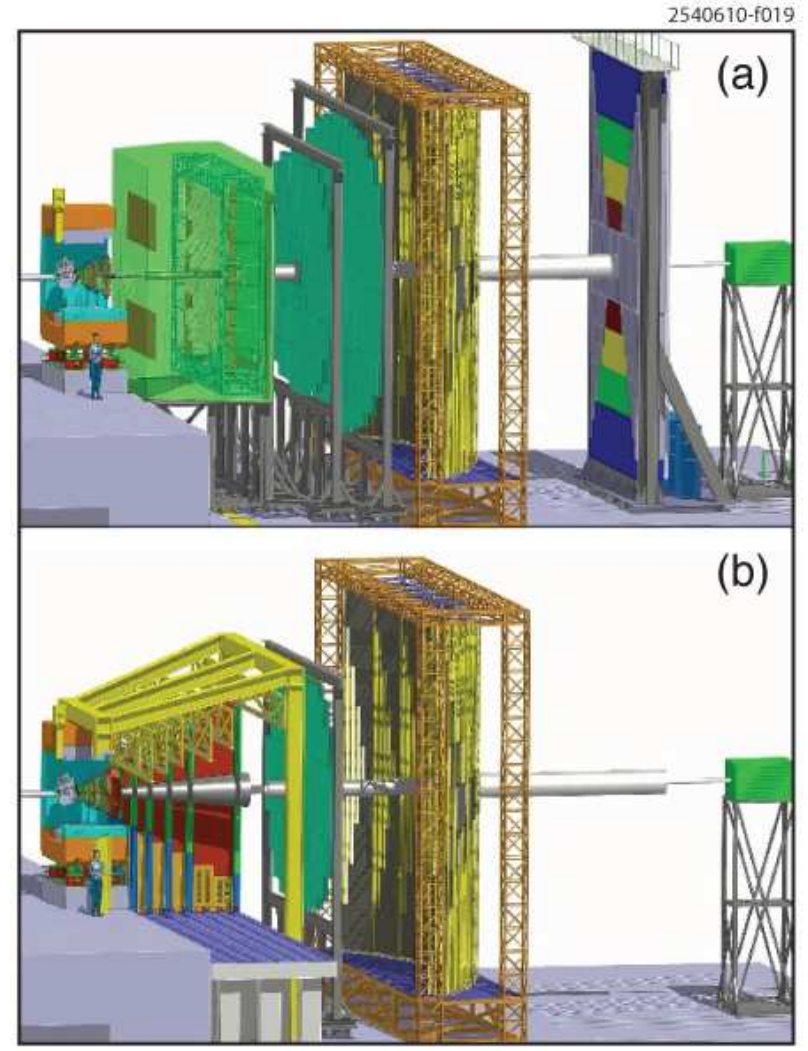

FIG. 107: The CBM experimental facility with (a) the RICH and TRD electron detectors, or (b) the muon detection system 
- $D \bar{D}$ mixing;

- search for CP violation in charm decays;

- study of rare and forbidden decays of open charm mesons;

- high-precision study of regular charmonia and charmonium-like states;

- tests of the Standard Model in $\tau$-lepton decays;

- search for lepton flavor violation;

- search for $\mathrm{CP} / \mathrm{T}$ violation in $\tau$-lepton decays;

- extensive study of light-quark $(u, d, s)$ states between 1 and $3 \mathrm{GeV}$ using ISR;

- production of polarized antinucleons.

This experimental program would be carried out at a facility with the following basic features [1063]:

- collision energy varying from 2-5 GeV;

- luminosity of $\sim 5 \times 10^{34} \mathrm{~cm}^{-2} \mathrm{~s}^{-1}$ at $2 \mathrm{GeV}$ and more than $10^{35} \mathrm{~cm}^{-2} \mathrm{~s}^{-1}$ at the $\tau$-production threshold;

- a longitudinally polarized electron beam at the interaction point (IP) extending the experimental possibilities of the facility;

- extensive use of superconducting wigglers allowing control of damping parameters and tuning for optimal luminosity in the whole energy range;

- $e^{+} e^{-}$center-of-mass energy calibration with relative accuracy of $\approx 5 \times 10^{-4}$, achieved with the Compton backscattering technique.

The planned factory has separate rings for electrons and positrons and one interaction region. Each ring features $\sim 800 \mathrm{~m}$ circumference and a racetrack shape, with two arcs and two long $(\sim 100 \mathrm{~m})$ straight sections to accommodate the injection and radio-frequency (RF) equipment for the machine and the interaction region for the experiment. The injection facility includes a full-energy $(2.5 \mathrm{GeV})$ linear accelerator equipped with the polarizedelectron source and a positron complex with a $500 \mathrm{MeV}$ linac, converter, and accumulating ring. The injection facility operates at $50 \mathrm{~Hz}$ and can produce $\geq 10^{11}$ positrons per second. High luminosity is provided by the Crab Waist collision approach, which assumes the beam intersection at large Piwinski angle and local focusing of the beams at the IP by means of two Crab Sextupoles with properly matched betatron phase advance in between. Local focusing rotates the vertical waist at IP according to the horizontal displacement of each individual particle, decouples the betatron oscillations, and therefore effectively reduces the beam-beam coupling betatron resonances.

\subsection{Charmonium photoproduction facilities}

The mechanism of charmonia photoproduction and their interactions with hadrons and nuclei have been the subject of much interest since their discovery (see Sect. 4.3). Generally, because of the small size of these heavy mesons on the hadronic scale of $\sim 1 \mathrm{fm}$, it is expected that one can apply QCD to describe their interactions with hadronic matter. Heavy quarkonium production thus probes the local color fields in the target and can reveal properties such as their response to momentum transfer and their spatial distribution, which are of fundamental interest for understanding nucleon structure in QCD. While this interpretation is valid at all energies, the details (what mechanism produces the relevant color fields, which configurations in the target are their main source) vary considerably between high energies and the near-threshold region, calling for detailed experimental and theoretical study of this fascinating landscape.

\subsection{1. $J / \psi$ photoproduction at high energy}

The mechanism of exclusive $J / \psi$ photoproduction is well-understood at high energies $(W>10 \mathrm{GeV})$ and $|t|<1 \mathrm{GeV}$, where the coherence length is large compared to the nucleon size, $l_{\text {coh }} \gg 1 \mathrm{fm}$ : the process takes the form of the scattering of a small-size color dipole off the target (Fig. 108(a)). The leading interaction in the small-size expansion is via two-gluon exchange with the target. The nucleon structure probed in this case is the gluon generalized parton distribution (or GPD), which describes the two-gluon form factor of the target and is normalized to the usual gluon density in the zero momentum-transfer limit. In the process shown in Fig. 108(a), the transverse momenta of the exchanged gluons are large, $\left|\boldsymbol{k}_{T 1,2}\right| \sim m_{J / \psi}$, but their difference can be small, resulting in a small invariant momentum transfer to the target $|t| \sim\left|\boldsymbol{k}_{T 1}-\boldsymbol{k}_{T 2}\right|^{2}$. Hence the reaction can leave a proton or a nuclear target in its ground state or a slightly excited state. Experiments in exclusive $J / \psi$ photo- and electro-production at HERA [751, 752] have confirmed this picture through detailed measurements of the $Q^{2}$-independence of $t$-slopes in electroproduction, energy dependence of the cross section, and comparison with other exclusive vector meson channels (universality of the gluon GPD); see [1066] for a review. They have also measured the $t$-slope of the differential cross section and its change with energy, which allows one to infer the average transverse radius of gluons in the nucleon and its change with $x$ (Fig. 109). This information represents an essential input to small- $x$ physics (initial condition of evolution equations) and the phenomenology of high-energy $p p$ collisions with hard processes [1067].

Of particular interest is elastic or quasi-elastic scattering of a charmed dipole on nuclei (Fig. 108(a)), with a subsequent transformation of the $c \bar{c}$ into a $J / \psi$. The momentum transfer is measurable in this reaction be- 

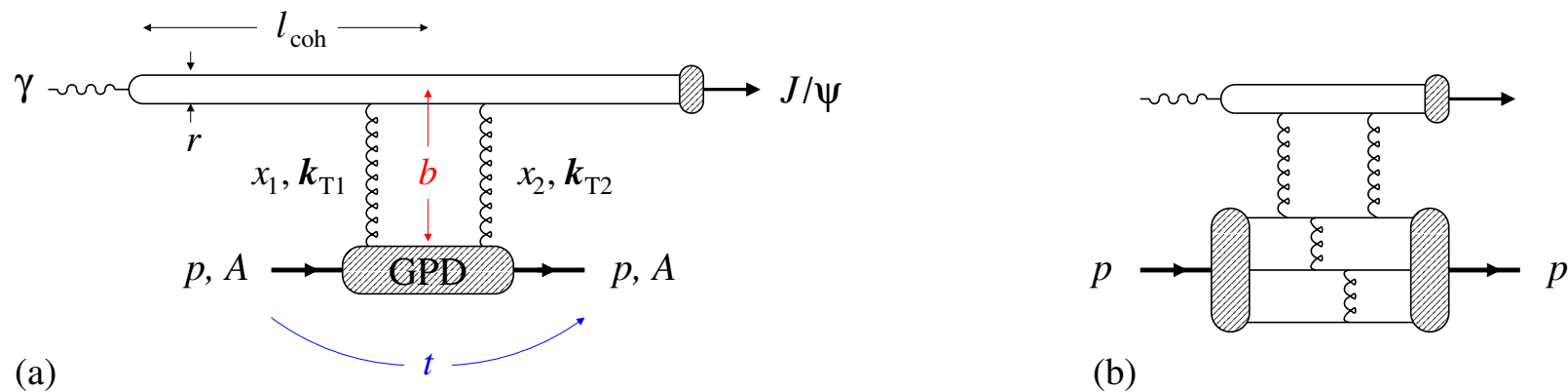

(a)

(b)

FIG. 108: Dynamical mechanisms of $J / \psi$ photoproduction. (a) Two-gluon exchange mechanism at high and intermediate energies, $W-W_{\mathrm{th}}>$ few $\mathrm{GeV}$, based on QCD factorization for hard exclusive meson production. The space-time evolution in the target rest frame corresponds to the dipole picture of high-energy scattering $[1064,1065]$, where $l_{\text {coh }}$ is the coherence length, $r$ the transverse size of the $c \bar{c}$ dipole and $b$ its impact parameter with the target. Here $x_{1,2}$ and $\boldsymbol{k}_{T 1,2}$ denote the longitudinal momentum fractions and transverse momenta of the exchanged gluons. The invariant momentum transfer to the target proton or nucleus is small and of the order of the inverse target size, $|t| \sim\left|\boldsymbol{k}_{T 1}-\boldsymbol{k}_{T 2}\right|^{2} \sim R_{\mathrm{target}}^{-2}$. (b) Coherent multi-gluon exchange proposed for near-threshold production on the proton, analogous to the hard scattering mechanism for high- $t$ elastic form factors. Near threshold the minimum value of the invariant momentum transfer $\left|t_{\min }\right|$ is large

cause it is the difference between the transverse momentum of the incoming virtual photon and the final meson. Caldwell and Kowalski [1068] propose investigation of the properties of nuclear matter by measuring the elastic scattering of $J / \psi$ on nuclei with high precision. The $J / \psi$ mesons are produced from photons emitted in highenergy electron-proton or electron-nucleus scattering in the low- $x$ region. Over the next few years, some relevant data should be available from RHIC, where STAR is collecting data on $J / \psi$ photoproduction. Based on the recent RHIC $\mathrm{Au}+\mathrm{Au}$ luminosity, a sample of order 100 events might be expected. Such measurements could be performed at the future ENC (Electron-Nucleon Collider, GSI), Electron-Ion Collider (EIC, Brookhaven National Lab, or Jefferson Lab (JLab)), or LHeC (Large Hadronelectron Collider, CERN) facilities. The advantage of $J / \psi$ photoproduction compared to the electroproduction of light vector mesons is its high cross section and small dipole size, even at $Q^{2}=0$. In addition, the momenta of the decay products of $J / \psi \rightarrow \mu^{+} \mu^{-}$or $e^{+} e^{-}$can be precisely measured. The smallness of the dipole in low$x$ reactions assures that the interaction is mediated by gluon exchange only. Thus the deflection of the $J / \psi$ directly measures the intensity and the spatial distribution of the nuclear gluon field.

The measurement of $J / \psi$ scattering on nuclei could become an important source of information on nuclear structure and high-density QCD. In the absence of nuclear shadowing, the interaction of a dipole with a nucleus can be viewed as a sum of dipole scatterings of the nucleons forming the nucleus. The size of the $c \bar{c}$ dipole in elastic $J / \psi$ scattering is around $0.15 \mathrm{fm}$, i.e., it is much smaller than the nucleon radius. It is therefore possible that dipoles interact with smaller objects than nucleons; e.g., with constituent quarks or hot spots. The conventional assumption is that the nucleus consists of nucleons and that dipoles scatter on an ensemble of nucleons according to the Woods-Saxon [1069] distribution,

$$
\begin{aligned}
\rho_{\mathrm{WS}}(r) & =\frac{N}{1+\exp \left(\frac{r-R_{A}}{\delta}\right)}, \\
R_{A} & \equiv 1.12 A^{\frac{1}{3}}-0.86 A^{-\frac{1}{3}} \mathrm{fm},
\end{aligned}
$$

where $R_{A}$ is the nuclear radius for atomic number $A$, $\delta=0.54 \mathrm{fm}$ is the skin depth, and $N$ is chosen to normalize $\int d^{3} \vec{r} \rho_{\mathrm{WS}}(r)=1$. Under the foregoing assumption (or slight variations thereon), the dipole model predicts the coherent and incoherent nuclear cross sections shown in Fig. 110. Deviations from the predicted $|t|$-distribution reveal the effects of nuclear shadowing, which depend on the effective thickness of the target and thus change with the dipole impact parameter, $b$. In the coherent process, the nucleus remains in its ground state. In the incoherent process, the nucleus gets excited and frequently breaks into nucleons or nucleonic fragments. Experimentally we expect to be able to distinguish cases where the nucleus remains intact and cases where the nucleus breaks up. In the nuclear breakup process, there are several free neutrons and protons in the final state, as well as other fragments. The number of free nucleons could depend on the value of the momentum transfer. The free nucleons and fragments have high momenta and different charge-tomass ratios than the nuclear beam and should therefore be measurable in specialized detectors.

The transverse momenta of the $J / \psi$ can be determined, in a TPC detector with $2 \mathrm{~m}$ radius and $3.5 \mathrm{~T}$ magnetic field, with a precision of $O(1) \mathrm{MeV}$. The momenta of the breakup protons can be precisely measured in the forward detector. Therefore a measurement of $t$-distributions together with a measurement of nuclear debris could become a source of invaluable information about the inner structure of gluonic fields of nuclei [1068]. 


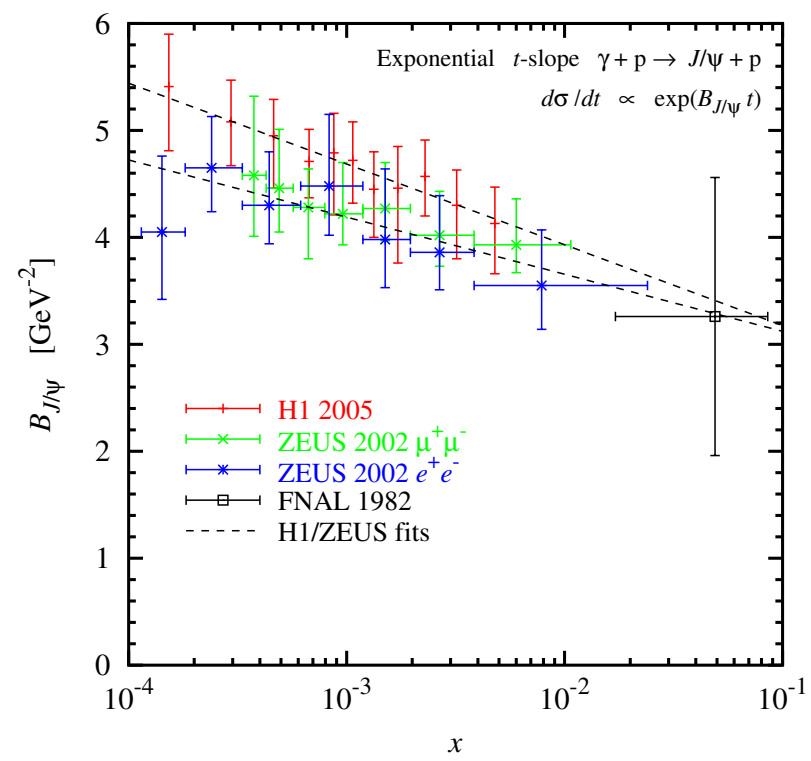

FIG. 109: The exponential $t$-slope, $B_{J / \psi}$, of the differential cross section of exclusive $J / \psi$ photoproduction measured in the FNAL E401/E458 [1070], HERA H1 [751], and ZEUS [753] experiments, as a function of $x=$ $m_{J / \psi}^{2} / W^{2}$ [1109]. (In the H1 and ZEUS results, the quoted statistical and systematic uncertainties were added linearly.) The dashed lines represent the published two-dimensional fits to the $\mathrm{H} 1$ and ZEUS data. The average squared transverse radius of gluons with momentum fraction $x$ in the nucleon can be inferred from the measured slope as $\left\langle b^{2}\right\rangle_{g}=2\left(B_{J / \psi}-\Delta B\right)$, where $\Delta B \approx 0.3-0.6 \mathrm{GeV}^{-2}$ accounts for the finite transverse size of the $c \bar{c}$ pair in the production amplitude [1064, 1065]. The data show that the nucleon's gluonic transverse radius at $x \sim 10^{-1}$ is smaller than the transverse charge (Dirac) radius and increases slowly toward small $x$; see [1065, 1109] for details

\subsection{2. $J / \psi$ photoproduction at low energy}

Measurements of exclusive $J / \psi$ photoproduction at lower energies were performed in the Fermilab broadband beam experiment [1070], which detected the recoiling proton, as well as several other experiments [1071, 1072]. The few existing data suggest that the two-gluon exchange mechanism of Fig. 108(a) continues to work in this region, and the gluonic size of the nucleon observed in these experiments consistently extrapolates to the HERA values (Fig. 109) [1073]. However, no precise differential measurements are available for detailed tests of the reaction mechanism. New data in this region are expected from the COMPASS experiment at CERN and a future Electron-Ion Collider (EIC).

Charmonium photoproduction near threshold will be studied in experiments at JLab. The Continuous Electron Beam Accelerator Facility (CEBAF) at JLab delivers $500 \mathrm{MHz}$ electron beams with energies up to $6 \mathrm{GeV}$ to three experimental halls. The ongoing up-

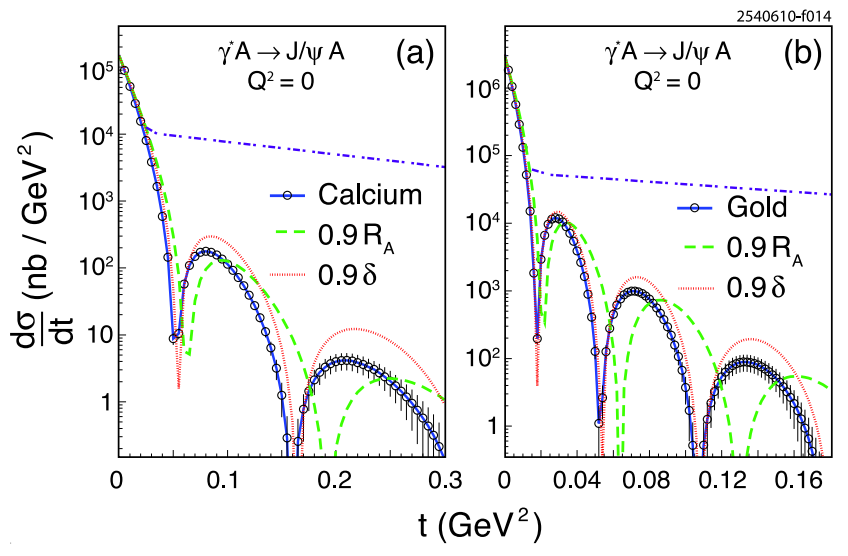

FIG. 110: The prediction [1068] of the dipole model for the $t$ distribution of coherent $J / \psi$ photoproduction on (a) calcium and (b) gold nuclei, assuming that the single nucleon distribution can be identified with the Woods-Saxon distribution, parametrized by the nuclear radius $R_{A}$ and skin depth $\delta$ as in Eq. (197). The simulated measurements with nominal $R_{A}$ and $\delta$ (open circles and solid curve) have statistical error bars based on an assumed collected sample of $10^{6}$ events. Somewhat smaller values of $R_{A}$ or $\delta$ (by $10 \%$ or $20 \%$, respectively) result in the dashed and dotted curves, respectively. The dotdashed curve shows the sum of the coherent and incoherent processes in the case of no correlations

grade will increase the maximum energy to $12 \mathrm{GeV}$. The first beam delivery is planned for 2014. Halls A and $\mathrm{C}$ are equipped with small-acceptance, high-resolution spectrometers and able to receive beam currents up to $100 \mu \mathrm{A}$. Hall B is equipped with a large acceptance toroidal spectrometer (CLAS) and is able to receive up to $0.1 \mu \mathrm{A}$. A new Hall $\mathrm{D}$, being built as a part of the upgrade, will use a tagged-photon beam with a $12 \mathrm{GeV}$ endpoint and intensity up to $100 \mathrm{MHz} / \mathrm{GeV}$.

In exclusive $J / \psi$ photoproduction near threshold $\left(E_{\gamma, \mathrm{thr}}=8.21 \mathrm{GeV}\right.$ for the free nucleon $)$, to be studied with JLab $12 \mathrm{GeV}$, the reaction mechanism is expected to change in several important aspects. First, the minimum invariant momentum transfer to the nucleon becomes large: $\left|t_{\min }\right|=2.23 \mathrm{GeV}^{2}$ at threshold, and $\left|t_{\min }\right|=1.3-0.3 \mathrm{GeV}^{2}$ at $E_{\gamma}=8.5-12 \mathrm{GeV}$. This requires more exceptional high-momentum configurations in the target to bring about an exclusive transition, similar to elastic $e N$ scattering at large $t$. It is expected that, in this situation, color correlations in the wave functions play an essential role, suggesting a new avenue for their experimental study. Second, while at high energies the production amplitude is mostly imaginary (absorptive), near threshold the real part plays an essential role, rendering the partonic interpretation of the production process more complex.

Two possible scenarios for near-threshold exclusive production on the nucleon have been proposed. One scenario assumes that the two-gluon exchange mechanism of Fig. 108(a) continues to apply, justified by the small 
size of the $J / \psi$, and extends this description to the nearthreshold region [1073]. Here the challenge lies in modeling the gluon GPD in the "extreme" near-threshold region, characterized by large $t_{\min }$ and large "skewness" (difference in momentum gluon fractions $x_{1} \neq x_{2}$ ). Some support for this picture comes from the fact that the exclusive $\phi$ electroproduction data at JLab with $6 \mathrm{GeV}$ beam energy [1074, 1075] are well described by a dynamical model based on gluon GPDs [1076]. The other scenario is based on analogy with the hard scattering mechanism for high- $t$ elastic form factors. It assumes that the production process happens predominantly in the valence (3-quark) configuration of the nucleon and that the momentum transfer is balanced via hard-gluon exchange [1077] as illustrated in Fig. 108(b). The two basic pictures make different predictions for the energy dependence of the cross section and $t$-slope near threshold, and can be tested with the expected JLab $12 \mathrm{GeV}$ data. The quantitative implementation of the above scenarios, and a possible unified description, are the subjects of ongoing theoretical research. Independently of the details, the expected JLab data will greatly advance our knowledge of color correlations and the gluonic response of the nucleon at low energies.

The cross section for exclusive $J / \psi$ photoproduction at $11 \mathrm{GeV}$ is expected to be $0.2-0.5 \mathrm{nb}$, rapidly falling toward the threshold. The JLab experiment plans to map out the $J / \psi$ differential cross section in the region from 9 to $12 \mathrm{GeV}$.

Another objective of $J / \psi$ production experiments is to study the interaction of the produced system with nuclear matter at low energies. The small coherence and formation lengths allow extraction of the $J / \psi$-nucleon cross sections from the $A$-dependence of the $J / \psi$ photoproduction cross section with minimal corrections to the colortransparency effects. The only such experiment done at sufficiently low energies [1078] obtained $3.5 \pm 0.9 \mathrm{mb}$; the signal was extracted from a single-muon transverse momentum spectrum and the background level was not well understood. A new experiment at JLab can reduce the statistical and systematic errors by a factor of three.

A proposal to study $J / \psi$ photoproduction close to threshold in Hall $\mathrm{C}$ has been conditionally approved by the JLab Program Advisory Committee. The lepton decay modes of $J / \psi$ will be detected. In spite of a small acceptance of $\sim 3 \times 10^{-4}$ to $J / \psi$ decay products, the expected rate of detected $J / \psi$ is $150-200$ per hour in a $2.2 \%$ radiation-length-thick liquid hydrogen target. The effective photon flux of the $50 \mu \mathrm{A}$ electron beam will be increased due to a radiator in front of the target which has a thickness of $7 \%$ of a radiation length. The high resolution of the Hall $\mathrm{C}$ spectrometers will provide strong background suppression.

\subsection{Proposed $\bar{p} p$ project at Fermilab}

A uniquely capable and cost-effective multipurpose experiment could be mounted by adding a magnetic spectrometer to the existing Fermilab E760 lead-glass calorimeter [1079] using an available BESS solenoid [1080], fine-pitch scintillating fibers (SciFi), the DØ SciFi readout system [1081], and hadron ID via fast timing [1082]. If the relevant cross sections are as large as expected, this apparatus could produce world-leading measurements of $X(3872)$ properties, along with those of other charmonium and nearby states, as is now proposed $^{23}$ to Fermilab $[1083,1084]$. The Fermilab Antiproton Accumulator's $8 \mathrm{GeV}$ maximum kinetic energy and ability to decelerate down to $\approx 3.5 \mathrm{GeV}$ suit it well for studies in this mass region. If approved, the experiment could start about a year after completion of the Tevatron Collider run.

Antiproton Accumulator experiments E760 and E835 made the world's most precise $(\lesssim 100 \mathrm{keV})$ measurements of charmonium masses and widths [1085, 1086], thanks to the precisely known collision energy of the stochastically cooled $\bar{p}$ beam (with its $\approx 0.02 \%$ energy spread) with a hydrogen cluster-jet target [1087]. Significant charmonium-related questions remain, most notably the nature of the mysterious $X(3872)$ state [32] and improved measurements of the $h_{c}(1 P)$ and $\eta_{c}(2 S)$ [1]. The width of the $X$ may well be $\ll 1 \mathrm{MeV}$ [122]. This unique $\bar{p} p$ precision would have a crucial role in establishing whether the $X(3872)$ is a $D^{* 0} \bar{D}^{0}$ molecule [1088], a tetraquark state [285], or something else entirely.

The $\bar{p} p \rightarrow X(3872)$ formation cross section may be similar to that of the $\chi_{c}$ states $[721,1089]$. The E760 $\chi_{c 1}$ and $\chi_{c 2}$ detection rates of 1 event $/ \mathrm{nb}^{-1}$ at the mass peak [1090] and the lower limit $\mathcal{B}(X(3872) \rightarrow$ $\left.\pi^{+} \pi^{-} J / \psi\right)>0.042$ at $90 \%$ CL [124] imply that, at the peak of the $X(3872)$, about 500 events/day can be observed. (Although CDF and DØ could also amass $\sim 10^{4}$ $X(3872)$ decays, backgrounds and energy resolution limit their incisiveness.) Large samples will also be obtained in other modes besides $\pi^{+} \pi^{-} J / \psi$, increasing the statistics and improving knowledge of $X(3872)$ branching ratios.

While the above may be an under- or overestimate, perhaps by as much as an order of magnitude, it is likely that a new experiment at the Antiproton Accumulator could obtain the world's largest clean samples of $X(3872)$, in perhaps as little as a month of running. The high statistics, event cleanliness, and unique precision available in the $\bar{p} p$ formation technique could enable the world's smallest systematics. Such an experiment could provide a definitive test of the nature of the $X(3872)$.

\footnotetext{
23 The experiment would also address nonquarkonium topics, such
} as charm mixing and $C P$ violation. 


\subsection{Future linear collider}

A high-energy $e^{+} e^{-}$linear collider provides excellent possibilities for precision and discovery, within the Standard Model as well as for new physics. The International Linear Collider ILC [1091] is a proposed machine based on superconducting RF cavities that will provide centerof-mass energies of up to $500 \mathrm{GeV}$, with the possibility for an upgrade to $1 \mathrm{TeV}$. At the design energy of $500 \mathrm{GeV}$, it will deliver a luminosity of $2 \times 10^{34} \mathrm{~cm}^{-2} \mathrm{~s}^{-1}$, providing high-statistics datasets for precision studies. Two mature ILC detector concepts, ILD [1092] and SID [1093] exist, both sophisticated general-purpose detectors with excellent tracking and vertexing capabilities and unprecedented jet energy resolution achieved with highly granular calorimeter systems and particle flow reconstruction algorithms. In parallel, the technology for the Compact Linear Collider CLIC [1094], using a two-beam acceleration scheme to reach center-of-mass energies up to $3 \mathrm{TeV}$, is being developed. In a staged construction, such a machine would initially run at energies comparable to the design goals for the ILC before reaching the multi-TeV regime. The detector concepts for CLIC are based on the already-mature ILC detectors, with some modifications to account for the higher final collision energy. These planned colliders are excellent tools for top physics. The precise control of the beam energy at a linear $e^{+} e^{-}$collider allows a scan of the $t \bar{t}$ production threshold to determine the top mass, width, and production cross section [1095].

There are several interesting observables in the $t \bar{t}$ threshold region that can be used for these measurements. These are the total cross section, top momentum distribution, top forward-backward asymmetry and the lepton angular distribution in the decay of the top quark. The total cross section as a function of $\sqrt{s}$ rises sharply below the threshold and peaks roughly at the position of the would-be $1 S t \bar{t}$ resonance mass [1096]. The normalization at the peak is proportional to the square of the resonance wave function at the origin and inversely proportional to the top quark decay width. Theoretically, the resonance mass (hence the peak position) can be predicted very accurately as a function of $m_{t}$ and $\alpha_{\mathrm{s}}$, so that the top quark mass can be determined by measuring the peak position accurately. We may determine the top quark width and the top quark Yukawa coupling from the normalization, since the exchange of a light Higgs boson between $t$ and $\bar{t}$ induces a Yukawa potential, which affects the resonance wave function at the origin [1097].

The top quark momentum distribution is proportional to the square of the resonance wave function in momentum space [1098, 1099]. The wave function of the wouldbe $1 S$ toponium resonance can be measured, since the top quark momentum can be experimentally reconstructed from the final state, unlike in the bottomonium or charmonium cases. The wave function is also predicted theoretically and is determined by $\alpha_{\mathrm{s}}$ and $m_{t}$. The top quark forward-backward asymmetry below threshold is gener- ated as a result of interference between the $S$-wave and $P$-wave resonance states [1100]. This interference is sizable, since the top quark width is not very different from the level splitting between the $1 S$ and $1 P$ states, and since the width is much larger than the $S$ - $P$ splittings of the excited states. Thus, by measuring the forwardbackward asymmetry, one can obtain information on the level structure of the $S$ and $P$-wave resonances in the threshold region. Both the top momentum distribution and forward-backward asymmetry provide information on $t \bar{t}$ dynamics, which are otherwise concealed because of the smearing due to the large top decay width.

It is known that the charged lepton angular distribution is sensitive to the top quark spin. By measuring the top quark spin in the threshold region [1101], it is possible to extract the chromoelectric and electric dipole moments of the top quark, which are sensitive to $C P$ violations originating from beyond-the-standard-model physics [1102].

By combining the collision energy dependence of these observables, precise measurements of the top parameters are possible. Figure 111 shows simulations of the sensitivity of the cross section, the peak of the top momentum distribution and of the forward-backward asymmetry to the top mass in a threshold scan at a linear collider [1103]. (See [1104] for an earlier, similar analysis.) To illustrate the sensitivity, three different input masses, spaced by $200 \mathrm{MeV}$, are shown. From a simultaneous fit to these three observables, a statistical uncertainty of $19 \mathrm{MeV}$ on $m_{t}$ was obtained, neglecting sources of systematic errors such as the determination of the luminosity spectrum of the collider or theoretical uncertainties. To achieve the highest possible precision, detailed understanding of the beam energy spectrum, of beamstrahlung and of initial state radiation is crucial. The measured cross section in such a scan is theoretically well described, as discussed in detail in Sect. 6 of [1] and references therein. Using NNLO QCD calculations, an overall experimental and theoretical error of $100 \mathrm{MeV}$ on the top mass is reachable with a threshold scan at a linear collider [1105]. Further important improvements in precision arise from NNNLO QCD calculations, the summation of large logarithms at NNLL order and a systematic treatment of electroweak and top quark finite-lifetime effects. Also, the determination of corrections beyond NLO for differential observables such as the momentum distribution and the forward-backward asymmetry used in the study described above would be required to fully exploit the precision of the expected data.

A method has been developed to improve accuracies of kinematic variables in reconstructed top-quark events in the threshold region [1106]. This method would improve measurements of the top quark observables mentioned above.

An alternative to the threshold-region measurements described above is reconstruction of decay products in $t \bar{t}$ pair production far above threshold. This approach has the advantage that the top measurement is possi- 


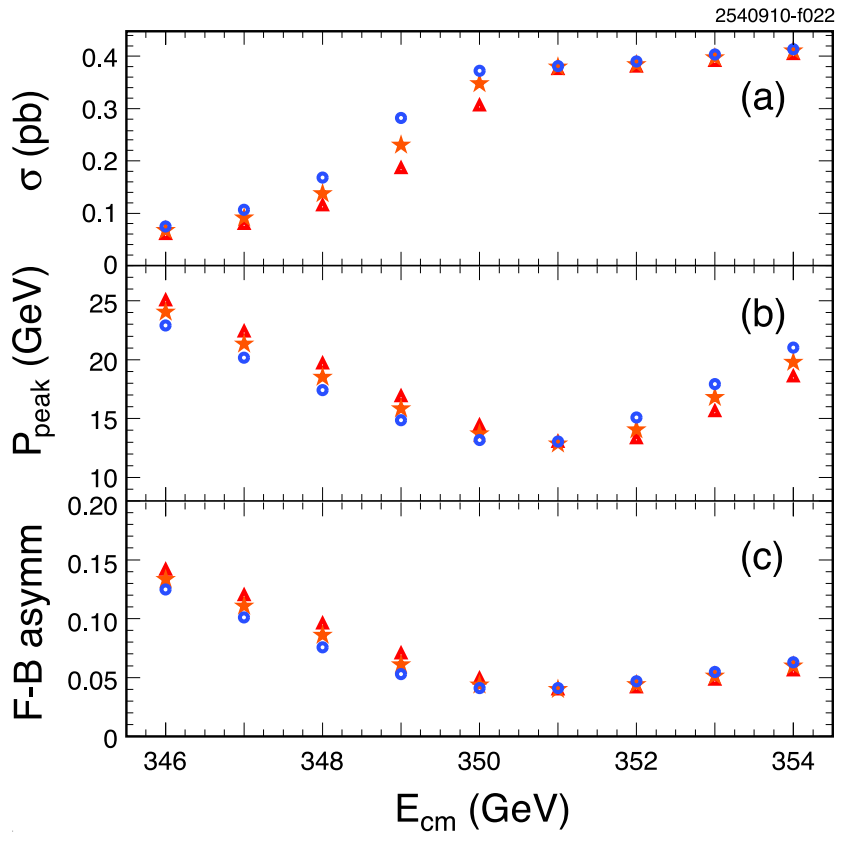

FIG. 111: Sensitivity of three important observables to the top mass from a simulated threshold scan at a linear collider: (a) the top production cross section, (b) the peak of the top momentum distribution $P_{\text {peak }}$, and (c) the forward-backward asymmetry. The solid triangles (open circles) correspond to input MC generated with top mass that is $200 \mathrm{MeV}$ higher (lower) than that used for the MC sample with nominal top mass, represented by stars. Adapted from [1103] with kind permission, copyright (2003) Springer-Verlag

ble at full collider energy in parallel with other measurements, resulting in much higher integrated luminosity. The top quarks decay essentially exclusively into a $b$ quark and a $W$ boson, giving rise to two $b$ jets and additional jets or leptons from the $W$ decays in the final state. The expected performance of the ILC detectors leads to very high precision in the invariant mass determination. Due to the clean environment in $e^{+} e^{-}$collisions, a measurement in the full-hadronic six-jet mode, which has the highest branching fraction, is possible. Figure 112 shows the reconstructed top quark invariant mass in the fully hadronic decay mode obtained from a simulation for the ILD detector concept [1092] at an energy of $500 \mathrm{GeV}$ and corresponding to an integrated luminosity of $100 \mathrm{fb}^{-1}$. The simulation uses a complete detector modeling and standard model backgrounds. The statistical error on the mass reconstruction is $90 \mathrm{MeV}$ in this channel alone. With a higher integrated luminosity of $500 \mathrm{fb}^{-1}$ and by combining measurements in fully hadronic and in semileptonic top decays, a statistical precision of $30 \mathrm{MeV}$ seems reachable, comparable to the experimental precision of a threshold scan. However, the theoretical interpretation of the invariant mass is considerably more complicated than in the case of threshold measurements. Recently, a systematic formal-

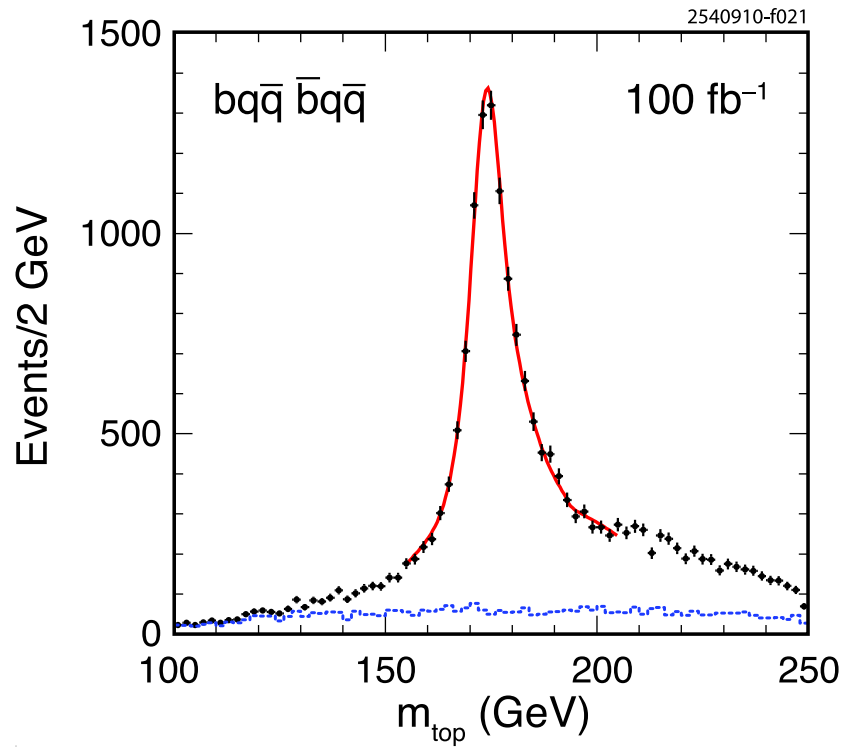

FIG. 112: Distribution of the reconstructed top quark invariant mass for the fully hadronic $t \bar{t} \rightarrow b q \bar{q} \bar{b} q \bar{q}$ decay channel in the ILD detector concept for an integrated luminosity of $100 \mathrm{fb}^{-1}$, including the full non-top background, indicated in the dotted histogram. The solid curve represents a fit for the top mass. From [1092]

ism to relate the measured invariant mass to theoretically meaningful parameters has been established [1107, 1108]. This formalism has the potential to compete with the threshold-scan calculations in terms of achievable precision. The computations were carried out to NLL. Further higher-order calculations are needed to reduce the present theoretical uncertainties and fully exploit the experimental precision achievable at a future linear collider. See also Sect. 2.8.3.

Beyond precision measurements of the top mass and width, a Linear Collider would also provide excellent conditions for a wealth of other top-related studies, such as the measurement of the top-Yukawa coupling in the process $e^{+} e^{-} \rightarrow t \bar{t} H$ and the search for anomalous couplings in decays. 


\section{CONCLUSIONS AND PRIORITIES}

Below we present a summary of the most crucial developments in each of the major topics and suggested directions for further advancement.

Spectroscopy: An overview of the last decade's progress in heavy quarkonium spectroscopy was given in Sect. 2 .

With regard to experimental progress, we conclude:

1. New measurements of inclusive hadronic cross sections (i.e., $R$ ) for $e^{+} e^{-}$collisions just above open $c \bar{c}$ and $b \bar{b}$ flavor thresholds have enabled improved determinations of some resonance parameters but more precision and fine-grained studies are needed to resolve puzzles and ambiguities. Likewise, progress has been made studying exclusive open-flavor two-body and multibody composition in these regions, but further data are needed to clarify the details. Theory has not yet been able to explain the measured exclusive two-body cross sections.

2. Successful observations were made (Table 4) of 6 new conventional heavy quarkonium states $(4 c \bar{c}, 2$ $b \bar{b})$; of these, only the $\eta_{b}(1 S)$ lacks a second, independent $5 \sigma$ confirmation. Improved measurement of $\eta_{c}(1 S)$ and $\eta_{c}(2 S)$ masses and widths would be quite valuable. Unambiguous observations and precise mass and width measurements are needed for $\eta_{b}(2 S), h_{b}\left({ }^{1} P_{1}\right), \Upsilon\left(1^{3} D_{1}\right)$, and $\Upsilon\left(1^{3} D_{3}\right)$ in order to constrain theoretical descriptions.

3. Experimental evidence has been gathered (Table 9) for up to 17 unconventional heavy quarkonium-like states. All but $Y_{b}(10888)$ are in the charmonium mass region, and all but 5 remain unconfirmed at the $5 \sigma$ level. Confirmation or refutation of the remaining 12 is a high priority.

4. Theoretical interpretations for the unconventional states (Table 20) range from coupled-channel effects to quark-gluon hybrids, mesonic molecules, and tetraquarks. More measurements and theoretical investigations are necessary to narrow the possibilities. In particular, high-resolution measurements of lineshapes promise deeper insights into the nature of various of those states.

5 . The $X(3872)$ was the first unexpected state to be observed and has generated the most experimental and theoretical attention. Its sub-MeV proximity to $D^{* 0} \bar{D}^{0}$-threshold (Tables 10-11) and dominant $D^{0} \bar{D}^{0} \pi^{0}$ branching fraction suggest a $D^{* 0} \bar{D}^{0}$ molecular component, although this interpretation is not universally shared. The $X(3872)$ has been confirmed in four decay modes (Table 12). The discovery mode, $\pi^{+} \pi^{-} J / \psi$, is still the best measured, and has a branching fraction comparable in size to that of $\omega J / \psi ; D^{0} \bar{D}^{0} \pi^{0}$ is ten times more common and $\gamma J / \psi$ three times less. The $X(3872)$ quantum numbers have been narrowed to $1^{++}$or $2^{-+}$.

6 . The charged $Z$ states observed in $Z^{-} \rightarrow \pi^{-} \psi(2 S)$ and $\pi^{-} \chi_{c 1}$ would be, if confirmed, manifestly exotic. Hence their confirmation or refutation is of the utmost importance.

With regard to lattice QCD calculations:

7. Lattice QCD technology has progressed to the point that it may provide accurate calculations of the energies of quarkonium states below the open flavor threshold, and also provide information about higher states.

8. Precise and definitive calculations of the $c \bar{c}$ and $b \bar{b}$ meson spectra below threshold are needed. Unquenching effects, valence quark annihilation channels and spin contributions should be fully included.

9. Unquenched calculations of states above the openflavor thresholds are needed. These would provide invaluable clues to the nature of these states.

10. The complete set of Wilson loop field strength averages entering the definition of the nonperturbative $Q \bar{Q}$ potentials must be calculated on the lattice.

11. Calculations of local and nonlocal gluon condensates on the lattice are needed as inputs to weaklycoupled pNRQCD spectra and decay calculations.

12. NRQCD matching coefficients in the lattice scheme at one loop (or more) are needed.

13. Higher-order calculations of all the relevant quantities due to the lattice-to- $\overline{M S}$ scheme change are required in order to relate lattice and continuum results in the EFT.

14. Lattice calculations of the overlap between quarkonia and heavy-light states in the threshold region, as well as with hybrids or exotic states, should be performed.

15. A better determination of the $r_{0}$ lattice scale and a nonperturbative determination of $\Lambda_{\overline{\mathrm{MS}}}$ with $2+1$ or $2+1+1$ sea quarks is needed.

With regard to effective field theories (EFTs),

16. Higher-order perturbative EFT calculations of static energies, static potentials, and relativistic corrections to the potentials and energy levels have appeared recently for different heavyquark/antiquark configurations. Further efforts in this direction are needed, and the emerging patterns of renormalons should be studied in relation to the behavior of the bound states perturbative series. 
17. We have described simulation studies for a future linear collider which demonstrate that precise determinations of the top mass and the top Yukawa coupling can be obtained from a $t \bar{t}$ production scan near threshold. To at least match this expected precision, a complete NNLL computation is necessary to obtain a firmer grasp of the theoretical uncertainties. The complete NNNLO computation is also desirable to assess the importance of the resummation of logarithms. The electroweak and non-factorizable corrections may be calculated by developing an effective theory description for unstable particles.

18. Better experimental data for $b \bar{b}$ production above threshold would diminish the impact of the experimental error in nonrelativistic sum rules determinations of the bottom quark mass.

19. The $2 \sigma$ discrepancy between the EFT calculation and measurements of the $\eta_{b}(1 S)$ mass needs to be resolved.

Decay: Section 3 described the enormous progress on heavy quarkonium decays, showing that many branching fraction, width, and spectra measurements have attained high precision. Some of these results provide crucial anchors for theoretical approaches while others have just scratched the surface of what may be attainable with more data and improved techniques. Accomplishments and priorities are:

20. Precise measurements of the dileptonic and total widths in charmonium (Tables 28-31) and bottomonium (Tables 32-35) below the respective openflavor thresholds have been performed.

21. A novel and empirical measurement of the nontrivial radiative photon lineshape in $J / \psi$ and $\psi(2 S)$ decays to $\eta_{c}(1 S)$, which in turn enabled determination of much-improved branching fractions, has appeared. This has stimulated theoretical activity to explain the photon spectral shape and raised the importance of measuring the corresponding spectra for $\psi(2 S) \rightarrow \gamma \eta_{c}(2 S), \psi(2 S) \rightarrow \gamma \chi_{c J}(1 P)$, $\Upsilon(n S) \rightarrow \gamma \eta_{b}(1 S, 2 S)$, and $\Upsilon(n S) \rightarrow \gamma \chi_{b J}(n P)$. Precise measurement of the $\psi(2 S) \rightarrow \gamma \chi_{c J}(1 P)$ lineshapes will be essential in quantifying the branching fraction for the nonresonant $\psi(2 S) \rightarrow$ $\gamma \gamma J / \psi$ decay.

22. There are new branching fraction measurements for decays of $J / \psi, \psi(2 S)$, and $\Upsilon$ to $\gamma \eta\left(^{\prime}\right)$ that present a puzzling and unexplained pattern (Table 26).

23. Measurements of the $\gamma g g$ spectra and branching fractions (Table 25) for $\Upsilon(1 S, 2 S, 3 S), J / \psi$, and $\psi(2 S)$ all have been published. Considerably more attention to measurements, in which backgroundsubtraction uncertainties have limited the precision of charmonium results much more severely than for bottomonium, and predictions, which have had mixed success on predicting the radiative photon spectra, is needed.

24. First measurements of the two- and three-photon partial widths of $\chi_{c 0,2}$ (Table 27) and $J / \psi$, respectively, have been performed. Although the experimental relative uncertainties on these widths are not yet below the $10 \%$ and $30 \%$, respectively, they already present challenges to the theory. Better experimental precision for the two-photon couplings of $\eta_{c}(1 S)$ and $\eta_{c}(2 S)$ would be very useful to constrain cross-particle branching fraction measurements as well as relevant theoretical descriptions.

25. Detailed and provocative measurements of dipion and $\eta$ transitions for the $\psi$ (Table 37) and $\Upsilon$ (Tables 38-39) systems below the respective openflavor thresholds challenge theoretical rate and dipion-mass spectra predictions, while the surprisingly high rates observed above these thresholds remain a mystery.

26. Measurements of $\psi(3770) \rightarrow$ non- $D \bar{D}$ (Table 40 ) conflict with one another. Definitive measurements of exclusive decays can best supply confidence in such a rate being more than a few percent, because indirect or aggregate (Table 41) comparisons appear to be quite challenging.

27. A multitude of measurements has been accumulated in the realm of exclusive hadronic decays of heavy quarkonia. These have deepened theoretical mysteries (e.g., $\rho \pi$ puzzle, conflicting measurements of non- $D \bar{D}$ decays of $\psi(3770))$ but whetted the community's appetite for more information on such decays of $\chi_{c J}, \chi_{b J}, \eta_{c}(1 S), \eta_{c}(2 S), \eta_{b}(1 S)$, $h_{c}\left({ }^{1} P_{1}\right)$, and $h_{b}\left({ }^{1} P_{1}\right)$.

28. Initial but nevertheless intriguing measurements of the rate for deuterons to appear in $\Upsilon$ decays and the mechanism of baryon-number compensation therein have been reported. Further experimental and theoretical attention in $\Upsilon$ decays, the LHC, and future facilities are warranted. This information is useful for tuning MC generators and may be relevant for the molecular interpretation of $X(3872)$ (Sect. 2.9.3) and other loosely-bound states.

29. A new measurement (Table 22) has resolved the longstanding discrepancy between experiment and theory on multipole amplitudes in $\psi(2 S) \rightarrow \gamma \chi_{c J}$, $\chi_{c J} \rightarrow \gamma J / \psi$.

30. A theoretical understanding of the photon energy spectrum from $J / \psi \rightarrow \gamma \eta_{c}(1 S)$ and $\psi(2 S) \rightarrow$ $\gamma \eta_{c}(1 S)$ (see item \#21) is required (Sects. 3.1.1 and $3.1 .2)$. 
31. It would be important to have a coherent EFT treatment for all magnetic and electric transitions. In particular, a rigorous treatment of the relativistic corrections contributing to the E1 transitions and a nonperturbative analysis of the M1 transitions is missing. The first is relevant for transitions involving $P$ states, the second for any transition from above the ground state.

32. New resummation schemes for the perturbative expressions of the quarkonium decay widths should be developed. At the moment, this is the major obstacle to precise theoretical determinations of the $\Upsilon(1 S)$ and $\eta_{b}(1 S)$ inclusive and electromagnetic decays (Sect. 3.2.1).

33. More rigorous techniques to describe abovethreshold quarkonium decays and transitions, whose descriptions still rely upon models, should be developed (Sects. 3.3.1 and 3.4).

Production: The theoretical and experimental status of production of heavy quarkonia was given in Sect. 4. Conclusions and priorities are as follows:

34. It is very important either to establish that the NRQCD factorization formula is valid to all orders in perturbation theory or to demonstrate that it breaks down at some fixed order.

35. A more accurate treatment of higher-order corrections to the color-singlet contributions at the Tevatron and the LHC is urgently needed. The re-organization of the perturbation series that is provided by the fragmentation-function approach (Sect. 4.1.5) may be an important tool.

36. An outstanding theoretical challenge is the development of methods to compute color-octet longdistance NRQCD production matrix elements on the lattice.

37. If NRQCD factorization is valid, it likely holds only for values of $p_{T}$ that are much greater than the heavy-quark mass. Therefore, it is important for experiments to make measurements of quarkonium production, differentially in $p_{T}$, at the highest possible values of $p_{T}$.

38. Further light could be shed on the NRQCD velocity expansion and its implications for low-energy dynamics by comparing studies of charmonium production and bottomonium production. The higher $p_{T}$ reach of the LHC may be particularly important for studying bottomonium production at values of $p_{T}$ that are much greater than the bottomonium mass.

39. It would be of considerable help in disentangling the theoretical issues in production of the $J / \psi$ and $\Upsilon$ if experimental measurements could separately quantify direct and feeddown contributions. Ideally, the direct production cross sections and polarizations would both be measured differentially in $p_{T}$.

40. It is important to resolve the apparent discrepancy between the CDF and D $\varnothing$ measurements of the $\Upsilon$ polarization, which were performed for different rapidity ranges, $|y|<0.6(\mathrm{CDF})$ and $|y|<1.8(\mathrm{D} \varnothing)$. A useful first step would be for the two experiments to provide polarization measurements that cover the same rapidity range.

41. It would be advantageous to measure complete quarkonium polarization information in a variety of spin-quantization frames and to make use of frameinvariant quantities to cross-check measurements in different frames [722, 723, 1030]. Care should be taken in comparing different polarization measurements to insure that dependences on the choices of frame and the kinematic ranges of the experiments have been taken into account.

42. Measurements of inclusive cross sections, charmonium angular distributions, and polarization parameters for $P$-wave charmonium states would provide further important information about quarkonium production mechanisms.

43. Studies of quarkonium production at different values of $\sqrt{s}$ at the Tevatron and the LHC, studies of hadronic energy near to and away from the quarkonium direction at the Tevatron and the LHC, and studies of the production of heavy-flavor mesons in association with a quarkonium at $e^{+} e^{-}, e p, p \bar{p}$, and $p p$ machines could give information that is complementary to that provided by traditional observations of quarkonium production rates and polarizations.

44. Theoretical uncertainties in the region near the kinematic endpoint of maximum quarkonium energy might be reduced through a systematic study of resummations of the perturbative and velocity expansions in both ep and $e^{+} e^{-}$quarkonium production.

45. In predictions for exclusive and inclusive quarkonium production in $e^{+} e^{-}$annihilation, large corrections appear at $\mathrm{NLO}$. An important step would be to identify the origins of these large corrections. It might then be possible to improve the convergence of perturbation series by resumming specific large contributions to all orders in $\alpha_{\mathrm{s}}$.

46. The central values of the Belle and $B A B A R$ measurements of $\sigma\left(e^{+} e^{-} \rightarrow J / \psi+\eta_{c}(1 S)\right) \times \mathcal{B}_{>2}$, where $\mathcal{B}_{>2}$ is the branching fraction for the $\eta_{c}(1 S)$ to decay into a final state with more than two charged particles, differ by about twice the uncertainty of 
either measurement, suggesting that further experimental attention would be valuable. Comparisons with theory would be more informative if the uncertainty from the unmeasured branching fraction $\mathcal{B}_{>2}$ were reduced or eliminated.

47. The central values for the prompt $J / \psi$ inclusive production cross section that were obtained by $B A B A R$ and Belle differ by more than a factor of two. It would be very desirable to clear up this discrepancy.

48. Belle has presented results for $\sigma\left(e^{+} e^{-} \rightarrow J / \psi+\right.$ $c \bar{c})$ and $\sigma\left(e^{+} e^{-} \rightarrow J / \psi+X_{\text {non }-c \bar{c}}\right)$. It would be beneficial to have similar results from BABAR.

49. Measurements of quarkonium production in $\gamma p$ and $\gamma \gamma$ collisions could provide additional information about production mechanisms and should be carried out at the next opportunity at an $e^{+} e^{-}$or $e p$ collider. It may also be possible to measure quarkonium production in $\gamma \gamma$ and $\gamma p$ collisions at the LHC [828].

50. The observation and study of the $B_{c}$ mesons and their excitations are new and exciting components of the quarkonium-physics plans for both the Tevatron and the LHC.

51. Theoretical progress should always be mirrored by the development of simulation tools for experiment. A particularly important goal would be to develop experiment-friendly simulation tools that incorporate the state-of-the-art theory for inclusive and associative quarkonium production.

In Medium: The status of heavy quarkonium production in cold and hot matter was presented in Sect. 5 . Conclusions are, for cold matter:

52. Studies are now attempting to place a limit on the allowed level of quark energy loss in next-to-leading order Drell-Yan dilepton production. These will determine the maximum amount of gluon energy loss that can be applied to $J / \psi$ production and suppression models of $p A$ interactions.

53. A limit on the level of energy loss apparent in $J / \psi$ production as a function of nuclear mass, $A$, and longitudinal momentum, expressed either as a function of $x_{F}$ or rapidity, $y$, will put constraints on the nuclear absorption cross section. These constraints will help determine the importance of formation-time effects and feeddown on the quarkonium absorption cross section. Ultimately, the cold-nuclear-matter baseline should include different asymptotic absorption cross sections based on their final-state radii and formation times.

For hot matter:
54. It is important to calculate the quarkonium spectral functions at nonzero temperature using an effective field theory approach with different hierarchies of relevant scales amended with lattice QCD calculations of the relevant correlation functions.

55. We would like to be able to compare the hot-matter effects more directly to heavy-ion data. To do this, more realistic dynamical models of quarkonium production in heavy-ion collisions must be developed that rely on state-of-the-art calculations of the quarkonium spectral functions.

Important future RHIC measurements include:

56. The open charm and open bottom cross sections as a function of rapidity and centrality in $\mathrm{d}+\mathrm{Au}$ and $\mathrm{Au}+\mathrm{Au}$ collisions, as well as a measurement of the rapidity distribution in $p p$ collisions. Such a measurement is complementary to quarkonium production and necessary to establish whether the reduced $J / \psi$ production cross section at forward rapidity, manifested in the larger effective absorption cross shown in Figs. 78, 82 and 85, is associated with reduced charm production or is particular to boundstate formation. This should be feasible with the upgraded vertex detectors being installed in 2011 and 2012, in conjunction with another $\mathrm{d}+\mathrm{Au}$ run.

57. The $J / \psi$ elliptic flow, $v_{2}$, in $\mathrm{Au}+\mathrm{Au}$ collisions. Statistical recombination models predict strong secondary $J / \psi$ production in heavy-ion collisions. This measurement will be an important test of this recombination picture.

58. Higher-statistics measurements of $\psi$ and $\Upsilon$ production in $\mathrm{d}+\mathrm{Au}$ and $\mathrm{Au}+\mathrm{Au}$ collisions, which can be expected with increasing RHIC luminosity. These will provide further tests of the quarkonium production and suppression mechanisms in cold and hot matter.

Experimental outlook: Section 6 gives an overview of newly-commissioned, under-construction, and onlyplanned experimental facilities and what their activities relating to heavy quarkonium will be:

59. BESIII operating at BEPCII will continue where BESII and CLEO-c left off, with a robust program of charmonium spectroscopy and decay investigations. Initial datasets at the $J / \psi$ and $\psi(2 S)$ already exceed previous accumulations, with additional data acquisition at $\psi(3770)$ and the $D \bar{D}^{*}$ peak at $\sqrt{s}=4170 \mathrm{MeV}$ planned for the near future. Fine scans above open-charm threshold are likely. At its highest energy, BEPCII will directly produce $Y(4260)$ for much-needed further study.

60. ALICE, ATLAS, CMS, and LHCb are being commissioned along with the LHC, and have potent 
heavy quarkonium programs underway, planning important production and polarization measurements in both $p p$ and heavy-ion collisions. The four experiments have distinct and complementary experimental strengths and specialties, but also will have significant overlaps in many measurements for cross-checking of results.

61. The $\overline{\mathrm{P} A N D A}(p \bar{p})$ and CBM (nucleus-nucleus) experiments at the FAIR facility at GSI will complement the activities at other laboratories.

62. The knowledge of open-charm cross section in proton-antiproton annihilations is extremely important to shape the initial physics program of PANDA at FAIR. A collaboration should be formed to prepare a proposal to perform inclusive measurements at Fermilab with $\bar{p} p$ collisions in the charmonium energy region using existing detector elements.

63. Lepton-hadron colliders have significant role in advancing the heavy-quarkonium-physics agenda, with the energy, intensity, and experimental upgrades at JLab likely to contribute before other similar proposed facilities.

64. A tau-charm factory and/or a more flexible superflavor factory have been proposed to continue the giant strides in heavy quarkonium physics taken at $e^{+} e^{-}$machines, from Mark-I at SPEAR to the very recent landmark results from both $B$ factories, CLEO-c, and BESIII. The factory-level luminosities combined with sophisticated detectors and well-defined intitial-state energy-momentum and quantum numbers give $e^{+} e^{-}$collisions many important advantages.

65. A future linear collider (CLIC or ILC) would offer important opportunities to measure top quark properties in the $t \bar{t}$ threshold region (see item \#17 above).

\section{Acknowledgments}

The authors appreciate and acknowledge support for work on this document provided, in part or in whole, by

- the U.S. Department of Energy (DOE), under contracts DE-FG02-91-ER40690 (P. Artoisenet),

DE-AC02-06-CH11357 (G. T. Bodwin), DE-AC05-06-OR23177 (E. Chudakov and C. Weiss) DE-AC02-07-CH11359, through FNAL, which is operated for DOE by the Fermi Research Alliance, LLC, under Grant No. DE-FG02-91-ER40676 (E. Eichten and V. Papadimitriou),

DE-AC02-76-SF00515 (A. Gabareen Mokhtar and J. P. Lansberg),

DE-AC02-05-CH11231 (S. R. Klein),

DE-AC02-98-CH10886 (P. Petreczky and J. W. Qiu), DE-FG02-96-ER41005 (A. A. Petrov),
DE-AC52-07-NA27344f (R. Vogt), and DE-FG02-94-ER40823 (M. Voloshin);

- the German Research Foundation (DFG) Collaborative Research Center 55 (SFB) and the European Union Research Executive Agency (REA) Marie Curie Initial Training Network (www.physik.uniregensburg.de/STRONGnet), under Grant Agreement PITN-GA-2009-238353 (G. Bali);

- the European Union Marie Curie Research Training Network (RTN) Flavianet, under Contract MRTN-CT2006-035482, and the German Research Foundation (DFG) Cluster of Excellence Origin and Structure of the Universe (www.universe-cluster.de)

(N. Brambilla and A. Vairo);

- the Polish Ministry of Science and Higher Education (J. Brodzicka);

- the National Natural Science Foundation of China (NSFC) under Grants 10875155 and 10847001 (C.-H. Chang), 10721063 (K.-T. Chao), 10920101072 and 10845003 (W. Qian), and 10775412, 10825524, and 10935008 (C.-Z. Yuan);

- the Ministry of Science and Technology of China, under Grant 2009CB825200 (K.-T. Chao);

- The German Research Foundation (DFG) under grant GZ 436 RUS 113/769/0-3 and the Russian Foundation for Basic Research (RFBR) under grants 08-02-13516 and 08-02-91969 (S. Eidelman);

- the U.S. National Science Foundation (NSF), under contracts PHY-07-56474 (A. D. Frawley), PHY-07-58312 and PHY-09-70024 (B. K. Heltsley), CAREER Award PHY-05-47794 (A. Petrov), and PHY-05-55660 (R. Vogt),;

- Science and Engineering Research Canada (NSERC) (X. Garcia i Tormo);

- the Helmholtz Association, through funds provided to the virtual institute Spin and strong QCD (VH-VI231), the German Research Foundation (DFG) (under grants SFB/TR 16 and 436 RUS 113/991/0-1) and the European Community-Research Infrastructure Integrating Activity Study of Strongly Interacting Matter (acronym HadronPhysics2, Grant Agreement 227431) under the European Union Seventh Framework Programme (C. Hanhart);

- the Belgian American Educational Foundation and the Francqui Foundation (J. P. Lansberg);

- the Belgian Federal Science Policy (IAP 6/11) (F. Maltoni);

- the Brazil National Council for Scientific and Technological Development $(\mathrm{CNPq})$ and Foundation for Research Support of the State of São Paulo (FAPESP) (F. S. Navarra and M. Nielson);

- the World Class University (WCU) project of the National Research Foundation of Korea, under contract R32-2008-000-10155-0 (S. Olsen);

- the Ministry of Education and Science of the Russian Federation and the State Atomic Energy Corporation "Rosatom" (P. Pakhlov and G. Pakhlova); 
- the France-China Particle Physics Laboratory (FCPPL) (W. Qian);

- the French National Research Agency (ANR) under Contract "BcLHCb ANR-07-JCJC-0146" (P. Robbe);

- the Spanish Ministry of Science and Innovation (MICNN), under grant FPA2008-02878 and Generalitat Valenciana under grant GVPROMETEO2010056 (M. A. Sanchis-Lozano);

- the Portuguese Foundation for Science and Technology (FCT), under contracts SFRH/BPD/42343/2007 and SFRH/BPD/42138/2007 (P. Faccioli and H. K. Wöhri)

N. Brambilla, A. D. Frawley, C. Lourenço, A. Mocsy, P. Petreczky, H. K. Wöhri, A. Vairo, and R. Vogt acknowledge the hospitality of Institute for Nuclear Theory at the University of Washington and the U.S. Department of Energy for partial support during their attendance at the CATHIE-INT miniprogram on heavy quarkonium in Seattle.

We thank Hee Sok Chung for preparing Fig. 59, and Roman Mizuk for his perspective on the $Z^{+}$analyses. We thank Eric Braaten for his feedback on the manuscript, and Dave Besson, Mathias Butenschön, and Bernd Kniehl for useful discussions. We thank Jeanne Butler for assistance with preparation of the figures.
[1] N. Brambilla et al., CERN-2005-005, (CERN, Geneva, 2005), arXiv:hep-ph/0412158

[2] D. C. Hom et al., Phys. Rev. Lett. 36 (1976) 1236

[3] H. D. Snyder et al., Phys. Rev. Lett. 36 (1976) 1415

[4] S. W. Herb et al., Phys. Rev. Lett. 39 (1977) 252

[5] C. Edwards et al., Phys. Rev. Lett. 48 (1982) 70

[6] S. B. Athar et al. (CLEO Collaboration), Phys. Rev. D 70 (2004) 112002, arXiv:hep-ex/0408133

[7] J. Siegrist et al. (Mark I Collaboration), Phys. Rev. Lett. 36 (1976) 700

[8] P. A. Rapidis et al. (MARK I Collaboration), Phys. Rev. Lett. 39 (1977) 526 [Erratum-ibid. 39 (1977) 974]

[9] W. Bacino et al. (DELCO Collaboration), Phys. Rev. Lett. 40 (1978) 671

[10] R. Brandelik et al. (DASP Collaboration), Phys. Lett. B 76 (1978) 361

[11] R. H. Schindler et al. (MARK II Collaboration), Phys. Rev. D 21 (1980) 2716

[12] A. Osterheld et al. (Crystal Ball Collaboration), SLAC-PUB-4160, 1986

[13] J. Z. Bai et al. (BES Collaboration), Phys. Rev. Lett. 88 (2002) 101802, arXiv:hep-ex/0102003

[14] K. K. Seth, Phys. Rev. D 72 (2005) 017501

[15] M. Ablikim et al. (BES Collaboration), Phys. Lett. B 660 (2008) 315, arXiv:0705.4500 [hep-ex]

[16] D. Cronin-Hennessy et al. (CLEO Collaboration), Phys. Rev. D 80 (2009) 072001, arXiv:0801.3418 [hepex]

[17] S. Eidelman et al. (Particle Data Group), Phys. Lett. B 592 (2004) 1

[18] C. Amsler et al. (Particle Data Group), Phys. Lett. B 667 (2008) 1

[19] M. Ablikim et al., Phys. Rev. Lett. 101 (2008) 102004

[20] K. Todyshev (KEDR Collaboration), presentation at ICHEP2010, the 35th International Conference on High Energy Physics, July 22-28, 2010, Paris, France

[21] G. Pakhlova et al. (Belle Collaboration), Phys. Rev. D 77 (2008) 011103 arXiv:0708.0082 [hep-ex]

[22] G. Pakhlova et al. (Belle Collaboration), Phys. Rev. Lett. 98 (2007) 092001, arXiv:hep-ex/0608018

[23] G. Pakhlova et al. (Belle Collaboration), Phys. Rev. Lett. 100 (2008) 062001, arXiv:0708.3313 [hep-ex]

[24] G. Pakhlova et al. (Belle Collaboration), Phys. Rev. D 80 (2009) 091101, arXiv:0908.0231 [hep-ex]

[25] G. Pakhlova et al. (Belle Collaboration), Phys. Rev.
Lett. 101 (2008) 172001, arXiv:0807.4458 [hep-ex]

[26] G. Pakhlova et al. (Belle Collaboration), Phys. Rev. D 83, 011101(R) (2011), arXiv:1011.4397 [hep-ex]

[27] B. Aubert et al. (BABAR Collaboration), Phys. Rev. D 76 (2007) 111105, arXiv:hep-ex/0607083

[28] B. Aubert et al. (BABAR Collaboration), Phys. Rev. D 79 (2009) 092001, arXiv:0903.1597 [hep-ex]

[29] P. del Amo Sanchez et al. (BABAR Collaboration), Phys. Rev. D 82 (2010) 052004, arXiv:1008.0338 [hepex]

[30] S. Dobbs et al. (CLEO Collaboration), Phys. Rev. D 76 (2007) 112001, arXiv:0709.3783 [hep-ex]

[31] T. Barnes, S. Godfrey, and E. S. Swanson, Phys. Rev. D 72 (2005) 054026, arXiv:hep-ph/0505002

[32] E. J. Eichten, K. Lane, and C. Quigg, Phys. Rev. D 73 (2006) 014014 [Erratum-ibid. D 73 (2006) 079903], arXiv:hep-ph/0511179

[33] E. S. Swanson, Phys. Rept. 429 (2006) 243, arXiv:hep$\mathrm{ph} / 0601110$

[34] E. Eichten, K. Gottfried, T. Kinoshita, K. D. Lane, and T. M. Yan, Phys. Rev. D 21 (1980) 203

[35] W. M. Yao et al. (Particle Data Group), J. Phys. G 33 (2006) 1

[36] B. Aubert et al. (BABAR Collaboration), Phys. Rev. Lett. 102 (2009) 012001, arXiv:0809.4120 [hep-ex]

[37] K.-F. Chen et al. (Belle Collaboration), Phys. Rev. D 82 (2010) 091106 (R), arXiv:0808.2445 [hep-ex]

[38] D. Besson et al. (CLEO Collaboration), Phys. Rev. Lett. 54 (1985) 381

[39] D. M. J. Lovelock et al., Phys. Rev. Lett. 54 (1985) 377

[40] N. A. Tornqvist, Phys. Rev. Lett. 53 (1984) 878

[41] A. Drutskoy et al. (Belle Collaboration), Phys. Rev. D 81 (2010) 112003, arXiv:1003.5885 [hep-ex]

[42] Yu. A. Simonov and A. I. Veselov, JETP Lett. 88 (2008) 5, arXiv:0805.4518 [hep-ph]

[43] L. Lellouch, L. Randall, and E. Sather, Nucl. Phys. B 405 (1993) 55, arXiv:hep-ph/9301223

[44] P. Rubin et al. (CLEO Collaboration), Phys. Rev. D 72 (2005) 092004, arXiv:hep-ex/0508037

[45] J. L. Rosner et al. (CLEO Collaboration), Phys. Rev. Lett. 95 (2005) 102003, arXiv:hep-ex/0505073

[46] S. Dobbs et al. (CLEO Collaboration), Phys. Rev. Lett. 101 (2008) 182003, arXiv:0805.4599 [hep-ex]

[47] M. Ablikim et al. (BESIII Collaboration), Phys. Rev. 
Lett. 104 (2010) 132002, arXiv:1002.0501 [hep-ex]

[48] M. Andreotti et al. (E835 Collaboration), Phys. Rev. D 72 (2005) 032001

[49] S. K. Choi et al. (Belle collaboration), Phys. Rev. Lett. 89 (2002) 102001 [Erratum-ibid. 89 (2002) 129901], arXiv:hep-ex/0206002

[50] B. Aubert et al. (BABAR Collaboration), Phys. Rev. Lett. 92 (2004) 142002, arXiv:hep-ex/0311038

[51] D. M. Asner et al. (CLEO Collaboration), Phys. Rev. Lett. 92 (2004) 142001, arXiv:hep-ex/0312058

[52] H. Nakazawa (Belle Collaboration), Nucl. Phys. Proc. Suppl. 184 (2008) 220

[53] B. Aubert et al. (BABAR Collaboration), Phys. Rev. D 72 (2005) 031101, arXiv:hep-ex/0506062

[54] K. Abe et al. (Belle Collaboration), Phys. Rev. Lett. 98 (2007) 082001, arXiv:hep-ex/0507019

[55] S. Uehara et al. (Belle Collaboration), Phys. Rev. Lett. 96 (2006) 082003, arXiv:hep-ex/0512035

[56] B. Aubert et al. (BABAR Collaboration), Phys. Rev. D 81 (2010) 092003, arXiv:1002.0281 [hep-ex]

[57] T. Aaltonen et al. (CDF Collaboration), Phys. Rev. Lett. 100 (2008) 182002, arXiv:0712.1506 [hep-ex]

[58] V. M. Abazov et al. (DØ Collaboration), Phys. Rev. Lett. 101 (2008) 012001, arXiv:0802.4258 [hep-ex]

[59] B. Aubert et al. (BABAR Collaboration), Phys. Rev. Lett. 101 (2008) 071801 [Erratum-ibid. 102, 029901 (2009)], arXiv:0807.1086 [hep-ex]

[60] G. Bonvicini et al. (CLEO Collaboration), Phys. Rev. D 81 (2010) 031104, arXiv:0909.5474 [hep-ex]

[61] B. Aubert et al. (BABAR Collaboration), Phys. Rev. Lett. 103 (2009) 161801, arXiv:0903.1124 [hep-ex]

[62] G. Bonvicini et al. (CLEO Collaboration), Phys. Rev. D 70 (2004) 032001, arXiv:hep-ex/0404021

[63] P. del Amo Sanchez et al. (BABAR Collaboration) Phys. Rev. D 82 (2010) 111102, arXiv:1004.0175 [hep-ex]

[64] J. S. Whitaker et al. (Mark I Collaboration), Phys. Rev. Lett. 37 (1976) 1596

[65] C. J. Biddick et al., Phys. Rev. Lett. 38 (1977) 1324

[66] W. M. Tanenbaum et al. (Mark I Collaboration), Phys. Rev. D 17 (1978) 1731

[67] W. Bartel et al., Phys. Lett. B 79 (1978) 492

[68] D. Cronin-Hennessy et al. (CLEO Collaboration), Phys. Rev. D 81 (2010) 052002, arXiv:0910.1324 [hepex]

[69] R. E. Mitchell et al. (CLEO Collaboration), Phys. Rev. Lett. 102 (2009) 011801, arXiv:0805.0252 [hep-ex]

[70] H. Nakazawa (Belle Collaboration), presentation at ICHEP2010, the 35th International Conference on High Energy Physics, July 22-28, 2010, Paris, France

[71] J. J. Dudek, R. Edwards, and C. E. Thomas, Phys. Rev. D 79 (2009) 094504, arXiv:0902.2241 [hep-ph]

[72] K. Ackerstaff et al. (OPAL Collaboration), Phys. Lett. B 420 (1998) 157, arXiv:hep-ex/9801026

[73] F. Abe et al. (CDF Collaboration), Phys. Rev. Lett. 81 (1998) 2432, arXiv:hep-ex/9805034

[74] I. F. Allison et al. (HPQCD, Fermilab Lattice, and UKQCD Collaborations), Phys. Rev. Lett. 94 (2005) 172001, arXiv:hep-lat/0411027

[75] A. Abulencia et al. (CDF Collaboration), Phys. Rev. Lett. 97 (2006) 012002, arXiv:hep-ex/0603027

[76] T. S. Nigmanov, K. R. Gibson, M. P. Hartz, and P. F. Shepard (CDF Collaboration), arXiv:0910.3013 [hep-ex]

[77] V. M. Abazov et al. (DØ Collaboration), Phys. Rev.
Lett. 102 (2009) 092001, arXiv:0805.2614 [hep-ex]

[78] S. Brandt, C. Peyrou, R. Sosnowski, and A. Wroblewski, Phys. Lett. 12 (1964) 57

[79] M. Artuso et al. (CLEO Collaboration), Phys. Rev. Lett. 94 (2005) 032001, arXiv:hep-ex/0411068

[80] B. Fulsom (BABAR Collaboration), presentation at ICHEP2010, the 35th International Conference on High Energy Physics, July 22-28, 2010, Paris, France

[81] E. Eichten, S. Godfrey, H. Mahlke, and J. L. Rosner, Rev. Mod. Phys. 80 (2008) 1161, arXiv:hep$\mathrm{ph} / 0701208$

[82] S. Godfrey and S. L. Olsen, Ann. Rev. Nucl. Part. Sci. 58 (2008) 51, arXiv:0801.3867 [hep-ph]

[83] T. Barnes and S. L. Olsen, Int. J. Mod. Phys. A 24 (2009) 305

[84] G. V. Pakhlova, P. N. Pakhlov, and S. I. Eidelman, Phys. Usp. 53 (2010) 219 [Usp. Fiz. Nauk 180 (2010) 225]

[85] S. K. Choi et al. (Belle Collaboration), Phys. Rev. Lett. 91 (2003) 262001, arXiv:hep-ex/0309032

[86] I. Adachi et al. (Belle Collaboration), arXiv:0809.1224 [hep-ex]

[87] B. Aubert et al. (BABAR Collaboration), Phys. Rev. D 77 (2008) 111101, arXiv:0803.2838 [hep-ex]

[88] D. E. Acosta et al. (CDF II Collaboration), Phys. Rev. Lett. 93 (2004) 072001, arXiv:hep-ex/0312021

[89] A. Abulencia et al. (CDF Collaboration), Phys. Rev. Lett. 98 (2007) 132002, arXiv:hep-ex/0612053

[90] T. Aaltonen et al. (CDF Collaboration), Phys. Rev. Lett. 103 (2009) 152001, arXiv:0906.5218 [hep-ex]

[91] V. M. Abazov et al. (DØ Collaboration), Phys. Rev. Lett. 93 (2004) 162002, arXiv:hep-ex/0405004

[92] K. Abe et al. (Belle Collaboration), arXiv:hepex/0505037

[93] P. del Amo Sanchez et al. (BABAR Collaboration), Phys. Rev. D 82 (2010) 011101, arXiv:1005.5190 [hepex]

[94] G. Gokhroo et al. (Belle Collaboration), Phys. Rev. Lett. 97 (2006) 162002, arXiv:hep-ex/0606055

[95] T. Aushev et al., Phys. Rev. D 81 (2010) 031103, arXiv:0810.0358 [hep-ex]

[96] B. Aubert et al. (BABAR Collaboration), Phys. Rev. D 77 (2008) 011102, arXiv:0708.1565 [hep-ex]

[97] B. Aubert et al. (BABAR Collaboration), Phys. Rev. D 74 (2006) 071101, arXiv:hep-ex/0607050

[98] B. Aubert et al. (BABAR Collaboration), Phys. Rev. Lett. 102 (2009) 132001, arXiv:0809.0042 [hep-ex]

[99] V. Bhardwaj (Belle Collaboration), presentation at the International Workshop on Heavy Quarkonium May 18-21, 2010, Fermilab, Batavia, IL

[100] K. Abe et al. (Belle Collaboration), Phys. Rev. Lett. 94 (2005) 182002, arXiv:hep-ex/0408126

[101] B. Aubert et al. (BABAR Collaboration), Phys. Rev. Lett. 101 (2008) 082001, arXiv:0711.2047 [hep-ex]

[102] S. Uehara et al. (Belle Collaboration), Phys. Rev. Lett. 104 (2010) 092001, arXiv:0912.4451 [hep-ex]

[103] P. Pakhlov et al. (Belle Collaboration), Phys. Rev. Lett. 100 (2008) 202001, arXiv:0708.3812 [hep-ex]

[104] C. Z. Yuan et al. (Belle Collaboration), Phys. Rev. Lett. 99 (2007) 182004, arXiv:0707.2541 [hep-ex]

[105] R. Mizuk et al. (Belle Collaboration), Phys. Rev. D 78 (2008) 072004 arXiv:0806.4098 [hep-ex]

[106] T. Aaltonen et al. (CDF Collaboration), Phys. Rev. Lett. 102 (2009) 242002, arXiv:0903.2229 [hep-ex] 
[107] Kai Yi (for the CDF Collaboration), arXiv:1010.3470 [hep-ex]

[108] B. Aubert et al. (BABAR Collaboration), Phys. Rev. Lett. 95 (2005) 142001, arXiv:hep-ex/0506081

[109] B. Aubert et al. (BABAR Collaboration), arXiv:0808.1543v2 [hep-ex]

[110] Q. He et al. (CLEO Collaboration), Phys. Rev. D 74 (2006) 091104, arXiv:hep-ex/0611021

[111] T. E. Coan et al. (CLEO Collaboration), Phys. Rev. Lett. 96 (2006) 162003, arXiv:hep-ex/0602034

[112] C. P. Shen et al. (Belle Collaboration), Phys. Rev. Lett. 104 (2010) 112004, arXiv:0912.2383 [hep-ex]

[113] B. Aubert et al. (BABAR Collaboration), Phys. Rev. Lett. 98 (2007) 212001, arXiv:hep-ex/0610057

[114] X. L. Wang et al. (Belle Collaboration), Phys. Rev. Lett. 99 (2007) 142002, arXiv:0707.3699 [hep-ex]

[115] S. K. Choi et al. (Belle Collaboration), Phys. Rev. Lett. 100 (2008) 142001, arXiv:0708.1790 [hep-ex]

[116] R. Mizuk et al. (Belle Collaboration), Phys. Rev. D 80 (2009) 031104, arXiv:0905.2869 [hep-ex]

[117] K. F. Chen et al. (Belle Collaboration), Phys. Rev. Lett. 100 (2008) 112001, arXiv:0710.2577 [hep-ex]

[118] S. Barlag et al. (ACCMOR Collaboration), Z. Phys. C 46 (1990) 563

[119] C. Cawlfield et al. (CLEO Collaboration), Phys. Rev. Lett. 98 (2007) 092002, arXiv:hep-ex/0701016

[120] V. V. Anashin et al. (KEDR Collaboration), Phys. Lett. B 686 (2010) 84, arXiv:0909.5545 [hep-ex]

[121] E. Braaten and M. Lu, Phys. Rev. D 76 (2007) 094028, arXiv:0709.2697 [hep-ph]

[122] E. Braaten and J. Stapleton, Phys. Rev. D 81 (2010) 014019, arXiv:0907.3167 [hep-ph]

[123] M.L. Goldberger and K. Watson, Collision Theory, Wiley, New York (1964)

[124] B. Aubert et al. (BABAR Collaboration), Phys. Rev. Lett. 96 (2006) 052002, arXiv:hep-ex/0510070

[125] B. Aubert et al. (BABAR Collaboration), Phys. Rev. D 71 (2005) 031501, arXiv:hep-ex/0412051

[126] S. Godfrey and N. Isgur, Phys. Rev. D 32 (1985) 189

[127] Yu. S. Kalashnikova and A. V. Nefediev, Phys. Rev. D 82 (2010) 097502, arXiv:1008.2895 [hep-ph]

[128] Z. Q. Liu, X. S. Qin, and C. Z. Yuan, Phys. Rev. D 78 (2008) 014032, arXiv:0805.3560 [hep-ex]

[129] X. H. Mo et al., Phys. Lett. B 640 (2006) 182, arXiv:hep-ex/0603024

[130] K. Abe et al. (Belle Collaboration), Phys. Rev. Lett. 93 (2004) 051803, arXiv:hep-ex/0307061

[131] B. Aubert et al. (BABAR Collaboration), Phys. Rev. D 79 (2009) 112001, arXiv:0811.0564 [hep-ex]

[132] M. Neubert, Phys. Rept. 245 (1994) 259, arXiv:hep$\mathrm{ph} / 9306320$

[133] A. V. Manohar and M. B. Wise, Camb. Monogr. Part. Phys. Nucl. Phys. Cosmol. 10 (2000) 1

[134] N. Brambilla, A. Pineda, J. Soto, and A. Vairo, Rev. Mod. Phys. 77 (2005) 1423, arXiv:hep-ph/0410047

[135] N. Brambilla, D. Gromes, and A. Vairo, Phys. Lett. B 576 (2003) 314, arXiv:hep-ph/0306107

[136] N. Brambilla, D. Gromes, and A. Vairo, Phys. Rev. D 64 (2001) 076010, arXiv:hep-ph/0104068

[137] W. E. Caswell and G. P. Lepage, Phys. Lett. B 167 (1986) 437

[138] G. T. Bodwin, E. Braaten, and G. P. Lepage, Phys. Rev. D 51 (1995) 1125 [Erratum-ibid. D 55 (1997) 5853], arXiv:hep-ph/9407339
[139] A. Pineda and J. Soto, Nucl. Phys. Proc. Suppl. 64 (1998) 428, arXiv:hep-ph/9707481

[140] N. Brambilla, A. Pineda, J. Soto, and A. Vairo, Nucl. Phys. B 566 (2000) 275, arXiv:hep-ph/9907240

[141] N. Brambilla, X. Garcia i Tormo, J. Soto, and A. Vairo, Phys. Lett. B 647 (2007) 185, arXiv:hep-ph/0610143

[142] N. Brambilla, A. Vairo, X. Garcia i Tormo, and J. Soto, Phys. Rev. D 80 (2009) 034016, arXiv:0906.1390 [hepph]

[143] A. V. Smirnov, V. A. Smirnov, and M. Steinhauser, Phys. Lett. B 668 (2008) 293, arXiv:0809.1927 [hep$\mathrm{ph}]$

[144] C. Anzai, Y. Kiyo, and Y. Sumino, Phys. Rev. Lett. 104 (2010) 112003, arXiv:0911.4335 [hep-ph]

[145] A. V. Smirnov, V. A. Smirnov, and M. Steinhauser, Phys. Rev. Lett. 104 (2010) 112002, arXiv:0911.4742 [hep-ph]

[146] N. Brambilla, X. G. i. Tormo, J. Soto, and A. Vairo, Phys. Rev. Lett. 105 (2010) 212001, arXiv:1006.2066 [hep-ph]

[147] Y. Sumino, Phys. Rev. D 76 (2007) 114009, arXiv:hepph/0505034

[148] C. Anzai, Y. Kiyo, and Y. Sumino, Nucl. Phys. B 838 (2010) 28, arXiv:1004.1562 [hep-ph]

[149] B. A. Kniehl, A. A. Penin, Y. Schroder, V. A. Smirnov, and M. Steinhauser, Phys. Lett. B 607 (2005) 96, arXiv:hep-ph/0412083

[150] N. Brambilla, J. Ghiglieri, and A. Vairo, Phys. Rev. D 81 (2010) 054031, arXiv:0911.3541 [hep-ph]

[151] N. Brambilla, A. Vairo, and T. Rosch, Phys. Rev. D 72 (2005) 034021, arXiv:hep-ph/0506065

[152] S. Fleming and T. Mehen, Phys. Rev. D 73 (2006) 034502, arXiv:hep-ph/0509313

[153] H. Suganuma, A. Yamamoto, N. Sakumichi, T. T. Takahashi, H. Iida, and F. Okiharu, Mod. Phys. Lett. A 23 (2008) 2331, arXiv:0802.3500 [hep-ph]

[154] A. Yamamoto, H. Suganuma, and H. Iida, Prog. Theor. Phys. Suppl. 174 (2008) 270, arXiv:0805.4735 [hep-ph]

[155] J. Najjar and G. Bali, PoS LAT2009 (2009) 089, arXiv:0910.2824 [hep-lat]

[156] N. Brambilla, A. Pineda, J. Soto, and A. Vairo, Phys. Lett. B 470 (1999) 215, arXiv:hep-ph/9910238

[157] B. A. Kniehl and A. A. Penin, Nucl. Phys. B 563 (1999) 200, arXiv:hep-ph/9907489

[158] M. B. Voloshin, Nucl. Phys. B 154 (1979) 365

[159] H. Leutwyler, Phys. Lett. B 98 (1981) 447

[160] A. Pineda, Phys. Rev. D 65 (2002) 074007, arXiv:hepph/0109117

[161] A. Pineda and J. Soto, Phys. Lett. B 495 (2000) 323, arXiv:hep-ph/0007197

[162] A. H. Hoang and I. W. Stewart, Phys. Rev. D 67 (2003) 114020, arXiv:hep-ph/0209340

[163] A. V. Manohar and I. W. Stewart, Phys. Rev. D 62 (2000) 014033, arXiv:hep-ph/9912226

[164] X. Garcia i Tormo and J. Soto, Phys. Rev. Lett. 96 (2006) 111801, arXiv:hep-ph/0511167

[165] J. L. Domenech-Garret and M. A. Sanchis-Lozano, Phys. Lett. B 669 (2008) 52, arXiv:0805.2916 [hep-ph]

[166] M. Beneke, Y. Kiyo, and K. Schuller, Nucl. Phys. B 714 (2005) 67, arXiv:hep-ph/0501289

[167] A. A. Penin, V. A. Smirnov, and M. Steinhauser, Nucl. Phys. B 716 (2005) 303, arXiv:hep-ph/0501042

[168] A. A. Penin and M. Steinhauser, Phys. Lett. B 538 
(2002) 335, arXiv:hep-ph/0204290

[169] B. A. Kniehl, A. A. Penin, V. A. Smirnov, and M. Steinhauser, Nucl. Phys. B 635 (2002) 357, arXiv:hep-ph/0203166

[170] N. Brambilla and A. Vairo, Phys. Rev. D 62 (2000) 094019, arXiv:hep-ph/0002075

[171] N. Brambilla, Y. Sumino, and A. Vairo, Phys. Rev. D 65 (2002) 034001, arXiv:hep-ph/0108084

[172] N. Brambilla, Y. Sumino, and A. Vairo, Phys. Lett. B 513 (2001) 381, arXiv:hep-ph/0101305

[173] A. Abulencia et al. (CDF Collaboration), Phys. Rev. Lett. 96 (2006) 082002, arXiv:hep-ex/0505076

[174] N. Brambilla and A. Vairo, Phys. Rev. D 71 (2005) 034020, arXiv:hep-ph/0411156

[175] B. A. Kniehl, A. A. Penin, A. Pineda, V. A. Smirnov, and M. Steinhauser, Phys. Rev. Lett. 92 (2004) 242001 [Erratum-ibid. 104 (2010) 199901], arXiv:hep$\mathrm{ph} / 0312086$

[176] S. Recksiegel and Y. Sumino, Phys. Lett. B 578 (2004) 369, arXiv:hep-ph/0305178

[177] A. A. Penin, A. Pineda, V. A. Smirnov and M. Steinhauser, Phys. Lett. B 593 (2004) 124 [Erratumibid. 677 (2009) 343] [Erratum-ibid. 683 (2010) 358], arXiv:hep-ph/0403080

[178] N. Brambilla, A. Pineda, J. Soto, and A. Vairo, Phys. Rev. D 63 (2001) 014023, arXiv:hep-ph/0002250

[179] A. Pineda and A. Vairo, Phys. Rev. D 63 (2001) 054007 [Erratum-ibid. D 64 (2001) 039902], arXiv:hep$\mathrm{ph} / 0009145$

[180] A. Laschka, N. Kaiser, and W. Weise, PoS CONFINEMENT8 (2008) 168, arXiv:0901.2260 [hep-ph]

[181] N. Brambilla, D. Eiras, A. Pineda, J. Soto, and A. Vairo, Phys. Rev. D 67 (2003) 034018, arXiv:hepph/0208019

[182] C. T. H. Davies, K. Hornbostel, I. D. Kendall, G. P. Lepage, C. McNeile, J. Shigemitsu, and H. Trottier (HPQCD Collaboration), Phys. Rev. D 78 (2008) 114507, arXiv:0807.1687 [hep-lat]

[183] Y. Namekawa et al. (PACS-CS Collaboration), PoS LATTICE2008 (2008) 121, arXiv:0810.2364 [hep-lat]

[184] Y. Taniguchi (PACS-CS Collaboration), PoS LAT2009 (2009) 208, arXiv:0910.5105 [hep-lat]

[185] M. Göckeler et al. (QCDSF Collaboration), PoS LAT2009 (2009) 125, arXiv:0912.0167 [hep-lat]

[186] M. Creutz, Phys. Lett. B 649 (2007) 230, arXiv:heplat/0701018

[187] M. Creutz, PoS CONFINEMENT8 (2008) 016, arXiv:0810.4526 [hep-lat]

[188] C. Bernard, M. Golterman, Y. Shamir and S. R. Sharpe, Phys. Lett. B 649 (2007) 235, arXiv:hep-lat/0603027

[189] M. Golterman, PoS CONFINEMENT8 (2008) 014, arXiv:0812.3110 [hep-ph]

[190] G. P. Lepage, L. Magnea, C. Nakhleh, U. Magnea, and K. Hornbostel, Phys. Rev. D 46 (1992) 4052, arXiv:hep-lat/9205007

[191] A. X. El-Khadra, A. S. Kronfeld, and P. B. Mackenzie, Phys. Rev. D 55 (1997) 3933, arXiv:hep-lat/9604004

[192] A. S. Kronfeld, Phys. Rev. D 62 (2000) 014505, arXiv:hep-lat/0002008

[193] X. Liao and T. Manke, arXiv:hep-lat/0210030

[194] M. Okamoto et al. (CP-PACS Collaboration), Phys. Rev. D 65 (2002) 094508, arXiv:hep-lat/0112020

[195] J. Harada, A. S. Kronfeld, H. Matsufuru, N. Naka- jima and T. Onogi, Phys. Rev. D 64 (2001) 074501, arXiv:hep-lat/0103026

[196] H. W. Lin et al. (Hadron Spectrum Collaboration), Phys. Rev. D 79 (2009) 034502, arXiv:0810.3588 [heplat]

[197] J. J. Dudek, R. G. Edwards, N. Mathur, and D. G. Richards, Phys. Rev. D 77 (2008) 034501, arXiv:0707.4162 [hep-lat]

[198] J. J. Dudek and E. Rrapaj, Phys. Rev. D 78 (2008) 094504, arXiv:0809.2582 [hep-ph]

[199] M. Göckeler et al., Phys. Rev. D 82, 114511 (2010), arXiv:1003.5756 [hep-lat]

[200] C. Ehmann and G. Bali, PoS LAT2007 (2007) 094, arXiv:0710.0256 [hep-lat]

[201] C. Ehmann and G. S. Bali, PoS LATTICE2008 (2008) 114, arXiv:0903.2947 [hep-lat]

[202] L. Levkova and C. E. DeTar, PoS LATTICE2008 (2008) 133, arXiv:0809.5086 [hep-lat]

[203] G. Bali and C. Ehmann, PoS LAT2009 (2009) 113, arXiv:0911.1238 [hep-lat]

[204] G. Z. Meng et al. (CLQCD Collaboration), Phys. Rev. D 80 (2009) 034503, arXiv:0905.0752 [hep-lat]

[205] T. W. Chiu and T. H. Hsieh (TWQCD Collaboration), Phys. Lett. B 646 (2007) 95, arXiv:hep-ph/0603207

[206] S. Prelovsek, Acta Phys. Polon. Supp. 3 (2010) 975, arXiv:1004.3636 [hep-lat]

[207] C. W. Bernard et al., Phys. Rev. D 64 (2001) 054506, arXiv:hep-lat/0104002

[208] G. P. Lepage, Phys. Rev. D 59 (1999) 074502, heplat $/ 9809157$

[209] T. Burch et al. (Fermilab Lattice and MILC Collaborations), Phys. Rev. D 81 (2010) 034508, arXiv:0912.2701 [hep-lat]

[210] L. Liu, H. W. Lin, K. Orginos, and A. Walker-Loud, Phys. Rev. D 81 (2010) 094505, arXiv:0909.3294 [heplat]

[211] A. Gray et al. (HPQCD and UKQCD Collaborations), Phys. Rev. D 72 (2005) 094507, arXiv:hep-lat/0507013

[212] S. Meinel, Phys. Rev. D 79 (2009) 094501, arXiv:0903.3224 [hep-lat]

[213] C. Allton et al. (RBC-UKQCD Collaboration), Phys. Rev. D 78 (2008) 114509, arXiv:0804.0473 [hep-lat]

[214] S. Meinel, W. Detmold, C. J. Lin, and M. Wingate, PoS LAT2009 (2009) 105, arXiv:0909.3837 [hep-lat]

[215] E. B. Gregory et al., Phys. Rev. Lett. 104 (2010) 022001, arXiv:0909.4462 [hep-lat]

[216] E. B. Gregory et al., PoS LAT2009 (2009) 092, arXiv:0911.2133 [hep-lat]

[217] S. Meinel, Phys. Rev. D 82 (2010) 114514, arXiv:1008.3154 [hep-lat]

[218] G. S. Bali, H. Neff, T. Düssel, T. Lippert, and K. Schilling (SESAM Collaboration), Phys. Rev. D 71 (2005) 114513, arXiv:hep-lat/0505012

[219] A. Yamamoto, H. Suganuma, and H. Iida, Phys. Rev. D 78 (2008) 014513, arXiv:0806.3554 [hep-lat]

[220] W. Detmold, K. Orginos, and M. J. Savage, Phys. Rev. D 76 (2007) 114503, arXiv:hep-lat/0703009

[221] Y. Koma, M. Koma, and H. Wittig, Phys. Rev. Lett. 97 (2006) 122003, arXiv:hep-lat/0607009

[222] Y. Koma and M. Koma, Nucl. Phys. B 769 (2007) 79, arXiv:hep-lat/0609078

[223] Y. Koma and M. Koma, PoS LAT2009 (2009) 122, arXiv:0911.3204 [hep-lat]

[224] A. A. Penin, arXiv:0905.4296 [hep-ph] 
[225] S. Meinel, Phys. Rev. D 82 (2010) 114502, arXiv:1007.3966 [hep-lat]

[226] K. Maltman, D. Leinweber, P. Moran, and A. Sternbeck, Phys. Rev. D 78 (2008) 114504, arXiv:0807.2020 [hep-lat]

[227] C. McNeile, C. T. H. Davies, E. Follana, K. Hornbostel, and G. P. Lepage, Phys. Rev. D 82 (2010) 034512, arXiv:1004.4285 [hep-lat]

[228] I. Allison et al. (HPQCD Collaboration), Phys. Rev. D 78 (2008) 054513, arXiv:0805.2999 [hep-lat]

[229] S. Bethke, Eur. Phys. J. C 64 (2009) 689, arXiv:0908.1135 [hep-ph]

[230] D. Besson et al. (CLEO Collaboration), Phys. Rev. D 74 (2006) 012003, arXiv:hep-ex/0512061

[231] X. Garcia i Tormo and J. Soto, Phys. Rev. D 72 (2005) 054014, arXiv:hep-ph/0507107

[232] N. Brambilla, X. Garcia i Tormo, J. Soto, and A. Vairo, Phys. Rev. D 75 (2007) 074014, arXiv:hep-ph/0702079

[233] G. T. Bodwin, J. Lee, and D. K. Sinclair, Phys. Rev. D 72 (2005) 014009, arXiv:hep-lat/0503032

[234] X. Garcia i Tormo and J. Soto, Phys. Rev. D 69 (2004) 114006, arXiv:hep-ph/0401233

[235] D. Besson et al. (CLEO Collaboration), Phys. Rev. D 78 (2008) 032012, arXiv:0806.0315 [hep-ex]

[236] J. Libby et al. (CLEO Collaboration), Phys. Rev. D 80 (2009) 072002, arXiv:0909.0193 [hep-ex]

[237] X. Garcia i Tormo and J. Soto, arXiv:hep-ph/0701030

[238] P. Colangelo and A. Khodjamirian, arXiv:hep$\mathrm{ph} / 0010175$

[239] P. Colangelo, P. Santorelli, and E. Scrimieri, arXiv:0912.1081 [hep-ph]

[240] K. G. Chetyrkin, J. H. Kuhn and C. Sturm, Eur. Phys. J. C 48 (2006) 107, arXiv:hep-ph/0604234

[241] R. Boughezal, M. Czakon, and T. Schutzmeier, Phys. Rev. D 74 (2006) 074006, arXiv:hep-ph/0605023

[242] B. A. Kniehl and A. V. Kotikov, Phys. Lett. B 642 (2006) 68, arXiv:hep-ph/0607201

[243] A. Maier, P. Maierhofer, and P. Marqaurd, Phys. Lett. B 669 (2008) 88, arXiv:0806.3405 [hep-ph]

[244] A. Maier, P. Maierhofer, P. Marquard, and A. V. Smirnov, Nucl. Phys. B $\mathbf{8 2 4}$ (2010) 1, arXiv:0907.2117 [hep-ph]

[245] A. H. Hoang, V. Mateu, and S. Mohammad Zebarjad, Nucl. Phys. B $\mathbf{8 1 3}$ (2009) 349, arXiv:0807.4173 [hepph]

[246] Y. Kiyo, A. Maier, P. Maierhofer, and P. Marquard, Nucl. Phys. B 823 (2009) 269, arXiv:0907.2120 [hep$\mathrm{ph}]$

[247] J. H. Kuhn, M. Steinhauser, and C. Sturm, Nucl. Phys. B 778 (2007) 192, arXiv:hep-ph/0702103

[248] K. G. Chetyrkin, J. H. Kuhn, A. Maier, P. Maierhofer, P. Marquard, M. Steinhauser, and C. Sturm, Phys. Rev. D 80 (2009) 074010, arXiv:0907.2110 [hep-ph]

[249] A. Hoang, presentation at Euroflavour2010, the Fifth Workshop of the European Flavour Physics Network FLAVIAnet

[250] A. Pineda and A. Signer, Phys. Rev. D 73 (2006) 111501, arXiv:hep-ph/0601185

[251] A. Signer, Phys. Lett. B 672 (2009) 333, arXiv:0810.1152 [hep-ph]

[252] S. Narison, Phys. Lett. B 693 (2010) 559, arXiv:1004.5333 [hep-ph]

[253] M. Beneke, Y. Kiyo, and K. Schuller, Phys. Lett. B 658 (2008) 222, arXiv:0705.4518 [hep-ph]
[254] M. Beneke, Y. Kiyo, and A. A. Penin, Phys. Lett. B 653 (2007) 53, arXiv:0706.2733 [hep-ph]

[255] M. Beneke and Y. Kiyo, Phys. Lett. B 668 (2008) 143, arXiv:0804.4004 [hep-ph]

[256] P. Marquard, J. H. Piclum, D. Seidel, and M. Steinhauser, Nucl. Phys. B 758 (2006) 144, arXiv:hep$\mathrm{ph} / 0607168$

[257] P. Marquard, J. H. Piclum, D. Seidel, and M. Steinhauser, Phys. Lett. B 678 (2009) 269, arXiv:0904.0920 [hep-ph]

[258] D. Eiras and M. Steinhauser, Nucl. Phys. B 757 (2006) 197, arXiv:hep-ph/0605227

[259] Y. Kiyo, D. Seidel, and M. Steinhauser, JHEP 0901 (2009) 038, arXiv:0810.1597 [hep-ph]

[260] A. Pineda and A. Signer, Nucl. Phys. B 762 (2007) 67, arXiv:hep-ph/0607239

[261] A. H. Hoang and M. Stahlhofen, Phys. Rev. D 75 (2007) 054025, arXiv:hep-ph/0611292

[262] A. H. Hoang and C. J. Reisser, Phys. Rev. D 71 (2005) 074022, arXiv:hep-ph/0412258

[263] A. H. Hoang, C. J. Reisser, and P. Ruiz-Femenia, Phys. Rev. D 82 (2010) 014005, arXiv:1002.3223 [hep-ph]

[264] M. Beneke, B. Jantzen, and P. Ruiz-Femenia, Nucl. Phys. B 840 (2010) 186, arXiv:1004.2188 [hep-ph]

[265] Tevatron Electroweak Working Group and CDF and DØ Collaborations, arXiv:0903.2503 [hep-ex]

[266] V. S. Fadin, V. A. Khoze, and T. Sjostrand, Z. Phys. C 48 (1990) 613

[267] K. Hagiwara, Y. Sumino, and H. Yokoya, Phys. Lett. B 666 (2008) 71, arXiv:0804.1014 [hep-ph]

[268] Y. Kiyo, J. H. Kuhn, S. Moch, M. Steinhauser, and P. Uwer, Eur. Phys. J. C 60 (2009) 375, arXiv:0812.0919 [hep-ph]

[269] Y. Sumino and H. Yokoya, JHEP 1009 (2010) 034, arXiv:1007.0075 [hep-ph]

[270] A. Vairo, Int. J. Mod. Phys. A 22 (2007) 5481, arXiv:hep-ph/0611310

[271] N. Brambilla, A. Vairo, A. Polosa, and J. Soto, Nucl. Phys. Proc. Suppl. 185 (2008) 107

[272] D. Horn and J. Mandula, Phys. Rev. D 17 (1978) 898

[273] P. Hasenfratz, R. R. Horgan, J. Kuti, and J. M. Richard, Phys. Lett. B 95 (1980) 299

[274] K. J. Juge, J. Kuti, and C. Morningstar, Phys. Rev. Lett. 90 (2003) 161601, arXiv:hep-lat/0207004

[275] G. S. Bali and A. Pineda, Phys. Rev. D 69 (2004) 094001, arXiv:hep-ph/0310130

[276] S. L. Zhu, Phys. Lett. B 625 (2005) 212, arXiv:hep$\mathrm{ph} / 0507025$

[277] E. Kou and O. Pene, Phys. Lett. B 631 (2005) 164, arXiv:hep-ph/0507119

[278] F. E. Close and P. R. Page, Phys. Lett. B 628 (2005) 215, arXiv:hep-ph/0507199

[279] G. Chiladze, A. F. Falk, and A. A. Petrov, Phys. Rev. D 58 (1998) 034013, arXiv:hep-ph/9804248

[280] A. Pineda, JHEP 0106 (2001) 022, arXiv:hepph/0105008

[281] N. A. Tornqvist, Phys. Rev. Lett. 67 (1991) 556

[282] S. Dubynskiy and M. B. Voloshin, Phys. Lett. B 666 (2008) 344, arXiv:0803.2224 [hep-ph]

[283] C. F. Qiao, Phys. Lett. B 639 (2006) 263, arXiv:hepph/0510228

[284] R. L. Jaffe, Phys. Rev. D 15 (1977) 267

[285] L. Maiani, F. Piccinini, A. D. Polosa, and V. Riquer, Phys. Rev. D 71 (2005) 014028, arXiv:hep-ph/0412098 
[286] D. Ebert, R. N. Faustov, and V. O. Galkin, Phys. Lett. B 634 (2006) 214, arXiv:hep-ph/0512230

[287] Yu. S. Kalashnikova, Phys. Rev. D 72 (2005) 034010, arXiv:hep-ph/0506270

[288] E. Braaten and M. Kusunoki, Phys. Rev. D 69 (2004) 074005, arXiv:hep-ph/0311147

[289] H. Høgaasen, J. M. Richard, and P. Sorba, Phys. Rev. D 73 (2006) 054013, arXiv:hep-ph/0511039

[290] F. Buccella, H. Hogaasen, J. M. Richard, and P. Sorba, Eur. Phys. J. C 49 (2007) 743, arXiv:hep-ph/0608001

[291] N. A. Törnqvist, Z. Phys. C 61 (1994) 525, arXiv:hep$\mathrm{ph} / 9310247$

[292] N. A. Tornqvist, arXiv:hep-ph/0308277

[293] E. S. Swanson, Phys. Lett. B 588 (2004) 189, arXiv:hep-ph/0311229

[294] E. S. Swanson, Phys. Lett. B 598 (2004) 197, arXiv:hep-ph/0406080

[295] S. Pakvasa and M. Suzuki, Phys. Lett. B 579 (2004) 67, arXiv:hep-ph/0309294

[296] M. B. Voloshin, Phys. Lett. B 579 (2004) 316, arXiv:hep-ph/0309307

[297] M. B. Voloshin, Phys. Lett. B 604 (2004) 69, arXiv:hep-ph/0408321

[298] C. Quigg, Nucl. Phys. Proc. Suppl. 142 (2005) 87, arXiv:hep-ph/0407124

[299] S. Weinberg, Phys. Rev. 130 (1963) 776

[300] S. Weinberg, Phys. Rev. 131 (1963) 440

[301] S. Weinberg, Phys. Rev. 137 (1965) B672

[302] V. Baru, J. Haidenbauer, C. Hanhart, Yu. Kalashnikova, and A. E. Kudryavtsev, Phys. Lett. B 586 (2004) 53, arXiv:hep-ph/0308129

[303] V. Baru, J. Haidenbauer, C. Hanhart, A. E. Kudryavtsev, and U. G. Meissner, Eur. Phys. J. A 23 (2005) 523, arXiv:nucl-th/0410099

[304] D. Gamermann, J. Nieves, E. Oset, and E. Ruiz Arriola, Phys. Rev. D 81 (2010) 014029, arXiv:0911.4407 [hep-ph]

[305] D. Morgan, Nucl. Phys. A 543 (1992) 632

[306] F. K. Guo, C. Hanhart, and U. G. Meissner, Phys. Lett. B 665 (2008) 26, arXiv:0803.1392 [hep-ph]

[307] A. De Rujula, H. Georgi, and S. L. Glashow, Phys. Rev. Lett. 38 (1977) 317

[308] M. B. Voloshin and L. B. Okun, JETP Lett. 23 (1976) 333 [Pisma Zh. Eksp. Teor. Fiz. 23 (1976) 369]

[309] E. van Beveren and G. Rupp, the Phys. Rev. Lett. 91 (2003) 012003, arXiv:hep-ph/0305035

[310] E. E. Kolomeitsev and M. F. M. Lutz, Phys. Lett. B 582 (2004) 39, arXiv:hep-ph/0307133

[311] T. E. Browder, S. Pakvasa, and A. A. Petrov, Phys. Lett. B 578 (2004) 365, arXiv:hep-ph/0307054

[312] F. K. Guo, C. Hanhart, S. Krewald, and U. G. Meissner, Phys. Lett. B 666 (2008) 251, arXiv:0806.3374 [hep-ph]

[313] A. Martinez Torres, K. P. Khemchandani, D. Gamermann, and E. Oset, Phys. Rev. D 80 (2009) 094012, arXiv:0906.5333 [nucl-th]

[314] M. T. AlFiky, F. Gabbiani, and A. A. Petrov, Phys. Lett. B 640 (2006) 238, arXiv:hep-ph/0506141

[315] S. Fleming, M. Kusunoki, T. Mehen, and U. van Kolck, Phys. Rev. D 76 (2007) 034006, arXiv:hep-ph/0703168

[316] S. Fleming and T. Mehen, Phys. Rev. D 78 (2008) 094019, arXiv:0807.2674 [hep-ph]

[317] S. Fleming and T. Mehen, AIP Conf. Proc. 1182 (2009) 491, arXiv:0907.4142 [hep-ph]
[318] E. Braaten and M. Lu, Phys. Rev. D 74 (2006) 054020, arXiv:hep-ph/0606115

[319] E. Braaten and M. Kusunoki, Phys. Rev. D 72 (2005) 014012, arXiv:hep-ph/0506087

[320] E. Braaten and M. Lu, Phys. Rev. D 79 (2009) 051503, arXiv:0712.3885 [hep-ph]

[321] G. Cotugno, R. Faccini, A. D. Polosa, and C. Sabelli, Phys. Rev. Lett. 104 (2010) 132005, arXiv:0911.2178 [hep-ph]

[322] F. K. Guo, C. Hanhart, and U. G. Meissner, Phys. Rev. Lett. 102 (2009) 242004, arXiv:0904.3338 [hep-ph]

[323] C. Hanhart, Yu. S. Kalashnikova, and A. V. Nefediev, Phys. Rev. D 81 (2010) 094028, arXiv:1002.4097 [hepph]

[324] F. K. Guo, J. Haidenbauer, C. Hanhart, and U. G. Meissner, Phys. Rev. D 82 (2010) 094008, arXiv:1005.2055 [hep-ph]

[325] C. Hanhart, Yu. S. Kalashnikova, A. E. Kudryavtsev, and A. V. Nefediev, Phys. Rev. D 76 (2007) 034007, arXiv:0704.0605 [hep-ph]

[326] Yu. S. Kalashnikova, and A. V. Nefediev, Phys. Rev. D 80 (2009) 074004, arXiv:0907.4901 [hep-ph]

[327] D. L. Canham, H. W. Hammer, and R. P. Springer, Phys. Rev. D 80 (2009) 014009, arXiv:0906.1263 [hep$\mathrm{ph}]$

[328] C. Bignamini, B. Grinstein, F. Piccinini, A. D. Polosa, and C. Sabelli, Phys. Rev. Lett. 103 (2009) 162001, arXiv:0906.0882 [hep-ph]

[329] T. Aaltonen et al. (CDF Collaboration), Phys. Rev. D 80 (2009) 031103, arXiv:0905.1982 [hep-ex]

[330] G. Corcella et al., JHEP 0101 (2001) 010, arXiv:hep$\mathrm{ph} / 0011363$

[331] T. Sjostrand, P. Eden, C. Friberg, L. Lonnblad, G. Miu, S. Mrenna, and E. Norrbin, Comput. Phys. Commun. 135 (2001) 238, arXiv:hep-ph/0010017

[332] P. Artoisenet and E. Braaten, Phys. Rev. D 81 (2010) 114018, arXiv:0911.2016 [hep-ph]

[333] P. Artoisenet and E. Braaten, arXiv:1007.2868 [hep$\mathrm{ph}]$

[334] D. M. Asner et al. (CLEO Collaboration), Phys. Rev. D 75 (2007) 012009, arXiv:hep-ex/0612019

[335] T. J. Burns, F. Piccinini, A. D. Polosa, and C. Sabelli, Phys. Rev. D 82 (2010) 074003, arXiv:1008.0018 [hepph]

[336] T. Sjostrand, private communication to C. Sabelli

[337] P. L. Cho and M. B. Wise, Phys. Rev. D 51 (1995) 3352, arXiv:hep-ph/9410214

[338] R. L. Jaffe and F. Wilczek, Phys. Rev. Lett. 91 (2003) 232003, arXiv:hep-ph/0307341

[339] L. Maiani, F. Piccinini, A. D. Polosa, and V. Riquer, Phys. Rev. Lett. 93 (2004) 212002, arXiv:hep$\mathrm{ph} / 0407017$

[340] G. 't Hooft, G. Isidori, L. Maiani, A. D. Polosa, and V. Riquer, Phys. Lett. B 662 (2008) 424, arXiv:0801.2288 [hep-ph]

[341] C. Amsler, T. Gutsche, S. Spanier, N. A. Tornqvist, "Note on Scalar Mesons," in [447]

[342] G. 't Hooft, arXiv:hep-th/0408148

[343] C. Alexandrou, Ph. de Forcrand, and B. Lucini, Phys. Rev. Lett. 97 (2006) 222002, arXiv:hep-lat/0609004

[344] N. V. Drenska, R. Faccini, and A. D. Polosa, Phys. Rev. D 79 (2009) 077502, arXiv:0902.2803 [hep-ph]

[345] L. Maiani, V. Riquer, F. Piccinini, and A. D. Polosa, Phys. Rev. D 72 (2005) 031502, arXiv:hep-ph/0507062 
[346] B. Aubert et al. (BABAR Collaboration), Phys. Rev. D 74 (2006) 091103, arXiv:hep-ex/0610018

[347] M. Ablikim et al. (BES Collaboration), Phys. Rev. Lett. 100 (2008) 102003, arXiv:0712.1143 [hep-ex]

[348] C. P. Shen et al. (Belle Collaboration), Phys. Rev. D 80 (2009) 031101, arXiv:0808.0006 [hep-ex]

[349] N. V. Drenska, R. Faccini, and A. D. Polosa, Phys. Lett. B 669 (2008) 160, arXiv:0807.0593 [hep-ph]

[350] A. D. Polosa, private communication

[351] L. Maiani, A. D. Polosa, V. Riquer, and C. A. Salgado, Phys. Lett. B 645 (2007) 138, arXiv:hep-ph/0606217

[352] A. Ali, C. Hambrock, and M. J. Aslam, Phys. Rev. Lett. 104 (2010) 162001, arXiv:0912.5016 [hep-ph]

[353] A. Ali, C. Hambrock, I. Ahmed, and M. J. Aslam, Phys. Lett. B 684 (2010) 28, arXiv:0911.2787 [hep-ph]

[354] D. Ebert, R. N. Faustov, and V. O. Galkin, Eur. Phys. J. C 58 (2008) 399, arXiv:0808.3912 [hep-ph]

[355] M. B. Voloshin, Prog. Part. Nucl. Phys. 61 (2008) 455, arXiv:0711.4556 [hep-ph]

[356] M. E. Peskin, Nucl. Phys. B 156 (1979) 365

[357] G. Bhanot and M. E. Peskin, Nucl. Phys. B 156 (1979) 391

[358] A. B. Kaidalov and P. E. Volkovitsky, Phys. Rev. Lett. 69 (1992) 3155

[359] A. Sibirtsev and M. B. Voloshin, Phys. Rev. D 71 (2005) 076005, arXiv:hep-ph/0502068

[360] M. B. Voloshin, Mod. Phys. Lett. A 19 (2004) 665, arXiv:hep-ph/0402011

[361] J. Erlich, E. Katz, D. T. Son, and M. A. Stephanov, Phys. Rev. Lett. 95 (2005) 261602, arXiv:hepph/0501128

[362] A. Karch, E. Katz, D. T. Son, and M. A. Stephanov, Phys. Rev. D 74 (2006) 015005, arXiv:hep-ph/0602229

[363] S. Dubynskiy, A. Gorsky, and M. B. Voloshin, Phys. Lett. B 671 (2009) 82, arXiv:0804.2244 [hep-th]

[364] M. A. Shifman, A. I. Vainshtein, and V. I. Zakharov, Nucl. Phys. B 147 (1979) 385

[365] L. J. Reinders, H. Rubinstein, and S. Yazaki, Phys. Rept. 127 (1985) 1

[366] S. Narison, Camb. Monogr. Part. Phys. Nucl. Phys. Cosmol. 17 (2002) 1, arXiv:hep-ph/0205006

[367] R. D. Matheus, F. S. Navarra, M. Nielsen, and R. Rodrigues da Silva, Phys. Rev. D 76 (2007) 056005, arXiv:0705.1357 [hep-ph]

[368] R. D. Matheus, S. Narison, M. Nielsen, and J. M. Richard, Phys. Rev. D 75 (2007) 014005, arXiv:hep-ph/0608297

[369] S. H. Lee, M. Nielsen, and U. Wiedner, arXiv:0803.1168 [hep-ph]

[370] T. V. Brito, F. S. Navarra, M. Nielsen, and M. E. Bracco, Phys. Lett. B 608 (2005) 69, arXiv:hep$\mathrm{ph} / 0411233$

[371] F. S. Navarra and M. Nielsen, Phys. Lett. B 639 (2006) 272, arXiv:hep-ph/0605038

[372] R. D. Matheus, F. S. Navarra, M. Nielsen, and C. M. Zanetti, Phys. Rev. D 80 (2009) 056002, arXiv:0907.2683 [hep-ph]

[373] J. Sugiyama, T. Nakamura, N. Ishii, T. Nishikawa, and M. Oka, Phys. Rev. D 76 (2007) 114010, arXiv:0707.2533 [hep-ph]

[374] M. Nielsen and C. M. Zanetti, Phys. Rev. D 82 (2010) 116002, arXiv:1006.0467 [hep-ph]

[375] R. M. Albuquerque and M. Nielsen, Nucl. Phys. A 815 (2009) 53, arXiv:0804.4817 [hep-ph]
[376] S. H. Lee, K. Morita, and M. Nielsen, Nucl. Phys. A 815 (2009) 29, arXiv:0808.0690 [hep-ph]

[377] G. J. Ding, Phys. Rev. D 79 (2009) 014001, arXiv:0809.4818 [hep-ph]

[378] S. H. Lee, A. Mihara, F. S. Navarra, and M. Nielsen, Phys. Lett. B 661 (2008) 28, arXiv:0710.1029 [hep-ph]

[379] M. E. Bracco, S. H. Lee, M. Nielsen, and R. Rodrigues da Silva, Phys. Lett. B 671 (2009) 240, arXiv:0807.3275 [hep-ph]

[380] K. m. Cheung, W. Y. Keung, and T. C. Yuan, Phys. Rev. D 76 (2007) 117501, arXiv:0709.1312 [hep-ph]

[381] M. Nielsen, F. S. Navarra, and S. H. Lee, Phys. Rept. 497 (2010) 41, arXiv:0911.1958 [hep-ph]

[382] J. R. Zhang and M. Q. Huang, Phys. Rev. D 80 (2009) 056004, arXiv:0906.0090 [hep-ph]

[383] Y. b. Dong, A. Faessler, T. Gutsche, and V. E. Lyubovitskij, Phys. Rev. D 77 (2008) 094013, arXiv:0802.3610 [hep-ph]

[384] Y. Dong, A. Faessler, T. Gutsche, S. Kovalenko, and V. E. Lyubovitskij, Phys. Rev. D 79 (2009) 094013, arXiv:0903.5416 [hep-ph]

[385] I. W. Lee, A. Faessler, T. Gutsche, and V. E. Lyubovitskij, Phys. Rev. D 80 (2009) 094005, arXiv:0910.1009 [hep-ph]

[386] T. Branz, T. Gutsche, and V. E. Lyubovitskij, Phys. Rev. D 80 (2009) 054019, arXiv:0903.5424 [hep-ph]

[387] T. Branz, T. Gutsche and V. E. Lyubovitskij, Phys. Rev. D 82 (2010) 054025, arXiv:1005.3168 [hep-ph]

[388] W. S. Hou, Phys. Rev. D 74 (2006) 017504, arXiv:hep$\mathrm{ph} / 0606016$

[389] M. Drees and K.-i. Hikasa, Phys. Rev. D 41 (1990) 1547

[390] E. Fullana and M. A. Sanchis-Lozano, Phys. Lett. B 653 (2007) 67, arXiv:hep-ph/0702190

[391] F. Domingo, U. Ellwanger, E. Fullana, C. Hugonie, and M. A. Sanchis-Lozano, JHEP 0901 (2009) 061, arXiv:0810.4736 [hep-ph]

[392] U. Ellwanger, C. Hugonie, and A. M. Teixeira, Phys. Rept. 496 (2010) 1, arXiv:0910.1785 [hep-ph]

[393] S. Schael et al., (ALEPH, DELPHI, L3, and OPAL Collaborations), Eur. Phys. J. C 47 (2006) 547, arXiv:hep-ex/0602042

[394] R. Dermisek and J. F. Gunion, Phys. Rev. D 73 (2006) 111701, arXiv:hep-ph/0510322

[395] R. Dermisek and J. F. Gunion, Phys. Rev. D 75 (2007) 075019, arXiv:hep-ph/0611142

[396] F. Domingo, U. Ellwanger, and M. A. Sanchis-Lozano, Phys. Rev. Lett. 103 (2009) 111802, arXiv:0907.0348 [hep-ph]

[397] B. Aubert et al. (BABAR Collaboration), Phys. Rev. Lett. 103 (2009) 181801, arXiv:0906.2219 [hep-ex]

[398] M. A. Sanchis-Lozano, Mod. Phys. Lett. A 17 (2002) 2265, arXiv:hep-ph/0206156

[399] C. Balazs, M. S. Carena, A. Menon, D. E. Morrissey, and C. E. M. Wagner, Phys. Rev. D 71 (2005) 075002, arXiv:hep-ph/0412264

[400] M. Drees and M. M. Nojiri, Phys. Rev. D 49 (1994) 4595, arXiv:hep-ph/9312213

[401] K. Hagiwara, K. Kato, A. D. Martin, and C. K. Ng, Nucl. Phys. B 344 (1990) 1

[402] S. P. Martin, Phys. Rev. D 77 (2008) 075002, arXiv:0801.0237 [hep-ph]

[403] S. P. Martin and J. E. Younkin, Phys. Rev. D 80 (2009) 035026, arXiv:0901.4318 [hep-ph] 
[404] J. E. Younkin and S. P. Martin, Phys. Rev. D 81 (2010) 055006, arXiv:0912.4813 [hep-ph]

[405] B. McElrath, Phys. Rev. D 72 (2005) 103508, arXiv:hep-ph/0506151

[406] L. N. Chang, O. Lebedev, and J. N. Ng, Phys. Lett. B 441 (1998) 419, arXiv:hep-ph/9806487

[407] P. Fayet, Phys. Lett. B 84 (1979) 421

[408] P. Fayet and J. Kaplan, Phys. Lett. B 269 (1991) 213

[409] P. Fayet, Phys. Rev. D 74 (2006) 054034, arXiv:hepph/0607318

[410] G. K. Yeghiyan, Phys. Rev. D 80 (2009) 115019, arXiv:0909.4919 [hep-ph]

[411] A. L. Fitzpatrick, D. Hooper, and K. M. Zurek, Phys. Rev. D 81 (2010) 115005

[412] D. E. Kaplan, M. A. Luty, and K. M. Zurek, Phys. Rev. D 79 (2009) 115016, arXiv:0901.4117 [hep-ph]

[413] T. Cohen, D. J. Phalen, A. Pierce, and K. M. Zurek, Phys. Rev. D 82 (2010) 056001, arXiv:1005.1655 [hep$\mathrm{ph}]$

[414] H. Albrecht et al. (ARGUS Collaboration), Phys. Lett. B 179 (1986) 403

[415] P. Rubin et al. (CLEO Collaboration), Phys. Rev. D 75 (2007) 031104, arXiv:hep-ex/0612051

[416] O. Tajima et al. (Belle Collaboration), Phys. Rev. Lett. 98 (2007) 132001, arXiv:hep-ex/0611041

[417] B. Aubert et al. (BABAR Collaboration), Phys. Rev. Lett. 103 (2009) 251801, arXiv:0908.2840 [hep-ex]

[418] M. Ablikim et al. (BES Collaboration), Phys. Rev. Lett. 100 (2008) 192001, arXiv:0710.0039 [hep-ex]

[419] P. Fayet, Phys. Rev. D 81 (2010) 054025, arXiv:0910.2587 [hep-ph]

[420] B. Aubert et al. (BABAR Collaboration), arXiv:0808.0017 [hep-ex]

[421] J. Insler et al. (CLEO Collaboration), Phys. Rev. D 81 (2010) 091101, arXiv:1003.0417 [hep-ex]

[422] N. Brambilla, Y. Jia, and A. Vairo, Phys. Rev. D 73 (2006) 054005, arXiv:hep-ph/0512369

[423] G. Feinberg and J. Sucher, Phys. Rev. Lett. 35 (1975) 1740

[424] J. Sucher, Rept. Prog. Phys. 41 (1978) 1781

[425] E. Eichten, K. Gottfried, T. Kinoshita, K. D. Lane, and T. M. Yan, Phys. Rev. D 17 (1978) 3090 [Erratum-ibid. D 21 (1980) 313]

[426] J. S. Kang and J. Sucher, Phys. Rev. D 18 (1978) 2698

[427] K. J. Sebastian, Phys. Rev. D 26 (1982) 2295

[428] G. Karl, S. Meshkov, and J. L. Rosner, Phys. Rev. Lett. 45 (1980) 215

[429] H. Grotch and K. J. Sebastian, Phys. Rev. D 25 (1982) 2944

[430] P. Moxhay and J. L. Rosner, Phys. Rev. D 28 (1983) 1132

[431] R. McClary and N. Byers, Phys. Rev. D 28 (1983) 1692

[432] H. Grotch, D. A. Owen, and K. J. Sebastian, Phys. Rev. D 30 (1984) 1924

[433] Fayyazuddin and O. H. Mobarek, Phys. Rev. D 48 (1993) 1220

[434] T. A. Lahde, Nucl. Phys. A 714 (2003) 183, arXiv:hep$\mathrm{ph} / 0208110$

[435] D. Ebert, R. N. Faustov, and V. O. Galkin, Phys. Rev. D 67 (2003) 014027, arXiv:hep-ph/0210381

[436] A. Y. Khodjamirian, Phys. Lett. B 90 (1980) 460

[437] A. Le Yaouanc, L. Oliver, O. Pene, and J. C. Raynal, "Hadron Transitions In The Quark Model", ed. Gordon and Breach (New York, 1988)
[438] J. J. Dudek, R. G. Edwards, and D. G. Richards, Phys. Rev. D 73 (2006) 074507, arXiv:hep-ph/0601137

[439] N. Brambilla, P. Roig and A. Vairo, arXiv:1012.0773 [hep-ph]

[440] G. Li and Q. Zhao, Phys. Lett. B 670 (2008) 55, arXiv:0709.4639 [hep-ph]

[441] V. V. Anashin et al., arXiv:1002.2071 [hep-ex]

[442] S. Uehara et al. (Belle Collaboration), Eur. Phys. J. C 53 (2008) 1, arXiv:0706.3955 [hep-ex]

[443] J. P. Lees et al. (BABAR Collaboration), Phys. Rev. D 81 (2010) 052010, arXiv:1002.3000 [hep-ex]

[444] B. Aubert et al. (BABAR Collaboration), Phys. Rev. D 78 (2008) 012006, arXiv:0804.1208 [hep-ex]

[445] N. E. Adam et al. (CLEO Collaboration), Phys. Rev. Lett. 94 (2005) 232002, arXiv:hep-ex/0503028

[446] H. Mendez et al. (CLEO Collaboration), Phys. Rev. D 78 (2008) 011102, arXiv:0804.4432 [hep-ex]

[447] K. Nakamura et al. (Particle Data Group), Journal of Physics G 37 (2010) 075021

[448] G. Li (BESIII Collaboration), presentation at ICHEP2010, the 35th International Conference on High Energy Physics, July 22-28, 2010, Paris, France

[449] X.-R. Lu (BESIII Collaboration), presentation at MESON2010, the 11th International Workshop on Meson Production, Properties and Interaction Kraków, Poland 10-15 June 2010

[450] M. Artuso et al. (CLEO Collaboration), Phys. Rev. D 80 (2009) 112003, arXiv:0910.0046 [hep-ex]

[451] J. L. Rosner, Phys. Rev. D 64 (2001) 094002, arXiv:hep-ph/0105327

[452] E. J. Eichten, K. Lane, and C. Quigg, Phys. Rev. D 69 (2004) 094019, arXiv:hep-ph/0401210

[453] T. E. Coan et al. (CLEO Collaboration), Phys. Rev. Lett. 96 (2006) 182002, arXiv:hep-ex/0509030

[454] R. A. Briere et al. (CLEO Collaboration), Phys. Rev. D 74 (2006) 031106, arXiv:hep-ex/0605070

[455] S. Godfrey and J. L. Rosner, Phys. Rev. D 64 (2001) 074011 [Erratum-ibid. D 65 (2002) 039901], arXiv:hep$\mathrm{ph} / 0104253$

[456] G. T. Bodwin, E. Braaten, and G. P. Lepage, Phys. Rev. D 46 (1992) 1914, arXiv:hep-lat/9205006

[457] N. Brambilla, E. Mereghetti, and A. Vairo, JHEP 0608 (2006) 039, arXiv:hep-ph/0604190

[458] N. Brambilla, E. Mereghetti, and A. Vairo, Phys. Rev. D 79 (2009) 074002, arXiv:0810.2259 [hep-ph]

[459] A. Vairo, Eur. Phys. J. A 31 (2007) 728, arXiv:hepph/0610251

[460] A. A. Penin, A. Pineda, V. A. Smirnov, and M. Steinhauser, Nucl. Phys. B 699 (2004) 183, arXiv:hepph/0406175

[461] G. T. Bodwin and Y. Q. Chen, Phys. Rev. D 64 (2001) 114008, arXiv:hep-ph/0106095

[462] Y. Kiyo, A. Pineda, and A. Signer, arXiv:1006.2685 [hep-ph]

[463] R. D. Field, Phys. Lett. B 133 (1983) 248

[464] S. G. Karshenboim, Phys. Rept. 422 (2005) 1, arXiv:hep-ph/0509010

[465] W. Kwong, P. B. Mackenzie, R. Rosenfeld, and J. L. Rosner, Phys. Rev. D 37 (1988) 3210

[466] A. Petrelli, M. Cacciari, M. Greco, F. Maltoni, and M. L. Mangano, Nucl. Phys. B 514 (1998) 245, arXiv:hep-ph/9707223

[467] A. Abele et al. (Crystal Barrel Collaboration), Phys. Lett. B 411 (1997) 361 
[468] R. Partridge et al., Phys. Rev. Lett. 44 (1980) 712

[469] G. S. Adams et al. (CLEO Collaboration), Phys. Rev. Lett. 101 (2008) 101801, arXiv:0806.0671 [hep-ex]

[470] M. Ablikim et al. (BES Collaboration), Phys. Rev. D 73 (2006) 052008, arXiv:hep-ex/0510066

[471] T. K. Pedlar et al. (CLEO Collaboration), Phys. Rev. D 79 (2009) 111101, arXiv:0904.1394 [hep-ex]

[472] S. B. Athar et al. (CLEO Collaboration), Phys. Rev. D 76 (2007) 072003, arXiv:0704.3063 [hep-ex]

[473] K. T. Chao, Nucl. Phys. B 335 (1990) 101

[474] J. P. Ma, Phys. Rev. D 65 (2002) 097506, arXiv:hep$\mathrm{ph} / 0202256$

[475] B. A. Li, Phys. Rev. D 77 (2008) 097502, arXiv:0712.4246 [hep-ph]

[476] J. L. Rosner, Phys. Rev. D 27 (1983) 1101

[477] J. L. Rosner, Proceedings of the 1985 Int. Symp. on Lepton and Photon Interactions at High Energies, Kyoto, Japan, Aug 19-24, 1985; ed. by M. Konuma and K. Takahashi, published by Kyoto Univ., Research Inst. Fund. Phys., 448 (1986)

[478] F. J. Gilman and R. Kauffman, Phys. Rev. D 36 (1987) 2761 [Erratum-ibid. D 37 (1988) 3348]

[479] J. Z. Bai et al. (BES Collaboration), Phys. Rev. D 58 (1998) 097101, arXiv:hep-ex/9806002

[480] F. Ambrosino et al. (KLOE Collaboration), Phys. Lett. B 648 (2007) 267, arXiv:hep-ex/0612029

[481] R. Escribano and J. Nadal, JHEP 0705 (2007) 006, arXiv:hep-ph/0703187

[482] J. Libby et al. (CLEO Collaboration), Phys. Rev. Lett. 101 (2008) 182002, arXiv:0806.2344 [hep-ex]

[483] B. Aubert et al. (BABAR Collaboration), Phys. Rev. D 74 (2006) 012002, arXiv:hep-ex/0605018

[484] J. L. Rosner, Phys. Rev. D 79 (2009) 097301, arXiv:0903.1796 [hep-ph]

[485] H. Nakazawa et al. (Belle Collaboration), Phys. Lett. B 615 (2005) 39, arXiv:hep-ex/0412058

[486] C. C. Kuo et al., Phys. Lett. B 621 (2005) 41, arXiv:hep-ex/0503006

[487] W. T. Chen et al., Phys. Lett. B 651 (2007) 15, arXiv:hep-ex/0609042

[488] S. Uehara et al. (Belle Collaboration), Phys. Rev. D 78 (2008) 052004, arXiv:0810.0655 [hep-ex]

[489] S. Uehara et al. (Belle Collaboration), Phys. Rev. D 79 (2009) 052009, arXiv:0903.3697 [hep-ex]

[490] S. Dobbs et al. (CLEO Collaboration), Phys. Rev. D 73 (2006) 071101, arXiv:hep-ex/0510033

[491] S. Uehara et al. (Belle Collaboration), Phys. Rev. D 82 (2010) 11403, arXiv:1007.3779 [hep-ex]

[492] K. M. Ecklund et al. (CLEO Collaboration), Phys. Rev. D 78 (2008) 091501, arXiv:0803.2869 [hep-ex]

[493] L. Landau, Phys. Abstr. A 52 (1949) 125

[494] C. N. Yang, Phys. Rev. 77 (1950) 242

[495] R. Barbieri, R. Gatto, and R. Kogerler, Phys. Lett. B 60 (1976) 183

[496] J. Wicht et al. (Belle Collaboration), Decays at Phys. Lett. B 662 (2008) 323, arXiv:hep-ex/0608037

[497] B. Aubert et al. (BABAR Collaboration), Phys. Rev. D 69 (2004) 011103, arXiv:hep-ex/0310027

[498] G. S. Adams et al. (CLEO Collaboration), Phys. Rev. D 73 (2006) 051103, arXiv:hep-ex/0512046

[499] Z. Li et al. (CLEO Collaboration), Phys. Rev. D 71 (2005) 111103, arXiv:hep-ex/0503027

[500] N. E. Adam et al. (CLEO Collaboration), Phys. Rev. Lett. 96 (2006) 082004, arXiv:hep-ex/0508023
[501] V. V. Anashin et al. (KEDR Collaboration), Phys. Lett. B 685, 134 (2010), arXiv:0912.1082 [hep-ex]

[502] M. Ablikim et al. (BES Collaboration), Phys. Rev. Lett. 97 (2006) 121801, arXiv:hep-ex/0605107

[503] M. Ablikim et al. (BES Collaboration), Phys. Lett. B 659 (2008) 74

[504] M. Ablikim et al., Phys. Rev. D 74 (2006) 112003

[505] R. Brandelik et al. (DASP Collaboration), Phys. Lett. B 73 (1978) 109

[506] J. Z. Bai et al. (BES Collaboration), Phys. Rev. D 65 (2002) 052004, arXiv:hep-ex/0010072

[507] V. V. Anashin et al., JETP Lett. 85 (2007) 347

[508] D. Besson et al. (CLEO Collaboration), Phys. Rev. Lett. 96 (2006) 092002 [Erratum-ibid. 104 (2010) 159901], arXiv:hep-ex/0512038

[509] M. Ablikim et al. (BES Collaboration), Phys. Lett. B 652 (2007) 238, arXiv:hep-ex/0612056

[510] G. S. Adams et al. (CLEO Collaboration), Phys. Rev. Lett. 94 (2005) 012001, arXiv:hep-ex/0409027

[511] J. L. Rosner et al. (CLEO Collaboration), Phys. Rev. Lett. 96 (2006) 092003, arXiv:hep-ex/0512056

[512] D. Besson et al. (CLEO Collaboration), Phys. Rev. Lett. 98 (2007) 052002, arXiv:hep-ex/0607019

[513] B. Aubert et al. (BABAR Collaboration), Phys. Rev. D 72 (2005) 032005, arXiv:hep-ex/0405025

[514] K. Gottfried, Phys. Rev. Lett. 40, 598 (1978)

[515] G. Bhanot, W. Fischler, and S. Rudaz, Nucl. Phys. B 155, 208 (1979)

[516] T. M. Yan, Phys. Rev. D 22, 1652 (1980)

[517] Y. P. Kuang and T. M. Yan, Phys. Rev. D 24 (1981) 2874

[518] W. Buchmuller and S. H. H. Tye, Phys. Rev. D 24, 132 (1981)

[519] L. S. Brown and R. N. Cahn, Phys. Rev. Lett. 35, 1 (1975)

[520] S. Dubynskiy and M. B. Voloshin, Phys. Rev. D 76, 094004 (2007), arXiv:0707.1272 [hep-ph]

[521] Y. P. Kuang, Front. Phys. China 1, 19 (2006), arXiv:hep-ph/0601044

[522] B. L. Ioffe and M. A. Shifman, Phys. Lett. B 95, 99 (1980)

[523] C. Cawlfield et al. (CLEO Collaboration), Phys. Rev. D 73 (2006) 012003, arXiv:hep-ex/0511019

[524] W. Kwong and J. L. Rosner, Phys. Rev. D 38, 279 (1988)

[525] Z. G. He, Y. Fan, and K. T. Chao, Phys. Rev. D 81, 074032 (2010), arXiv:0910.3939 [hep-ph]

[526] Y. P. Kuang and T. M. Yan, Phys. Rev. D 41, 155 (1990)

[527] Y. P. Kuang, Phys. Rev. D 65 (2002) 094024, arXiv:hep-ph/0201210

[528] H. W. Ke, J. Tang, X. Q. Hao, and X. Q. Li, Phys. Rev. D 76, 074035 (2007), arXiv:0706.2074 [hep-ph]

[529] P. Moxhay, Phys. Rev. D 37, 2557 (1988)

[530] Y. P. Kuang, S. F. Tuan, and T. M. Yan, Phys. Rev. D 37, 1210 (1988)

[531] Yu. A. Simonov and A. I. Veselov, Phys. Rev. D 79, 034024 (2009), arXiv:0804.4635 [hep-ph]

[532] P. Moxhay, Phys. Rev. D 39 (1989) 3497

[533] H. Y. Zhou and Y. P. Kuang, Phys. Rev. D 44 (1991) 756

[534] F. K. Guo, C. Hanhart, and U. G. Meissner, Phys. Rev. Lett. 103 (2009) 082003, arXiv:0907.0521 [hep-ph]

[535] F. K. Guo, C. Hanhart, G. Li, U. G. Meissner, 
and Q. Zhao, Phys. Rev. D 82 (2010) 034025 , arXiv:1002.2712 [hep-ph]

[536] G. S. Adams et al. (CLEO Collaboration), Phys. Rev. D 80 (2009) 051106, arXiv:0906.4470 [hep-ex]

[537] J. Z. Bai et al. (BES Collaboration), Phys. Lett. B 605 (2005) 63, arXiv:hep-ex/0307028

[538] M. B. Voloshin, Phys. Rev. D 71 (2005) 114003, arXiv:hep-ph/0504197

[539] Q. He et al. (CLEO Collaboration), Phys. Rev. Lett. 101 (2008) 192001, arXiv:0806.3027 [hep-ex]

[540] D. Cronin-Hennessy et al. (CLEO Collaboration), Phys. Rev. Lett. 92 (2004) 222002, arXiv:hepex/0311043

[541] M. B. Voloshin, Mod. Phys. Lett. A 18 (2003) 1067, arXiv:hep-ph/0304165

[542] D. Cronin-Hennessy et al. (CLEO Collaboration), Phys. Rev. D 76 (2007) 072001, arXiv:0706.2317 [hepex]

[543] M. B. Voloshin and V. I. Zakharov, Phys. Rev. Lett. 45 (1980) 688.

[544] S. R. Bhari et al. (CLEO Collaboration), Phys. Rev. D 79 (2009) 011103, arXiv:0809.1110 [hep-ex]

[545] B. Aubert et al. (BABAR Collaboration), Phys. Rev. D 78 (2008) 112002, arXiv:0807.2014 [hep-ex]

[546] B. Aubert et al. (BABAR Collaboration), Phys. Rev. Lett. 96 (2006) 232001, arXiv:hep-ex/0604031

[547] A. Sokolov et al. (Belle Collaboration), Phys. Rev. D 79 (2009) 051103, arXiv:0901.1431 [hep-ex]

[548] Yu. A. Simonov and A. I. Veselov, Phys. Lett. B 671 (2009) 55, arXiv:0805.4499 [hep-ph]

[549] C. Meng and K. T. Chao, Phys. Rev. D 77 (2008) 074003, arXiv:0712.3595 [hep-ph]

[550] C. Meng and K. T. Chao, Phys. Rev. D 78 (2008) 034022, arXiv:0805.0143 [hep-ph]

[551] X. Liu, B. Zhang and X. Q. Li, Phys. Lett. B 675 (2009) 441, arXiv:0902.0480 [hep-ph]

[552] Y. J. Zhang, G. Li, and Q. Zhao, Phys. Rev. Lett. 102 (2009) 172001, arXiv:0902.1300 [hep-ph]

[553] D. Y. Chen, Y. B. Dong, and X. Liu, Eur. Phys. J. C 70 (2010) 177, arXiv:1005.0066 [hep-ph]

[554] T. Appelquist and H. D. Politzer, Phys. Rev. Lett. 34 (1975) 43

[555] A. De Rujula and S. L. Glashow, Phys. Rev. Lett. 34 (1975) 46

[556] M. E. B. Franklin et al., Phys. Rev. Lett. 51 (1983) 963

[557] J. Z. Bai et al. (BES Collaboration), Phys. Rev. D 69 (2004) 072001, arXiv:hep-ex/0312016

[558] J. Z. Bai et al. (BES Collaboration), Phys. Rev. Lett. 92 (2004) 052001, arXiv:hep-ex/0310024

[559] M. Ablikim et al. (BES Collaboration), Phys. Rev. D 70 (2004) 112007 [Erratum-ibid. D 71 (2005) 019901], arXiv:hep-ex/0410031

[560] M. Ablikim et al. (BES Collaboration), Phys. Rev. D 70 (2004) 112003, arXiv:hep-ex/0408118

[561] M. Ablikim et al. (BES Collaboration), Phys. Lett. B 614 (2005) 37, arXiv:hep-ex/0407037

[562] M. Ablikim et al. (BES Collaboration), Phys. Lett. B 648 (2007) 149, arXiv:hep-ex/0610079

[563] M. Ablikim et al. (BES Collaboration), Phys. Rev. D 74 (2006) 012004, arXiv:hep-ex/0605031

[564] N. E. Adam et al. (CLEO Collaboration), Phys. Rev. Lett. 94 (2005) 012005, arXiv:hep-ex/0407028

[565] C. Z. Yuan, AIP Conf. Proc. 814 (2006) 65, arXiv:hep- ex/0510062

[566] L. Chen and W. M. Dunwoodie (MARK-III Collaboration), SLAC-PUB-5674 (1991)

[567] M. Ablikim et al. (BES Collaboration), Phys. Lett. B 619 (2005) 247

[568] M. Ablikim et al. (BES Collaboration), Phys. Rev. D 71 (2005) 072006, arXiv:hep-ex/0503030

[569] R. A. Briere et al. (CLEO Collaboration), Phys. Rev. Lett. 95 (2005) 062001, arXiv:hep-ex/0505101

[570] T. K. Pedlar et al. (CLEO Collaboration), Phys. Rev. D 72 (2005) 051108, arXiv:hep-ex/0505057

[571] X. H. Mo, C. Z. Yuan, and P. Wang, arXiv:hep$\mathrm{ph} / 0611214$

[572] M. Ablikim et al. (BES Collaboration), Phys. Lett. B 630 (2005) 7, arXiv:hep-ex/0506045

[573] D. M. Asner et al. (CLEO Collaboration), Phys. Rev. D 79 (2009) 072007, arXiv:0811.0586 [hep-ex]

[574] P. Naik et al. (CLEO Collaboration), Phys. Rev. D 78 (2008) 031101, arXiv:0806.1715 [hep-ex]

[575] S. B. Athar et al. (CLEO Collaboration), Phys. Rev. D 75 (2007) 032002, arXiv:hep-ex/0607072

[576] M. Ablikim et al., Phys. Rev. D 74 (2006) 072001, arXiv:hep-ex/0607023

[577] M. Ablikim et al. (BES Collaboration), Phys. Rev. D 70 (2004) 092002, arXiv:hep-ex/0406079

[578] M. Ablikim et al. (BES Collaboration), Phys. Rev. D 72 (2005) 092002, arXiv:hep-ex/0508050

[579] M. Ablikim et al. (BES Collaboration), Phys. Lett. B 630 (2005) 21, arXiv:hep-ex/0410028

[580] M. Ablikim et al. (BES Collaboration), Phys. Lett. B 642 (2006) 197, arXiv:hep-ex/0607025

[581] Q. He et al. (CLEO Collaboration), Phys. Rev. D 78 (2008) 092004, arXiv:0806.1227 [hep-ex]

[582] M. Ablikim et al., Phys. Rev. D 73 (2006) 052006, arXiv:hep-ex/0602033

[583] Q. Zhao, Phys. Lett. B 659 (2008) 221, arXiv:0705.0101 [hep-ph]

[584] M. Ablikim et al. (BES Collaboration), Phys. Lett. B 641 (2006) 145, arXiv:hep-ex/0605105

[585] M. Ablikim et al., Phys. Rev. D 76 (2007) 122002

[586] M. Ablikim et al. (BES Collaboration), Phys. Lett. B 650 (2007) 111, arXiv:0705.2276 [hep-ex]

[587] M. Ablikim et al. (BES Collaboration), Phys. Lett. B 656 (2007) 30, arXiv:0710.0786 [hep-ex]

[588] M. Ablikim et al. (BES Collaboration), Eur. Phys. J. C 52 (2007) 805, arXiv:0710.2176 [hep-ex]

[589] M. Ablikim et al. (BES Collaboration), Phys. Lett. B 670 (2008) 179, arXiv:0810.5608 [hep-ex]

[590] M. Ablikim et al. (BES Collaboration), Phys. Lett. B 670 (2008) 184, arXiv:0810.5611 [hep-ex]

[591] M. Ablikim et al. (BES Collaboration), Eur. Phys. J. C 64 (2009) 243

[592] M. Ablikim et al. (BES Collaboration), Eur. Phys. J. C 66 (2010) 11

[593] G. S. Huang et al. (CLEO Collaboration), Phys. Rev. Lett. 96 (2006) 032003, arXiv:hep-ex/0509046

[594] D. Cronin-Hennessy et al. (CLEO Collaboration), Phys. Rev. D 74 (2006) 012005 [Erratum-ibid. D 75 (2007) 119903], arXiv:hep-ex/0603026

[595] G. S. Adams et al. (CLEO Collaboration), Phys. Rev. D 73 (2006) 012002, arXiv:hep-ex/0509011

[596] S. Dubynskiy and M. B. Voloshin, Phys. Rev. D 78 (2008) 116014, arXiv:0809.3780 [hep-ph]

[597] H. H. Gutbrod et al., Phys. Rev. Lett. 37 (1976) 667 
[598] H. Sato and K. Yazaki, Phys. Lett. B 98 (1981) 153.

[599] H. Albrecht et al. (ARGUS Collaboration), Phys. Lett. B 236 (1990) 102

[600] G. Gustafson and J. Hakkinen, Z. Phys. C 61 (1994) 683

[601] S. J. Brodsky, arXiv:0904.3037 [hep-ph]

[602] R. A. Briere et al. (CLEO Collaboration), Phys. Rev. D 78 (2008) 092007, arXiv:0807.3757 [hep-ex]

[603] R. Barbieri, M. Caffo, and E. Remiddi, Phys. Lett. B 83 (1979) 345

[604] G. T. Bodwin, E. Braaten, D. Kang, and J. Lee, Phys. Rev. D 76 (2007) 054001, arXiv:0704.2599 [hep-ph]

[605] B. Aubert et al. (BABAR Collaboration), Phys. Rev. D 81 (2010) 011102, arXiv:0911.2024 [hep-ex]

[606] D. Kang, T. Kim, J. Lee, and C. Yu, Phys. Rev. D 76 (2007) 114018, arXiv:0707.4056 [hep-ph]

[607] Y. J. Zhang and K. T. Chao, Phys. Rev. D 78 (2008) 094017, arXiv:0808.2985 [hep-ph]

[608] D. M. Asner et al. (CLEO Collaboration), Phys. Rev. D 78 (2008) 091103, arXiv:0808.0933 [hep-ex]

[609] M. B. Einhorn and S. D. Ellis, Phys. Rev. D 12(1975) 2007

[610] S. D. Ellis, M. B. Einhorn, and C. Quigg, Phys. Rev. Lett. 36 (1976) 1263

[611] C. E. Carlson and R. Suaya, Phys. Rev. D 14 (1976) 3115

[612] C. H. Chang, Nucl. Phys. B 172 (1980) 425

[613] E. L. Berger and D. L. Jones, Phys. Rev. D 23 (1981) 1521

[614] R. Baier and R. Ruckl, Phys. Lett. B 102 (1981) 364

[615] R. Baier and R. Ruckl, Nucl. Phys. B 201 (1982) 1

[616] R. Baier and R. Ruckl, Z. Phys. C 19 (1983) 251

[617] G. A. Schuler, arXiv:hep-ph/9403387

[618] P. Artoisenet, J. P. Lansberg, and F. Maltoni, Phys. Lett. B 653 (2007) 60, arXiv:hep-ph/0703129

[619] J. M. Campbell, F. Maltoni, and F. Tramontano, Phys. Rev. Lett. 98 (2007) 252002, arXiv:hep-ph/0703113

[620] P. Artoisenet, J. M. Campbell, J. P. Lansberg, F. Maltoni, and F. Tramontano, Phys. Rev. Lett. 101 (2008) 152001, arXiv:0806.3282 [hep-ph]

[621] H. Fritzsch, Phys. Lett. B 67 (1977) 217

[622] F. Halzen, Phys. Lett. B 69 (1977) 105

[623] M. Gluck, J. F. Owens, and E. Reya, Phys. Rev. D 17 (1978) 2324

[624] V. D. Barger, W. Y. Keung, and R. J. N. Phillips, Phys. Lett. B 91 (1980) 253

[625] J. F. Amundson, O. J. P. Eboli, E. M. Gregores, and F. Halzen, Phys. Lett. B 372 (1996) 127, arXiv:hep$\mathrm{ph} / 9512248$

[626] J. F. Amundson, O. J. P. Eboli, E. M. Gregores, and F. Halzen, Phys. Lett. B 390 (1997) 323, arXiv:hepph/9605295

[627] G. T. Bodwin, E. Braaten, and J. Lee, Phys. Rev. D 72 (2005) 014004, arXiv:hep-ph/0504014

[628] F. Abe et al. (CDF Collaboration), Phys. Rev. Lett. 79 (1997) 578

[629] G. C. Nayak, J. W. Qiu, and G. Sterman, Phys. Lett. B 613 (2005) 45, arXiv:hep-ph/0501235

[630] G. C. Nayak, J. W. Qiu, and G. Sterman, Phys. Rev. D 72 (2005) 114012, arXiv:hep-ph/0509021

[631] Z. B. Kang, J. W. Qiu, and G. Sterman, in preparation.

[632] E. Braaten, S. Fleming, and T. C. Yuan, Ann. Rev. Nucl. Part. Sci. 46, 197 (1996), arXiv:hep-ph/9602374

[633] J. C. Collins and D. E. Soper, Nucl. Phys. B 194 (1982)
445

[634] E. L. Berger, J. W. Qiu, and X. f. Zhang, Phys. Rev. D 65, 034006 (2002), arXiv:hep-ph/0107309

[635] G. C. Nayak, J. W. Qiu, and G. Sterman, Phys. Rev. Lett. 99 (2007) 212001, arXiv:0707.2973 [hep-ph]

[636] G. C. Nayak, J. W. Qiu, and G. Sterman, Phys. Rev. D 77 (2008) 034022, arXiv:0711.3476 [hep-ph]

[637] P. Hagler, R. Kirschner, A. Schafer, L. Szymanowski, and O. V. Teryaev, Phys. Rev. Lett. 86 (2001) 1446, arXiv:hep-ph/0004263

[638] Ph. Hagler, R. Kirschner, A. Schafer, L. Szymanowski, and O. V. Teryaev, Phys. Rev. D 63 (2001) 077501, arXiv:hep-ph/0008316

[639] F. Yuan and K. T. Chao, Phys. Rev. Lett. 87 (2001) 022002, arXiv:hep-ph/0009224

[640] S. P. Baranov, Phys. Rev. D 66 (2002) 114003

[641] S. P. Baranov and N. P. Zotov, JETP Lett. 86 (2007) 435, arXiv:0707.0253 [hep-ph]

[642] S. P. Baranov and A. Szczurek, Phys. Rev. D 77 (2008) 054016, arXiv:0710.1792 [hep-ph]

[643] G. T. Bodwin, X. Garcia i Tormo, and J. Lee, Phys. Rev. Lett. 101 (2008) 102002, arXiv:0805.3876 [hep$\mathrm{ph}]$

[644] G. T. Bodwin, X. Garcia i Tormo, and J. Lee, Phys. Rev. D 81 (2010) 114014, arXiv:1003.0061 [hep-ph]

[645] J. P. Ma and Z. G. Si, Phys. Rev. D 70 (2004) 074007, arXiv:hep-ph/0405111

[646] A. E. Bondar and V. L. Chernyak, Phys. Lett. B 612 (2005) 215, arXiv:hep-ph/0412335

[647] V. V. Braguta, A. K. Likhoded, and A. V. Luchinsky, Phys. Rev. D 72 (2005) 074019, arXiv:hep-ph/0507275

[648] V. V. Braguta, A. K. Likhoded, and A. V. Luchinsky, Phys. Lett. B 635 (2006) 299, arXiv:hep-ph/0602047

[649] V. V. Braguta, Phys. Rev. D 78 (2008) 054025, arXiv:0712.1475 [hep-ph]

[650] F. Abe et al. (CDF Collaboration), Phys. Rev. Lett. 79 (1997) 572

[651] J. P. Lansberg, Int. J. Mod. Phys. A 21 (2006) 3857, arXiv:hep-ph/0602091

[652] J. P. Lansberg, Eur. Phys. J. C 61 (2009) 693, arXiv:0811.4005 [hep-ph]

[653] M. Kramer, Prog. Part. Nucl. Phys. 47 (2001) 141, arXiv:hep-ph/0106120

[654] J. P. Lansberg et al., AIP Conf. Proc. 1038 (2008) 15, arXiv:0807.3666 [hep-ph]

[655] S. Abachi et al. (DØ Collaboration), Phys. Lett. B 370 (1996) 239

[656] A. A. Affolder et al. (CDF Collaboration), Phys. Rev. Lett. 85 (2000) 2886, arXiv:hep-ex/0004027

[657] D. E. Acosta et al. (CDF Collaboration), Phys. Rev. D 71 (2005) 032001, arXiv:hep-ex/0412071

[658] A. Abulencia et al. (CDF Collaboration), Phys. Rev. Lett. 99 (2007) 132001, arXiv:0704.0638 [hep-ex]

[659] A. Adare et al. (PHENIX Collaboration), Phys. Rev. Lett. 98 (2007) 232002, arXiv:hep-ex/0611020

[660] S. S. Adler et al. (PHENIX Collaboration), Phys. Rev. Lett. 92 (2004) 051802, arXiv:hep-ex/0307019

[661] E. T. Atomssa (PHENIX Collaboration), Eur. Phys. J. C 61 (2009) 683, arXiv:0805.4562 [nucl-ex]

[662] A. Adare et al. (PHENIX Collaboration), Phys. Rev. D 82 (2010) 012001, arXiv:0912.2082 [hep-ex]

[663] C. L. da Silva (PHENIX Collaboration), Nucl. Phys. A 830 (2009) 227C, arXiv:0907.4696 [nucl-ex]

[664] B. I. Abelev et al. (STAR Collaboration), Phys. Rev. 
C 80 (2009) 041902, arXiv:0904.0439 [nucl-ex]

[665] P. L. Cho and M. B. Wise, Phys. Lett. B 346 (1995) 129, arXiv:hep-ph/9411303

[666] A. K. Leibovich, Phys. Rev. D 56 (1997) 4412, arXiv:hep-ph/9610381

[667] E. Braaten, B. A. Kniehl, and J. Lee, Phys. Rev. D 62 (2000) 094005, arXiv:hep-ph/9911436

[668] A. A. Affolder et al. (CDF Collaboration), Phys. Rev. Lett. 84 (2000) 2094, arXiv:hep-ex/9910025

[669] D. E. Acosta et al. (CDF Collaboration), Phys. Rev. Lett. 88 (2002) 161802

[670] V. M. Abazov et al. (DØ Collaboration), Phys. Rev. Lett. 94 (2005) 232001 [Erratum-ibid. 100 (2008) 049902], arXiv:hep-ex/0502030

[671] V. M. Abazov et al. (DØ Collaboration), Phys. Rev. Lett. 101 (2008) 182004, arXiv:0804.2799 [hep-ex]

[672] B. Gong and J. X. Wang, Phys. Rev. Lett. 100 (2008) 232001, arXiv:0802.3727 [hep-ph]

[673] B. Gong and J. X. Wang, Phys. Rev. D 78 (2008) 074011, arXiv:0805.2469 [hep-ph]

[674] B. Gong, X. Q. Li and J. X. Wang, Phys. Lett. B 673 (2009) 197, arXiv:0805.4751 [hep-ph]

[675] Y. Q. Ma, K. Wang, and K. T. Chao, arXiv:1002.3987 [hep-ph]

[676] S. J. Brodsky and J. P. Lansberg, Phys. Rev. D 81 (2010) 051502, arXiv:0908.0754 [hep-ph]

[677] M. Kramer, Nucl. Phys. B 459 (1996) 3, arXiv:hepph/9508409

[678] V. G. Kartvelishvili, A. K. Likhoded, and S. R. Slabospitsky, Sov. J. Nucl. Phys. 28 (1978) 678 [Yad. Fiz. 28 (1978) 1315]

[679] E. Braaten and T. C. Yuan, Phys. Rev. D 52 (1995) 6627, arXiv:hep-ph/9507398

[680] V. A. Khoze, A. D. Martin, M. G. Ryskin, and W. J. Stirling, Eur. Phys. J. C 39 (2005) 163, arXiv:hep-ph/0410020

[681] J. P. Lansberg, J. R. Cudell, and Yu. L. Kalinovsky, Phys. Lett. B 633 (2006) 301, arXiv:hep-ph/0507060

[682] H. Haberzettl and J. P. Lansberg, Phys. Rev. Lett. 100 (2008) 032006, arXiv:0709.3471 [hep-ph]

[683] P. Artoisenet and E. Braaten, Phys. Rev. D 80 (2009) 034018, arXiv:0907.0025 [hep-ph]

[684] Z. G. He, R. Li, and J. X. Wang, Phys. Rev. D 79 (2009) 094003, arXiv:0904.2069 [hep-ph]

[685] Z. G. He, R. Li, and J. X. Wang, arXiv:0904.1477 [hep$\mathrm{ph}]$

[686] Y. Fan, Y. Q. Ma, and K. T. Chao, Phys. Rev. D 79 (2009) 114009, arXiv:0904.4025 [hep-ph]

[687] P. Artoisenet, AIP Conf. Proc. 1038 (2008) 55

[688] B. Gong, J. X. Wang, and H. F. Zhang, arXiv:1009.3839 [hep-ph]

[689] T. Shears (on behalf of the CDF Collaboration), Eur. Phys. J. C 33 (2004) S475

[690] J. P. Lansberg, arXiv:1006.2750 [hep-ph]

[691] A. Abulencia et al. (CDF Collaboration), Phys. Rev. Lett. 98 (2007) 232001, arXiv:hep-ex/0703028

[692] Y. Q. Ma, K. Wang, and K. T. Chao, arXiv:1009.3655 [hep-ph]

[693] M. Butenschoen and B. A. Kniehl, arXiv:1009.5662 [hep-ph]

[694] CMS collaboration, CMS Physics Analysis Summary, CMS PAS BPH-10-002 (2010)

[695] M. Butenschoen and B. A. Kniehl, Phys. Rev. Lett. 104 (2010) 072001, arXiv:0909.2798 [hep-ph]
[696] F. D. Aaron et al. (H1 Collaboration), Eur. Phys. J. C 68 (2010) 401, arXiv:1002.0234 [hep-ex]

[697] C. Adloff et al. (H1 Collaboration), Eur. Phys. J. C 25 (2002) 25, arXiv:hep-ex/0205064

[698] G. C. Nayak, M. X. Liu, and F. Cooper, Phys. Rev. D 68 (2003) 034003, arXiv:hep-ph/0302095

[699] H. S. Chung, S. Kim, J. Lee, and C. Yu, Phys. Rev. D 81 (2010) 014020, arXiv:0911.2113 [hep-ph]

[700] J. P. Lansberg, Phys. Lett. B 695 (2011) 149, arXiv:1003.4319 [hep-ph]

[701] T. Aaltonen et al. (CDF Collaboration), Phys. Rev. Lett. 102 (2009) 242001, arXiv:0902.1271 [hep-ex]

[702] V. A. Khoze, A. D. Martin and M. G. Ryskin, Eur. Phys. J. C 19 (2001) 477 [Erratum-ibid. C 20 (2001) 599], arXiv:hep-ph/0011393

[703] F. Yuan, Phys. Lett. B 510 (2001) 155, arXiv:hep$\mathrm{ph} / 0103213$

[704] S. R. Klein and J. Nystrand, Phys. Rev. Lett. 92 (2004) 142003, arXiv:hep-ph/0311164

[705] V. A. Khoze, A. D. Martin, M. G. Ryskin, and W. J. Stirling, Eur. Phys. J. C 35 (2004) 211, arXiv:hep-ph/0403218

[706] V. P. Goncalves and M. V. T. Machado, Eur. Phys. J. C 40 (2005) 519, arXiv:hep-ph/0501099

[707] A. Bzdak, Phys. Lett. B 619 (2005) 288, arXiv:hep$\mathrm{ph} / 0506101$

[708] A. Bzdak, L. Motyka, L. Szymanowski and J. R. Cudell, Phys. Rev. D 75 (2007) 094023, arXiv:hep$\mathrm{ph} / 0702134$

[709] W. Schafer and A. Szczurek, Phys. Rev. D 76 (2007) 094014, arXiv:0705.2887 [hep-ph]

[710] L. Motyka and G. Watt, Phys. Rev. D 78 (2008) 014023, arXiv:0805.2113 [hep-ph]

[711] Z. Conesa del Valle (PHENIX Collaboration), Nucl. Phys. A 830 (2009) 511C, arXiv:0907.4452 [nucl-ex]

[712] S. Klein and J. Nystrand, Phys. Rev. C 60 (1999) 014903, arXiv:hep-ph/9902259

[713] A. J. Baltz, S. R. Klein, and J. Nystrand, Phys. Rev. Lett. 89 (2002) 012301, arXiv:nucl-th/0205031

[714] J. Nystrand, Nucl. Phys. A 752 (2005) 470, arXiv:hep$\mathrm{ph} / 0412096$

[715] M. Strikman, M. Tverskoy and M. Zhalov, Phys. Lett. B 626 (2005) 72, arXiv:hep-ph/0505023

[716] V. P. Goncalves and M. V. T. Machado, J. Phys. G 32 (2006) 295, arXiv:hep-ph/0506331

[717] V. P. Goncalves and M. V. T. Machado, arXiv:0706.2810 [hep-ph]

[718] Yu. P. Ivanov, B. Z. Kopeliovich, and I. Schmidt, arXiv:0706.1532 [hep-ph]

[719] A. L. Ayala Filho, V. P. Goncalves, and M. T. Griep, Phys. Rev. C 78 (2008) 044904, arXiv:0808.0366 [hep$\mathrm{ph}]$

[720] G. Bauer (CDF II Collaboration), Int. J. Mod. Phys. A 20 (2005) 3765, arXiv:hep-ex/0409052

[721] E. Braaten, Phys. Rev. D 73 (2006) 011501, arXiv:hepph/0408230

[722] P. Faccioli, C. Lourenço, J. Seixas, and H. K. Wohri, Phys. Rev. Lett. 102 (2009) 151802, arXiv:0902.4462 [hep-ph]

[723] P. Faccioli, C. Lourenço, and J. Seixas, Phys. Rev. D 81 (2010) 111502, arXiv:1005.2855 [hep-ph]

[724] R. Li and J. X. Wang, Phys. Lett. B 672 (2009) 51, arXiv:0811.0963 [hep-ph]

[725] J. P. Lansberg, Phys. Lett. B 679 (2009) 340, 
arXiv:0901.4777 [hep-ph]

[726] E. Braaten, D. Kang, J. Lee, and C. Yu, Phys. Rev. D 79 (2009) 054013, arXiv:0812.3727 [hep-ph]

[727] C. N. Brown et al. (FNAL E866 Collaboration and NuSea Collaboration), Phys. Rev. Lett. 86 (2001) 2529, arXiv:hep-ex/0011030

[728] T. H. Chang et al. (FNAL E866/NuSea collaboration), Phys. Rev. Lett. 91 (2003) 211801, arXiv:hepex/0308001

[729] J. C. Collins and D. E. Soper, Phys. Rev. D 16 (1977) 2219

[730] I. Abt et al. (HERA-B Collaboration), Eur. Phys. J. C 60 (2009) 517, arXiv:0901.1015 [hep-ex]

[731] K. Gottfried and J. D. Jackson, Nuovo Cim. 33 (1964) 309

[732] A. D. Martin, R. G. Roberts, W. J. Stirling, and R. S. Thorne, Eur. Phys. J. C 4 (1998) 463, arXiv:hep$\mathrm{ph} / 9803445$

[733] H. L. Lai et al. (CTEQ Collaboration), Eur. Phys. J. C 12 (2000) 375, arXiv:hep-ph/9903282

[734] CDF Collaboration, CDF public note 9966

[735] E. Braaten and J. Lee, Phys. Rev. D 63 (2001) 071501, arXiv:hep-ph/0012244

[736] Rapidity interval communicated privately by J. Lee

[737] CDF Collaboration, CDF public plot

[738] Berkeley Workshop on Physics Opportunities with Early LHC Data

[739] A. C. Kraan, AIP Conf. Proc. 1038 (2008) 45, arXiv:0807.3123 [hep-ex]

[740] C. Albajar et al. (UA1 Collaboration), Phys. Lett. B 200 (1988) 380

[741] C. Albajar et al. (UA1 Collaboration), Phys. Lett. B 256 (1991) 112

[742] Z. Tang (STAR Collaboration), Nucl. Phys. A 834 (2010) $282 \mathrm{C}$

[743] P. Artoisenet, F. Maltoni, and T. Stelzer, JHEP 0802 (2008) 102, arXiv:0712.2770 [hep-ph]

[744] J. Alwall et al., JHEP 0709 (2007) 028, arXiv:0706.2334 [hep-ph]

[745] P. Artoisenet, In the Proceedings of 9th Workshop on Non-Perturbative Quantum Chromodynamics, Paris, France, 4-8 Jun 2007, pp 21, arXiv:0804.2975 [hep-ph]

[746] M. Klasen and J. P. Lansberg, Nucl. Phys. Proc. Suppl. 179-180 (2008) 226, arXiv:0806.3662 [hep-ph]

[747] F. D. Aaron et al. (H1 Collaboration), JHEP 1005 (2010) 032, arXiv:0910.5831 [hep-ex]

[748] S. Kananov (ZEUS Collaboration), Nucl. Phys. Proc. Suppl. 184 (2008) 252

[749] S. Chekanov et al. (ZEUS Collaboration), Nucl. Phys. B 718 (2005) 3, arXiv:hep-ex/0504010

[750] J. Breitweg et al. (ZEUS Collaboration), Phys. Lett. B 487 (2000) 273, arXiv:hep-ex/0006013

[751] A. Aktas et al. (H1 Collaboration), Eur. Phys. J. C 46 (2006) 585, arXiv:hep-ex/0510016

[752] S. Chekanov et al. (ZEUS Collaboration), Nucl. Phys. B 695 (2004) 3, arXiv:hep-ex/0404008

[753] S. Chekanov et al. (ZEUS Collaboration), Eur. Phys. J. C 24 (2002) 345, arXiv:hep-ex/0201043

[754] A. Aktas et al. (H1 Collaboration), Phys. Lett. B 568 (2003) 205, arXiv:hep-ex/0306013

[755] S. Chekanov et al. (ZEUS Collaboration), JHEP 1005 (2010) 085, arXiv:0910.1235 [hep-ex]

[756] C. Adloff et al. (H1 Collaboration), Phys. Lett. B 541 (2002) 251, arXiv:hep-ex/0205107
[757] C. Adloff et al. (H1 Collaboration), Phys. Lett. B 483 (2000) 23, arXiv:hep-ex/0003020

[758] S. Chekanov et al. (ZEUS Collaboration), Phys. Lett. B 680 (2009) 4, arXiv:0903.4205 [hep-ex]

[759] I. P. Ivanov, N. N. Nikolaev, and A. A. Savin, Phys. Part. Nucl. 37 (2006) 1, arXiv:hep-ph/0501034

[760] S. Chekanov et al. (ZEUS Collaboration), Eur. Phys. J. C 27 (2003) 173, arXiv:hep-ex/0211011

[761] S. Chekanov et al. (ZEUS Collaboration), JHEP 0912 (2009) 007, arXiv:0906.1424 [hep-ex]

[762] S. Fleming, A. K. Leibovich, and T. Mehen, Phys. Rev. D 74 (2006) 114004, arXiv:hep-ph/0607121

[763] B. A. Kniehl, D. V. Vasin, and V. A. Saleev, Phys. Rev. D 73 (2006) 074022, arXiv:hep-ph/0602179

[764] A. D. Martin, M. G. Ryskin, and G. Watt, Eur. Phys. J. C 66 (2010) 163, arXiv:0909.5529 [hep-ph]

[765] M. Kramer, J. Zunft, J. Steegborn, and P. M. Zerwas, Phys. Lett. B 348 (1995) 657, arXiv:hep-ph/9411372

[766] P. Artoisenet, J. M. Campbell, F. Maltoni, and F. Tramontano, Phys. Rev. Lett. 102 (2009) 142001, arXiv:0901.4352 [hep-ph]

[767] C. H. Chang, R. Li, and J. X. Wang, Phys. Rev. D 80 (2009) 034020, arXiv:0901.4749 [hep-ph]

[768] M. Butenschoen, Nucl. Phys. Proc. Suppl. 191 (2009) 193

[769] M. Butenschoen and B. A. Kniehl, PoS D IS2010 (2010) 157, arXiv:1006.1776 [hep-ph]

[770] B. A. Kniehl and G. Kramer, Eur. Phys. J. C 6 (1999) 493, arXiv:hep-ph/9803256

[771] M. Beneke, M. Kramer, and M. Vanttinen, Phys. Rev. D 57 (1998) 4258, arXiv:hep-ph/9709376

[772] S. P. Baranov, JETP Lett. 88 (2008) 471

[773] P. Artoisenet, private communication.

[774] F. Maltoni et al., Phys. Lett. B 638 (2006) 202, arXiv:hep-ph/0601203

[775] E. J. Eichten and C. Quigg, Phys. Rev. D 52 (1995) 1726 arXiv:hep-ph/9503356

[776] P. Nason et al., arXiv:hep-ph/0003142

[777] G. T. Bodwin, H. S. Chung, D. Kang, J. Lee, and C. Yu, Phys. Rev. D 77 (2008) 094017, arXiv:0710.0994 [hep-ph]

[778] K. Abe et al. (Belle Collaboration), Phys. Rev. Lett. 89 (2002) 142001, arXiv:hep-ex/0205104

[779] K. Y. Liu, Z. G. He, and K. T. Chao, Phys. Lett. B 557 (2003) 45, arXiv:hep-ph/0211181

[780] E. Braaten and J. Lee, Phys. Rev. D 67 (2003) 054007 [Erratum-ibid. D 72 (2005) 099901], arXiv:hepph/0211085

[781] K. Hagiwara, E. Kou, and C. F. Qiao, Phys. Lett. B 570 (2003) 39, arXiv:hep-ph/0305102

[782] K. Abe et al. (Belle Collaboration), Phys. Rev. D 70 (2004) 071102, arXiv:hep-ex/0407009

[783] G. T. Bodwin, D. Kang, T. Kim, J. Lee, and C. Yu, AIP Conf. Proc. 892 (2007) 315, arXiv:hep$\mathrm{ph} / 0611002$

[784] Z. G. He, Y. Fan, and K. T. Chao, Phys. Rev. D 75 (2007) 074011, arXiv:hep-ph/0702239

[785] G. T. Bodwin, J. Lee, and C. Yu, Phys. Rev. D 77 (2008) 094018, arXiv:0710.0995 [hep-ph]

[786] Y. J. Zhang, Y. j. Gao, and K. T. Chao, Phys. Rev. Lett. 96 (2006) 092001, arXiv:hep-ph/0506076

[787] B. Gong and J. X. Wang, Phys. Rev. D 77 (2008) 054028, arXiv:0712.4220 [hep-ph]

[788] M. Gremm and A. Kapustin, Phys. Lett. B 407 (1997) 
323, arXiv:hep-ph/9701353

[789] G. T. Bodwin, D. Kang, and J. Lee, Phys. Rev. D 74 (2006) 014014, arXiv:hep-ph/0603186

[790] V. V. Braguta, A. K. Likhoded, and A. V. Luchinsky, Phys. Lett. B 646 (2007) 80, arXiv:hep-ph/0611021

[791] V. V. Braguta, A. K. Likhoded, and A. V. Luchinsky, Phys. Rev. D 79 (2009) 074004, arXiv:0810.3607 [hepph]

[792] G. T. Bodwin, D. Kang, and J. Lee, Phys. Rev. D 74 (2006) 114028, arXiv:hep-ph/0603185

[793] M. Beneke, A. Signer, and V. A. Smirnov, Phys. Rev. Lett. 80 (1998) 2535, arXiv:hep-ph/9712302

[794] A. Czarnecki and K. Melnikov, Phys. Lett. B 519 (2001) 212, arXiv:hep-ph/0109054

[795] B. Aubert et al. (BABAR Collaboration), Phys. Rev. Lett. 87 (2001) 162002, arXiv:hep-ex/0106044

[796] K. Abe et al. (BELLE Collaboration), Phys. Rev. Lett. 88 (2002) 052001, arXiv:hep-ex/0110012

[797] F. Yuan, C. F. Qiao, and K. T. Chao, Phys. Rev. D 56 (1997) 321, arXiv:hep-ph/9703438

[798] P. L. Cho and A. K. Leibovich, Phys. Rev. D 54 (1996) 6690, arXiv:hep-ph/9606229

[799] S. Baek, P. Ko, J. Lee, and H. S. Song, J. Korean Phys. Soc. 33 (1998) 97, arXiv:hep-ph/9804455

[800] G. A. Schuler, Eur. Phys. J. C 8 (1999) 273, arXiv:hep$\mathrm{ph} / 9804349$

[801] V. V. Kiselev, A. K. Likhoded, and M. V. Shevlyagin, Phys. Lett. B 332 (1994) 411, arXiv:hep-ph/9408407

[802] K. Y. Liu, Z. G. He, and K. T. Chao, Phys. Rev. D 68 (2003) 031501, arXiv:hep-ph/0305084

[803] K. Y. Liu, Z. G. He, and K. T. Chao, Phys. Rev. D 69 (2004) 094027, arXiv:hep-ph/0301218

[804] K. Hagiwara, E. Kou, Z. H. Lin, C. F. Qiao, and G. H. Zhu, Phys. Rev. D 70 (2004) 034013, arXiv:hep$\mathrm{ph} / 0401246$

[805] A. V. Berezhnoy and A. K. Likhoded, Phys. Atom. Nucl. 67 (2004) 757 [Yad. Fiz. 67 (2004) 778 ] arXiv:hep-ph/0303145

[806] D. Kang, J. W. Lee, J. Lee, T. Kim, and P. Ko, Phys. Rev. D 71 (2005) 094019, arXiv:hep-ph/0412381

[807] S. Fleming, A. K. Leibovich, and T. Mehen, Phys. Rev. D 68 (2003) 094011, arXiv:hep-ph/0306139

[808] Z. H. Lin and G. h. Zhu, Phys. Lett. B 597 (2004) 382, arXiv:hep-ph/0406121

[809] A. K. Leibovich and X. Liu, Phys. Rev. D 76 (2007) 034005, arXiv:0705.3230 [hep-ph]

[810] P. Pakhlov et al. (Belle Collaboration), Phys. Rev. D 79 (2009) 071101, arXiv:0901.2775 [hep-ex]

[811] Y. J. Zhang and K. T. Chao, Phys. Rev. Lett. 98 (2007) 092003, arXiv:hep-ph/0611086

[812] Y. Q. Ma, Y. J. Zhang, and K. T. Chao, Phys. Rev. Lett. 102 (2009) 162002, arXiv:0812.5106 [hep-ph]

[813] B. Gong and J. X. Wang, Phys. Rev. D 80 (2009) 054015, arXiv:0904.1103 [hep-ph]

[814] B. Gong and J. X. Wang, Phys. Rev. Lett. 102 (2009) 162003, arXiv:0901.0117 [hep-ph]

[815] C. Peterson, D. Schlatter, I. Schmitt, and P. M. Zerwas, Phys. Rev. D 27 (1983) 105.

[816] Z. G. He, Y. Fan, and K. T. Chao, Phys. Rev. D 81 (2010), 054036, arXiv:0910.3636 [hep-ph]

[817] Y. Jia, Phys. Rev. D 82 (2010) 034017, arXiv:0912.5498 [hep-ph]

[818] E. Braaten and Y. Q. Chen, Phys. Rev. Lett. 76 (1996) 730, arXiv:hep-ph/9508373
[819] Y. J. Zhang, Y. Q. Ma, K. Wang, and K. T. Chao, Phys. Rev. D 81 (2010) 034015, arXiv:0911.2166 [hepph]

[820] S. Todorova-Nova, arXiv:hep-ph/0112050

[821] J. Abdallah et al. (DELPHI Collaboration), Phys. Lett. B 565 (2003) 76, arXiv:hep-ex/0307049

[822] J. P. Ma, B. H. J. McKellar, and C. B. Paranavitane, Phys. Rev. D 57 (1998) 606, arXiv:hep-ph/9707480

[823] G. Japaridze and A. Tkabladze, Phys. Lett. B 433 (1998) 139, arXiv:hep-ph/9803447

[824] R. M. Godbole, D. Indumathi, and M. Krämer, Phys. Rev. D 65 (2002) 074003, arXiv:hep-ph/0101333

[825] M. Klasen, B. A. Kniehl, L. Mihaila, and M. Steinhauser, Nucl. Phys. B 609 (2001) 518, arXiv:hepph/0104044

[826] M. Klasen, B. A. Kniehl, L. N. Mihaila, and M. Steinhauser, Phys. Rev. Lett. 89 (2002) 032001, arXiv:hepph/0112259

[827] M. Klasen, B. A. Kniehl, L. N. Mihaila, and M. Steinhauser, Nucl. Phys. B $\mathbf{7 1 3}$ (2005) 487, arXiv:hepph/0407014

[828] J. de Favereau de Jeneret et al., arXiv:0908.2020 [hep$\mathrm{ph}]$

[829] CDF collaboration, CDF public note 9294

[830] CDF collaboration, CDF public note 7649

[831] CDF collaboration, CDF public note 9740

[832] W. Beenakker, H. Kuijf, W. L. van Neerven, and J. Smith, Phys. Rev. D 40 (1989) 54

[833] P. Nason, S. Dawson, and R. K. Ellis, Nucl. Phys. B 327 (1989) 49 [Erratum-ibid. B 335 (1990) 260]

[834] C. H. Chang and Y. Q. Chen, Phys. Rev. D 48 (1993) 4086

[835] C. H. Chang, Y. Q. Chen, G. P. Han, and H. T. Jiang, Phys. Lett. B 364 (1995) 78, arXiv:hep-ph/9408242

[836] K. Kolodziej, A. Leike, and R. Ruckl, Phys. Lett. B 355 (1995) 337, arXiv:hep-ph/9505298

[837] A. V. Berezhnoy, V. V. Kiselev, and A. K. Likhoded, Z. Phys. A 356 (1996) 89

[838] S. P. Baranov, Phys. Rev. D 56 (1997) 3046

[839] A. V. Berezhnoi, V. V. Kiselev, and A. K. Likhoded, Phys. Atom. Nucl. 60 (1997) 100 [Yad. Fiz. 60 (1997) 108]

[840] S. Frixione, M. L. Mangano, P. Nason, and G. Ridolfi, Adv. Ser. Direct. High Energy Phys. 15 (1998) 609, arXiv:hep-ph/9702287

[841] E. Braaten, K. m. Cheung, and T. C. Yuan, Phys. Rev. D 48 (1993) 5049, arXiv:hep-ph/9305206

[842] K. m. Cheung, Phys. Lett. B 472 (2000) 408, arXiv:hep-ph/9908405

[843] C. H. Chang, Y. Q. Chen, and R. J. Oakes, Phys. Rev. D 54 (1996) 4344, arXiv:hep-ph/9602411

[844] C. H. Chang, C. F. Qiao, J. X. Wang, and X. G. Wu, Phys. Rev. D 73 (2006) 094022, arXiv:hep-ph/0601032

[845] C. H. Chang, C. F. Qiao, J. X. Wang, and X. G. Wu, Phys. Rev. D 72 (2005) 114009, arXiv:hep-ph/0509040

[846] M. A. G. Aivazis, J. C. Collins, F. I. Olness, and W. K. Tung, Phys. Rev. D 50 (1994) 3102, arXiv:hepph/9312319

[847] M. A. G. Aivazis, F. I. Olness, and W. K. Tung, Phys. Rev. D 50 (1994) 3085, arXiv:hep-ph/9312318

[848] F. I. Olness, R. J. Scalise, and W. K. Tung, Phys. Rev. D 59 (1999) 014506, arXiv:hep-ph/9712494

[849] J. Amundson, C. Schmidt, W. K. Tung, and X. Wang, JHEP 0010 (2000) 031, arXiv:hep-ph/0005221 
[850] C. H. Chang, J. X. Wang, and X. G. Wu, Phys. Rev. D 70 (2004) 114019, arXiv:hep-ph/0409280

[851] C. H. Chang, C. F. Qiao, J. X. Wang, and X. G. Wu, Phys. Rev. D 71 (2005) 074012, arXiv:hep-ph/0502155

[852] C. H. Chang, J. X. Wang, and X. G. Wu, Comput. Phys. Commun. 175 (2006) 624, arXiv:hep$\mathrm{ph} / 0604238$

[853] T. Sjostrand, S. Mrenna, and P. Z. Skands, JHEP 0605 (2006) 026, arXiv:hep-ph/0603175

[854] C. H. Chang, J. X. Wang, and X. G. Wu, Phys. Rev. D 77 (2008) 014022, arXiv:0711.1898 [hep-ph]

[855] E. V. Shuryak, Phys. Rept. 61 (1980) 71

[856] P. Petreczky, Nucl. Phys. A 830 (2009) 11C, arXiv:0908.1917 [hep-ph]

[857] Z. Fodor and S. D. Katz, arXiv:0908.3341 [hep-ph]

[858] C. DeTar and U. M. Heller, Eur. Phys. J. A 41 (2009) 405, arXiv:0905.2949 [hep-lat]

[859] P. Petreczky, Nucl. Phys. Proc. Suppl. 140 (2005) 78, arXiv:hep-lat/0409139

[860] H. Satz, Rept. Prog. Phys. 63 (2000) 1511, arXiv:hep$\mathrm{ph} / 0007069$

[861] B. Muller and J. L. Nagle, Ann. Rev. Nucl. Part. Sci. 56 (2006) 93, arXiv:nucl-th/0602029

[862] T. Matsui and H. Satz, Phys. Lett. B 178 (1986) 416

[863] CERN press release New State of Matter created at CERN, Feb. 10, 2000

[864] P. Faccioli, C. Lourenço, J. Seixas, and H. K. Woehri, JHEP 0810 (2008) 004, arXiv:0809.2153 [hep-ph]

[865] R. Vogt, Nucl. Phys. A 700 (2002) 539, arXiv:hep$\mathrm{ph} / 0107045$

[866] K. J. Eskola, H. Paukkunen, and C. A. Salgado, JHEP 0904 (2009) 065, arXiv:0902.4154 [hep-ph]

[867] A. D. Frawley, T. Ullrich, and R. Vogt, Phys. Rept. 462 (2008) 125, arXiv:0806.1013 [nucl-ex]

[868] D. Kharzeev and K. Tuchin, Nucl. Phys. A 735 (2004) 248, arXiv:hep-ph/0310358

[869] D. Kharzeev, E. Levin, M. Nardi, and K. Tuchin, Phys. Rev. Lett. 102 (2009) 152301, arXiv:0808.2954 [hep$\mathrm{ph}]$

[870] D. Kharzeev and H. Satz, Phys. Lett. B 366 (1996) 316, arXiv:hep-ph/9508276

[871] J. P. Blaizot and J. Y. Ollitrault, Phys. Lett. B 217 (1989) 386

[872] B. Alessandro et al. (NA50 Collaboration), Eur. Phys. J. C 48 (2006) 329, arXiv:nucl-ex/0612012

[873] B. Alessandro et al. (NA50 Collaboration), Eur. Phys. J. C 33 (2004) 31

[874] M. J. Leitch et al. (FNAL E866/NuSea Collaboration), Phys. Rev. Lett. 84 (2000) 3256, arXiv:nuclex/9909007

[875] C. Lourenço, R. Vogt, and H. K. Woehri, JHEP 0902 (2009) 014, arXiv:0901.3054 [hep-ph]

[876] K. J. Eskola, V. J. Kolhinen, and P. V. Ruuskanen, Nucl. Phys. B 535 (1998) 351, arXiv:hep-ph/9802350

[877] K. J. Eskola, V. J. Kolhinen, and C. A. Salgado, Eur. Phys. J. C 9 (1999) 61, arXiv:hep-ph/9807297

[878] J. Pumplin, D. R. Stump, J. Huston, H. L. Lai, P. M. Nadolsky, and W. K. Tung, JHEP 0207 (2002) 012, arXiv:hep-ph/0201195

[879] D. Stump, J. Huston, J. Pumplin, W. K. Tung, H. L. Lai, S. Kuhlmann, and J. F. Owens, JHEP 0310 (2003) 046, arXiv:hep-ph/0303013

[880] J. Badier et al. (NA3 Collaboration), Z. Phys. C 20 (1983) 101
[881] I. Abt et al. (HERA-B Collaboration), Eur. Phys. J. C 60 (2009) 525, arXiv:0812.0734 [hep-ex]

[882] E. Scomparin (NA60 Collaboration), Nucl. Phys. A 830 (2009) 239c, arXiv:0907.3682 [nucl-ex]

[883] A. Adare et al. (PHENIX Collaboration), Phys. Rev. C 77 (2008) 024912 [Erratum-ibid. C 79 (2009) 059901], arXiv:0711.3917 [nucl-ex]

[884] A. D. Frawley, presentation at the ECT* workshop on Quarkonium Production in Heavy-Ion Collisions, Trento (Italy), May 25-29, 2009 and at the Joint CATHIE-INT mini-program "Quarkonia in Hot QCD", June 16-26, 2009

[885] D. M. Alde et al., Phys. Rev. Lett. 66 (1991) 2285

[886] R. Vogt, Phys. Rev. C 61 (2000) 035203, arXiv:hepph/9907317

[887] B. Povh and J. Hufner, Phys. Rev. Lett. 58 (1987) 1612

[888] R. Vogt, Phys. Rev. C 71 (2005) 054902, arXiv:hep$\mathrm{ph} / 0411378$

[889] R. Arnaldi, P. Cortese, and E. Scomparin, Phys. Rev. C 81 (2010) 014903, arXiv:0909.2199 [hep-ph]

[890] R. Gavai, D. Kharzeev, H. Satz, G. A. Schuler, K. Sridhar, and R. Vogt, Int. J. Mod. Phys. A 10 (1995) 3043, arXiv:hep-ph/9502270

[891] E. G. Ferreiro, F. Fleuret, J. P. Lansberg, and A. Rakotozafindrabe, Phys. Lett. B 680 (2009) 50, arXiv:0809.4684 [hep-ph]

[892] M. Beneke and I. Z. Rothstein, Phys. Rev. D 54 (1996) 2005 [Erratum-ibid. D 54 (1996) 7082], arXiv:hep$\mathrm{ph} / 9603400$

[893] X. F. Zhang, C. F. Qiao, X. X. Yao, and W. Q. Chao, arXiv:hep-ph/9711237

[894] T. Umeda, K. Nomura, and H. Matsufuru, Eur. Phys. J. C 39S1 (2005) 9, arXiv:hep-lat/0211003

[895] M. Asakawa and T. Hatsuda, Phys. Rev. Lett. 92 (2004) 012001, arXiv:hep-lat/0308034

[896] S. Datta, F. Karsch, P. Petreczky, and I. Wetzorke, Phys. Rev. D 69 (2004) 094507, arXiv:hep-lat/0312037

[897] A. Jakovác, P. Petreczky, K. Petrov, and A. Velytsky, Phys. Rev. D 75 (2007) 014506, arXiv:hep-lat/0611017

[898] G. Aarts et al., Phys. Rev. D 76 (2007) 094513, arXiv:0705.2198 [hep-lat]

[899] S. Digal, P. Petreczky, and H. Satz, Phys. Rev. D 64 (2001) 094015, arXiv:hep-ph/0106017

[900] C. Y. Wong, Phys. Rev. C 72 (2005) 034906

[901] Á. Mócsy and P. Petreczky, Phys. Rev. D 73 (2006) 074007, arXiv:hep-ph/0512156

[902] A. Mocsy and P. Petreczky, Eur. Phys. J. C 43 (2005) 77, arXiv:hep-ph/0411262

[903] W. M. Alberico, A. Beraudo, A. De Pace, and A. Molinari, Phys. Rev. D 75 (2007) 074009

[904] D. Cabrera and R. Rapp, Phys. Rev. D 76 (2007) 114506, arXiv:hep-ph/0611134

[905] Á. Mócsy and P. Petreczky, Phys. Rev. D 77 (2008) 014501, arXiv:0705.2559 [hep-ph]

[906] Á. Mócsy and P. Petreczky, Phys. Rev. Lett. 99 (2007) 211602, arXiv:0706.2183 [hep-ph]

[907] M. Laine, O. Philipsen, and M. Tassler, JHEP 0709 (2007) 066, arXiv:0707.2458 [hep-lat]

[908] M. Laine, JHEP 0705 (2007) 028, arXiv:0704.1720 [hep-ph]

[909] M. Laine, Nucl. Phys. A 820 (2009) 25C, arXiv:0810.1112 [hep-ph] 
[910] N. Brambilla, J. Ghiglieri, A. Vairo, and P. Petreczky, Phys. Rev. D 78 (2008) 014017, arXiv:0804.0993 [hepph]

[911] Y. Aoki, Z. Fodor, S. D. Katz, and K. K. Szabo, Phys. Lett. B 643 (2006) 46, arXiv:hep-lat/0609068

[912] M. Cheng et al., Phys. Rev. D 77 (2008) 014511, arXiv:0710.0354 [hep-lat]

[913] Y. Aoki, S. Borsanyi, S. Durr, Z. Fodor, S. D. Katz, S. Krieg, and K. K. Szabo, JHEP 0906 (2009) 088, arXiv:0903.4155 [hep-lat]

[914] A. Bazavov et al., Phys. Rev. D 80 (2009) 014504, arXiv:0903.4379 [hep-lat]

[915] M. Cheng et al., Phys. Rev. D 81 (2010) 054504, arXiv:0911.2215 [hep-lat]

[916] A. Bazavov and P. Petreczky, PoS LAT2009 (2009) 163, arXiv:0912.5421 [hep-lat]

[917] A. Bazavov and P. Petreczky (HotQCD Collaboration), J. Phys. Conf. Ser. 230, 012014 (2010), arXiv:1005.1131 [hep-lat]

[918] A. Bazavov and P. Petreczky, arXiv:1009.4914 [hep-lat]

[919] S. Borsanyi et al. (Wuppertal-Budapest Collaboration), JHEP 1009, 073 (2010), arXiv:1005.3508 [heplat]

[920] S. Borsanyi et al., JHEP 1011 (2010) 077, arXiv:1007.2580 [hep-lat]

[921] A. Bazavov, P. Petreczky, and A. Velytsky, arXiv:0904.1748 [hep-ph]

[922] P. Petreczky, Eur. Phys. J. C 43 (2005) 51, arXiv:heplat/0502008

[923] O. Kaczmarek, F. Karsch, P. Petreczky, and F. Zantow, Phys. Lett. B 543, 41 (2002), arXiv:heplat/0207002

[924] S. Digal, S. Fortunato, and P. Petreczky, Phys. Rev. D 68, 034008 (2003), arXiv:hep-lat/0304017

[925] P. Petreczky and K. Petrov, Phys. Rev. D 70 (2004) 054503, arXiv:hep-lat/0405009

[926] O. Kaczmarek and F. Zantow, Phys. Rev. D 71 (2005) 114510, arXiv:hep-lat/0503017

[927] O. Kaczmarek, PoS C POD07 (2007) 043, arXiv:0710.0498 [hep-lat]

[928] P. Petreczky, J. Phys. G 37 (2010) 094009, arXiv:1001.5284 [hep-ph]

[929] P. Petreczky, arXiv:0906.0502 [nucl-th]

[930] F. Karsch, E. Laermann, P. Petreczky, and S. Stickan, Phys. Rev. D 68, 014504 (2003), arXiv:heplat/0303017

[931] A. Mocsy, Eur. Phys. J. C 61 (2009) 705, arXiv:0811.0337 [hep-ph]

[932] P. Petreczky and D. Teaney, Phys. Rev. D 73 (2006) 014508, arXiv:hep-ph/0507318

[933] T. Umeda, Phys. Rev. D 75 (2007) 094502, arXiv:heplat/0701005

[934] P. Petreczky, Eur. Phys. J. C 62 (2009) 85, arXiv:0810.0258 [hep-lat]

[935] M. Laine, O. Philipsen, P. Romatschke, and M. Tassler, JHEP 0703 (2007) 054, arXiv:hepph/0611300

[936] Y. Burnier, M. Laine, and M. Vepsalainen, JHEP 0801 (2008) 043, arXiv:0711.1743 [hep-ph]

[937] A. Beraudo, J. P. Blaizot, and C. Ratti, Nucl. Phys. A 806 (2008) 312, arXiv:0712.4394 [nucl-th]

[938] M. A. Escobedo and J. Soto, Phys. Rev. A 78 (2008) 032520, arXiv:0804.0691 [hep-ph]

[939] M. A. Escobedo and J. Soto, Phys. Rev. A 82 (2010)
042506, arXiv:1008.0254 [hep-ph]

[940] A. Vairo, PoS C CONFINEMENT8 (2008) 002, arXiv:0901.3495 [hep-ph]

[941] E. Braaten and R. D. Pisarski, Nucl. Phys. B 337 (1990) 569

[942] E. Braaten and R. D. Pisarski, Nucl. Phys. B 339 (1990) 310

[943] E. Braaten and R. D. Pisarski, Phys. Rev. D 45 (1992) 1827

[944] J. Frenkel and J. C. Taylor, Nucl. Phys. B 334 (1990) 199

[945] N. Brambilla, M. A. Escobedo, J. Ghiglieri, J. Soto and A. Vairo, JHEP 1009 (2010) 038, arXiv:1007.4156 [hep-ph]

[946] O. Jahn and O. Philipsen, Phys. Rev. D 70, 074504 (2004), arXiv:hep-lat/0407042

[947] N. Brambilla, J. Ghiglieri, P. Petreczky, and A. Vairo, Phys. Rev. D 82 (2010) 074019, arXiv:1007.5172 [hep$\mathrm{ph}]$

[948] Y. Burnier, M. Laine, and M. Vepsalainen, JHEP 1001, 054 (2010), arXiv:0911.3480 [hep-ph]

[949] A. Rothkopf, T. Hatsuda and S. Sasaki, PoS LAT2009 (2009) 162, arXiv:0910.2321 [hep-lat]

[950] G. Aarts, S. Kim, M. P. Lombardo, M. B. Oktay, S. M. Ryan, D. K. Sinclair, and J. I. Skullerud, arXiv:1010.3725 [hep-lat]

[951] A. Dumitru, Y. Guo, and M. Strickland, Phys. Lett. B 662 (2008) 37, arXiv:0711.4722 [hep-ph]

[952] O. Philipsen and M. Tassler, arXiv:0908.1746 [hep-ph]

[953] A. Dumitru, Y. Guo, and M. Strickland, Phys. Rev. D 79 (2009) 114003, arXiv:0903.4703 [hep-ph]

[954] Y. Burnier, M. Laine, and M. Vepsalainen, Phys. Lett. B 678 (2009) 86, arXiv:0903.3467 [hep-ph]

[955] A. Dumitru, Y. Guo, A. Mocsy, and M. Strickland, Phys. Rev. D 79 (2009) 054019, arXiv:0901.1998 [hep$\mathrm{ph}]$

[956] M. Asakawa, S. A. Bass, and B. Muller, Phys. Rev. Lett. 96 (2006) 252301, arXiv:hep-ph/0603092

[957] A. Andronic, P. Braun-Munzinger, K. Redlich, and J. Stachel, Nucl. Phys. A $\mathbf{7 8 9}$ (2007) 334, arXiv:nuclth/0611023

[958] X. Zhao and R. Rapp, Phys. Lett. B 664 (2008) 253, arXiv:0712.2407 [hep-ph]

[959] F. Karsch, D. Kharzeev, and H. Satz, Phys. Lett. B 637 (2006) 75, arXiv:hep-ph/0512239

[960] C. Young and E. Shuryak, Phys. Rev. C 79 (2009) 034907, arXiv:0803.2866 [nucl-th]

[961] D. Teaney, arXiv:0905.2433 [nucl-th]

[962] G. D. Moore and D. Teaney, Phys. Rev. C 71 (2005) 064904, arXiv:hep-ph/0412346

[963] H. van Hees and R. Rapp, Phys. Rev. C 71 (2005) 034907, arXiv:nucl-th/0412015

[964] R. Rapp and H. van Hees, arXiv:0903.1096 [hep-ph]

[965] C. Young and E. Shuryak, Phys. Rev. C 81, 034905 (2010), arXiv:0911.3080 [nucl-th]

[966] R. Arnaldi et al. (NA60 Collaboration), Phys. Rev. Lett. 99 (2007) 132302

[967] R. Arnaldi (NA60 Collaboration), Nucl. Phys. A 830 (2009) 345c, arXiv:0907.5004 [nucl-ex]

[968] B. Alessandro et al. (NA50 Collaboration), Eur. Phys. J. C 39 (2005) 335, arXiv:hep-ex/0412036

[969] B. Alessandro et al. (NA50 Collaboration), Phys. Lett. B 553 (2003) 167,

[970] R. Arnaldi et al. (NA60 Collaboration), Eur. Phys. J. 
C 59 (2009) 607, arXiv:0810.3204 [nucl-ex]

[971] K. J. Eskola, H. Paukkunen, and C. A. Salgado, JHEP 0807 (2008) 102, arXiv:0802.0139 [hep-ph]

[972] D. Kharzeev, E. Levin, and M. Nardi, Nucl. Phys. A 730 (2004) 448 [Erratum-ibid. A 743 (2004) 329], arXiv:hep-ph/0212316

[973] L. Hulthen and M. Sagawara, Handbüch der Physik, 39 (1957)

[974] S. Afanasiev et al. (PHENIX Collaboration), Phys. Lett. B 679 (2009) 321, arXiv:0903.2041 [nucl-ex]

[975] E. T. Atomssa (PHENIX Collaboration), Nucl. Phys. A 830 (2009) 331c, arXiv:0907.4787 [nucl-ex]

[976] J. P. Lansberg and H. Haberzettl, AIP Conf. Proc. 1038 (2008) 83, arXiv:0806.4001 [hep-ph]

[977] A. Adare et al. (PHENIX Collaboration), Phys. Rev. Lett. 101 (2008) 122301, arXiv:0801.0220 [nucl-ex]

[978] L. A. Linden Levy, Nucl. Phys. A 830 (2009) 353c, arXiv:0908.2361 [nucl-ex]

[979] M. J. Leitch, J. Phys. G 34 (2007) S453, arXiv:nuclex/0701021

[980] B. I. Abelev et al. (STAR Collaboration), Phys. Rev. D 82 (2010) 012004, arXiv:1001.2745 [nucl-ex]

[981] H. Liu (STAR Collaboration), Nucl. Phys. A 830 (2009) 235c, arXiv:0907.4538 [nucl-ex]

[982] A. Adare et al. (PHENIX Collaboration), arXiv:1010.1246 [nucl-ex]

[983] A. Adare et al. (PHENIX Collaboration), Phys. Rev. Lett. 98 (2007) 232301, arXiv:nucl-ex/0611020

[984] R. Vogt, Phys. Rev. C 81 (2010) 044903, arXiv:1003.3497 [hep-ph]

[985] R. Arnaldi (NA60 Collaboration), presentation at the ECT* Workshop on Quarkonium Production in HeavyIon Collisions, Trento (Italy), May 25-29, 2009

[986] C. A. Bertulani, S. R. Klein, and J. Nystrand, Ann. Rev. Nucl. Part. Sci. 55 (2005) 271, arXiv:nuclex/0502005

[987] G. Baur, K. Hencken, D. Trautmann, S. Sadovsky, and Y. Kharlov, Phys. Rept. 364 (2002) 359, arXiv:hep$\mathrm{ph} / 0112211$

[988] K. Hencken et al., Phys. Rept. 458 (2008) 1, arXiv:0706.3356 [nucl-ex]

[989] M. G. Ryskin, Z. Phys. C 57 (1993) 89

[990] V. Rebyakova, M. Strikman and M. Zhalov, Phys. Rev. D 81 (2010) 031501, arXiv:0911.5169 [hep-ph]

[991] L. Frankfurt, M. Strikman, and M. Zhalov, Phys. Rev. C 67 (2003) 034901, arXiv:hep-ph/0210303

[992] L. Frankfurt, M. Strikman, and M. Zhalov, Phys. Lett. B 537 (2002) 51, arXiv:hep-ph/0204175

[993] B. I. Abelev et al. (STAR Collaboration), Phys. Rev. C 77 (2008) 034910, arXiv:0712.3320 [nucl-ex]

[994] C. Adler et al. (STAR Collaboration), Phys. Rev. Lett. 89 (2002) 272302, arXiv:nucl-ex/0206004

[995] B. I. Abelev et al. (STAR Collaboration), Phys. Rev. C 81 (2010) 044901, arXiv:0912.0604 [nucl-ex]

[996] L. Frankfurt, M. Strikman, and M. Zhalov, Phys. Lett. B 670 (2008) 32, arXiv:0807.2208 [hep-ph]

[997] L. Frankfurt, M. Strikman, and M. Zhalov, Phys. Lett. B 640 (2006) 162, arXiv:hep-ph/0605160

[998] S. R. Klein and J. Nystrand, Phys. Rev. Lett. 84 (2000) 2330, arXiv:hep-ph/9909237

[999] B. I. Abelev et al. (STAR Collaboration), Phys. Rev. Lett. 102 (2009) 112301, arXiv:0812.1063 [nucl-ex]

[1000] V. P. Goncalves and W. K. Sauter, Phys. Rev. D 81 (2010) 074028, arXiv:0911.5638 [hep-ph]
[1001] B. E. Cox, J. R. Forshaw, and R. Sandapen, JHEP 0906 (2009) 034, arXiv:0905.0102 [hep-ph]

[1002] D. M. Asner et al., Int. J. of Mod. Phys. A 24 (2009) 1, arXiv:0809.1869 [hep-ex]

[1003] E. Klempt and A. Zaitsev, Phys. Rept. 454 (2007) 1, arXiv:0708.4016 [hep-ph]

[1004] T. Feldmann and P. Kroll, Phys. Rev. D 62 (2000) 074006, arXiv:hep-ph/0003096

[1005] V. L. Chernyak and A. R. Zhitnitsky, Phys. Rept. 112 (1984) 173

[1006] B. Q. Li and K. T. Chao, Phys. Rev. D 79 (2009) 094004, arXiv:0903.5506 [hep-ph]

[1007] K. Aamodt et al. (ALICE Collaboration), JINST 3 (2008) S08002

[1008] F. Carminati et al. (ALICE Collaboration), J. Phys. G 30 (2004) 1517

[1009] B. Alessandro et al. (ALICE Collaboration), J. Phys. G 32 (2006) 1295

[1010] J. Alme et al., Nucl. Instrum. Meth. A 622 (2010) 316, arXiv:1001.1950 [physics.ins-det]

[1011] S. Grigoryan and A. De Falco, ALICE-INT-2008-01

[1012] M. Bedjidian et al., arXiv:hep-ph/0311048

[1013] W. Sommer, C. Blume, F. Kramer, and J. F. GrosseOetringhaus (ALICE Collaboration), Int. J. Mod. Phys. E 16 (2007) 2484, arXiv:nucl-ex/0702045

[1014] D. Stocco et al., ALICE-INT-2006-029

[1015] P. Gonzalez, P. Ladron de Guevara, E. Lopez Torres, A. Marin, and E. Serradilla (ALICE Collaboration), Eur. Phys. J. C 61 (2009) 899 [Erratum-ibid. C 61 (2009) 915], arXiv:0811.1592 [hep-ex]

[1016] ATLAS Collaboration, CERN-LHCC-99-014

[1017] ATLAS Collaboration, CERN-LHCC-99-015

[1018] G. Aad et al. (ATLAS Collaboration), arXiv:0901.0512 [hep-ex]

[1019] G. Aad et al. (ATLAS Collaboration), JINST 3 (2008) S08003

[1020] R. Adolphi et al. (CMS Collaboration), JINST 3 (2008) S08004

[1021] F. Ragusa and L. Rolandi, New J. Phys. 9 (2007) 336

[1022] W. Adam et al. (CMS Collaboration), JINST 3 (2008) P07009

[1023] S. Chatrchyan et al. (CMS Collaboration), JINST 5 (2010) T03022

[1024] CMS Collaboration, Dimuons in CMS at 900 and 2360 $\mathrm{GeV}$, CMS performance note (2010) DP-2010/005

[1025] M. Bargiotti and V. Vagnoni, CERN-LHCB-2007-042

[1026] T. Sjostrand, L. Lonnblad, S. Mrenna, and P. Skands, arXiv:hep-ph/0308153

[1027] CMS Collaboration, First two months of data taking at $7 \mathrm{TeV}: J / \psi \rightarrow \mu \mu, W \rightarrow \mu \nu, Z \rightarrow \mu \mu$ mass plots and displays of $Z \rightarrow \mu \mu$ candidates, CMS performance note (2010) DP-2010/016

[1028] D. Acosta et al. (CMS Collaboration), CERN/LHCC 2006-001, CMS-TDR-008-1

[1029] CMS Collaboration, Feasibility study of a $J / \psi$ cross section measurement with early CMS data, CMS PAS BPH-07-02

[1030] P. Faccioli, C. Lourenço, J. Seixas, and H. K. Wohri, arXiv:1006.2738 [hep-ph]

[1031] A. A. Alves et al. (LHCb Collaboration), JINST 3 (2008) S08005

[1032] D. J. Lange, Nucl. Instrum. Meth. A 462 (2001) 152

[1033] S. Agostinelli et al. (GEANT4 Collaboration), Nucl. Instrum. Meth. A 506 (2003) 250 
[1034] M. Needham (LHCb Collaboration), CERN-LHCBCONF-2009-011

[1035] Y. Gao, W. Qian, P. Robbe, and M.-H. Schune, LHCBPUB-2010-011

[1036] G. Sabatino (LHCb Collaboration), HEP 2009, CERNPoster-2009-121

[1037] L. Nicolas, EPFL thesis \#4530, Lausanne, October 2009

[1038] J. He (LHCb Collaboration), CERN-LHCB-CONF2009-013

[1039] Y. Gao, J. He, and Z. Yang, CERN-LHCB-2008-059

[1040] O. Yushchenko, CERN-LHCb-2003-113

[1041] C. H. Chang, C. Driouichi, P. Eerola, and X. G. Wu, Comput. Phys. Commun. 159 (2004) 192, arXiv:hep$\mathrm{ph} / 0309120$

[1042] Y. Gao, J. He, and Z. Yang, CERN-LHCB-2008-077

[1043] I. P. Gouz, V. V. Kiselev, A. K. Likhoded, V. I. Romanovsky, and O. P. Yushchenko, Phys. Atom. Nucl. 67 (2004) 1559 [Yad. Fiz. 67 (2004) 1581], arXiv:hep$\mathrm{ph} / 0211432$

[1044] H. W. Ke and X. Q. Li, Sci. China G53 (2010) 2019, arXiv:0910.1158 [hep-ph]

[1045] S. Descotes-Genon, J. He, E. Kou, and P. Robbe, Phys. Rev. D 80 (2009) 114031, arXiv:0907.2256 [hep-ph]

[1046] S. Sakai (STAR Collaboration), J. Phys. G 36 (2009) 064056

[1047] S. Hashimoto et al., KEK-REPORT-2004-4

[1048] M. Bona et al., arXiv:0709.0451 [hep-ex]

[1049] K. Oide, Prog. Theor. Phys. 122 (2009) 69

[1050] P. Raimondi, D. N. Shatilov, and M. Zobov, arXiv:physics/0702033

[1051] T. E. Browder, T. Gershon, D. Pirjol, A. Soni, and J. Zupan, Rev. Mod. Phys. 81 (2009) 1887, arXiv:0802.3201 [hep-ph]

[1052] B. Aubert et al. (BABAR Collaboration), Nucl. Instrum. Meth. A 479 (2002) 1, arXiv:hep-ex/0105044

[1053] A. Abashian et al. (Belle Collaboration), Nucl. Instrum. Meth. A 479 (2002) 117

[1054] D. G. Hitlin et al., arXiv:0810.1312 [hep-ph]

[1055] FAIR Baseline Technical Report, 2006

[1056] H. Staengle et al., Nucl. Instrum. Meth. A 397 (1997) 261

[1057] M. F. Lutz, B. Pire, O. Scholten, and R. Timmermans (PANDA Collaboration), arXiv:0903.3905 [hep-ex]

[1058] V. Flaminio et al., CERN-HERA 70-03 (1970)

[1059] A. Lundborg, T. Barnes, and U. Wiedner, Phys. Rev. D 73 (2006) 096003, arXiv:hep-ph/0507166

[1060] R. Arnaldi et al. (NA60 Collaboration), Eur. Phys. J. C 43 (2005) 167

[1061] Eds. V. Friese and W.F.J. Müller, CBM Progress Report 2008, GSI Report 2009-03, ISBN 978-3-98112986-1

[1062] P. Raimondi, presentation at the 2nd SuperB Workshop, Frascati, March 2006

[1063] E. Levichev, Phys. Part. Nucl. Lett. 5 (2008) 554

[1064] L. Frankfurt, W. Koepf, and M. Strikman, Phys. Rev. D 57 (1998) 512, arXiv:hep-ph/9702216

[1065] A. Caldwell and H. Kowalski, Phys. Rev. C 81 (2010) 025203.

[1066] L. Frankfurt, M. Strikman, and C. Weiss, Ann. Rev. Nucl. Part. Sci. 55 (2005) 403, arXiv:hep-ph/0507286

[1067] L. Frankfurt, C. E. Hyde, M. Strikman, and C. Weiss, Phys. Rev. D 75 (2007) 054009, arXiv:hep-ph/0608271

[1068] A. Caldwell and H. Kowalski, arXiv:0909.1254 [hep-ph]
[1069] A. Bohr and B. R. Mottelson, Nuclear Structure (Benjamin, New York, 1969)

[1070] M. E. Binkley et al., Phys. Rev. Lett. 48 (1982) 73

[1071] B. Gittelman, K. M. Hanson, D. Larson, E. Loh, A. Silverman, and G. Theodosiou, Phys. Rev. Lett. 35 (1975) 1616

[1072] U. Camerini et al., Phys. Rev. Lett. 35 (1975) 483

[1073] L. Frankfurt and M. Strikman, Phys. Rev. D 66 (2002) 031502, arXiv:hep-ph/0205223

[1074] K. Lukashin et al. (CLAS Collaboration), Phys. Rev. C 63 (2001) 065205, arXiv:hep-ex/0101030

[1075] J. P. Santoro et al. (CLAS Collaboration), Phys. Rev. C 78 (2008) 025210, arXiv:0803.3537 [nucl-ex]

[1076] S. V. Goloskokov and P. Kroll, Eur. Phys. J. C 50 (2007) 829, arXiv:hep-ph/0611290

[1077] S. J. Brodsky, E. Chudakov, P. Hoyer, and J. M. Laget, Phys. Lett. B 498 (2001) 23, arXiv:hep-ph/0010343

[1078] R. L. Anderson et al., Phys. Rev. Lett. 38 (1977) 263

[1079] L. Bartoszek et al., Nucl. Instrum. Meth. A 301 (1991) 47

[1080] A. Moiseev et al. (BESS Collaboration), Astrophys. J. 474 (1997) 479

[1081] V. M. Abazov et al. (DØ Collaboration), Nucl. Instrum. Meth. A 565 (2006) 463, arXiv:physics/0507191

[1082] See http://psec.uchicago.edu/

[1083] D. M. Asner et al., P-986 Letter of Intent: MediumEnergy Antiproton Physics at Fermilab (2009)

[1084] See http://capp.iit.edu/hep/pbar/

[1085] T. A. Armstrong et al. (E760 Collaboration), Phys. Rev. D 47 (1993) 772

[1086] M. Andreotti et al. (Fermilab E835 Collaboration), Phys. Lett. B 654 (2007) 74, arXiv:hep-ex/0703012

[1087] G. Garzoglio et al., Nucl. Instrum. Meth. A 519 (2004) 558

[1088] N. A. Tornquist, Phys. Lett. B 590 (2004) 209, arXiv:hep-ph/0402237

[1089] E. Braaten, Phys. Rev. D 77 (2008) 034019, arXiv:0711.1854 [hep-ph]

[1090] T. A. Armstrong et al. (E760 Collaboration), Nucl. Phys. B 373 (1992) 35

[1091] J. Brau et al. (ILC Collaboration), arXiv:0712.1950 [physics.acc-ph]

[1092] T. Abe et al. (ILD Concept Group - Linear Collider Collaboration), arXiv:1006.3396 [hep-ex]

[1093] H. Aihara et al., arXiv:0911.0006 [physics.ins-det]

[1094] R. W. Assmann et al., CERN-2000-008

[1095] G. Aarons et al. (ILC Collaboration), arXiv:0709.1893 [hep-ph]

[1096] V. S. Fadin and V. A. Khoze, Sov. J. Nucl. Phys. 48 (1988) 309 [Yad. Fiz. 48, 487 (1988)]

[1097] M. Jezabek and J. H. Kuhn, Phys. Lett. B 316 (1993) 360

[1098] Y. Sumino, K. Fujii, K. Hagiwara, H. Murayama, and C. K. Ng, Phys. Rev. D 47 (1993) 56

[1099] M. Jezabek, J. H. Kuhn, and T. Teubner, Z. Phys. C 56 (1992) 653

[1100] H. Murayama and Y. Sumino, Phys. Rev. D 47 (1993) 82

[1101] R. Harlander, M. Jezabek, J. H. Kuhn, and T. Teubner, Phys. Lett. B 346 (1995) 137, arXiv:hepph/9411395

[1102] M. Jezabek, T. Nagano, and Y. Sumino, Phys. Rev. D 62 (2000) 014034, arXiv:hep-ph/0001322 
[1103] M. Martinez and R. Miquel, Eur. Phys. J. C 27 (2003) 49, arXiv:hep-ph/0207315

[1104] K. Fujii, T. Matsui, and Y. Sumino, Phys. Rev. D 50 (1994) 4341

[1105] A. H. Hoang et al., Eur. Phys. J. direct C 2 (2000) 1, arXiv:hep-ph/0001286

[1106] K. Ikematsu, K. Fujii, Z. Hioki, Y. Sumino, and T. Takahashi, Eur. Phys. J. C 29 (2003) 1, arXiv:hepph/0302214
[1107] S. Fleming, A. H. Hoang, S. Mantry, and I. W. Stewart, Phys. Rev. D 77, 074010 (2008), arXiv:hepph/0703207

[1108] S. Fleming, A. H. Hoang, S. Mantry, and I. W. Stewart, Phys. Rev. D 77, 114003 (2008), arXiv:0711.2079 [hep$\mathrm{ph}]$

[1109] L. Frankfurt, M. Strikman, and C. Weiss, arXiv:1009.2559 [hep-ph] 